
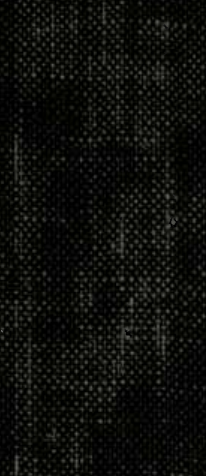

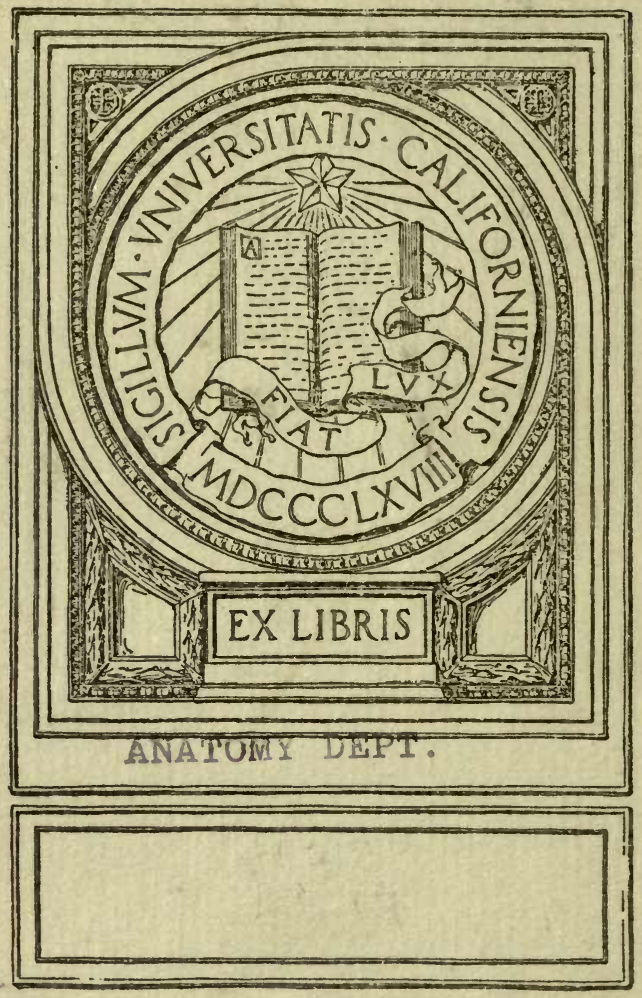






\title{
THE MICROTOMIST'S VADE-MECUM
}

\section{A HANDBOOK OF THE METHODS OF MICROSCOPIC ANATOMY}

BY

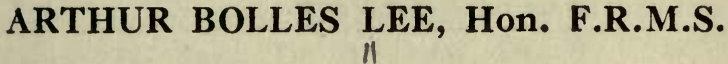

\author{
EIGHTH EDITION
}

EDITED BY

\section{J. BRONTË GATENBY,}

B.A., B.Sc., D.Phil. (Oxon.), D.Sc. (Lond.), F.R.M.S., SOMETIME LECTURER IN HISTOLOGY, OXFORD; LECTURER IN CYTOLOGY AND SENIOR ASSISTANT IN ZOOLOGY, UNIVERSITY COLLEGE, LONDON, AND SENIOR DEMY, MAGDALEN COLLEGE, OXFORD.

With the collaboration of

W. M. BAYLISS, M.A., D.Sc.(Oxon.), F.R.S., F.R.M.S., PROFESSOR OF GENERAL PHYSIOLOGY IN UNIVERSITY COLLEGE, LONDON;

$$
\text { C. DA FANO, M.D., F.R.M.S., }
$$

L.D. ON MORBID ANATOMY, UNIVERSITY OF PAVIA (ITALY) ; LECTURER IN HISTOLOGY, KING'S COLLEGE, LONDON;

A. DREW, D.Sc. (Lond.), F.R.M.S.

IMPERIAL CANCER RESEARCH FUND, LONDON;

W. CRAMER, Ph.D., D.Sc., M.R.C.S., L.R.C.P., F.R.M.S., IMPERIAL CANCER RESEARCH FUND, LONDON;

AND

J. THORNTON CARTER, F.R.M.S., F.Z.S.

HON. RESEARCH ASSISTANT, UNIVERSITY COLLEGE, LONDON.

\section{PHILADELPHIA}

P. BLAKISTON'S SON \& CO. 


$$
\begin{aligned}
& <4 \\
& 1921
\end{aligned}
$$

aunt. dept

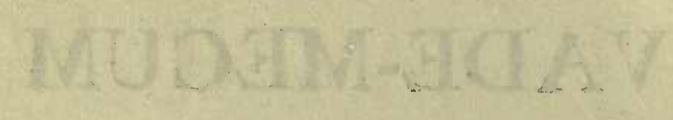

30.

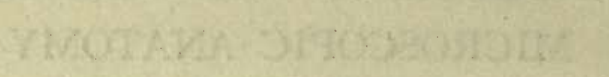

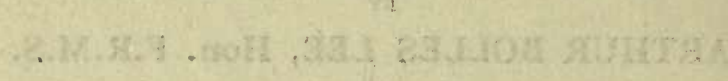

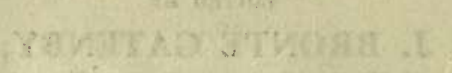

Anatomy Dept 


\section{PREFACE TO EIGHTH EDITION.}

IN the preparation of this new edition of Dr. Bolles Lee's wellknown book I have received the assistance of Professor W. M. Bayliss, Dr. C. Da Fano, Dr. A. Drew, Dr. W. Cramer, and Mr. J. Thornton Carter. It has been almost entirely. due to these workers that my plans for the new edition have been able to be carried out in the way I wished. Thanks to them, this book may be considered a most complete and stimulating book of reference for the research worker.

Professor W. M. Bayliss, to whom I am especially grateful, has rewritten Chapter XI. on "Staining." Dr. C. Da Fano has revised, and in some cases almost completely rewritten, the difficult chapters on "Neurological Techniques," which constitute indeed a special branch of microtomy. Dr. A. Drew has completely rewritten the valuable section on "Protozoa"; while from Dr. W. Cramer's pen has come most of the article on "Fatty Substances," which will bring to the notice of embryologists and histologists the newest advances in the micro-chemistry of this subject. Mr. J. Thornton Carter has revised the section on "Teeth and Bone." I have personally been responsible for the rest of the book, besides having written special chapters or sections on "Chromatin, Chromosomes, Nucleoli, Glycogen, Iron, Yolk, Fat, Mitochondria, Golgi Apparatus and Benzidine Dyes "; I have completely rewritten the section on "Mammalian Embryological Methods." In the sections dealing with the cytoplasmic inclusions, most of the various structures, known as " attraction sphere rodlets," "idiozome," "Golgi-Kopsch apparat," "nebenkern batonettes," etc., are grouped under the term "Golgi apparatus," as now seems justifiable in view of the results of modern researches on the subject.

A small, yet very important, addition to the present volume has been the inclusion of two new methods for staining bacteria in tissue $(\S \S 689,761)$. The histologist and cytologist are often puzzled to know whether certain enigmatic bodies they find are or are not bacterial in nature. 


\section{PREFACE.}

An addition which is likely to be welcomed, and to make the book more useful, is a special chapter for students (Chapter XXXVII.).

I had especially desired to have a chapter dealing with "Tissue Culture," and had asked my friend Mr. H. M. Carleton, of Oxford, to undertake it; but as illness prevented his carrying out the work, I was myself obliged at the eleventh hour to write the article. Dr. A. Drew was most helpful here, and Dr. Strangeways, of Cambridge, lent me some literature on the subject.

For advice and encouragement in this arduous task $I$ have to thank my many friends. Dr. Bolles Lee sent me his blessing and three hundred references; Professor J. P. Hill placed his notebooks and experience at my disposal; Dr. J. A. Murray, of the Cancer Research Fund, proved a perfect mine of suggestions, and I dug freely at this source. I am very grateful to both Dr. Murray and his amiable staff, of whom Dr. Drew and Dr. Cramer have helped me most. Professor Starling, of University College, Professor E. S. Goodrich, of Oxford, Professor Sherrington, of Oxford, Professor Boycott, of University College Medical School, Professor E. B. Wilson, of Columbia University, and Professor D. M. S. Watson, of University College, London, helped me in one way or another. Professor W. M. Bayliss and Dr. Oscar Brady gave me valuable information on certain chemical aspects. My friends at the Rothamsted Station, especially Mr. Ward Cutler and. Dr. Imms, were very helpful. Dr. Allen, of Plymouth, and Dr. Orton kindly answered inquiries.

Great care has been taken in the preparation of the Index, and I have to thank my sister for much assistance.

In writing a book of this kind, it is difficult at times to ascertain exactly who did originate some one method, and if we have occasionally overlooked the original source we will be glad to have such lapsus calami rectified in some future edition.

Finally, it must be remarked that the arrangement and method of exposition of the new material closely follows that of Dr. Bolles Lee, while the bulk of the book is still largely due to him.

\section{J. BRONTË GATENBY.}

UNIVERSITY COLLEGE, IJONDON. 


\section{CONTENTS.}

PART I.

CHAPTER I.

PAGE

INTRODUCTORY

CHAPTER II.

KILLING

CHAPTER III.

Fixing AND HARDENING . . . . . . . . 18

CHAPTER IV.

Fixing and Hardening Agents-Mineral Acids and their SALTS . . . . . . . .

CHAPTER V.

Fixing and Hardening Agents-Chlorides, Organic Acids, AND OTHERS

CHAPTER VI.

De-alcoholisation and Clearing Agents

CHAPTER VII.

IMBEddING METHODS-INTRODUCTION . . . . . 71

CHAPTER VIII.

Imbedding Methods : Paraffin and other Fusion Masses • 76 Paraffin, 76 ; Gelatin, 92.

\section{CHAPTER IX.}

Collodion (Celloidin) ANd other Imbedding Methods • 95 Collodion or Celloidin, 95 ; other Cold Masses, 106 ; Grind. ing Masses, 108 ; Freezing, 109. 
CHAPTER $\mathrm{X}$.

Serial Section Mounting

Methods for Paraffin Sections, 111; Methods for Watery Sections, 116 ; Methods for Celloidin Sections, 116.

CHAPTER XI.

StanNing

CHAPTER XII.

Carmine and Cochineal Stains .

Theory of Carmine Staining, 135 ; Aqueous Carmines, Acid, 136 ; Neutral and Alkaline, 140 ; Alcoholic Carmines and Cochineals, 141.

\section{CHAPTER XIII.}

Hamateïn (Hamatoxylin) Stains

Theory of Staining with Hæmatoxylin, 145 ; Iron-hæmateïn Lakes, 147; Aluminium-hæmateïn Lakes, 151 ; other Hæmateïn Compounds, 155.

CHAPTER XIV.

Nuclear Stains with Coal-tar Dyes .

Progressive Stains, 159; Regressive Stains, 162.

CHAPTER XV.

Plasma Stains with Coal-tar Dyes

CHAPTER XVI.

Metiylen BLue.

CHAPTER XVII.

Metallic Stains (Impregnation Methods).

Silver, 198; Gold, 202 ; other Metallic Stains, 208.

\section{CHAPTER XVIII.}

Other Stains and Combinations .

Carmine Combinations, 212 ; Hæmateïn Combinations, 213.

\section{CHAP'TER XIX.}

Examination and Preservation Media

Aqueous Liquids, 217 ; Mercurial Liquids, 220 ; other Fluids, 220 ; Glycerin Media, 222; Glycerin Jellies, 223 ; High Refractive Liquids, 224 ; Resinous Media, 225. 
CONTENTS.

CHAPTER XX.

PAGE

Cements and Varnishes

229

\section{PART II.}

SPECIAL METHODS AND EXAMPLES.

\section{CHAPTER XXI.}

INJECTION-GELATIN MASSES (WARM) • • • • • 232 Carmine, 234 ; Blue, 236 ; other Colours, 237.

CHAPTER XXII.

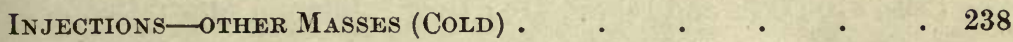

CHAPTER XXIII.

Maceration, Digestion, and Corrosion

Maceration, 243 ; Digestion, 248 ; Corrosion, 249.

\section{CHAPTER XXIV.}

\section{Decalcification, Desilicification, and Bleaching}

Decalcification, 251 ; Desilicification, 255 ; Bleaching, 255.

\section{CHAPTER XXV.}

\section{Embryological Methods}

Mammalia, 263 ; Aves, 271 ; Reptilia, 274 ; Amphibia, 275 ;

Pisces, 279 ; Tunicata, 281 ; Bryozoa, 282 ; Mollusca, 282 ;

Arthropoda, 284 ; Vermes, 288.

\section{CHAPTER XXVI.}

Cytological Methods .

Glycogen, 294 ; Iron, 297 ; Chromosomes, 303 ; Cytoplasmic

Inclusions, 316.

\section{CHAPTER XXVII.}

Tegumentary ORgans

\section{CHAPTER XXVIII.}

Muscle and Tendon (Nerve-endings).

Striated Muscle, 344 ; Electric Organs, 345 ; Teudon, 347 ;

Smooth Muscle, 348. 
Connective Tissues

Connective Tissue, 350; Elastic, 352; Plasma Cells 354;

Fatty Substances, 356; Bone and Cartilage, 369; Skeletons of Embryos, 377.

CHAPTER XXX.

BLOOD AND GLaNdS

Blood, 379 ; Benzidine Dyes, 388 ; Glands, 391.

CHAPTER XXXI.

Nervous System-General Methods . $. \quad . \quad . \quad .397$

\section{CHAPTER XXXII.}

Nervous System-Special Methods, chiefly Cytological • 410 Cells, 410 ; Cells and Fibres, 416 ; Golgi's Internal Apparatus, 435 ; Medullary Sheath, 439.

CHAPTER XXXIII.

MYelin Stains (WEIGERT AND OTHERS)

CHAPTER XXXIV.

Axis-Cylinder and Dendrite Stains (Golgi and others)

CHAPTER XXXV.

Neuroglia and Sense Organs

Neuroglia, 479 ; Retina, 493 ; Inner Ear, 496.

\section{CHAPTER XXXVI.}

METHODS FOR INVERTEBRATES

Tunicata, 499 ; Molluscoida, 500 ; Mollusca, 500 ; Arthropoda, 504 ; Vermes, 509 ; Echinodermata, 518 ; Cœlenterata, 521 ; Porifera, 525 ; Protozoa, 526.

\section{CHAPTER XXXVII.}

Cultivation of Tissue "in vitro" and its Technique . $\quad 550$

CHAPTER XXXVIII.

A Guide For Students of Microtomy 


\title{
THE MICROTOMIST'S VADE-MECUM.
}

\author{
PAR'T I. \\ CHAPTER I. \\ INTRODUCTORY.
}

1. The General Method.-The methods of modern microscopic anatomy may be roughly classed as General and Special. There is a General or Normal method which consists in carefully fixing the structures to be examined, staining them with a nuclear stain, dehydrating with alcohol, and mounting series of sections of the structures in balsam. It is by this method that the work is blocked out and very often finished. Special points are then studied, if necessary, by Special Methods, such as examination of the living tissue elements, in situ or in "indifferent" media; fixation with special fixing agents ; staining with special stains; dissociation by teasing or maceration; injection; impregnation; and the like.

There is a further distinction which may be made, and which may help to simplify matters. The processes of the preparation of tissues may be divided into two stages, Preliminary Preparation and Ulterior Preparation. Now the processes of preliminary preparation are essentially identical in all the methods, essential divergences being only found in the details of ulterior preparation. By preliminary preparation is meant that group of processes whose object it is to get the tissues into a fit state for passing unharmed through all the ulterior processes to which it may be desired to submit them. It comprehends the operations of (1) killing; (2) fixing; (3) the washing and other manipulations necessary for removing the fixing agent from the tissues, and substituting for it the preservative liquid or other reagents which it is desired to employ. Ulterior preparation comprehends the processes sketched out in $\$ 33$ et seq.

2. Preliminary Preparation.- The first thing to be done with any structure is to fix its histological elements. (This statement applies equally to all classes of objects, whether it be desired to cut them into sections or to treat them in any other special way.) Two 


\section{INTRODUCTORY.}

things are implied by the word "fixing" : first, the rapid killing of the element, so that it may not have time to change the form it had during life, but is fixed in death in the attitude it normally had during life ; and second, the hardening of it to such a degree as may enable it to resist without further change of form the action of the reagents with which it may subsequently be treated. Without good fixation it is impossible to get good stains or good sections, or preparations good in any way.

The structure, having been duly fixed by one of the processes described in the chapter on Fixing Agents, is, except in special cases, washed in order to remove from the tissues as far as possible all traces of the fixing reagent.

The kind of liquid with which washing out is done is not a matter of indifference. If corrosive sublimate (for instance), or osmic acid, or a solution into which chromic acid or a chromate enters, have been used for fixing, the washing may be done with water. But if certain other agents, such as picric acid, have been used, the washing should be done with alcohol. The reason for this difference is that the first-named reagents (and, indeed, all the compounds of the heavy metals used for fixing) cause certain of the elements of the tissues to become less reactive, and partly or wholly insoluble in water. The combinations of picric acid and the elements of the tissues, on the other hand, are generally much more soluble in watery fluids.

These operations having been duly performed, two roads become open. The object may be further prepared by what may be termed the wet method, in which all subsequent operations are performed by means of aqueous media. Or it may be further prepared by the dehydration method, which consists in treatment with successive alcohols of gradually increasing strength, final dehydration with absolute alcohol, imbibition with an essential oil or other so-called clearing agent which serves to remove the alcohol, and lastly either mounting at once in balsam or other resinous medium or imbedding in paraffin for the purpose of making sections. The dehydration method is the course which is generally preferred, chiefly because of its great superiority as regards the preservation of tissues. For the presence of water is the most important factor in the conditions that bring about the decomposition of organic matter, and its complete removal is the chief condition of permanent preservation.

3. Dehydration.-The further course of preparation by the dehydration method is as follows:-At the same time that the superfluous fixing agent is being removed from the tissues, or as soon as that is done, the water of the tissues must be removed. This is 
necessary for two reasons : first, in the interest of preservation, as above explained; and secondly, because all water must be removed in order to allow the tissues to be impregnated with the imbedding material necessary for section-cutting, or with the balsam with which they are to be finally preserved. This dehydration is performed as follows:-The objects are brought into weak alcohol, and are then passed through successive alcohols of gradually increased strength, remaining in each the time necessary for complete saturation, and the last bath consisting of absolute or at least very strong alcohol.

In dealing with delicate objects, it may be necessary to take special precautions in order to avoid injury to them through the violent diffusion-currents that are set up in the passage from water to alcohol, or from one bath of alcohol to another of considerably different density. Some kinds of diffusion-apparatus may conveniently be used in these cases. The objects may be placed with some of their liquid in a tube corked at one end and closed at the other by a diaphragm of muslin or chamois skin or other suitable membrane, the tube being then immersed in a vessel containing the grade of alcohol that it is desired to add to the liquid in the tube, and the whole allowed to remain until by diffusion through the diaphragm the two liquids have become of equal density. Or, Совв's differentiator (Proc. Linn. Soc., N.S.W., v, 1890, p. 157 ; Journ. Roy. Mic. Soc., 1890, p: 821) may be employed. Or, the apparatus of HASWELL (Proc. Linn. Soc., N.S.W., vi, 1891, p. 433 ; Journ. Roy. Mic. Soc., 1892, p. 696). Or that of Cheatle, described in Journ. Pathol. and Bacteriol., i, 1892, p. 253, or Journ. Roy. Mic. Soc., 1892, p. 892. See also Schultze (Zeit. wiss. Mik., ii, 1885, p. 537); and SUSUKI, ibid., 1909, p. 211 ; KOLSTER (ibid., xvii, 1900, p. 294).

The "Siebdosen," or sieve-dishes of STEINACH, Zimmermann, and SuchanneK (vide Zeit. wiss. Mik., iv, 1887, p. 433, and vii, 1890, p. 158), are useful for many purposes. See also Tischatkin, ibid., xxiii, p. 45. FAIRCHILD's perforated porcelain cylinders for washing (ibid., xii, 1896, p. 301) seem to be a very neat idea. See also the similar device of SchafFer (ibid., xvi, 1900, p. 422 ; Journ. Roy. Mic. Soc., 1900, p. 394). For EwALD's section-washing apparatus, see Zeit. Biol., xxxiv, 1897, p. 264.

That of Schoebel (ibid., xx, 1903, p. 168) is simple and efficient ; as also that of KREIGBAUM (ibid., xxvii, 1910, p. 504).

A capillary siphon for the aspiration of liquids in the fixing, staining, and washing of suspended blood-corpuscles, sperm-cells, protozoa, and the like, is described by Ewald, ibid., p. 253.

It is sometimes stated that it is necessary that the last alcoholbath should consist of absolute alcohol. This, however, is incorrect, a strength of 95 per cent. being sufficient in most cases. For the small amount of water that remains in the tissues after treatment with these grades of alcohol is efficiently removed in the bath of 
clearing agent if a good clearing agent be employed. Oil of cedar will remove the remaining water from tissues saturated with 95 per cent. alcohol; oil of bergamot will "clear" from 90 per cent. alcohol, and anilin oil will clear from 70 per cent. alcohol.

I am not aware of any substance that can entirely take the place of alcohol for dehydration and preservation. Acetone and methylal have been substituted for alcohol in the dehydration of methylenblue preparations (PARKer, Zool. Anz., 403, 1892, p. 376), and anilin oil can be made to dehydrate watery sections if they be first mopped up with blotting-paper; but a really efficient substitute for alcohol in general work remains yet to be discovered.

4. Preservation.-Considered as a mere dehydrating agent, alcohol fulfils its functions fairly well. But considered as a histological preservative agent, it is far less satisfactory. If tissues be left in alcohol for only a few days before further preparation, injurious effects will perhaps not be very disagreeably evident. But it is otherwise if they are put away in it for many weeks or months before the final preparation is carried out. The dehydrating action of the alcohol being continuously prolonged, the minute structure of tissues is sometimes considerably altered by it ; they become overhard and shrink, and become brittle, and their capacity for taking stains well becomes seriously diminished. KuLtschitzky (Zeit. wiss. Mik., iv, 1887, p. 349) has proposed to remedy this by putting up objects after fixation and washing out with alcohol in ether, xylol, or toluol. Flemming (Arch. mik. Anat., xxxvii, 1891, p. 685) advises putting up objects after fixation in a mixture of alcohol, glycerin, and water, in about equal parts, pointing out that objects thus preserved may be at any moment either prepared for sectioning by treatment with pure alcohol or softened for dissection or teasing by a little soaking in water, and that they do not become so hard and brittle as alcohol specimens, and retain their staining power much better. After extensive experience of this plan I can recommend it, and would only further suggest that the action of the liquid seems to me to be in many cases much improved by addition of a little acetic acid (say 0.5 to 0.75 per cent.).

For material that is intended only for section-cutting, I find that by far the best plan is to clear (next $\S$ ) and imbed at once in paraffin. This affords, as far as I can see, an absolutely perfect preservation. Cedar-wood oil is, I find, nearly, if not quite, as good as paraffin, so far as the preservation of the tissues is concerned, but of course it is not so handy for storage. 
5. Removal of Alcohol; Clearing.-The water having been sufficiently removed, as described in $\S 3$, the alcohol is in its turn removed from the tissues, and its place taken by some anhydrous substance, generally an essential oil, which is miscible with the material used for imbedding or mounting. This operation is generally known as Clearing. It is very important that the passage from the last alcohol to the clearing agent be a gradual one. This is effected by placing the clearing medium under the alcohol. A sufficient quantity of alcohol is placed in a tube (a watch-glass will do, but tubes are generally better), and then with a pipette a sufficient quantity of clearing medium is introduced at the bottom of the alcohol. Or you may first put the clearing medium into the tube, and then carefully pour the alcohol on to the top of it. The two fluids mingle but slowly. The objects to be cleared, being now quietly put into the supernatant alcohol, float at the surface of separation of the two fluids, the exchange of fluids takes place gradually, and the objects slowly sink down into the lower layer. When they have sunk to the bottom, the alcohol may be drawn off with a pipette, and after some further lapse of time the objects will be found to be completely penetrated by the clearing medium.

This method of making the passage from one fluid to another applies to all cases in which objects have to be transferred from a lighter to a denser fluid-for instance, from alcohol, or from water, to glycerin.

This is a convenient stage for carrying out minute dissections, if any such have to be done, a drop of clearing agent being a most helpful medium for carrying out such dissections (see $\S 9$ ).

At this point the course of treatment follows one of two different roads, according as the object is to be mounted direct in balsam $(\S 8)$, or is first to be sectioned $(\S 6)$.

6. Imbedding, and Treatment of Sections.-The objects are now imbedded. They are removed from the clearing medium, and soaked until thoroughly saturated in the imbedding medium. This is, for small objects, generally paraffin, liquefied by heat, and for large objects either paraffin or a solution of collodion or " celloidin" (in this last case the clearing may be omitted and the tissues be imbedded direct from the alcohol). The imbedding medium containing the object is then made to solidify, and sections are made with a microtome through the imbedding mass and the included objects. The sections are then mounted on a slide by one of the 
methods described in the chapter on Serial Section Methods, the imbedding material is removed from them (in the case of paraffin), they are stained in situ on the slide, dehydrated with alcohol, cleared, and mounted in balsam or damar. Or they may be stained, washed, dehydrated, and cleared in watch-glasses, and afterwards mounted as desired-the imbedding medium being first removed if desirable.

Or, the material may be stained in bulk, before cutting the sections. In this case the object, after having been fixed and washed out, is taken from the water, or while still on its way through the lower alcohols (it should not be allowed to proceed to the higher grades of alcohol before staining, if that can be avoided), and passed through a bath of stain, then dehydrated with successive alcohols, passed through a clearing medium into paraffin, cut, and treated as above described, the sections in this case being mounted direct from the chloroform, xylol, or other solvent with which the paraffin is removed, If aqueous staining media be applied (and this is sometimes desirable), the structures should either be stained in toto immediately after fixing and washing out, or sections may be stained on the slide, the objects, if delicate, being passed through successive baths of alcohol of gradually decreasing strength before being put into the aqueous stain.

In my opinion it is generally advisable not to stain in bulk material that is intended to be sectioned; by staining it as sections the staining can be much better controlled, and many excellent stains can in this way be employed that are not available for staining in bulk; and of course sections can be stained much more rapidly than material in bulk.

Balsam mounts of which the stain has faded, or which it may be desired to submit to some other staining process, or mount in some other medium, may often with great advantage be re-stained and re-mounted. All that is necessary is to put the slide into a tube of xylol or benzol till the cover falls off (about two days), wash well for some hours in clean xylol, and pass through alcohol into the new stain. Since this was pointed out to me by Dr. Henneguy I have unmounted and re-stained a large number of old preparations, and have succeeded in every case with series of sections mounted on Mayer's albumen, or by the water method. For shellac-mounted series, see E. Meyer, Biol. Centralb., x, 1890, p. 509, or last edition.

The most convenient vessels, I find, in which to perform the various operations of staining, differentiating, dehydrating, clearing, etc., on the slide, are flat-bottomed corked glass tubes. I have mine made 10 
centimetres high and 27 millimetres internal diameter. Each of these will then take two slides, English size, placed back to back.

\%. Résumé of the General Method.-To sum up, you may either fix, wash out, stain, wash, dehydrate, clear, imbed, cut sections, clear and mount them in balsam; or fix, wash, dehydrate, clear, imbed, cut, stain, wash, dehydrate, clear, and mount-according to choice.

8. Preparation of Entire Objects, or of Material that is not to be sectioned.-The treatment of objects which can be studied without being cut into sections is identical with that above described, with the omission of those passages that relate to imbedding processes. Its normal course may be described as fixation, washing out, staining, treatment with successive alcohols of gradually increasing strength, final dehydration with absolute alcohol, clearing, and mounting in balsam.

In the preparation of entire objects or structures that are intact and covered by an integument not easily permeable by liquids, special care must be taken to avoid swelling from endosmosis on the passage of the objects from any of the liquids employed to a liquid of less density, or shrinkage from exosmosis on the passage to a liquid of greater density. This applies most specially to the passage from the last alcohol into the clearing medium. A slit should be made in the integument, if possible, so that the two fluids may mingle without hindrance. And in all cases the passage is made gradual by placing the clearing medium under the alcohol, as described (§5). Fluids of high diffusibility should be employed as far as possible in all the. processes. Fixing agents of great penetrating power (such as picric acid or alcoholic sublimate solution) should be employed where the objects present a not easily permeable integument. Washing out is done with successive alcohols, water being used only in the case of fixation by osmic acid, or the chromic mixtures or other fixing solutions that render washing by water imperative. Staining is done by preference with alcoholic staining media. The stains most to be recommended are Grenacher's borax-carmine, or one of Mayer's alcoholic carminic acid or hæmatein stains. Aqueous stains are more rarely indicated, though there are many cases in which they are admissible, and some in which they are preferable.

9. Minute Dissections.-These are best done, if necessary, in a drop of clearing agent. I recommend cedar-wood oil for this purpose as it gives to the tissues a consistency very favourable for dissection, whilst its viscosity serves to lend support to delicate structures. Clove oil has a tendency to make tissues that have lain in it for some 
time very brittle. The brittleness is, however, sometimes very helpful in minute dissections. Another property of clove oil is that it does not easily spread itself over the surface of a slide, but has a tendency to form very convex drops, and this also makes it frequently a very convenient medium for making minute dissections in.

If it be desired to dissect in a watery fluid, such as glycerin, it may be well to prepare the slide by spreading on it a thin layer of MAYER's albumen, and on this place a small drop of glycerin, or other dissecting medium. As soon as the dissection has. been accomplished, a cover may be let fall, horizontally, on to the preparation to keep the parts in place, and a weight placed on it. Then the mount may be filled up with glycerin, or other mounting medium, run in under the cover, and closed, if desired, or instead of the albumen a solution of gelatin may be taken, and hardened in formol with the objects on it. For a balsam mount, after clove or cedar oil, ScHÄLliBaUM's collodion may be taken, and the organs fixed in situ on this by adding xylol.

10. Instruments.-For all that concerns the mechanism and manipulation of the Microscope, see vol. i of CARPENTER's The Microscope, eighth edition, 1891; paying particular attention to all that is said concerning the English and the Continental Models, pp. 254 to 261, the Substage, pp. 184 to 189, Condensers, pp. 289 to 316, and Tube Length, pp. 158 to 159.

For information concerning the principles of construction and - manipulation of the Microtome, see also CARPenter's The Microscope. Microtomes are instruments for the accurate production of thin slices of tissues. They are used both for cutting tissues that have acquired a certain favourable consistency through having been imbedded in paraffin, and also for cutting tissues that have been imbedded in softer masses, such as collodion, and tissues that have not been imbedded at all. Not all microtomes are equally well adapted for all these three classes of work. The microtome of the zoologist should at all events be one that is well adapted for cutting imbedded material.

Now there are two methods of imbedding in general use-the paraffin method and the celloidin method. In the paraffin method the object is cut $d r y$, frequently with the knife set square to the line of section. In the celloidin method, as in the cutting of unimbedded tissues, it is generally cut wet, and always with the knife set slanting. Some microtomes that are well adapted for the paraffin method are ill adapted for the celloidin method or the cutting of unimbedded 
material, and vice versâ. It may be well to possess the two sorts of instrument; but if only one can be afforded it should be such as will give good work in either way.

Microtomes fall further into two classes according as the knife and the surface of section of the object are (A) in a horizontal plane, or (В) in a vertical plane. The former offer greater facility for the orientation of the plane of section, which is an important point for the zoologist and embryologist. Amongst these may be mentioned (a) The "Sliding" Microtomes, in which the knife is carried on a sledge and moved against the object (those of Thoma, Schanze, ReICHERT, and others). The Thoma, of medium size, as made by R. Jung, Hebelstrasse, Heidelberg (No. 56 of his catalogue for 1911, which may be obtained from Mr. C. Baker, 244, High Holborn, London), is very suitable for the zoologist. It works equally well with either paraffin or celloidin, and can be adapted as a freezing microtome. But this (as is the case with the others above mentioned) will not always furnish work of the highest accuracy ; for the knife being only clamped at one end is liable to spring, and to give sections of unequal thickness. This defect is remedied in $(b)$, a type of sliding microtomes in which the knife is clamped at both ends and is a fixture, the object being carried on a sledge and moved against it (CAmbridge Scientific Instrument Company's large microtome, the Mrnot precision microtome, LeITz's, DE Groot's, JunG's "Tetrander." This last seems to be near perfection; see the description by MaYer in Zeit. wiss. Mik., xxvii, 1910, p. 52; but is more cumbrous than is desirable for ordinary work.

Class A also includes some instruments in which the knife is carried on a horizontal arm and swung against the object by a rotary movement (Jung, Roy, Fromme, Reichert, Thate, and others). I know nothing of these personally, but doubt their constant accuracy.

Class B contains some very fine instruments, admirably adapted for the production of continuous ribbons of sections by the paraffin method, but not so well adapted for celloidin or other work in the wet way, or for soft objects. Amongst these are the New and Old Rocking Microtome, made by The Scientific Instrument Co., Cambridge, or by SwIFT \& Son, or by JUNG, or by VAN DER STAD, Amsterdam; the Minot, made by Bausch \& Lomb and the Spencer Model, or by Becker (Göttingen), or by Zimmermann (21, Emilienstrasse, Leipzig); the Reinhold-Giltay, made by J. W. Giltay, Delft.

For descriptions of the multitudinous models on the market see 
the reports in the Zeit. wiss. Mik. and Journ. Roy. Micr. Soc., and the price lists of the instrument makers.

11. Staining Reagents and Chemicals.-For some years it has been difficult to obtain good dyes, but recently several new firms have succeeded in manufacturing materials suitable for the most critical work. The pre-war continental dyes were generally impure products, and since the new dyes being made are mostly purer, it is often necessary to make some slight alteration in the quantity of dye added to a staining mixture before the optimum staining condition is reached. Details of such alteration necessary for British dyes should be sent to the Journal of the Royal Microscopical Society. I advise the reader to get his reagents and dyes from some well-known British firm which has specialised in the matter.

Great Britain.-Flatters and Garnett, Ltd., 309, Oxford Road, Manchester (all sorts of chemicals, dyes and apparatus ; makers of Gilson's "Euparal "); the British Drug Houses, Ltd., Graham Street, City Road, London, N. 1 (makers of many sorts of dyes) ; The British Dyestuffs Corporation, Ltd., 70, Spring Gardens, Manchester (important makers of dyes ; London agents are, Baird and Tatlock, 14, Cross Street, Hatton Garden, E.C. 1); Hawksley \& Sons, 83, Wigmore Street, Cavendish Square, W. 1; and Charles Baker, 244, High Holborn, W.C. (both firms are agents for Grübler, and for most microscope and accessory apparatus makers). For incubators, etc., for embedding and tissue-culture work, Charles Hearson \& Co., Ltd., 235, Regent Street, W. 1.

United States of America.-Eimer and Amend, 205-211, Third Avenue, New York; Palo Company, 90, Maiden Lane, New York ; Edward Pennock, 3609, Woodland Avenue, Philadelphia, Pa.; General Biological Supply House, 5508, Kimback Avenue, Chicago, Ill. ; The Will Corporation, Rochester, N.Y.; Paul Weiss, 1620, Arapahoe Street, Denver, Colorado.

Germany.-G. Grübler and Hollborn, Chemiker, Leipzig, Germany. 


\section{CHAPTER II.}

\section{KILLING.}

12. In the majority of cases, the first step in the preparation of an organ or organism consists in exposing it as rapidly and as completely as possible to the action of one of the Fixing Agents that are discussed in the next chapter. The organ or organism is thus taken in the normal living state; the fixing agent serves to bring about at the same time, and with sufficient rapidity, both the death of the organism and that of its histological elements.

It should be noted that narcotisation generally implies some change in the cells, and most narcotics have to be applied for a long time. Such treatment is absolutely barred in material destined for careful cytological study. This applies especially to ether and chloroform, which are extremely injurious to cells: in the case of larger mammals like the cat and dog a preliminary treatment in ether or chloroform may be necessary, but directly after anæsthesia the animals' throats should be cut or they should be killed by a blow, if possible. Coal gas chambers are good for killing all mammals, and I do not believe carbon monoxide is hurtful to cells. Amphibians killed by chloroform are often completely spoilt for cytological purposes; if the brain is not wanted, pith the animal. For birds the time-honoured custom of wringing their necks is recommended. In the case of small lizards, newts and such live stock it is a good plan to cut off their heads quickly with strong scissors. If the material is wanted for chromosome or mitochondria work look up these sections for special directions.

But these methods are by no means applicable to all cases. There are many animals, especially such as are of a soft consistence, and deprived of any rigid skeleton, but possessing a considerable faculty of contractility, which if thus treated contract violently, and die in a state of contraction that renders them unfit for study. In these cases special methods of killing must be resorted to. Speaking generally, there are two ways of dealing with these difficult cases. You may kill the animal so suddenly that it has not time to contract: or you may paralyse it by narcotics before killing it. See also under "Chromosomes," § 652, and "Mitochondria," § 673. 


\section{Sudden Killing.}

13. Heat.-The application of Heat affords a means of killing suddenly. By it the tissues are more or less fixed at the same time that somatic death is brought about.

The difficulty consists in hitting off the right temperature, which is of course different for different objects. I think that $80^{\circ}$ to $90^{\circ} \mathrm{C}$. will generally be amply sufficient, and that very frequently it will not be necessary to go beyond $60^{\circ} \mathrm{C}$. An exposure to heat for a few seconds will generally suffice.

Small objects (Protozoa, Hydroids, Bryozoa) may be brought into a drop of water in a watch-glass or on a slide, and heated over the flame of a spirit-lamp. For large objects, the water or other liquid employed as the vehicle of the heat may be heated beforehand and the animals thrown into it.

As soon as it is supposed that the protoplasm of the tissues is coagulated throughout, the animals should be brought into alcohol (30 to 70 per cent. alcohol) (if water be employed as the heating agent).

An excellent plan for preparing many marine animals is to kill them in hot fresh water. Some of the larger Nemertians are better preserved by this method than by any other with which I am acquainted.

14. Slowly Contracting Animals.-Animals that contract but slowly, such as Alcyonium and Veretillum, and some Tunicates, such as Pyrosoma, are very well killed by throwing them into some very quickly acting fixing liquid, used either hot or cold. Glacial or very strong acetic acid (VAN BENEDEN's method) is an excellent reagent for this purpose; it may be used, for example, with some Medusæ. After an immersion of a few seconds or a few minutes, according to the size of the animals, they should be brought into alcohol of at least 50 per cent. strength. Lemon juice employed in this way has given me very good results with small Annelids and Hirudinea. Corrosive sublimate is another excellent reagent for this purpose.

\section{Narcotisation.}

15. Narcotisation is performed by adding some anæsthetic substance very gradually, in very small doses, to the water containing the animals, and waiting patiently for it to take effect slowly.

Menthol.- -Now used with great success for anæsthetising large marine animals. Place latter in clean vessel, and sprinkle over surface of water, menthol crystals. As the latter dissolve the animals expand. In from twelve to twenty-four hours they may be transferred to a fixer. Very good for Anemones, Holothuria, Ascidia and many Mollusca. (Personal communication from Dr. E. J. Allen, Plymouth.)

16. Nicotin in solution (Andres, Atti R. Accad. dei Lincei, v, 1880, p. 9). Andres employs a solution of 1 gramme of nicotin in a litre 
of sea water. The animal is placed in a jar containing half a litre of sea water, and the solution of nicotin is gradually conducted into it by means of a thread, acting as a syphon, of such a thickness as to be capable of carrying over the whole of the solution of nicotin in twentyfour hours. See also Mitth. Zool. Stat. Neapel, Bd. ii, 1880, p. 123.

17. Chloroform may be employed either in the liquid state or in the state of vapour. The animals being extended, a watch-glass containing chloroform may be floated on the surface of the water in which they are contained, and the whole covered with a bell-glass. As soon as they have become insensible they are killed by means of hot sublimate or chromic acid solution plentifully poured on to them. (Konotnefr, Mitth. Zool. Stat. Neapel, v, 1884, p. 233.)

Liquid chloroform is employed by squirting it in small quantities on to the surface of the water containing the animals. A syringe or pipette having a very small orifice, so as thoroughly to pulverise the chloroform, should be employed. Small quantities only should be projected at a time, and the dose should be repeated every five minutes until the animals are anæsthetised.

I have seen large Medusæ very completely anæsthetised in extension in an hour or two by this method. ANDREs finds that it does not succeed with Actiniæ, as with them maceration of the tissues supervenes before anæsthesia is established.

Preyer (Mitth. Zool. Stat. Neapel, Bd. vii, 1886, p. 27) recommends chloroform water for star-fishes.

WADDINGTON employs a mixture of equal parts of 1 per cent. sol. of cocaine (or eucain) and saturated sol. of chloroform in water (sea or fresh), according to the habitat.

18. Ether and Alcohol may be administered in the same way. ANDREs has obtained good results with Actiniæ by the use of a mixture (invented by SALVATORE LO BIANCO) containing 20 parts of glycerine, 40 parts of 70 per cent. alcohol, and 40 parts of sea water. This mixture should be carefully poured on to the surface of the water containing the animals, and allowed to diffuse quietly through it. Several hours are sometimes necessary for this.

EIsig (Fauna u. Flora Golf. Neapel, xvi, 1887, p. 239) benumbs Capitellidæ by putting them into a mixture of 1 part of 70 per cent. alcohol with 9 parts of sea water.

Oestergren (Zeit. wiss. Mik., xix, 1903, p. 300) makes a saturated (7 to 8 per cent.) solution of ether in sea or soft water, and uses it either concentrated or diluted to about 1 per cent., and finds it to succeed with all classes of aquatic animals. 
CoRI (Zeit. wiss. Mik., vi, 1890, p. 438) recommends a mixture composed of 10 c.c. methyl-alcohol (of 96 per cent. strength), 90 c.c. water (fresh or sea water), and $0.6 \mathrm{grm}$. of sodium chloride (to be added only when fresh water is taken, the addition of the salt having for its object to prevent maceration). It may be well to add to this mixture a very few drops of chloroform (for Cristatella; Zeit. wiss. Zool., lv, 1893, p. 626).

19. Chloreton (Aceton Chloroform) is recommended for invertebrates and larvæ of Rana by RANDOLPH (Zool. Anz., xxiii, 1900, p. 436). KRECKer (Zeit. wiss. Zool., xcv, 1910, p. 383) takes solutions of $\frac{1}{3}$ to 1 per cent. for Oligochæta. Sulima (Zeit. Biol. Techn., Strasburg, i, 1909, p. 379) takes a mixture of 99 parts of sea water and 1 of 10 per cent. sol. of chloreton in absolute alcohol, for Scyllium and Anguilla.

For Bryozoa, see Bessie Green, Journ. Roy. Mic. Soc., 1914.

20. Hydrate of Chloral.-Foetringer (Arch. de Biol., vi, 1885, p. 115) operates by dropping crystals of chloral into the water containing the animals. For Alcyonella he takes 25 to 80 centigrammes of chloral for each hundred grammes of water. It takes about three-quarters of an hour to render a colony sufficiently insensible. He has obtained satisfactory results with marine and fresh-water Bryozoa, with Annelida, Mollusca, Nemertians, Actiniæ, and with Asteracanthion. He did not succeed with Hydroids.

Lo Bianco (Mitth. Zool. Stat. Neapel, Bd. ix, 1890, p. 442) employs for various marine animals freshly prepared solutions of chloral in sea water, of from $\frac{1}{10}$ to $\frac{1}{5}$ per cent. strength.

I have never had the slightest success with Nemertians.

VERWORN (Zeit. wiss. Zool., xlvi, 1887, p. 99) puts Cristatella for a few minutes into 10 per cent. solution of chloral, in which the animals sooner or later become extended.

KÜKenthal (Jena Zeit. Naturw., Bd. xx, 1887, p. 511) has obtained good results with some Annelids by means of a solution of 1 part of chloral in 1,000 parts of sea water.

The chloral method gives rise to maceration with some subjects, as I can testify, and has been said to distort nuclear figures.

21. Cocaine (Richards, Zool. Anz., cxevi, 1885, p. 332).--Richards puts a colony of Bryozoa into a watch-glass with 5 c.c. of water, and adds gradually 1 per cent. solution of hydrochlorate of cocaine in water. After five minutes the animals are somewhat numbed; half a cubic centimetre of the solution is added, and ten minutes later the animals should be found to be dead in a state of extension.

This method is stated to succeed with Bryozoa, Hydra, and 
certain worms. It is the best method for Rotifers (Rousselet). It has also been recommended for Aplysia.

It has been pointed out (by CorI, in the paper quoted $\S 18$ ) that, unfortunately, when fixing agents, such as sublimate solution, are added to the animals, the cocaine is thrown down on them as a white precipitate. This precipitate, however, may be redissolved afterwards in alcohol (EIsIG).

Cocaine solutions cannot be depended on to keep for more than a few days.

22. Eucain. Harris (Journ. Roy. Mic. Soc., 1900, p. 404) recommends a 1 per cent. solution of eucain hydrochloride, as giving far better results, with Vorticellidæ, Rotatoria, and Vermes. Rousselet (ibid.) reports favourably as to its action on Flosculariæ. It is stated to be perfectly stable in aqueous media. It dissolves in sea water to about 0.5 per cent.

23. Hydroxylamin.-Hofer (Zeit. wiss. Mik., vii, 1890, p. 318). Either the sulphate or, preferably, the hydrochlorate may be used. This should be dissolved in water (spring or sea water, according to the habitat) and exactly neutralised by addition of carbonate of soda. The organisms are placed in a solution diluted to about 0.1 per cent., for thirty minutes or less (as for Infusoria), to $\mathbf{0 . 2 5}$ per cent., for from fifteen minutes to one hour ( $H y d r a), 1$ per cent., one half to two hours (Hirudo), or as much as ten to twenty hours (Helix and Anodonta).

Hydroxylamin is a powerful reducing agent, and should therefore be well washed out before treating with easily reducible fixing agents.

24. Chloride or Sulphate of Magnesium.-Tuldberg (Arch. Zool. Expér. et Gen., x, 1892, p. 11). For Actiniæ, a 33 per cent. solution of the chloride should be very slowly added to the water containing the expanded animal, until the vessel contains 1 per cent. of the salt (thus for 1 litre of sea water 33 c.c. of the solution must be added). The addition must be completed within half an hour, and thirty minutes later the animal may be fixed.

For terrestrial and fresh-water Invertebrates rather stronger solutions should be used.

Redenbaugh (Amer. Natural., xxix, 1895, p. 399) takes the sulphate, either added in crystals to the sea water containing the animals until a saturated solution is obtained, or in the shape of a saturated solution into which they are thrown (Annelids).

See also Mayer, Biol. Bull. Wood's Hole, xvii, 1909, p. 341 (puts direct into sol. of $\frac{3}{8}$ strength).

25. Poisoning by small doses of some fixing agent is sometimes good. Lo Branco kills Ascidia and Rhopalcea in an extended state (Mitth. Zool. Stat. Neapel, ix, 1890, p. 471) by pouring a little 1 per cent. 
chromic acid on to the surface of the water containing them, and allowing it to diffuse slowly into it. About twelve to twenty-four hours is necessary. He kills Ciona in a similar way with a mixture of 1 part of 1 per cent. chromic acid and 9 parts of 49 per cent. acetic acid.

Osmic acid, or Kleinenberg's solution, is sometimes employed in the same way.

I have seen Medusæ killed in a satisfactory manner by means of crystals of corrosive sublimate added to the water containing them.

Morphia, Curare, Strychnin, Prussic Acid, and other paralysing drugs, have also been employed.

26. Asphyxiation may be sometimes successfully practised. Terrestrial Gastropods may be killed for dissection by putting them into a jar quite full of water that has been deprived of its air by boiling, and hermetically closing it. After from twelve to twentyfour hours they are generally found dead and extended. The effect is obtained somewhat quicker if a little tobacco be added to the water.

Good results are sometimes obtained with aquatic animals by simply leaving them to exhaust the oxygen of the water in which they are contained. I have sometimes succeeded with Holothuriæ and other Echinoderms in this way. WARD (see Amer. Nat., xxv, 1891 , p. 398) has succeeded with Hydroids, Actiniæ, and similar forms, and UexкüLL (Mitth. Zool. Stat. Neapel, xii, 1896, p. 463) with Echinids.

Marine animals are sometimes successfully killed by simply putting them into spring water.

Warm Water will sometimes serve to immobilise and even kill both marine and fresh-water organisms.

Carbonic Acid Gas has been recommended (by Fol, Zool. Anz., cxxviii, 1885 , p. 698). The water containing the animals should be saturated with the gas. The method is stated to succeed with most Cœlenterata and Echinodermata, but not with Molluses or Fishes. I have had most excellent results with small Annelids and Hirudinea. It is not necessary to employ a generator for obtaining the gas. It suffices to take an ordinary "soda-water" syphon, and squirt its contents into the water containing the animals.

Narcotisation is very rapidly obtained with very small animals, but much more slowly with larger ones. For instance, Stylaria proboscidea, I find, is paralysed in a few seconds; a small Nephelis of 15 or 20 millimetres in length, will require about five minutes; and a large Nephelis, of from 10 to 15 centimetres, will require as many hours.

UExkÜLL (Mitth. Zool. Stat. Neapel, xii, 1896, p. 463) has paralysed 
Echinids very rapidly with carbonic acid, likewise a small Teleostean fish; whilst Scyllium and Crustaceans were affected much more slowly, and mussels not at all.

2\%. Peroxide of Hydrogen.-Volk (Zool. Anz., xix, 1896, p. 294) kills Rotatoria by means of one or two drops of a 3 per cent. solution added to 1 c.c. of the water containing them. 


\section{CHAPTER III.}

FIXING AND HARDENING.

28. The Functions of Fixing Agents.-The meaning of the term "fixing" has been explained above (§ 2). Here is an example showing the necessity of fixation. If a portion of living retina be placed in aqueous humour, serum, or other so-called " indifferent" medium, or in any of the media used for permanent preservation, it will be found that the rods and cones will not preserve the appearance they have during life for more than a very short time; after a few minutes a series of changes begins to take place, by which the outer segments of both rods and cones become split into discs, and finally disintegrate so as to be altogether unrecognisable, even if not totally destroyed. Further, in an equally short time the nerve-fibres become varicose, and appear to be thickly studded with spindleshaped knots ; and other post-mortem changes rapidly occur. If, however, a fresh piece of retina be treated with a strong solution of osmic acid, the whole of the rods and cones will be found perfectly preserved after twenty-four hours' time, and the nerve-fibres will be found not to be varicose. After this preliminary hardening, portions of the retina may be treated with water (which would be ruinous to the structures of a fresh retina), they may even remain in water for days without harm; they may be stained, acidified, hardened, imbedded, cut into sections, and mounted in either aqueous or resinous media without suffering.

This example shows that one of the objects aimed at in fixing is to impart to tissues the degree of hardening necessary to enable them to offer such mechanical resistance to post-mortem change and to the processes of after-treatment as not to suffer change of form. Another important function of fixing is to render insoluble elements of cells and tissues that would otherwise be more or less dissolved out by the liquids employed in the after-treatment. A third and highly important function of fixing agents consists in producing optical differentiation in structures. By coagulating the elements of tissues and cells, fixing agents alter their indices of refraction, raising them in varying degrees. They do not act in an equal degree on all the constituent elements of cells and tissues, but raise the index of 
some more than that of others, thus producing optical differentiation where there was little or none before. Compare the aspect of the epithelium of the tail of a living tadpole, observed in water, with its aspect after the action of a little diluted solution of Flemming. In the living state the protoplasm of its cells has a refractive index little superior to that of water, and consequently so low an index of visibility that hardly any structure can be made out in the object. But as soon as the protoplasm has been sufficiently coagulated by the reagent the refractive indices of some of its elements will have been raised to above that of balsam, the chromatin of the nuclei will be brought out, and other structures be revealed where none was visible before.

29. The Action of Fixing Agents consists in coagulating and rendering insoluble certain of the constituents of tissues. This is effected sometimes without any chemical action being involved, as when alcohol is employed, which acts by simple withdrawal of the water of the tissues. But in the majority of cases the fixing agents enter into chemical combination with certain of the elements of the tissues. The compounds thus formed are sometimes unstable and soluble, so that they are removable by washing, as is the case with several of those formed by picric acid. It is found in practice, however, that those formed by chromic acid and its salts, and the salts of the heavy metals, as mercury, iron, platinum, gold, and silver, are mostly insoluble.

The insolubility of these bodies is an advantage in that it ensures that the tissues shall not be robbed of their essential constituents, nor deprived of their desired consistency and optical differentiation, by the reagents subsequently employed. It is also sometimes an advantage in that certain of the compounds in question have the property of combining with certain colouring matters, and thus affording important stains which could not otherwise be obtained; or in other words, of acting as mordants.

But it is sometimes a disadvantage, inasmuch as these same compounds which render possible the production of some stains are hindrances to the production of others. Tissues that have been fixed with osmic or chromic acid or their salts are in general not easily to be stained with carmine or similar colouring matters, unless the metals have been previously removed by special chemical treatment ; though they may generally be stained with hæmalum, or, after sectioning, with iron hæmatoxylin or tar colours.

According to Fischer (Fixirung, Färbung, und Bau des Proto- 
plasmas, Jena, G. Fischer, 1899), the coagulation which constitutes fixation is, in the case of the liquid and semi-liquid constituents of tissues, always a phenomenon of precipitation. The more solid constituents (such as fibrils that are visible during life, nucleoli, and the like) he admits may be acted on by fixing reagents without the formation of any visible precipitates. But all the liquid ones, in so far as they are fixed at all, are visibly precipitated in special precipitation forms, which vary according to the precipitant. Each fixing agent gives its own characteristic fixation image, which may be more or less lifelike, but can never be absolutely so. Fischer gives copious descriptions of the precipitation forms of the chief organic compounds found in tissues, and of the precipitation powers of the chief fixing agents, which the reader will do well to study.

It seems to be a consequence of Fischer's theory of fixation by precipitation that the most energetic fixing agents should always be found amongst the most energetic precipitants. But on the showing of his experiments this is not so. For instance, it is allowed on all hands that osmic acid is a most energetic fixative. But Fischer finds (op. cit., pp. 12-14,27) that it is a very incomplete and weak precipitant. Or, to take a contrary instance, he finds that picric acid is an energetic precipitant of the majority of cell constituents ; but surely every cytologist must admit that it is not a highly energetic fixative!

It would seem to follow, from these instances and from other similar ones, that Fischer's tables of precipitating power cannot be taken as a measure of the fixing power of the reagents. And further, the study of the fixation images of tissues afforded by osmic acid, formaldehyde, and other reagents, seems to show that the coagulation brought about by them is in part accompanied by the formation of visible precipitates, but in part not so, and that they may do their work to a larger extent than he seems to admit through a homogeneous coagulation. Fischer, studying the effects of certain fixatives on albumose, states that mixing 10 per cent. slightly acid deuteroalbumose with Altmann's bichromate-osmic fluid causes a precipitate of granules of from 1 to $3 \mu$ in diameter, while corrosive sublimate of 7 per cent. causes granules of 0.4 to $1 \mu$ in size; one might be led away, as was Fischer, to consider that Altmann's fluid used on cells therefore causes artifacts to appear. As a matter of fact corrosive sublimate is much more dangerous than Altmann's fluid, in this respect, while Altmann's fluid merely preserves cell granules which are visible intra vitam. The ground protoplasm after corrosive is more granular and coarsely reticulate than after Altmann; this 
does not apply to mitochondria or Golgi elements which are often very badly preserved with corrosive.

Fischer (op. cit.) says, "Many kinds of cell contents, indeed the majority, have an alkaline reaction, and are thereby quite inaccessible to the precipitating action of certain agents, such as osmic acid, or bichromate; and the action of certain other fixatives, such as platinum chloride or chromic acid, is more or less hindered by the presence of free alkalies. For neither the chromic acid (of the Flemming), nor the platinum chloride (of the Hermann) would be adequate to act as acidifiers to the osmic acid of the mixtures."

My own experience is directly contrary to these conclusions of Fischer : it is common knowledge among modern workers that a cell fixed in acetic acid-containing solutions has a more " raked out" appearance than when the acetic acid is omitted: this applies not only to cell granules, but to the appearance of the ground cytoplasm, nucleoli, and chromatin filaments. The statement that osmic acid must be acidified before it will fix all parts of the cell is also contrary to general experience. Formalin neutralised gives a gentler and more precise fixation than acid formalin. While Fischer's results may be excellent so far as concern his theoretical conclusions on the fixation of weak solutions of egg-white, etc., too much attention should not be paid to one who is not thoroughly acquainted with practical cytology and histology. But from his very suggestive observations it certainly appears that the formation of visible precipitates is a very widespread, if not universal concomitant of fixation; and that the wider the precipitating power of a fixative (i.e. the greater the number of organic liquids that it can precipitate), the greater will be the number of artifacts to which it can give rise.

It has lately been pointed out by some workers (e.g. UnNa, Arch. f. Mikr. Anat., Ixxviii), that many of the fixing reagents come under the category of oxidisers; Unna places great importance on the fact that some of the most successful fixers are oxidisers, e.g. $\mathrm{OsO}_{4}, \mathrm{~K}_{2} \mathrm{Cr}_{2} \mathrm{O}_{7}, \mathrm{CrO}_{3}$; but formalin, admittedly a splendid reagent, is a reducer, while picric acid and corrosive sublimate are feeble oxidisers, and that only under special conditions.

Of the ten common reagents used for fixing, only four are marked oxidisers, and Unna's generalities with reference to the significance of oxidisers may not be the correct explanation. Generalities such as made by Unna with reference to the rôle of oxidisers in fixation resemble like claims which have been made with reference to the supposed necessity for the constant use of an acid in fixatives (and preferably acetic acid which is really a dangerous reagent). 
With regard to the relative values of oxidisers or reducers in fixing cytoplasm or"nucleus, formalin (reducer)* and $\mathrm{OsO}_{4}$ (oxidiser) are both famous cytoplasm fixers, while acetic acid (neither oxidiser nor reducer), or alcohol (reducer) and $\mathrm{CrO}_{3}$ (oxidiser) are well-known nuclear fixatives.

Helly's fluid, formol-bichromate or formol-Flemming are all splendid fixatives, and mixtures of both oxidisers and reducers ; it is difficult to see how Unna's theories can apply here. In the case of Flemming fluid, without acetic acid, it is certain that the fixation process in so far as it concerns the $\mathrm{OsO}_{4}$ in this mixture, is not solely an oxidising process, at least of the same nature as the fixation reaction by the chromic acid $\left(\mathrm{CrO}_{3}\right)$. In a word, fixation of the cell by various kinds of chemical reagents is an extremely complicated matter concerning a large number of organic substances whose reactions to the chemical used are probably different in most cases.

30. The Characters of the Usual Fixing Agents.-These agents are as follows :-

Chloroform and urea are also used. In the group marked $\mathrm{A}$ are arranged the more valuable reagents, in B the less valuable or destructive ones. Good fixatives can be made from the substances in group A without using any of the reagents in group B. The latter contain most of the reagents useful for chromosome work, the former, reagents useful for fixing the cytoplasm and "resting" nucleus.

From group A have been made the following mixtures: Altmann, Champy, and Flemming and Hermann-without-acetic acid; these. are among the best mixtures known. Then there are formol ( 5 per cent, to 10 per cent.), Regaud, Helly, formol-Müller and formolFlemming, which are so good for mammals. Good general microanatomical fixatives from both groups A and B, are Zenker, Bouin,

* See, however, Blum, Enzykl. d. mikr. Tech., 1910. 
Gilson-Petrunkewitsch and corrosive acetic, but these all destroy much of the cell-contents, and give an incorrect picture of the cell, excepting of chromatinic structures, for which they are indicated.

A good fixing agent should first of all preserve all the elements it is desired to fix. But that is not enough ; it should also give good optical differentiation, and should have sufficient power of penetration to ensure that small pieces of tissue be equally fixed by it throughout. No single substance or chemical compound fulfils all that is required of a good fixing agent; hence it is that all the best fixing agents are mixtures. Osmic acid, for instance, fulfils some of these conditions, but not all of them. It kills rapidly and preserves admirably the elements of cytoplasm, but nuclei not so well. But the optical differentiation that it gives, though sometimes good, is often very inferior. For osmic acid, by coagulating in nearly equal degrees alike the spongioplasm (the plastin reticulum) and the hyaloplasm (the enchylema) of the cell-body, and the chromatin of nuclei, raises alike the refractive indices of all of them; so that if the fixing action have been in the least degree overdone, the cells acquire a homogeneous aspect in which the finer details are obscured by the general refractivity of the whole. If now, instead of using it pure, it be used in combination with chromic acid, a better differentiation is obtained ; for chromic acid, whilst enhancing, and at all events not interfering with the fixation of chromatin, serves to facilitate penetration and to counteract the excessive action of the osmic acid on the protoplasm, so that the cells come out less homogeneous and with more detail observable in them.

Descriptive embryologists .often use strange illogical mixtures containing both reducible substances and violent reducers, both fat-solvents and fat-preservers, mixed together without regard for the chemistry of fixation. It is only the logically planned fixative that is found generally useful, and which stands the test of time. Fixation falls under three broad headings :-

1. Micro-anatomical, in which correct preservation of cell aggregates, without shrinkage or expanding, is the desideratum. Such is the aim of most descriptive embryologists.

2. Cytological from the point of view of the chromosome or nucleus.

3. Cytological from the point of view of fixing the cell in a state which most resembles its condition when alive; also so as to identify the cell elements, especially in the cytoplasm.

In most cases the results attained by workers belonging to sections 1 and 2 can truly be said to give a caricature of the cell intra vitam. I give below a general classification of fixatives, those in $(a)$ being 
fixatives causing the maximum disturbance and destruction in the individual cell, those in $(c)$ the least.

A great deal, however, depends on the accessibility of the cells to the fixative, and as to whether vertebrate or invertebrate material is being used.

(a) Carnoy, Petrunkewitsch, alcohol, Gilson, picro-nitric, etc.

Fat, mitochondria, Golgi apparatus, and often delicate yolk discs do not show after these. (Using alcohols and xylol subsequently.)

(b) Bouin, Zenker, corrosive acetic, Flemming-with-acetic acid, etc.

Mitochondria and Golgi apparatus rarely show after these, except possibly in mammals, where these cell inclusions are more resistant than in invertebrata. Fats show with the last-mentioned fixative.

(c) Osmic acid, Flemming-without-acetic, Champy, Altmann, formalin, Mann's mercury-osmic liquid, Sjövall's method, etc. Preserve all formed granules (except glycogen). (Using fluids subsequently as above.)

In section $(c)$ the formol alone will not preserve fat; but see Sjövall's method ( $\$ 696)$.

The fixatives have not been classed according to how they themselves alone affect the contents of the cell, but according to how they preserve the cell preparatory to its treatment in the liquids necessary for embedding and sectioning.

Injurious liquids which should never be used in cytological fixation (3, vide supra) are acetic acid, chloroform and alcohol. Acetic acid is nearly the most destructive to delicate lipins, and its use, except where chromosomes are being studied, is rarely indicated; any worker who uses acetic acid in his fixing mixtures cannot hope to get a correct picture of any part of his cell, possibly excepting the chromosomes (not the resting nucleus). The most valuable fixatives are osmium - tetroxide, bichromate of potassium, chromium - trioxide, and formaldehyde, possibly in the order named; the most valuable mixtures are Müller-formol (or Helly), Flemming-without-acetic, Altmann, and Champy; the three latter approach as near perfection as present-day technique allows. Altmann's fluid $\left(\mathrm{K}_{2} \mathrm{Cr}_{2} \mathrm{O}_{7}+\right.$ $\mathrm{OsO}_{4}$ ) I find tn be a splendid mixture. In no case, except in small invertebrates, do these fixatives (excluding formol) give a true fixation of cell aggregates ; this is due to their inferior penetrating powers, and to an unevenness of penetration. Small invertebrates, both marine and fresh-water, and small pieces of tissue, are usually exquisitely preserved in chrome-osmium mixtures, but are not then generally suitable for staining and mounting whole, especially for staining in carmine mixtures. 
For routine zoological work Bouin's picro-formalin-acetic is recommended. Gilson-Petrunkewitsch is a fixative which is easy to work and generally better than corrosive sublimate acetic.

For routine vertebrate histological work Zenker and Helly's Zenker-formol are indicated.

I think the beginner should avoid such things as liquid of FLEMming and similar mixtures.

Picric acid gives a fair though weak fixation, with very good penetration, is easy to manage, and does not make tissues brittle, which sublimate easily may do. Pure formol is not bad, and very easy to manage.

Speaking generally, osmic acid, chromic acid, bichromates, chloride of platinum, and the majority of the compounds of the heavy metals, are hindrances to staining; whilst heat, alcohol, trichloracetic acid, formol, corrosive sublimate, picric acid, and acetic acid, are neutral, or even favourable, in this respect.

31. The Practice of Fixation.- See that the structures are perfectly living at the instant of fixation, otherwise you will only fix pathological states or post-mortem states.

Some observers have made special observations on the effect of delay in fixation; J. Thornton Carter (Phil. Trans. Roy. Soc., Series B, vol. ceviii, 1917) has made some interesting experiments on the finely granular - ameloblasts in the developing teeth of the pike. He noticed that the cytoplasm gave evidence of marked changes unless fixed within three minutes of "death" ; these changes were manifested by the behaviour of the eytoplasmic granules to stains; the selectivity" of the latter was progressively altered as the rapid post-mortem changes were set in action.

Fixation is generally performed by immersion of the objects in the fixing liquid. In this case, everything should be done to facilitate the rapid penetration of the fixing agent. To this end let the structures be divided into the smallest portions that can conveniently be employed, and if entire organs or organisms are to be fixed whole, let openings, as large as possible, be first made in them.

The penetration of reagents is greatly facilitated by heat. You may warm the reagent and put it with the object to be fixed in the paraffin stove, or you may even employ a fixing agent heated to boiling-point (as boiling sublimate solution for certain corals and Hydroids, or boiling absolute alcohol for certain Arthropods with very resistent integuments). But this should only be done as a last resource.

Let the quantity of fixing agent employed be many times 
the volume of the objects to be fixed. If this precaution be not observed the composition of the fixing liquid may be seriously altered by admixture of the liquids or of the soluble substances of the tissues thrown into it. For a weak and slowly acting fixing agent, such as picric acid, the quantity of liquid employed should be in volume about one hundred times that of the object to be fixed. Reagents that act very energetically, such as Flemming's solution, may be employed in smaller proportions.

But fixation may also be performed by injection of the fixing liquid into the objects, thus ensuring a more rapid and thorough penetration of voluminous objects. See for this practice the methods of fixation by injection of GolgI, DE QuervaIN, MaNN, and others, given under Nervous System.

Braus and Druener (Jena Zeit. Naturw., Bd. xxix, 1895, p. 435) fix fishes by injection through the bulbus aorta. The vessels are first washed out with normal salt solution, and the fixing liquid is then thrown in.

Kolmer (Anat. Anz., xlii, 1912, p. 47) fixes thus even large mammals (Chimpanzee, Goat). He first washes out with RINGER's solution.

It is well not to leave specimens in fixing liquids longer than is sufficient to obtain the desired reaction. Sublimate, for instance, soon makes tissues brittle. But long immersion may be necessary to produce the desired optical differentiation with some reagents.

Careful washing out (by which is meant the removal from the tissues of the excess of uncombined fixative) is necessary in order to get tissues to stain properly. But it is not always equally imperative. Alcohol and formaldehyde do not require washing out before staining; acetic and picric acid only for some stains ; sublimate will allow of staining even if not washed out, but allows of a sharper stain if well washed out; all osmic, chromic, and platinic liquids require very thorough washing out.

It is important to use the appropriate liquid for washing out the fixing agent after fixation. It is frequently by no means a matter of indifference whether water or alcohol be employed for washing out. Sometimes water will undo the whole work of fixation (as with picric acid). Sometimes alcohol causes precipitates that may ruin the preparations. Objects fixed in alcohol, formol, acetic acid, picric acid, or nitric acid require to be washed out with alcohol, or at least with some hardening liquid, whilst those that have been fixed with osmic or chromic acid, or with one of the other compounds of the heavy metals, require in general to be washed out with water. Sublimate, however, is best washed out with alcohol. 
Use liberal quantities of liquid for washing.

Change the liquid as often as it becomes turbid, if that should happen.

The process of washing out is greatly facilitated by heat. Picric acid, for instance, is nearly twice as soluble in alcohol warmed to $40^{\circ} \mathrm{C}$. as in alcohol at the normal temperature (Fol).

32. Fixation of Marine Animals.-The tissues of marine organisms are as a general rule more refractory to the action of reagents than those of corresponding fresh-water or terrestrial forms, and fixing solutions should in consequence be stronger (about two to three times).

Marine animals ought to be freed from the sea water adherent to their surface before treating them either with alcohol or any fixing reagent that precipitates the salts of sea water. If this be not done, the precipitated salts will form on the surfaces of the organisms a crust that prevents the penetration of reagents to the interior. Fixing solutions for marine organisms should therefore be such as serve to keep in a state of solution, and finally remove, the salts in question. If alcohol be employed, it should be acidified with hydrochloric or some other appropriate acid. Picro-nitric acid is a fixing reagent that fulfils the conditions here spoken of. (On this subject see Mayer, in Mitth. Zool. Stat. Neapel, ii (1881), pp. 1 et seq., and Allen and Browne in "Science of the Sea," John Murray, 1912).

33. Hardening. - The process of hardening is distinguished from that of fixing as being directed to the attainment of a degree of consistency sufficient to allow of soft tissues being cut into sections without imbedding. It is an after-process, and only ranks as a special method.

Methods of imbedding have now been brought to such a degree of perfection that the thorough hardening of soft tissues that was formerly necessary in order to cut thin sections from them is, in the majority of cases, no longer necessary. But there are some exceptions. Such are, for instance, the cases in which it is desired to cut very large sections, such as sections of the entire human brain.

The reagents employed for hardening are for the most part of the same nature as those employed for fixing. But it does not follow that all fixing agents can be employed for hardening. Corrosive sublimate, for instance, would be most inappropriate as a hardening agent.

34. The Practice of Hardening.- Employ in general a relatively large volume of hardening liquid, and change it very frequently. If 
the volume of liquid be insufficient, its composition will soon become seriously altered by the diffusion into it of the soluble substances of the tissues ; and the result may be a macerating instead of a hardening liquid. Further, as soon as, in consequence of this diffusion, the liquid has acquired a composition similar in respect of the proportions of colloids and crystalloids contained in it to that of the liquids of the tissues, osmotic equilibrium will become established, and diffusion will cease; that is to say, the hardening liquid will cease to penetrate. This means, of course, maceration of internal parts. On the other hand, it appears that a certain slight proportion of colloids in the hardening liquid is favourable to the desired reaction, as it gives a better consistency to the tissues by preventing them from becoming brittle. Hence the utility of employing a certain proportion of hardening agent.

Hardening had better be done in tall cylindrical vessels, the objects being suspended by a thread, or muslin bag, or otherwise, at the top of the liquid. This has the advantage of allowing diffusion to take place as freely as possible, whilst any precipitates that may form fall harmlessly to the bottom ; or, they may be laid on a layer of cotton-wool, or filter-paper, or spun glass.

In general, begin hardening with a weak reagent, increasing the strength gradually, as fast as the tissues acquire a consistency that enables them to support a more energetic action of the reagent.

Let the objects be removed from the hardening fluid as soon as they have acquired the desired consistency. 
CHAPTER IV.

FIXING AND HARDENING AGENTS-MINERAL ACIDS AND THEIR

SALTS.

35. Osmic Acid.-The tetroxide of osmium $\left(\mathrm{OsO}_{4}\right)$ is the substance commonly known as osmic acid, though it does not possess acid properties. It is extremely volatile, and in the form of an aqueous solution becomes partially reduced with great readiness in presence of the slightest contaminating particle of organic matter. It is generally believed that the aqueous solutions are reduced by light alone, but this is not the case : they may be exposed to the light with impunity if dust be absolutely denied access to them.

The solution of osmic acid in chromic acid solution is not, like the solution in pure water, easily reducible, but may be kept without any special precautions. I therefore keep the bulk of my osmium in the shape of a 2 per cent. solution of osmic acid in 1 per cent. aqueous chromic acid solution. This solution serves for fixation by osmium vapours, and for making up solution of Flemming, which is the form in which osmium is most generally employed. A small quantity of osmic acid may also be made up in 1 per cent. solution in distilled water, and kept in a drop-bottle with grooved stopper, from which quantities can be obtained when required without removing the stopper.

ConI (Zeit. wiss. Mik., vi, 1890, p. 442) finds that solutions in distilled water keep perfectly if there be added to them enough permanganate of potassium to give a very slight rosy tint to the liquid. From time to time, as the solution becomes colourless, further small quantities of the salt should be added, so as to keep up the rosy tint.

Busch finds that the addition of sodium iodate hinders reduction (Neurol. Centralb., xvii, 1898, p. 476).

PintNer finds that a slight addition of corrosive sublimate has the same effect, e.g. 10 drops of 5 per cent. solution of sublimate added to 100 c.c. of 1 per cent. solution of osmic acid.

For the Kopsch, Mann-Kopsch and Sjövall methods the osmic acid solution must be free from all traces of chrome and platinum salts, etc. 
For the so-called "regeneration" of reduced solutions, see previous editions.

Osmic acid is met with in commerce in the solid form in sealed tubes. The assigned weights should be checked, as they may vary greatly.

Fixation by the Vapours. - This is indicated in most of the cases in which it is possible to expose the tissues directly to the action of the vapour. The tissues are treated as described in $\S 695$. Very small objects, such as isolated cells, are simply placed on a slide, which is inverted over the mouth of the bottle. They remain there until they begin to turn brown (isolated cells will generally be found to be sufficiently fixed in thirty seconds : whilst in order to fix the deeper layers of relatively thick objects, such as retina, an exposure of several hours may be desirable). It is well.to wash the objects with water before staining, but a very slight washing will suffice. For staining, methyl-green may be recommended for objects destined for study in an aqueous medium, and, for permanent preparations, alum-carmine, picro-carmine, or hæmatoxylin.

In researches on nuclei, it may be useful to employ the vapours of a freshly prepared mixture of osmic and formic or acetic acid (Gilson, La Cellule, i, 1885, p. 96).

The reasons for preferring fixation by the vapour are that osmic acid is more highly penetrating in vapour than in solution; that the arduous washing out required by the solutions is done away with; and that all possibility of deformation through osmosis is elminated. See also under "Cramer's Method," § 695.

Fixation by Solutions.-Osmic acid is now very seldom used pure in the shape of solutions. When, however, it is so employed it is used in strengths varying from $\frac{1}{20}$ to 2 per cent. I should say myself that, as a rule, not more than $0 \cdot 1$ per cent., and never more than 2 per cent., should be used.

On account of its feeble penetrating power the objects to be fixed should be as small as possible.

The solutions should be kept protected from bright light during the immersion of tissues. (This precaution is not necessary if Flemming's or Hermann's solution be used.) If the immersion is to be a long one the tissues must be placed with the solution in wellclosed glass-stoppered vessels. The objects may be deemed to be fixed as soon as they have become brown throughout. But see "Mann-Kopsch Methods," § 693.

After-Treatment.-The excess of osmic acid must be well washed out before proceeding to any further steps in preparation; water should be used for washing. Notwithstanding the greatest care in 
soaking, it frequently happens that some of the acid remains in the tissues, and causes them to blacken in time, and in any case hinders staining. To obviate this blackening it has been advised to wash them out in ammonia-carmine or picro-carmine (not very effectual), or to soak them for twenty-four hours in a solution of bichromate of potash (Müller's solution or Erlicki's will do), or in 0.5 per cent. solution of chromic acid, or in Merkel's solution. The treatment with bichromate solutions has the great advantage of highly facilitating staining with carmine or hæmatoxylin. Max Schultze recommended washing, and mounting permanently in acetate of potash; Fol, treatment with a weak solution of carbonate of ammonia. But the best plan of all is to properly bleach the preparations. See "Bleaching." This may be done by means of peroxide of hydrogen. Overton (Zeit. wiss. Mik., vii, 1890, p. 10) finds that it is completed in a few minutes in a mixture of 1 part commercial peroxide with 10 to 25 parts 70 per cent. alcohol. (The commercial peroxide, slightly acidulated with $\mathrm{HCl}$, will keep well in the dark; but the mixture with alcohol must be made fresh for use.) According to Bristol (Amer. Natural, xxvii, 1893, p. 176) the peroxide acts best in the sun. BINET (Journ. de l'Anat. et de la Physiol., xxx, 1894, p. 449) has successfully used permanganate of potash. ManN (Methods, etc., p. 83) takes a solution of 0.25 per cent., and treats the browned tissues with 1 part of saturated solution of sulphurous acid to 9 of normal salt solution. . . MöNCKEBERG and BetHe (Arch. Mik. Anat., liv, 1899, p. 135) have succeeded in satisfactorily restoring the staining susceptibility of osmium material by means of sulphurous acid (obtained by adding hydrochloric acid to bisulphite of sodium, 2 to 4 drops of the acid added to 10 c.c. of a 2 per cent. solution of the salt).

For (Lehrb., p. 174) recommends a weak aqueous solution of ferricyanide of potassium.

I find the sulphate of iron solution used in Benda's hæmatoxylin stain has a marked bleaching effect, and so also, though in a less degree, the iron alum of Heidenhain's process.

Altmann (Die Elementarorganismen, pp. 33 and 35) puts sections overnight into gold chloride of 2 per cent., and reduces in formic acid in the sun, and removes the gold by iodised alcohol.

But perhaps the best plan is the chlorine method of MAYER, or his magnesium peroxide, for both of which see "Bleaching."

The same stains recommended for objects fixed by the vapours will be found useful here. For sections, of course, in both cases safranin and other anilin stains may be employed with advantage, as may hæmatoxylin. 
In general, osmic acid, especially when used in the form of vapour, fixes protoplasm very faithfully, nuclei badly. It is pre-eminently a fixative of the hyaloplasm or enchylema of cells. The penetrating power of the solution is very low, so that if any but very small pieces of tissue be taken the outer layers become over-fixed before the reagent has penetrated to the deeper layers. Over-fixed cells have a certain homogeneous, glassy, or colloid look, and are unfit for study, and attention should be confined to cells four or five layers deeper down, which will generally be found to present the required intensity of fixation. In these the fixation is admirable, with no shrinkage and next to no swelling of anything.

36. The Osmium Tetroxide Reaction.-ManN believed that during the osmic reaction on fatty substances the $\mathrm{OsO}_{4}$ was reduced to osmium tetra-hydroxide $\mathrm{Os}(\mathrm{OH})_{4}$. Other observers have assumed the reaction to be the reduction of the $\mathrm{OsO}_{4}$ to some lower oxide. The matter has recently been reviewed by Professor J. R. Partington and Mr. D. B. Huntingford, who find that the reduced substance is a hydrated form of $\mathrm{OsO}_{2}$, possibly $\mathrm{OsO}_{2}, 5 \mathrm{H}_{2} \mathrm{O}$, or $\mathrm{OsO}_{2}, 6 \mathrm{H}_{2} \mathrm{O}$. In all probability, Professor Partington informs me, the amount of water is not definite. (See also $\S 768$ on "Fat.")

37. Osmic Mixtures.-Nicolas (Intern. Monatsschr., 1891, p. 3) adds $\frac{1}{2}$ per cent. of osmic acid to nitric acid of 3 per cent. I have employed a similar mixture and not had good results, though I find the mixture keeps perfectly.

Busch (Neurol. Centralb., xvii, 1898, No. 10, p. 476; Zeit. wiss. Mik., $\mathrm{xv}, \mathrm{p}$. 373) finds that the penetration of osmic acid is enhanced by combining it with iodate of sodium, which by hindering its too rapid decomposition in the tissues ensures a more energetic action in the deeper layers. He adds 3 per cent. of sodium iodate to a 1 per cent. solution of osmic acid.

Unna (Monatschr. prakt. Derm., xxvi, 1898, p. 602) adds 1 per cent. of alum to a 1 per cent. solution. For some mixtures of Kolossow, see 5 th ed., or Zeit. wiss. Mikr., v, 1888, p. 51, and ix, 1892, p. 39. See also under "Cytology," $\$ 677$.

38. Chromic Acid.-Chromic anhydride, $\mathrm{CrO}_{3}$, is found in commerce in the form of red crystals that dissolve readily in water, forming chromic acid, $\mathrm{H}_{2} \mathrm{CrO}_{4}$. These crystals are very deliquescent, and it is therefore well to keep the acid in stock in the shape of a 1 per cent. solution. Care must be taken not to allow the crystals to be contaminated by organic matter, in the presence of which the anhydride is readily reduced into sesquioxide.

Chromic acid is generally employed in aqueous solution. Some 
observers (Klein; Urban Pritchard; Perényi) have recommended alcoholic solutions; but this is evidently irrational. For in the presence of alcohol chromic acid has a great tendency to become reduced to chromous oxide or sesquioxide, neither of which appears to have any fixing power.

The most useful strengths in which it is employed in aqueous solution are from $0 \cdot 1$ to 1.0 per cent. for a period of immersion of a few hours (structure of cells and ova). For nerve tissues weaker solutions are taken, $\frac{1}{50}$ to $\frac{1}{8}$ per cent. for a few hours. Stronger solutions, such as 5 per cent., should only be allowed to act for a few seconds.

Washing out.-The general practice is to wash out very thoroughly with water (by preference running water, for many hours) before bringing into alcohol or any staining liquid. For if the objects are put direct into alcohol it is found that after a short time a fine precipitate is thrown down on the surface of the preparations, thus forming an obstacle to the further penetration of the alcohol. Previous washing by water does not prevent the formation of this precipitate, and changing the alcohol does not prevent it from forming again and again. It has, however, been found by HaNs VIRCHOw (Arch. Mik. Anat., xxiv, 1885, p. 117) that it may be entirely prevented by simply keeping the preparations in the dark. The alcohol becomes yellow as usual (and should be changed as often as this takes place), but no precipitate is formed. If this precaution be taken, previous washing with water may be omitted, or at all events greatly abridged.

MAYER (Grundzüge, 1st ed., p. 28) proceeds as follows:-The fixed material is merely rinsed in water and brought direct into 70 per cent. alcohol. It is washed therein, preferably in the dark, until after several changes the alcohol remains colourless. It is then either passed through higher alcohols and imbedded in paraffin, the chromous oxide (or whatever chrome compound it may be that is present in the tissues) being removed from the sections after these are made; or this necessary removal is performed at once. If this be preferred, the material is broughtinto sulphuric acid diluted with twenty volumes of water, or into nitric acid diluted with ten volumes of water. After at most a few hours therein, it will have become of a light greyish green, and on removal of the acid may be readily stained. If it be preferred to treat the sections, it is sufficient to put them into the usual hydrochloric acid alcohol (4 to 6 drops of $\mathrm{HCl}$ to 100 c.c. of 70 per cent. alcohol), in which after a short time they become almost white, and will stain excellently with any of the 
usual stains. So also EdINGER (Zeit. wiss. Mik., i, 1884, p. 126 ; nitric acid 1 : 20 for five minutes). UnNa (Arch. Mik. Anat., xxx, 1887 , p. 47) holds that the chrome is present in the tissues in the form of chromic chromate, and removes it by treatment with peroxide of hydrogen. Overton (Zeit. wiss. Mik., vii, 1890, p. 9) employs a weak solution of sulphurous acid, which converts it into a sulphate. See also the directions for bleaching osmic acid preparations, § 35 .

Tissues that have been fixed in chromic acid may be stained in aqueous solutions, as water does not have an injurious effect on them.

The best stain for chromic material that has not been treated by Mayer's special process, or by a similar one, is hæmatoxylin, or, for sections, the basic tar colours.

Chromic acid is not a very penetrating reagent, and for this reason, as well as for others, is now seldom used pure for fixing.

For prolonged hardening it is generally employed in strengths of $\frac{1}{5}$ to $\frac{1}{2}$ per cent., the immersion lasting a few days or a few weeks, according to the size and nature of the object. Mucous membrane, for instance, will harden satisfactorily in a few days ; brain will require some six weeks.

Large quantities of the solution must be taken (at least 200 grammes for a piece of tissue of 1 centimetre cube-Ranvier).

In order to obtain the best results you should not employ portions of tissue of more than an inch cube. For a human spinal cord you should take 2 litres of solution, and change it for fresh after a few days. Six weeks or two months are necessary to complete the hardening.

I think it is frequently useful to add a little glycerin; there is less brittleness.

The solution should be taken weak at first, and the strength increased after a time. The objects should be removed from the solution as soon as they have acquired the desired consistency, as if left too long they will become brittle. They may be preserved till wanted in alcohol (95 per cent.). It is well to wash them out in water for twenty-four or forty-eight hours before putting them into the alcohol. After a time they generally become green in the alcohol. They may be bleached if desired.

Chromic acid is a most powerful and rapid hardening agent. (By it you may obtain in a few days a degree of hardening that you would hardly obtain in as many weeks with bichromate, for instance.) It has the defect of a great tendency to cause brittleness. 
39. Chromo-acetic Acid (Flemming, Zellsbz., Kern. u. Zellth., p. 382).

Chromic acid

Acetic acid
- 0.2 to $0 \cdot 25$ per cent.

- $0 \cdot 1$ per cent. in water.

Flemming found this the best reagent for the study of the achromatic elements of karyokinesis. You can stain with hæmatoxylin, or the basic anilin dyes.

The following has been recommended for Annelids by EHLERs :To 100 c.c. of chromic acid of 0.5 to 1 per cent. add from 1 to 5 drops of glacial acetic acid. The acetic acid is said to be sufficient to counteract any shrinkage due to the chromic acid. Fix overnight, wash out several hours in water.

Similar to this is the "chromo-acetic acid, No. 1," of Lo BIANco (Mitth. Zool. Stat. Neapel, ix, 1890, p. 443), viz. 1 part 50 per cent. acetic acid and 20 parts 1 per cent. chromic acid, which is found very useful for fixing marine animals.

40. Chromo-formic Acid (RABL, Morph. Jahrb., x, 1884, pp. 215, 216).-Four or 5 drops of concentrated formic acid are added to 200 c.c. of 0.33 per cent. chromic acid solution. The mixture must be freshly prepared at the instant of using. Fix for twelve to twenty-four hours, wash out with water. Used by Rabl for the study of karyokinesis.

41. Chromo-aceto-osmic Acid (Flemming, Zellsubstanz, Kern und Zelltheilung, 1882, p. 381). FIRST or WEAK formula :

Chromic acid

Osmic acid .

Glacial acetic acid
$0 \cdot 25$ per cent. )

. $0 \cdot 1 \quad$ in water.

$0 \cdot 1$

Meves (Encycl. mikr. Techn., 1, p. 475) sometimes adds 1 per cent. of sodium chloride.

Fol (Lehrb. d. vergl. mik. Anat., 1884, p. 100) recommends the following variant :

1 per cent. chromic acid

1 per cent. osmic acid

2 per cent. acetic acid

Water

-that is to say, a mixture weaker in osmium than Flemming's.

A mixture still weaker than this in osmium, viz. with 1 vol. osmic acid solution, instead of 2 , has been recommended by CorI (Zeit. wiss. Mik., vi, 1890, p. 441).

SECONd or Strong formula (Zeit. wiss. Mik., J, 1884, p. 349):

1 per cent. chromic acid . . . . 15 parts.

2 per cent. osmic acid . . . . 4 "

Glacial acetic acid . . . . . 1 part.

If this mixture be kept in stock in large quantities, it may go bad, on account of the large proportion of organic acid contained in it. 
I therefore recommend that the osmic and chromic acid be kept ready mixed in the proportions given, and 5 per cent. of acetic acid added at the moment of using.

Weaker Formula.-More recently, Flemming has been making up the mixture with only 2 parts of the osmic acid instead of 4 , and has spoken of this modification as "weaker osmium mixture" (Meves, in Encycl. Mikr. Techn., p. 476).

Meves (loc. cit.) takes for delicate objects 15 parts of chromic acid of only 0.5 per cent., 2 or 4 of osmic acid of 2 per cent., and 1 of acetic acid, and thus gets less shrinkage.

Under "Cytology" Sections, §678, see Benda and Gatenby modifications.

PoDwyssozki recommends (for glands especially) the following modification :

1 per cent. $\mathrm{CrO}_{3}$ dissolved in 0.5 per cent. solution of corrosive sublimate

2 per cent. osmic acid solution

Glacial acetic acid

15 c.c.

4 c.c.

6 to 8 drops.

The sublimate is said to augment the penetration of the osmium, but is unfavourable to staining (ZIEGLER's Beiträge z. path. Anat., i, 1886 ; Zeit. wiss. Mik., iii, 1886, p. 405).

The first or weak liquid is the better for very small objects, the second or strong one for larger ones, as it has better penetration. These liquids may be allowed to act for many hours or days, or according to some workers even weeks or months; but this exaggerated fixation is clearly only justifiable in very special cases, if at all. For chromosome studies some workers fix for only one hour. Others recommend cooling the Flemming on ice before using. Wash out very thoroughly in water (running, twenty-four hours), or treat as directed for chromic acid, $\S 38$. Stain with alum hæmatoxylin if you wish to stain in toto (staining in this way with other reagents is possible, but. difficult). Stain sections with safranin or other basic coal-tar colour, or with iron hæmatoxylin.

For fixing with the strong mixture you need only take a bulk of liquid of some 4 times the volume of the objects (but with the weak mixture the proportion should be increased). Both of them are first-rate fixatives of cellular structures, both as regards their preservation and as regards their optical differentiation. But they must be properly used, and not applied to objects for which they are not fitted. For instance, their power of penetration is extremely bad; they will not fix properly, even in a loose-celled tissue, through more than a layer of about five cells thick. They are therefore suitable 
only for very small objects or for very small pieces of tissue, such as suffice for cytological or histological work. The strong liquid especially has not the character of a general reagent. As a matter of fact it was recommended by FLemming in the first instance merely for a very special purpose, the hunting for karyokinetic figures, and not for general purposes. It is still very much used, but in my opinion unadvisedly. In most cases, Bouin's picroformol will do all that it is intended to do, without its disadvantages.

It may be used for prolonged hardening, e.g. of small pieces of nervous tissue, and is very good for that purpose.

Fat is blackened (or browned) by it. See $\$ 35$. Chromatin is mordanted by it for basic anilin dyes, enabling them to give peculiarly sharp and powerful stains.

42. Osmic Acid and Bichromate--Altmann (Die Elementarorganismen, Leipzig, 1890) takes for his "bioblasts" a mixture of equal parts of 5 per cent. solution of bichromate of potash and 2 per cent. solution of osmic acid. The bichromate ought not to contain any free chromic acid. Refer to $\S 680$.

Lo Bianco (Mitth. Zool. Stat. Neapel, ix, 1890, p. 443) employs for marine animals a mixture of 100 c.c. of 5 per cent. solution of bichromate and 2 c.c. of 1 per cent. osmic acid.

Hoenu (Arch. Anat. Phys., Anat. Abth., 1896, p. 31) recommends a mixture of 80 c.c. of 3 per cent. bichromate, 20 c.c. of 1 per cent. osmic acid, and 2 c.c. of glacial acetic acid.

43. Bichromate-chromic-osmic Acid.-ChAMPy (Arch. de Zool. Expér., 1913).-Mixture of 7 parts of 3 per cent. bichromate of potash, 7 parts of 1 per cent. chromic acid, 4 parts of 2 per cent. osmium tetroxide.

This mixture keeps well. Fix for from six to twenty-four hours. Wash out in running water about the same time.

You can stain in iron hæmatoxylin, or less well in Altmann or BENDA. See $\S 681$ for a description of mordanting after Champy's fluid. This fluid is extremely useful, and I nearly always use it in addition to FlemMing.

44. Osmic, Bichromate, and Platinic Mixture (Lindsay Johnson's Mixture).--Latest formula, 1895, communicated by Dr. Lindsay Johnson :

Bichromate of potash $(2.5$ per cent.) .

70 parts.

Osmic acid (2 per.cent.)

10 ,

Platinum chloride ( 1 per cent.).

Acetic or formic acid 
Henneguy, who has worked a great deal with this reagent, and recommends it highly, says (Lecons sur la Cellule, p. 61) that it is well only to add the acetic or formic acid just before using, as it frequently reduces the osmium and platinum very rapidly and energetically. He finds that it contracts the more spongy sorts of protoplasm less than mixture of Flemming. I think highly of itfor certain objects. Twelve hours is probably the optimum time for fixation. Wash out in water.

45. Platino-aceto-osmic Acid (Hermann's) Solution (Arch. Mik. Anat., xxxiv, 1889, p. 58).-One per cent. platinum chloride 15 parts, glacial acetic acid 1 part, and 2 per cent, osmic acid either 4 parts or only 2 parts. Hermann found that protoplasm structures are thus better preserved than with the chromic mixture. As with Flemming, the optimum time is from twelve to sixteen hours. Wash out at least three hours in running water.

The after-treatment and staining should be the same as for objects treated with Flemming's solution. Renger (Zeit. wiss. Zool., lxiii, 1898, p. 454) washes out for half an hour to an hour with saturated aqueous sol. of picric acid, which he thinks facilitates the staining, especially of nuclei.

The action of this fixative is, roughly, similar to that of Flemming's. Like Flemming's, it mordants chromatin for staining with "basic" colours, with which it affords equally fine nuclear stains. But, owing to the platinum in it, it diminishes more than Flemming's the colorability of tissues with " acid" colours, so that. it is extremely difficult to obtain good plasma stains after its action. It causes a notable shrinkage in chromatin. It gives a full fixation of cytoplasm, to which it gives a much more fine-grained aspect than liquid of Flemming does.

Leaving out the acetic acid, the solution may be used for mitochondria as in $\$ 679$.

46. Rawitz (Zeit. wiss. Mikr., xxv, 1909, p. 386) takes 4 parts of Kahlbaum's Phospho-Tungstic acid, 5 of alcohol, and 1 of acetic acid, added just beiore use, fixes for twenty-four hours, and washes out the sections before staining with water containing a little calcium acetate.

47. Nitric Acid (Altmann, Arch. Anat. Phys., 1881, p. 219).Altmann employs for fixing embryos dilute nitric acid, containing from 3 to $3 \frac{1}{2}$ per cent. pure acid. Such a solution has a sp. gr. of about 1.02 . Stronger solutions have been used, but do not give such good final results. After extensive trial I find Altmann's solution to be a secondrate reagent, giving a weak and thin fixation.

HIs (ibid., 1877, p. 115) recommended a 10 per cent. solution. 
Flemming at one time employed solutions of 40 to 50 per cent. for the ova of Invertebrates.

Tellyesniczky (Arch. mik. Anat., lii, 2, 1898, p. 222) thinks that "for general cell-fixing " the proper strength is 2 to $2 \frac{1}{2}$ per cent., as stronger grades act too energetically on the superficial layers.

MAYER has had good results with 5 per cent. solution.

Nitric acid has the valuable property of hardening yolk without making it brittle.

Pure water should in no case be used for washing out ; the prepara. tions should be brought direct into alcohol. Some persons take absolute, but I should say 70 per cent. is more generally indicated. Rabl has employed a 1 or 2 per cent. solution of alum.

For prolonged hardening, strengths of from 3 to 10 per cent. are sometimes employed. A strength of 12 per cent., allowed to act for two or thrse weeks, is said to afford very tough preparations of the encephalon.

BENdA (Verh. Anat. Ges., 1888 ; Ergeb. d. Anat., i, 1891, p. 7) fixes for twenty-four to forty-eight hours in 10 per cent. nitric acid, and then hardens in bichromate of potash.

Fol's Mixture (verbally communicated to me).-Three vols. of nitric acid, with 97 vols. of 70 per cent. alcohol.

48. Chromo-nitric Acid (Perényi's formula, Zool. Anzeig., v, 1882, p. 459) :

4 parts 10 per cent. nitric acid.

3 parts alcohol.

3 parts 0.5 per cent. chromic acid.

Fix for four to five hours and pass into alcohol of 70 per cent.

This mixture has been criticised (see previous editions) as irrational, the alcohol reducing the chromic acid and itself becoming etherised by the nitric acid. Some workers reject it, especially for ova, for which it is specially intended. But others speak highly of it. I myself have used it extensively for preparing objects for dissection and museum specimens, and found it admirable for these purposes. But preparations made to test its value from a eytological point of view have given me only second-rate results. It is now little used.

49. Chromic Acid and Platinum Chloride (Merkel's Macula lutea des Menschen, Leipzig, 1870 , p. 19).-Equal volumes of 1.400 solution of chromic acid and 1.400 solution of platinum chloride. Objects should remain in it for several hours or even days. After washing out with alcohol of 50 to 70 per cent., objects stain excellently. If objects that have been fixed by osmic acid be put into it for some hours, blackening is said to be effectually prevented.

This is an excellent hardening medium for delicate objects. Merkel allowed from three to four days for the action of the fluid. for the retina ; for Annelids Eisig employs an immersion of three to five hours, and transfers to 70 per cent. alcohol; for small leeches 
Whitman finds one hour sufficient, and transfers to 50 per cent. alcohol.

A similar mixture, with the addition of $0 \cdot 25$ to $0 \cdot 1$ per cent. of acetic acid, is recommended by BRAss for Protozoa; and LAVDowsky has used for nuclei a mixture of 10 parts of 1 per cent. chromic acid, 5 of 1 per cent. platinum chloride, and 100 of 5 per cent. acetic acid.

Whitman recommends for the hardening of pelagic fish ova a stronger mixture (due, I believe, to Eisig), viz.-

$0 \cdot 25$ per cent. solution of platinum chloride . . 1 vol.

1 per cent. solution of chromic acid . . . 1 ,

The ova to remain in it one or two days (Whitman, Methods in Micro. Anat., p. 153).

\section{Salts.}

50. Chromates.-The chromates are amongst the oldest and best tried of hardening agents. The bichromate of potash especially was at one time universally employed for hardening all sorts of tissues.

Flemming (Arch. mik. Anat., xviii, 1880, p. 352) pointed out that though it preserves cytoplasm well it causes chromatin to swell, and therefore should not be employed for the study of nuclei. But, duly corrected with acetic acid, it affords a correct and fine fixation of nuclei ; whilst preserving hyaloplasm and its inclusions, secretions, etc., much better than chromic acid.

For an elaborate study of the action of chrome salts on nucleus and cytoplasm, see Burckhardt, La Cellule, xii, 1897, p. 335. He finds that the bichromates of sodium, ammonium, magnesium, strontium, and zinc have the same destructive action on nuclei that the bichromate of potassium has ; but that the bichromates of barium, calcium, and copper have not. He concludes that acetic acid ought always to be added, not only to ensure the correct fixation of nuclei, but also to enhance penetration and the good preservation of cytoplasm.

The following is recommended by him as a good combination for the fixation both of eytoplasm and nucleus :

Bichromate of barium, 4 per cent. solution . . 60 vols.

Bichromate of potassium, 5 per cent. solution . . 30 ,

Glacial acetic acid . . . . . . 5 ,

(Instead of the barium you may take 4 per cent. solution of bichromate of calcium, or 6 per cent. solution of bichromate of copper.)

For the demonstration of the achromatic figure of cell division he recommends-

Chromic acid, 1 per cent. solution . . . . 60 vols.

Bichromate of potassium, 5 per cent. solution . . 30 ,

Glacial acetic acid . . . . . . . 5 , 
51. Bichromate of Potash.-Perhaps the most important of all known hardening agents, sensu stricto. It hardens slowly, much more so than chromic acid, but it gives an incomparably better consistency to the tissues. They may remain almost indefinitely exposed to its action without much hurt.

The strength of the solutions employed is from 2 to 5 per cent. As with chromic acid, it is extremely important to begin with weak solutions and proceed gradually to stronger ones. About three weeks will be necessary for hardening a sheep's eye in solutions gradually raised from 2 to 4 per cent. Spinal cord requires from three to six weeks, a brain at least as many months.

After hardening, the objects should be well soaked out in water before being put into alcohol, or be treated as directed for chromic acid, $\S 38$. They had better be kept in the dark when in alcohol. See § 38. (Вӧнм and OpPeL [T'aschenbuch, 3 Auf., 1896, p. 22] fix in the dark.) If you wish to have a good stain with carmine you should not put the objects into alcohol at all, even for a second, until they have been stained.

You may stain either with carmine or hæmatoxylin, as well as with tar colours.

Bichromate objects have an ugly yellow colour which cannot be removed by mere soaking in water. It is said that it can be removed by washing for a few minutes in a 1 per cent. solution of chloral hydrate.

Prof. GILson writes me that alcoholic solution of sulphurous anhydride $\left(\mathrm{SO}_{2}\right)$ is very convenient for the rapid decoloration of bichromate objects. A few drops suffice. See also $\S 38$, and "Bleaching."

To facilitate staining with hæmatoxylin, WolfF (Zeit. wiss. Mik., xv, 3,1899, p. 311) first stains in Boehmer's hæmatoxylin for twenty-four hours, and then for a few minutes in the same hæmatoxylin to which has been added 1 drop per watch-glassful of 5 per cent. solution of oxalic acid.

The simple aqueous solution of bichromate is hardly to be recommended as a fixing agent, because not only does it not preserve nuclei properly, but also because it penetrates very slowly. The first of these defects may be overcome entirely, the second to some extent by addition of acetic acid; whence the liquid of TeluyesNICZKY, next $\S$.

52. Acetic Bichromate (Tellyesniczky, Arch. mik. Anat., lii, 1889, p. 242) :

Bichromate

Glacial acetic acid .

Water

3 grms.

5 c.c.

100 ,

Smaller objects to remain in the fluid for one or two days, larger 
ones longer. Wash well in plenty of water, and pass through alcohols of increasing strength, beginning with 15 per cent.

Mixtures of bichromate with osmic acid have been given above, $\$ \$ 42$, 43 and 44.

53. MÜLleR's Solution.-

Bichromate of potash

Sulphate of soda

Water

The duration of the reaction is about the same as with the simple solution of chromic salt.

Recent authors find the action of this liquid to be identical with that of plain bichromate, and doubt whether the sulphate in it has any effect whatever as regards its hardening properties. Fol says that for mammalian embryos, for which it has been recommended, it is worthless.

54. ERLicki's Solution (Warschauer med. Zeit., xxii, Nos. 15 and 18 ; Progrès Médical, 1897, No. 31):

Bichromate of potash

Sulphate of copper .

Water

Here the addition of the cupric sulphate is intelligible, for this salt is itself a hardening agent of some energy. As a matter of fact, "Erlicki" hardens very much more rapidly than either simple bichromate or Müller's solution. A spinal cord may be hardened in it in four days at the temperature of an incubator, and in ten days at the normal temperature (Fol, Lehrb. d. vergl. mik. Anat., p. 106). Human embryos of several months may be conveniently hardened in it.

Nerve-centres that have been hardened in Erlicki's fluid frequently contain dark spots with irregular prolongations, simulating ganglioncells. These are now known to consist of precipitates formed by the fluid. They may be removed by washing with hot water, or with water slightly acidified with hydrochloric acid, or by treating the specimens with 0.5 per cent. chromic acid before putting them into alcohol (Tschisch, Virchow's Arch., Bd. xevii, p. 173 ; EDINGER, Zeit. wiss. Mik., ii, p. 245 ; Loewenthal, Rev. méd. de la Suisse romande, 6 me année, i, p. 20).

55. Kultschitzky's Solution (Zeit. wiss. Mik., iv, 1887, p. 348).-A saturated solution of bichromate of potash and sulphate of copper in 50 per cent. alcohol, to which is added at the instant of using a little acetic acid, 5 or 6 drops per 100 c.c.

To make the solution, add the finely powdered salts to the alcohol in 
excess, and leave them together in total darkness, for twenty-four hours.

Fix for twelve to twenty-four hours in the dark. Then treat with strong alcohol for twelve to twenty-four hours.

56. Dekhurzen's Liquids ( $C . R$. Acad. Sci., exxxvii, 1903, pp. 415 and 445 ).-(A) 250 c.c. of 2.5 per cent. sol. of bichromate in sea-water, 25 c.c. of $6 \cdot 3$ per cent. nitric acid, and 54 c.c. of 2 per cent. osmic acid. For general use with marine animals:

(в) 173.1 c.c. of the bichromate sol. and 26.9 of 2 per cent. sol. of osmic acid. For objects containing calcareous elements that it is desired to preserve.

These liquids are stated to be isotonic with sea-water.

57. Bichromate and Sublimate (Kultschitzkx, Arch. f. mik. Anat., xlix, 1897, p. 8). - Two parts bichromate, $\frac{1}{4}$ part corrosive sublimate, 50 parts 2 per cent. acetic acid, and 50 parts 96 per cent. alcohol. The mixture should be filtered after twenty-four hours. Tissues of vertebrates may remain in it for four to six days. LAVDowsKY (Zeit. wiss. Mik., xvii, 1900, p. 301) takes 500 c.c. of 1 per cent. acetic acid, 20 to 25 grms. bichromate, and 5 to 10 c.e. saturated solution of sublimate in water.

58. Bichromate of Ammonia.-This salt is in considerable favour for hardening. Its action is very similar to that of the potassium salt. Fol says that it penetrates somewhat more rapidly, and hardens somewhat more slowly. It should be employed in somewhat stronger solutions, up to 5 per cent.

59. Neutral Chromate of Ammonia is preferred by some. It is used in the same strength as the bichromate. Klein has recommended it for intestine, which it hardens, in 5 per cent. solution, in twenty-four hours.

60. Bichromate of Calcium.-Sonnenbrodt (Arch. mikr. Anat., ]xxii, 1908, p. 416) fixes ovaries of Gallus in 20 parts of 2 per cent. sol. of calcium bichromate with 10 of 2 per cent. sol. of sublimate and 1 of acetic acid.

61. Bichromates and Alcohol.-Mixtures of bichromate of potash or ammonia with alcohol may be employed, and have a more rapid action than the aqueous solution. Thus HamiLton takes for hardening brain a mixture of 1 part methylated spirits with 3 parts of solution of Müller; see also KuLtschitzky's Mixture, ante, § 55). Preparations should be kept in the dark during the process of hardening in these mixtures.

62. Sulphurous Acid.-Waddington (Journ. Roy. Mic. Soc., 1883, p. 185) uses a saturated solution of sulphurous acid in alcohol for fixing infusoria. Overton (Zeit. wiss. Mik., vii, 1890, p. 9) uses the vapours of an aqueous solution for fixing algæ. 


\section{CHAPTER V.}

FIXING AND HARDENING AGEN'TS-CHLORIDES, ORGANIC ACIDS, AND OTHERS.

\section{Chlorides.}

63. Bichloride of Mercury (Corrosive Sublimate)-Corrosive sublimate is soluble in about 16 parts of cold and 3 of boiling distilled water. It is more soluble in alcohol $(1: 3)$ or in ether $(1: 4)$ than in water. Its solubility in all these menstrua is augmented by the addition of hydrochloric acid, ammonious chloride, or camphor. With sodium chloride it forms a more easily soluble double salt; hence sea-water may dissolve over 15 per cent.

The simple aqueous solutions should always be made with distilled -not spring-water. The $\mathrm{HgCl}_{2}$ in them is partly split up by hydrolysis into $\mathrm{Cl}, \mathrm{H}$, and $(\mathrm{HgCl})_{2}$, or $\mathrm{HgClOH}$ (see Chem. Centralb., 1904, i, p. 571 ; the statements of MANN [Methods, pp. 22, 77] are incorrect). These solutions should give an acid reaction with litmus paper, whilst those made with strong sodium chloride solution are neutral.

For fixing, corrosive sublimate may be used pure; but in most cases a finer fixation will be obtained if it be acidified with acetic acid, say about 1 per cent. of the glacial acid. I find that a saturated solution in 5 per cent. glacial acetic acid is a very good formula for marine animals ; for others I should take the acid weaker. KaISER's solution consists of 10 grms. sublimate, 3 c.c. glacial acetic acid, and 300 c.c. distilled water (from Zeit. wiss. Mik., xi, p. 378). VAN BENEDEN has used a saturated solution in 25 per cent. acetic acid, and Lo Bianco (Mitth. Zool. Stat. Neapel, ix, 1890, p. 443). a mixture of 2 parts saturated solution with 1 part of 49 per cent. acetic acid.

It is sometimes advisable to take the most concentrated solution obtainable. For some very contractile forms (coral polypes, Planaria), a concentrated solution in warm or even boiling water should be employed. For Arthropoda alcoholic solutions are frequently indicated. Delicate objects, however, may require treatment with weak solutions. 
Objects should in all cases be removed from the fixing bath as soon as fixed, that is, as soon as they are seen to have become opaque throughout, which may be in a few minutes or even seconds.

Wash out with water or alcohol. Alcohol is almost always preferable. Alcohol of about 70 per cent. may be taken, and (MAYER, Intern. Monatsschr. Anat. Phys., iv, 1887, p. 43) a little tincture of iodine may be added to the liquid, either alcohol or water, used for washing, enough to make it of a good port-wine colour, and the mixture be changed until it no longer becomes discoloured by the objects/Apáthy (Mikrotechnik, p. 148) takes a 0.5 per cent. solution of iodine in strong alcohol, leaves the objects in it (suspended) until they have become of about the colour of the solution, and then washes for twenty-four hours in pure alcohol.

In obstinate cases solution of iodine in iodide of potassium (e.g. Lugol's) may be taken. MaYer (Zeit. wiss. Mik., xiv, 1897, p. 28) makes it by dissolving 5 grms. of iodide of potassium in 5 e.c. of distilled water and mixing this with a solution of $0.5 \mathrm{grm}$. of iodine in 45 c.c. of 90 per cent. alcohol, but seldom uses the mixture concentrated, merely adding as much of it as is required to the alcohol or water containing the objects. The important point is, that the iodine and iodide be employed together. The iodine may be washed out in obstinate cases with magnesia water. Similarly APÁTHY (Mitth. Zool. Stat. Neapel, xii, 1897 , pp. 729,730 ).

It has been objected to this process that iodine in potassic iodide precipitates corrosive sublimate instead of dissólving it. That is true, but the precipitate is soluble in excess of the precipitant.

The iodide of potassium process should be employed with care, for the iodide may partly redissolve the precipitated compounds formed by the sublimate with the albuminoids, ete., of the tissues, and it may be well not to begin adding the iodine till the objects have been brought into fairly strong alcohol, 70 or 80 per cent.

It is important that the sublimate be thoroughly removed from the tissues, otherwise they become brittle, and will not stain so well. They will also become brittle if they are kept long in alcohol.

It may happen that if the extraction of the excess of sublimate from the tissues in bulk has been insufficient, crystals may form in the sections after they have been mounted in balsam. This may easily be prevented by treating the sections themselves with tincture of iodine for a quarter of an hour before mounting. Some workers hold that this does away with the necessity of treating the tissues in bulk with iodine, which is frequently a very long process. Thus, MaNN (Zeit. wiss. Mik., xi, 1894, p. 479) prefers treating the sections rather than the tissues in bulk, on the ground that the iodine makes them 'soft, so that they shrink on coming into paraffin. SCHAPER 
(Anat. Anz., xiii, 1897, p. 463), however, has shown that neglect to extract the sublimate from the.tissues in bulk may give birth to serious artifacts, which appear to arise during the imbedding process. So also Loyez (Arch. Anat. Micr., viii, 1905, p. 71). Heidenuain (Zeit. wiss. Mik., xxv, 1909, p. 398) removes the iodine from sections by means of sodium thiosulphate.

You may stain in any way you like. Carmine stains are peculiarly brilliant after sublimate.

The solutions must not be touched with iron or steel, as these produce precipitates that may hurt the preparations. To manipulate the objects, wood, glass or platinum may be used ; for dissecting them, hedgehog spines, or quill pens, or cactus spines.

When properly employed, sublimate is for general work undoubtedly a most useful fixing agent. It is applicable to most classes of objects. It is perhaps less applicable, in the pure form, to Arthropods, as it possesses no great power of penetrating chitin. For cytological work it is, according to my experience, not to be trusted, and only to be recommended where more precise fixing agents are counter-indicated by reason of their lack of penetration, or the like. Amongst other defects it has that of frequently causing very serious shrinkage of cells.

64. Sublimate with Salt.-A solution containing 5 grms. sublimate, $0.5 \mathrm{grm}$. sodium chloride, and 100 c.c. water has been quoted as "solution of GaUle."

A $\frac{1}{2}$ per cent. aqueous solution of sodium chloride saturated whilst hot with sublimate was much recommended by Heidennain (Festschrift f. Koelliker, 1892, p. 109).

The addition of sodium chloride allows a stronger solution to be obtained than can be made with pure water, and also, it is stated, enhances the penetration of the sublimate. But the fixation-precipitates (§29) formed by the double salt are (according to SPULER, Eneyl. mik. Technik., p. 1274) for the most part soluble in water, thus giving rise to imperfect preservation.

Concentrated (i.e. over 20 per cent.) solution in sea-water is recommended for some marine animals.

Stoelzner (Zeit. wiss. Mikr., xxiii, 1906, p. 25) recommends saturated solution of sublimate in sugar solution of $4 \frac{1}{2}$ per cent., as isotonic (for warm-blooded animals).

Liquid of Lang (Zool. Anzeiger, 1878, i, p. 14).-For Plunaria.Distilled water . $\quad . \quad+\quad . \quad 100$ parts.

Chloride of sodium . . . . . 6 to 10 ,"

Acetic acid . . . . 6 to 8 ,

Bichloride of mercury - ․ . - . 3 to 12 "

(Alum, in some cases . . . . . $\frac{1}{2}$ part.)

65. Alcoholic Solutions.-ApátHy (Mikrotechnik, p. 111) recom- 
mends a solution of 3 to 4 grms. of sublimate and $0.5 \mathrm{grm}$. sodium chloride in 100 c.c. of 50 per cent. alcohol for general purposes.

OHLMacher (Journ. Exper. Medicine, ii, 6, 1897, p. 671) takesAbsolute alcohol . . . . . 80 parts. Chloroform . . . . . . 15 , Glacial acetic acid . . . . . 5 , Sublimate to saturation (about 20 per cent.).

"Ordinary pieces" of tissue are sufficiently fixed in fifteen to thirty minutes. Entire human cerebral hemispheres, subdivided by Meynert's section, take eighteen to twenty-four hours.

For liquids containing a much higher proportion of acetic acid, see Acetic Alcohol.

66. Acetone Solution.-Hesd (Arch. Anat. Phys., Anat. Abth., 1897, p. 227) fixes nerve-tissue in a 1 per cent. solution of sublimate in 40 per cent. acetone, and washes out through increasingly concentrated grades of acetone.

67. Phenol Solution.-Pappenherm (Arch. Path. Anat., elvii, 1899, p. 23) shakes up carbolic acid with aqueous sublimate solution and filters.

68. Ciaccio (Arch. Ital. Anat. Embr., vi, 1907, p. 486) has an irrational mixture of sublimate, iodine, and formol.

69. Mercuro-nitric Mixtures.-Frenzel (Arch. mik. Anat., xxvi, 1885, p. 232) recommends a half-saturated solution of sublimate in 80 per cent. alcohol, to which is added nitric acid in the proportion of 1 drop to 1 or 2 c.c. Objects of the size of a pea to be fixed in it for five or ten minutes, then hardened in the same sublimate alcohol without the acid, and finally in 90 per cent. alcohol. It is said that the nitric acid renders after-treatment with iodine unnecessary.

GILson's Mixture (GILson, in litt. 1895).

Nitric acid of $46^{\circ}$ strength (this would be sp. gr. $1 \cdot 456$, or 80 per cent., nearly) 15 c.c.

Glacial acetic acid

Corrosive sublimate

60 per cent. alcohol

Distilled water

4,

20 grms.

100 c.c.

880 ,

When required for marine animals add a few crystals of iodine, which will prevent the formation of precipitates of sea salts. If in any case the preparations should show a granular precipitate, this may be removed by washing with water containing a little tincture of iodine. 
I find that it affords in general a faithful and delicate fixation, and gives to tissues an excellent consistency. Objects may remain in it for a considerable time without hurt. It has a high degree of penetration. A treatment for a few days with it will serve to remove the albumen from the ova of Batrachians. This liquid may be recommended to beginners, as it is very easy to work with. For some objects, as I found, the proportion of sublimate may be increased with advantage.

Kostanecki and Siedlecki (Arch. mik. Anat., xlviii, 1896, p. 181) take a mixture of saturated sublimate solution and 3 per cent. nitric acid in equal parts, or a mixture of equal parts of sublimate solution, 3 per cent. nitric acid, and absolute alcohol, fix for twenty-four hours, and wash out in iodine-alcohol.

Petrunkewitsch (Zool. Jahrb. Abth. Morph., xiv, 1901, p. 576) takes water 300 , absolute alcohol 200 , glacial acetic acid 90 , nitric acid 10, and sublimate to saturation. Both this and Gilson's have been much used lately.

70. Picro-sublimate Mixtures.-RABL's (Zeit. wiss. Mik., xi, 1894, p. 165).- Sublimate, saturated solution in water, 1 vol. ; a similar solution of picric acid, 1 vol.; distilled water, 2 vols. Embryos may be left in it for twelve hours, washed for two hours in water, and brought into weak alcohol.

O. vom Rath (Anat. Anz., xi, 1895, p. 268) takes cold saturated solution of picric acid, 1 part; hot saturated solution of sublimate, 1 part; glacial acetic acid, $\frac{1}{2}$ to 1 per cent. Also the same with the addition of 10 per cent. of 2 per cent. osmic acid solution.

y1. Osmio-sublimate Mixtures.-Mann's (Zeit. wiss. Mik., xi, 1894, p. 481) consists of a freshly prepared mixture of equal parts of 1 per cent. osmic acid solution and saturated solution of sublimate in normal salt solution (for nerve-centres). See "Mann-Kopsch Method," § 693.

72. Chromo-sublimate.-Lo Bianco (Mitth. Zool. Stat. Neapel, ix, 3, 1890, p. 443).-Concentrated sublimate solution, 100 parts ; 1 per cent. chromic acid, 50 parts.

ManN (Verh. Anat. Ges., 12, 1898, p. 39) takes for nerve-cells equal parts of 5 per cent. sublimate and 5 per cent. chromic acid.

93. Sublimate and Bichromate.-ZENKER's Mixture (Münchener med. Wochenschr., xxiv., 1894, p. 534 ; quoted from Mercier, Zeit. wiss. Mik., xi, 4, 1894, p. 471).-Five per cent. of sublimate and 5 per cent. of glacial acetic acid dissolved in solution of MüLLER. 
Fix for several hours or overnight, wash out with water, treat the tissues in bulk, or the sections with alcohol containing tincture of iodine. Refer to $\S 684$.

See also Retterer, Journ. Anat. Phys., xxxiii, 1897, p. 463, and xxxvii, 1901, p. 480.

If the objects be allowed to remain too long in the fluid there may be formed precipitates, which it is very difficult to remove. SPULER (Encycl. mik. Technik., 1st ed., p. 1280) says that they may be avoided by removing the objects as soon as penetrated, and completing the hardening in liquid of MüLler. I recommend this method.

Helly (Zeit. wiss. Mik., xx, 1904, p. 413) omits the acetic acid, and adds, immediately before use, 5 per cent. of formol. This is a splendid fixative for vertebrate material. Fix overnight, wash out in running water for several hours. See footnote to $\S 684$.

Maximow (ib., xxvi, 1909, p. 179) adds 10 per cent. of formol and sometimes 10 per cent. of osmic acid of 2 per cent. (fix in the dark).

Fò̀ (Quart. Journ. Mic. Sci., 1895, p. 287) takes equal parts of saturated solution of sublimate in normal salt solution, and of liquid of Müller, or 5 per cent. solution of bichromate.

Hoyer (Arch. Mikr. Anat., liv, 1899, p. 97) takes 1 part 5 per cent. sublimate and 2 of 3 per cent. bichromate.

KoHN (ib., lxx, 1907, p. 273) takes 5 parts 5 per cent. sublimate, 15 parts $3 \frac{1}{2}$ per cent. bichromate, and 1 part acetic acid.

74. Sublamin (Ethylendiamin Sulphate of Mercury) is recommended in 5 per cent. solution by KLINGMÜLLER and VeIEL (Zeit. wiss. Mikr., xxi, 1904, p. 58).

75. Platinum Chloride.-The substance used and intended by the authors who have recommended this reagent is not the true platinic chloride, or tetrachloride, $\mathrm{PtCl}_{4}$, but the compound $\mathrm{H}_{2} \mathrm{PtCl}_{6}$, that is, platinochloric, or hydro-chloro-platinic acid, by custom called platinum chloride. It occurs as brown-red crystals, easily soluble in water and very deliquescent. For this reason it had better be stocked in the form of a 10 per cent. solution, kept in the dark (weak solutions - 0.5 per cent.-may be kept in the light).

It appears that some authors have stated that they were using platinous chloride, $\mathrm{PtCl}_{2}$, but that is not possible, as this salt is not soluble in water.

RABL (Morph. Jahrb., x, 1884, p. 216) employed an aqueous solution of $1: 300$. The objects remained in it for twenty-four hours, and were then washed out with water. Well-washed preparations give good chromatin stains with the "basic" tar colours; but I find, as do others, that plasma-staining with the "acid" colours is 
rendered extremely difficult. It causes a certain shrinkage of chromatin.

It is now almost always employed in the form of mixtures. For these see $\$ \S 44,45,49,76$, as well as the mixtures given under "Picric Acid" and "Formol."

96. RABL (Zeit. wiss. Mikr., xi, 1894, p. 165) takes for embryos of vertebrates, and also for other objects, 1 vol. of 1 per cent. platinum chloride, 1 of saturated sublimate, and 2 of water.

Lenнossín (Arch. mikr. Anat., li, 1898, p. 220) takes 20 parts of 1 per cent. platinum chloride, 20 of 5 per cent. sublimate, and 1 of acetic acid.

77. Palladium Chloride (Schulze, Arch. mik. Anat., iii, 1867, p. 477).- Used by Schulze as a hardening agent in a 1:800 solution, acidified with hydrochloric acid.

CATtaneo has used it in solutions of $1: 300,1: 600$, or $1: 800$ strength, for from one to two minutes, for Infusoria.

Frenkel (Anat. Anz., viii, 1893, p. 538) recommends for connective tissue a mixture of 15 parts 1 per cent. palladium chloride, 5 parts 2 per cent. osmic acid, and a few drops of acetic acid.

78. Iridium Chloride (EISEN, Zeit. wiss. Mik., xiv, 1897, p. 195).Solution of $\frac{1}{2}$ or $\frac{1}{5}$ per cent., acidified with 1 per cent. of glacial acetic acid.

With the ovotestis of the snail, I have obtained about the worst fixation I have ever seen, but with the testis of Triton much better results.

79. Osmium Chloride (EISEN, Journ. of Morph., xvii, 1900).-Solution of $\frac{1}{2}$ to $\frac{1}{10}$ per cent. From specimens I have seen I should say it is useless.

80. Perchloride of Iron (FoL, Zeit. wiss. Zool., xxxviii, 1883, p. 491, and Lehrb. d. vergl. mik. Anat., p. 102). - Fol recommends 1 vol. of Tinct. Ferri Perchlor. B.P. diluted with 5 to 10 vols. of 70 per cent. alcohol.

The tincture diluted with 3 to 4 vols. of either alcohol or water has been recommended for fixing medullated nerve by PLATNER (Zeit. wiss. Mik., vi, 1889, p. 187).

81. Iron Alum.--Strong (Journ. comp. Neur., xiii; 1903, p. 296) fixes (and decalcifies) heads of young Acanthias in 9 parts of 5 per cent. solution of iron alum with 1 of formol, for about two weeks.

82. Chloride of Zine is sometimes used for hardening brain (see Part II). GILson ( $L a$ Cellule, vi, 1890, p. 122) has used it as a fixative for the silk glands of Lepidoptera, as follows :

Glacial acetic acid .

5 c.c.

Nitric acid of $46^{\circ}$ (or 80 per cent. nearly)

5,

Alcohol of 80 per cent.

Distilled water

100

Dry chloride of zinc

300 ,"

20 grms. 
83. Iodine.-KeNt (Manual of the Infusoria, 1881, p. 114) uses it for fixing Infusoria. Prepare a saturated solution of potassic iodide in distilled water, saturate this solution with iodine, filter, and dilute to a brown-sherry colour. A very small portion only of the fluid is to be added to that containing the Infusoria.

Or you may use Lugol's solution :

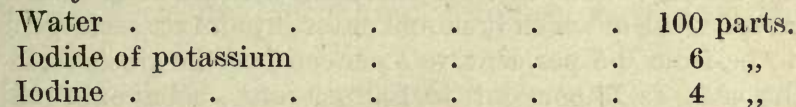

Or for small marine animals, a solution of iodine in sea-water.

Personally I have found it very useful for the examination of sperma- tozoc. See also under Goodrich's Iodine-Bouin method.

Very small objects may be instantaneously fixed by means of vapour of Iodine. Crystals of iodine may be heated in a test-tube till the vapours are given off ; then on inclining the tube the heavy vapours may be made to flow over the objects arranged on a slide. The slide should then be warmed to about $40^{\circ} \mathrm{C}$. for one to three minutes in order to evaporate the iodine from the objects, which may then be mounted or otherwise treated as desired (Overton, Zeit. wiss. Mik., vii, 1890, p. 14).

\section{Organic Acids, and other Agents.}

84. Acetic Acid.-A substance most injurious to the finer elements of the cytoplasm; in some cases it is indicated for a study of the nuclear elements. Flemming, who has made a special investigation of its action on nuclei, finds (Zellsubstanz, etc., p. 380) that the best strength is from 0.2 to 1 per cent. Strengths of 5 per cent. and more bring out the nuclein structures clearly at first, but after a time cause them to swell and become pale, which is not the case with the weaker strengths (ibid., p. 103). The strong acid is, however, a valuable fixative of certain objects, which it kills with the utmost rapidity, and leaves fixed in a state of extension.

The modus operandi of VAN BENEDEN is as follows:-Pour glacial acetic acid in liberal quantity over the organisms, leave them until they are penetrated by it-which should be in five or six minutes, as the strong acid is a highly penetrating reagent-and wash out in frequent changes of alcohol of gradually increasing strength. Some persons begin with 30 per cent. alcohol, but this appears to me rather weak, and I think 70 per cent. or at least 50 per cent. should be preferred.

Other energetic reagents may be combined with the glacial acetic acid if desired. Dr. Lindsay Johnson (in litt.) has found that one of the best fixatives for retina is a mixture of equal parts glacial acetic acid and 2 per cent. osmic acid. S. Lo Bianco adds to his "concen. trated "(49 per cent.) acid one-tenth of a 1 per cent. solution of chromic acid. He finds that even this small proportion of chromic 
acid serves to counteract in a marked degree the softening action of the acetic acid.

Acetic acid, used alone, is only a fixative for a limited time. If its action be prolonged, it becomes a swelling agent. Its function in mixtures is, besides that of killing, the valuable one of counteracting the shrinking action of the ingredients with which it is combined, and by its swelling action enhancing the penetration of the mixture; whilst by clarifying tissues it adds to the optical differentiation of their elements.

The proportions in which it should enter into mixtures in general seem to me to be from 0.5 per cent. to 5 per cent. of the glacial acid; higher strengths, such as 25 per cent. to 100 per cent., being only indicated in cases in which the highest possible penetration is the chief consideration.

Throughout this work, wherever acetic acid is mentioned, it is the glacial acid that is meant unless the contrary is stated.

All liquids containing a large proportion of this acid (e.g., $\S \$ 85$, 86) should only be allowed to act for a very short time.

85. Acetic Alcohol (CARnoy, La Cellule, iii, 1886, p. 6 ; and ibid., 1887, p. 276 ; v. Beneden et Neyt, Bull. Ac. Sci. Belg., xiv, 1887, p. 218 ; Zacharias, Anat. Anz., iii, 1888, pp. 24-27; v. GehuchTEN, ibid., 8, p. 227).-CARNOY has given two formulæ for this important reagent. The first is-

Glacial acetic acid . . . . 1 part.

Absolute alcohol . . . . 3 parts.

The second is-

Glacial acetic acid . . . . 1 part.

Absolute alcohol . . . 6 parts.

Chloroform . . . . 3 ,

The addition of chloroform is said to render the action of the mixture more rapid.

V. Beneden and Neyt take equal volumes of glacial acid and absolute alcohol.

ZACHARIAS takes-

Glacial acetic acid . . . . 1 part.

Absolute alcohol . . . . 4 parts.

Osmic acid . . . . a few drops.

Acetic alcohol is one of the most penetrating and quickly acting fixatives known. It preserves both nuclei and cytoplasm, and admits of staining in any way that may be preferred. It was employed by all of the authors quoted for the ova of Ascarisproverbially one of the most difficult objects to fix,-but I have found that it is applicable to many other objects. Wash out with alcohol, and avoid aqueous liquids as far as possible in the aftertreatment. 
86. Acetic Alcohol with Sublimate--CARNoy and LeBRUn ( $L a$ Cellule, xiii, 1, 1887, p. 68, due to GiLson).

Absolute alcohol .

Glacial acetic acid

Chloroform .

Sublimate to saturation.

(The mixture does not keep long, forming ethyl acetate, which precipitates).

Isolated ova of Ascaris, even though furnished with a shell, are fixed in twenty-five to thirty seconds. Entire oviducts take about ten minutes. The liquid is therefore one of the most penetrating and rapidly acting of any, if not the most.

Wash out with alcohol until all traces of odour of the acetic acid have disappeared (I myself wash out with alcohol containing tincture of iodine). I consider this a very fine reagent.

For Ohlmacher's mixture see $\S 65$.

G. S. SANsom's Carnoy Modification.-

Absolute alcohol

Glac. acetic acid

Chloroform .

Corr. subl. to saturation. Leave ten minutes to half an hour; wash in iodine absolute, then absolute. (Personal communication.)

Eminently suitable for study of vertebrate material. I have seen some really brilliant results obtained by the use of this fluid ( $\$ 590)$.

87. Trichlor-acetic Acid (Holmgren, Anat. Hefte, xviii, 1901, H. 2).-Five per cent. solution in water. Fix (nerve-cells) for eight to twenty-four hours, wash out with alcohol. See also Heidennuin, Zeit. wiss. Mikr., xxii, 1905, p. 321, and xxv, 1909, p. 405, who makes a mixture of 6 per cent. sublimate solution with 2 per cent. of trichloracetic and 1 per cent. of acetic acid, which he calls "Subtriessig."

88. Trichlor-acetic Fluid for Batrachia (Champy, Arch. d. Zool. Expér. et Gén., t. lii, 1913).-

Carbolic acid cryst. in sat. aq. sol. . . 15 parts.

Formol, 40 per cent.

Trichlor-acetic acid, 20 per cent. $\quad$. 1.5,

Outside of tissue often bad, inner parts better.

89. Salicylic Acid (Heidenhain, Arch. mik. Anat., liv, 1899, p. 186).Saturated solution in one-third alcohol. A trial has given me simply atrocious results.

90. Chloride and Acetate of Copper (Ripart et Petit's Liquid, Carnoy, La Biologie Cellulaire, p. 94).- -

Camphor water (not saturated)

Distilled water

75 grms.

Crystallised acetic acid

Acetate of copper

1 grm.

Chloride of copper

0.30 " 
This is a very moderate and delicate fixative, extremely useful for objects that are to be studied in as fresh a state as possible in aqueous media. Objects fixed in it stain instantaneously and perfectly with methyl green. Osmic acid may be added to the liquid to increase the fixing action. For cytological researches a valuable medium.

91. Nitrate of Copper (GIlson, from GELDERD, La Cellule, xxv, 1909 , p. 12). -Nitrate of copper 200, formol 500, sea-water 200. Seven parts of this solution to be diluted with 100 of sea-water. For Crustacea.

92. Acetate of Uranium (Schenk, Mitth. Embryol. Inst. Wien, 1882, p. 95 ; cf. Gilson, $L a$ Cellule, i, 1885, p. 141) has a mild fixing action, and a high degree of penetration, and may be combined with methyl green.

Friedenthal (Sitzb. Ges. Nat. Freunde Berlin, 1907, p. 209) recommends equal parts of saturated solution of the acetate and trichlor-acetic acid of 50 per cent.

93. Picric Acid.-Picric acid in aqueous solution should be employed in the form of a strong solution whenever it is desired to make sections or other preparations of tissues with the elements in situ, as weak solutions macerate; but for dissociation preparations or the fixation of isolated cells, weak solutions may be taken. Flemming finds that the fixation of nuclear figures is equally good with strong or weak solutions. The saturated solution is the one most employed. (One part of picric acid dissolves in about 86 parts of water at $15^{\circ} \mathrm{C}$. ; in hot water it is very much more soluble.) Objects should remain in it for from a few seconds to twenty-four hours, according to their size. For Infusoria one to at most two minutes will suffice, whilst objects of a thickness of several millimetres require several hours.

Picric acid should always be washed out with alcohol, that of 70 per cent. being mostly indicated. Staining should be performed by means of alcoholic solutions, or if with aqueous, then with such as are themselves weak hardening agents, such as hæmalum, carmalum, methyl green.

Washing out is facilitated by heat, the extraction being about twice as rapid at $40^{\circ} \mathrm{C}$. as at the normal temperature (FoL).

It has been found by JELINEK (Zeit. wiss. Mik., xi, 1894, p. 242) that the extraction is greatly quickened by the addition of a base to the wash-alcohol. He recommends carbonate of lithia. A few drops of a saturated solution of the salt in water are added to the alcohol; a precipitate is formed. The objects are put into the turbid alcohol, which becomes clear and yellow in proportion as the picrin is extracted. Further quantities of carbonate are added from time to time until the colour has been entirely extracted. 
Tissues fixed in picric acid can be perfectly stained in any stain. It is seldom necessary to remove the picric acid by washing out before staining. Paracarmine, Boraxcarmine, or Hæmacalcium may be recommended for entire objects.

The most important property of picric acid is its great penetration. This renders it peculiarly suitable for the preparation of chitinous structures.

94. Picric Alcohol (GAGe, Proc. Amer. Soc. Micr., 1890, p. 120).Alcohol (95 per cent.), 250 parts ; water, 250 parts ; picric acid, 1 part.

95. Picro-acetic Acid.-BoverI (Zellenstudien, 1, 1887, p. 11) dilutes a concentrated aqueous solution of picric acid with two volumes of water and adds 1 per cent. of acetic acid. According to my experience, the results are miserable.

ZIMMER's mixture (from DeEgener, Zool. Jahrb., Abth. Morph., xxvii, 1909 , p. 634). - Saturated aqueous solution of picric acid, 10 parts; absolute alcohol, 9 ; acetic acid, 1.

96. Piero-sulphuric Acid (Kleinenberg, Quart. Journ. Mic. Sci., April, 1879, p. 208 ; MAYER, Mitt. Zool. Stat. Neapel, ii, 1880, p. 2).MAYER takes distilled water, 100 vols.; sulphuric acid, 2 vols. ; picric acid, as much as will dissolve.

Liquid of KLEINENBERG is made by diluting the concentrated picrosulphuric acid prepared as above with three times its volume of water.

I hold that the concentrated solution is generally preferable. This particularly applies to marine organisms.

Wash out with successive alcohols, beginning with 70 per cent., never with water.

Warm alcohol extracts the acid much more quickly than cold, without which weeks may be required to fully remove the acid from chitinous structurès.

This liquid may still be useful for Arthropoda, on account of its great power of penetrating chitin; and for some embryological purposes. For a fuller account see early editions.

9\%. Picro-nitric Acid (MaYer, Mitth. Zool. Stat. Neapel, 1881, p. 5)-

Water

Nitric acid (of 25 per cent. $\mathrm{N}_{2} \mathrm{O}_{5}$ )

Picric acid, as much as will dissolve.

Properties of this fluid similar to those of picro-sulphuric acid, with the advantage of avoiding the formation of gypsum crystals, and the disadvantage that it is much more difficult to soak out of the tissues. Mayer states that with eggs containing a large amount of yolk material, like those of Palinurus, it gives better results than nitric, picric, or picro-sulphuric acid. I myself consider it distinctly superior to picro-sulphuric for most things. See Hill's fluid, $§ 586$. 
98. Picro-hydrochloric Acid (MAYER, ibid.).-

Water . . . . . 100 vols.

Hydrochloric acid (of 25 per cent. $\mathrm{HCl}$ ) . 8 ,

Picric acid, as much as will dissolve.

99. Picro-chromic Acid (FoL, Lehrb., p. 100).-

Picric acid, sol. sat. in water . . . 10 vols.

1 per cent. chromic acid solution - . 25 ",

Water . . . . . 65 ,

I have seen Fol's formula, with the addition of a trace of acetic acid, quoted as "liquid of Haensel."

Lo BIANco takes equal parts of picro-sulphuric acid and chromic acid of 1 per cent.

RAwITz (Leitfaden, 1895, p. 24) takes 1 part of picro-nitric acid, and 4 parts 1 per cent. chromic acid. Wash out in 70 per cent. alcohol.

100. Picro-osmic Acid.-Flemming (Zells. Kern u. Zellth., p. 381) has experimented with mixtures made by substituting picric for chromic acid in the chromo-osmic mixtures $(\$ 42)$, and finds the results identical, so far as regards the fixation of nuclei. The fixation of cytoplasm is in my preparations decidedly inferior.

O. vom RATH (Anat. Anz., xi, 1895, p. 289) adds to 200 c.c. of saturated aqueous solution of picric acid, 12 c.c. of 2 per cent. solution of osmic acid, and 2 c.c. of glacial acetic acid.

RAwITZ (Leitfaden, p. 24) takes picro-nitric acid, 6 vols. ; 2 per cent. osmic acid, 1 vol. Fix for half to three hours. Transfer direct to 70 per cent. alcohol.

101. Picro-platinic and Picro-platin-osmic Mixtures.-0. vом Rati (loc. cit., last $\S$, pp. 282, 285) makes a picro-platinic mixture with 200 c.c. saturated aqueous solution of picric acid, $1 \mathrm{grm}$. of platinic chloride (dissolved in 10 c.c. of water), and 2 c.c. of glacial acetic acid.

The picro-platin-osmic mixture, which is, in my opinion, much superior, is made by adding to the foregoing 25 c.c. of 2 per cent. osmic acid.

Other Picric Mixtures. See $\S \S 70$ and 110 to 112.

\section{Other Fixing and Hardening Agents.}

102. Alcohol.-For fixing only two grades of alcohol should be employed-very weak, or absolute. Absolute alcohol ranks as a fixing agent because it kills and hardens with such rapidity that structures have hardly time to get deformed in the process; very weak, because it possesses a sufficiently energetic coagulating action and yet contains enough water to have but a feeble dehydrating action. The intermediate grades do not realise these conditions, and therefore should not be employed alone for fixing. But they may be very useful in combination with other fixing agents by 
enhancing their penetrating power ; 70 per cent. is a good grade for this purpose.

Table for diluting alcohol (after GAY-LUSSAC).-To use this table, find in the upper horizontal row of figures the percentage of the alcohol that it is desired to dilute, and in the vertical row to the left the percentage of the alcohol it is desired to arrive at. Then follow out the vertical and horizontal rows headed respectively by these figures, and the figure printed at the point of intersection of the two rows will show how many volumes of water must be taken to reduce one hundred volumes of the original alcohol to the required grade.

\begin{tabular}{|c|c|c|c|c|c|c|c|c|c|}
\hline \multirow{2}{*}{$\begin{array}{c}\text { Weaker } \\
\text { grade } \\
\text { required. }\end{array}$} & \multicolumn{9}{|c|}{ Original Grade. } \\
\hline & $\begin{array}{c}90 \\
\text { p. } 100 .\end{array}$ & $\begin{array}{c}85 \\
\text { p. } 100 .\end{array}$ & $\begin{array}{c}80 \\
\text { p. } 100 .\end{array}$ & $\begin{array}{c}75 \\
\text { p. } 100 .\end{array}$ & $\begin{array}{c}70 \\
\text { p. } 100 .\end{array}$ & $\begin{array}{c}65 \\
\text { p. } 100 .\end{array}$ & $\begin{array}{c}60 \\
\text { p. } 100 .\end{array}$ & $\begin{array}{c}55 \\
\text { p. } 100 .\end{array}$ & $\begin{array}{c}50 \\
\text { p. } 100 .\end{array}$ \\
\hline $\begin{array}{c}\text { p. } 100 . \\
85\end{array}$ & $6 \cdot 56$ & & & & & & & & \\
\hline 80 & $13 \cdot 79$ & $6 \cdot 83$ & & & & & & & \\
\hline 75 & $21 \cdot 89$ & $14 \cdot 48$ & $7 \cdot 20$ & & & & & & \\
\hline 70 & $31 \cdot 05$ & $23 \cdot 14$ & $15 \cdot 35$ & $7 \cdot 64$ & & & & & \\
\hline 65 & $41 \cdot 53$ & $33 \cdot 03$ & $24 \cdot 66$ & $16 \cdot 37$ & $8 \cdot 15$ & & & & \\
\hline 60 & $53 \cdot 65$ & $44 \cdot 48$ & $35 \cdot 44$ & $26 \cdot 47$ & $17 \cdot 58$ & $8 \cdot 76$ & & & \\
\hline 55 & $67 \cdot 87$ & $57 \cdot 90$ & $48 \cdot 07$ & $38 \cdot 32$ & $28 \cdot 63$ & $19 \cdot 02$ & $9 \cdot 47$ & & \\
\hline 50 & $84 \cdot 71$ & $73 \cdot 90$ & $63 \cdot 04$ & $52 \cdot 43$ & $41 \cdot 73$ & $31 \cdot 25$ & $20 \cdot 47$ & $10 \cdot 35$ & \\
\hline 45 & $105 \cdot 34$ & $93 \cdot 30$ & $81 \cdot 38$ & $69 \cdot 54$ & $57 \cdot 78$ & $46 \cdot 09$ & $34 \cdot 46$ & $22 \cdot 90$ & $11 \cdot 41$ \\
\hline 40 & $130 \cdot 80$ & $117 \cdot 34$ & $104 \cdot 01$ & $90 \cdot 76$ & $77 \cdot 58$ & $64 \cdot 48$ & $51 \cdot 43$ & $38 \cdot 46$ & $25 \cdot 55$ \\
\hline 35 & $163 \cdot 28$ & $148 \cdot 01$ & $132 \cdot 88$ & $117 \cdot 82$ & $102 \cdot 84$ & $87 \cdot 93$ & 73.08 & $58 \cdot 31$ & $43 \cdot 59$ \\
\hline 30 & $206 \cdot 22$ & $188 \cdot 57$ & $171 \cdot 05$ & $153 \cdot 61$ & $136 \cdot 04$ & 118.94 & $101 \cdot 71$ & $84 \cdot 54$ & $67 \cdot 45$ \\
\hline
\end{tabular}

Alcohol is an easily oxidisable substance. Chromic acid, for instance, easily oxidises it, first into aldehyde, and then into acetic acid. It follows that alcohol should not be combined in mixtures with oxidising agents of notable energy. Further, alcohol is a reducing agent, and therefore should not be combined with easily reducible substances. These remarks particularly apply to chromic acid. See $\S \S 38,39,48$.

For fixing, alcohol is a very third-class reagent, only to be used alone where better ones cannot be conveniently employed, though it enters as a useful ingredient into many mixtures, in which it serves to enhance the power of penetration. For hardening it is an im- 
portant one. 90 to 95 per cent. is the most generally useful strength. Weaker alcohol, down to 70 per cent., is often indicated. Absolute alcohol is seldom advisable. You ought to begin with weak, and proceed gradually to stronger, alcohol. Large quantities of alcohol should be taken. The alcohol should be frequently changed, or the tissue should be suspended near the top of it ( $\$ 34)$. Many weeks may be necessary for hardening large specimens. Small pieces of permeable tissue, such as mucous membrane, may be sufficiently hardened in twenty-four hours.

103. Absolute Alcohol.-This is sometimes valuable on account of its great penetrating power. Mayer finds that boiling absolute alcohol is often the only means of killing certain Arthropoda rapidly enough to avoid maceration.

It is important to employ for fixing a very large proportion of alcohol. Alum-carmine is a good stain for small specimens so fixed. For preservation, the object should be put into a weaker alcohol, 90 per cent. or less.

As to the supposed superiority of absolute alcohol over ordinary strong alcohol, see last $\S$; and amongst authors upholding its superiority, see besides Ranvier, Mayer (Mitth. Zool. Stat. Neapel, ii, 1880, p. 7); BRÜEL (Zool. Jahrb., Abth. Morph., x, 1897, p. 569); and van ReES (ibid., iii, 1888, p. 10).

Absolute alcohol is a product that it is almost impossible to preserve in use, on account of the rapidity with which it hydrates on exposure to air. Fol recommends that a little quicklime be kept in it. This absorbs part at least of the moisture drawn by it from the air.

Ranvier prepares a sufficiently "absolute" alcohol as follows:Strong (95 per cent.) alcohol is treated with calcined cupric sulphate, with which it is shaken up and allowed to remain for a day or two. It is then decanted and treated with fresh cupric sulphate, and the operation is repeated until the fresh cupric sulphate no longer becomes conspicuously blue on contact with the alcohol; or until, on a drop of the alcohol being mixed with a drop of turpentine, no particles of water can be seen in it under the microscope. The cupric sulphate is prepared by calcining common blue vitriol in a porcelain capsule over a flame until it becomes white, and then reducing it to powder (see Proc. Acad. Nat. Sci. Philad., 1884, p. 27 ; Journ. Roy. Mic. Soc., 1884, pp. 322 and 984).

Test for the presence of water (Yvon, $C . R$. Acad. Sci., 1897, p. 1181).Add coarsely powdered calcium carbide; the merest trace of water will cause an evolution of acetylene gas, and on agitation the alcohol will become turbid.

104. One-third Alcohol.-The grade of weak alcohol that is generally held to be most useful for fixing is one-third alcohol, or RANVIER's ALCOHOL. It consists of two parts of water and one part 
of alcohol of 90 per cent. (and not of absolute alcohol). See the Traité Technique of Ranvier, p. 241, et passim.

Objects may be left for twenty-four hours in this alcohol; not more, unless there be no reason for avoiding maceration, which will generally occur after that time. You may conveniently stain with picro-carmine, alum-carmine, or methyl green.

This reagent is a very mild fixative. Its hardening action is so slight that it is not at all indicated for the fixing of objects that are intended to be sectioned. Its chief use is for extemporaneous and dissociation preparations.

105. Pyridin.-Pyridin has been recommended as a hardening agent (by A. DE SouzA). It hardens, dehydrates, and clears at the same time. It is said to harden quickly, and to give particularly good results with brain. See Comptes Rendus hebd. de la Soc. de Biologie, 8 sér., t. iv, 1887, p. 622.

This substance is strongly alkaline, and, either pure or diluted with water, dissolves many albumens and fats. It causes considerable shrinkage of nuclei (not so much of cytoplasm). It is now in much use in certain neuro-fibril stains, see BIELSCHOWSKY and RAMón. It is soluble in water and in alcohol. Pure, it will harden and dehydrate small brains in a week.

106. Acetone is said to harden very rapidly. It precipitates lipins, and may yet prove an important reagent. ScHolz (Zeit. wiss. Mikr., xxii, 1905, p. 415) fixes small objects in warm acetone for half an hour to an hour and brings them direct, or through alcohol and ether, into celloidin.

Similarly Fuss (Arch. path. Anat., clxxxv, 1906, p. 5), using it cold, and LinTwarew (ibid., ccvi, 1911, p. 36) for erythrocytes, in which it preserves the hæmoglobin.

10\%. Lucidol. - This is a proprietary name applied to peroxide of benzol $\left(\mathrm{C}_{6} \mathrm{H}_{5}-\mathrm{CO}\right)_{2} \mathrm{O}_{2}$. It is a white powder insoluble in water, but soluble in acetone and pyridin; heated, the white powder first of all melts and then explodes. The introduction of "Lucidol " is due to EHRIICH, who suggested it to SzECSI. "Lucidol" must share with its solvents, acetone and pyridin, their defects as fixing agents. Acetone, especially tends to tear cells to pieces, owing to its attraction for water. So far, there have been very few references to the use of "Lucidol" in histological literature. LANGERON (C. R. Soc. de Biol., lxxvi, 1914) claims that "Lucidol" is especially convenient for work on blood, fæces and cell smears, and two of Szecsi's methods are given below. It should be pointed out that the Lucidol-acetone solution gives a very active penetration, and unlike CARNOY's or SCHAUDINN's fluids, does not dissolve away lipins. 
In all probability some method of mordanting the fixed material in either $\mathrm{K}_{2} \mathrm{Cr}_{2} \mathrm{O}_{7}$ or $\mathrm{CrO}_{3}$ dissolved in a suitable solvent might help to " set" the lipins, and so avoid their dissolution when the material or smears are brought into alcohols.

Szecsi (Deutsche med. Woch., 1913) uses two solutions: one consists of 10 grms. of "Lucidol" to 100 c.c. of acetone, the other 12 grms. of "Lucidol" to 100 c.c. of pyridin. Both solutions are used for smears, or for pieces of tissue. After fixation, the latter are washed out in a mixture of acetone 3 parts, and xylol or toluol 2 parts; and then transferred to methyl alcohol or pure xylol according to whether one is dealing with smears, or tissue for embedding in wax.

For tissues proceed as follows :--Fix small pieces at room temperature in the acetone solution for about four or six hours, or in the pyridin solution for ten to twelve hours. In each case transfer to the above-mentioned acetone xylol mixture for several hours, but not longer than ten. Clear in pure xylol or toluol, embed in paraffin wax. Sections can be stained as desirable.

For the treatment of smears, etc., see under "Blood."

108. Formaldehyde, Formic Aldehyde, Methyl Aldehyde (Formol, Formalin, Formalose).--Formaldehyde is the chemical name of the gaseous compound $\mathrm{HCOH}$, obtained by the oxidation of methylalcohol. "Formol," "Formalin," and "Formalose" are commercial names for the saturated (40 per cent.) solution of this in distilled water. This quickly loses in strength through contact with air, and laboratory solutions rarely contain more than 38 per cent. of formaldehyde.

Much confusion has been caused by indiscriminate use of the terms " formaldehyde" and "formol." The proper way is evidently either to state the strengths of solutions in terms of formaldehyde, and say so; or to say "formol-or formalin-with so many volumes of water." The majority of writers seem to state in terms of formol.

Solutions of formaldehyde sometimes decompose partially or entirely, with formation of a white deposit of paraformaldehyde. Fish says that to avoid this the solution should be kept in darkened bottles in the cool, or, according to some, it suffices to add glycerin to them.

The solutions almost always have an acid reaction, due to the presence of formic acid; but that is, as a rule, rather an advantage. But some observers hold that neutral or feebly alkaline solutions fix better than acid ones. Solutions may be neutralised by saturation 
with magnesium or sodium earbonate; some workers use lithium carbonate, but this should not be used for BouIN mixtures: it will generally suffice to make them up with spring water.

It was said above that formaldehyde possesses certain hardening and preserving qualities. It hardens gelatine, for instance, and certain albuminoids ; but others, on the contrary, are not hardened by it, but sometimes even rendered more soluble than they are naturally. For some theoretical considerations concerning its action on tissues, see F. Blum, in Anat. Anz., xi, 1896, p. 718 ; Benedecenti, in Arch. Anat. u. Phys., Abth, 1897, p. 219 ; Gerota, in Intern. Monatschr. Anat., xiii, 1896, p. 108; Zeit. wiss. Mik., xiii, p. 311 ; SJöbring in Anat. Anz., xvii, 1900, p. 274 ; and Blum, in Encycl. mik. Technik., p. 393. It seems to be generally admitted that this action consists in the formation of methylene compounds with the substances of the tissues.

I find that, used pure, it is far from a first-class fixative. For it over-fixes and shrinks some things, and swells and vacuolates others. But notwithstanding this it is frequently very convenient on account of its compatibility with the most various stains. It has a high degree of penetration, and is a valuable ingredient in many mixtures.

It is a powerful reducing agent, and therefore incompatible with such reagents as chromic acid or osmic acid and the like, which it very rapidly decomposes.

For fixing I find that a strength of about 4 per cent. (1 vol. formol to 9 of water, or to 8 of water if the formol has been long kept) is generally about right; and this is the strength used by most writers. For cytological purposes a fixation of at least two days seems indicated: this applies especially to gonads which are notoriously difficult to preserve in formol. The strengths used in CAJAL's and DA FANO's formol silver nitrate GoLGI apparatus methods, generally give fine results for tissues other than genital. For these, injection fixation may be indicated. See also §141. MaYer takes 1 of formol to 9 of sea-water, for marine animals. Few workers use much stronger solutions. Only one (Hoyer, Anat. Anz., ix, 1894, p. 236. Erganzungsheft) seems to have used concentrated solutions, I think this exaggerated, for I have found enormous over-fixation with solutions of 1 to 2 vols. of water. Wash out with alcohol (of 50 per cent. or more), not water.

For hardening, the same strengths may be taken. Hardening is more rapid than with alcohol. For prolonged hardening, considerable volumes of liquid should be taken, and the liquid should be 
renewed from time to time; for the formaldehyde fixes itself on the tissues with which it comes in contact, deserting the solution, which thus becomes progressively weaker. The specimens should be suspended in the liquid or otherwise isolated from contact with the containing vessel. The hardening obtained is gentle and tough, giving an elastic and not a brittle consistency. It varies greatly with different tissues. Mucin is not precipitated and remains transparent. Fat is not dissolved (see $\S \S 768$ et seq.). Micro-organisms retain their specific staining reactions. Formaldehyde is said to harden celloidin as well as gelatin, and to be useful for celloidinimbedding (Blum, Anat. Anz., xi, 1896, p. 724).

Several of the following mixtures are irrational, becoming reduced more or less quickly, but may give good results all the same.

109. Alcoholic Formol (Lavdowskr, Anat. Hefte, iv, 1894, p. 361).Water 40 parts, 95 per cent. alcohol 20 , formol 6 , acetic acid 1 ; or water 30 , alcohol 15, formol 5, acetic acid 1 .

GUlland (Zeit. wiss. Mikr., xvii, 1900, p. 222) takes (for blood) 1 part formol and 9 parts of alcohol.

Bles (Trans. Roy. Soc. 'Edinburgh, xli, 1905, p. 792) takes 7 of formol, 90 of alcohol of 70 per cent., and 3 of acetic acid.

TellyesnicZKY (Encycl. mikr. Techn., i, p. 472) takes 5 of formol, 100 of alcohol of 70 per cent., and 5 of acetic acid.

110. Picro-Formol.-P. BouIn (Phénomènes cytologiques anormaux dans L'Histogenèse, etc., Nancy, 1897, p. 19) recommends-

Picric acid, saturated aqueous sol. . . 75 parts.

Formol

Acetic acid

Wash out with alcohol, first of 50 per cent., then 70 per cent. till the picric acid is mostly removed. I consider this to be for most purposes the most valuable fixative yet made known. I have satisfied myself that the proportions are exactly what they should be and cannot be changed without hurt. It is rather a strong fixative, and should not be allowed to act for more than 18 hours. If a weaker mixture be desired, dilute the whole with water. The penetration is great, the fixation equable, delicate detail well preserved, staining qualities admirable, especially with iron-hematoxylin and Saürefuchsin. See also "Cytology" sections, § 656, under heading of "Chromosomes and Urea," and Garnier, Bibl. Anat., v, 1898, p. 279.

The formulæ of Graf (State Hosp. Bull. New York, 1897 ; Journ. Roy. Mic. Soc., 1898, p. 492) are in my view too weak.

Moreaux (Bibl. Anat., 1910, p. 265) takes 15 parts formol, 85 of trichlor-acetic acid of 3 per cent., and picric acid to saturation. 
111. Picro-platinic Formol (M. and P. Bours, Bibl. Anat., 1898, f. 2, p. 2).-

Platinum chloride, 1 per cent. sol.

Picric acid, saturated sol. .

Formol.

Formic or acetic acid

The mixture does not keep more than a day or two, and it is probably inferior.

Bourv also (Arch. Biol., xvii, 1900, p. 211) simply substitutes formol for the osmic acid in Hermans's mixture, § 45.

112. Sublimate Formol (M. and P. Bourn, loc. cit.).-A similar mixture, in which sublimate of 1 per cent. is substituted for the platinum chloride.

Another formula of the same authors (Arch. Biol., xvii, 1900, p. 211) is 1 part of formol to 3 of saturated aqueous sublimate. Rinse with water and bring into alcohol of 70 per cent.

SPULER (Encycl. mik. Technik., 1st ed., p. 1280) adds to sublimate of 3 per cent. or more 1 per cent. of glacial acetic acid and 10 per cent. of formol.

Mann (Verh. Anat. Ges., 1898, p. 39) takes for nerve-cells $2 \frac{1}{2}$ grms. sublimate, $1 \mathrm{grm}$. picric acid, 5 c.c. formol, and 100 c.c. water, or (Methods, etc., p. 97) for all tissues $2 \frac{1}{2}$ grms. sublimate, 20 c.c. formol, and 80 c.c. water.

Branca (Journ. Anat. et Phys., xxxv, 1899, p. 767) adds 10 parts of formol and 1 of acetic acid to 60 parts of saturated solution of picric acid in șaturated aqueous sublimate.

NowaK (Anat. Anz., Xx, 1901, p. 244) takes 30 parts of saturated sublimate, 30 of 1 per cent. chromic acid, 27 of water, 3 of acetic acid; and 10 of formalin.

113. Formol-Müller.-This is the name given by ORTH (Berl. klin. Wochenschr., 1896, No. 13) to a mixture of 1 part of formol with 10 of liquid of Müller ( $\$ 53$ ). It should be freshly made up. Fix for three hours in the stove, or twelve at normal temperature, wash out with running water. Much used, especially for nervous tissues.

Moeluer (Zeit. wiss. Zool., lxvi, 1899, p. 85) takes 1 vol. of formol and 4 of 3 per cent. bichromate (for the intestine of mammals).

Held (Abk. Sächs. Ges. Wiss., xxxi, 1909, p. 196) takes 3 per cent. sol. of bichromate with 4 per cent. of formol and 5 per cent. of acetic acid (for inner ear). See also Morel and BASSAL, Journ. Anat. Phys., xlv, 1909, p. 632, and HeLly and MAXıMow formulæ. Look up section on "Mitochondria," especially paragraphs on Regaud and Schridde, $\S \S 685$ to 687.

114. Chromic Acid Formol.-Lo Bianco fixes marine animals for half to one hour in 10 parts of 1 per cent. chromic acid with 1 of formol and 9 of sea-water, and passes into graded alcohols. 
Marchoux (from Pérez, Arch. Zool. Exper., v, 1910, p. 11) takes 11 parts 1 per cent. chromic acid, 1 of acetic acid, 4 of water, and 16 of formol (added just before using).

These mixtures are neither so good nor reliable as Bouin's picro. formol.

115. Copper Formol.-Nelis (Bull. Acad. Se. Belg., 1899 (1900), p. 726) fixes spinal ganglia for twenty-four hours in 1 litre of 7 per cent. formol with 5 c.c. of acetic acid, 20 grms. of cupric sulphate, and sub. limate to saturation.

StAPPERS (La Cellule, xxv, 1909, p. 356) used (for Sympoda) a mixture of GiLson's : 100 parts of formol of 5 per cent. with 2 of nitrate of copper.

Strong (Journ. Comp. Neur., xiii, 1903, p. 296) fixes the head of Acanthias by injecting a mixture of equal parts of formol and 5 per cent. solution of bichromate of copper.

116. Nitric Acid Formol.-Wilhelmi (Fauna u. Flora Golf. Neapel, xxxii, 1909, p. 15) fixes Triclads in Apáthy's mixture of equal parts of 6 per cent. nitric acid and 6 per cent. formol, and brings them direct into strong alcohol.

117. Acetone Formol.-Bing and Ellermann (Arch. Anat. Phys., Phys. Abth., 1901, p. 260) fix medullated nerves in 9 parts of acetone with 1 of formol. 


\section{CHAPTER VI.}

\section{DE-ALCOHOLISATION AND CLEARING AGENTS.}

118. Introduction.-De-alcoholisation agents are liquids employed for the purpose of getting rid of the alcohol which has been employed for dehydrating tissues ( $\S 3$ ), and facilitating the penetration of the paraffin used for imbedding, or the balsam or other resinous medium in which preparations are, in most cases, finally mounted. Hence all of them must be capable of expelling alcohol from tissues, and must be at the same time solvents of Canada balsam and the other resinous mounting media. The majority of them are essential oils.

Clearing agents are liquids whose function it is to make microscopic preparations transparent by penetrating amongst the highly refracting elements of which the tissues are composed, the clearing liquids themselves having an index of refraction superior, or equal, or, at all events, not greatly inferior, to that of the tissues to be cleared. Hence all clearing agents are liquids of high index of. refraction.

The majority of de-alcoholisation agents being also liquids of high refraction, it follows that they serve at the same time for de-alcoholisation and for clearing; and in consequence it has come about that de-alcoholisation agents are generally spoken of as clearing agents. But that practice is not strictly correct, for not all clearing agents are solvents of the resins, and not all de-alcoholising agents can serve as clearers. I shall, however, still in many cases continue to use the term "clearing " to signify "de-alcoholising," for the sake of brevity.

Neelsen and Schiefferdecker (Arch. Anat. Phys., 1882, p. 206) examined a large series of ethereal oils (prepared by Schimmel \& Co., Leipzig), with the object of finding a not too expensive substance that should combine the properties of clearing quickly alcohol preparations, not dissolving out anilin colours, clearing celloidin without dissolving it, and not evaporating too quickly.

Of these, the following three fulfil the conditions:-Cedar-wood, Origanum, Sandal-wood. 
To these should be added the others recommended in the following paragraphs.

See also the paper of JoRdan (Zeit. wiss. Mik., xv, 1898, p. 50) as to the behaviour of some essential oils towards celloidin.

119. The Practice of De-alcoholisation or Clearing. - The old plan was to take the object out of the alcohol and float it on the surface of the de-alcoholising or clearing medium in a watch-glass. This plan was faulty, because the alcohol escapes from the surface of the object into the air quicker (in most instances) than the de-alcoholising or clearing agent can get into it ; hence the object must shrink. To avoid this cause of shrinkage, the operation is now generally done by the method suggested by Mayer and Giesbrecht, which consists in putting the clearing medium under the alcohol containing the object, as described $\S 5$. The objects should not be considered to be perfectly penetrated by the clearing medium until the wavy refraction-lines caused by the mixture of the two liquids at their surface have ceased to form, and they should not be mounted or imbedded until they have first been soaked for some time in a fresh quantity of clearing medium, to remove any alcohol that has got into the first bath.

The penetration of all clearing media may be hastened by using them warm.

It frequently happens that the essential oil with which objects are being treated in a watch-glass or on a slide becomes cloudy after a short time, and fails to clear the tissues. This is owing to a combination between the essential oil and moisture, derived, I think, rather from the air than from the objects. themselves. The cloudiness can usually be removed by warming (as pointed out by HATCHETT JACKsON, Zool. Anzeig., 1889, p. 630), but in certain moist states of the atmosphere it may persist, notwithstanding continued warming. It is for this reason that I advise that clearing be done, whenever possible, in shallow corked tubes, under which conditions the phenomenon rarely occurs. In any case, be careful not to breathe on the liquid.

120. Choice of a De-alcoholisation or Clearing Agent.-I advise the beginner to keep on his table the following:-Oil of cedar, for general use and for preparing objects for imbedding in paraffin ; clove oil, for making minute dissections in (\$122), and for much work with safranin, etc.; oil of bergamot, which will clear from 90 per cent. alcohol, and which does not extract coal-tar colours; carbolic acid, for rapidly clearing very imperfectly dehydrated objects.

For special clearers for celloidin sections see Chapter IX. 
121. Cedar Oil (Neelsen and SchiefFerdecker, loc. cit., § 118).Clears readily tissues in 95 per cent. alcohol without shrinkage; does not extract anilin colours. Celloidin sections are cleared in five to six hours.

The observer should be careful as to the quality of the cedar oil he obtains. I have examined the clearing properties of a sample, obtained from a celebrated firm, which totally failed to clear absolute alcohol objects after many days.

Cedar oil is very penetrating, and for this and other reasons is, in my experience, the very best of all media for preparing objects for paraffin imbedding. I find it to be less hurtful to cells than any other medium known to me. Tissues may remain in it for any length of time without hurt. If it should become milky through keeping, filter.

122. Clove Oil. - Samples of clove oil of very different shades of colour are met with in commerce. It is frequently recommended that only the paler sorts should be employed in histology. Doubtless it is, in general, best to use a pale oil, provided it be pure; but it is not always easy to obtain a light-coloured oil that is pure. Clove oil passes very readily from yellow to brown with age, so that in choosing a colourless sample you run great risk of obtaining an adulterated sample, for clove oil is one of the most adulterated substances in commerce.

Clove oil does not easily spread itself over the surface of a slide, but has a tendency to form very convex drops. This property makes it a very convenient medium for making minute dissections in. It also has the property of making tissues that have lain in it for some time very brittle. This brittleness is also sometimes very helpful in minute dissections.

These qualities may be counteracted if desired by mixing the clove oil with bergamot oil.

This is one of the most useful of clearers. According to BeHrens (Tabellen, 3rd ed., 1898, p. 33), it will clear from alcohol of 74 per cent.

It has a high index of refraction, and clears objects more than balsam mounting media. It dissolves celloidin (or collodion), and therefore should not be used for clearing sections cut in that medium without special precautions. New clove oil washes out basic tar colours more quickly than old.

123. Cinnamon (or Cassia) Oil greatly resembles clove oil, but is in general thinner, and is more highly refractive. An excellent medium, which I particularly recommend. 
124. Oil of Bergamot (SchinfFerdecker, Arch. Anat. Phys., 1882 [Anat. Abth.], p. 206).-Clears 95 per cent. alcohol preparations and celloidin preparations quickly, and does not extract anilin colours.

Bergamot oil is, I believe, the least refractive of these essences, having a lower index than even oil of turpentine.

SuchaNneK (Zeit.wiss. Mik., vii, 1890, p. 158) says that bleached, colourless bergamot oil will not take up much water, whereas a green oil will take up as much as 10 per cent.

VAN DER STRICHT (Arch. de Biol., xii, 1892, p. 741) says that bergamot oil will, with time, dissolve out the fatty granules of certain ova.

125. Oil of Origanum (Neelsen and Schiefferdecker, Arch. Anat. Phys., 1882, p. 204).-Ninety-five per cent. alcohol preparations are cleared quickly, and so are celloidin sections, without solution of the celloidin. Anilin colours are somewhat extracted.

For work with celloidin sections care should be taken to obtain Ol. Origani Cretici ("Spanisches Hopfenöl"), not Ol. Orig. Gallici (v. Gieson; see Zeit. wiss. Mik., iv, 1887, p. 482). Specimens of origanum oil vary greatly in their action on celloidin sections.

SquIRe, in his Methods and Formulce, etc., p. 81, says that origanum oil (meaning the commercial product) is nothing but oil of white thyme more or less adulterated (see next §), and that the product sold as Ol. Origani Cretici is probably oil of marjoram.

126. Oil of Thyme.-Fish (Proc. Amer. Mic. Soc., 1893 ; Zeit. wiss. Mik., xi, p. 503), following Bumpus, says that for most of the purposes for which origanum oil has been recommended, oil of thyme will do just as well if not better. The red oil is just as efficient as the white for clearing.

Schimmel \& Co., in their Report of October, 1895, p.•69, state that in France white oil of thyme is adulterated with oil of turpentine to the extent of as much as 50 per cent.

127. Oil of Gaultheria.-Used by UnNa (Monatschr. prakt. Derm., Ergänzungsh, 1885, p. 53) for thinning balsam. The artificial oil, methyl salicylate, is recommended by Guíguen (Comp. Rend. Soc. Biol., v, 1898, p. 285) both as a de-alcoholisation and clearing agent and as a solvent of paraffin. The refractive index is $1 \cdot 53$. It is, unfortunately, very sensitive to water.

128. Sandal-wood Oil (NEELSEN and SCHIEFFERDECKER, loc. cit.).Very useful, but its high price is prohibitive.

129. Oil of Cajeput.-Now much used. I have used it myself and found it to clear well, but to be rather thin. CARNOY and LEBRUN 
(La Cellule, xiii, 1897, p. 71) have found it useful for clearing celloidin sections. It dissolves celloidin very slowly and clears without shrinkage.

130. Oil of Turpentine. - Generally used for dissolving out the paraffin from sections; but many other reagents, such as xylol and benzol, are preferable for this purpose. If used for alcohol objects, it causes considerable shrinkage, and alters the structure of cells more than any other elearing agent known to me. Turpentine has, I believe, the lowest index of refraction of all the usual clearing agents except bergamot oil ; it clears objects less than balsam.

131. Terpinol (liquid, from Schimmel \& Co.) is recommended by MAYER, Zeit. wiss. Mikr., xxvi, 1910, p. 523. Clears from alcohol of 90 per cent., or even 80 per cent. One part xylol and 4 terpinol has been much used lately.

132. Carbolic Acid.-Best used in concentrated solution in alcohol. Clears instantaneously, even very watery preparations. This is a very good medium, but it is better avoided for preparations of soft parts which it is intended to mount in balsam, as they generally shrink by exosmosis when placed in the latter medium. It is, however, a good medium for celloidin sections.

GAGE's Mixture (Proc. Amer. Soc. Micr., 1890, p. 120).-Carbolic acid crystals melted, 40 c.e. ; oil of turpentine, 60 c.c.

133. Creosote.-Much the same properties as carbolic acid. Beech-wood creosote is the sort that should be preferred for many purposes,--amongst others, for clearing celloidin sections, for which it is a very good medium.

134. Anilin Oil.-Common anilin oil will readily clear sections from 70 per cent. alcohol, and with certain precautions (for which see the paper of SUCHANNEK quoted below) objects may be cleared from watery media without the intervention of alcohol at all. This renders it valuable in certain cases as a medium for preparing for paraffin imbedding. For ordinary work the usual commercial anilin will suffice; and it is immaterial whether it be colourless or have become brown through oxidation. For difficult work it is well to use a perfectly anhydrous oil. For directions for preparing this see Suchannek, Zeit. wiss. Mik., vii, 1890, p. 156, or the third edition of this work.

Anilin is chiefly used for clearing celloidin sections. It ought however to be soaked out before mounting by something else (chloroform or xylol for instance for some hours), as if not removed it will brown both the tissues and the mounting medium. 
135. Xylol, Benzol, Toluol, Chloroform.-Too volatile to be recommendable as clearing agents in which it is desired to examine specimens, but very useful for preparing paraffin sections for balsam. Of the three first-mentioned liquids, benzol is the most volatile, then toluol, and xylol is the least volatile, in the proportion of $4: 5: 9$ (Squire, Methods and Formulce, p. 20). Chloroform is injurious to some delicate stains, but is in other respects an excellent de-alcoholisation agent, as it will take up a good deal of water, if any be left in the preparations. I consider it too volatile to be safe to use before balsam. Xylol is the best of these in that respect.

Both xylol and toluol are liable to become acid if kept in only partially filled vessels. 


\section{CHAPTER VII.}

IMBEDDING METHODS-INTRODUCTION.

136. Imbedding Methods.-The processes known as Imbedding Methods are employed for a twofold end. Firstly, they enable us to surround an object, too small or too delicate to be firmly held by the fingers or by any instrument, with some plastic substance that will support it on all sides with firmness but without injurious pressure, so that by cutting sections through the composite body thus formed the included object may be cut into sufficiently thin slices without distortion. Secondly, they enable us to fill out with the imbedding mass the natural cavities of the object, so that their lining membranes or other structures contained in them may be duly cut in situ; and, further, they enable us not only to surround with the supporting mass each individual organ or part of any organ that may be present in the interior of the object, but also to fill with it each separate cell or other anatomical element, thus giving to the tissues a consistency they could not otherwise possess, and ensuring that in the thin slices cut from the mass all the minutest details of structure will precisely retain their natural relations of position.

These ends are usually attained in one of two ways. Either the object to be imbedded is saturated by soaking with some material that is liquid while warm and solid when cold, which is the principle of the processes here called Fusion Imbedding Methods; or the object is saturated with some substance which whilst in solution is sufficiently fluid to penetrate the object to be imbedded, whilst, after the evaporation or removal by other means of its solvent, it acquires and imparts to the imbedded object sufficient firmness for the purpose of cutting. The methods founded on this principle are here called Evaporation Imbedding Methods.

In any of these processes the material used for imbedding is technically termed an " imbedding mass."

There are two chief methods of imbedding - the paraffin method and the celloidin or collodion method.

The paraffin method is the one in most use; for it is the more rapid, requiring only hours where the celloidin process requires days or weeks; and it is the one which the most readily affords very thin 
sections. But this only applies to fairly small objects : with objects of much over half an inch in diameter you cannot easily get with paraffin much thinner sections than you can with celloidin; and if you try to cut in paraffin objects of still greater size, say an inch and upwards, it will frequently happen that you will not get perfect sections at all, blocks of paraffin of this size having a tendency to split under the impact of the knife. This defect is, however, much reduced by the employment of a softer paraffin than is usual. In this way Strasser (Zeit. wiss. Mik., ix, 1892, p. 7) has obtained series of frontal sections $30 \mu$ thick through the entire human brain, in paraffin blocks measuring $10 \times 15 \mathrm{~cm}$. And MAYER, with the Tetrander microtome, has obtained series of only $7 \cdot 5 \mu$ with a surface of $4 \frac{1}{2} \times 3 \mathrm{~cm}$.

For very large objects celloidin is safer, because it does not split, and presents advantages for the manipulation of the sections obtained. For all classes of objects it has the advantages of affording a transparent mass (which facilitates orientation of the object) and of producing less shrinkage than paraffin (paraffin unavoidably shrinks on cooling to at least 12 per cent.). It is for these two reasons that celloidin is so frequently preferred by embryologistseven for small objects.

Aqueous masses, such as gum or gelatin, may render great service in cases in which it is desired to avoid dehydrating tissues, and to. apply chemical tests to them.

13\%. Imbedding Manipulations.-Imbedding in a melted mass, such as paraffin, is performed in one of the following ways. A little tray or box or thimble is made out of paper, some melted mass is poured into it, and the object placed in the midst of it. Or, the paper tray being placed on cork, the object may be fixed in position in it whilst empty by means of pins and the tray filled with melted mass at one pour. The pins are removed when the mass is cold.

In either case, when the mass is cold the paper is removed from it before cutting.

To make paper trays proceed as follows. Take a piece of stout paper or thin cardboard, of the shape of the annexed figure (Fig. 1) ; thin (foreign) post-cards do very well indeed. Fold it along the lines $a a^{\prime}$ and $b b^{\prime}$, then along $c c^{\prime}$ and $d d^{\prime}$, taking care to fold always the same way. Then make the folds $A A^{\prime}, B B^{\prime}, C C^{\prime}, D D^{\prime}$, still folding the same way. To do this you apply $A c$ against $A a$, and pinch out the line $A A^{\prime}$, and so on for the remaining angles. This done, you have an imperfect tray with dogs' ears at the angles. To 
finish it, turn the dogs' ears round against the ends of the box, turn down outside the projecting flaps that remain, and pinch them down. A well-made post-card tray will last through several imbeddings, and will generally work better after having been used than when new.

Another method of folding the paper (MAYER) is described in the Grundzüge, LeE and MAYer, 4th ed., p. 77.

GIESBRECHT now makes trays of photographic films, which, being transparent, facilitate orientation under the dissecting microscope.

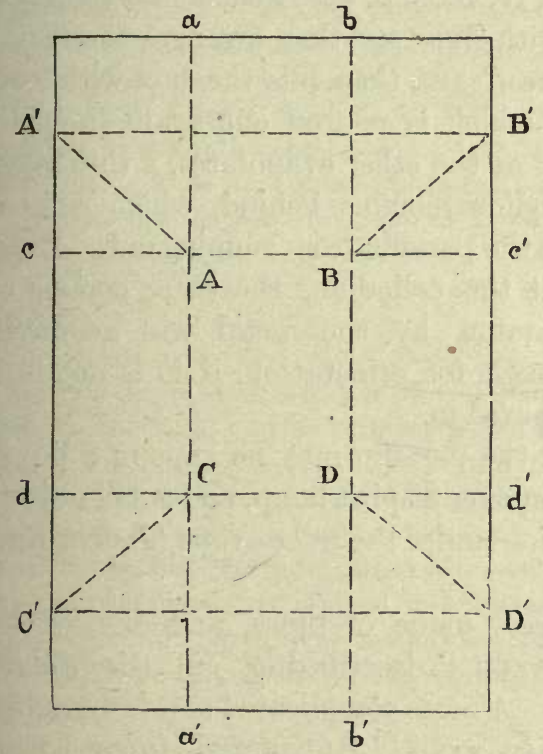

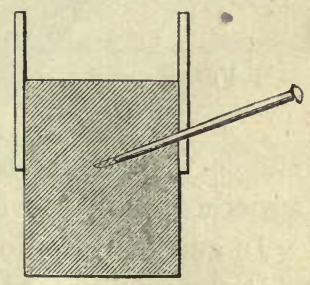

Fig. 2.

Fig. 1.

To make paper thimbles, take a good cork, twist a strip of paper several times round it so as to make a projecting collar, and stick a pin through the bottom of the paper into the cork. For work with fluid masses, such as celloidin, the cork may be loaded at the bottom by means of a nail or piece of lead, to prevent it from floating when the whole is thrown into spirit or other liquor for hardening (Fig. 2). Or you may use short lengths of solid lead rod instead of cork.

LEUCKHART's Imbedding Boxes are made of two pieces of typemetal (Fig. 3). Each of these pieces has the form of a carpenter's "square" with the end of the shorter arm triangularly enlarged outwards. The box is constructed by placing the two pieces together on a plate of glass which has been wetted with glycerine and gently warmed. The area of the box will vary according to the 
position given to the pieces, but the height can be varied only by using different sets of pieces. Two sets will be sufficient for most

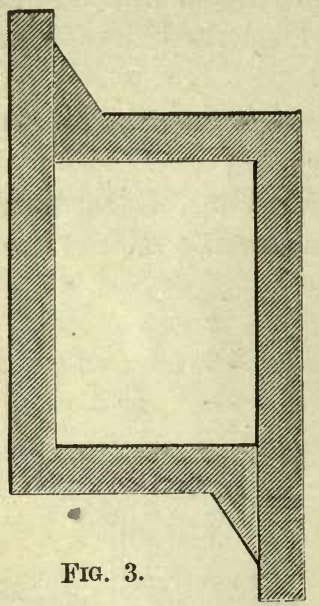
work, one set of 1 centimetre in height, and one of 2 centimetres, each being 8 centimetres in length, and 3 in breadth. To make the box paraffin-tight, so that it will hold the melted paraffin long enough in the liquid state to permit of the objects being carefully orientated in it, Mayer (Mitth. Zool. Stat. Neapel, iv, 1883, p. 429) first smears the glass plate with glycerine, then arranges the metal "squares," and then fills the box with collodion, which is poured out again immediately. As the ether evaporates, a thin layer of collodion remains behind, which suffices to keep the paraffin from running out. Even without the collodion, the mere cooling of the paraffin by the metal will generally suffice to keep it in long enough for orientation, if it is not in a superheated state when it is poured in.

In such a collodionised box the paraffin may be kept in a liquid state by warming now and then over a spirit lamp, and small objects be placed in any desired position under the microscope (Journ. Roy. Mic. Soc. [N.S.], ii, p. 880).

A lighter form of "squares," made of brass, and devised by Andres, Giesbrecht, and Mayer, is described loc. cit. (See Journ. Roy. Mic. Soc., 1883, p. 913.) A more complicated sort is described by Wruson in Zeit. wiss. Mik., xxvii, 1910, p. 228, for use with imbedded threads to serve as orientation guides. See "Orientation."

Frankr (Zeit. wiss. Mik., xiii, 1897, p. 438) builds up boxes with rectangular blocks of glass, which may be found convenient, but are more expensive than the metal squares.

SELENKa has described and figured another sort of apparatus having the same object. It consists of a glass tube, through which a stream of warm water may be passed and changed for cold as desired, the object being placed in a depression in the middle of the tube (see Zool. Anz., 1885, p. 419). A simple modification of this apparatus, which any one may make for himself, is described by ANDRews in Amer. Natural., 1887, p. 101 ; and a more complicated imbedding and orienting box, seldom necessary, is described by JORDAN in Zeit. wiss. Mil., xvi, 1899, p. 32 .

To imbed in a watch-glass, the object, previously saturated with paraffin, is put into a (preferably very concave) watch-glass con- 
taining molten paraffin.-After this has been solidified by cooling (see next chapter), a block containing the object is cut out of it, and mounted on the object-holder of the microtome (this is, of. course, applicable to other masses, such as celloidin).

For imbedding very small objects in this way certain precautions may be necessary in order not to lose them. SAMTER (Zeit. wiss. Mik., xi, 1894, p. 469) saturates small unstained objects with paraffin that has previously been strongly coloured with alkanna extract, and then imbeds them in pure paraffin. RHumbLer (ibid., xii, 1895, p. 312, and xiii, 1896, p. 303) stains previously the objects themselves with eosin dissolved in strong alcohol, and removes the stain from the sections with weak alcohol. See also ibid., xiii, p. 200, a paper by SCHYDLOwskI ; and in Zeit. wiss. Zool., lviii, 1897, p. 144, a process of BoRGERT.

BORGERT (Zeit. wiss. Zool., lviii, 1897, p. 144) allows paraffin to solidify in a watch-glass, bores a hole in it, and places the objects in the hole with a little benzol, and puts the whole for a short time into a stove.

A watch-glass provided at the bottom with a groove or trough, in which small objects may be made to collect, is described by LEFÈvRE, Journ. App. Mic., v, 1902, p. 280 (see Journ. Roy. Mic. Soc., 1903, p. 233).

LAAUTERBorn (Zeit. wiss. Zool., lix, 1895, p. 170) brings the objects through chloroform into paraffin in a small glass tube, and after cooling breaks the tube and so obtains a cylinder of paraffin with the objects ready for cutting.

HOYER (Arch. mik. Anat., liv, 1899, p. 98) performs all the operations in a glass cylinder ( 5 centimetres long and 7 millimetres wide), open at both ends, but having a piece of moist parchment paper tied over one of the openings. It is then not necessary to break the cylinder; by removing the parchment paper the paraffin can be pushed out of it in the shape of a cylinder containing the objects imbedded at one end of it.

MAYER (Zeit.wiss. Mikr., xxiv, 1907, p. 130). takes the gelatin capsules used by chemists ; after cooling in water the gelatin swells and is easily removed.

Meves (Arch. mikr. Anat., Ixxx, Abth. ii, 1912, p. 85) employs wedgeshaped capsules made by G. Pohl, Schönbaum, Bez, Dantzig. 


\section{CHAPTER VIII.}

IMBEDDING METHODS-PARAFFIN AND OTHER FUSION MASSES.

138. Saturation with a Solvent.-The first stage of the paraffin method consists in the saturation of the object with some substance which is a solvent of paraffin. The process is sometimes called "clearing," since many of the substances used for infiltration are also " clearing" agents.

The process of saturation should be carefully performed with well-dehydrated objects in the manner described in $\S 119$.

Saturation liquids being liquids that are, on the one hand, miscible with alcohol, and on the other hand good solvents of paraffin, are not quite as numerous as could be wished.

According to Graefe (Chem. Centralb., 1906, p. 874), at $20^{\circ} \mathrm{C}$. petroleum ether (1 c.c.) dissolves $200 \mathrm{mg}$. of paraffin, chloroform 246, benzol 285, carbon tetrachloride 317. And according to APÁTHy, at $20^{\circ} \mathrm{C}$. benzol dissolves 8 parts per cent., chloroform 10 , toluol 10, xylol 12, oil of turpentine 8 , cedar oil 4 to 6 , bergamot oil 0.5 to 3 , creosote and clove oil hardly any. Acetone, according to MAYer, dissolves hardly any.

Turpentine I do not recommend, because in my experience it is of all others the clearing agent that is the most hurtful to delicate structures.

Clove oil mixes very imperfectly with paraffin, and quickly renders tissues brittle.

Oil of bergamot mixes still more imperfectly with paraffin.

Benzol has been recommended by Brass (Zeit. wiss. Mik., ii, 1885, p. 301), and is now much used.

Toluol (or toluen) has been recommended by Hols (Zool. Anz., 1885, p. 223).

Xylol is said by M. Heidenhain (Kern. und Protoplasm, p. 114) to be a cause of shrinkage in cells. So it is, if you use it to dealcoholise the specimens. But used after oil of cedar, or the like, it is very good, as it is one of the best of solvents of paraffin.

Chloroform is deficient in penetrating power, so that it requires an excessive length of time for clearing objects of any size; and it must be very thoroughly got rid of by evaporation in the paraffin 
bath, or by successive baths of paraffin, as if the least trace of it remmains in the paraffin used for cutting it will make it soft. The process of removal requires a very long time, in some cases days. It ought therefore to be reserved for small and easily penetrable objects.

Naphtha has been recommended by Webster (Journ. Anat. and Physiol., xxv, 1891, p. 278).

FIELD and MarTiN (Zeit. wiss. Mik., xi, 1894, p. 10) recommend a light petroleum known as "petroleum-æther." It is highly volatile, and thus a cause of shrinkage.

Sulphide of carbon has been recommended by Heidenniain (Zeit. wiss. Mik., xviii, 1901, p. 166) as being a very powerful solvent of paraffin. Most workers have found it to be much too disagreeable and dangerous a reagent for ordinary work, and not necessary even for delicate work. See under "Teeth" and "Chitin."

Carbon tetrachloride has been recommended by PLEČnIK (op. cit, , xix, 1903, p. 328) and Pranter (ibid., p. 329) on the ground of not dissolving out osmium-blackened fats.

MAYER finds it no better than benzol.

As a general thesis, the best of all these are cedar oil, benzol, and chloroform.

Cedar-wood oil is, according to my continued experience, for the reasons stated by me in Zool. Anz., 1885, p. 563, for general work the very best clearing agent for paraffin imbedding. . It penetrates rapidly, preserves delicate structure better than any clearing agent known to me, does not make tissues brittle, even though they may be kept for weeks or months in it, and has the great advantage that if it be not entirely removed from the tissues in the paraffin bath it will not seriously impair the cutting consistency of the mass; indeed, I fancy it sometimes improves it by rendering it less brittle.

139. The Paraffin Bath.-The objects having been duly saturated with a solvent, the next step is to substitute melted paraffin for the saturating medium.

Some authors lay great stress on the necessity of making the passage from the saturating agent to the paraffin as gradual as possible, by means of successive baths of mixtures of solvent and paraffin kept melted at a low temperature, say $35^{\circ} \mathrm{C}$. With oil of cedar, at all events, this is not necessary. I simply put the objects into melted paraffin kept just at its melting-point, and keep them there till they are thoroughly saturated; the paraffin being changed once or twice for fresh only if the objects are sufficiently voluminous to have brought over with them a notable quantity of clearing agent. If the objects have been for a very long time-months or 
years-in the cedar oil, so that this has become thick, I remove it partially or entirely by soaking in xylol (thirty minutes to several hours) before putting into the paraffin. But with fresh oil of cedar I find no advantage in doing so.

Giesbrecht's method (Zool. Anz., 1881, p. 484), is as follows :Objects to be imbedded are saturated with chloroform, and the chloroform and objects are gradually warmed up to the melting-point of the paraffin employed, and during the warming small pieces of paraffin are by degrees added to the chloroform. So soon as it is seen that no more bubbles are given off from the objects, the addition of paraffin may cease, for that is a sign that the paraffin has entirely displaced the chloroform in the objects. This displacement having been a gradual one, the risk of shrinkage of the tissues is reduced to a minimum.

MAYER (Grundzüge, LeE and MAYER, 1910, p. 84) first saturates the objects with benzol, and then adds to the benzol some small pieces of paraffin, and lets them dissolve in the cold. After several hours (up to eighteen) the whole is brought in an open vessel on to the cold water-bath, the bath is then warmed gradually so as to attain a temperature of $60^{\circ} \mathrm{C}$. in about two hours, and as fast as the benzol evaporates melted paraffin is added to it. Lastly, the paraffin is changed once before the definitive imbedding. $\mathrm{He}$ rarely leaves objects overnight in the water-bath.

АРÁтнY (Mikrotechnik, pp. 149, 150) first clears with oil of cedar, then brings the objects (by the process described $\S 119$ ) into a solution of paraffin in chloroform saturated at the temperature of the laboratory. The objects remain in the chloroform-paraffin solution for from one to three hours, without warming, until all the cedar oil is soaked out of them. The whole is then warmed on the waterbath or oven to a few degrees above the melting-point of the paraffin intended to be used for imbedding, and the object is brought into a mixture of equal parts of paraffin and chloroform, being suspended therein near the top on a bridge made of hardened filter paper (or in a special apparatus to the same end, not yet described). It remains in this mixture, at the temperature of the oven, for one to three hours, and lastly is brought (still on the paper bridge or in the apparatus) into pure paraffin, where it remains for. half an hour to two hours.

Denne (in litt., 1907) points out that the objects ought at first to be at the bottom of the mixture. For this mixture is not a true solution, and the lower section of the contents of the tube is comparatively free from paraffin while the upper part is nearly pure 
paraffin. He moves the holder up in the tube at intervals, and the infiltration proceeds gradually with the minimum risk of shrinkage. Lastly, he removes the objects, on the holder, to the top of a tube of pure paraffin.

The practice of giving successive baths first of soft and then of hard paraffin, which has been frequently advised, appears to me entirely illusory.

It is important to keep the paraffin $d r y$-that is, protected from vapour of water during the bath.

It is still more important to keep it as nearly as possible at meltingpoint. If it be heated for some time to a point much over its normal melting-point, the melting-point will rise, and you will end by having a harder paraffin than you set out with. And as regards the preservation of tissues, of course, the less they are heated the better. Overheating, as well as prolonged heating, tends, amongst other things, to make tissues brittle.

The duration of the bath must, of course, vary according to the size and nature of the object. An embryo of 2 to 3 millimetres in thickness ought to be thoroughly saturated after an hour's bath, or often less. Many workers habitually give much longer baths, I think often longer than necessary. But some objects, such as ova of Crustacea, may require three or four days (HeIdEcke, Jena. Zeit., xxxviii, 1904, p. 506 ; Mayer, Grundzüge, Lee and Mayer, 1910, p. 85; Brinkmann, Mitth. Zool. Stat. Neapel, xvi, 1903, p. 367, three to five days for uterus of Selachians; Müller, Arch. mikr. Anat., lxix, 1906, p. 3, for lungs of mammals ; Poso, Esperienze microtechniche, Napoli, 1910 , p. 29, five to twelve days for uterus and placenta of Homo). I take as a guide, generally, the length of time the object has taken to clear in the cedar oil, assuming that the warm melted paraffin ought to penetrate at least as quickly as the cold oil ; and then allowing somewhat longer, say as much again, in order to be on the right side.

140. Water-baths and Ovens. - It is important that the paraffin should not be exposed to a moist atmosphere whilst it is in the liquid state. If a water-bath be used for keeping it at the required temperature provision should be made for protecting the paraffin from the steam of the heated water.

A very convenient apparatus for this purpose is that of Paul Mayer, or "Naples water-bath," which will be found described at p. 146 of Journ. Roy. Mic. Soc., 1883, or CARPENTER's The Microscope, p. 452. An extremely simple stove, which any one can make for himself, is described in Centralbl. Bakt., xlv, 1907, p. 191 (see Journ. Roy. Mic. Soc., 1908, p. 109). For others, see the price-lists of the instrument makers, 
especially Jung, and GRÜBLER and HOLLBORN ; and the descriptions in the technical journals.

141. Imbedding IN VACU0.-There are objects which, on account of their consistency or their size, cannot be penetrated by paraffin in the ordinary way, even after hours or days in the bath. For such objects the method of imbedding under a vacuum (strictly, under diminished atmospheric pressure) renders the greatest service. It not only ensures complete penetration in a very short time - a few minutes-but it has the further advantage of preventing any falling in of the tissues, such as may easily happen with objects possessing internal cavities if it be attempted to imbed them in the ordinary way. It is realised by means of any arrangement that will allow of keeping paraffin melted under a vacuum.

That of HofFmann is described and figured at p. 230 of Zool. Anz., 1884. In this arrangement the vacuum is produced by means of a pneumatic water aspiration pump, the vessel containing the paraffin being placed in a desiccator heated by a water-bath and furnished with a tube that brings it into communication with the suction apparatus.

Francotte (Bull.. Soc. Belg. Mic., 1884, p. 45) produces the requisite vacuum by the condensation of steam.

FOL (Lehrb., p. 121) employs the vacuum apparatus of Hoffmann, but simplifies the arrangement for containing the paraffin. The paraffin is contained in a stout test-tube furnished with a rubber stopper traversed by a tube that puts it into communication with the pump. The lower end of the test-tube dips into a water-bath. You pump out the air once or twice, wait a few minutes, then turn out the object with the paraffin (which by this time will have become abnormally hard), and re-imbed in fresh paraffin.

See also Pringle, in Journ. Path. and Bacteriol., 1892, p. 117, or Journ. Roy. Mic. Soc., 1892, p. 893 ; Kolster, in Zeit. wiss. Mik., xviii, 1901, p. 170 ; B́erg, Zeit. wiss. Mik., xxvi, 1909, p. 209 ; Fuhrmann, ibid., xxi, 1904, p. 462 ; Kolmer and WolfF, ibid., xix, 1902, p. 148 ; Gemmill, Journ. Roy. Mic. Soc., 1911, p. 26.

142. Imbedding and Orientation.-As soon as the objects are thoroughly saturated with paraffin they should be imbedded by one of the methods given above (§137), and the paraffin cooled as described next $\S$.

But it may be desirable to have the object fixed in the cooled paraffin in a precisely arranged position, and, above all, in a precisely marked position. Very small objects may be oriented as follows :The object is removed from the melted paraffin, and placed on a cylinder of solid paraffin. A needle or piece of stout iron wire is now heated in the flame of a lamp, and with it a hole is melted in the end of the cylinder; the specimen is pushed into the melted paraffin, and placed in any desired position. The advantages of the method lie in the quickness and certainty with which it can be performed. 
In using the needle it is important to melt as little paraffin as possible at one time, in order that that which is melted may cool again as rapidly as possible.

KERR (Quart. Journ. Micr. Sc., xlv, 1901, p. 4) employs an electrically heated needle.

The method of Patten (Zeit. wiss. Mik., xi, 1894, p. 13) is useful when one desires to orient large numbers of small objects. You get some writing paper of the sort that is made with two sets of raised parallel lines running at right angles to each other ("linen cloth paper "). Small strips are cut from this, and at suitable intervals along them small drops of a mixture of collodion and clove oil, of about the consistency of thick honey, are arranged close together along one of the ribs that run lengthwise. The objects to be imbedded are cleared in clove oil or oil of bergamot. They are taken one by one on the point of a knife, and after the excess of oil has been drawn off, are transferred each to a drop of the collodion mixture, in which they will stay in any required position. When half a dozen or more objects have been oriented in reference to the cross lines (which are to be parallel to the section planes) the whole thing is placed in turpentine. This washes out the clove oil and fixes the objects very firmly to the paper. 'The paper with the attached objects is now passed through the bath of paraffin and imbedded in the usual way. After cooling on water the block is trimmed and the paper peeled off, leaving the objects in the paraffin close to the under-surface of the block. This surface is now seen to be marked by the orienting lines of the ribbed paper, and also by any record numbers which may before imbedding have been written with a soft pencil on the paper.

KNowen (Journ. Morph., xvi, 1900, p. 507) takes smooth paper and engraves parallel lines on it with a needle, and takes xylol instead of turpentine.

A somewhat more complicated form of this process has been described by Woodworth, Bull. Mus. Comp. Zool., xxxviii, vol. xxv, 1893, p. 45.

A similar process has also been described by FIELD and Martin in Zeit. wiss. Mik., xi, 1894, p. 11, small strips of gelatin being used instead of paper.

MAYER also (Grundzüge, LEe and MAYER, 1910, p. 89) takes strips of photographic gelatin, and lets the collodion set in benzol.

HofFManN (Zeit. wiss. Mik., xv, 1899, p. 312, and xvii, 1901, p. 443) takes, instead of the ribbed paper, glass slips ruled with a diamond, and completely imbeds the objects in large drops of clove oil collodion (equal parts), allowed to stand for twenty-four hours in an open vessel. The drops are caused to set in xylol. See also SAMTER, ibid., xiii, 1897, p. 441 ; Jordan, ibid., xvi, 1899, p. 33 ; and Peter, Verh. Anat. Ges., xiii Vers., 1899, p. 134.

Entz (Arch. Protistenk., xv, 1909, p. 98) orients in clove oil collodion on a cover-glass coated with paraffin, and puts the whole into chloroform in which the mixture sets into a sheet which can be detached.

Denne (Journ. Appl. Mic., iii, 1902, p. 888) imbeds on disks of paper held at the bottom of glass tubes containing the paraffin by bent wires, 
by means of which a cylinder of paraffin containing the object may be lifted out as soon as cool.

Wilson (Zeit. wiss. Mik., xvii, 1900, p. 169) makes orientation lines by imbedding alongside the objects strands of osmium-blackened nervefibres. See also a further development by Wilson, ibid., xxvii, 1910, pp. 228 and 231.

143. Cooling the Mass.-Whatever method of imbedding and orientation in the molten paraffin has been employed, the important point now to be attended to is that the paraffin be cooled rapidly. The object of this is to prevent crystallisation of the paraffin (which may happen if it be allowed to cool slowly) and to get as homogeneous a mass as possible.

If the definitive imbedding has been done in a watch-glass, hold it on the top of cold water until all the paraffin has solidified, and then let it sink to the bottom. When thoroughly cool, cut out blocks containing the objects. If the watch-glass has been smeared with a drop of a mixture of equal parts of glycerine and water before putting the paraffin into it, the solidified paraffin will generally detach itself in a single cake and float up in a few minutes, or hours at any rate. Do not attempt to remove it entire by warming the bottom of the watch-glass. Similarly with the paper trays or metal imbedding boxes. Or you may put them to cool on a cold slab of metal or stone.

SELENKa cools the mass by passing a stream of cool water through the imbedding tube described above ( $\$ 137)$. MAYER cools the mass in the paraffin-tight moulds ( $\$ 137$ ) by passing cold water through a special movable water-bath, which allows of the arrangement of the objects by transmitted light under a dissecting microscope, see Mitth. Zool. Stat. Neapel, iv, 1883, p. 429 ; Intern. Monatsschr. Anat. Hist., iv, 1887, p. 39. A complicated apparatus for the same purpose is described by MeIssner (Zeit. wiss. Mik., xviii, 1902, p. 286). Similarly, HAHN, ibid., xxv, 1908, p. 184, and KAPPERS, ibid., xxiv, 1907, p. 254. See also FARKAS, ibid., $\mathrm{xxx}, 1913, \mathrm{p} .168$, for experiments on cooling methods.

The paraffin blocks with the objects are now mounted on the carrier of the microtome in position for cutting, and pared to the proper shape (next §). If any bubbles or cavities or opaque spots be present, prick with a heated needle till all is smooth and homogeneous. The same should be done if any cavities present themselves in the course of cutting. In bad cases, re-imbed.

144. Shape and Orientation of the Block of Mass to be cut.-These differ accordingly as the cutting is done with a slanting knife or a square-set knife (see next §). In the first case, the block is best trimmed to a three-sided prism, and orientated as in Fig. 4, so that 
the knife enters it at the angle $a$ and leaves it at the angle $c$. When the section is cut it will adhere to the knife only by the angle $c$, and can thus most readily be removed by means of a brush or needle. The object itself should come to lie in the block close to the line $b c$, so that the knife at first cuts only paraffin, and that if the section begins to roll it may be caught and held down by a brush or sectionstretcher before the object itself is reached. For the square-set knife the block is best trimmed to a four-sided prism, and orientated as in the first case, so that the knife first touches one angle, if only isolated sections are to be cut. But if ribbons ( $(148)$ are to be cut, the block must be orientated with one of its sides parallel to the knife-edge, and the opposite side must be strictly parallel to this one.

An object which is not approximately isodiametrical but gives a section which is wider in one direction than another should be orientated end on, that is, so as to present its narrowest diameter to the knife-edge : for it is in this position that it will offer the least resistance to the blade, and tend the least to make the edge bend away or dig into it. This is specially important with longitudinal sections of worms, Amphioxus, embryos of vertebrates, and the like. Most especially with a square-set knife should the narrowest diameter of the object be presented to the knife; and only when

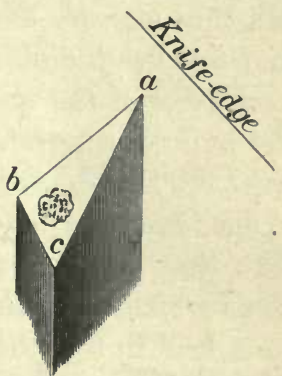

Fig. 4. the object is particularly hard, or otherwise difficult to cut, should it be turned so as not to let the whole of that diameter be attacked at once by the knife, but only a corner of it. And as far as possible arrange that the hardest part of an object be the last to be touched by the knife.

For NoAck's simple apparatus for accurately orientating small blocks, see Zeit. wiss. Mik., xv, 1899, p. 438, or Journ. Roy. Mic. Soc., 132, 1899, p. 550.

For ETERNOD's machine for trimming blocks to true cubes, see Zeit. wiss. Mik., xv, p. 421, and for that of SCHAFFER, ibid., xvi, 1900, p. 417.

145. Knife Position.-The position to be given to the knife may be considered under two heads, viz., its slant and its tilt.

By the slant of the knife is meant the angle that its edge mak s with the line of section: that is, with the line along which it is drawn through the object (or along which the object moves across it in the case of microtomes with fixed knives). The position is 
transverse when the edge makes an angle of $90^{\circ}$ with the line of section, or the knife in that case is said to be set square. It is oblique or slanting when it makes a smaller angle with that line. The difference between the effect of the two positions is that the oblique position affords a more acute-angled wedge than the transverse one.

It does so for the following reasons:-Neglecting for the moment the distinction between the cutting-facets and the surfaces of the blade (which are distinct usually because they are not ground to the same angle), ${ }^{*}$ it is clear that the knife itself is a wedge, the angle of which depends on the relation between the height of its base and the distance from the base to the edge. With the same base the angle becomes more acute the greater the distance from edge to base. Now by slanting the knife we can effect what is equivalent to an increase in the distance from edge to base; for we can thus increase the distance between the point of the edge which first touches the object; and the point of the back (strictly, of the back edge of the under cutting-facet) which last leaves it. When the knife is set transversely, the line along which any point of it traverses the object is the shortest possible from edge to base of the wedge, and the effective angle of wedge is the least acute obtainable with that knife. But if it is set as obliquely as possible, the line along which any point of it traverses the object traverses the knife from heel to toe, that is, along the greatest possible distance from edge to base, and therefore affords practically a much more acuteangled wedge than in the first case ; and so on, of course, for intermediate positions. (See the stereometrical constructions of these relations by SCHIEFFERDECKER, op. cit., p. 115 ; and also with more instructive figures, АрÁтHy, "Ueber die Bedeutung des Messerhalters in der Mikrotomie," in Sitzber. med.-naturw. Section d. Siebenbürgischen Museumvereins, Bd. xix, Heft 7. p. 1 (Kolozsvár, 1897, A. K. Ajtai).

For honing knives see Ssobolew, Zeit. wiss. Mik., xxvi, 1909, p. 65 ; LendvaI, ibid., p. 203 ; Funck, ibid., xxvii, 1910, p. 75.

Very large objects are best cut with the slanting knife, and so are all objects of very heterogeneous consistency, such as tissues

* The edge of a microtome knife is eomposed of two plane surfacesthe upper and lower cutting-facets, which meet one another at an acute angle, the cutting-edge, and posteriorly join on to the upper and lower surfaces of the blade (see some good figures of differently shaped knives in BeHrens, Kossel und SchiefFerdecker, Das Mikroskop., p. 115, et seq.; and in APÁtry's paper quoted below). It will be seen that the two facets together form a wedge welded on to the blade by the base. 
that contain much chitin or much muscular tissue ; and better with a slowly working sliding microtome than with a quick-working Rocker or the like. Soft masses such as gelatin or celloidin cut wet, can only be cut with the slanting knife. The slanting position causes less compression of sections than the transverse one. It has the defect of producing rolling in paraffin sections more easily than the transverse position. The latter is the proper position for cutting ribbons of sections from paraffin.

By the tilt of the knife is meant the angle that a plane passing through its back and edge makes with the plane of section: or, practically, the greater or less degree of elevation of the back above the edge (it is not to be confounded with the inclination of the long axis of the knife to the horizon; any accidental inclination that this may have is a matter of no moment).

The question of the proper tilt to be given to the knife under different circumstances has been investigated by Apáthy, loc. cit. supra. He concludes - (1) The knife should always be tilted somewhat more than enough to bring the back of the under cutting-facet clear of the object. (2) It should in general be less tilted for hard and brittle objects than for soft ones; therefore, coteris paribus, less for paraffin than for celloidin. (3) The extent of useful tilt varies between $0^{\circ}$ and $16^{\circ}$ or occasionally $20^{\circ}$. (4) Excessive tilt causes rifts (longitudinal) in the paraffin, also furrows that in bad cases split up the section into narrow ribbons. It also makes sections roll. Also it may cause the knife not to bite, thus causing sections to be missed. Or it may give an undulatory surface to the sections, owing to vibrations set up in the knife, which may be heard as a deep humming tone. Further, I would add, excessive tilt may cause the knife to act as a scraper, carrying away portions of tissue bodily from their places. Excessive tilt may often be recognised by the knife giving out a short metallic sound just as it leaves the object. For knives with plane under-surfaces it is seldom advisable to give less than $10^{\circ}$ tilt. Knives with concave undersurfaces, on the contrary, may require to be placed almost horizontal. Jung's knife-holders give mostly a tilt of about $9^{\circ}$, which is only enough for cutting ribbons with hard paraffin.

A knife with too little tilt will often cut a second section, or fragments of one, without the object being raised, showing that during the first cut the object was pressed down by the knife, and recovered itself afterwards. This fault is denoted by the ringing tone given out by the knife on passing back over the object before the latter is raised. Such a knife gives out a dull rattling sound whilst 
cutting. Too little tilt causes folding or puckering of sections, and does not allow of the cutting of the thinnest possible sections, as the edge does not bite enough. It is thus frequently a cause of sections being missed, or coming off thicker at one end than the other.

A slanting knife should have more tilt given to it than a squareset one.

Ribbon section-cutting ( $\$ 148$ ) requires a relatively hard paraffin and less tilt. With celloidin it is very important to avoid insufficient tilt, as the elastic celloidin yields before an insufficiently tilted knife and is not cut.

The tilt of the knife is given to a certain extent by the knifeholder sold with the microtome. With plane-concave knives it can be regulated to a certain extent by simply turning the blade over. It is more accurately regulated by means of mechanical contrivances, of which the most simple are the horseshoe-shaped wedges of Neumayer (see Jung's price-list). A pair of these, each ground to the same angle, is taken, and one of them placed (thin end towards the operator) under, and the other (thick end towards the operator) over, the clamping-arm of the knife-holder. Three pairs, having different degrees of pitch, are supplied, and are sufficient for most work. Other contrivances to the same end consist of knife-holders that permit of rotating the knife on its long axis, and, though more costly, will be found a great convenience where much sectioncutting has to be done. For these see Jung's price-list, and various recent papers in Zeit. wiss. Mik., also that of APÁTHy, in the paper quoted above (very complicated), and especially the description of the two latest of Jung, viz., his model $l$ and model $n$, by MAYER and Schozber, in Zeit. wiss. Mik., xvi, 1899, p. 29 (see figure of model $l$ in Journ. Roy. Mic. Soc., 132, 1899, p. 546). Also CarPenter's The Microscope, p. 463.

146. Cutting and Section-stretching.-Paraffin sections are cut $d r y$,-that is, with a knife not moistened with alcohol or other liquid. By this means better sections are obtained, but a difficulty generally arises owing to the tendency of sections so cut to curl up on the blade of the knife. It is sometimes difficult by any means to unroll a thin section that has curled. To prevent sections from rolling, the following points should be attended to.

First and foremost, the paraffin must not be too hard, see $\S 151$.

If, after cutting has begun, the paraffin be found to be too hard, it may be softened by placing a lamp near the imbedded object. But then, the paraffin being warmed most on the side nearest the lamp. 
becomes softer on that side, and the sections have a tendency to become compressed and puckered-in on that side.

If, on the contrary, the paraffin be found too soft, it may be hardened by exposing it to the cooling influence of a lump of ice.

It is often sufficient to moderate the temperature of the room by opening or closing the window, stirring the fire, setting up a screen, or the like.

For other devices for warming or cooling the paraffin see HELD, Arch. Anat. Phys., Anat. Abth., 1897, p. 345 ; Van Walsem, Zeit. wiss. Mik., xi, 1894, p. 218 ; LeNDENFELd, ibid., xviii, 1901, p. 18 ; Krause, ibid., xxv, 1908, p. 299 ; Foot and Strobell, Biol. Bull. Wood's Hole, ix, 1905, p. 281.

Secondly, the knife should be set square, for the oblique position encourages rolling, and the more the knife is oblique the more do the sections roll.

Thirdly, it is better to cut ribbons than disconnected sections; ribbons of sections will often cut flat, when the same mass will only give rolled sections if cut disconnectedly.

Rolling may often be lessened or suppressed by cutting the sections thinner.

Mechanical means may be employed. The simplest of these is as follows :

During the cutting the edge of the section that begins to curl is caught and held down on the blade of the knife by means of a small camel-hair brush with a flat point, or by a small spatula made by running a piece of paper on to the back of a scalpel. Or, which is much better, the section is held down by means of an instrument called a "section-stretcher." This consists essentially of a little metallic roller suspended over the object to be cut in such a way as to rest on its free surface with a pressure that can be delicately regulated so as to be sufficient to keep the section flat without in any way hindering the knife from gliding beneath it.

See the descriptions of various forms of section-stretchers, Zool. Anzeig., vol. vi, 1883, p. 100 (Schultze); Mitth. Zool. Stat. Neapel, iv, 1883 , p. 429 (MAYer, ANDres, and Giesbrecht); Arch. mik. Anat., xxiii, 1884, p. 537 (DECKER); Bull. Soc. Belg. Mic., x, 1883, p. 55 (FRANсотте); The Microscope, February, 1884 (GAGE and SMITH); WhitMaN's Meth. in Mic. Anat., 1885, p. 91 ; Zeit. wiss. Mik., iv, 1887, p. 218 (STRASSER) ; ibid., x, 1893, p. 157 (BORN). The best are those of Mayer and Born.

I find that MAYER's, beautifully made by JUNG, works admirably and is most valuable.

Another plan is to allow the sections to roll, but to control the rolling. To this end, the block of paraffin is pared to the shape of a 
wedge five or six times as long as broad, the object being contained in the broad part, and the edge turned towards the knife (see Fig. 4). The sections are allowed to roll and come off as coils, the section of the object lying in the outermost coil, which will be found to be a very open one-indeed, very nearly flat. Lay the coil on a slide with this end downwards, warm gently, and the part containing the object will unroll completely and lie quite flat.

ANILe (Glandole duodenali, Napoli, 1903, p. 51) and VastariniCResi (Mon. Zool. Ital., 1906, p. 164) lay a strip of wet filter-paper on the block.

A defect opposite to that of the rolling of sections is the compression and the crumpling or puckering of sections, indicating that the paraffin has been compressed by the knife instead of being merely cut true by it. Such sections, besides showing creases or folds, have a smaller area than that of the block from which they are cut. This is a bad fault, for the compression may obliterate important cavities or efface important limits between cell-layers, etc. It may be caused by a badly cutting knife, and is very easily caused by the paraffin being too soft. To prevent it, correct the knife or cool the paraffin, or re-imbed in harder paraffin.

Very large sections tend to form folds on the knife, and are difficult to remove from it. MAYER (Grundzüge, LeE and MAYER, p. 94) gets them to wrap themselves round a glass or gelatin tube laid on the block just in front of the knife-edge and rolled forwards as it progresses. When cut, the section is rolled off on to the surface of water.

14\%. Cutting Brittle Objects (Collodionisation).- Some objects are by nature so brittle that they break or crumble before the knife, or furnish sections so friable that it is impossible to mount them in the ordinary way. Ova are frequently in this case. A remedy for this state of things consists in covering the exposed surface of the object just before cutting each section with a thin layer of collodion, which serves to hold together the loose parts ; and will enable the operator to cut sections considerably thinner than can be obtained in the usual way.

The primitive form of the process was to place a drop of collodion on the free surface of each section just before cutting it. But this practice has two defects; the quantity of collodion employed sensibly softens the paraffin, and the thick layer of collodion when dry causes the sections to roll.

MARK (Amer. Natural., 1885, p. 628 ; cf. Journ. Roy. Mic. Soc., 1885, p. 738) gives the following directions :

"Have ready a little very fluid collodion in a small bottle, through 
the cork of which passes a small camel-hair brush, which just dips into the collodion with its tip. The collodion should be of such a consistency that when applied in a thin layer to a surface of paraffin it dries in two or three seconds without leaving a shiny surface. It must be diluted with ether as soon as it begins to show signs of doing so.

"Take the brush out of the collodion, wipe it against the neck of the bottle, so as to have it merely moist with collodion, and quickly pass it over the free surface of the preparation. Care must be taken not to let the collodion touch the vertical surfaces of the paraffin, especially not the one which is turned towards the operator, as that will probably cause the section to become stuck to the edge or undersurface of the knife. As soon as the collodion is dry, which ought to be in two or three seconds, cut the section, withdraw the knife, and pass the collodion brush over the newly exposed surface of the paraffin. Whilst this last layer of collodion is drying, take up the section from the knife and place it with the collodionised surface downwards on a slide prepared with fixative of Schaellibaum. Then cut the second section, and repeat the manipulations just described in the same order."

Henking (Zeit. wiss. Mik., iii, 1886, p. 478) takes instead of collodion a solution of paraffin in absolute alcohol.

For extremely brittle objects, such as ova of Phalangida, he recommends a thin (light yellow) solution of shellac in absolute alcohol.

HeIDER (Embryonalentw. v. Hydrophilus, 1889, p. 12 ; cf. Zeit. wiss. Mik., viii, 1892, p. 509) employs a solution made by mixing a solution of gum mastic in ether, of a syrupy consistency, with an equal volume of collodion, and diluting the. mixture with ether until quite thin and liquid.

RABL (ibid., xi, 2, 1894, p. 170) employs superheated paraffin (of about $100^{\circ} \mathrm{C}$.). This has the advantage of filling up any cavities there may be in the objects, and also of preventing the sections from rolling. A complicated development of this process is described by LENDENFELD in Zeit. wiss. Mik., xviii, 1901, p. 18.

APÁthy (Mikrotechnik, p. 183) employs a 1 per cent. solution of celloidin, allows the sections to roll, and unrolls them by the waterprocess (§ 149).

Jordan (Zeit. wiss. Mik.) adds 5 drops of oil of cedar to 15 c.c. of the solution of celloidin, and finds that rolling is prevented.

148. Ribbon Section-cutting.-If a series of paraffin sections be cut in succession and not removed from the knife one by one as cut, but allowed to lie undisturbed on the blade, it not unfrequently happens that they adhere to one another by the edges so as to form a chain or ribbon which may be taken up and transferred to a slide 
without breaking up, thus greatly lightening the labour of mounting a series. For the production of a ribbon, the paraffin must be of a melting-point having the right relation to the temperature of the laboratory, see $§ 151$. Secondly, the knife should be set square. Thirdly, the block of paraffin should be trimmed so as to present a straight edge parallel to the knife-edge; and the opposite edge should also be parallel to this. It is by no means necessary to have recourse to special mechanical contrivances, as in the so-called ribbon microtomes; the Thoma microtome is sufficient. But the automatic microtomes, and amongst them the Cambridge Rocking Microtome and the Minot, are certainly most advantageous for this purpose.

If the paraffin is very hard, it is necessary for sections of $10 \mu$, and advisable for thinner ones, to coat the block with softer paraffin. To do this, take paraffin of about $40^{\circ} \mathrm{C}$. melting-point, melt it, heat it to about $80^{\circ}$ on the water-bath, dip the block into it for an instant, and rapidly turn it over so that the fluid paraffin may run down away from the top part as much as possible. Allow it to cool, and pare away again the soft paraffin from the two sides that are not to be arranged parallel to the knife. Or, as I frequently prefer, simply plaster a wall of soft paraffin (superheated) on to the fore and aft faces of the block with a small spatula. Large blocks may have two coatings given them.

It sometimes happens that the ribbon becomes electrified during the cutting, and twists and curls about in the air in a most fantastic and undesirable manner. It may be got flat by warming slightly.

149. Section Flattening.-The sections having been obtained may be cleared and mounted at once if they are quite perfect, that is, neither rolled nor creased nor compressed. But should they in the least degree show any of these defects, they must first be unrolled or smoothed, or expanded to their proper dimensions.

The most efficacious plan is combined treatment with fluid and heat. The sections are either floated on to the surface of warm water or warm alcohol contained in a suitable dish, which causes them to flatten out perfectly, and are then transferred to a slide, by floating them into position, or otherwise. Or the slide has a layer of water spread over it, the sections are laid on the water, and the slide is heated (to somewhat below the melting-point of the paraffin) until the sections flatten out, which happens in a few seconds.

A special water-bath for flattening sections is described by Nowak in Zeit. wiss. Mik., xir, 1896, p. 447. 
150. Clearing and Mounting.-The sections having been duly smoothed by one of these processes, and duly fixed to the slide (Chapter X), unless it is desired to keep them loose, all that now remains is to get rid of the paraffin and mount or stain as the case may be. Many solvents have been recommended for this purpose :Turpentine, warm turpentine, a mixture of 4 parts of essence of turpentine with 1 of creasote, creasote, a mixture of turpentine and oil of cloves, benzin, toluol, xylol, thin solution of Canada balsam in xylol (only applicable to very thin sections), hot absolute alcohol, naphtha, or any other paraffin oil of low boiling-point. Of these xylol and toluol are generally in most respects the best. Benzol and chloroform are too volatile for safe manipulation.

If the slide be warmed to the melting-point of the paraffin, a few seconds will suffice to remove the paraffin if the slide be plunged into a tube of xylol or toluol. For thin sections, 10 to $15 \mu$, it is not necessary to warm at all. The sections may be mounted direct from the xylol, or the slide may be brought into a tube of alcohol to remove the solvent for staining.

Paraffin sections can be stained without removal of the paraffin, so that after-treatment with alcohol can be suppressed, but this is only very exceptionally advantageous.

151. Pure Paraffin.- It is now almost universally admitted that pure paraffin is superior for ordinary work to any of the many mixtures with wax and the like that used to be recommended. Paraffin varies enormously in hardness according to the temperature of its surroundings. It should therefore be taken of a meltingpoint suitable to the temperature of the laboratory. A paraffin melting at $50^{\circ} \mathrm{C}$. or a little harder, is that which in my experience gives the best results so long as the temperature of the laboratory is between $15^{\circ}$ and $17^{\circ} \mathrm{C}$. For higher temperatures a harder paraffin is required, and for lower temperatures a softer one.

Many workers of undoubted competence prefer masses somewhat harder than this ; so, for instance, Heidenhain $\left(58^{\circ}\right)$, Apáthy $\left(55^{\circ}\right)^{\circ}$, Rabl $\left(56^{\circ}\right)$, Mayer $\left(58^{\circ}\right.$ to $60^{\circ}$ in summer ; in winter about $56^{\circ}$, but never less than $50^{\circ}$ ). Mayer points out that at Naples the temperature during five months of the summer and autumn is over $22^{\circ} \mathrm{C}$. in the laboratory, sometimes over $30^{\circ}$. Temperatures such as these are seldom realised in the British Isles, and, whilst I quite admit that such hard paraffin may have its raison d'être for Naples, I hold that for that very reason it is in general unnecessarily hard for cooler climates.

My recommendation of a relatively soft paraffin refers to work with the Thoma sliding microtome. Microtomes with fixed knives, 
such as the Cambridge, the Minot, or the Reinhold-Giltay, will give good results with much harder paraffin, and, in fact, require such.

Stout knives of hard steel will take a harder paraffin than thin ones of soft steel; but the latter may be preferable for soft masses.

For thin sections a harder paraffin is required than for thick ones.

Hard objects require a harder paraffin than soft ones.

Brass (Zeit. wiss. Mik., ii, 1885, p. 300) recommends paraffin that has been kept for some years, as it has less tendency to crystallise than new paraffin.

Paraffin of various melting-points is easily found in commerce. Intermediate sorts may be made by mixing hard and soft paraffin. I find that 2 parts of paraffin melting at $50^{\circ}$ with 1 of paraffin melting at $36^{\circ} \mathrm{C}$. give a mass melting at $48^{\circ} \mathrm{C}$, and a mixture of 1 part of that melting at $53^{\circ}$ with 1 part of that melting at $45^{\circ}$ gives a mass melting at $50^{\circ} \mathrm{C}$.

According to E. Burchardt (Jena Zeit. Naturw., xxxiv, 1900, p. 719) mixtures of paraffins of different melting-points give better results than an unmixed paraffin of the same melting-point as the mixture. He recommends 10 parts of $40^{\circ}$ paraffin +1 of $45^{\circ}+1$ of $52^{\circ}+1$ of $58^{\circ}+6$ of $60^{\circ}$.

For methods for ascertaining melting-points see Kissling, Chem. Centralb. ii, 1901, p. 507.

152. Overheated Paraffin.-SPEE (Zeit. wiss. Mik., ii, 1885, p. 8) takes paraffin of about $50^{\circ} \mathrm{C}$. melting-point and heats it in a porcelain capsule by means of a lamp until it has become brownish-yellow, and after cooling shows an unctuous or soapy surface on being cut. This mass may be obtained ready prepared from Grübler. The object of this preparation is to make the mass stickier, in view of cutting ribbons.

Van Walsem (Verh. Alcad. Wetensch. Amsterdam, 1899, p. 132) still recommends the addition of 5 per cent. of yellow wax to paraffin of $52^{\circ}$ to $57^{\circ}$ melting-point (for large sections of central nervous system).

Johnston (Journ. Appl. Micr., vi, 1903, p. 2662) adds 1 per cent, of india-rubber in very small pieces, dissolved by heating to $100^{\circ} \mathrm{C}$. for twenty-four hours, or several days to $60^{\circ} \mathrm{C}$. Clear with xylol. For very brittle objects.

153. Soap Masses.-These have never been much used, and are now entirely discarded. But see early editions, or PölzAм (Morph. Jahrb., iii, 1877, p. 558); KADYI (Zool. Anz., 1879, vol. ii, p. 477) ; DöL.KEN (Zeit. wiss. Mik., xiv, 1897, p. 32).

\section{Gelatin Masses.}

154. Gelatin Imbedding is a method that has the advantage of being applicable to tissues that have not been in the least degree dehydrated. 
The modus operandi is, on the whole, the same as for other fusion masses, with the difference that the objects are prepared by saturation with water instead of alcohol or a clearing agent. After the cooling of the mass it may sometimes be cut at once, but it is generally necessary to harden it. This may be done by treatment for a few minutes with absolute alcohol (KAIsER), or for a few days with 90 per cent. alcohol (KLEBS) or chromic acid (KLEBS) or formaldehyde (NICOLAS), or it may be frozen (SolLAS).

The mass can be removed from the sections by meains of warm water.

155. Glycerin Gelatin, KLeBs' (Arch. mik. Anat., v, 1869, p. 165).A concentrated solution of isinglass mixed with half its volume of glycerin.

KaISER's (Bot. Centralb., i, 1880, p. 25).-One part by weight of gelatin is left for about two hours in 6 parts by weight of water; 7 parts of glycerin are added, and for every $100 \mathrm{grms}$. of the mixture $1 \mathrm{grm}$. of concentrated carbolic acid. The whole is warmed for ten to fifteen minutes, stirring all the while, until the whole of the flakes produced by the carbolic acid have disappeared.

GerLACH's (Unters. a. d. Anat. Inst. Erlangen, 1884 ; Journ. Roy. Mic. Soc., 1885, p. 541). - Take gelatin, 40 grms.; saturated solution of arsenious acid, 200 c.c.; glycerin, 120 c.c. Clarify with white of egg. The objects to be prepared for imbedding by a bath of one-third glycerin.

Apátuy (Mitth. Z. Stat. Neapel, xii, 1897, p. 718, and Zeit. wiss. Mikr., xxix, 1913, p. 472) soaks small objects first in glycerin and water (equal parts) and then for at least twenty-four hours at $40^{\circ} \mathrm{C}$. in a solution of 1 part of gelatin in 3 of glycerin and 6 of water. They are then arranged in some of this in an imbedding box, and the whole is warmed (over calcium chloride) in a stove at $45^{\circ}$ to $60^{\circ} \mathrm{C}$. until the mass has evaporated down to one-half, losing 5 of its 6 volumes of water (as I understand - the description is not clear). Blocks are then cut out and hardened in absolute alcohol (suspended therein) for several days (one day per millimetre of thickness), cleared in terpinol (one day per millimetre), and cut with a knife wetted with the same. Said to give sections of $3 \mu$, without the least shrinkage.

Brunotrti's Gold Gelatin Mass (Journ. de Botan., vi, 1892, p. 194). - Twenty grms. gelatin dissolved with heat in 200 c.c. distilled water, and 30 to 40 c.c. of glacial acetic acid with 1 grm. corrosive sublimate added after filtering. Objects are prepared by soaking in some of the mass diluted with 2 to 3 volumes of water, then imbedded in the undiluted mass. The mass is then hardened in spirit or bichromate of potash, picric acid, or the like. No heat at all is required in this process.

Nicolas's Method (Bibliogr. Anat., Paris, 3 année, 1896, p. 274).Preparations are first soaked for one or two days in a 3 to 
4 per cent. aqueous solution of gelatin kept at $25^{\circ} \mathrm{C}$., then for the same time in a 10 per cent. solution, and then for two or three days more in a 20 to 25 per cent. solution containing 8 to 10 per cent. of glycerin and kept at $35^{\circ} \mathrm{C}$. They are then imbedded in some of the same mass in paper trays, and as soon as the gelatin has set are thrown into a mixture of formol 1 part, water 7. After a few days therein the gelatin has become hard and insoluble, and may be cut or preserved for months in weak formol solution, or dilute alcohol or glycerin, or even in pure water. Sections must be very gradually passed through successive alcohols for dehydration, as they curl up very easily. They, however, flatten out at once on being brought from absolute alcohol into cresylol, and may then be mounted in balsam. To mount in glycerin is of course easy.

Burzynski (Polu. Arch. Biol. Med. Wiss., i, 1901, p. 39) finds that alkaline formol hardens gelatin better than acid.

Gaskell (Journ. Path. Bact., July, 1912, p. 58) soaks in pure gelatin, melted s.a., for two to five hours at $37^{\circ} \mathrm{C}$, and hardens the mass in vapour of formol, for three or more days. To cut, he freezes. He mounts in glycerin jelly, to avoid dehydration and shrinkage. 


\section{CHAPTER IX.}

COLLODION (CELLOIDIN) AND OTHER IMBEDDING METHODS.

156. Introduction.--Collodion (or celloidin) masses do not require the employment of heat. They do not require that the objects should be cleared before imbedding, and that is an advantage in the case of very large objects. They are more or less transparent, which facilitates orientation. And they are specially indicated for very large objects, for the soaking in collodion, being quite inoffensive to the most delicate elements, may be prolonged if necessary for weeks. Lastly, the mass being quite transparent after mounting, it is not necessary to remove it from the sections before staining and mounting them; it may remain, and fulfil the function of an admirable support to the tissues, holding in their places brittle or detached elements that without that help would fall to pieces and be lost.

There are disadvantages. One is that the process is a very long one; as usually practised, it requires some three days for the imbedding of an object that can be imbedded in paraffin in an hour. Another is that it is impossible to obtain with celloidin sections quite so thin as those furnished by paraffin.

In the older celloidin method the mass is cut wet, before clearing. I strongly recommend the more recently introduced practice of clearing before cutting, and cutting dry as described in \$§ 168-170.

15\%. Collodion, Celloidin, Parlodion and Photoxylin.-The collodion method is due to Duval (Journ. de l'Anat., 1879, p. 185).

Celloidin, recommended later on by Merkel and SchiefferDECKER (Arch. Anat. Phys., 1882, p. 200), is merely a patent collodion. It may be obtained from almost all of the usual dealers in histological reagents. It is sent out in the form of tablets. These tablets may, if desired, be dissolved at once in ether, or a mixture of ether and alcohol, to make a collodion of any desired strength. But it is better, as recommended by APÁTHY, to cut them up into thin shavings, which should be allowed to dry in the air until they become yellow, transparent, and of a horny consistency, and that these be then dissolved in alcohol and ether (sulphuric, free from acid). The solutions thus prepared are free from the excess of water that is present in the undried celloidin, and give after hardening a mass 
that is more transparent and of a better consistency for cutting (Zeit. wiss. Mik., vi, 1889, p. 164).

Imbedding masses of excellent quality can be prepared with ordinary collodion, but celloidin furnishes more readily solutions of known concentration. Otherwise there is but little to choose between the two, and therefore in this work the terms collodion and celloidin are used indifferently.

According to Unna (Monatschr. p. Dermatol., xxx, 1900, pp. 422 and 476 ; Zeit. wiss. Mik., xviii, 1901, p. 32) a more inelastic, and therefore better, mass is obtained by adding to celloidin 2 per cent. of oil of turpentine, stearate of soda, or (best of all) castor oil. Celloidin with this addition has been put on the market under the name of "Celloidinum inelasticum," by the Chemische Fabrik vorm. E. Schering, in Berlin.

Photoxylin (Krysinsky, Virchow's Archiv, cviii, 1887, p. 217 ; Busse, Zeit. wiss. Mik., ix, 1892, p. 47) is a dry substance, of the aspect of cotton-wool, and chemically nearly related to celloidin. It can be obtained from GRÜBLER. It gives a clear solution in a mixture of equal parts of ether and absolute alcohol, and should be used in exactly the same way as celloidin. It has the advantage of affording a mass which after hardening in 85 per cent. alcohol remains perfectly transparent. Some writers say that it gives a better consistency, but others deny this (ApÁthY, e.g.)

TschernischefF (Zeit. wiss. Mik., xvii, 1900, p. 449) recommends Colloxylin (10 grms. dissolved in 10 grms. of engenol or clove oil, with the addition of 50 c.c. of ether and 1 of absolute alcohol).

\section{The Older Celloidin Method.}

158. Preparation of Objects. - The objects must first be very thoroughly dehydrated with absolute alcohol. They are then soaked till thoroughly penetrated in ether, or, which is better, in a mixture of ether and absolute alcohol. Duval (loc. cit.) takes for this purpose a mixture of ten parts of ether to one of alcohol ; ScHIEFFERDECKER (and the majority of workers) a mixture of equal parts of ether and alcohol ; Tuвby (in Nature, November 17th, 1892, p. 51) advises a mixture of four parts of ether and one of alcohol. FisH advises acetone, see next $\S$. MANN (Methods, etc., p. 172) takes equal parts of ether and methyl alcohol. So also Pavlow, Zeit. wiss. Mikr., xxi, 1904, p. 15.

This stage may be omitted if the objects are of a sufficiently permeable nature, and they may be brought direct from alcohol into the collodion bath.

159. The Collodion Bath.-The secret of success here is to infiltrate the objects first with thin solutions, then with the definitive thick 
one. (A thin solution may be taken to mean one containing from 4 to 6 per cent. of celloidin [dried as described in $\$ 157$ ]; a thick solution, one containing 10 to 12 per cent.)

If collodion be taken, the thin solutions may be made by diluting it with ether. If photoxylin or celloidin be taken, the solutions are made in a mixture of ether and absolute alcohol in equal parts.

The dried celloidin shavings dissolve very slowly in the mixture. Elschnig (Zeit. wiss. Mikr., x, 1893, p. 443) has found that solution is obtained much quicker if the shavings be first allowed to swell up for twenty-four hours in the necessary quantity of absolute alcohol, and the ether be added afterwards.

Busse (op. cit., ix, 1892, p. 47) gives the following proportions for the successive baths:-No. 1, 10 parts by weight of photoxylin or perfectly dried celloidin to 150 parts of the ether and alcohol mixture ; No. 2, 10 parts of photoxylin or celloidin to 105 of the mixture ; No. 3, 10 parts to 80 of the mixture (already-used solution may be employed for the first bath).

I generally use only two solutions : one weak one, and one strong one corresponding approximately to Busse's No. 2. His No. 3 is so thick that excessive time is required to obtain penetration by it.

Mann (Methods, p. 172) uses solutions of $2 \frac{1}{2}$ and 10 per cent.

Apáthy (Behrens, Tabellen., 1898, p. 82) takes 2 per cent. and 4 per cent. for the first baths, 8 per cent. for the last.

Myers (Arch. Anat. Phys., Anat. Abth., 1902, p. 370) takes $1 \frac{1}{2}$ per cent., 6 per cent., and 16 per cent.

See also Neumayer, Zeit. wiss. Mik., XXV, 1908, p. 38 ; De Vecchi, ibid., xxiii, 1906, p. 312 ; and FuHrmann, Zeit. wiss. Zool., Ixxviii, 1905, p. 524 .

Fish (Journ. Appl. Microscop., ii, 1899, p. 323) first infiltrates with acetone (which he says may be used as a fixing and dehydrating agent at the same time), then with a 4 per cent. solution of pyroxylin (guncotton) in acetone, and, lastly, in an 8 per cent. acetone solution of the same. See for other solutions $\$ \$ 170$ and 171 .

The objects ought to remain in the first bath until very thoroughly penetrated;-days, even for small objects,-weeks or months for large ones (human embryos of from six to twelve weeks, for instance).

When the object is duly penetrated by the thin solution, or solutions, if more than one have been employed, it should be brought into the thickest one. This may be done (as first described in this work, 1st ed., 1885, p. 194) by allowing the thin solution to concentrate slowly (the stopper of the containing vessel being raised, for instance, by means of a piece of paper placed under it), and making up the loss from evaporation with thick solution. 
AРÁtHy (Mikrotechnik, p. 121) holds that it is preferable to transfer to fresh thick solution, as he finds that a better consistency after hardening is thus obtained.

160. Imbedding.- The objects must now, if it has not been done before, be imbedded-that is, arranged in position in the thick collodion in the receptacle in which they are to be hardened. For the usual manipulations see $\S 137$. If paper thimbles be taken for imbedding, the bottoms should be made of soft wood in preference to cork. See $\S 165$. They should be prepared for the reception of the object by pouring into them a drop of collodion, which is allowed to dry. The object of this is to prevent bubbles coming up through the wood or cork and lodging in the mass. Watch-glasses, deep porcelain water-colour moulds, and the like, also make convenient imbedding receptacles. Care should be taken to have them perfectly dry.

It not infrequently happens that during these manipulations bubbles make their appearance in the mass. Before proceeding with the hardening these should be got rid of by exposing the whole for an hour or two to the vapour of ether in a desiccator or other well-closed vessel. Care should be taken that the ether (which may be poured on the bottom of the vessel) does not wet the mass (Busse, Zeit. wiss. Mik., viii, 1892, p. 467).

161. Orientation.-Celloidin being more or less transparent, it is seldom necessary to resort to special aids to orientation.

Aрáthy (Zeit. wiss. Mik., v, 1888, p. 47) arranges objects on a small rectangular plate of gelatin, placed on the bottom of the imbedding-recipient. The gelatin is turned out with the mass after hardening, and cut with it. The edges of the gelatin form good orientation lines.

HALle and Born (Zeit. wiss. Mik., xii, 1896, p. 364) use plates of hardened white of egg, in which a shallow furrow for the reception of the objects has been cut by means of a special instrument. See also $\S 142$.

For the complicated method of EycLeSHYMER (Amer. Nat., xxvi, 1892 , p. 354) see previous editions.

See also the article "Rekonstruction" in the Encycl. mik. Technik.

162. Hardening, Preliminary.-The objects being imbedded, the treatment should be as follows:-The receptacles or supports are set with the mass under a glass shade, allowing of just enough communication with the air to set up a slow evaporation. Or porcelain moulds or small dishes may be covered with a lightly fitting cover. 
As soon as the added thick collodion (of which only just enough to cover the object should have been taken) has so far sunk down that the object begins to lie dry, fresh thick solution is added, and the whole is left as before. (If the first layer of collodion has become too dry, it should be moistened with a drop of ether before adding the fresh collodion.) Provision should be again made for slow evaporation, either in one of the ways above indicated, or-which is perhaps better-by setting the objects under a hermetically fitting bell-jar, which is lifted for a few seconds only once or twice a day. I have frequently found it advantageous to set the objects under a bell-jar, together with a dish containing alcohol, so that the evaporation is gone through in an atmosphere of alcohol. This is especially indicated for very large objects. The whole process of adding fresh collodion and placing the objects under the required conditions of evaporation is repeated every few hours for, if need be, two or three days.

When the mass has attained a consistency such that the ball of a finger (not the nail) no longer leaves an impress on it, it should be scooped out of the dish or mould, or have the paper removed if it has been imbedded in paper, and be submitted to the next stage of the hardening process. (If the mass is found to be not quite hard enough to come away safely, it should be put for a day or two into weak alcohol, 30 to 70 per cent.)

163. Hardening, Definitive.-Several methods are available for the definitive hardening process. One of these is the chloroform method, due to Vialiannes (Rech. sur l'Hist. et le Dév. des Insectes, 1883, p. 129).

It consists in bringing the objects into chloroform. In some cases a few hours' immersion is sufficient to give the requisite consistence. In no case have my specimens required more than three days. The collodion frequently becomes opaque on being put into the chloroform, but regains its transparency after a time.

Small objects may be hardened by chloroform without preliminary hardening by evaporation. All that is necessary is to expose the mass to the air for a few seconds until a membrane has formed on it, and then bring it into chloroform. If the mass is in a test-tube this may be filled up with chloroform and left for two or three days if need be. By this time the collodion mass will be considerably hardened, and also somewhat shrunk, so that it can be shaken out of the tube. It is then brought into fresh chloroform in a larger vessel, where it remains for a few more days until it is ready for cutting. But sufficient hardening is sometimes obtained in a few hours. 
Good chloroform is a necessity.

The above processes are excellent, but I regard them as primitive forms of the chloroform method. I now almost always harden in vapour of chloroform. All that is necessary is to put the liquid mass (after having removed bubbles as directed in § 160), with its recipient, into a desiccator on the bottom of which a few drops of chloroform have been poured. The action is very rapid, and the final consistency of the mass at least equal to that obtained by alcohol hardening.

The more commonly employed hardening method is the alcohol method. The objects are thrown into alcohol and left there until they have attained the right consistency (one day to several weeks). The bottle or other vessel containing the alcohol ought not to be tightly closed, but should be left at least partly open.

The strength of the alcohol is a point on which the practice of different writers differs greatly. Busse (Zeit. f. wiss. Mikr., ix, 1,1892 , p. 49) has found, as I also have done, that alcohol of about 85 per cent. is the best, both as regards the cutting consistency and the transparency of the mass. (Care must be taken to keep masses hardened in this grade of alcohol moist while cutting, as they dry by evaporation very quickly.)

Some workers use lower grades, 70 to 80 per cent., or even lower. AРÁTHY (Microtechnik, p. 185) mentions " glycerin-alcohol," but without giving details. BLUM (Anat. Anz., xi, 1896, p. 724) mentions "weak spirit with formol added to it," saying that formol hardens celloidin.

Lastly, the mass may be frozen. After preliminary hardening by alcohol, it is soaked for a few hours in water, in order to get rid of the greater part of the alcohol (the alcohol should not be removed entirely, or the mass may freeze too hard). It is then dipped for a few moments into gum mucilage in order to make it adhere to the freezing plate, and is frozen. If the mass have frozen too hard, cut with a knife warmed with warm water.

Florman (Zeit. wiss. Mik., vi, 1889, p. 184) recommends that the definitive hardening should be done without the aid of alcohol or chloroform, by simply cutting out the blocks, turning them over, and carefully continuing the evaporation process in the way described above. I described this process myself in the first edition of this work. I doubt whether it is possible in this way to carry the hardening much beyond the point attained by the chloroform or alcohol method without incurring a very undesirable degree of shrinkage.

164. Preservation.-The hardened blocks of collodion may be preserved till wanted in weak alcohol (70 per cent.), or dry, by dipping 
them into melted paraffin (АРÁTHY, Zeit. wiss. Mikr., v, 1888, p. 45), or, after rinsing with water, in glycerine-jelly, which may be removed with warm water before cutting (АРÁTHy, Mitth. Zool. Stat. Neapel, xii, 1897, p. 372).

Reference numbers may be written with a soft lead pencil on the bottom of the paper trays, or with a yellow oil pencil on the bottom of the watch-glasses in which the objects are imbedded. On removal of the paper from the collodion after hardening, the numbers will be found impressed on the collodion.

165. Cutting. - If the object has not been stained before imbedding, it may form so transparent a mass with the collodion that the arrangement of the object and sections in the right position may be rendered very difficult. It is, therefore, well to stain the collodion lightly, just enough to make its outlines visible in the sections. This may be done by adding picric acid or other suitable colouring matter dissolved in alcohol to the collodion used for imbedding, or to the oil used for clearing.

To fix a collodion block to the microtome take a piece of soft wood, or, for very small objects, pith, of a size and shape adapted to fit the holder of the microtome. Cover it with a layer of collodion, which you allow to dry. Take the block of collodion or the infiltrated and hardened but not imbedded object, and cut a slice off the bottom, so as to get a clean surface. Wet this surface first with absolute alcohol, then with ether (or allow it to dry); place one drop of very thick collodion on the prepared wood or pith and press down tightly on to it the wetted or dried surface of the block or object. Then throw the whole into weak (70 per cent.) alcohol for a few hours, or even less, or, better, into chloroform, or vapour of chloroform, for a few minutes, in order that the joint may harden.

LINDSAY JoHnson prefers a mixture of beeswax, 1 part; rosin, 2 parts. To use it you must get the block of celloidin perfectly dry at the bottom, then warm the object-holder slightly, if possible over a flame; drop on to it a few drops of melted cement, and press on to it the block of collodion, which will be firmly fixed as soon as the cement is cool-that is, in a few seconds.

For objects of any considerable size it is best not to use cork for mounting on the microtome, if the object-holder be a vice; for cork bends under the pressure of the holder, and the elastic collodion bends with it, deforming the object. If the object-holder be of the cylinder type, a good cork may be used; but even then, I think, wood is safer. GAGE has recommended bits of glass cylinders. JELINEK (Zeit. wiss. Mik., xi., 1894, p. 237) recommends a sort of 
vulcanite known as "Stabilit," which is manufactured for electrical insulation purposes. It is supplied in suitable blocks by JUNG, and by GRÜBLER. Wood is liable to swell in alcohol so that it no longer fits into the object-holder. BABCock (Journ. R. Micr. Soc., 1901, p. 339) uses a block of hard paraffin, with the surface corrugated.

Sections (from such masses as have not been cleared before cutting) are cut with a knife kept abundantly wetted with alcohol (of 50 to 85 or even 95 per cent.). ApátHy recommends that the knife be smeared with yellow vaseline; it cuts better, is protected from the alcohol, and the mobility of the alcohol on the blade is lessened.

The knife is set in as oblique a position as possible.

Very brittle sections may be collodionised as explained $\S 147$.

The sections are either brought into alcohol (of 50 to 85 or 95 per cent.) as fast as they are made, or if it be desired to mount them in series, they are treated according to one of the methods described below, in Chapter X.

Masses that have been cleared before cutting with cedar oil or the like may be cut $d r y, \S 170$.

166. Staining.-The sections may now be stained as desired, either loose, or mounted in series on slides or on paper as described in Chapter X. It is not in general necessary, nor indeed desirable, to remove the mass before staining, as it usually either remains colourless, or gives up the stain on treatment with alcohol. But if it be desired, the mass may be removed by treating the sections with absolute alcohol or ether.

16\%. Clearing and Mounting.-You may mount in glycerin without removing the mass, which remains as clear as glass in that medium. You may mount in balsam, also, without removing the mass, which does no harm, and serves the useful purpose of holding the parts of the sections together during the manipulations. Dehydrate in alcohol of 95 or 96 per cent. (not absolute, as this attacks the collodion). NIKIforow (Zeit. wiss. Mik., viii, 1891, p. 189) recommends a mixture of equal parts of alcohol and chloroform. Clear with a substance that does not dissolve collodion. The clearing agents most recommended are origanum oil (Ol. Origan. Cretici, it is said, should be taken, not Ol. Orig. Gallici; but see as to this reagent the remarks in $\S 125$ ), bergamot oil (said to make sections shrink somewhat), oil of sandal-wood, lavender oil, oil of cedar-wood (safe and gives excellent results, but acts rather slowly), chloroform, xylol, or benzol (may make sections shrink if not well dehydrated), 
or Dunham's mixture of 3 or 4 parts of white. oil of thyme with 1 part of oil of cloves. (As to oil of thyme, see also $\$ \S 125,126$. )

Fish (Proc. Amer. Mik. Soc., 1893) advises a mixture of 1 part of red oil of thyme with 3 parts of castor oil, the latter being added in order to counteract the volatility of the thyme oil. But later (June, 1895), writing to me, Dr. Fish says he has substituted the white oil of thyme for the red, and finds it an advantage in orientating. See also § 126, and under "Euparal."

Some specimens of clove oil dissolve collodion very slowly, and may be used, but I would not be understood to recommend it. The action of origanum oil varies much, according to the samples; some sorts do not clear the collodion, others dissolve it, others pucker it. Minot (Zeit. wiss. Mik., iii, 1886, p. 175) says that Dunham's mixture " clarifies the sections very readily, and softens the celloidin just enough to prevent the puckering which is so annoying with thyme alone."

Carbolic acid has been recommended. WEIGERT (Zeit. wiss. Mik., iii, 1886, p. 480 ) finds that a mixture of 3 parts of xylol with 1 part of carbolic acid (anhydrous) clears well. But it must not be used with the basic anilin stains, as it discolours them. For these anilin oil may be used with xylol in the place of carbolic acid.

Anilin oil cleąrs well (it will clear from 70 per cent. alcohol), but unless thoroughly removed the preparation becomes yellowish-brown, see § 134. See van Greson, Amer. Mon. Mic. Journ., 1887, p. 49, or Journ. Roy. Mic. Soc., 1887, p. 519, for a review of these clearing agents.

Beech-wood creasote has been recommended (by M. Flesch).

EyCleshyMer (Amer. Nat., xxvi, 1892, p. 354) advises a mixture of equal parts of bergamot oil, cedar oil, and carbolic acid.

For oil of cajeput see $\S 129$; and for this and other clearers see also JoRdan, Zeit. wiss. Mik., xv, 1898, p. 51, who recommends, amongst other things, oil of Linaloa, which remains colourless.

\section{The Newer Celloidin Method.}

168. The New Method, by Clearing before Cutting.-This process is due, I believe, in the first instance to E. Meyer (Biol. Centralb., x, 1890, p. 508), who advised soaking blocks before cutting for twenty-four hours in glycerin. Bumpus (Amer. Anat., xxvi, 1892, p. 80) advises clearing the mass, after hardening in chloroform, with white oil of thyme or other suitable clearing agent. See $\S 167$. The knife is wetted with the clearing oil, and the same oil is employed for covering the exposed surface of the object after each cut. Similar recommendations are made by EYcleshyMer (op. cit., pp. 354,563$)$, carbolic acid, or glycerin, or the mixture given $\$ 167$, being suggested for clearing; and GILson has for a long time past 
adopted the practice of clearing before cutting with cedar oil, as described in the next $\S$.

FisH (loc. cit., § 167) also advocates the practice of clearing in the mass, recommending the clearing mixture there given. Similarly GAGE, Trans. Amer. Mik. Soc., xvii, 1896, p. 361.

All the authors above quoted cut in the wet way, that is to say, with a knife wetted with the clearing liquid.

169. GiLson's Rapid Process (communicated April, 1892).-The object is dehydrated, soaked in ether, and brought into a test-tube with collodion or thin celloidin solution. The tube is dipped into a bath of melted paraffin, and the collodion allowed to boil (which it does at a very low temperature) until it has become of a syrupy consistence. (It should be boiled down to about one-third of its volume.) The mass is then turned out, mounted on a block of hardened celloidin, and the whole hardened in chloroform or in a mixture of chloroform and cedar oil for about an hour. It is then cleared in cedar oil (if hardened in pure chloroform : special clearing will not be necessary if it has been hardened in the mixture). It may now be fixed in the microtome and cut, using.cedar oil to wet the knife, and cover the exposed surface of the object after each cut.

This process is very much more rapid than the old process : small objects can be duly infiltrated in an hour, where days would be required by the old process. As collodion boils at a very low temperature, very little heat is required, and there is no risk of the tissues suffering on that head.

19. The Dry Cutting Method.-I recommend the following as a further improvement. Infiltrate with collodion or celloidin either by Gilson's process, or by soaking in the cold in the usual way, $\S 159$. Imbed as usual. Harden in vapour of chloroform for from one hour (generally sufficient for small objects) to overnight. This is done by putting the object (definitively imbedded in the final thick solution, but without any preliminary hardening in the air) into a Steinach's sieve-dish or into a desiccator, on the bottom of which a teaspoonful of chloroform has been poured. (The objects may remain for months in the chloroform vapour if desired.) As soon as the mass has attained sufficient superficial hardness, it is, of course, well to turn it out of its recipient, and turn it over from time to time, in order that it may be equally exposed on all sides to the action of the vapour. When fairly hard throw it into GiLson's mixture. This should be at first a mixture of 1 part of chloroform with 1 or 2 parts of cedar oil. From time to time more cedar oil 
should be added, so as to bring the mixture up gradually to nearly pure cedar oil. As soon as the object is cleared throughout, the mass may be exposed to the air, and the rest of the chloroform will evaporate gradually. The block may now either be mounted on the holder of the microtome, $\S 165$, and cut at once, or may be preserved indefinitely without change in a stoppered bottle. Cut $d r y$, the cut surface will not dry injuriously under several hours. The cutting quality of the mass is often improved by allowing it to evaporate in the air for some hours.

The hardening may be done at once in the chloroform and cedar oil mixture, instead of the chloroform vapour, but I find the latter preferable. And clearing may be done in pure cedar oil instead of the mixture, but then it will be very slow, whereas in the mixture it is extremely rapid.

StePanow (Zeit. wiss. Mik., xvii, 1900, p. 185) soaks and imbeds in a solution of celloidin in a mixture of equal parts of ether and clove oil, hardens in alcohol or vapour of chloroform, or in benzol, and cuts either wet or dry.

See also Tschernischeff, ibid., p. 449.

JORDAN, ibid., p. 193, imbeds in a mixture of 5 parts of 8 per cent. celloidin solution with 1 of oil of cedar, hardens first in vapour of chloroform and then in a mixture of 5 parts of chloroform with 1 of oil of cedar, and cuts wet or dry.

171. Double Imbedding in Collodion and Paraffin.-This is sometimes employed for objects of which it is desired to have very thin sections, and which are too brittle to give good sections by the plain paraffin process.

KultschiTzKy's Method (Zeit. wiss. Mik., iv, 1887, p. 48).After the collodion bath, the object is soaked in oil of origanum (Oleum Origani vulg.). It is then brought into a mixture of origanum oil and paraffin heated to not more than $40^{\circ} \mathrm{C}$., and lastly into a bath of pure paraffin.

The mass may be preserved in the dry state, and may be cut dry.

RYDER (Queen's Micr. Bull., 1887, p. 43; Journ. Roy. Micr. Soc., 1888 , p. 512) modified the process by substituting chloroform for the origanum oil.

IDE (La Cellule, vii, 1891, p. 347, and viii, 1, 1892, p. 114) imbeds in collodion in a tube by GrLson's process $(\$ 169)$; the collodion is boiled for forty minutes, then brought for fifteen minutes (this is for small objects) into chloroform heated to $30^{\circ} \mathrm{C}$. containing $\frac{1}{4}$ part of paraffin dissolved in it, then for ten minutes into pure melted paraffin.

Field and Martin (Bull. Soc. Zool. de France, 1894, p. 48) make a solution of dried celloidin in a mixture of equal parts of absolute alcohol and toluene, of about the consistency of clove oil. This solution is 
saturated with paraffin, added in shavings at a temperature not exceeding $20^{\circ}$ to $23^{\circ} \mathrm{C}$. The tissues are prepared by soaking in some of the mixture of alcohol and toluene, and are then penetrated with the celloidin-paraffin solution. The mass is hardened in a saturated solution of paraffin in chloroform or in toluene, and is finally imbedded in pure paraffin in the usual way.

STEPANOw imbeds in paraffin after clearing with benzol, last §.

JORDAN, after imbedding as in last §, passes through a bath of paraffin dissolved in chloroform into pure paraffin.

Wilhelmi (Fauna Flora Golf. Neapel, xxxii, 1909, p. 17), following APÁTHY, imbeds in celloidin, hardens in chloroform, then adds benzol to the chloroform, and passes through pure benzol (half an hour to an hour) into paraffin, and cuts dry.

Similarly, BrECKNer, Zeit. wiss. Mik., xxv, 1908, p. 29.

SterLing (Jena Zeit., 1909, p. 253) soaks for two or three days in equal parts of clove oil and collodion, puts for a couple of hours (until clear) into xylol, and imbeds in paraffin.

See also Dahlgren, Journ. Appl. Microsc., 1898, p. 97 ; Sabussow, Mitth. Zool. Stat. Neapel, xii, 1896, p. 353 ; MEYER, ibid., xiv, 1901, p. 295 ; Mitropinanow, Arch. Zool. Expér. (3), 3, 1896, p. 617 ; Federici, Anat. Anz., xxi, 1907, p. 602 ; Bordage, Bull. Sci. France Belg., xxxix, 1905, p. 385 ; GaNDOLFI, Zeit. wiss. Mik., xxv, 1909, p. 421 ; MAYER, ibid., Xxiv, 1907, p. 132.

\section{Other Cold Masses.}

172. Lead-Gum Imbedding Nethod of J. SALKind (C. R. Soc. de Biol., t. Ixxix, 1916, No. 16).- The principle of this method is that an aqueous solution of gum treated by acetate of lead, when exposed to the action of ammonia, is transformed into a gel, sufficiently stiff to allow of thin sections being cut.

(1) Dissolve a quantity of gum of cherry (white for preference) in double its weight of $a q$. dest. After filtration, add to the solution one-third its volume of the liquid subacetate of lead (extract of Saturne), to which has been added 5 per cent. of glacial acetic acid. This gives a kind of thin collodion-like solution, in which you place the pieces at room temperature, to be imbedded, after a fixation, for which see below.

(2) Leave about twelve hours for pieces about a millimetre in thickness : larger pieces must be left longer. After the correct period has elapsed, you let the lead gum solution evaporate in the air till the solution reaches the consistency of a thick celloidin solution.

(3) Arrange the pieces to be imbedded in a paper box (or on a piece of paper), in a large drop of the thick solution. Expose to strong ammonia vapour for about five minutes till the block hardens to the consistency of cartilage. 
(4) Trim the block, and fasten it on to the plate of a microtome by means of some of the thick lead-gum (hardened afterwards in ammonia vapour). Cut sections with an oblique knife, the block being moistened with a solution 1 per cent. sodium chloride in $a q$. dest. The sections are placed in the same solution, in which they must not stay more than one hour.

(5) Sticking the sections to the slide is done by a modification of OLT's method, $\S 182$. Cover the slide with albumen, then with gelatin, arrange the sections, press down with a cloth, and harden in formol vapour. See also J. A. Murray, below.

(6) The lead-gum is then dissolved away in 5 per cent. acetic acid. After washing you stain and mount in any way desired.

Neither gum arabic (acacia), plum, nor apricot give quite such good results as cherry gum. SALKIND recommends two fixatives to precede this method of imbedding.

A. Formol, acetic acid, sub-acetate of lead, 1 part each. Aq. lest., 5 parts.

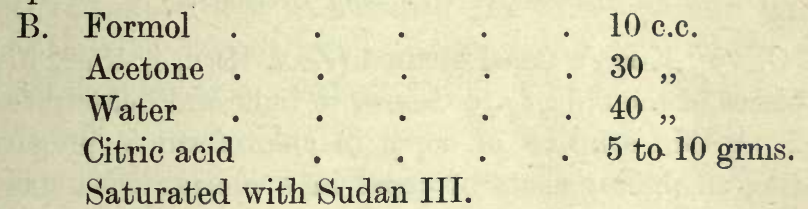

After A, it is not necessary to wash out. After B, and the majority of such fixatives as bichromate especially, you must wash out in running water.

J. A. MURRAY (Report of Imper. Cancer Bureau, 1919) fixes cartilage in 10 per cent. formol-salt solution for at least twenty-four hours. After Salkind's lead-gum imbedding, cuts sections $10-15 \mu$ thick with sliding microtome. Transfers sections for from ten minutes to one hour in 1 per cent. $\mathrm{NaCl}$ solution. Special slides prepared beforehand by coating in 1 per cent. gelatin and allowing to dry.

The prepared slide is immersed in the salt solution ( $\mathrm{NaCl})$, sections arranged with a smooth-pointed glass rod, superfluous liquid drained off, and a wetted cigarette paper carefully lowered over the sections. Firm pressure with several layers of filter paper makes the sections adhere to the slide. Withdraw the cigarette paper and expose to formol vapour for a few minutes. Transfer to 10 per cent. formol five minutes, then treat in the 5 per cent. acetic to remove the lead-gum. Stain.

173. Joliet's Gum and Glycerin Method (Arch. Zool. Expér. et Gén., $\mathrm{x}, 1882$, p. xliii).-Pure gum arabic dissolved in water to the consistency of a thick syrup. Pour a little of the solution into a watch-glass, and add from 6 to 10 drops of pure glycerin. In the winter or in rainy weather less glycerin should be taken than in the summer or dry weather.

The object is imbedded in the mass in the watch-glass and the whole 
left to dry for from one to four days. When it has assumed a cartilaginous consistency, a block containing the object is cut out, turned over, and allowed to dry again until wanted for use. A stove, or the sun, may be employed for drying, but it is best to dry slowly at the normal temperature.

174. Stricker's Gum Method (Hdb. $d$. Gewebel., p. xxiv).-A concentrated solution of gum arabic. The object is imbedded in the gum in a paper case. The whole is thrown into alcohol, and after two or three days may be cut. The alcohol should be of about 80 per cent. (MAYER).

I have seen masses of sufficiently good consistency prepared by this simple method.

175. Hyatt's Shellac Method, see Am. M. Mic. Journ., i, 1880, p. 8 ; Journ. Roy. Mic. Soc., iii, 1880, p. 320. For sections through hard chitinous organs consisting of several pieces, such as stings and ovipositors, retaining all the parts in their natural positions.

176. BRunotTr's Cold Gelatin Mass has been given, § 155 .

\section{Masses for Grinding Sections.*}

19\%. G. von Koch's Copal Method (Zool. Anz., i, 1878, p. 36).Small pieces of the object are stained in bulk and dehydrated with alcohol. A thin solution of copal in chloroform is prepared by triturating small fragments of copal in a mortar with fine sand, pouring on chloroform to the powder thus obtained and filtering. The objects are brought into a capsule filled with the copal solution. The solution is now slowly evaporated by gently heating the capsule on a tile by means of a common night-light placed beneath it. As soon as the solution is so far concentrated as to draw out into threads that are brittle after cooling, the objects are removed from the capsule and placed to dry for a few days on the tile in order that they may more quickly become hard. When they have attained such a degree of hardness that they cannot be indented by a fingernail, sections are cut from them by means of a fine saw. The sections are rubbed down even and smooth on one side with a hone, and cemented, with this side downwards, to a slide, by means either of Canada balsam or copal solution. The slide is put away for a few days more on the warmed tile. As soon as the cement is perfectly hard the sections are rubbed down on a grindstone, and then on a hone, to the requisite thinness and polish, washed with water, and mounted in balsam.

The process may be varied by imbedding the objects unstained,

* For the manipulations of section-grinding. see CARPEnter's The Microscope. 
removing the ce pal from the sections by soaking in chloroform, decalcifying them if necessary, and then staining.

It is sometimes a good plan, after removing the copal, to cement, a section to a slide by means of hard Canada balsam, then decalcify cautiously the exposed half of the specimen, wash, and stain it.

This method was invented in order to enable the hard and soft parts of corals to be studied in their natural relations, and is valuable for this and similar purposes.

178. Ehrenbaum's Colophonium and Wax Method (Zeit. wiss. Mik., 1884, p. 414).-Ehrenbaum recommends a mass consisting of 10 parts of colophonium to 1 of wax. The addition of wax makes the mass less brittle. Sections are obtained by grinding in the usual way. The mass is removed from them by means of turpentine followed by chloroform.

179. Johnstone-Lavis and Vosmaer's Balsam Method (Journ. Roy. Mic. Soc., 1887, p. 200).-Alcohol material is carefully and gradually saturated, first with benzol, and then with thin and thick solution of benzol-balsam. It is then dried for a day in the air and for several days more in a hot-air bath. When hard it is ground in the usual way.

180. WeIL's Canada Balsam Method, see Zeit. wis8. Mik., v, 1888, p. 200.

181. GIESBRECHT's Shellac Method.-For hard parts only, spines of Echinus, shell, etc., see Morph. Jahrb., vi, 1880, p. 95, or the abstract in LEE und MAYER, Grundzüge.

\section{Congelation Masses.}

182. The Methods of Freezing.-For the requisite manipulations and means of producing the requisite degree of cold, see CARPENTER's The Microscope (ether spray); JoHne, Zeit. wiss. Mik., xiv, 1897, p. 370 (liquid carbonic acid); WoLFF, ibid., xxv, 1908, p. 175 (ethyl chloride); Krause, ibid., p. 289 (solid carbonic acid); Jung, Verh. Ges. Naturf. Aertze, lxix, 1898, p. 129 (ethyl chloride); BRISsY, C. R. Soc. Biol., lxii, 1907, p. 1115 (liquid air).

Fresh tissues may be, and are, frequently frozen without being included in any mass. But the formation of ice crystals frequently causes tearing of delicate elements, and it is better to infiltrate the tissues with a mass that does not crystallise in the freezing mixture, but becomes simply hard and tough, such as one of those given below.

When sections have been obtained, it is difficult to manipulate them. Out (Zeit. wiss. Mik., xxiii, 1906, p. 327) puts them into a 1 per cent. solution of gelatin, brings them therein on to a slide, 
hardens for an hour in vapour of formaldehyde, and soaks for a few minutes in formol of 10 per cent. ANITschKow (ibid., xxvii, 1910, p. 73) puts them into alcohol of 50 per cent., gets them on to a slide prepared with Mayer's albumen, presses down with paper, puts into alcohol of 98 per cent., and thence through lower grades into water.

183. Gum and Syrup Masses.-HamiLton (Journ. of Anat. and Phys., xii, 1878, p. 254) soaked tissues in syrup made with double refined sugar, 2 ounces; water, 1 fluid ounce; then washed the superfluous syrup from the surface, and put into ordinary gum mucilage for an hour or so, and then imbedded in the freezing microtome with mucilage in the usual way.

CoLE (Methods of Microscopical Research, 1884, p. xxxix) takes gum mucilage (B. P.), 5 parts ; syrup, 3 parts. (For brain and spinal cord, retina, and all tissues liable to come in pieces put 4 parts of syrup to 5 of gum.) Add 5 grains of pure carbolic acid to each ounce of the medium.

(Gum mucilage [B. P.] is made by dissolving 4 ounces of picked gum acacia in 6 ounces of water. The syrup is made by dissolving 1 pound of loaf sugar in 1 pint of water and boiling.)

The freezing is conducted as follows:-The gum and syrup is removed from the outside of the object by means of a cloth; the spray is set going and a little gum mucilage painted on the freezing plate ; the object is placed on this and surrounded with gum mucilage ; it is thus saturated with gum and syrup, but surrounded when being frozen with mucilage only. This combination prevents the sections from curling up on the one hand, or splintering from being too hard frozen on the other. Should freezing have been carried too far, wait for a few seconds.

WebB (The Microscope, ix, 1890, p. 344 ; Journ. Roy. Mic. Soc., 1890, p. 113) takes thick solution of dextrin in solution of carbolic acid in water ( 1 in 40 ).

184. Gelatin (Sollas, Quart. Journ. Mic. Soc., xxiv, 1884, pp. 163, 164). Gum Gelatin (JACOBS, Amer. Natural., 1885, p. 734). White of Egg (Rolletr, Denskschr. math. naturw. Kl. k. Acad. Wiss. Wien, 1885 ; Zeit. wiss. Mik., 1886, p. 92). - Small portions of tissue brought in the white of a freshly laid egg on to the freezing stage, frozen and cut. Oil of Aniseed (Kunne, Centralb. f. Bakteriol., xii, 1892, p. 28 ; Journ. Roy. Mic. Soc., 1892, p. 706 ; V. A. Moone, Amer. Mon. Mic. Journ., 1894, p. 373 ; Journ. Roy. Mic. Soc., 1895, p. 247). Anethol (anise camphor) (STEPANOW, Zeit. wiss. Mik., xvii, 1900, p. 181).

For details of these see previous editions.

For DöLLKEN's method of solidifying formol by means of resorcin, see Zeit. wiss. Mik., xiv, 1, 1897, p. 33. 


\section{CHAPTER X.}

SERIAL SECTION MOUNTING.

185. Choice of a Method.-I recommend the following:-For general work with paraffin sections, the combined water and albumen method, § 188. For very delicate work, the water method. For collodion sections, the albumen method; for large collodion sections, Graham KerR's seems the most convenient.

\section{Methods for Paraffin Sections.}

186. The Water or Desiccation Method.-Gaule (Arch. Anat. Phys., Phys. Abth., 1881, p. 156); Suchannek (Zeit. wiss. Mik., vii, 1891, p. 464); Gulland (Journ. Anat. and Phys., xxvi, 1891, p. 56); SchIEFFERdecker (Zeit. wiss. Mik., ix, 1892, p. 202); Heidenhain (Kern. und Protoplasma, p. 114); Nusbaum (Anat. Anz., xii, 2, 1896, p. 52) ; MAYER in the Grundzüge, LeE und MAYer, 1898 , p. 113 ; DE Groot (Zeit. wiss. Mik., xv, 1898, p. 62), and others.-The principle of this method is that the sections are made to adhere to the slide without the intervention of any cementing substance, being brought into intimate contact with the glass by being slowly drawn down by the evaporation of a layer of water on which they are floated. It is now practised, with unessential variations, as follows :

(a) For sections that are large and not numerous. The sections are flattened out on water by one or other of the processes described in $§ 149$. The slide is then drained and put away to dry until every trace of water has completely evaporated away from under the sections. This drying may be performed at the temperature of the laboratory, in which case many hours will be necessary (to be safe it will generally be necessary to leave the sections overnight). Or it may be performed in a stove or on a water-bath at a temperature a few degrees below the melting-point of the paraffin (best not above $40^{\circ} \mathrm{C}$.), in which case fixation will be much more rapid, large thin sections being often sufficiently fixed in an hour, though thick ones will require half a dozen hours or more. The paraffin must not be allowed to melt before the sections are perfectly dry; the sections are sure to become detached if it does. Perfectly dry sections have a 
certain brilliant transparent look that is easily recognisable. As soon as dry the paraffin may be removed, and they may be further treated as desired. To remove the paraffin all that is requisite is to put the slide into a tube of xylol or other good solvent, which in a few seconds, or minutes at most, removes the paraffin perfectly. Most workers first melt the paraffin, but I find this is not necessary.

(b) For series of numerous small sections. Clean a slide perfectly, so that water will spread on it without any tendency to run into drops (see below). Breathe on it, and with a brush draw on it a streak of water as wide as the sections and a little longer than the first row of sections that it is intended to mount. With a dry brush arrange the first row of sections (which may be either loose ones or a length of a ribbon) on this streak. Breathe on the slide again, draw on it another streak of water under the first one and arrange the next row of sections on it, and so on until the slide is full. Then breathe on the slide again, and with the brush add a drop of water at each end of each row of sections, so as to enable them to expand freely; then warm the slide so as to flatten out the sections, taking care not to melt the paraffin. Some persons do this by holding it over a small flame for a few seconds. I prefer to lay it on a slab of thick glass, warmed, watching the flattening of the sections through a lens if necessary. As soon as they are perfectly flat, draw off the excess of water from one corner of the mount with a dry brush, and put aside to dry as before $(a)$.

In order to succeed in this method it is absolutely essential that the sections be perfectly expanded and come into close contact with the slide at all points. And to ensure this it is necessary that the slide should be perfectly free from grease, so that the water may wet it equally everywhere. The test for this is, firstly, to breathe on the slide; the moisture from the breath should condense on it evenly all over, and disappear evenly. Secondly, streaks of water drawn on it with a brush should not run. To obtain a slide that will fulfil these conditions, clean it well in the usual way, place a drop of water on it and rub it in thoroughly with a damp cloth and try the tests. If this does not suffice, take a turn of a corner of the cloth round a finger and rub it with a piece of chalk, then damp the cloth and rub the slide with it, finishing up with a clean part of the cloth and clean water (DE GRoot, loc. cit., supra). If after performing this operation twice the slide still refuses to take the water thoroughly it should be rejected as incorrigible; for there are apparently some sorts of glass that can never be got to wet properly. Mayer finds carbonate of magnesia or soda useful. 
Gudernatsch (Zeit. wiss. Mikr., xxiv, 1908, p. 358) washes the slide well with potash soap, and arranges the sections on it whilst still wet. Helly (ibid., 1906, p. 330) passes it two or three times over the flame of a Bunsen burner.

Tap water seems preferable to distilled water; it seems to spread better and give a stronger adhesion. NUSBAUM adds a trace of gum arabic (1 or 2 drops of mucilage to a glass of water); АРÁTHY (Microtechnik, p. 126) adds 1 per cent. of Mayer's albumen ( $\$ 187)$; and HENNEGUY (Leçons sur la Cellule, 1896, p. 62) takes a $1: 5,000$ solution of gelatin, with a trace of bichromate of potash, added just before using, and dries the slides exposed to light. Similarly, Burchardt (Jeina Zeit., xxxiv, 1900, p. 719).

Some workers have used alcohol (50 or 70 per cent.) instead of water; but this I believe to be now generally abandoned.

This is the most elegant method of any, as there is nothing on the slide except the sections that can stain, or appear as dirt in the mount. Tissues do not suffer from the drying, provided the material has been properly imbedded. Sections stick so fast by this method that they will stand watery or other fluids for weeks, so long as they are not alkaline. When successfully performed it is quite safe, provided that the sections are of a suitable nature. They must be such as to afford a sufficient continuous surface, everywhere in contact with the slide. Sections of parenchymatous organs stick well ; sections of thin-walled tubular organs stick badly. Sections of chitinous organs are very unsafe. The larger and thinner sections are, the better do they stick, and vice versâ. Sections from chromic or osmic material adhere less well than sections from alcohol or sublimate material.

By taking a staining solution instead of pure water for expanding, the sections can be got to stain at the same time, and so be brought into balsam without passing through alcohol; see MaYer, Mitth. Zool. Stat. Neapel, xii, 1896, p. 320 ; SCHMORL, Path.-hist. Untersuchungsmethoden, 1897, p. 38 ; SмIтн, Journ. Anat. Phys., xxxiv, 1899, p. 151.

18\%. MAYER's Albumen (Mitth. Zool. Stat. Neapel, iv, 1883 ; Internat. Monatschr.f. Anat., iv, 1887, p. 42).-White of egg, 50 c.c. ; glycerin, 50 c.c.; salicylate of soda, 1 grm. Shake them well together, and filter into a clean bottle. The filtering may take days or a week, but the preparation does not spoil meanwhile.

Francotte shakes up the albumen with a few drops of acetic acid before adding the other ingredients, and finds the filtering greatly quickened. So do I. Be careful with the acid.

A very thin layer of the mixture is spread on a slide with a fine brush and well rubbed in with the finger ( $I$ prefer a small rubber 
"squeegee"). The sections are laid on it and pressed down lightly with a brush (if they will bear it). The slide may then be warmed for some minutes on a water-bath, and the paraffin removed with a solvent.

It is not necessary to warm the slide at all; the paraffin can be removed in the cold if desired by putting the slide into toluol, xylol, or the like. But the slide must, in any case, be treated with alcohol after removal of the paraffin, in order to get rid of the glycerin, which will cause cloudiness if not perfectly removed.

This method allows of the staining of sections on the slide with perfect safety, both with alcoholic and aqueous stains, provided they be not alkaline.

According to my experience, the albumen method is absolutely safe, provided that alkaline fluids be avoided in the after-treatment. It has the defect that certain plasma stains (not chromatin stains) colour the albumen very strongly, and cannot be removed from it, and that sections are not expanded by it.

It sometimes happens that the mixture after it has stood for some time becomes turbid, and at last coagulates, passing into a caseous state; or it may undergo a hyaline coagulation, drying up like amber. But up to the very last it does not in general lose its adhesive properties. I have, however, found it to do so, after keeping for five or six years, so that, to be on the safe side, it may be well to make it up fresh every six months.

Heidenhain (Zeit. wiss. Mikr., xxii, 1905, p. 331) makes it up with 1 grm. of blood albumen dissolved in 25 c.c. of water, and an equal volume of 50 per cent. alcohol.

188. The Albumen and Water Method (Henneguy, Journ. de l'Anat. et de la Physiol., 1891, p. 398).-A drop of water is spread on a slide painted with Mayer's white-of-egg mixture, the sections are arranged on it, the whole is warmed (not to the melting-point of the paraffin) until the sections flatten out; the water is then evaporated off at a temperature of about $40^{\circ} \mathrm{C}$., and as soon as it has sufficiently disappeared, which at that temperature will be in about ten to fifteen minutes, the slide is further treated as described last §.

This is a most valuable method. It is quicker than the water method, and, for difficult material, safer.

See also Ohlmacher, Journ. Amer. Med. Assoc., April, 1893.

The so-called "Japanese" method, attributed to IKEDA by REINKE (Zeit. wiss. Mik., xii, 1895, p. 21), is merely that of HENNEGUY.

MANN (Anat. Anz., viii, 1893, p. 442) shakes up white of egg with 
water, coats slides with it and dries them. He flattens sections on water at $40^{\circ} \mathrm{C}$., lifts them out on a prepared slide, and dries for five minutes at $35^{\circ} \mathrm{C}$.

189. Garlic-water-Hollande (Arch. d'Anat. Micr., xiii, 1911, p. 171) gives the following as more adhesive than albumen :- 50 grms. of crushed and chopped garlic are rubbed up with 80 c.c. of chloroformwater (Codex, A.C.) and filtered after twenty-four hours. Use as albumen.

190. SCHÄLlibaum's Collodion (Arch. mikr. Anat., xxii, 1883, p. 565). - One part of collodion shaken up with 3-4 parts of clove or lavender oil. Use as albumen. Sections can be treated with alcohol (not absolute) and divers staining fluids. I do not find it safe for this. RABL, however (Zeit. wiss. Mik., xi, 1894, p. 170), finds that it is if you take 2 parts of collodion to 3 of clove oil, and make up fresh every four or five days.

191. OBREgIA's Method for Paraffin or Celloidin Sections (Neurologisches Centralb., ix, 1890, p. 295 ; Gulland, Journ. of Path., February, 1893).--Slides, or glass plates of any size, are coated with a solution made of-

Syrupy solution of powdered candy-sugar made with boiling distilled water . . . . 30 c.c. 95 per cent. alcohol . . . . . 20 , Transparent syrupy solution of pure dextrin made by boiling with distilled water . . . 10 ,

They are dried slowly for two or three days until the surface is just sticky to the moist finger. Paraffin sections are arranged and heated for a few minutes to a temperature slightly above the meltingpoint of the paraffin. The paraffin is removed by some solvent, and this in turn by absolute alcohol. The alcohol is poured off, and the sections are covered with solution of celloidin. The plates are left to evaporate for ten minutes in a horizontal position, then brought into water, in which the sheet of celloidin with the sections soon becomes detached, and may be further treated as desired, e.g., as in Weigert's process, $\S 198$. The evaporation must not be artificially hastened.

Dimmer (Zeit. wiss. Mik., xvi, 1899, p. 44) coats the slides with a solution of about 16 parts of gelatin in 300 of warm water, and dries them (two days), and proceeds in other respects as above.

A good method for large sections, equally applicable to paraffin sections, to celloidin sections, and to sections of material that has not been imbedded at all.

For Blochman's modification of Weigert's process, by means of which large sections can be preserved unmounted, see Zeit. wiss. Mik., xiv, 1897, p. 189. 
192. Strasser's Collodion Paper Method (ibid., iii, 1886, p. 346).This is an extremely complicated modification of Weigert's method for celloidin sections, and is only adapted for use with STRASSER's automatic ribbon-microtome. See Zeit. wiss. Mik., iii, 1886, p. 346 ; vi, 1889, p. 154 ; vii, 1890 , pp. 290 and 304 ; ix, 1892, p. 8 ; xii, 1895, p. 154 ; and xiv, 1897, p. 39 ; also SchoenemanN, ibid., xix, 1903, p. 333 ; Strasser, ibid., p. 337 ; and RupPricht, ibid., xxviii, 1912, p. 281.

\section{Methods for Watery Sections.}

193. Fol's Gelatin (Fol, Lehrb., p. 132).-Four grammes of gelatin are dissolved in 20 c.c. of glacial acetic acid by heating on a water-bath and agitation. To 5 c.c. of the solution add 70 c.c. of 70 per cent. alcohol and 1 to 2 c.c. of 5 per cent. aqueous solution of chrome-alum. Pour the mixture on to the slide and allow it to dry. In a few hours the gelatin passes into the insoluble state. It retains, however, the property of swelling and becoming somewhat sticky in presence of water. The slide may then be immersed in water containing the sections; these can be slid into their places, and the whole lifted out; the sections will be found to be fixed.

This method is specially intended for sections made under water, large celloidin sections amongst others.

Similarly, Ruppricht, loc. cit., last $\S$, with the needless complication of a seriation on Strasser's collodionised paper.

STRASSER (loc. cit., last §) also employs a dry gelatin film which he makes sticky by means of carbol-xylol.

\section{Methods for Celloidin Sections.}

194. The Albumen Method.-I find that celloidin sections may be mounted on Mayer's albumen, and have the celloidin removed, if desired, by putting them into ether-alcohol. Care must be taken to press them down very thoroughly on to the albumen; and it is well not to have them too wet.

Similarly, Jordan (Zeit. wiss. Mik., xv, 1898, p. 54), and ARGUTINsky (ibid., xvii, 1900, p. 37). See also Jordan, ibid., 192-194; DantschakofF, ibid., xxv, 1908, p. 35 ; Maximow, ibid., xxvi, 1909, p. 184 ; АNiтschкоw, ibid., xxvii, 1910, p. 68 ; WeBer, ibid., xxix, 1912, p. 186 ; Rubaschin Anat. Anz., xxxi, 1907, p. 30. Weber paints over the series on the albumen with a layer of thin collodion, and puts into alcohol of 50 per cent., then into a mixture of equal parts of chloroform and absolute alcohol. After staining, pure absolute alcohol must be avoided.

195. Summers' Ether Method (Amer. Mon. Mic. Journ., 1887, p. 73).-Place the sections in 95 per cent. alcohol for a minute or 
two, arrange on the slide, and then pour over the sections sulphuric ether vapour, from a bottle partly full of liquid ether. The colloidin will immediately soften and become perfectly transparent. Place the slide in 80 per cent. alcohol, or even directly in 95 per cent. if desired. I have not myself found this method safe.

Instead of pouring the ether vapour over the slide, it may, of course, be treated with ether vapour in a preparation glass or similar arrangement, which I think preferable.

GAGE (Proc. Amer. Soc. Mic., 1892, p. 82) advises that the slide be one that has been previously coated with a 0.5 per cent. solution of white of egg and dried ; the collodion adheres much more strongly to an albuminised surface.

Auburtin (Anat. Anz., xiii, 1897, p. 90) arranges on a clean slide, dehydrates the sections with blotting-paper and treatment with absolute alcohol, then drops on to them a mixture of alcohol and ether which dissolves out the celloidin from the sections, then allows the thin collodion thus formed to evaporate into a thin sheet on the slide. Then 70 per cent. alcohol and other desired reagents.

Similarly, MAIER (Münch. med. Wochenschr., lvii, 1910, No. 12 ; Zeit. wiss. Mik., xxvii, 1910, p. 385), but adding a treatment for ten to fifteen minutes with sulphide of carbon.

See also Mrers, Arch. Anat. Phys., Anat. Abth., 1902, p. 371 (complicated).

196. AрÁTHY's Oil of Bergamot Method (Mitth. Zool. Stat. Neapel, 1887, p. 742 ; Zeit. wiss. Mik., v, 1888, pp. 46 and 360, and vi, 1889, p. 167).-Cut with a knife smeared with yellow vaseline and wetted with 95 per cent. alcohol. Float the sections, as cut, on bergamot oil (must be green, must mix perfectly with 90 per cent. alcohol, and must not smell of turpentine), or on carbolxyol (Mikrotechnik, p. 176). The sections flatten themselves out on the surface of the oil, and are then transferred to a slide which (АР́́тну, Mikrotechnik, pp. 127 and 176) has been previously collodionised and dried.

If the sections are to be stained, the slide after removal of the bergamot oil, by a cigarette paper, is exposed for a few minutes to the vapour of a mixture of ether and alcohol, then brought into 90 per cent. alcohol, and after a quarter of an hour therein may be stained in any fluid that contains 70 per cent. alcohol or more.

If it be desired to stain in a watery fluid, care must have been taken when arranging the sections to let the celloidin of each section overlap that of its neighbours at the edges, so that the ether vapour may fuse them all into one continuous plate. This will become detached from the slide in watery fluids, and may then be treated as a single section. Terpinol may be taken instead of bergamot oil. 
19\%. АрÁthy's Series-on-the-Knife Method (Zeit. wiss. Mik., vi, 1888 , p. 168).-The knife is well smeared with yellow vaseline, rubbed evenly on, and is wetted with alcohol of 70 to 90 per cent. As fast as the sections are cut they are drawn with a needle or small brush to a dry part of the blade, and there arranged in rows, the celloidin of each section overlapping or at least touching that of its neighbours. When a series (or several series, if you like) has been thus completed, the sections are dried by laying blotting-paper on them, and the series is painted over with some of the thinnest celloidin solution used for imbedding, is allowed to evaporate for five minutes in the air, and the knife is then removed and brought for half an hour into 70 per cent. alcohol. This hardens the celloidin around the sections into a continuous lamella, which can be easily detached by means of a scalpel, and stained, or further treated as desired.

198. WeIgERT's Collodion Method (Zeit. wiss. Mikr., 1885, p. 490).-Slides, or larger plates of glass, are prepared by coating them with collodion in a thin layer, as photographers do, and allowing them to dry (they may be kept thus in stock). Sections (cut wet with alcohol) are got on to one of these (by a roundabout process, not essential), and arranged in order, and gently pressed down with paper.

Now remove with blotting-paper any excess of alcohol that may remain on or around the sections, pour collodion over them, and get it to spread in an even layer. As soon as this layer is dry at the surface you may write any necessary indications on it with a small brush charged with methylen blue (the colour will remain fast throughout all subsequent manipulations).

The plate may now be either put away till wanted in 80 per cent. alcohol, or may be brought into a staining fluid. The watery fluid causes the double sheet of collodion to become detached from the glass, holding the sections fast between its folds. It is then easy to stain, wash, dehydrate, and mount in the usual way, merely taking care not to use alcohol of more than 90 to 96 per cent. for dehydration. Weigert recommends for clearing the mixture of xylol and carbolic acid ( $\$ 167)$.

The series should be cut into the desired lengths for mounting whilst in the alcohol.

A good method for large and thick sections.

For BLochman's modification see § 191.

STRASSER takes gummed paper instead of the glass plates used in this process. See the papers quoted $\S 192$. 
See also Wintersteiner (Zeit. wiss. Mik., x, 1893, p. 316) and КUво (Arch. mik. Anat., lxx, 1907, p. 173).

199. OBRegIA’s Method. - Slides are prepared as directed (§ 191), the sections are arranged on them and covered with celloidin or photoxylin and evaporated as described, § 191.

For Dimmen's modification see also § 191.

200. Collodion Film Method.*-Graham KerR (in litt., 1908) seriates on Kodak films. A film has the emulsion removed by hot water. The sections are arranged on a dry film, and the application of a drop of absolute alcohol and ether (or an atmosphere of alcohol and ether) suffices to weld them into a mass with the film. The sheet may then be stained and mounted, or rolled up and stored in cedar oil.

Other Methods for Celloidin Sections.-See $\S 193$ (FoL) and 182 (OLt).

* The late Dr. S. G. Scott used mica sheets, upon which he stuck paraffin sections. These could be distributed to a class of students by simply cutting out pieces of mica supporting the sections. 


\section{CHAPTER XI.*}

\section{STAINING.}

201. Dyes.-In practice, the staining materials are usually of the nature of neutral salts. But in the so-called "basic" dyes it is the base or the cation that consists of the complex organic compound possessing colour; in the " acidic" dyes it is the acid or anion that is the staining agent. In the former case the colour-base is combined with a simple acid, generally hydrochloric or sulphuric, but sometimes acetic. In the latter case the colour-acid is combined with an inorganic base, usually sodium. For example, the dye called fuchsin is the hydrochloride of the base rosaniline, and its staining properties are clearly due to the latter. Acid-fuchsin, on the other hand, is the sodium salt of a sulphonic acid derived from fuchsin, and its coloured constituent is present as the acid. The free colour-base or colour-acid is in most cases insoluble in water, although it may exist therein in the colloidal state. These are frequently colourless in themselves. It follows that the addition of acids to formulæ for "acidic" dyes or bases to "basic" dyes is devoid of a rational foundation.

Although the terms " acidic" and "basic" serve to indicate an important difference between dye-salts, their careless use may lead to unwarranted conclusions. Thus, if a cell-constituent takes up a basic dye, it does not follow that this body has the chemical nature of an acid. It may have, but substances other than acids are stained by basic dyes, as we shall see below.

A " neutral " dye-salt may clearly also be formed by combination between a colour-base and a colour-acid. These compounds are for the most part insoluble in water, although soluble in alcohol. Being of high molecular dimensions, they have the properties of colloids, amongst others, that of forming permanent colloidal solutions in the presence of excess of either component. Moreover, the composition of the dye that is precipitated varies according to the relative proportion of the two reagents in the solution. It is, therefore, not a simple salt, but rather a "colloidal-complex" or "adsorptioncompound." These dyes have limited use, more especially in

* W. M. Bayliss. 
distinguishing between various kinds of leucocytes in blood (see Ehrlich and Lazarus, Die Anaemie, Wien, 1898).

Since the acidic dyes are salts of fairly strong sulphonic acids with strong bases, they are electrolytically dissociated in solution to a large extent. Thus their solutions contain coloured anions, colourless cations (usually $\mathrm{Na}$ ), together with undissociated salt. They are only hydrolytically dissociated to a negligible degree, if at all. The basic dyes, on the other hand, are salts of weak bases (amino- or imino-derivatives) with strong acids. Although electrolytically dissociated as salts, so that their solutions contain coloured cations and undissociated salt together with colourless anions, they also undergo hydrolytic dissociation to a notable degree. Thus they contain free colour base and free colourless acid in addition to their ions.

It is important to remember that commercial samples of dyes contain mineral salts, as a rule, sodium chloride or sulphate, sometimes as much as 30 per cent. or more. This must be borne in mind in statements as to their properties. For instance, it is often said that the Congo-red dyes are direct dyes for cotton fibre. This is only the case in the presence of salts, as will be shown presently.

Details of the chemical composition of different dyes are beyond the scope of this book. The reader is referred to CAIN and THORPE's Synthetic Dyestuffs, 1913.

202. The Nature of the Staining Process.-From what has been said in the preceding paragraph it will be realised that a solution of a dye is a complex system from a physico-chemical standpoint. Moreover, the structures to be stained are present as separate phases, solid or liquid, of a heterogeneous system. It is clear, therefore, that the properties of boundary surfaces must be taken into consideration, in addition to differences of chemical composition and of colloidal state. Much discussion has taken place with respect to the process of dyeing, and various theories of its nature as being essentially chemical or essentially physical, in the sense of adsorption, mechanical or electrical, or in the sense of solid solution, involving partition between the solution and the tissue elements according to relative solubility of the dye therein, have been advocated. It is probable that all these factors play their part in varying proportion and that no one theory alone can explain all the facts.

We shall be in a better position to appreciate the complexity of the conditions present if we examine, to begin with, the case of a pure substance, cellulose, in relation to pure solutions of an acidic and a basic dye respectively. 
We take, then, a piece of the purest analytical filter paper, wash it with distilled water to remove possible traces of acid, and place it in a dilute solution of Congo red, freed from foreign salts. It is scarcely stained at all. Add next a very small amount of a neutral salt, say, sodium chloride. The paper is deeply stained. How are these facts to be explained? In view of the chemical inertness of cellulose, it seems unlikely that a chemical combination occurs between the dye and the paper under the influence of a neutral salt at ordinary temperatures. Moreover, the same behaviour is shown by such different substances as charcoal, silk, alumina, silica, and so on. The process must be one of adsorption or deposition of the dye on the surface by some means. In other words, it must be associated with the decrease of surface energy of some kind. In the absence of foreign electrolytes, adsorption may be due to decrease of surface energy of the ordinary kind, shown as surface tension. This is confirmed by the fact that the dye, in the absence of electrolytes, can be washed out again by water. But since the degree of staining is very small, there must be some influence at work restricting the mechanical adsorption. There is, indeed, another property of the boundary surfaces between phases which demands attention here. This is the electrical charge, nearly always present. If we test paper in water, we find that it has a negative charge. Similarly, by appropriate means, we find that the dye itself has a negative charge. Whether this is that of the coloured anion or of complex aggregates of these ions with undissociated salt is not certain, but, according to Freundlich, " acidic" dyes are adsorbed as a whole. In the present case it is immaterial, because the material to be adsorbed has a negative charge in either case. This being so, there are repellent forces acting between the dye and the paper. Or, if we take the point of view of energetics, the adsorption of electro-negative dye would increase the negative charge on the paper, with an increase of free energy, which is contrary to the Second Law of Thermodynamics. Suppose, however, that we have also present the ions into which a neutral salt dissociates. The cations, being positively charged, are deposited on the surface of the paper, decreasing or annulling its negative charge and reducing the free energy. There is now little or no obstacle to the adsorption of the dye. From the work of PerRin we know that an ion may be adsorbed on an oppositely charged surface to so great an extent that the charge on this surface may actually be reversed in sign. This occurs, as it appears, when surface energy of some kind other than electrical is diminished by the presence of such ions, and is found mainly with 
plurivalent or organic ions. But since colloids are readily precipitated by such ions, owing to neutralisation of their charges, it may happen that a dye such as Congo red is neutralised and precipitated before it has attached itself to the adsorbing surface by virtue of its charge.

Since the amount adsorbed is dependent on the magnitude of the electric charge, we have an explanation of the fact that alcohol diminishes the effect of electrolytes. The charge on a surface is proportional to the dielectric constant of the liquid phase in which it is immersed, and the dielectric constant of alcohol is less than that of water.

It is a well-known fact that colloids of the suspensoid class, such as gold and coagulated egg white, are much more sensitive to the action of electrolytes than are those of the emulsoid class, in which the colloidal particles themselves contain water and differ from the medium in which they are suspended merely by the smaller quantity of water which they contain. Such are gum arabic, starch, colloidal silica, and proteins in general, gelatin, raw egg white, and so on. Faraday showed that colloidal gold could be protected from precipitation by salt if a trace of gelatin was added. This is explained by the adsorption of a coating of gelatin over the gold particles, which are thereby converted into the emulsoid variety so far as their surfaces are concerned. We find similar phenomena in the staining of paper by Congo red. It is protected from the dye even in the presence of salts. But the conditions are made more complex by the possibility of using in the experiment either an electro-positive or electro-negative protein. A trace of acid or alkali respectively has this effect on proteins, by the production of dissociated salts. Now the former are more powerfully adsorbed by the negative paper than the latter are, while at the same time they reduce, instead of increasing, the electric charge. Actually the latter effect preponderates, so that the presence of electropositive protein increases the depth of staining.

Turning now to the basic dyes, we find that the paper is the more deeply stained the lower the concentration of salt present. According to Freundlich, it is the colour base that is chiefly adsorbed in this case. As was pointed out above, these dyes are hydrolytically dissociated, so that free base is present. This free base, being insoluble, is in the colloidal state, and, like colloidal bases in general, has a positive charge, due to electrolytic dissociation of the surface of the particles. See HARDY in Van Bemmelen Gedenkboek, p. 188. Thus, not only are the coloured ions, in this case the 
cations, strongly adsorbed by the negative paper, but the free base is also. Since foreign electrolytes diminish the charge on the paper, their effect on staining by basic dyes is naturally of the opposite kind to that described in the case of the acidic dyes. The effect of alcohol is in the same direction as that of electrolytes, since it also decreases the electric charge and, therefore, the amount of dye adsorbed. Facts of the kind referred to in the preceding statements have given rise to an "Electrical Theory of Dyeing," which probably accounts for a larger number of them than any other single theory is able to do. For further particulars of theories and facts relating to dyeing and staining; the reader is referred to Alfred Fischer's Fixirung, Färbung und Bau des Protoplasmas, Jena, 1899 ; PeletJoLIVET's Theorie des Farbeprozesses, Dresden, 1910; First Report on Colloid Chemistry, Brit. Ass., 1917 ; Gee and Harrison, Trans. Faraday Soc., vol. vi, 1910; Harrison, Journ. Soc. Dyers and Colourists, December, 1911 ; BAyliss, Biochem. Journ., vol. i, 1906 , p. 175.

The reducing properties, in the chemical sense, of cell constituents play an important part in certain special staining reactions, particularly those with metallic salts. Osmic acid (osmium tetroxide) is reduced to a lower oxide or perhaps to the metallic state by unsaturated fats. Gold and silver salts are reduced by nervous tissue under appropriate conditions and deposited in the colloidal state. All the various colours of colloidal gold in different degrees of dispersion may be seen in tissues prepared by the gold impregnation methods. Details of these methods will be found in other chapters of this book.

Effect of Heat.-Since surface tension is decreased by rise of temperature, it would naturally be expected that, if this factor is concerned in the process of staining, the depth of the stain would be lessened at a higher temperature. This is actually the case with simple substances like cellulose up to $50^{\circ} \mathrm{C}$. or thereabouts. At first sight this would seem to be at variance with the frequent practice of fixing stains by heating the preparation. But the temperatures used are much higher than those referred to, and, in point of fact, if filter paper is stained in a Congo red solution at $100^{\circ} \mathrm{C}$., the dye is firmly fixed, and cannot readily be removed by washing. It is difficult to say what actually happens here. Chemical combination suggests itself, but the nature of the compound formed is not easy to conceive. The temperature at which a material possesses the maximum electric charge, as shown by GEE and HARRISON, also plays a part in the phenomena. 
Chemical combination obviously occurs in some cases when substances stained by adsorption are heated to $100^{\circ} \mathrm{C}$. Thus Bayliss (Proc. Roy. Soc., B., vol. Ixxxiv, 1911, p. 83) showed that various insoluble hydroxides, such as that of aluminium, are stained blue by the free acid of Congo red, which is its own colour. When heated to $100^{\circ} \mathrm{C}$, combination takes place with the formation of the usual red colour of the salts of this dye. Silk behaves in the same way, and even crystals of leucine, doubtless to be explained by the formation of salts with the fairly strong acid.

Conditions similar to this, however, do not arise in histological staining, where dye salts are used. It is difficult, moreover, to see how the conditions for reaction by double decomposition could arise in the cell, since acids and bases sufficiently strong to displace hydrochloric acid or sodium hydroxide are required. Reactions with precipitation might occur, but these would give rise to the appearance of new solid structures in the cell. There is no evidence that such precipitates are produced in a simple staining process, although they are undoubtedly formed by fixing agents (HARDY, Journ. of Physiology, vol. xxiv, p. 158).

The following experiment by Martin Heidenhain is sometimes given as evidence of the formation of salts of dyes with proteins. A solution of Congo red, as is well known, turns blue when made acid with acetic acid, owing to the separation of the free acid. If such a blue solution is added to an acidulated solution of serum albumin, a red solution is obtained. Since this is the characteristic colour of the salts of Congo red, it is natural to interpret it as a salt of the dye acid with the protein base. But the fact that it exists in a solution sufficiently acid to decompose the sodium salt of the dye shows that the colour acid is more firmly combined with protein than with sodium, a view that it is difficult or impossible to hold. Moreover, it appears that even 5 per cent. sulphuric acid is unable to split off the acid from some of these protein " compounds." It is clear that the phenomena must have a different interpretation. It may be that the free colour acid exists in two forms, a true and a pseudoacid-the former of a red colour and ionised; the latter, blue, insoluble and non-ionised. When adsorbed by protein, for some reason or other, the acid may be for the most part in the former condition. See the work of Wo. Ostwald on Congo-rubin (Koll. Chem. Beihefte, B. $x, 1919)$. But further evidence is required.

203. Removal of Dyes.-When a stain is fixed by adsorption of the ordinary, non-electrical type, it can be removed by frequent washing with distilled water. This is very difficult if the dye is held 
by electrical forces. The reason is, in all probability, that the dye can only be set free by reversing the sign of the charge on the surface. This cannot be done by pure water alone. It can be done, however, by acid or alkali in the appropriate case. For example, if an acidic dye has been fixed on a negative surface by the aid of cations, which convert the charge to a positive one, $\mathrm{OH}^{\prime}$ ions, provided by alkali, are powerful enough to change the sign of the charge back again to negative and thus free the dye, whereas $\mathrm{H}^{\circ}$ ions from an acid only increase the positive charge and fix the dye more firmly. Hence the statement that acidic dyes are fast to acids. A basic dye, adsorbed by a negative surface, is removed by acids and intensified by alkalies. A corresponding explanation holds. Thus, $\mathrm{H}^{*}$ ions from acids make the surface more positive, hence the dye is released. $\mathrm{OH}^{\prime}$ ions make it more negative, hence the dye is held faster. In all cases, if the acid or alkali is strong enough, any dye-salt adsorbed is decomposed, sometimes with change of colour.

The process of " differentiation" by alcohol or other agent, to be referred to below, is an application of these facts. Alcohol removes a " basic" dye because it reduces the negative charge of the tissue elements and thus releases part of the positively charged constituent of the dye adsorbed.

204. "Specific" Stains.-Certain tissue elements and cellconstituents have the property of staining deeply with particular dyes. That of nervous structures with methylen blue and of mitochondria with dyes containing di-ethyl-safranin, such as Janusgreen, may be given as examples. The property may be shown either by their taking up the stain from a dilute solution more rapidly than other structures present do ("progressive" staining), or by their holding on to it more tightly when excess of general stain is washed away by appropriate treatment. This latter process is sometimes known as "differentiation" or as "regressive" staining.

It is natural to interpret this behaviour as due to a chemical combination of a special kind, as did EHRLICH in his well-known theory of "chemo-receptors," according to which certain " sidechains" of protoplasmic molecules have special affinities for particular groups in the dye molecules. While this may be the case in isolated instances, there are many facts which show that it cannot be accepted as a general law. It is difficult to see what purely chemical relationship can exist between complex, substituted, diazo-sulphonates, as a large number of these specific dyes are, and the chemical components of cells. Moreover, although methylen 
blue and other thiazines are specific vital stains for nervous tissue, certain safranin azo-dyes - diazin-green, for example-which have no chemical relationship to the former, are also vital nerve stains ; while similar compounds of the safranin series itself have no such property. See Mrchaelis, Chemie der Farbstoffe, 1902, p. 104. We must remember also that the conception of large protoplasmic molecules with side-chains in the chemical sense, the so-called "biogens," is becoming more and more discredited. See Hopkins, Address to Physiology Section, Brit. Assoc., 1913. We have already seen how complex are the physical factors that intervene in such a simple case as the staining of paper, and to these may be added questions of solid solution, distribution between phases, diffusibility, and so forth. Indeed, it would seem that each individual case of specific staining requires investigation by itself. Evans (Amer. Journ. of Physiol., vol. xxxvii., p. 255) in an investigation of " macrophages" comes to the conclusion that chemo-receptors are not responsible for vital staining with the benzidine and related series of dyes.

Alfred Fischer (op. cit., pp. 107-150) gives interesting cases of staining differentially particles of the same substance by different dyes. Particles can be made to take up either dye, according to their size, the order in which the different dyes are applied and the degree of differentiation by removal of stain.

205. Some Applications of the Theory of Staining.-It is of interest to see how some histological facts are explained in the theory sketched above. Most of the structures in the living cell have negative electric charges, probably on account of the slightly alkaline nature of the surrounding fluids. This fact accounts for the ease with which tissues in general are stained by basic dyes. It is remarkable that hæmoglobin is one of the few constituents that have a. positive charge. Accordingly, it is stained by acidic dyes, such as eosin or acid fuchsin. The effect of electrolytes is shown in the experiments of Mayr (Hofmeister's Beitrage, vol. vii., p. 560). He finds that the affinity of Nissl bodies for basic dyes is abolished by previous treatment with neutral salts.

206. Objects of Staining. - Most constituents of cells are, in their natural state, either colourless or only faintly coloured. Thus they are only visible if their refractive indices differ from those of the media in which they are immersed. Such, for example, are fatty globules and the granules of many secreting cells. But, as seen thus, it is not an easy matter to judge of their true forms. This is 
greatly facilitated by staining them either more deeply than or of a different colour from their surroundings. If colourless glass beads, although they are easily seen by refraction, could only be observed from the direction of a line through the hole in the centre, the recognition of their true form would be difficult. Immersing them in a medium of the same refractive index as themselves would render them invisible. But if they were made of coloured glass and immersed in such a medium, they would be readily detected and their shape recognised.

The chief object of histological staining is then to cause certain constituents of the cells to take on a different intensity of tint from others. This may be done in various ways, as will be seen later. It is usual to distinguish two kinds of selective staining, histological and cytological selection. In the former an entire tissue or group of tissue elements is prominently stained, the elements of other kinds present remaining colourless or being differently stained, as in the impregnation of nerve endings by the silver and gold reduction methods. In the latter the stain is taken up or retained by some constituent element of the cell, such as the chromatin of the nucleus or an element of the cytoplasm.

The nuclear stains are of importance in marking out the contours and relations of the tissues making up regions or organs as a whole and are thus of special value to the embryologist and morphologist.

At one time, it was thought to be possible to distinguish between "basophilous" and "acidophilous" tissue elements, according to their affinity for basic or acidic dyes. EHRLICH (Du Bois Reymond's Archiv., 1879, p. 571) thought that the basic dyes have a special affinity for the chromatin of nuclei and the acidic dyes for the cytoplasm and intercellular substances. But we have already seen that the same substance may take up either kind of dye, according to the conditions present. Most staining processes are undertaken on cells which have been acted on by fixing reagents or by the socalled "mordants," and these may reverse the natural behaviour to dyes. EHRLICH's statement only applies in fact to cover-glass preparations fixed and dried by heat, without the action of reagents. The acidic colours, orange and acid fuchsin, although they stain cytoplasm, may give good chromatin differentiation when used as regressive stains. Methylen blue is basic, but stains nerves. The widely used carmin and hæmatoxylin are both acidic dyes, but in combination with alum they give nuclear stains. Other instances might be given, but these will suffice. 
20\%. Intra-vitam Staining. - It is clear that unless the cell-membrane of a living cell is permeable to a dye, no constituent of the cell can be stained by it. Most dyes appear to be more or less toxic if they enter the cell. But, while alive, the latter is to a large extent protected, since the dye does not obtain entrance. A living Amoba is stained by very few dyes. Neutral red, however, passes through the membrane and stains various structures, while having no apparent effect on the activities of the organism. The auricle of the frog's heart can also be stained with this dye, while continuing its normal contractions. Used in this way, the dye is applied in very dilute solution. Since neutral red is a very sensitive indicator just about the neutral point, the fact of its permeability and non-toxicity makes it a valuable test for the presence of acid or alkali within the cell.

When a dye enters a living cell, it usually stains various granules and structures contained therein, while at the same time it is uniformly diffused through the liquid phase of the protoplasm. If the process of staining is conditioned by phenomena at boundary surfaces, simple undifferentiated protoplasm in the living state should be incapable of staining, and this seems to be the general experience. As regards the question of permeability to a given dye, unless the cell is able to show that it is still alive by movement or by contractility, it is clearly a matter of difficulty to be certain that, when a particular dye enters, it does so during life or only after it has destroyed the normal properties of the cell-membrane. The nucleus itself seems to be very resistant to dyes while alive, and it has been stated that the appearance of stain in it is a sure indication of death. BoLLES LEE made a large number of observations and came to the conclusion that most of the "intra-vitam" stains are either due to mere diffusion through the liquid protoplasm or that the stained constituents were not really living, being food particles or products of cell activity.

At the same time, many of the methods which come under this heading are of much value. Methylen blue may be injected into the living animal and frequently gives very successful staining of nervous structures, owing to the fact of its being conveyed into intimate contact with the cells by means of the blood vessels.

The various methods of preserving the stain in the structures to which it was localised during life obviously depend on the adequacy of the means used to fix and maintain these structures and to retain the properties owing to which the stain was taken up. This is by no means a simple matter. Moтt describes in living nerve cells a 
number of minute particles which stain on the outside with methylen blue. In fixed cells, as is well known, these particles aggregate together to form the "Nissl granules." MichaELis found similar granules in liver cells. As the cells die, the stain leaves the granules and passes into the nucleus.

The behaviour of the living nucleus to methyl green has given rise to some discussion. It appears that no uni-cellular organism in which the nucleus was stained has been observed to move, whereas the chlorophyll grains may take up the stain while the cell is normally motile. No convincing case of staining of the living nucleus has in fact been described.

The question as to whether the cell elements which stain during life are to be described as living or not is scarcely putting the problem from the right point of view. If a dye obtains contact with the interfaces between constituents of a cell, it will in all probability be deposited there to a degree depending on the various properties of the interface described previously. This may occur independently of the fact as to whether one or both of the phases is living.

Apart, however, from these questions, it must be conceded that these so-called "vital stains" are frequently very useful. According to BOLLES LEE's experience, methylen blue is the most generally useful of them. It has (with Bismarck brown, Congo red, and neutral red) the valuable point that it is sufficiently soluble in saline solutions, and may therefore be employed with marine organisms by simply adding it to sea-water. The others are not thus soluble to a practical extent, but BoLles LeE finds that gentian and dahlia become so if a trace of chloral hydrate -0.25 per cent. is ample enough - be added to the saline solution. Any of these reagents may be rubbed up with serum, or other "indifferent" liquid.

Methylen blue may be fixed in the tissues, and permanent preparations made, by one or other of the methods described in Chap. XVI. Bismarck brown stains may be fixed with 0.2 per cent. chromic acid or with sublimate solution (MAYER), or 1 per cent. osmic acid (LOISEL, Journ. de l'A nat. et de la Phys., 1898, No. 2, p. 212-a work that contains a good deal of information on the subject of intra-vitam stains), and the preparations may be stained with safranin, care being taken not to expose them too long to the action of alcohol. For the study of cellgranules, neutral red is perhaps the best.

Fiscres (Unters. ueb. vitale Faerbungen, Leipzig, 1908) finds that alizarin is specific for nerves. Add excess of alizarin to boiling water, boil and filter, and add 1 vol. of the filtrate to the water containing the organisms (Cladocera). The stain takes several hours.

For sulphorhodamin, which is selective for many organs (kidney, liver, uterus, skin, lymph-glands, etc.), see ANDREEw, in Virchow's Arch., eciv, 1911, p. 447.

The details of the various methods used for intra-vital staining 
and the fixation of the results are described in other parts of this book. The reader may be referred to the work of Goldman (Unters. ueber die Sekretion des Organismus im Lichte der "vitalen Färbung," Laupp : Tübingen, 1912) for certain aspects of the problem.

208. Dead Cells.-Changes take place in cells in consequence of which they cease to respond to external stimuli and are said to be " dead." Their staining reactions are naturally different in this state both from those during life and from those after their structures have been "fixed" by treatment with some appropriate solution. Although it seems likely that valuable information would be obtained from the investigation of their behaviour in the dead but unfixed state, very little work appears to have been done. DoGIEL obtained various differentiations in nerve cells by observations at various stages after death.

209. Fixed Tissues.-The majority of staining methods are undertaken on tissues that have been fixed and hardened by reagents. It is sufficient to mention here that some of these reagents merely serve to coagulate or precipitate the constituents of cells without marked changes in their chemical nature, although their physical state is more or less altered. Alcohol is one of these agents. Other fixing fluids, of which those containing chromic acid are representatives, produce what seem to be compounds of cell proteins with the reagent. In this latter case, we have what is known as a "mordant" present.

The distinction is often made between "substantive" and "adjective" staining. These expressions are really based on a particular theory of the staining process and are somewhat difficult to justify logically. When a substance takes up a dye without the necessity of the presence of any additional reagent, the staining is said to be "substantive." When a mordant is required, it is "adjective." The only true case of the former process is when an electro-negative surface adsorbs a basic dye. Such a surface is practically unstained by an " acidic" dye, when pure. The presence of an electrolyte is necessary. But sodium chloride may suffice, and the question arises whether this should be called a mordant. Mordants are usually understood to be agents which form insoluble compounds with dyes and in this way cause their fixation in places from which otherwise they might be washed out by the subsequent treatment with dehydrating agents, etc. Such a substance may clearly be either already present in the fixed preparation when the dye is added, or it may be added together with or subsequently to 
the staining agent. It will readily be understood that the production of an insoluble dye salt renders solution in water more difficult, but this alone is not enough. The compound must also be firmly attached to the surface. Thus, the barium salts of colour acids are insoluble, but barium salts cannot be used as mordants for acidic dyes ; the compound formed is readily washed off mechanically.

The insoluble compounds between a dye and a mordant are known as "lakes." But the chemical nature of these substances is by no means clear-especially when they are produced in situ in stained tissues. In fact, they do not behave as simple compounds of the dye and the mordant. They resist the action of strong acids and bases in moderately strong solutions. So that the stains obtained by this method are characterised by durability and " fastness."

On the whole, we must conclude that there are factors still unknown, but probably related to the electrical properties of the surface, which play an important part in these reactions with mordants. A simple chemical theory does not account for all the phenomena. Changes in the properties of the surface by the action of the mordant are concerned. In this connection, we may call to mind how frequently multivalent cations are used as mordants. Alum is one of the commonest of these.

A further mention may here be made of the "progressive" and "regressive" methods, between which there is a more definite distinction than that between "substantive" and "adjective" staining. A preparation may be placed in a very dilute solution of a dye and the action stopped when the elements with the greatest " affinity" for the dye have taken it up. The staining of nuclei with dilute hæmatoxylin-alum may be mentioned. If the action is prolonged, various other constituents of the cell, besides the nucleus, take on the colour. As a rule, no great differentiation is obtained by this method. Better results are given by the "regressive" method, in which a general overstaining is followed by a partial decoloration, in which certain elements retain the stain, owing to special chemical or physical properties, after it has been removed from the rest. Thus safranin stains the whole section of a deep red colour; acting on it with alcohol removes the stain from all but the chromatin and the nucleoli. This action of alcohol may be explained, as already pointed out, by its effect on the magnitude of the electric charge, since its dielectric constant is lower than that of water. Other differentiating agents are also used. Iron-alum, in the ironhæmatoxylin method, serves both as preliminary mordant and as 
differentiating agent. This double action is not easy to explain and confirms what was said above as to the complexity of the process.

210. Metachromasy. - There are a few dyes, mostly of the basic aniline series, which stain certain elements in the colour of the ordinary solutions of the dye, other elements in that of the free colour-base. Safranin stains nuclei red; mucin and the ground substance of cartilage, orange. Methyl violet stains "amyloid," and mucin red.

Although in a few cases this behaviour may be due to the dye being really a mixture of two dyes, as in the case of iodine green, there is no doubt that this is not the explanation of genuine cases. According to MichaELIS, the appearance of the colour of the base is not due to the alkalinity of the elements in question. The fact that the red stain given to mucin by thionin can be changed into blue by alcohol and back to red by water shows that the change is not one involving great alterations of chemical structure, and a tautomeric one is naturally suggested. It would appear that the change is one by which an amino-group becomes freed from its combination with the mineral acid of the salt. In the case of thionin, the acid is supposed to change its connection to the nitrogen which unites the two benzene rings. What are the conditions which regulate the change from one form to the other is unknown. A similar difficulty is met with in the case of iodine, which is brown in solution in alcohol, violet in chloroform.

We must, however, not overlook the changes in colour shown by substances in the colloidal state merely in consequence of a decrease in their degree of dispersion or increase in size of particles. Gold is a notable case. It.may be red, violet, blue or green. All of these tints are met with in its use as a histological reagent. Whether similar phenomena may occur in the adsorption of dyes is uncertain, but cannot be dismissed without further evidence.

211. The Use of Stains in Practice.-Stains for special purposes are described in other pages of this book. It will be obvious from the contents of the present chapter that caution must be exercised in making deductions as to chemical composition from behaviour to dyes.

In general, the dyes of the anilin series, although of great value for particular purposes, are apt to be less permanent than the old logwood and carmine stains. The Canada balsam used must be as pure as possible if the stain is to. last for any length of time. 
The most distinctive processes, involving the use of mordants and regressive differentiation, can only be undertaken on sections. Staining in bulk is useful when the general anatomy is the object of study. For cytological work it is of little value.

As remarked, the main object of staining is to obtain better information of the appearance of the structures present in the cell. The fact, however, that this appearance is not necessarily that of the living state should never be allowed to escape remembrance. Without special investigation of the case, it is not permissible to draw conclusions as to the chemical nature of a cell constituent from its behaviour to dyes.

212. Choice of a Stain.-Mr. Bolles LeE believes that the following may be recommended to the beginner for general work:-For sections, MAYER's homalum; or, for chromosmium objects more especially, BENDA's or HeIDENHAIN's iron hæmatoxylin.

For staining in toto Grenacher's alcoholic borax-carmine, or Mayer's carmalum, or hæmalum, unless the object be so impermeable as to require a very highly alcoholised stain, in which case take Mayer's paracarmine, or for chromic acid objects Mayer's hoemacalcium.

For fresh tissues or small entire objects, methyl green, if it is not important to have permanent preparations ; if it is, take carmalum or alum-carmine (but both of these may give precipitates with marine animals). 


\section{CHAPTER XII.}

\section{CARMINE AND COCHINEAL STAINS.}

213. Carmine.-Carmine is by no means merely carminic acid with at most certain impurities. According to the analysis of Liebermann (Ber. d. Chem. Ges., Jahrg. 18, 1886, pp. 1969-1975) it is a very peculiar alumina-lime-protein compound of carminic acid, a true chemical compound from which at all events aluminium and calcium can no more be absent than sodium from salt. It results from the researches of MAYER (Mitth. Zool. Stat. Neapel, x, 1892, p. 480) that in the processes of histological staining (not of industrial dyeing) the active factors of the compound are, besides the carminic acid, always the alumina, and in some cases the lime. The other bases are inactive; the nitrogenous matters, so far as they have any influence at all, are an obstacle, as it is they that give rise to the wellknown putrefaction of the solutions.

This being so, it follows that carminic acid may, if desired, be taken as the basis of staining solutions instead of carmine. Staining solutions thus prepared do not give essentially better stains than those made with carmine; but have the advantage of being of more constant composition. For carmine is a product which varies greatly from sample to sample.

Carminic acid of sufficient purity is furnished by GRüBLER and Hollborn (or C. A. F. Kahlbaum, in Berlin). It is soluble in water and weak alcohol (that of 70 per cent. only dissolves less than 3 per cent.). It cannot be used alone for staining, as it only gives in this way a weak and diffuse stain.

214. Cochineal.-According to Mayer (Mitth. Zool. Stat. Neapel, $\mathrm{x}, 1892, \mathrm{p} .496$ ), the active principle of extract or tincture of cochineal (as used in histology) is not free carminic acid, but carminic acid chemically combined with a base which is not lime, but some alkali. The watery extract made with alum, or cochineal-alum carmine $(\S 216)$, owes its staining power to the formation of carminate of alumina (last $\S$ ). The tincture made with pure alcohol, on the other hand, contains only the above-mentioned carminate of some alkali. This carminate alone stains weakly and diffusely (like carminic acid alone). But if in the tissues treated with it it meets with lime salts, 
alumina or magnesia salts, or even metallic salts capable of combining with it and forming insoluble coloured precipitates in the tissues, then a strong and selective stain may result. And if the necessary salts be added to the tincture itself, there results a solution containing the necessary elements for affording a strong and selective stain with all classes of objects. Hence Mayer's new formula, $\S 236$.

215. General Remarks.-Carmine stains are chiefly used for staining entire objects, or tissues in bulk. In most cases this can be done more satisfactorily by means of carmine than by means of any other known agent. For most hæmateïn solutions have a disastrous tendency to overstain; and the tar-colours are generally inapplicable to staining in bulk.

Grenacher's alcoholic borax-carmine may be recommended to the beginner as being the easiest of these stains to work with: or paracarmine, for objects which require a highly alcoholic solution. Carmalum, or one of the alum-carmines, is also an easy and safe reagent.

Overstains may in all cases be washed out with weak $\mathrm{HCl}$ (e.g. 0.1 per cent.). Alum-solution will often suffice, or, according to Henneguy (Journ. de l'Anat. et de la Physiol., xxvii, 1891, p. 400), permanganate of potash. All carmine stains, with the exception of aceto-carmine, are permanent in balsam. The alum-carmines are fairly permanent in glycerin. None of the acid stains, nor any of Grenacher's fluids, should be used with calcareous structures that it is wished to preserve, unless they be taken in a state of extreme dilution.

\section{a. Aqueous Carmine Stains.}

\section{Acid.}

216. Alum-carmine (Grenacher, Arch. mik. Anat., xvi, 1879, p. 465).-An aqueous solution (of 1 to 5 per cent. strength, or any other strength that may be preferred) of common or ammonia alum is boiled for ten to twenty minutes with $\frac{1}{2}$ to 1 per cent. of powdered carmine. (It is perhaps the safer plan to take the alum solution highly concentrated in the first instance, and after boiling the carmine in it dilute to the desired strength.) When cool, filter.

This stain must be avoided in the case of calcareous structures that it is wished to preserve.

Trzzoni (Bull. Se. Med. Bologna, 1884, p. 259), Pisenti (Gazz. degli Ospetali, No. 24; Zeit. wiss. Mik., ii, 1885, p. 378), and GrIEB (Mem. 
Soc. Ital. Sci., t. vi, No. 9, 1887 ; Zeit. wiss. Mik., vii, 1, 1890, p. 47) have given modifications of Grenacher's formula which do not appear to me rational.

MAYER (ibid., xiv, 1897, p. 29) makes a stronger stain by taking 2 grms. carmine, 5 grms. alum, and 100 c.c. water, and boiling for an hour.

Alum-carmine is an excellent stain. It is particularly to be recommended to the beginner, as it is easy to work with; it is hardly possible to overstain with it. Its chief defect is that it is not very penetrating, and therefore unsuitable for staining objects of considerable size in bulk.

21\%. Acetic Acid Alum-Carmine (Hennegur, in Traité des Méth. Techn., Lee et Henneguy, 1887, p. 88).-Excess of carmine is boiled in saturated solution of potash alum. After cooling add 10 per cent. of glacial acetic acid, and leave to settle for some days, then filter.

For staining, enough of the solution is added to distilled water to give it a deep rose tint. In order to ensure rapid diffusion it is well to bring the tissues into the stain direct from alcohol. Stain for twenty-four to forty-eight hours, and wash for an hour or two in distilled water. Mount in balsam. You can mount in glycerin, but the preparations do not keep so well.

The advantage of this carmine is that it has much greater power of penetration than the non-acidified alum-carmine.

218. Cochineal Alum-Carmine (РARTsch, Arch. mik. Anat:, xiv, 1877, p. 180).-Powdered cochineal is boiled for some time in a 5 per cent. solution of alum, the decoction filtered, and a little salicylic acid added to preserve it from mould.

Another method of preparation has been given by Czokor (ibid., xviii, 1880, p. 413).-Mayer finds that Partsch's is the more rational, the proportion of alum in it being exactly right, whilst in Czokor's it is insufficient. Partsch's fluid also keeps better.

RABL (Zeit. wiss. Mik., xi, 2, 1894, p. 168) takes 25 grms. each of cochineal and alum, 800 c.c. of water, and boils down to 600 c.c. He prefers this because it is not so purely nuclear a stain as the others.

These solutions give a stain that is practically identical with that of alum-carmine made from carmine, with perhaps even more delicate differentiations.

Rawitz (Zeit. wiss. Mik., xxv, 1909, p. 392) takes cochineal 4 grms., nitrate of aluminium (or ammonio-sulphate of cobalt) 4 grms., water 100 c.c., and glycerin 100 c.c. Only for sections.

219. MAYeR's Carmalum (Mitth. Zool. Stat. Neapel, x, 1892, p. 489).-Carminic acid, 1 grm.; alum, 10 grms. ; distilled water, 
200 c.c. Dissolve with heat (if necessary). Decant or filter. Add some antiseptic, either 1 c.c. formol, or $0 \cdot 1$ per cent. salicylic acid, or 0.5 per cent. salicylate of soda. The solution will then keep. It stains well in bulk, even osmium objects. If washed out with distilled water only, the plasma will remain somewhat stained. If this be not desired, wash out carefully with alum solution, or, in difficult cases with weak acid, followed in either case with water. The general effect is that of an alum-carmine stain.

A weaker solution may be made by taking from three to five times as much alum and five times as much water, and dissolving in the cold.

With either solution the objects to be stained should not have an alkaline reaction.

RAwitz (Anat. Anz., xv, 1899, p. 438) takes 2 grms. carminic acid, 20 grms. ammonia-alum, 150 c.c. water, and 150 c.c. glycerin. A strongly staining solution, which is said to keep well. Only for sections.

$A l l$ solutions prepared with alum tend to precipitate. Carmalum made up with 500 c.c. of water instead of 200 , and with glycerin or 10 per cent. of formol or pyroligneous acid added, keeps well.

220. Mayer's Aqueous Aluminium-Chloride Solution (Mitth. Zool. Stat. Neapel, x, 1902, p. 490).-Carminic acid, 1 grm.; chloride of aluminium, 3 grms.; water, 200 c.c. Add an antiseptic, as for carmalum.

Use as carmalum. The stain is of a blue-violet colour, very powerful, and elective, but not so purely nuclear as carmalum. It is recommended only as a substitute for carmalum in cases in which the latter is counter-indicated on account of the alum in it or the like.

221. Alum-Carmine and Picric Acid.-Alum-carmine objects may be double-stained with picric acid. LEGAL (Morph. Jahrb., viii, p. 353) combines the two stains by mixing 10 vols. of alum-carmine with 1 of saturated picric acid solution. I find this very recommendable.

Aceto-Carmine (Acetic Acid Carmine) Schneider (Zool. Anzeig., 1880 , p. 254). - To boiling acetic acid of 45 per cent. strength add carmine until no more will dissolve, and filter. (Forty-five per cent. acetic acid is, according to Schneider, the strength that dissolves the largest proportion of carmine.)

To use the solution you may either dilute it to 1 per cent. strength, and use the dilute solution for slow staining; or a drop of the concentrated solution may be added to a fresh preparation under the coverglass. If you use the concentrated solution it fixes and stains at the same time, and hence may render service for the study of fresh objects. It is very penetrating. The stain is a pure nuclear one. Unfortunately the preparations cannot be preserved, and for this and other reasons the stain is of very restricted applicability. 
A similar stain has been prepared with formic acid by Pianese (see Zeit. wiss. Mik., x, 4, 1894, p. 502).

For BuRchardT's pyroligneous-acid carmines see Arch. mik. Anat., liii, 1898, p. 232 ; and Jena Zeit. Naturw., xxxiv, 1900, p. 720.

222. Iron Carmine.-I recommend trial of the following, which I have already published in the Traité des Méth. Techniques, LEE et Hennegur, 1902. Sections (I have not tried material in bulk) are mordanted (a few hours will suffice) in sulphate of iron (Benda's liquor ferri, as for iron hæmatoxylin), washed, and stained for an hour or so in 0.5 per cent. solution of carminic acid in alcohol of 50 per cent. Wash in alcohol of 50 per cent.; no differentiation is necessary. When successful, an almost pure chromatin stain, quite as shorp as iron hæmatoxylin, but somewhat weak.

Iron Carmine.-PFeiffer voN Wellheim (Zeit. wiss. Mik., xv, 1898, p. 123) mordants for six to twelve hours in a very weak solution of chloride of iron in 50 per cent. alcohol, washes in 50 per cent. alcohol, and stains as above. Overstains may be corrected with 0.1 to 0.5 per cent. $\mathrm{HCl}$ alcohol. I find this good, but not so good as the last.

Iron Carmine (Zacharias, Zool. Anz., 1894, p. 62).- Stain for several hours in an aceto-carmine (made by boiling $1 \mathrm{grm}$. of carmine with 150 to 200 c.c. of acetic acid of 30 per cent., for twenty minutes, and filtering). Rinse the objects with dilute acetic acid, and bring them (taking care not to touch them with metallic instruments) into a 1 per cent. solution of ammoniated citrate of iron. Leave them, for as much as two or three hours if need be, till thoroughly penetrated and blackened (with sections this happens in a few minutes). Wash for several hours in distilled water. A chromatin and plasma stain.

Hollande's Chlorcarmin Staining Method (C. R. Soc. Biol., 1916, Ixxix, p. 662, and Jour. Roy. Micr. Soc., 1920).-Place 5 c.c. pure hydrochloric acid in a porcelain dish; add little by little 14 grms. powdered carmine, stirring constantly to make a homogeneous doughy mass. Allow to digest for twenty-four hours; add 250 c.e. aq. dest., bring to the boil, and keep boiling for half an hour. Filter; make up to 180 c.c. with aq. dest., and then add enough 75 per cent. alcohol to make a total volume of 200 c.c. Stain sections or pieces of tissue for two to twenty-four hours. Rinse in aq. dest. or 30 per cent. alcohol; immerse in 3 per cent. iron alum solution, in which the sections become black, and are then slowly decolourised; when differentiation is complete, rinse in a 1 per cent. pyridin solution, and wash under the tap for ten to fifteen minutes. Counterstain and mount as desired. This is a very intense stain suitable for mitochondria and cell granules.

223. Iron Carmalum (DE GRoot, Zeit. wiss. Mik., xx, 1903, p. 21).Dissolve $0.1 \mathrm{grm}$. of ferric alum in 20 c.c. distilled water and add $1 \mathrm{grm}$. carminic acid. Dissolve, add 180 c.c. of water, warm, add 5 grms. potash alum, dissolve, cool, filter, and add 2 drops of hydrochloric acid. To be used as carmalum, and said to give a stronger stain. 
224. Iron Cochineal (SPULER, Encyclopcedie d. mik. Technik, 1903, p. 153 , and 1910 , p. 240). - Stain for forty-eight hours in a stove, in extract of cochineal (made in a highly complicated way), wash with water, put into solution of ferric alum of $\frac{3}{1}$ per cent. strength for twentyfour hours or more. If the stain is not sufficiently intense, the whole process may be repeated.

Peter (Zeit. wiss. Mik., xxi, 1904, p. 314) stains material in bulk for forty-eight hours (sections eighteen to twenty-four) in an incubator, in a similar extract, acidified with $\mathrm{HCl}$, treats with iron-alum of $2 \frac{1}{2}$ per cent. for one hour to one day (sections half to two minutes), then alcohol, xylol, paraffin, or balsam. Chromatin black, protoplasm grey, yolk granules red.

HANSEN (ibid., xxii, 1905, p. 85) stains sections or entire objects in a solution of 5 to 10 grms. cochineal, 8 grms. ferric alum, 250 c.c. water, and 25 c.c. sulphuric acid of 10 per cent., boiled for fifteen to twenty minutes.

\section{B. So-called "Neutral" and Alkaline.}

225. Ammonia-Carmine.-Best made by the method of RANviER. Make a simple solution of carmine in water with a slight excess of ammonia, and expose it to the air in a deep crystallising dish until it is entirely dried up. It should be allowed to putrefy if possible. Dissolve the dry deposit in pure water, and filter.

VAN WiJhe (Vers. Akad., Amsterdam, viii, Deel, p. 507) takes an old strong solution of carmine in ammonia (or boils carmine with ammonia and peroxide of hydrogen), then precipitates it by adding alcohol to excess, washes the precipitate with alcohol, and dries it.

226. Soda-Carmine appears to be still used by some for central nervous system (see CuccATr, Zeit. wiss. Mik., iv, 1887, p. 50). It can be obtained from GrÜBLER \& HollborN (Natron-Carmin).

226a. ORTH's Lithium-Carmine (see early editions) macerates strongly, and is superfluous. For that of BEst, see Zeit. wiss. Mik., xxiii, 1906, p. 322 .

227. Magnesia-Carmine (MAYER, Zeit. wiss. Mik., xiv, 1897, p. 23). -Take $1 \mathrm{grm}$. carmine, $0.1 \mathrm{grm}$. magnesia usta, and 50 c.c. distilled. water, boil for five minutes, filter, and add 3 drops of formol. This is the stock solution. A weak solution may be made by boiling $0 \cdot 1 \mathrm{grm}$. carmine for half an hour in 50 c.c. of magnesia water (made by leaving $0 \cdot 1 \mathrm{grm}$. of magnesia usta in contact with 100 c.c. of spring water for a week with frequent agitation, and decanting when required for use). Said to be less injurious to tissues than the other alkaline carmines.

228. As to Picro-Carmine.-The term "picro-carmine" is commonly used to denote a whole tribe of solutions in which carmine, ammonia, and picric acid exist uncombined in haphazard proportions. These solutions do not contain a double salt of picric and carminic acid and ammonia, or picro-carminate of ammonia. They are always alkaline, and frequently injurious to tissues. The 
raison d'être of picro-carmine does not lie in its capacity of affording a double stain, but in that the picric acid in it is supposed to neutralise the ammonia, which it only does imperfectly. See MAYer in Zeit. wiss. Mik., xiv, 1897, p. 18.

229. RanvieR's Picro-Carmine, Original Formula (Traité, p. 100). -To a saturated solution of picric acid add carmine (dissolved in ammonia) to saturation. Evaporate down to one-fifth the original volume in a drying oven, and separate by filtration the precipitate that forms in the liquid when cool. Evaporate the mother liquid to dryness, and you will obtain the picro-carmine in the form of a crystalline powder of the colour of red ochre. It ought to dissolve completely in distilled water; a 1 per cent. solution is best for use.

For slow staining, dilute solutions may advantageously have 1 or 2 per cent. of chloral hydrate added to them.

Overstains may be washed out with hydrochloric acid, say 0.5 per cent. in water, alcohol, or glycerin.

Preparations should be mounted in balsam, or if in glycerin, this should be acidulated with 1 per cent. of acetic acid, or better, formic acid.

RANviER's Newer Formula does not give a more constant product (see previous editions).

230. Van WiJhe dissolves 0.5 per cent. of the dry ammonia-carmine, $\S 225$, in a 1 per cent. solution of neutral picrate of ammonia, boils until the vapour ceases to blue reddened litmus paper, and adds 1 per cent. of chloral hydrate. Gives an almost neutral preparation.

231. MAYER's Picro-Magnesia Carmine (Zeit. wiss. Mik., xiv, 1897, p. 25) is relatively constant and innocuous to tissues. It consists of 1 vol. of the stock solution of magnesia-carmine ( $\$ 227$ ), and 10 vols. of a 0.6 per cent. solution of picrate of magnesia, or of equal parts of the weak solution and the picrate solution. The picrate may be obtained from GRÜBLER \& HOLLBORN, or the solution may be made by heating $0.25 \mathrm{grm}$. of carbonate of magnesia in 200 c.c. of 0.5 per cent. solution of picric acid, allowing to settle, and filtering.

DE GROoT's picro-magnesia carmine (ibid., xxix, 1912, p. 184) contains ammonia, which is bad, and seems to me superfluous.

232. Other Formulæ for Picro-Carmine and Other Aqueous Carmines (Acid and Alkaline).- I have tried most of them, and found no real advantage in any of them (see previous editions).

\section{B. Alcoholic Carmine Stains.}

233. Alcoholic Borax-Carmine (Grenacher, Arch. mik. Anat., xvi, 1879, pp. 466 et seq.). -Make a concentrated solution of carmine in borax solution ( 2 to 3 per cent. carmine to 4 per cent. borax) by boiling for half an hour or more (or allowing it to stand, with occasional stirring, for two or three days); dilute it with about an 
equal volume of 70 per cent. alcohol, allow it to stand some time and filter.

Preparations should remain in the stain until they are thoroughly penetrated (for days if necessary), and then be brought (without first washing out) into alcohol of 70 per cent. acidulated with 4 to 6 drops of hydrochloric acid to each 100 c.c. of alcohol. They are left in this until they have taken on a bright transparent look (which may require days), and may then be washed or hardened in neutral alcohol. Four drops of $\mathrm{HCl}$ is generally enough. Three drops I find not quite sufficient.

For delicate objects, and for very impermeable objects, it may be well to increase the proportion of alcohol in the stain; it may conveniently be raised to about 50 per cent. It should not exceed 60 per cent. in any case (MAYER).

This stain used to be the most popular of any for staining in bulk. It is easy to use, and gives a most splendid coloration. But it is not so penetrating as is commonly supposed, and has the defect of sometimes forming precipitates in the cavities of bulky objects which cannot be removed by washing out. And the fluid is alkaline, and therefore may not be suitable for certain delicate work.

234. Mayer's Paracarmine (Mitth. Zool. Stat. Neapel, x, 3, 1892, p. 491).-Carminic acid, 1 grm. ; chloride of aluminium, $0.5 \mathrm{grm}$.; chloride of calcium, 4 grms. ; 70 per cent. alcohol, 100 c.c. Dissolve cold or warm, allow to settle, and filter.

Objects to be stained should not have an alkaline reaction, nor contain any considerable amount of carbonate of lime (spicules or skeletal parts of corals, etc.) which would give rise to precipitates. Wash out sections or objects intended to be sectioned, with pure 70 per cent. alcohol. Objects intended to be mounted whole may be washed out with a weak solution of aluminium chloride in alcohol, or if this be not sufficient, with 5 per cent. common acetic acid (or 2.5 per cent. glacial acetic acid) in alcohol. This may also be done with section material, if it is desired to obtain a more purely nuclear stain.

For staining bulky objects with large cavities, such as Salpa, the solution should be diluted (with alcohol); and as this may cause precipitates to form during the staining, especially if the objects are not very clean, it is advisable to slightly acidify the dilute solutions.

Instead of calcium chloride, which is very hygroscopic, strontium chloride may be taken.

Paracarmine is less hurtful to delicate tissues than borax carmine; it is more highly alcoholic, therefore more penetrating; and has less 
tendency to form precipitates in the interior of objects. But, in my hands, it does not give quite so fine a stain.

234a. Alcoholic Hydrochloric-Acid Carmine.-GrenaCher's receipt (Arch.f. Mik. Anat., xvi, 1879, p. 468) is troublesome. That of MAYER (Mitth. Zool. Stat. Neapel, iv, 1883, p. 521 ; Intern. Monatsschr.f. Anat., etc., 1897, p. 43) is better : Carmine 4 grms.; water, 15 c.c.; hydrochloric acid, 30 drops. Boil till the carmine is dissolved, add 95 c.c. of 85 per cent. alcohol, and neutralise by adding ammonia until the carmine begins to precipitate.

If it be desired to dilute the solution, it should be done with alcohol not water, and alcohol of 80 to 90 per cent. should be taken for washing out.

A very powerful stain, which I have found useful. If it be desired to have a purely nuclear stain, the alcohol must be very slightly acidulated with $\mathrm{HCl}$.

For a complicated receipt of Loewenthal see Zeit. wiss. Mik., xix, 1902, p. 56 .

235. Alcoholic Cochineal, Mayer's Old Formula (Mitth. Zool. Stat. Neapel, ii, 1881, p. 14).-Cochineal in coarse powder is macerated for several days in alcohol of 70 per cent. For each gramme of the cochineal there is required 8 to 10 c.c. of the alcohol. Stir frequently. Filter.

The objects to be stained must previously be saturated with alcohol of 70 per cent., and alcohol of the same strength must be used for washing out or for diluting the staining solution. The washing out must be repeated with fresh alcohol until the latter takes up no more colour. Warm alcohol acts more rapidly than cold. Overstaining seldom happens; it may be corrected by means of 70 per cent. alcohol, containing $\frac{1}{10}$ per cent. hydrochloric or 1 per cent. acetic acid.

Small objects and thin sections may be stained in a few minutes ; larger animals require hours or days.

A nuclear stain, slightly affecting protoplasm. The colour varies with the reaction of the tissues, and the presence or absence of certain salts in them. Crustacea with thick chitinous integuments are generally stained red, most other organisms blue. The stain is also often of different colours in different tissue elements of the same preparation. Glands or their secretion often stain grey-green.

Acids lighten the stain and make it yellowish-red. Caustic alkalies turn it to a deep purple.

All acids must be carefully washed out from the objects before staining, or a diffuse stain will result. The stain is permanent in oil of cloves and balsam.

Very penetrating and especially useful for Arthropoda. 
It has over the new fluid (next $\S$ ) the advantage of being more highly alcoholic; and it does not contain free acid, so that it can be used with calcareous structures which it is wished to preservewhich the new fluid cannot. For specimens of Pluteus, for instance, I find it excellent. But it only gives good results with such objects as contain the necessary salts, $\S 212$.

236. MAYER's Alcoholic Cochineal, New Formula (Mitth. Zool. Stat. Neapel, x, 1892, p. 498).-Cochineal, 5 grms.; chloride of calcium, 5 grms. ; chloride of aluminium, 0.5 grm.; nitric acid of $1.20 \mathrm{sp}$. gr., 8 drops; 50 per cent. alcohol, 100 c.c. Powder the cochineal and rub up with the salts, add the alcohol and acid, heat to boiling-point, leave to cool, leave for some days standing with frequent agitation, filter.

Use as the old tincture, the objects being prepared and washed out with 50 per cent. alcohol. Mayer only recommends it as a succedaneum of paracarmine.

Since this fluid contains in itself all the necessary salts (\$212), it gives good results with all classes of objects. 


\section{CHAPTER XIII.}

\section{HAMATEIN (H EMATOXYLIN) STAINS.}

23\%. Introduction.-Homatoxylin is a dye extracted from logwood. It is a substance that oxidises very readily, thus becoming converted into hoematein, or, as often happens, into other more highly oxidised products. It appears to be now thoroughly well established (see NiETzki, Chemie der organischen Färbstoffe, Berlin, Springer, 1889, pp. 215 - 217, and MAYER, Mitth. Zool. Stat. Neapel, $\mathrm{x}, 1891, \mathrm{p} .170)$ that the colouring agent in solutions of logwood or hæmatoxylin is not the hæmatoxylin itself, but hæmatein formed in them (or, in some cases, one of the higher oxidation products).

Hæmatein is an acid body, a "colour acid" (\$\$ 201, 206). Substantively employed, it is a very weak plasma stain. But combined with appropriate mordants it becomes basic, and can be made to give a powerful nuclear stain, or at the same time a nuclear and a selective plasma stain. The mordants employed in histology are aluminium, chrome, iron, copper, and (rarely) vanadium and molybdenum. Aluminium and iron are the mordants most employed, the former furnishing lakes used for progressive staining of material in bulk, the latter forming in most cases in the tissues a lake that requires differentiation, and is only applicable to the staining of sections.

The presence of a sufficient amount of hæmatein in staining solutions was formerly brought about by allowing solutions of hæmatoxylin to oxidate spontaneously by exposure to air. The change thus brought about in the solutions is known as "ripening," and until it has taken place the solutions are not fit to use for staining.

It was discovered by MAYER and UnNa independently (see MAYER in Mitth. Zool. Stat. Neapel, x, 1891, pp. 170-186; UnNa in Zeit. wiss. Mik., viii, 1892 , p. 483) that nothing is easier than to bring about this change artificially; all that is necessary being, for instance, to add to a solution of hæmatoxylin containing alum a little neutralised solution of peroxide of hydrogen or other powerful 
oxidising agent.* The solution becomes almost instantaneously dark blue, "ripe" and fit for staining. Other methods of " ripening," or of preparing hæmatein separately, are given further on, and constitute a great progress. For under the old practice of leaving staining solutions to "ripen " by the action of the air, it is necessary to wait for a long time before the reaction is obtained. During all this time, it may be weeks or months, there is no means, except repeated trial, of ascertaining whether the solution at any moment contains sufficient hæmatein to afford a good stain. And here a second difficulty arises: the oxidising process continuing, the solutions become "over-ripe"; the hæmatein, through further oxidation, passes over into colourless compounds, and the solutions begin to precipitate. They are therefore, in reality, a mixture in constantly varying proportions of "unripe," "ripe," and " overripe" constituents (the first and last being useless for staining purposes), and, in consequence, their staining power is very inconstant.

Logically, therefore, as concluded by MAYER, not hæmatoxylin, but hoematein, should be taken in the first instance for making the staining solution.

But this is not always indicated; for such solutions may easily over-oxidise, either in the bottle or on contact with the tissues. So that it is always preferable to start from hæmatoxylin. In this case, it should not be done by dissolving the hæmatoxylin straight away in the other ingredients of the staining solution. The solutions should be made up from a strong stock solution made by dissolving hæmatoxylin crystals in absolute alcohol: one in ten is a good proportion. This solution should be kept for a long time-months, at least, a year if possible; it gradually becomes of a vinous red, and should not be used till it has become quite dark. It has then become to a great extent oxidised into hæmatein, and the staining solutions made up from it will be at once fairly ripe.

Hæmatein (or hæmatoxylin) affords a stronger stain than carmine, and gives better results with tissues fixed in osmic or chromic mixtures. The alum solutions are indicated for staining in bulk, iron hæmatoxylin for sections.

238. Hæmatoxylin is found in commerce in the form of crystals, either colourless or browned by oxidation, easily soluble in either water, glycerin, or alcohol.

* Re-invented lately (Zeit. wiss. Mik., xxix, 1912, p. 69) by Piazza, who adds to Boehmer's solution about 20 per cent., to Delafield's about 7 per cent., to Ehrlich's about 12 per cent. of peroxide of hydrogen. 
239. Hæmatein is found in commerce as a brown powder, entirely, though with difficulty, soluble in distilled water and in alcohol, giving a yellowish-brown solution, which remains clear on addition of acetic acid. Alkalies dissolve it with a blue-violet tint. (See also last edition.)

240. Iron Hæmatoxylin, Generalities.-This method is due to Benda (Verh. Phys. Ges., 1885-1886, Nos. 12, 13, 14; Arch. Anat. Phys., 1886, p. 562 ; third ed. of this work, p. 365).

The method was independently worked out about the same time by M. Heidenhain. The method is almost universally practised in the form given by Heidenhain, not on account of any essential difference between the two, for there is none, but chiefly because Heidenhain has given more precise instructions concerning the process.

After carefully comparing Heidenhain's process with Benda's later process (next §), I find that the two give an absolutely identical stain; that is to say, that if you mordant in Benda's liquor ferri (next $\S$ ), and differentiate in the same, you will get exactly the same effect as by mordanting in ferric alum and differentiating in the same. But you may vary the results somewhat by varying the differentiation. Benda has pointed out (Verb. Anat. Ges., xv, 1901, p. 156) that you may differentiate either by an agent which simply dissolves the lake - such as acetic or hydrochloric acid; or by an oxidising agent, such as chromic acid, or the liquor ferri or the ferric alum. The former, he thinks, are the best for the demonstration of nuclear structures, the latter for cytoplasmic structures. For these he greatly recommends WEIGERT's borax-ferricyanide mixture, as being the easiest and safest to employ.

For myself, I find that differentiation in the iron salt (§ 241 or $\S 242$ ) is sufficient for almost all purposes. Acetic acid of 30 per cent. acts much too quickly to be safe, and causes swelling of the tissues.

VAN GIEson's picro-säurefuchsin has been recommended as a differentiation fluid by Benda (Deutsch. med. Wochenschr., 1898, No. 30). I find it gives very delicate differentiations, but acts very slowly, requiring nearly as many hours as the iron alum solution does minutes. The addition of the säurefuchsin to the picric acid is, I find, not necessary, and may prove an injurious complication.

In these processes hæmatoxylin is generally used for the stain, not homatein, the iron salt oxidising it into hæmatein, or into a higher oxidation product. I have obtained some good stains with 
hæmatein, but also some very bad ones; presumably the solutions easily over-oxidise on contact with the iron salt.

The hæmatoxylin is generally dissolved in water. I frequently prefer alcohol, of 50 per cent., as less injurious to tissues.

The method is a regressive one. It has been proposed to stain progressively, which I have tried, and had extremely bad results.

The differentiation requires to be carefully timed. For this reason the method is only applicable to sections, which should be thin, best not over $10 \mu$.

Iron hæmatoxylin is one of the most important of stains. It enables us to stain elements which cannot be selectively stained in any other way. The stain is very powerful, and of a certain optical quality that is peculiarly suited to the employment of high powers ; it will allow of the use of deeper eye-pieces than other stains. It will take effect on any material, and is quite permanent. Further details as to the characters of the stain are given in $\S 242$.

241. BendA's later Iron Hæmatoxylin (Verb. d. Anat. Ges., vii, 1, 1893 , p. 161).- Sections are mordanted for twenty-four hours in liquor ferri sulphurici oxidati, P.G.,* diluted with one or two volumes of water. They are then well washed, first with distilled water, then with tap water, and are brought into a 1 per cent. solution of hæmatoxylin in water, in which they remain till they have become thoroughly black. They are then washed and differentiated. The differentiation may be done either in 30 per cent. acetic acid, in which case the progress of the decoloration must be watched; or in a weaker acid, which will not require watching; or in the sulphate solution strongly diluted with water.

I find that if the iron solution be taken for the differentiation, it should be taken extremely diluted (of a very pale straw-colour, about $1: 30$ of water), and the progress of the differentiation watched; as if it be only diluted about tenfold, for instance, the decoloration is extremely rapid. See also last §.

I also find that Benda's mordant is unnecessarily, sometimes harmfully, strong, and that the liquor ferri may be diluted tenfold with advantage. The duration of the bath in the mordant is also for most purposes excessive as directed by Benda. I find that three to six hours in the solution diluted tenfold is generally sufficient with favourable material.

* This preparation consists of sulphate of iron, 80 parts ; water, 40 ; sulphuric acid, 15 ; and nitric acid, 18, and contains 10 per cent. of Fe. Donbtless the ferri persulphatis liquor B. P. will do instead; the point is, to have a per-salt, and not a proto-salt. 
242. Heidenhain's Iron Hæmatoxylin (M. Heidenhain, "Uber Kern und Protoplasma," in Festschr. für Kölliker, 1892, p. 118).Sections are treated from half an hour to at most two or three hours with a 1.5 to 4 per cent. solution of ferric alum (ammonio-ferric sulphate). By this is always meant in histology the double salt of ammonium and sesquioxide of iron $\left(\mathrm{NH}_{4}\right)_{2} \mathrm{Fe}_{2}\left(\mathrm{SO}_{4}\right)_{4}$, in clear violet crystals ; the double salt of the protoxide, or salt of МонR in green crystals, will not serve. If the crystals have become yellow and opaque, they have gone bad, and should be rejected. They ought to be kept in a stoppered bottle, and the solution should be made in the cold (Arch. mik. Anat., xliii, 1894, pp. 431, 435). The sections are then washed with water and stained for half an hour in an aqueous solution (of about 0.5 per cent.) of hæmatoxylin. They are then rinsed with water, and again treated with the iron solution, which slowly washes out the stain. The progress of the differentiation ought to be controlled under the microscope. The sections should to this end be removed from time to time from the alum solution, and put into tap-water whilst they are being examined. This is favourable to the stain. As soon as a satisfactory differentiation has been obtained, the preparations are washed for at least a quarter of an hour in running water, but not more than an hour, and mounted. The results differ according to the duration of the treatment with the iron and the stain. If the baths have been of short duration, viz. not more than half an hour in the iron and as much in the stain, blue preparations will be obtained. These show a very intense and highly differentiated stain of nuclear structures, cytoplasmic structures being pale. If the baths in the iron and in the stain have been prolonged (twelve to eighteen hours), and the subsequent differentiation in the second iron bath also duly prolonged, black preparations will rasult. These show chromosomes stained, central corpuscles stained intensely black, cytoplasm sometimes colourless, sometimes grey, in which case achromatic spindlefibres and cell-plates are stained, connective-tissue fibres black, red blood-corpuscles black, micro-organisms sharply stained, striated muscle very finely shown.

Later (Zeit. wiss. Mik., xiii, 1896, p. 186) Heidenhain gives further instructions for the employment of this stain in the study of central corpuscles. All alcohol should be removed from the tissues " by means of distilled water before bringing them into the mordant.

* Why? I find my iron-alum solution, as well as the liquor ferri sulph. oxid., last $\S$, mix clear with alcohol without the least precipitate forming. 
This should be a $2 \frac{1}{2}$ per cent. solution of ferric alum, not weaker. Leave the sections therein (fixed to slides by the water method, $\S 186$ ) for six to twelve hours, or at least not less than three. Keep the slides upright in the mordant, not lying flat. Wash out well with water before staining. Stain in a " ripened" hæmatoxylin solution, i.e. one that has stood for four weeks [of course, if you make it up with the ripened brown alcoholic solution recommended $\S 237$ sub. fin., this will be superfluous]. Stain from twenty-four to thirty-six hours. Use the same staining solution over and over again until it becomes spoilt; for the solution after having been used gives a more energetic stain, owing to its containing a trace of iron brought over by the sections. Differentiate in a $2 \frac{1}{2}$ per cent. solution of ferric alum. Rinse for ten minutes in running water, clear with xylol, not with any essential oil, and mount in xylol-balsam. See also under " Centrosomes," and "Chromosomes," etc.

Bielaszewics (Bull. Acad. Cracovie, 1909, 2 serié, p. 152) differentiates with very weak solution of calcium chloride; GUARNIERI (Mon. Zool. Ital., xvii, 1906, p. 44) with saturated solution of picric acid.

GURWITSCH (Zeit. wiss. Mik., xviii, 1902, p. 291) floods sections on the slide with mordant, warms on a water-bath till bubbles are given off or the mordant becomes turbid, then stains with the hæmatoxylin in the same way. The whole process takes about ten minutes.

HeLd (Arch. Anat. Phys., Anat. Abth., 1897, p. 277) adds to the staining bath a very little of the iron-alum solution until a scarcely perceptible precipitate is produced. A dangerous practice. I find it is not even safe to add a little of an over-used bath (supra).

Francotte (Arch. Zool. Expér., vi, 1898, p. 200) mordants with tartrate of iron, MALLORY (Journ. Exper. Med., v. 1900, p. 15) with chloride.

243. Iron Hæmatoxylin (BÜTSCHLI, Unters. über mikroskopische Schaume u. das Protoplasma, etc., 1892, p. 80). - Sections treated with a weak brown aqueous solution of ferric acetate, washed with water, and stained in 0.5 per cent. aqueous solution of hæmatoxylin. A stain of extraordinary intensity, used by Bütschli for sections, $1 \mu$ in thickness, of Protozoa.

244. Weigert's Iron Hæmatoxylin Mixture (Zeit. wiss. Mik., xxi, 1904, p. 1).-Mix 1 part of a 1 per cent. solution of hæmatoxylin in alcohol of 96 per cent. with 1 of a solution containing 4 c.c. of liq. ferri sesquichlor., 1 c.c. of officinal hydrochloric acid (sp. gr. 1.124) and 95 of water. The mixture may be kept for some days (until it begins to smell of ether), but is best used fresh. Stain sections for a few minutes ; no differentiation is necessary.

For an earlier process of WeIGERT's (Allg. Zeit. Psychiatr., 1894, p. 245) see last edition.

MOREL and BASSAL (Journ. Anat. Phys., xlv, 1909, p. 632) stain in bulk in Weigert's mixture with the addition of 1 c.c. of 4 per cent. solution of acetate of copper. 
245. JANSSENS' Iron Hæmatoxylin ("Hématoxyline noire"; $L a$ Cellule, xiv, 1897, p. 207).-A similar mixture to that of DeLAFIELD, ferric alum being taken instead of ammonia alum, the rest as in Delafield's. A progressive stain, nuclear : for yeast cells.

246. HANSEN's Iron Hæmatoxylin (Zeit. wiss. Mik., xxii, 1905, p. 55). -A solution of 10 grms. ferric alum in 150 c.c. water is added to a solution of $1.6 \mathrm{grm}$. hæmatoxylin in 75 c.c. water, the mixture heated to boiling-point and cooled without access of air. Filter before use. To get a pure nuclear stain, add dilute sulphuric acid.

\section{4\%. Aluminium Hæmatein (Alum Hæmatoxylin) Generalities.-} The mordant and dye are generally combined in a single staining bath, giving a progressive stain. The stain is in different tones of blue or red according to the composition of the staining solution. Neutral or alkaline solutions give a blue stain; acid solutions give a red one. In order to get a blue stain in preparations that have come out red through the acidity of the staining bath, it is a common practice to treat them with weak ammonia, in the belief that the blue colour is restored by neutralisation of the acid that is the cause of the redness. According to MAYER, the ammonia acts, not by neutralising the acid, but by precipitating the alumina, which carries down the hæmatein with.it (if no alumina were present the colour would be purple, not blue). The same result can generally be obtained by merely washing out with common tap-water, which is usually sufficiently alkaline, and can be obtained with certainty by treatment with bicarbonate of soda or acetate of soda or potash. And this is the preferable course, as ammonia is certainly a dangerous thing to treat delicate tissues with. See Scotr's tap-water substitute, $\S 676$. Of course this is a different question from that of neutralising with an alkali tissues that have been treated with an acid to correct over-staining. Here the neutralisation may be indicated in the interest of the preservation of the stain.

SQuire (Methods, p. 22) finds that sections can be blued in a few seconds by treatment with a 1:1000 solution of bicarbonate of soda in distilled water. MAYER holds that acetate of potash is the most inoffensive reagent to take; a strength of 0.5 to 1 per cent. may be taken.

Several of these solutions have a great tendency to over-stain. Over-stains may be corrected by washing out with weak acids (e.g. 0.1 to 0.2 or even 0.5 per cent. of hydrochloric acid, or with oxalic or tartaric acid), but this is not favourable to the permanence of the stain. Carnoy (La Cellule, xii, 2, 1897, p. 215) recommends iodised water. If acids be used, it is well to neutralise afterwards with ammonia or bicarbonate of soda $(0 \cdot 1$ per cent.). 
Bicarbonate of soda may be used for neutralisation with 70 per cent. alcohol as the vehicle (von Wistinghausen, Mitth. Zool. Stat. Neapel, x, 1891, p. 41).

Over-staining may be avoided by staining very slowly in dilute solutions. The purest chromatin stains are obtained by staining for a short time (sublimate sections half an hour, say) in solutions of medium strength, such as hæmalum diluted ten to twenty-fold with water. The stain obtained either with very strong solutions, or with the slow stain of the dilute solutions, is at the same time a plasma-stain, which of course may or may not be desired. MaYer says that very dilute solutions will give a pure nuclear stain if they have been diluted with alum-solution, or have been acidified. Chromosmium material will not yield a pure chromatin stain unless it is very fresh; it is consequently next to impossible to obtain the reaction with paraffin sections of such material; they constantly give a plasma-stain in addition to the chromatin stain, which is not the case with sublimate material.

The stain is fairly permanent in balsam, but is very liable to fade a little, and may fade a great deal. If acids have been used after staining, great care should be taken to wash them out thoroughly before mounting. In aqueous media the stain cannot be relied on to keep (this refers to the old solutions: MAYER finds that his hæmatein preparations have kept well for at least some months in glycerin, if not acid, and, with certain precautions, in balsam). Turpentine-balsam should not be used.

Formulæ $\S 248$ to 259 give aqueous solutions, and $\S \S 260$ to 263 alcoholic ones.

248. MAYER's Hæmalum, Newer Formula (Zeit. wiss. Mik., xx, 1903, p. 409).-Hoematoxylin, 1 grm. ; water, 1 litre. Dissolve, and add $0.2 \mathrm{grm}$. of iodate of sodium $\left(\mathrm{NaIO}_{3}\right)$ and $50 \mathrm{grms}$. of alum, dissolve and filter.

This is an amended formula. The original one (Mitth. Zool. Stat. Neapel, $\mathrm{x}, 1891, \mathrm{p} .172$ ) was : 1 grm. of homatein (or the ammonia salt, $\S \S 238,239$ ) dissolved with heat in 50 c.c. of 90 per cent. alcohol, and added to a solution of $50 \mathrm{grms}$. of alum in a litre of distilled water.

This solution does not keep very well, but may be made more stable by adding 50 grms. of chloral hydrate and $1 \mathrm{grm}$. of citric (or acetic) acid.

It stains equally well, either at first, or later. Concentrated, it stains sometimes almost instantaneously, or in any case very rapidly. (Spring water or tap-water containing lime must not be used for diluting; perhaps weak solution of alum in distilled water is the 
best means of all.) After staining, sections may be washed out either with distilled or common water. It is admirable for staining in bulk. Large objects will, however, require twenty-four hours' staining, and should be washed out for the same time (this should be done with 1 per cent. alum solution if a sharp nuclear stain be desired). All alum must be carefully washed out of the tissues before mounting in balsam; and it is well to blue the stain with tap-water or otherwise, $\$ 257$. The stain is generally a nuclear one ; in any case such may be obtained by washing out with alum-solution. Mayer's preparations have kept well in glycerin (care being taken not to have it acid), also in balsam. If oil of bergamot be used for clearing, it must be thoroughly removed by means of oil of turpentine before mounting, and oil of cloves is dangerous. It is best (Mayer, in litt.) to use only xylol, benzol, or chloroform, and to mount in xylol-balsam or chloroform-balsam or benzol-balsam.

Hæmalum may be mixed with alum-carmine, Säurefuchsin, or the like, to make a double staining mixture; but it seems preferable to use the solutions in succession.

249. Mayer's Acid Hæmalum (Mitth. Zool. Stat. Neapel, x, 1891, p. 174).-This is hæmalum with 2 per cent. glacial acetic acid (or 4 per cent. common acetic acid). To be used as the last, washing out with ordinary water in order to obtain a blue-violet tint of stain. The solution keeps better.

250. UnnA's Half-ripe Constant Stock Solution (Zeit. wiss. Mik., viii, 1892, p. 483).

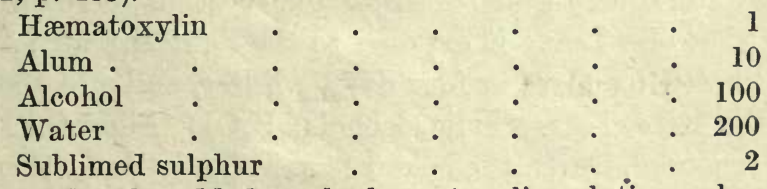

If the sulphur be added to the hæmatoxylin solution only when the latter has become somewhat strongly blue, i.e. after two or three days' time, the stage of oxidation attained by the solution will be fixed for some time by the sulphur, and according to Unna the solution will remain "constant" in staining power. MAYER (Mitth. Zool. Stat. Neapel, xii, 1896, p. 309) finds that the sulphur process does not preserve the solutions for long, whilst glycerin does. See below, "GLyChamaLUM."

251. MAYER's Glychæmalum (Mitth. Zool. Stat. Neapel, xii, 1896, p. 310).-Hæmatein (or hæmateate of ammonia), $0.4 \mathrm{grm}$. (to be rubbed up in a few drops of glycerin); alum, 5 grms.; glycerin, 30 ; distilled water, 70. The stain is not purely nuclear, but may be made so by washing out with alum solution or a weak acid. The solution keeps admirably. 
RAwitz (Leitfaden, 2nd ed., p. 63) takes 1 grm. hæmatein, 6 grms. ammonia alum, 200 grms. each of water and glycerin.

Or (Zeit. wiss. Mik., xxv, 1909, p. 391) 1 grm. hæmatein, 10 grms. of nitrate of aluminium, $250 \mathrm{grms}$. each of water and glycerin.

252. Hansen's Solution (Zool. Anz., 1895, p. 158).-See fourth edition.

253. HaRris's Solution (Micr. Bull., xv, 1898, p. 47 ; Journ. App. Mic., iii, p. 777).-Alum-hæmatoxylin solution ripened by addition of mercuric oxide. MAYER (Grundzüge, 1901, p. 171) finds the formula "gives too much hæmatein."

254. Böhmer's Hæmatoxylin (Arch. mik. Anat., iv, 1868, p. 345 ; Aerzt. Intelligenzbl., Baiern., 1865, p. 382).-Make (A) a solution of hæmatox. cryst. 1 part, alcohol (absolute) 12 parts, and (B) alum 1 part, water 240. For staining, add two or three drops of $\mathrm{A}$ to a watch-glassful of $\mathbf{B}$.

The alcoholic solution of hæmatoxylin ought to be old and dark (§ 237).

A. G. HoRnyold (Trans. Manch. Micr. Soc., 1915) prepares :Solution A : hæmatoxylin, $0.7 \mathrm{grm}$.; absolute alcohol, 20 c.c. Solution B : alum, 0.35 grm. ; aq. dest., 60 c.c. Mix A and B, expose to light in window for three or four days, then add 20 drops of tincture of iodine. Stain sections five to ten minutes till red-brown. Differentiate in 70 per cent. alcohol, to which add a few drops of acetic acid. The sections then turn blue. Good stain after osmic fixatives.

255. Delafield's Hæmatoxylin (Zeit. wiss. Mik., ii, 1885, p. 288 ; frequently attributed erroneously to GRENACHER or PRUDDEN).To 400 c.c. of saturated solution of ammonia-alum (that is about 1 to 11 of water) add 4 grms. of hæmatox. cryst. dissolved in 25 c.c. of strong alcohol. Leave it exposed to the light and air in an unstoppered bottle for three or four days. Filter, and add 100 c.c. of glycerin and 100 c.c. of methylic alcohol $\left(\mathrm{CH}_{4} \mathrm{O}\right)$. Allow the solution to stand (uncorked) until the colour is sufficiently dark, then filter.

This solution keeps for years. It is well to allow it to ripen for at least two months before using it.

For staining, enough of the solution should be added to pure water to make a very dilute stain. It is an extremely powerful stain.

It is still much used. I find that when well ripened-for years rather than months - it is yuite a first-class stain.

BÜTSCHLI (Unters. $\ddot{i b . ~ m i k r o s c o p i s c h e ~ S c h a ̈ u m e ~ u . ~ d a s ~ P r o t o p l a s m a, ~}$ etc., 1892) recommends, under the name of "acid hæmatoxylin," solution of Delafield very strongly diluted, and with enough acetic acid added to it to give it a decidedly red tint. This gives a sharper and more differentiated nuclear stain than the usual solution.

Martinottr (Zeit. wiss. Mik., xxvii, 1910, p. 31) makes it up with $0 \cdot 2$ per cent. of hoematein, and less alum ( 2 per cent.). 
256. Ehrlich's Acid Hæmatoxylin (Zeit. wiss. Mik., 1886, p. 150).Water 100 c.c., absolute alcohol 100, glycerin 100, glacial acetic acid 10, hæmatoxylin 2 grms., alum in excess.

Let the mixture ripen in the light (with occasional admission of air) until it acquires a dark red colour. It will then keep, with constant power, for years, if kept in a well-stoppered bottle. It is very appropriate for staining in bulk, as overstaining does not occur. I find it excellent.

MaNN (ibid., xi, 1895, p. 487) makes up this stain with an equal quantity of hæmatein instead of hæmatoxylin.

MaYer (Grundzüge, LeE and MaYer, 1st ed., p. 154) finds that this is too much and makes the mixture overstain; $0.4 \mathrm{grm}$. of hæmatein is quite enough.

For proper method of using EHRLICH's hæmatoxylin, see ScotT, $\S 669$.

257. Burcirardt's Pyroligneous Acid Hæmatoxylin (Arch. mik. Anat., liii, 1898, p. 232) would seem to be superfluous at least.

258. UnNA's Oxidised Hæmatoxylin (from Martinotri, Zeit. wiss. Mik., xxvii, 1910, p. 31).-Hæmatoxylin 0.5, alum 2, water 60 , alcohol 10 , glycerin 20 , peroxide of hydrogen solution 10 , carbonate of soda 0.05 .

MaRTinotTI, loc. cit., makes it up with homatein ( 0.2 grm.).

259. AрÁтнy's Hæmatein Mixture I A (Mitth. Zool. Stat. Neapel, xii, 1897, p. 712).-Make (A) a solution of 9 per cent. alum, 3 per cent. glacial acetic acid, and 0.1 per cent. salicylic acid in water, and (в) a 1 per cent. solution of hæmatoxylin in 70 per cent. alcohol, preserved for six to eight weeks in a bottle not quite full. Mix 1 part of A with 1 of $B$ and 1 of glycerin. Stains either sections or material in bulk. Apáthy uses it for staining neuro-fibrils.

260. KLeInenberG'S Hæmatoxylin (Quart. Journ. Micr. Sci., lxxiv, 1879 , p. 208).-Highly irrational and very inconstant in its composition and its effects ; see early editions ; also the criticism of MAYER (Mitth. Zool. Stat. Neapel, x, 1891, p: 174), and that of SQUIRE in his Methods and Formula, p. 25, and the alternative formulæ of SQUIRE (loc. cit.) and of von Wistinghausen (Mitth. Zool. Stat. Neapel, x, 1891, p. 41).

261. MayeR's Hæmacalcium (Mitth. Zool. Stat. Neapel, x, 1891, p. 182).-Hæmatein (or hæmateate of ammonia, §§ 238, 239), 1 grm. ; chloride of aluminium, 1 grm. ; chloride of calcium, 50 grms. ; glacial acetic acid, 10 c.c. (or common acetic acid, 20 c.c.); 70 per cent. alcohol, 600 c.c. Rub up finely together the first two ingredients, add the acid and alcohol, dissolve either cold or with heat; lastly add the chloride of calcium. 
If the objects stain in too red a tone they may be treated with a solution (of about 2 per cent.) of chloride of aluminium in 70 per cent. alcohol, or with a 0.5 to 1 per cent. solution of acetate of soda or potash in absolute alcohol; but washing with neutral alcohol will generally suffice.

With certain objects this solution does not penetrate well. This may be remedied by acidifying the solution, or, which is better, by leaving the objects for some time before staining in acid alcohol. Anyway objects ought not to have an alkaline reaction. If these precautions be taken, it will not be necessary to use acid for washing out.

The solution is not recommended as giving as good results as hæmalum, and Mayer recommends it merely as a substitute for Kleinenberg's, in cases in which an alcoholic hæmatein stain seems indicated, as being easy to prepare, and constant in its effects.

262. MAYER's Hæmastrontium (Grundzüge, LEE and MAYER, 1910 , p. 166).-1 grm. hæmatein, 1 grm. aluminium chloride, 50 grms. strontium chloride, 600 c.c. alcohol of 70 per cent., and (if desired) $0.25 \mathrm{grm}$. citric acid. Prepare and use as hæmacalcium.

263. DE GRoot's Alcoholic Hæmalum (Zeit. wiss. Mik., xxix, 1912, p. 182).-Mix 20 c.c. of glycerin with 240 of alcohol of 70 per cent. Take 4 c.c. of the mixture, 2 c.c. of hydrogen peroxide, and $0.5 \mathrm{grm}$. of hæmatoxylin, and dissolve with heat. Add 60 c.c. of the mixture, 4 grms. of calcium chloride, and 2 grms. of sodium bromide. Dissolve, add 3 grms. of alum, heat and add 100 c.c. of the mixture. When the alum is dissolved add $0.2 \mathrm{grm}$. of ferri-cyanide of potassium; dissolve and add 3 grms. more of alum and the rest of the mixture. Said to stain almost as well as hrmalum. Wash out with alcohol of 70 per cent.

264. Other Alumina-Hæmatein Solutions.-A large number of suppressed receipts will be found given in the earlier editions.

265. R. Heidenhain's Chrome Hæmatoxylin (Arch. mik. Anat, xxiv, 1884, p. 468, and xxvii, 1886, p. 383). - Stain for twelve to twentyfour hours in a $\frac{1}{3}$ per cent. solution of hæmatoxylin in distilled water. Soak for the same time in a 0.5 per cent. solution of neutral chromate of potash. Wash out the excess of chromate with water.

Objects that have been fixed in corrosive sublimate ought to be very carefully washed out with iodine, or the like, as neutral hæmatoxylin forms a black precipitate with any excess of sublimate that may remain in the tissues. See Tornier, in Arch. mik. Anat., 1886, p. 181.

The process is adapted to staining in bulk. You can decolour the objects to any extent by prolonging the soaking in the chromate. Bichromate will do instead of the neutral chromate.

266. Apáthy's Modification of Heidenhain's Process (Zeit. wiss. Mik., v, 1888, p. 47). - This is an alcoholic method. Stain in a 1 per 
cent. solution of hæmatoxylin in 70 or 80 per cent. alcohol. Differentiate sections of 10 to $15 \mu$, half the time of staining, sections of 25 to $40 \mu$ twice the time of staining, in 1 per cent. solution of bichromate of potash in 70 to 80 per cent. alcohol, and wash out in alcohol of 70 per cent. All these processes should be done in the dark.

For celloidin series of sections, Apáthy (ibid., 1889, p. 170) stains in the hæmatoxylin solution as above for ten minutes; then removes the excess of hæmatoxylin fluid from the sections by means of blotting. paper, and brings the series for five to ten minutes into 70 per cent. alcohol containing only a few drops of a strong (5 per cent.) solution of bichromate.

267. Schultze's Chrome Hæmatoxylin (Zeit. wiss. Mik., xxi, 1904, p. 5).- The tissues to be fixed for twelve or more hours in a bichromate or chromic acid solution, preferably an osmium-bichromate mixture or liquid of Flemming, then to be washed out for twenty-four hours in 50 per cent. alcohol in the dark and stained for twenty-four hours or more in 0.5 per cent. hæmatoxylin in alcohol of 70 per cent., then washed out in alcohol of 80 per cent.

268. Hansen's Chrome Hæmatoxylin (ibid., xxii, 1905, p. 64).Ten grms. of chrome alum boiled in 250 c.c. of water till green, and $1 \mathrm{grm}$. hæmatoxylin (dissolved in 15 c.c. of water) added; to the mixture when cold add 5 c.c. of sulphuric acid of 10 per cent. and (drop

- by drop) a solution of $0.55 \mathrm{grm}$. of bichromate of potash in 20 c.c. of water. Filter before use. Wash out with water free from air.

269. Vanadium Hæmatoxylin (HeidenhaIN, Encyclop. mik. Technik., 1903 , p. 518). - Add 60 c.c. of a 6 per cent. solution of hæmatoxylin to a 0.25 per cent. solution of vanadate of ammonium (quantity not stated; should be 30 c.c., see CoHN in Anat. Hefte, xv, 1895, p. 302). The mixture to be used after three or four days; it will not keep over eight days. To be used with sections of sublimate material. A strong plasma stain for special purposes, especially mucus glands.

270. BENDA's Copper Hæmatoxylin (Arch. mik. Anat., xxx, 1887, p. 49). - See fourth edition. According to my experience, not to be compared with iron hæmatoxylin, and superfluous.

2\%1. Mallory's Phospho-molybdic Acid Hæmatoxylin (Anat. Anz., 1891, p. 375).-One part 10 per cent. phospho-molybdic acid solution, 1 part hæmatoxylin, 100 parts water, and 6 to 10 parts chloral hydrate. Let the solution ripen for a week in sunlight, and filter. Chiefly for central nervous system. Sections should be stained for from ten minutes to one hour, and washed out in two or three changes of 40 to 50 per cent. alcohol. It is necessary that the solution should be saturated with hæmatoxylin in order to obtain the best results; if a good stain be not obtained at once, more hæmatoxylin must be added. Water must never be used for diluting it. 
See also RibBert (Centralb. allg. Path., vii, 1896, p. 427 ; Zeit. wiss. Mik., xv, 1898, p. 93), Patellani (Mon. Zool. Ital., xiii, 1902, p. 6), and Golovin (Zeit. wiss. Mik., xix, 1902, p. 184).

SARGENT (Anat. Anz., xv, 1898, p. 214) quotes this stain, preceded by mordanting for twenty-four hours in 5 per cent. sulphate of copper, as KENYON'S.

KoDis (Arch. mik. Anat., lix, 1901, p. 211) takes hæmatoxylin, 1 part ; molybdic anhydride, 1.5 ; water, $100 ; \mathrm{H}_{2} \mathrm{O}_{2}, 0 \cdot 5$, or a crystal of $\mathrm{HgO}$.

Police (Arch. Zool. Napoli, iv, 1909, p. 300) takes 0.35 grm. hæmatoxylin, 10 drops phospho-molybdic acid of 10 per cent., 10 grms. chloral liydrate, and 100 grms. alcohol of 70 per cent.

272. MALLoRy's Phospho-tungstic Hæmatoxylin (Journ. Exp. Med., v, 1900, p. 19; Zeit. wiss. Mik., xviii, 1901, p. 178) :

Hæmatoxylin . . . . . . $0 \cdot 1$

Water . . . . . $80 \cdot 0$

10 per cent. solution of (MERCK's) phosphotungstic acid . . . . . $20 \cdot 0$

Peroxide of hydrogen (U.S. Ph.) . . . 0.2

(Dissolve the hæmatoxylin, add the acid, then the peroxide.) Stain sections two to twenty-four hours, wash out with water. A polychromic stain, nuclei blue, intercellular substances pink. I consider this a fine stain.

273. Donaggio's Tin Hæmatoxylin (Ann. Nevrol. Napoli, xxii, 1904, p. 192).-A 1 per cent. solution of hæmatoxylin is poured slowly into an equal volume of 20 per cent. solution of pink-salt (ammonio-chloride of tin). Keep in the dark.

274. Osmium Hæmatoxylin.-Schultze (Zeit. wiss. Mik., xxvii, 1910, p. 465) treats tissues for twenty-four hours or more with osmic acid of 1 per cent., washes well with water, and puts for a couple of days into ripened 0.5 per cent. solution of hæmatoxylin in alcohol of 35 to 50 per cent. Wash out for a day or more with alcohol of 70 per cent. Intense plasma stain. 


\section{CHAPTER XIV.}

\section{NUCLEAR STAINS WITH COAL-TAR DYES.}

2\%5. Introduction.-Very few coal-tar dyes give a precise nuclear or chromatin stain by the progressive method (\$209). Two of them -methyl green and Bismarck brown-are pre-eminently progressive chromatin stains. Many of the others-for instance, safranin, gentian, and especially dahlia - may be made to give a progressive nuclear stain with fresh tissues by combining them with acetic acid; but in general are not so suitable for this kind of work as the two colours first named.

Again, very few coal-tar dyes give a pure plasmatic stain (one leaving nuclei unaffected). The majority give a diffuse stain, which in some few cases becomes by the application of the regressive method (§209) a most precise and splendid chromatin stain.

But plasma staining is generally done by the progressive method.

The basic anilin dyes were at one time greatly in vogue for the staining of chromatin in researches on the structure of nuclei. They have been little used for that purpose since the working out of the iron hæmatoxylin process, which gives a more energetic stain. But they may still be useful as a means of controlling the iron hæmatoxylin process, which frequently stains all sorts of things besides chromatin, which does not occur with the best tar colour stains.

The acid and neutral anilin dyes afford some of our best plasma stains.

I recommend-for staining nuclei of fresh tissues, methyl green ; for staining nuclei of fixed tissues by the regressive method, safranin for a red stain, and gentian violet or Thionin for a blue one; as a plasma stain for sections, Säurefuchsin ; for entire objects, picric acid.

\section{A. Progressive Stains.}

276. Methyl Green.-This is the most common in commerce of the "anilin" greens. It appears to go by the synonyms of Methylanilin green, Grünpulver, Vert Lumière, Lichtgrün; these two last are in reality the name of another colour. When first studied by Calberla, in 1874 (Morphol. Jahrb., iii, 1887, p. 625), it went by the 
name of Vert en cristaux. It is commonly met with in commerce under the name of more costly greens, especially under that of iodine green. It is important not to confuse it with the latter, nor with aldehyde green (Vert d'Eusèbe), nor with the phenylated rosanilins, Paris green, and Vert d'Alcali, or Vèridine.

Methyl green is the chloromethylate of zinc and penta-methyl rosanilin-violet. It is obtained by the action of methyl chloride on methyl violet. The commercial dye always contains unconverted methyl violet as a consequence of defective purification. It is sometimes adulterated with anilin blue (soluble blue). It is also sometimes adulterated with a green bye-product of the manufacture - the chloride of nona-methyl-para-leukanilin. See BENEDIKT and Knecht's Chemistry of the Coal-tar Colours. For tests for purity see Mayer, Mitth. Zool. Stat. Neapel, xii, 1896, p. 312, and Fischer, Fixirung, Färbung, u. Bau des Protoplasmas, p. 89.

Methyl green is extremely sensitive to alkalies. It is therefore important to use it only in acidified solutions and to use only acid, or at least perfectly neutral fluids for washing and mounting.

This is an extremely important histological reagent. Its chief use is as a chromatin stain for fresh, unfixed tissues. For this purpose it should be used in the form of a strong aqueous solution containing a little acetic acid (about 1 per cent. in general). The solutions must always be acid. If the tissues have been previously fixed with acetic acid you will not get a chromatin stain. The same applies to fixation with acetic acid sublimate : whilst pure sublimate will allow of a chromatin stain (BurckHardT, La Cellule, xii, 1897, p. 364). You may wash out with water (best acidulated) and mount in some acid aqueous medium containing a little of the methyl green in solution. The mounting medium, if aqueous, must be acidulated.

Employed in this way, with fresh unfixed tissues, methyl green is a pure chromatin stain, in the sense of being a precise colour reagent for chromatin. For in the nucleus it stains nothing but chromosomes or chromatin elements ; it does not stain plasmatic nucleoli (unless indeed these contain chromatin), nor caryoplasm, nor achromatic filaments. Outside the nucleus it stains some kinds of cytoplasm and some kinds of formed material, especially glandular secretions (silk, for instance, and mucin). The chromatin elements are invariably stained of a bright green (with the exception of the nuclein of the head of some spermatozoa), whilst extra-nuclear structures are in general stained in tones of blue or violet. But this metachromatic reaction is probably due to the methyl-violet impurity, and is not obtained with a chemically pure methyl green. 
Staining is instantaneous; overstaining never occurs. The solution is very penetrating, kills cells instantly without swelling or other change of form, and preserves their forms for at least some hours, so that it may be considered as a delicate fixative. It may be combined without precipitating with divers fixing or preserving agents. Osmic acid (of $0 \cdot 1$ to 1 per cent.) may be added to it, or it may be combined with solution of RIPART and PETIT (this is an excellent medium for washing out and mounting in).

Alcoholic solutions may also be used for staining. They also should be acidulated with acetic acid.

The stain does not keep easily. It is difficult to mount it satisfactorily in balsam, because the colour does not resist alcohol sufficiently (unless this be charged with the colour). The resistance of the colour to alcohol is, however (at all events if it be used in the EHRLICH-BIONDI combination), considerably increased by treating the sections for a few minutes with tincture of iodine before staining (M. Heidenhain).

Of preparations mounted with excess of colour in the usual aqueous media, I find the most fortunate only survive for a few months. Dr. Hennegur, however, writes to me that it keeps well in BRUN's glucose medium. P.221

It was first pointed out, I believe, by Heschl (Wiener med. Wochenschr., 2, 1879), that methyl green is a reagent for amyloid degeneration. His observations were confirmed by CuRschmanN (Virchow's Arch., vol. lxxix, 1880, p. 556), who showed that it colours amyloid substance of an intense violet; but this, as pointed out by Squire (Methods and Formula, etc., Churchill, 1892, p. 37), is undoubtedly due to its containing methyl violet as an impurity.

\section{2\%\%. Bismarck Brown (Manchester Brown, Phenylen Brown,} Vesuvin, La Phénicienne).-A fairly pure nuclear stain that will work either with fresh tissues or with such as have been hardened in chromic acid, or otherwise.

The colour is not very easily soluble in water. You may boil it in water, and filter after a day or two (WEIGERT, in Arch. mik. Anat., $\mathrm{xv}, 1878$, p. 258). You may add a little acetic or osmic acid to the solution. MaYsel (ibid., xviii, 1880, pp. 237, 250) dissolves the colour in acetic acid (this solution does not give a permanent stain). Alcoholic solutions may also be used, e.g. saturated aqueous solution diluted with one-third volume of 90 per cent. alcoholic; or CALBERLA's glycerin-and-alcohol mixture or dilute glycerin (say of 40 per cent. to 50 per cent.) may very advantageously be employed.

The watery solutions must be frequently filtered (but then much 
of the colour is retained on the filter). The addition to them of carbolic acid has been recommended (vide Journ. Roy. Mic. Soc., 1886 , p. 908). Bismarck brown stains rapidly, but never overstains. The stain is permanent both in balsam and in glycerin.

This colour may be used as a chromatin stain for fresh tissues in the same way as methyl green. Herla (Arch. Biol., xiii, 1893, p. 423) employs for ova of Ascuris a mixture of 0.25 parts vesuvin, 0.25 malachite green, 10 of glycerin and 100 of water, and washes out with weak glycerin.

The chief use of this colour is for progressive staining ; but it may be employed for staining by the regressive method (see $\S 289$ ), and also for intra-vitam staining ( $\$ 208$ ) (for this purpose it is necessary to see that the colour employed be pure and neutral).

278. Methyl Violet (Methylanilin Violet, Anilin Violet, Paris Violet).GraSer (Deutsche Zeit. Chirurgie, xxvii, 1888, pp. 538-584; Zeit. wiss. Mik., $\vee, 3,1888$, p. 378) stains sections from twelve to twenty-four hours in a solution so dilute that at the end of that time the sections will have taken up all the colour from the liquid. They are then washed out for a short time in acidulated alcohol, and then in pure alcohol. The method is applicable to objects fixed in Flemming's mixture.

279. Other Progressive Stains.-Most of the basic tar colours used for regressive staining will also give by the progressive method a nuclear stain of greater or less purity if used in solutions acidified with acetic acid. Amongst these may be mentioned thionin, which need not even be acidified; also, for fresh tissues especially, gentian violet, dahlia, and toluidin blue.

\section{B. Regressive Stains.}

280. The Practice of Regressive Staining : The Staining Bath.Sections only, or material that is thin enough to behave like sections, such as some membranes, can be stained by this method.

The solutions employed are made with alcohol, water, or anilin, or sometimes other menstrua, according to the solubility of the colour. There seems to be no special object in making them with alcohol if water will suffice, the great object being to get as strong a solution as possible. Indeed, the solutions made with strong alcohol are found not to give quite such good results as those made with water or weak alcohol. Alcohol of 50 per cent. strength, however, may be said to constitute a very generally desirable medium. The sections must be very thoroughly stained in the solution. As a general rule they cannot be left too long in the staining fluid. With the powerful solutions obtained with anilin a few minutes or half an hour will usually suffice, but to be on the safe side 
it is frequently well to leave the sections twelve to twenty-four hours in the fluid. Up to a certain point the more the tissues are stained the better do they resist the washing-out process, which is an advantage. Some workers, indeed, prefer weak solutions; so Heidenhain, Encycl. mik. Technik, i, pp. 433, 434; but the nature of the fixing agent should be taken into account.

Material fixed in chromic or chromo-osmic mixtures gives a sharper and more selective stain than material fixed in sublimate or the like. In fact, to ensure the best results, only material fixed in chromic mixtures (or Hermann's fluid) should be employed.

During the staining the tissues become overstained, that is, charged with colour in an excessive and diffuse manner. The stain must now be differentiated by removal of the excess of colour.

281. Differentiation,-This is generally done with alcohol, sometimes neutral, sometimes acidulated (with $\mathrm{HCl}$ ). The stained sections, if loose (celloidin sections), are brought into a watch-glassful of alcohol; if mounted in series on a slide, they are brought into a tube of alcohol (differentiation can be done by simply pouring alcohol on to the slide, but it is better to use a tube or other bath). It is in either case well to just rinse the sections in water, or even to wash them well in it, before bringing them into alcohol.

The sections in the watch-glass are seen to give up their colour to the alcohol in clouds, which are at first very rapidly formed, afterwards more slowly. The sections on the slide are seen, if the slide be gently lifted above the surface of the alcohol, to be giving off their colour in the shape of rivers running down the glass. In a short time the formation of the clouds or of the rivers is seen to be on the point of ceasing; the sections have become pale and somewhat transparent, and (in the case of chrom-osmium objects) have changed colour, owing to the coming into view of the general ground colour of the tissues. (Thus chrom-osmium-safranin sections turn from an opaque red to a delicate purple.) At this point the differentiation is complete, or nearly so.

It is generally directed that absolute alcohol be taken for differentiation. This may be well in some cases, but in general 95 per cent. is found to answer perfectly well. HeIDENHaIN (Encycl., i, p. 434) takes methyl alcohol.

The hydrochloric-acid-alcohol extracts the colour much more quickly from resting nuclei than from kinetic nuclei. Therefore, washing out should be done with neutral alcohol whenever it is desired to have resting nuclei stained as well as dividing nuclei ; the acid process serving chiefly to differentiate karyokinetic figures. 
The proportion of $\mathrm{HCl}$ with which the alcohol should be acidified for the acid process should be about 1: 1000, or less; seldom more.

The length of time necessary for differentiating to the precise degree required varies considerably with the nature of the tissues and the details of the process employed; all that can be said is that it generally lies between thirty seconds and two minutes. The acid process is vastly more rapid than the neutral process, and therefore of course more risky.

There exists also a method of differentiation known as substitutionone stain being made to wash out another. Thus methylen blue and gentian violet are discharged from tissues by aqueous solution of vesuvin or of eosin ; fuchsin is discharged from tissues by aqueous solution of methylen blue. The second stain "substitutes" itself for the first in the general "ground" of the tissues, leaving, if the operation has been successfully carried out, the nuclei stained with the first stain, the second forming a "contrast" stain. In the paper of RESEGOTTI in Zeit. wiss. Mik., v, 1888, p. 320, it is stated as a very general rule that colours that do not give a nuclear stain by the regressive method will wash out those that do. But RESEgotri used the second colour in alcoholic solution; so that it remains uncertain how far the differentiation should be attributed to the second colour itself, and how far to the alcohol used as a vehicle. The same remark applies to BendA's Safranin-andLichtgrün process.

282. Clearing.-After due differentiation, the extraction of the colour may be stopped by putting the sections into water; but the general practice is to clear and mount them at once.

You may clear with clove oil or anilin, which will extract some more colour from the tissues. Or you may clear with an agent that does not attack the stain (cedar oil, bergamot oil, xylol, toluol, etc. ; see the chapter on Clearing Agents). If you have used neutral alcohol for washing out, you had perhaps better clear with clove oil, as neutral alcohol does not always, if the staining have been very prolonged, extract the colour perfectly from extra-nuclear parts. But if you have not stained very long, and if you have used acidulated alcohol for washing out, clove oil is not necessary, and it may be better not to use it, as it somewhat impairs the brilliancy of the stain. A special property of clove oil is that it helps to differentiate karyokinetic figures, as it decolours resting nuclei more rapidly than those in division.

Some colours are much more sensitive to the action of clove oil than others; and much depends on the quality of this muchadulterated essence. New clove oil extracts the colour more quickly than old, and anilin than clove oil. 
Series of sections on slides are conveniently cleared by pouring the clearing agent over them.

After clearing you may either mount at once in damar or balsam, or stop the extraction of the colour, if clove oil have been used, by putting the sections into some medium that does not affect the stain (xylol, cedar oil, etc.). Chloroform should be avoided, either as a clearer or as the menstruum for the mounting medium.

283. General Results. - The results depend in great measure on the previous treatment of the tissues. If you have given them a prolonged fixation in Flemming's strong chromo-aceto-osmic mixture, and have differentiated after staining with acid alcohol and cleared with clove oil, you will get, with some special exceptions, nothing stained but nucleoli and the chromatin of dividing nuclei, that of resting nuclei remaining unstained. If you have given a lighter fixation, with Flemming's weak mixture or some other fixing agent not specially inimical to staining, and have differentiated after staining with neutral alcohol, you will get the chromatin of resting nuclei stained as well. Either process may also stain mucin, the ground-substance of connective tissues (especially cartilage), the bodies of Nissl in nerve-cells, and the yolk of ova.

284. Henneguy's Permanganate Method (Journ. de l'Anat. et de la Physiol., xxvii, 1891, p. 397). - Sections are treated for five minutes with 1 per cent. solution of permanganate of potassium. They are then washed with water and stained (for about half the time that would have been taken if they had not been mordanted with the permanganate) in safranin, rubin, gentian violet, vesuvin, or the like, and are differentiated with alcohol, followed by clove oil in the usual way.

The mordanting action of the permanganate is so energetic that if it has been overmuch prolonged before staining with safranin, or, still more, with rubin, it becomes almost impossible to differentiate the sections properly; it may be necessary to leave them for a month or more in clove oil.

285. Ohlmacher's Formaldehyde Process (Medical News, February 16th, 1895).- Ohlmacher states that formaldehyde is a powerful mordant for tar colours. Tissues may either be mordanted separately by treatment for a short time (one minute is enough for cover-glass preparations) with a 2 per cent. to 4 per cent. formalin solution; or the formalin may be combined with the stain. One grm. of fuchsin or methylen blue dissolved in 10 c.c. of absolute alcohol may be added to 100 c.c. of 4 per cent. formalin solution. Sections are said to stain in half a minute and to resist alcohol much more than is the case with those treated by the usual solutions.

286. Safranin.- One of the most important of these stains, on account of its power, brilliancy, and permanence in balsam, and the 
divers degrees of electivity that it displays for the nuclei and other constituent elements of different tissues.

The great secret of staining with safranin is to get a good safranin. In ordering it, from Grübler \& Hollborn or elsewhere, it is well to specify whether you want it for staining nuclei or for staining elastic fibres, or for what other purpose you may require it. There are presumably at least a score of sorts of safranin in the market, differing to a considerable extent in colour, weight, solubility, and histological action. Some are easily soluble in water and not so in alcohol, some the reverse, and some freely soluble in both. The brand I have been using for a long time, which gives good results, is the "Safranin O" of Grübler \& Co.

Staining.-The majority of safranins are not sufficiently soluble in water, so that solutions in other menstrua must be employed.

Pfitzner (Morph. Jahrb., vi, p. 478, and vii, p. 291) advised a solution of safranin 1 part, absolute alcohol 100 parts, and water 200 parts, the last to be added only after a few days.

Flemming (Arch. mik. Anat., xix, 1881, p. 317) used a concentrated solution in absolute alcohol, diluted with about one-half of water.

BABES (ibid., 1883, p. 356) used (A) a mixture of equal parts of concentrated alcoholic solution and concentrated aqueous solution (this is very much to be recommended), or (B) a concentrated or supersaturated aqueous solution made with the aid of heat.

Some people still employ simple aqueous solutions.

The anilin solution of BABES (Zeit. wiss. Mik., iv, 1887, p. 470) consists of water 100 parts, anilin oil 2 parts, and an excess of safranin. The mixture should be warmed to from $60^{\circ}$ to $80^{\circ} \mathrm{C}$., and filtered through a wet filter. This solution will keep for a month or two.

ZWAARDEMAKER (ibid., iv, 1887, p. 212) makes a mixture of about equal parts of alcoholic safranin solution and anilin water (saturated solution of anilin oil in water;-to make it, shake up anilin oil with water, and filter). This, I find, will keep for many months, perhaps indefinitely.

I myself use equal parts of saturated solution in anilin water and saturated solution in absolute alcohol.

Differentiation.-For general directions see $\$ \$ 281$ and 282.

Flemming's acid differentiation (Zeit. wiss. Mik., i, 1884, p. 350).Differentiate, until hardly any more colour comes away, in alcohol acidulated with about 0.5 per cent. of hydrochloric acid, followed by pure alcohol and clove oil. (You may use the $\mathrm{HCl}$ in watery 
solution if you prefer it.) Or you may use a lower strength, viz. 0.1 per cent. at most (see Arch. mik. Anat., xxxvii, 1891, p. 249); and this I find is generally preferable.

Objects are supposed to have been well fixed-twelve hours at least-in the strong chromo-aceto-osmic mixture, and stained for some hours. In this way you get kinetic chromatin and nucleoli alone stained.

Podwyssozki (Beitr. z. Path. Anat., i, 1886, p. 289) differentiates (for from a few seconds to two minutes) in a strongly alcoholic solution of picric acid, followed by pure alcohol. Same results (except that the stain will be brownish instead of pure red).

BABES recommends treatment with iodine, according to the method of Gram (see next section). This process has also been recommended by Prenant (Int. Monatsschr. Anat., etc., iv, 1887, . p. 368).

It has been shown by Ohlmacher (Journ. Amer. Med. Assoc., vol. xx, No. 5, February 4th, 1893, p. 111) that if tissues be treated with iodine or picric acid after staining with safranin, there may be produced in the tissue elements a precipitate of a dark red substance of a erystalline nature, but of lanceolate, semilunar, falciform, or navicellar forms. The precipitate is formed both in normal and pathological tissue, readily in carcinomatous tissues; and Ohlmacher concludes that many of the bodies that have been described as "coccidia," "sporozoa," or other "parasites" of carcinoma are nothing but particles of this precipitate.

See also the differentiation process of MARTINotTi and RESEGOTTI (Zeit. wiss. Mik., iv, 1887, p. 328) for alcohol-fixed material, and of . GARBINI (Zeit. wiss. Mik., v, 2, 1888, p. 170).

In preparations made with chromo-aceto-osmic acid, safranin stains, besides nuclei, elastic fibres, the cell bodies of certain horny epithelia, and the contents of certain gland-cells (mucin, under certain imperfectly ascertained conditions).

The stain is perfectly permanent.

28\%. Gentian Violet may be used in aqueous solution, or as directed for safranin.

In some cases it may be useful to employ the method devised by GRAM for the differentiation of bacteria in tissues (Fortschr. $d$. Medicin., ii, 1884, No. 6 ; British Med. Journ., September 6th, 1884, p. 486 ; Journ. Roy. Mic. Soc. [N.S.], iv, 1884, p. 817). In this the sections are treated, after staining, with a solution composed of-

Iodine

Iodide of potassium

Water
1 grm. 300

2 grms. 
for two or three minutes, until they become black. They are then differentiated with neutral alcohol, until they turn grey, and are then finally differentiated with clove oil.

By this process, in resting nuclei the nucleoli alone are stained, or the chromatin if stained is pale; in dividing nuclei the chromatin is stained with great intensity, being nearly black in the equatorial stage.

Gentian violet is an exceedingly powerful stain, quite as precise as safranin.

The stain keeps well. It is more or less dichroic, possibly owing to the fact that the dye is not a pure substance, but a mixture of "Krystallviolett" and methyl violet.

HeRMann (Arch. mik. Anat., xxxiv, 1889, p. 58) first stains for twentyfour hours or more in safranin, differentiates incompletely with alcohol, then stains for three to five minutes in the anilin-water gentian solution, treats with the iodine solution for one to three hours, and finally differentiates with absolute alcohol.

288. Thionin.-The hydrochloride of thionin, or violet of Lauth, is a colour chemically nearly allied to methylen blue. Its action is so selective from the first that it may almost be considered to be a progressive stain. If you stain for only a short time (a few minutes) in a concentrated aqueous solution, hardly anything but the chromatin will be found to be stained. If the staining be prolonged, plasmatic elements will begin to take up the colour. After a short stain no special differentiation is required; all that is necessary is to rinse with water, dehydrate, and mount. After a strong stain you differentiate with alcohol in the usual way, with this advantage, that the stain is so highly resistant to alcohol that there is no risk whatever of over-shooting the mark; the stain will not be more extracted in an hour than that of gentian or dahlia is in a minute, so that the process may be controlled under the microscope if desired. For this reason I think this stain may be useful to beginners, but I myself prefer gentian. It is a very powerful stain.

Thionin is a specific stain for mucin, $q . v$. Some observers have found the stain to fade. WolfF (Zeit. wiss. Mik., xv, 1899, p. 312) says that, to avoid this, preparations should be mounted in a little solid colo. phonium or balsam melted over a flame. FELIZAT and BRANCA (Journ. Anat. Phys., xxxiv, 1898, p. 590) mount without a cover. HenneguY (in litt.) clears with acetone.

KING (Anat. Record, iv, 1910, p. 236) stains with a saturated solution in carbolic acid of 1 per cent., and finds the stain permanent.

NiCOLlE's "thionine phéniquée" consists of 1 part of saturated solution in alcohol of 50 per cent., and 5 parts of 2 per cent. aqueous solution of carbolic acid. 
289. Other Regressive Stains.-The following may be useful :-

Dahlia, according to Flemming (Arch. mik. Anat., xix, 1881, p. 317), best used in aqueous solution, either neutral or acidified with acetic acid, and differentiated with neutral alcohol. A pure blue stain, which keeps well. See also Schuberc, in Zeit. wiss. Zool., lxxiv, 1903, p. 7, and lxxxvii, 1907, p. 557.

Victoria Blue (Victoriablau) (Lustgarten, Med. Jahrb. k. Ges.d. Aerzte zu Wien, 1886, pp. 285-291).-This dye ("Victoriablau 4 A ") has a special affinity for elastic fibres. For this object Lustgarten recommends an alcoholic solution of the dye diluted with 2 to 4 parts of water. Fixation in chrom-osmium, or at least in a chromic mixture, is, I believe, a necessary condition to this reaction. And you must stain for a long time.

Victoria has also a special affinity for mucus-cells, from which it is not washed out by alcohol, and for cartilage.

This stain keeps very well.

With Toluidin Blue I have had some superb stains of chromatin, unfortunately accompanied by a diffuse staining of cytoplasm.

MANN (Zeit. wiss. Mik., xi, 1894, p. 489) states that he has had good results by staining with it after eosin.

See further, as to the micro-chemical properties of this dye, Harris, The Philadelphia Medical Journal, May 14th, 1898. It much resembles methylen blue.

Metzner (Nagel's Handb. Phys., ii, 1907, p. 915) mordants sections, before staining, for three-quarters of an hour in iron alum.

Magdala Red (Naphthalin Red, Rose de Naphthaline).

Fuchsin (meaning the basic fuchsins, a series of Rosanilin salts having very similar reactions, and found in commerce under the names of Fuchsin, Anilin Red, Rubin, Rosein, Magenta, Solferino, Corallin).-Graser (Deutsche Zeit. Chirurgie, xxvii, 1888, pp. 538584; Zeit. wiss. Mik., v, 1888, p. 378) stains for twelve to twenty-four hours in a dilute aqueous solution, washes out for a short time in alcohol, stains for a few minutes in aqueous solution of methylen blue, and dehydrates with alcohol. A double stain. Chromatin and nucleoli red, all the rest blue.

ZieHL's Carbolic Fuchsin (Zeit. wiss. Mik., vii, 1890, p. 39) consists of fuchsin 1 grm., acid. carbol. crist. 5 grms., alcohol 10 grms., aq. dest. 100 grms. The stain is differentiated with alcohol followed by clove oil.

Kresofuchsin (Rothig, Arch. mik. Anat,, lvi, 1900, p. 354).-Its aqueous solution is red and stains mucus, cartilage, keratin, and nuclei red, whilst its alcoholic solution is blue and stains elastin blue. See also under "Connective tissues."

Bismarck Brown has this advantage, that being sufficiently resistant to alcohol it may be utilised for staining entire objects. 
KAISER (Biblioth. Zool., H. 7, 1 Halft, 1891 ; Zeit. wiss. Mik., viii, 1891, p. 363) stains for forty-eight hours, and at a temperature of $60^{\circ} \mathrm{C}$. in saturated solution of Bismarck brown in 60 per cent. alcohol (the solution to be made in boiling alcohol), and washes out (until all is decoloured except the karyokinetic figures) in 60 per cent. alcohol, containing 2 per cent. hydrochloric acid or 3 per cent. acetic acid.

Methyl Violet. See ante, $\S 278$.

Benzoazurin (Martin, Zeit. wiss. Mik., vi, 3, 1889, p. 193).-Stain for an hour or so in dilute aqueous solution, and wash out with $\mathrm{HCl}$ alcohol.

Methylen Blue.

Nigrosin (Errera, Proc.-Verb. Soc. Belge de Mik., 1881, p. 134) gives a good stain which resists alcohol well.

Methyl Green is sometimes useful in certain mixtures (see next chapter). 


\section{CHAPTER XV.}

PLASMA STAINS* WITH COAL-TAR DYES.

290. Introduction. - By a plasma stain is meant one that stains the extra-nuclear parts of cells and the formed material of tissues, or one of these.

The plasma stains described in this chapter are for the most part those obtained by means of " acid" dyes (§ 201); but some of them are obtained by means of "neutral" dyes ( $\$ 201$ ), and a few by "basic" dyes.

The mode of staining is generally progressive, almost always so when acid colours, used substantively ( $\$ 205)$, are employed. But the regressive method, with differentiation, is sometimes made use of, especially when a mordant has been used with the dye.

In some processes, e.g., Flemming's orange method, a basic and an acid dye (or vice versâ) being employed in succession, there is formed in the tissues a neutral colour (\$201) which effects the desired stain. These may be considered as adjective stains, the first colour serving as a mordant for the second. Not any two dyes taken at haphazard will behave in this way: they must be such as to form by combination a suitable neutral lake (cf. § 201). The basic dye may be made the primary stain, as in Flemming's process : or the contrary.

In such stains as Reinke's orange method, or the Ehrlich-Biondi mixture, and many others, one or more neutral colours are formed in the mixture and stain progressively.

Excepting Biebrich scarlet, I am not acquainted with any plasma stain that is thoroughly satisfactory for delicate work. In addition to Biebrich scarlet, I recommend for sections Säurefuchsin, either alone or in the form of Ehrlich-Biondi mixture, or Ehrlich's triacid: for material in bulk, picric acid (but only for rough work).

\section{Säurefuchsin (Acid Fuchsin, Fuchsin S, Acid Rubin, Rubin} S, Säurerubin, Acid Magenta, Magenta S).-The chemical descrip-

* This chapter includes only such stains as are used in ordinary work on tissues in bulk or sections, stains for special purposes being treated under "Nervous tissue," "Blood," etc. It includes some double or triple stains that affect nuclei as well as plasma, but in different hues. 
tion of this acid colour has been given ( $\$ 201)$ : it must not be confounded with basic fuchsin, as seems to have been done by some writers.

This dye is highly soluble in water, less so in alcohol. I use a 0.5 per cent. solution in water and allow it to act on sections for a few minutes in the case of easily stainable material, or twenty-four hours or more for chrom-osmium material. The stain is fast to neutral alcohol. It is very sensitive to alkalies, so that overstains can easily be removed by washing for a few minutes in tap-water. Acids strengthen the stain, so that it is frequently useful to treat sections after staining for a few seconds with acidulated water. A good stain should show the reticulum of cytoplasm, together with nuclear spindles and asters, stained red, and connective tissue strongly brought out. It may be advisable to acidify the staining bath very slightly. Successful stains are admirably sharp.

292. Pyronin.-A basic dye, red, only used (as far as I can find) in mixtures. Pappenheim (Arch. Path. Anat., clxvi, 1901, p. 427) takes 2 parts 1 per cent. solution of methyl green and 1 part 1 per cent. solution of pyronin, stains sections for five minutes, rinses, and differentiates in a solution of resorcin or hydroquinon in absolute alcohol. According to Corti and Ferrara, Mon. zool. Ital., xvi, 1905 , p. 319 , this mixture generally stains chromatin green and cytoplasm red, but in Flemming or Hermann material the reverse. It seems to me a coarse plasma stain, but likely to be sometimes useful.

UNNA's CARBOL-PYRONIN-METHYL GREEN modification (Encycl. Mik. Tech., 1910, ii, p. 412 : I am indebted for the formula to Dr. GaUdLITz) is as follows: Stain for five to ten minutes at $30^{\circ}$ to $40^{\circ} \mathrm{C}$. in methyl green $0 \cdot 15$ parts, pyronin $0 \cdot 25$, alcohol $2 \cdot 5$, glycerin 20 , and carbolic acid of 0.5 per cent. to make up 100 volumes. Cool rapidly, rinse, dehydrate, and pass through bergamot oil, or xylol or benzol (not clove-oil), into balsam. Brings out bacteria (red) in organic liquids. The mixture may be had from Grübler \& Hollborn.

293. Orange G.-This is the benzenazo-beta-naphthol-disulphonate of soda. As indicated by its chemical description, this is an "acid" colour.

It is easily soluble in water, less so in alcohol. Use as directed for Säurefuchsin. Almost, if not quite, as precise a stain as Säurefuchsin. It does not overstain, but may wash out other dyes.

294. Säurefuchsin and Orange G.-I have had good results by mixing the aqueous solutions of these two dyes, but unfortunately 
have not noted the proportions. SQuIRE (Methods and Formula, p. 42) takes 1 grm. Säurefuchsin, 6 grms. Orange $G$ in 60 c.c. of alcohol and 240 c.c. of water. See also under "Connective tissues."

295. Ehrlich-Biondi Mixture (or Ehrlich-Biondi-Heidenhain Mixture) (Pfluger's Arch., xliii, 1888, p. 40).

To 100 c.c. saturated aqueous solution of orange add with continual agitation 20 c.c. saturated aqueous solution of Säurefuchsin (Acid Fuchsin) and 50 c.c. of a like solution of methyl green.

(According to Krause (Arch. mik. Anat., xlii, 1893, p. 59), 100 parts of water will dissolve about 20 of Säurefuchsin (Rubin S), 8 of orange $G$ and 8 of methyl green.) The solutions must be absolutely saturated, which only happens after several days.

Dilute the mixture with 60 to 100 volumes of water. The dilute solution ought to redden if acetic acid be added to it; and if a drop be placed on blotting-paper it should form a spot bluish green in the centre, orange at the periphery. If the orange zone is surrounded by a broader red zone, the mixture contains too much fuchsin.

According to M. Heidenhain ("Ueber Kern u. Protoplasma," in Festschr.f. Kolliker, 1892, p. 115) the orange to be used should be " Orange G," the Acid Fuchsin or Säurefuchsin should be "Rubin $\mathrm{S}$ " ("Rubin" is a synonym of Fuchsin) and the methyl green should be "Methylgrün 00." And it is absolutely necessary that these ingredients be those prepared under those names by the Actienfabrik für Anilin-fabrikation in Berlin. They can be obtained from Grübler \& Hollborn, either separately, or as a mixture of the three dyes in powder (which I do not recommend).

The strong solutions directed to be taken readily precipitate on being mixed. To avoid this it is recommended by SquIRE (Methods and Formulce, etc., p. 37) to dilute them before mixing.

Other proportions for the mixture have been recommended by KraUSE (loc. cit. supra), viz. 4 c.c. of the Säurefuchsin solution, 7 of the orange Gr and 8 of the methyl green; the mixture to be diluted 50 to 100 -fold with water. Tноме (Arch. mik. Anat., lii, 1898, p. 820) gives the proportions $2: 5: 8$, and dilutes 100 -fold.

Stain sections (N.B. sections only) for six to twenty-four hours. Dehydrate with alcohol, clear with xylol, and mount in xylol balsam.

In the intention of the observers who have elaborated this stain it is a progressive stain, and not a regressive one. It does not require any differentiation, and the sections should be got through the alcohol into xylol as quickly as possible in order to avoid any extrac- 
tion of the methyl green, which easily comes away in the alcohol. DrüNer (Jena Zeit., xxix, 1894, p. 276) stains for ten minutes in the concentrated solution, treats for one minute with alcohol containing $0 \cdot 1$ per cent. of hydrochloric acid, and then with neutral alcohol.

The best results are obtained with sublimate material; chromosmium material, and the like, give a much inferior stain. Preparations made with the usual mixture, as given above, are liable to fade; by acidifying the mixture a stronger and more sharply selective stain is obtained, which does not fade. But too much acid must not be added, as this would cause a staining of the interfilar substances. According to the Encycl. mik. Technik, you may add 15 to 24 drops of 0.2 per cent. acetic acid to 100 c.c. of the diluted solution.

Another process of acidification is given by M. HeIDENHAIN (Ueber Kern und Protoplasma, p. 116); for this see fourth edition. See also Israel (Praktikum Path. Hist., 2 Aufl., Berlin, 1893, p. 69) ; Trambusti (Ricerche Lab. Anat. Roma, v, 1896, p. 82 ; Zeit. wiss. Mil., xiii, 1896, p. 357); and Thome (op. cit. supra). EISEN (Proc. Calif. Acad. (3), i, 1897, p. 8) acidifies with oxalic acid.

After acidification the solution must not be filtered, and if it has been kept for some time a little more acid must be added.

Before staining (M. HeIdenhaIN, loc. cit.), sections should be treated for a couple of hours with $0 \cdot 1$ per cent. acetic acid, then for ten to fifteen minutes with officinal tincture of iodine, and be rinsed with alcohol before bringing into the stain. The treatment with acid is necessary in order to ensure having the sections acid on mounting in balsam. The primary object of the iodine is to remove any sublimate from the preparations, but it also is said to enhance the power of staining of the chromatin with methyl green, and to produce a more selective staining of protoplasmic elements.

The stain is a very fine one when successful. But it is very capricious. The correct result should be a precise chromatin stain combined with a precise stain of the plastin element of cytoplasm by the Säurefuchsin. Now the least defect or excess of acidity causes the plasma stain of the Säurefuchsin to become a diffuse one, instead of being sharply limited to the plastin element. It is difficult to dehydrate the sections without losing the methyl green. For this reason the stain will only work with very thin sections; to be quite sure of good results, the sections should be of not more than $3 \mu$ in thickness, and if they are over 5 the desired results are almost hopeless. The stain keeps very badly. I admit that the 
method has its raison d'être for the very special objects for which it was imagined - for the researches on cell-granulations for which EHRLICH employed the three colours, or for the researches on the plastin element of cytoplasm for which Martin Heidenhain employed the mixture; for the study of gland cells; and for similar objects. But to recommend it, as has been done, as a general stain for ordinary work, is nothing but mischievous exaggeration. For it is far from having the qualities that should be possessed by a normal section stain. Workers have at length found this out, and it is now but little used except for the special purposes above indicated.

296. Ehrlich's "Triacid" Mixture.-This name would seem to indicate that the mixture contains three "acid" colours, which is not the case, methyl green being a strongly " basic" colour. Ehrlich explains in a letter to Mayer (see also EHrLich and Lazarus, Die Ancemie, 1898, p. 26) that it is so called " because in it all the three basic groups of the methyl green are combined with the acid dyestuffs." A very pretty' conundrum!

The latest receipt (op. cit., p. 28) is as follows:

Prepare separately saturated solutions of orange G, Säurefuchsin, and methyl green, and let them clarify by settling. Then mix, in the order given, using the same measure-glass, 13 to 14 c.c. of the orange, 6 to 7 of the Säurefuchsin, 15 of distilled water, 15 of alcohol, $12 \frac{1}{2}$ of the methyl green, 10 of alcohol, and 10 of glycerin. After adding the methyl green, shake well, but do not filter.

The mixture keeps well. I find its qualities and defects to be much those of the Ehrlich-Biondi mixture. The stain seems more powerful but less delicate, and the methyl green in it appears to have more resistance to alcohol, so that it is better adapted for ordinary work.

MAYER (Grundzüge, LEE and MAYER, p. 197) has simplified the formula thus : Take $1 \mathrm{~g}$. methyl green, $2 \mathrm{~g}$. orange, $3 \mathrm{~g}$. Säurefuchsin, and dissolve in a mixture of 45 c.c. water, 10 c.c. glycerin, and 20 c.c. alcohol of 90 per cent.

MOREL and DoLeris (C. R. Soc. Biol., liv, 1902, p. 1255) mix 1 vol, of the solution with one of 8 per cent. formalin and add 0.1 per cent. of acetic acid, and state that thus the methyl green is better fixed in the nuclei.

297. Pianese's Säurefuchsin-malachite Green (from Müller, Arch. Zellforsch., viii, 1912, p. 4) consists of $0.5 \mathrm{grm}$. malachite green, $0.1 \mathrm{grm}$. Säurefuchsin, and $0.01 \mathrm{grm}$. Martius yellow in 150 c.c. water and 50 c.c. alcohol. Stain for twenty-four hours, differentiate with alcohol, containing 1 to 2 drops of $\mathrm{HCl}$ per 200 c.c. 
298. Picric Acid.-Picric acid gives useful plasma stains after carmine and hæmatoxylin. The modus operandi consists merely in adding picric acid to the alcohols employed for dehydrating the objects.

Picric acid has considerable power of washing out other anilin stains; and in combination with hydrochloric acid it very greatly enhances the power with which this acid washes out carmine stains. It should, therefore, not be added to the acidulated alcohol taken for differentiating borax-carmine stains, or the like, but only to the neutral alcohol used afterwards. It has the great quality that it can be used for staining entire objects, and is much indicated for such objects as small Arthropods or Nematodes, mounted whole.

It can in some cases be employed by dissolving it in the solution of another dye (see Picro-carmine, LEGAL's alum-carmine, § 221, etc.); or (for sections) by dissolving it in the xylol or chloroform used for clearing.

Though picric acid is a useful ground stain, it is at most a rough one, being very diffuse. It stains, however, horn, chitin, muṣclę and erythrocytes, with special energy.

According to Frohlich (Zeit. wiss. Mik., xxvii, 1910, p. 349) picraminic acid (from Grübler \& Hollborn) has some advantages over picric acid.

299. Van Gieson's Picro-Säurefuchsin (from Zeit. wiss. Mik., xiii, 1896, p. 344).-To a saturated aqueous solution of picric acid are added a few drops of saturated aqueous solution of Säurefuchsin, until the mixture has become garnet-red. Or (Trans. Amer. Micr. Soc., xix, 1898, p. 105) to 100 parts of the picric acid solution add 5 parts of 1 per cent. solution of Säurefuchsin. After staining (sections only), rinse with water, dehydrate, and clear in oil of origanum.

Ohlmacher (Journ. Exper. Med., ii, 1897, p. 675) adds 0.5 per cent. of Säurefuchsin to a saturated solution of picric acid which has been diluted with an equal quantity of water. He uses this after previous staining with gentian violet.

RAMON y CAJAL recommends 0.1 grm. of Säurefuchsin to 100 of saturated solution of picric acid (ScHAFFER, Zeit. wiss. Zool., lxvi, 1899, p. 236).

Hansen (Anat. Anz., xv, 1898, p. 152) adds 5 c.c. of 2 per cent. solution of Säurefuchsin to 100 c.c. saturated solution of picric acid, and for staining adds to 3 c.c. of the mixture one-third of a drop of 2 per cent. acetic acid, stains for a few minutes or hours, rinses in 3 c.c. of water with 2 drops of the acidified stain added, dehydrates 
clears with xylol, and mounts in xylol-balsam. Connective-tissue red, elastin and all other elements yellow.

WeIGerT (Zeit. wiss. Mik., 1904, p. 3) adds 10 parts of 1 per cent. Säurefuchsin to 100 of saturated picric acid.

See also Möller, op. cit., xv, 1898, p. 172.

This stain is generally used as a contrast stain to follow hæmatoxylin. AрÁtнy (Behrens' Tabellen, 3rd ed., p. 129) takes for this purpose $1 \mathrm{grm}$. of Säurefuchsin in 500 c.c. of saturated solution of picrate of ammonia.

WrLhelmi (Fauna Flora Golf. Neapel, xxii, 1909, p. 18) takes $0 \cdot 2 \mathrm{grm}$. Säurefuchsin, $0.8 \mathrm{grm}$. picrate of ammonia, $10 \mathrm{grm}$. absolute alcohol, and $89 \mathrm{grm}$. water.

E. and T. SAvini (Zeit. wiss. Mik., xxvi, 1909, p. 31) use a formula due to BENDA. Ninety-five volumes of saturated solution of picrate of ammonia are mixed with 5 volumes of 1 per cent. solution of Säurefuchsin. For use, two to four drops of saturated solution of picric acid are added to 10 c.c. of the mixture. This neither overstains nor attacks the primary stain.

300. Flemming's Orange Method (Arch. mik. Anat., xxxvii, 1891, pp. 249 and 685). - Stain sections of Flemming or Hermann material in strong alcoholic safranin solution diluted with anilin water (§ 286); differentiate in absolute alcohol, containing at most 0.1 per cent. of hydrochloric acid, until hardly any more colour comes away; stain for one to three hours in gentian violet ( $\$ 287)$; wash for a short time in distilled water; treat with concentrated, or at least fairly strong, aqueous solution of orange G. After at most a few minutes, whilst pale violet clouds are still being given off from the sections on agitation, bring them into absolute alcohol until hardly any more colour comes away, clear in clove or bergamot oil, and mount in damar or balsam before the last pale clouds of colour have ceased to come away. The orange must be orange $G$.

WiniwaRter and SAINMont (Zeit. wiss. Mik., xxv, 1908, p. 157, and Arch. Biol., xxiv, 1909, p. 15) stain for twenty-four hours in the gentian, wash out after the orange for two to three hours in 100 c.c. absolute alcohol with 3 to 4 drops of $\mathrm{HCl}$, and differentiate finally with oil of cloves.

This is not a triple stain in the sense of giving three different colours in the result; it is a nuclear and plasmatic stain in mixed tones; the orange, apparently, combines with the gentian to form a "neutral" dye, soluble in excess of the orange $(\$ 203)$ which thus differentiates the stain.

See also Flemming in Arch. Anat. Phys. Anat. Abth., 1897, p. 175.

Never popular, this clumsy and uncertain process is now little used.

301. REINKE's Orange Method (Arch. mik.Anat., xliv, 2, 1894, p. 262), - To a concentrated aqueous solution of gentian violet are added " a few props " of a like solution of orange G. The solution precipitates in part. 
owing to the formation of an imperfectly soluble " neutral " colour, but becomes almost clear again if an excess of water be added. The solution is not to be filtered, but the sections are to be stained in the mixture made almost clear by addition of water. It is said that the "neutral " solution may be preserved for future use by adding to it one third of alcohol. After staining (sections previously stained with safranin), you differentiate rapidly with alcohol and clear with clove oil.

I have tried this process and obtained exactly the same results as with Flemming's process, and so have other workers.

ARNoLD's Orange Method (Arch. Zellforsch., iii, 1909, p. 434).Sections (of chrome material) are treated for five minutes with solution of equal parts of iodine and iodide of potassium in alcohol of 40 per cent., then washed and stained for four hours in saturated solution of safranin in alcohol of 75 per cent. : then washed and put for five to fifteen minutes into solution of 7 parts of methylen blue, 0.5 of carbonate of soda and 100 of water, washed, dehydrated, and treated until pale blue with solution of orange $\mathrm{G}$ in oil of cloves. Cytoplasmic reticulum blue on orange ground, nucleoli and centrosomes red. Instead of the safranin, basic fuchsin may be taken.

302. Bonney's Triple Stain (Virchow's Arch., cxciii, 1908, p. 547, and elsewhere).- Stain sections (of acetic alcohol or sublimate material, not chrome or formol material) for two minutes in a solution of 0.25 parts methyl violet and 1 part pyronin in 100 of water. Wipe slide dry, and flood twice with the following : 2 per cent. aqueous solution of orange G, boiled and filtered, is added drop by drop to 100 c.c. of acetone, with agitation, until there is formed a flocculent precipitate, which redissolves on further addition of the orange. Wash rapidly in pure acetone, and pass through xylol into balsam. Chromatin violet, cytoplasm red, connectivetissue yellow, keratin violet. Not adapted for blood films.

303. Bordeaux R.-An " acid " dye, giving a general stain taking effect both on chromatin and cytoplasm, and, I consider, a very good plasma stain. I use for chrom-osmium material a 1 per cent. solution, and stain for twelve to twenty-four hours. The stain is sufficiently fast.

304. Bordeaux R, Thionin, and Methyl Green (GRÄBERG, Zeit. wiss. Mik., xiii, 4, 1896, p. 460).

305. Congo Red (Congoroth) (see GrIEsBACH, in Zeit. wiss. Mik., see iii, 1866, p. 379).-An " acid" colour. Its solution becomes blue in presence of the least trace of free acid (hence Congo is a valuable reagent for demonstrating the presence of free acid in tissues; see the papers quoted loc. cit.). A stain much of the same nature as Säurefuchsin. It is useful for staining some objects during life (see ante, § 208). CARnoy (La Cellule, xii, 1897, p. 216) has had very 
good results with it after hæmatoxylin of DeLafield. He used 0.5 per cent. solution in water. Note that this colour is not to be confounded with other Congos, as Congo yellow, or brilliant Congo. It is one of the azo dyes.

306. Congo-Corinth.-Also an acid dye. Heidenharn (Zeit. wiss. Mik., xx, 1903, p. 179) recommends Congo-Corinth G (or the allied colour Benzopurpurin 6 B) (Elberfelder Farbwerke). Sections must be made alkaline before staining, by treating them with very weak sal ammoniac or caustic soda, in alcohol. After staining, pass through absolute alcohol into xylol. Used after alum hæmotoxylin, the stain of which it does not cause to fade.

307. Benzopurpurin.-According to GrIesbaCh (loc. cit., § 305), another "acid" colour very similar in its results to Congo red. See also ZsCHOKKe (ibid., v, 1888, p. 466), who recommends Benzopurpurin B, and says that weak aqueous solutions should be used for staining, which is effected in a few minutes, and alcohol for washing out. Deltapurpurin may be used in the same way.

See last $\S$ as to the necessity of alkalising the sections, which Heidenhain states is necessary with all dyes of this group.

308. Neutral Red (Neutralroth) (EHrLich, Allg. med. Zeit., 1894, pp. 2, 20; Zeit. wiss. Mik., xi, 1894, p. 250; Galeottr, ibid., p. 193):-A " basic" dye. The term " neutral " refers to the hue of its solution. Its neutral red tint is turned bright red by acids, yellow by alkalies. The stain in tissues is in general metachromatic, nuclei being red, cell-bodies yellow ( $f f$. Rosin, in Deutsche med. Wochenschr., xxiv, 1898, p. 615 ; Zeit. wiss. Mik., xvi, 2, 1899, p. 238). Up to the present this colour has chiefly been employed for intra-vitam staining. Tadpoles kept for a day or two in a solution of $1: 10,000$ or 100,000 absorb so considerable a quantity of the colour that all their tissues appear of a dark red. The stain is limited to cytoplasmic granules (EHRLICH), and to the contents of mucus cells (GALEOTTI).

According to Ehrlich and Lazarus (Spec. Pathol. und Therapie, herausgeg. von NothNAGEL, viii, 1,1898, p. 1 ; Zeit. f. wiss. Mik., $\mathrm{xv}, 3,1899, \mathrm{p} .338$ ) it may be used for intra-vitam staining of tissues in the same way as methylen blue, by injection or immersion with contact of air. It is especially a granule stain. Similar results are recorded by Arnold (Anat. Anz., xvi, 1899, p. 568, and xxi, 1902, p. 418). See also Ehrlich and Lazarus, Ancemie, i, 1898, p. 85 ; Loisel (Journ. de l'Anat. et de la Physiol., 1898, pp. 197, 210, 217) (intra-vitam staining of sponges); and Prowazek (Zeit. wiss. Zool., lxii, 1897, p. 187) (intra-vitam staining of Protozoa). I myself have had very good results with it as an intra-vitam stain.

According to Golovine (Zeit. wiss. Mik., xix, 1902, p. 176), the 
stain may be fixed in the tissues by means of sublimate, chromic acid, bichromates, picric acid, or platinum chloride, followed by molybdate of ammonium.

It has also been found useful for staining, in hardened material, the corpuscles of Nissu $(q . v$.) in nerve-cells. S. Mayer (Lotos, Prague, 1896, No. 2) states that it also stains degenerating myelin. The solutions that have been employed for staining fixed material are strong aqueous ones, 1 per cent. to concentrated.

309. Biebrich Scarlet (British Dyes, Ltd., Huddersfield).-A. K. Gordon (British Medical Journ., 1917, p. 828) finds this an excellent acid dye which never overstains and is not diffuse. Use in 1 per cent. solution, or as recommended by ScoTT ( $\$ 676)$.

I have used this stain a good deal and find it better than any other plasm dye that I have tried.

310. The Eosins, found in commerce under the names of Eosin, Saffrosin, Primerose Soluble, Phloxin, Bengal Rose, Erythrosin, Pyrosin B, Rose B, à l'Eau, etc., are all " acid " phthalein colours. They are not quite identicąl in their properties. Most of them are soluble both. in alcohol and in water, but some only in alcohol ("Primerose à l' Alcool").

They are all diffuse stains, formerly much used as contrast stains, less so now. Hansen (Anat. Hefte, xxvii, 1905, p. 620) adds 1 drop of acetic acid of 2 per cent, to 9 c.c. of 1 per cent. eosin, which makes the stain more selective.

For Bengal Rose see Griesbach, Zool. Anz., 1883, p. 172.

Eosin is a specific stain for red blood-corpuscles, and also for certain granules of leucocytes (see under "Blood").

The yolk of some ova takes the stain strongly, so that it is useful in some embryological researches.

311. EhrLICH's Indulin-Aurantia-Eosin, or Acidophilous Mixture, or Mixture C, or Mixture for Eosinophilous Cells (from the formula kindly sent me by Dr. GRÜBLER).--Indulin, aurantia, and eosin, of each 2 parts; glycerin, 30 parts. This gives a very thick, syrupy solution. To use it, cover-glass preparations may be floated on to it; or sections on slides may have a few drops poured on to them, the slide being laid flat till the stain has taken effect (twenty-four hours for Flemming material). I find that with Flemming material it gives a powerful and good stain, which is much more resistant to alcohol than that of the EHRLICH-Biondi mixture, and is, therefore, much more adapted to ordinary work. The stain keeps well.

Israel (Praktik. Path. Hist., Berlin, 1893, p. 68) gives a more complicated receipt. 
312. Methyl Green and Eosin (CAlberla, Morph. Jahrb., iii, 1877, Heft 3, p. 625 ; List, Zeit. wiss. Mik., ii, 1885, p. 147 ; Balbiani, Ann. Microgr., Paris, vii, 1895, p. 245 ; RHumbler, Zeit. wiss. Zool., lxi, 1895, p. 38).-See early editions.

313. Methylen Blue and Eosin (CHenzinsky, quoted from Zeit, wiss Mik., xi, 2, 1894, p. 269).

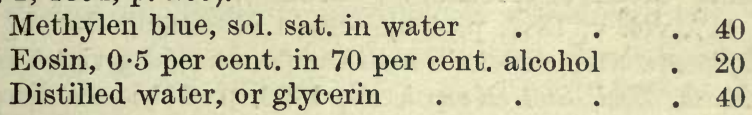

This solution will only keep for about eight days.

Pianese (ibid., xi, 1894, p. 345) adds a considerable proportion of carbonate of lithia.

See also the mixture of BrEmer (Arch. mik. Anat., xlv, 1895, p. 446).

I have tried Chenzinsky's mixture as a tissue stain, without good results ; but see Rosin, Berliner klin. Wochenschr., 1898, p. 251 ; Zeit. wiss. Mik., xvi, 1899, p. 223, and xvii, 1900, p. 333.

See also Laurent (Centralb. allg. Path., xi, 1900, p. 86 ; Zeit. wiss. Mik., xvii, 1900, p. 201).

314. Mallory's Eosin and Methylen Blue (Journ. Med. Research, January, 1904).- Sections of ZENKER material (other sublimate material not so good) are stained for half to three quarters of an hour at $56^{\circ} \mathrm{C}$. in 5 per cent. aqueous solution of eosin, rinsed and flooded with solution of 1 part of methylen blue, and 1 of potassium carbonate in 100 of water, diluted with about 7 parts of water. After forty minutes they are flooded (not washed) with water, and differentiated for aboult five minutes in alcohol of 95 per cent. Absolute alcohol, xylol, balsam.

315. Other Eosin and Methylen-blue Stains.-For some very important ones see under "Blood."

316. Light Green (Lichtgrün S. F.).-An " acid" colour, soluble in alcohol, and a good plasma stain.

Benda (Verh. physiol. Ges. Berlin, December 18th, 1891, Nos. 4 u. 5) stains sections for twenty-four hours in anilin-water safranin solution, then for about half a minute in a solution of $0.5 \mathrm{grm}$. Lichtgrün or Säureviolett (Grübler) in 200 c.c. of alcohol, dehydrates and mounts in balsam. This process gives a very elegant stain, but requires very thin sections, and there is always risk of the safranin being washed out. The Lichtgrün stain unfortunately does not keep at all well.

See also Prenant, Arch. mik. Anat., vii, 1905, p. 430, and Guieysse, C.R. Soc. Biol., lxii, 1907, p. 1212.

317. Janus Green (Mrciaelis, Arch. mik. Anat., lv, 1900, p. 565).Used in solution of $1: 30,000$ for staining certain granules (pancreas, -salivary glands, etc.) in the fresh state.

318. Malachite Green (syn. Solid Green, Victoria Green, New Green, Benzoyl Green, Fast Green).-A basic colour, which has been used as a 
plasma stain for the ova of Ascaris by VAN BENEDEN and NEYT. These authors used it for glycerin preparations; it can hardly be got into balsam.

Flemming (Arch. mik. Anat., xix, 1881, p. 324) attributes to it a special affinity for nucleoli.

319. Iodine Green ("HofmanN's Grün"), see Griesbacil (Zool. Anz., No. 117, vol. v, 1882, p. 406).-Stain essentially that of methyl green, but plasma often violet through the presence of a violet impurity (MAYER, Mitth. Zool. Stat. Nexpel, xii, 1896, p. 311 ; see also earlier editions). It is now only used by botanists.

320. Thiophen Green (Thiophengrün), see KRAUsE, Intern. Monatsschr. Anat., etc., iv, 1887, Heft 2.

321. Coerulein S., a green "acid" dye, is recommended for the staining of muscle-fibrils by M. v. LenHosséK (Anat. Anz., xvi, 1899, p. 339). See also HeIdenhain, ibid., xx, 1901, p. 37, and Rawitz, ibid., xxi, 1902, p. 554 .

322. Quinoleïn Blue (Cyanin, Chinolinblau; v. RAvvıer, Traité, p. 102).-Quinolein is said by Ranvier to have the property of staining fatty matters an intense blue.

It is useful for staining Infusoria, which in dilute solution it stains during life. See the methods of Certes.

From the reactions mentioned by Ranvier it would seem that his "bleu de quinoléine" is not the preparation that usually goes under that name. See Ehrlich, in Arch. mik. Anat., xiii, 1877, p. 266.

323. Indulin and Nigrosin.-Indulin, Nigrosin, Indigen, Coupier's Blue, Fast Blue R, Fast Blue B, Blackley Blue, Guernsey Blue, Indigo substitute are the names of brands of a group of dyes, mostly " acid," related to the base violanilin. According to Berresens the name Indulin is generally given to a bluish brand, and that of Nigrosin to a blacker one.

Nigrosin, used with sublimate material, I find stains both nuclei and cytoplasm, the chromatin strongly. It will not give the stain at all with chrom-osmium material.

According to CALBERLA (Morph. Jahrb., iii, 1877, p. 627) the concentrated aqueous solution of Indulin should be diluted with 6 volumes of water. Sections will stain in the dilute solution in five to twenty minutes. He also says that it never stains nuclei ; the remaining cellcontents and intercellular substance are stained blue. This seems to me to be, roughly, correct.

324. Safranin and Nigrosin (or Indigo-Carmine) (Kossinski, Zeit. wiss. Mik., vi, 1880, p. 61).--See early editions.

325. Picro-Nigrosin, Pfitzer (Deutsch. Botan. Gesellsch., 1883, p. 44) dissolves nigrosin in a saturated solution of picric acid in water, and uses it for fixing and staining at the same time, on the slide. See also under "Connective Tissues."

326. Anilin Blue.-Under this title are comprised various " basic" derivatives of the base rosanilin. They occur under the names 
Spirit Soluble Blue (Bleu Alcool), Gentian Blue 6 B, Spirit Blue 0, Opal Blue, Bleu de Nuit, Blue Lumière, Parma Bleu, Bleu de Lyon. Some authors give the name Bleu de Nuit and Gründstichblau as synonyms of Bleu de Lyon. The Encycl. mik. Technik. says it is "Anilinblau B-6 B," with many synonyms, or designations of brands, Parma blue being "Anilinblau R or 2 R." I find it a fairly good stain, giving very good differentiations of nerve-tissue and of cartilage (as has already been pointed out by BAUMGARTEN and by JaCOBy). MaURICE and Schulgin stain in bulk with it after boraxcarmine, using a very dilute alcoholic solution. BAUMGaRTEN and JACOBY stain sections in a 0.2 per cent. alcoholic solution.

Tonkoff (Arch. mik. Anat., lvi, 1900, p. 394) adds a little tincture of iodine to the solution of the dye, or mordants the sections with iodine.

Skrobansky (Intern. Monatsschr. Anat., xxi, 1904, p. 20) uses it in water with picric acid.

327. Carmine Blue (Bleu Carmin Aqueux, from Mesiter, Lucius, and Brunig, at Höchst-a-M.).-J JANssens (La Cellule, ix, 1893, p. 9) states that this colour possesses a special affinity for the parts of cytoplasm that are undergoing cuticular differentiation. He uses it in aleoholic solution acidified.

328. Methyl Blue.--Under this title are comprised some other derivatives of the base rosanilin. They are "acid" colours. Here belong Methyl Blue, Cotton Blue, Water Blue (Wasserblau), Methyl Water-Blue, China Blue (Chinablau), Soluble Blue.

Amongst these Water Blue (Wasserblau) possesses some useful properties. According to Mitrophanow (quoted from Zeit. wiss. Mik., v, 1888, p. 513), used in concentrated aqueous solution it gives a very good double stain with safranin. It is very resistant to alcohol. Using the Wasserblau first, and then the safranin, I have had some interesting results. The Wasserblau must be used first. With chrom-osmium material, twelve to twenty-four hours in the blue, and four or five in the safranin, may not be too much. My stains have not kept well.

ManN (Methods, etc., p. 216) uses a mixture of 35 parts 1 per cent. solution of eosin, 45 of methyl blue 1 per cent., and 100 of water. He has also (Zeit. wiss. Mik., xi, 1894, p. 490) used a similar mixture for nerve-cells.

329. Anilin Blue-black. - A preparation cited under this name has been recommended by BEvan LEwis and others for nervous tissue. The dye used by them cannot now be identified. Dr. GRÜBLER writes me that the anilin blue-black of his list is the oxyazo colour blue-black B or Azosciwarz; but that dye had not been discovered when Bevan 
Lewis wrote. See also HeIDENhaIn in Zeit. wiss. Mik., xx, 1903, p. 185, and $\mathrm{xxv}, 1909, \mathrm{p} .407$.

330. Violet B (or Methyl Violet B) (S. Mayer, Sitzb. k. k. Akad. wiss. Wien, iii, Abth., February, 1882).-Used in solutions of $1 \mathrm{grm}$. of the colour to 300 grms. of 0.5 per cent. salt solution, and with fresh tissues that have not been treated with any reagent whatever, this colour gives a stain so selective of the elements of the vascular system that favourable objects, such as serous membranes, appear as if injected. The preparations do not keep well; acetate of potash is the least unsatisfactory medium for mounting them in, or a mixture of equal parts of glycerin and saturated solution of picrate of ammonia (Anat. Anz., 1892, p. 221). See also under "Plasmafibrils."

The allied dye, Crystal Violet, has been employed for staining sections, e.g. by Kromayer and others. Benda (Neurol. Centralb., xix, 1900, p. 792) stains in a mixture of 1 vol. saturated sol. of the dye in 70 per cent. alcohol, 1 vol. 1 per cent. sol. of hydrochloric acid in 70 per cent. alcohol, and 2 vols. of anilin water, the liquid being warmed until vapour is given off, then cooled and the sections dried with blotting-paper, treated one minute with 30 per cent. acetic acid, dehydrated with alcohol and cleared with xylol.

331. Kresyl Violet.-An oxyazin dye, giving metachromatic stains. Herxhermer (Arch. mik. Anat., liii, 1899, p. 519, and liv, p. 289) stains sections of skin with Kresyl-echtviolett. Nuclei blue, plasma reddish. Similarly Fick (Centralb. allg. Path., xiii, 1902, p. 987 ; Zeit. wiss. Mik., $\mathrm{xx}, 1903, \mathrm{p} .223)$, staining for three or four minutes in a concentrated aqueous solution, and differentiating in alcohol until the connective tissue has become colourless. Keratohyalin violet-red to salmon. coloured.

332. Säureviolett, see $\S 316$.

333. Benzoazurin may be made to give either a diffuse or a nuclear stain, according to Martin (see Zeit. wiss. Mik., vi, 1889, p. 193).

334. RAwITz' "Inversion" Plasma Stains. - It has been discovered by RAwITZ that by means of appropriate mordants certain basic anilins, which by the usual methods of regressive staining are pure chromatin stains, may be made to afford a pure plasma stain, thus giving an "inversion" of the usual stain. The stain, in my opinion, is a vile one. For details see fourth edition, or RAwITz (Sitzb. Gesnaturf. Freunde, Berlin, 1894, p. 174 ; Zeit. wiss. Mik., xi, 1895, p. 503 ; and his Leitfaden f. hist. Untersuchungen, Jena, 1895, p. 76).

335. Artificial Alizarin (RAwITz, Anat. Anz., xi, 10, 1895, p. 295).A double stain by means of artificial Alizarin, or Alizarin-cyanin, requiring the use of special mordants supplied by the colour manufacturers, and very complicated. See fifth edition. 
RAWITZ (Zeit. wiss. Mik., 1909, pp. 393 and 395) also recommends a solution of $1 \mathrm{grm}$. of Säure-Alizarinblau B B (or Säuregrün G) (both from Höchst), 10 grms. ammonia alum, 100 c.c. glycerin, and 100 c.c. water.

SzÜTz (ibid., xxix, 1912, p. 289) fixes n a mixture of 15 c.c. 1 per cent. platinum chloride, 15 c.c. formol, and 30 c.c. saturated solution of sublimate, makes paraffin sections, and stains them with Heidenhats's iron-hæmatoxylin. They are then treated for five to six hours with 5 per cent. solution of aluminium acetate, rinsed, and stained for five to six hours with Benda's sulphalizarinate of soda (given under "Mitochondria"), and got into balsam. A red plasma stain, affecting plasma. fibrils. For intra-vitam stains with alizarin see $\$ 208$ (FISCHEL), and Nilsson, Zool. Anz., xxxv, 1909, p. 196.

336. For BendA's Alizarin Stains, see under "Centrosomes," "Mitochondria," and "Neuroglia." 


\section{CHAPTER XVI.}

\section{METHYLEN BLUE.}

33\%. Methylen Blue is a "basic" dye, being the chloride or the zinc chloride double salt of tetramethylthionin. It appears that some persons have confounded it with the " acid" dye methyl blue, to which it has not, histologically, any resemblance.

Commercial methylen blue sometimes contains as an impurity a small quantity of a reddish dye, which used to be taken to be methylen red. This impurity is present from the beginning in many brands of methylen blue, is frequently developed in solutions of the dye that have been long kept (so-called " ripened " solutions), and is still more frequently found in kept alkaline solutions. According to Nochт (Centralb. Bakteriol., xxv, 1899, pp. 764-769; Zeit. wiss. Mik., xvi, 1899, p. 225) it is not methylen red, nor methylen violet either, but a new colour, for which NocHt proposes the name "Roth aus Methylenblau."

According to Mich жLIs (Centralb. Bakteriol., xxix, 1901, p. 763, and xxx, 1901, p. 626 ; Zeit. wiss. Mik., xviii, 1902, p. 305, and xix, 1902, p. 68) confirmed later by Nocht, Reuter, and Giemsa, this dye is Methylenazur, an oxidation-product of methylen blue, already described by BERNTHSEN in 1885. It is an energetic dye, of markedly metachromatic action, and to it are due the metachromatic effects of methylen blue solutions (methylen blue itself is not metachromatic).

The presence of this dye as an impurity in methylen blue is not always an undesirable factor; on the contrary, it sometimes affords differentiations of elements of tissues or of cells that cannot be produced by any other means. Methylen blue that contains it is known as polychrome methylen blue, and is employed for staining certain cell-granules. UNNA (Zeit. wiss. Mik., viii, 1892, p. 483) makes this as follows: A solution of 1 part of methylen blue and 1 of carbonate of potash in 20 of alcohol and 100 of water is evaporated down to 100 parts. (It may be used at once, or after diluting with an equal volume of anilin water, for sections, which after staining may be differentiated with glycol, creosol, or Unna's glycerin-ether mixture-all of which, as well as the polychrome methylen blue, can be obtained from Grübler \& Hollborn.) МrсH жits 
(op. cit.) makes it as follows: 2 gr. of medicinal methylen blue are dissolved in 200 c.c. of water, and 10 c.c. of $\frac{1}{10}$ normal solution of caustic soda added. Boil for a quarter of an hour; after cooling add 10 c.c. of $\frac{1}{10}$ normal sulphuric acid, and filter.

Methylenazur is isolated from methylen blue by the prolonged action of an alkali or of silver oxide. It seems also that it is formed in certain mixtures of methylen blue with eosin (ROMANOwSKr, LAVERAN, GIEMSA and others), by means of the eosin, which in these mixtures acts chemically, and can be replaced by resorcin, hydroquinon, and the like. It is best procured from Grübler \& Hollborn, who supply it pure as "Azur I," and mixed with an equal quantity of methylen blue as "Azur II." See further as to this dye under "Stains for Blood." See also an important paper by Prowazek (Zeit. wiss. Mikr. Tech., 31).

There are several sorts of methylen blue sold, the most important being-"methylen blue, according to EHRLICH"; "methylen blue, according to $\mathrm{KocH}$ "; "methylen blue $\mathrm{BX}$, according to S. MAYER" ; "Methylenblau, medic. pur."

The colour to be employed for intra-vitam nerve staining should be as pure as possible. APÁtHy (Zeit. wiss. Mik., ix, 1893, p. 466) writes that the best-in fact, the only one that will give exactly the results described by him-is that of E. Merck, of Darmstadt, described as "medicinisches Methylenblau." DoGIEL (Encycl. mik. Technik., 1st edition, p. 811) has had his best results with "Methylenblau n. Ehrlich," or "BX," obtained from Grübler \& Hollborn.

338. The Uses of Methylen Blue.-As a histological reagent it is used for sections of hardened central nervous tissue, in which it gives a specific stain of medullated nerves. It gives more or less specific stains of the basophilous granulations of "Mastzellen" and plasma-cells, and the granules of NIssu in nerve-cells, also mucin. It is much used-in the form of mixtures affording methylen azurin the study of blood, blood parasites, and similar objects. For all of these see the respective sections in Part II. Further, it stains a large number of tissues intra vitam, with little or no interference with their vital functions. And last, not least, it can be made to furnish stains of nerve tissue, intercellular cement substances, lymph spaces; and the like, that are essentially identical with those furnished by a successful impregnation with gold or silver, and are obtained with greater ease and certainty; with this difference, however, that gold stains a larger number of the nervous elements that are present in a preparation, sometimes the totality of them; whilst methylen blue stains only a selection of them, so bringing them more prominently 
before the eye, and allowing them to be traced for greater distances. These two uses form the subject of this chapter.

339. Staining in toto during Life.-Small and permeable aquatic organisms may be stained during life by adding to the water in which they are confined enough methylen blue to give it a very light tint. After a time they will be found to be partially stainedthat is, it will be found that certain tissues have taken up the colour, others remaining colourless. If now you put back the animals into the tinted water and wait, you will find after a further lapse of time that further groups of tissues have become stained. Thus it was found by Ehruich (Biol. Centralb., vi, 1886, p. 214 ; Abh. k. Akad. Wiss. Berlin, February 25th, 1885) that on injection of the colour into living animals axis-cylinders of sensory nerves stain, whilst motor nerves remain colourless. [The motor nerves, however, will also stain, though later than the sensory nerves.] It might be supposed that by continuing the staining for a sufficient time, a point would be arrived at at which all the tissues would be found to be stained. This, however, is not the case. It is always found that the stained tissues only keep the colour that they have taken up for a short time after they have attained the maximum degree of coloration of which they are susceptible, and then begin to discharge the colour even more quickly than they took it up. According to EHRLich this decoloration is explained as follows: methylen blue, on contact with reducing agents in alkaline solution, can be reduced to a colourless body, its "leucobase." Now living or recently dead tissue elements are, or may be, both alkaline and very greedy of oxygen, and thus act on the dye as reducing agents. The leucobase thus formed is easily reoxidised into methylen blue by oxidising substances, or acids, or even by the mere contact of air-which latter property is taken advantage of in practice.

It follows that a total stain of all the tissues of a living intact organism can hardly be obtained under these conditions, but that a specific stain of one group or another of elements may be obtained in one of two ways. If the tissue to be studied be one that stains earlier than the others, it may be studied during life at the period at which it alone has attained the desired intensity of coloration. If it be one that stains later than the others, it may be studied at the period at which the earlier stained elements have already passed their point of maximum coloration and have become sufficiently decoloured, the later stained ones being at a point of desired intensity. Or the observer may fix the stain in either of these stages and preserve it for leisurely study by means of one of the processes given $\S 343$. 
The proper strength of the very dilute solutions to be employed for the staining of living organisms must be made out by experiment for each object. I think the tint is practically a sufficient guide, but it may be stated that when in doubt a strength of $1: 100,000$ may be taken, and increased or diminished as occasion may seem to require. ZoJa (Rendic. R. Ist. Lombardo, xxv, 1892 ; Zeit. wiss. Mik., ix, 1892, p. 208) finds that for Hydra the right strength is from $1: 20,000$ to $1: 10,000$.

The stain is capricious. It is not possible to predict without trial which tissues will stain first in any organism. The stain penetrates very badly, which is no doubt one cause of its capriciousness. Gland cells generally stain early ; then, in no definable order, other epithelium cells, fat cells, plasma cells, "Mastzellen," blood and lymph corpuscles, elastic fibres, smooth muscle, striated muscle. There are other elements that stain in the living state, but not when the staining is performed by simple immersion of intact animals in a dilute staining solution in the manner we are considering. Chief amongst these are nerve-fibres and ganglion-cells, which remain unstained in the intact organism. To get these stained, it is necessary to isolate them sufficiently, as explained in the following sections.

340. Staining Nervous Tissue* during Life.-It was made out by EHRLICH (op. cit., last §) that by injecting a solution of methylen blue into the vessels or tissues of living animals and shortly afterwards cutting out and examining small pieces of their tissues, these will be found to be intensely stained in some of their elements (chiefly nervous). If the tissues are mounted under a cover-glass, the stain will fade in a short time; but if the cover-glass be removed, so that oxygen can have access to the tissues, the stain will be restored, as explained last §. The chief elements stained in this way are peripheral nerves, and amongst these more especially axiscylinders of sensory nerves.

Ehrlich held that the stain so obtained is a product of a vital reaction of the tissues, and that it cannot be obtained with dead material. Dogiel, however (Arch. mik. Anat., xxxv, 1890, pp. 305 et seq.), found that muscle nerves of limbs of the frog could be stained as much as from three to eight days after the limbs had been removed from the animal. He concludes, indeed, that the reaction shows that the nerves were still living at that time. But it seems more natural to conclude with ApÁtHy (Zeit. wiss. Mik., ix, 1892, pp. 15 et seq.) that nerve-tissue can be stained after life has ceased. APÁtry 
has directly experimented on this point, and sums up the necessary conditions as follows: The tissue need not be living, but must be fresh; nothing must have been extracted from it chemically, and its natural state must not have been essentially changed by physical means. For example, the tissue must not have been treated with even dilute glycerin, nor with alcohol, though a treatment for a short time with physiological salt solution is not very hurtful ; it must not have been coagulated by heat. Michailow (ibid., xxvii, 1910, p. 7) prefers tissues that have lain from one and a half to two hours after the death of the subject in Ringer's salt solution.

As above explained, the primary stain obtained by injecting methylen blue, or immersing tissues in it, cnly lasts a very short time. In order to get it to last long enough for study, it must be re-blued by oxidation (see last $\S$ ). It is therefore the usual practice to dissect out the tissues to be examined, and leave them for some time exposed to the air. This is done in order that they may take up from the air the necessary oxygen. Another consideration that justifies the practice is that by exposure to air the preparations take up a trace of ammonia, and APÁTHY has experimentally established that this is an important factor in the sharpness of the stain. EHRLICH also (op. cit.) holds that an alkaline reaction of the tissues is a necessary condition to the stain. APÁtHy further holds that the stain is a regressive one, easily washed out by the surrounding liquid; and in order to prevent this washing-out being excessively rapid, it is desirable to have it go on in presence of as little liquid as possible.

341. The Modes of Staining. - The practice of the earlier workers at this subject was (following EHRLICH) to inject methylen blue into the vascular system or body-cavity of a living animal, wait a sufficient time, then remove the organ for further preparation and study. And there appears to have been a belief with some workers that it was essential that the stain should have been brought about by injection of the colouring matter into the entire animal. It is now known that the reaction can often be equally well obtained by removing an organ and subjecting it to a bath of the colouring matter in the usual way. But in some cases it seems that injection is preferable, if not necessary.

342. The Solutions employed.-The solutions used for injection are generally made in salt solution (physiological, or a little weaker); those for staining by immersion, either in salt solution or other " indifferent" liquid, or in pure water. The earlier workers generally took concentrated solutions. Thus Arnstein (Anat. Anz., 1887, 
p. 125) injected 1 c.c. of saturated (i.e. about 4 per cent.) solution into the vena cutanea magna of frogs, and removed the organ to be investigated after the lapse of an hour. BiEdermann (Sitzb. Akad. Wiss. Wien, Math. Nat. Cl., 1888, p. 8) injected 0.5 to 1 c.c. of a nearly saturated solution in 0.6 per cent. salt solution into the thorax of crayfishes, and left the animals for from two to four hours before killing them. S. MAYER (Zeit. wiss. Mik., vi, 1889, p. 423) took a strength of $1: 300$ or 400 of 0.5 per cent. salt solution. The solutions of RETzius are of the same strength. But the tendency of more recent practice is decidedly towards the employment of weaker solutions. APÁTHy (ibid., ix, 1892, pp. 25, 26 et seq.) finds that it is not only superfluous, but positively disadvantageous, to take solutions stronger than 1 : 1000. Dogiel (Encycl. Mik. Technik., 1st ed., p. 815) recommends $\frac{1}{8}$ to $\frac{1}{4}$ per cent., or at most $\frac{1}{2}$ per cent. For warm-blooded animals the solution should be warmed to $36^{\circ}$ or $37^{\circ} \mathrm{C}$, and before sending in the injection the blood-vessels should be well washed out with similarly warmed salt solution. The injected organs may be removed after twenty to thirty minutes. They should be placed on a thin layer of spun glass moistened with weak ( $\frac{1}{8}$ to $\frac{1}{15}$ per cent.) methylen blue, or simply spread out on a slide, and the whole placed in a Petri dish with a layer of the methylen blue on the bottom. The dish is best placed in a stove at $36^{\circ} \mathrm{C}$, and after fifteen to thirty minutes (if the pieces are thin) or one hour to one and a half hours (if they are thick) specimens may be removed for examination or preservation; or, without using the stove, specimens may be removed ten to twenty minutes after injection, placed on a slide, and moistened with weak methylen blue or salt solution, and brought under the microscope. Then as soon as the stain is sufficiently brought out (forty to sixty minutes) they may be fixed ( $\$ 343)$.

For staining by immersion the solutions should, if anything, be still weaker. DoGIEL (Arch. mik. Anat., xxxv, 1890, p. 305) places objects in a few drops of aqueous or vitreous humour, to which are added 2 or 3 drops of a $\frac{1}{16}$ to $\frac{1}{15}$ per cent. solution of methylen blue in physiological ( 0.75 per cent.) salt solution, and exposes them therein to the air. In thin pieces of tissues the stain begins to take effect in five or ten minutes, and attains its maximum in from fifteen to twenty minutes. For thicker specimens-retina, for instance - several hours may be necessary. The reaction is quickened by putting the preparations into a stove kept at $30^{\circ}$ to $35^{\circ} \mathrm{C}$. RougET (Compt. Rend., 1893, p. 802) employed a 0.05 per cent. solution in 0.6 per cent. salt solution (for muscles of Batrachia). ALLEN 
(Quart. Journ. Micr. Sci., 1894, pp. 461, 483) takes for embryos of the lobster a solution of 0.1 per cent. in 0.75 per cent. salt solution, and dilutes it with 15 to 20 volumes of sea-water. SeIdenmann (Zeit. wiss. Mik., xvii, 1900, p. 239) takes for the choroid a solution of 0.02 per cent. in 0.5 per cent. salt solution. Lavdowsky (ibid., xii, 1895, p. 177) takes $\frac{1}{10}$ to $\frac{1}{4}$ per cent. in white of egg, or serum. Similarly Young (ibid., xv, 1898, p. 253). MichaIlow (ibid., xxvii, 1910, p. 10) takes $\frac{1}{8}$ to $\frac{1}{32}$ per cent. in Ringer's salt solution (for nerves of Mammals).

Ара́тнy (Zeit. wiss. Mik., ix, 1892, p. 15 ; see also his Mikrotechnik, p. 172) proceeds as follows for Hirudinea and other invertebrates. A portion of the ventral cord is exposed, or dissected out. If it be desired to stain as many ganglion cells as possible, as well as fibres, the lateral nerves, as well as the connectives, should be cut through near a ganglion. The preparation is then treated with the stain. This is, for the demonstration chiefly of fibres in Hirudo and Pontobdella, either a $1: 1000$ solution in 0.5 to 0.75 per cent. salt solution, allowed to act for ten minutes; or a $1: 10,000$ solution allowed to act for an hour to an hour and a half; or a 1:100,000 solution allowed to act for three hours (Lumbricus requires twice these times; Astacus and Unio require three times; medullated nerves of vertebrates four times). For the demonstration of ganglion cells the stain is allowed to act three or four times as long.

The preparations from the $1: 1000$ solution are then washed in salt solution for an hour; those from the $1: 10,000$ solution for a quarter of an hour; those from the 1: 100,000 solution need not be washed at all. They are then treated with one of the ammoniacal fixing and differentiating liquids described in $\S 343$. This is done by pouring the liquid over them, and leaving them in it without moving them about in it for at least an hour, and by preference in the dark. The further treatment is as described in $\S 343$.

The object of the ammonia in these liquids is to differentiate the stain-to produce an artificial " secondary differentiation." It acts by washing out the absorbed colour from certain elements, others resisting longer.

See also, for Hirudinea, SÁnchez, in Trab. Lab. Invest. Biol. Univ. Madrid, vii, 1909, fasc. 1-4, or Zeit. wiss. Mik., xxvii, 1910, p. 393 (injection of solutions of $0 \cdot 2,0 \cdot 1$, or 0.05 per cent., with further treatment as Apáthy or Bethe).

343. Fixation of the Stain.-The stain obtained by any of these methods may be fixed, and more or less permanent preparations be made by one or other of the following methods : 
Arnstein (Anat. Anz., 1887, p. 551) puts the tissue for half an hour into saturated aqueous solution of picrate of ammonia.

S. Mayer (Zeit. wiss. Mik., vi, 1889, p. 422) preferred a mixture of equal parts of glycerin and saturated picrate of ammonia solution, which served to fix the colour and mount the preparations in. This was also in principle the method of ReTziUs (Intern. Monatsschr. Anat. Phys., vii, 1890, p. 328).

Dogres (Encycl. Mik. Techn., ii, p. 105) puts for two to twentyfour hours into saturated aqueous picrate of ammonia, and then into equal parts of glycerin and the picrate solution. (Thin membranes, and the like, may be fixed with 1 or 2 per cent. of 2 per cent. osmic acid solution added to the picrate solution and stained with picro-carmine before putting into the glycerin mixture.)

Other workers have employed saturated solution of iodine in iodide of potassium (so ARnstein) or picro-carmine (so FeIst, Arch. Anat. Entwickel., 1890, p. 116 ; cf. Zeit. wiss. Mik., vii, 1890, p. 231), the latter having the advantage of preserving the true blue of the stain if it be not allowed to act too long, and the preparation be mounted in pure glycerin.

Picric acid has been used by LAvDowsky, but this after careful study is rejected by DoGIEL.

АРÁtHy (op. cit., § 342 ) brings preparations either into a concentrated aqueous solution of picrate of ammonia free from picric acid, and containing 5 drops of concentrated ammonia for every 100 c.c. ; or, which is generally preferable, into a 1 to 2 per cent. freshly prepared solution of neutral carbonate of ammonia saturated with picrate. They remain in either of these solutions, preferably in the dark, for at least an hour. They are then brought into a small quantity of saturated solution of picrate of ammonia in 50 per cent. glycerin, where they remain until thoroughly saturated. They are then removed into a saturated solution of the picrate in a mixture of 2 parts 50 per cent. glycerin, 1 part cold saturated sugar solution, and 1 part similarly prepared gum-arabic solution. When thoroughly penetrated with this they are removed and mounted in the following gum-syrup medium (loc. cit., p. 37):

Picked gum-arabic

Cane-sugar (not candied)

Distilled water

Dissolve over a water-bath and add $0.05 \mathrm{grm}$. thymol. (This mounting medium sets quickly and as hard as balsam, so that no cementing of the mounts is necessary. Farrants' medium [with omission of the arsenious acid] will also do. In neither case should 
either ammonium picrate or methylen blue be added to the medium.) Preparations that have been fully differentiated ( $\$ 342)$ do not keep more than a few weeks; whilst those in which the differentiation has not been carried to the point of thorough tinctorial isolation of the neuro-fibrils have kept for five or six years (АрÁthy, Mitth. Zool. Stat. Neapel, xii, 1897, p. 712).

Pleschko (Anat. Anz., xiii, 1897, p. 16) fixes with picrate, and then puts into 10 per cent. formol for a few days.

The methods described next $\S$ are also available for material not destined to be sectioned.

344. Methods for Sections.-The preceding methods do not give preparations that will resist the operations necessary for imbedding in paraffin or mounting in balsam. A strong solution of platinum chloride is said to do this (see FeIsT, Arch. Anat. Entw., 1890, p. 116), but the preparations are not very satisfactory.

For the earlier method of PARKer (Zool. Anzeig., 1892, p. 375) with methylal see early editions. Later (Mitth. Zool. Stat. Neapel, xii, 1895, p. 4) he fixes the stain by dehydrating the objects in successive alcohols of $30,50,70,95$, and 100 per cent. strength, each containing 8 per cent. of corrosive sublimate, then brings them into a mixture of the last with an equal volume of xylol, and lastly into pure xylol.

For the earlier method of BETHE (Arch. mik. Anat., xliv, 1894, p. 585), see last edition.

Beтнe's later method (Anat. Anz., xii, 1896, p. 438) is as follows : After staining, pieces of tissue of 2 to $3 \mathrm{~mm}$. thickness are treated for ten to fifteen minutes with a concentrated aqueous solution of picrate of ammonia and then brought into a solution of $1 \mathrm{grm}$. of molybdate of ammonium, either in 20 of water, or in 10 of water and 10 of 0.5 per cent. osmic acid or 2 per cent. chromic acid; or into a solution of phosphomolybdate of sodium in the same proportions, each of these solutions having added to it 1 drop of hydrochloric acid, and if desired 1 grm. of peroxide of hydrogen. They remain in one of these solutions for three quarters to one hour (or from four hours to twelve in the osmic acid one), and are then passed through water, alcohol, xylol, balsam, or paraffin. (The objects that have been treated with one of the solutions of the sodium salt are not thoroughly resistant to alcohol, so that for them it is well to cool the alcohol to under $15^{\circ}$ C.) Sections may be after-stained with alum carmine, or " neutral " tar colours.

Slight modifications of this method are given by DoGIEL (Arch. 
mik. Anat., xlix, 1897, p. 772 ; liii, 1898, p. 237 ; Zeit. wiss. Zool., lxvi, 1899, p. 361 ; and Encycl. mik. Technik, 1903, p. 825, and 1910 , p. 108.) He omits the peroxide, the hydrochloric acid, and the cooling. Bethe (Zeit. wiss. Mik., xvii, 1900, p. 21) does not approve of these modifications.

Further modifications of the molybdenum method have been published by LeONTOwiтsch (Intern. Monatsschr. Anat., xviii, 1901, p. 142).

Michailow (Zeit. wiss. Mik., xxvii, 1910, p. 19) adds to 8 per cent. solution of molybdate 0.5 per cent. of formalin, leaves the objects in a large quantity of it (filtered) for twenty-four hours at $37^{\circ} \mathrm{C}$., washes with warm water, and passes through alcohol and xylol into xylol-damar (not balsam).

See also Schмidt (Arch. Ges. Phys., ciii, 1906, p. 522).

HARris (Philadelphia Medical Journ., May 14th, 1898), after staining, rinses with water, and brings into a saturated solution of either ferrocyanide or ferricyanide of potassium which has been cooled to within a few degrees of zero (a trace of osmic acid may be added to prevent maceration). They remain therein for three to twenty-four hours, and are then washed in distilled water for an hour, and are dehydrated in absolute alcohol kept at a low temperature, cleared in xylol or cedar oil, and imbedded in paraffin.

345. Impregnation of Epithelia, Lymph-spaces, etc. (DoGIEL, Arch. mik. Anat., xxxiii, 1889, pp. 440 et seq.).-Suitable pieces of tissue (thin membrane by preference) are brought fresh into a 4 per cent. solution of methylen blue in physiological salt solution (in the Encycl. mik. Technik, 1903, p. 827, Dogiel gives the strength of the methylen blue as $\frac{1}{2}$ to 1 per cent.). After a few minutes therein they are brought into saturated solution of picrate of ammonia, soaked therein for half an hour or more, then washed in fresh picrate of ammonia solution, and examined in dilute glycerin.

If it be wished only to demonstrate the outlines of endothelium cells, the bath in the stain should be a short one, not longer than ten minutes in general ; whilst if it be desired to obtain an impregna. tion of ground-substance of tissue, so as to have a negative image of juice canals or other spaces, the staining should be prolonged to fifteen or thirty minutes.

If it be desired to preserve the preparations permanently, they had better be mounted in glycerin saturated with picrate of ammonia, or (Encycl., 1910, ii, p. 110) fixed with ammonium molybdate and a trace of osmium. 
The effect is practically identical (except as regards the colour) with that of a negative impregnation with silver nitrate.

S. MAYer (Zeit. wiss. Mik., vi, 1889, p. 422) stains tissues for about ten minutes in a $1: 300$ or 400 solution of methylen blue in 0.5 per cent. salt solution, rinses in salt solution, and puts up in the glycerin-picrate of ammonia mixture given $\S 343$. The images are generally positive after injection of the colour into the vascular system; negative after immersion of the tissues.

TIMofeJew (Anat. Anz., xxxv, 1909, p. 296) impregnates for fifteen to twenty minutes in a solution of 1 : 300 or 400 strength, fixes with a very weak solution of ammonium picrate in salt solution, and puts up in a mixture of 50 c.c. glycerin, 50 c.c. water, and 35 c.c. saturated solution of the picrate : or fixes with ammonium molybdate of 8 per cent. and mounts in balsam.

346. Toluidin Blue or Thionin as succedanea of methylen blue.Harris (Philidelphia Med. Journ., May 14th, 1898) has found that there is no reaction of methylen blue that cannot be equally well obtained with toluidin blue or thionin. For staining pieces of tissue he takes:

Toluidin blue, $0 \cdot 1$ per cent. sol. in physiological salt solution . . . . . 2 parts.

Ammonium chloride $0 \cdot 25$ per cent. in water . 1 part.

Egg albumen

For injections he uses 1 part of the dye to 1000 of physiological salt solution.

Any of the methylen blue fixing methods may be employed and the whole technique is the same.

L. Martinotiti (Zeit. wiss. Mik., xxvii, 1910, p. 24) recommends a polychrome tolnidin blue, made by adding 0.5 per cent. of lithium carbonate to a 1 per cent. solution of the dye and keeping till a purple-red tone appears. Or, a stock solution made of $1 \mathrm{grm}$. toluidin blue, $0.5 \mathrm{grm}$. lithium carbonate, glycerin 20 grms., alcohol 5 grms., and water 75 . 


\section{CHAPTER XVII.}

\section{METALLIC STAINS (IMPREGNATION METHODS).}

34\%. The Characters of Impregnation Stains.-By impregnation is understood a mode of coloration in which a colouring matter is deposited in tissues in the form of a precipitate - the impregnated elements becoming in consequence opaque. By staining, on the other hand, is understood a mode of coloration in which the colouring matter is retained by the tissues as if in a state of solution, showing no visible solid particles under the microscope, the stained elements remaining in consequence transparent. But it is not right to draw a hard and fast line between the two kinds of coloration. Some of the metallic salts treated of in this chapter give, besides an impregnation, in some cases a true stain. And some of the dyes that have been treated of in the preceding chapters give, besides a stain, a true impregnation. Methylen blue, for instance, will give in one and the I same preparation an impregnation and a stain ; and in most chloride preparations the coloration is in places of the nature of a finely divided solid deposit, in others a perfectly transparent stain.

348. Negative and Positive Impregnations.-In a negative impregnation intercellular substances alone are coloured, the cells themselves remaining colourless or very lightly tinted. In a positive impregnation the cells are stained and the intercellular spaces are unstained. (A directly contrary statement, made in a recent Lehrbuch, is erroneous.)

Negative impregnation is generally held to be primary because brought about by the direct reduction of a metal in the intercellular spaces; positive impregnation to be secondary (in the case of silver nitrate at least) because it is brought about by the solution in the liquids of the tissues of the metallic deposit formed by a primary impregnation, and the consequent staining of the cells by the new solution of metallic salt thus formed. These secondary impregnations take place when the reduction of the metal in the primary impregnation is not sufficiently energetic (see on these points His, Schweizer Zeit. Heilk., ii, Heft 1, p. 1; GIERKe, Zeit. wiss. Mik., i, p. 393 ; RANvier, Traité, p. 107).

As to the nature of the black or brown deposit or stain formed in the intercellular spaces in cases of primary impregnation see ScHWaLbE, Arch. mik. Anat., vi, 1870, p. 5 ; GIERKE's Färberei zu mikroskopischen Zwecken, in vols. i and ii of Zeit.wiss. Mik.; JosePI, Sitzb. Akad. Wiss. 
Berlin, 1888 ; Zeit. wiss. Mik., xi, 1, 1894, p. 42 et seq. It evidently cannot consist of metallic silver, as it is soluble in hyposulphite of soda. See also Macallum, Proc. Roy. Soc., Ixxvi, 1905, p. 217, and Achard and Reynaud, C.R. Soc. Biol., lxi, 1906, p. 43.

349. Action of Light on Solutions of Metallic Salts.-Stock solutions of metallic salts are generally kept in the dark, or at least in coloured bottles, under the belief that exposure to light reduces them. It has been pointed out in $\S 35$ that in the case of osmic acid, not light, but dust is the reducing agent, and that solutions may be exposed to light with impunity if dust be absolutely denied access to them. I have now good evidence to the effect that the same is the case with other metallic solutions; and the point is raised whether such solutions are not positively improved for impregnation purposes by exposure to light! Dr. Lindsay JoHnson writes me as follows :

" One may (I find by experiment) state as a rule without exception that all the solutions of the chlorides and nitrates of the metals will keep indefinitely in clean white stoppered bottles in the sunlight; and as far as osmium, uranium, gold and silver, and platinum are concerned, actually improve or ripen by a good sunning. All photographers tell me their papers salt more evenly by old wellsunned silver nitrate than by a fresh solution kept in the dark; and I go so far as to say that this is one of the reasons why gold stains are so unsatisfactory."

Apáthy (Mitt. Zool. Stat. Neapel, xii, 1897, p. 722) leaves his gold solutions exposed to light, so long as there are no tissues in them.

350. State of the Tissues to be Impregnated.--The majority of stains given by dyes are only obtained with tissues that have been changed in their composition by the action of fixing and preservative reagents. With metallic impregnations the case is different; perfectly fresh tissues - that is, such as are either living, or at all events have not been treated by any reagent whatever-will also impregnate with the greatest ease and precision. Indeed, some impregnations will not succeed at all with tissues that are not fresh in the sense above explained.

\section{Silver.}

351. Silver Nitrate: Generalities.-The principles of its employment are given by RANviER (Traité, p. 105) as follows :

Silver nitrate may be employed either in solution or in the solid state. The latter method is useful for the study of the cornea and of fibrous tissues, but is not suitable for epithelia. For the cornea, 
for instance, proceed as follows: The eye having been removed, a piece of silver nitrate is quickly rubbed over the anterior surface of the cornea, which is then detached and placed in distilled water; it is then brushed with a camel's hair brush in order to remove the epithelium. The cornea is then exposed to the action of light. It will be found that the nitrate has traversed the epithelium and soaked into the fibrous tissue, on the surface of which it is reduced by the light. The cells of the tissues will be found unstained.

It is generally employed in solution, in the following manner: In the case of a membrane, such as the epiploön, the membrane must be stretched like a drum-head over a porcelain dish, ${ }^{*}$ and washed first with distilled water, and then washed with a solution of silver nitrate. In order to obtain a powerful stain it is necessary that this part of the operation be performed in direct sunlight, or at least in a very brilliant light. As soon as the tissue has begun to turn of a blackish grey the membrane is removed, washed in distilled water, and mounted on a slide in some suitable examination medium.

If the membrane were left in the water the cells would become detached, and would not be found in the finished preparation.

If the membrane had not been stretched as directed the silver would be precipitated not only in the intercellular spaces, but in all the small folds of the surface.

If the membrane had not been washed with distilled water before impregnation there would have been formed a deposit of silver on every spot on which a portion of an albuminate was present, and these deposits might easily be mistaken for a normal structure of the tissue. It is thus that impurities in the specimen have been described as stomata of the tissue.

If the solution be taken too weak-for instance, $1: 500$ or $1: 1000$, or if the light be not brilliant-a general instead of an interstitial stain will result; nuclei will be most stained, then protoplasm, and the intercellular substance will contain but very little silver. In general in a good "impregnation" the contents of the cells, and especially nuclei, are quite invisible.

The tissues should be constantly agitated in the silver-bath in

* The Hoggans Histological Rings will be found much more convenient. They are vulcanite rings made in pairs, in which one ring just fits into the other, so as to clip and stretch pieces of membrane between them. They will be found described and figured in Journ. Roy. Mic. Soc., ii, 1879, p. 357 , and in RoBIN's Journ. de l'Anat., 1879, p. 54. They may be obtained of Burge \& Warren, 42, Kirby Street, Hatton Garden, London, E.C. 
order to avoid the formation on their surfaces of deposits of chlorides and albuminates of silver.

These impregnations only succeed with fresh tissues.

352. Silver Nitrate: the Solutions to be employed (RANVIER).The solutions generally employed by RANviER vary in strength from $1: 300$ to $1: 500$. Thus $1: 300$ is used for the epiploön, pulmonary endothelium, cartilage, tendon; whilst a strength of $1: 500$ is employed for the phrenic centre, and the epithelium of the intestine. For the endothelium of blood-vessels (by injection) solutions of $1: 500$ to $1: 800$ are taken.

M. Duval (Précis, p. 229) takes solutions of 1, 2, or at most 3 per cent.

v. Recklinghausen used, for the cornea, a strength of from $1: 400$ to $1: 500$ (Die Lymphgefässe, etc., Berlin, 1862, p. 5).

Robinski (Arch. de Physiol., 1869, p. 451) used solutions varying between $0 \cdot 1$ and $0 \cdot 2$ per cent., which he allowed to act for thirty seconds.

Rouget (Arch. de Physiol., 1873, p. 603) employed solutions as weak as $1: 750$, or even $1: 1000$, exposing the tissues to their action several times over, and washing them with water after each bath.

The Hertwigs take, for marine animals, a 1 per cent. solution (Jen. Zeit. Naturk., xvi, pp. 313 and 324).

The Hoggans (Journ. of Anat. and Physiol., xv, 1881, p. 477) take for lymphatics a 1 per cent. solution.

Tourneux and Herrmann (RoBin's Journal de l'Anat., 1876, p. 200) took for the epithelia of Invertebrates $3: 1000$, and in some cases weaker solutions,-for one hour, washing out with alcohol of 90 per cent.

Hoyer (Arch. mik. Anat., 1876, p. 649) takes a solution of nitrate of silver, and adds ammonia to it until the precipitate that is formed just redissolves, then dilutes the solution until it contains from 0.75 to 0.50 per cent. of the salt. This ammonio-nitrate solution has the advantage of impregnating absolutely nothing but endothelium or epithelium ; connective tissue is not affected by it.

RANVIER's injection-mass for impregnating endothelium is given under "Injection."

Dekhuyzen (Anat. Anz., iv, 1889,.No. 25, p. 789) has applied to terrestrial animals the method of HARMER for marine animals (§356). For details see previous editions.

Regaud (Journ. Anat. et Phys., xxx, 1894, p. 719) recommends for the study of lymphatics a process devised by RENAUT, for the details of which see also previous editions. 
353. Other Salts of Silver.-AlfERow (Arch. Phys., i, 1874, p. 694) employs the picrate, lactate, acetate, and citrate, in solution of $1: 800$, and adds a small quantity of the acid of the salt taken (10 to 15 drops of a concentrated solution of the acid to 800 c.c. of the solution of the salt). This decomposes the precipitates formed by the action of the silver salt on the chlorides, carbonates, and other substances existing in the tissues.

Regaud and Dubreurl (C.R. Ass. Anat., 5 Sess. 1903, p. 122) take a fresh solution of protargol or a mixture of equal parts of 1 per cent. protargol and 1 per cent. osmic acid, thus avoiding precipitates.

354. Silver Nitrate: Reduction.-Reduction may be effected in media other than distilled water.

v. ReckuInghausen washed his preparations in salt solution before exposing them to the light in distilled water (Arch. path. Anat., xix, p. 451). Physiological salt solution (0.75 per cent.) is commonly used for these washings.

Müller (Arch.f. path. Anat., xxxi, p. 110), after impregnation by immersion for two or three minutes in a 1 per cent. solution of nitrate of silver in the dark, adds to the solution a small quantity of 1 per cent. solution of iodide of silver (dissolved by the aid of a little iodide of potassium). After being agitated in this mixture the preparations are washed with distilled water, and exposed to the light for two days in a 1 per cent. solution of nitrate of silver (see also GIERKE, in Zeit.wiss. Mik., i, 1884, p. 396).

Rouget (Arch. de Physiol., 1873, p. 603) reduces in glycerin; SzüTz (Zeit. wiss. Mik., xxix, 1912, p. 291) in glycerin with $\frac{1}{10}$ of formol.

Sattler (Arch. mik. Anat., xxi, p. 672) exposes to the light for a few minutes in water acidulated with acetic or formic acid. THANHOFFER (Das Mikroskop, 1880) employs a 2 per cent. solution of acetic acid.

KRAUSS brings his preparations, after washing, into a light red solution of permanganate of potash. Reduction takes place very quickly, even in the dark.

OPPITz puts for two or three minutes into a 0.25 or 0.50 per cent. solution of chloride of tin.

Jакiмovitch (Journ. de l'Anat., xxiii, 1888, p. 142) brings nerve preparations, as soon as they have become of a dark brown colour, into a mixture of formic acid 1 part, amyl alcohol 1 part, and water 100 parts, and exposes to the light for five to seven days, the mixture being renewed from time to time.

DEкHUYzeN (op. cit., last §) reduces in oil of cloves, after dehydration.

355. Fixation.-Legros (Journ. de l'Anat., 1868, p. 275) washes his preparations, after reduction, in hyposulphite of soda, to prevent after- 
blackening. According to Duval (Précis, p. 230) they should be washed for a few seconds only in 2 per cent. solution and then in distilled water.

Gerota (Arch. Anat. Phys., Phys. Abth., 1897, p. 428) reduces in a hydroquinone developing solution, followed by fixation in hyposulphite of soda, just as in photography.

356. Impregnation of Marine Animals.-On account of the chlorides that bathe the tissues of marine animals, tilese cannot be treated directly with nitrate of silver.

Hertwig (Jen. Zeit., xiv, 1880, p. 322) recommends fixing them with a weak solution of osmic acid, then washing with distilled water until the wash-water gives no more than an insignificant precipitate with silver nitrate, and then treating for six minutes with 1 per cent. solution of silver nitrate.

Harmer (Mitth. Zool. Stat. Neapel, v, 1884, p. 445) washes them for some time (half an hour) in a 5 per cent. solution of nitrate of potash in distilled water; they may then be treated with silver nitrate in the usual way. For some animals he recommends a 4.5 per cent. solution of sulphate of soda.

35\%. Double-staining Silver-stained Tissues.-... The nuclei of tissues impregnated with silver may be stained with the usual reagents, provided that solutions containing free ammonia be avoided. These stains will only succeed, however, with successful negative impregnations, as nuclei that have been impregnated will not take the second stain.

Impregnation with silver may be followed by impregnation with gold. In this case the gold generally substitutes itself for the silver in the tissues, and though the results are sharp and precise, the effect of a double stain is not produced. See hereon GERota, loc. cit., $\S 355$.

358. Impregnation of Nerve Tissue.-For this subject, which includes the important bichromate-and-silver method of GOLGI, and the neurofibril methods of BIELSCHOWsKY and RAMÓN Y CAJAL, see Part II. These give important results, not only with Nervous tissue, but with various forms of Connective tissue, mitochondrial formations, etc.

\section{Gold.}

359. The Characters of Gold Impregnations.-Gold chloride differs from nitrate of silver in that it generally gives positive ( $\$ 348$ ) impregnations only. It generally gives negative images only with such tissues as have first received a negative impregnation with 
silver, the gold substituting itself for the silver. In order to obtain these images you first impregnate very lightly with silver; reduce ; treat for a few minutes with a 0.5 per cent. solution of gold chloride, and reduce in acidulated distilled water.

This process, however, is in but little use, and except for certain special studies on the cornea and on connective tissue, the almost exclusive function of gold chloride is the impregnation of nervous tissue, for which it exhibits a remarkable selectivity.

360. Pre-impregnation and Post-impregnation.-Gold methods may be divided into two groups: viz. pre-impregnation methods, characterised by employing perfectly fresh tissues, and post-impregnation methods, characterised by the employment of fixed and hardened tissues. Both are chiefly used for rervous tissue. They give in some respects opposite results. Pre-impregnation gives nuclei unstained, cytoplasm rather strongly stained, axis-cylinders reddishviolet. Post-impregnation gives nuclei sharply stained, cytoplasm pale, axis-cylinders black, and (when successful) showing their neurofibrils sharply distinguished from the interfibrillar substance.

In ApÁthy's view (Mitth. Zool. Stat. Neapel, xii, 1897, p. 718) successful gold preparations should show a true stain, not an impregnation ( $\$ 347)$, the stain being brought about by the formation of gold oxide $(\mathrm{AuO})$ which combines with the tissue elements. $\mathrm{He}$ advises in consequence that preparations should not be moved about more than can be helped in the reducing bath, so that the colouring oxide may not be washed away from the tissues before the stain has taken effect.

361. As to the Commercial Salts of Gold.-SQuIRE's Methods and Formula, etc. (p. 43), says : "Commercial chloride of gold is not the pure chloride, $\mathrm{AuCl}_{3}$, but the crystallised double chloride of gold and sodium, containing 50 per cent. of metallic gold.

" Commercial chloride of gold and sodium is the above crystallised double chloride mixed with an equal weight of chloride of sodium, and contains 25 per cent. of metallic gold."

This, however, appears not to be the case in Germany. Dr. GrübleR, writing to MAYer (see the Grundzüge, Lee und Mayer, p. 215), says: "Aurum chloratum fuscum contains about 53 per cent. $\mathrm{Au}$, the flavum about 48 per cent. ; in both of them there should be only water and hydrochloric acid besides the gold, no sodium chloride. Pure Auronatrium chloratum contains 14.7 per cent. of sodium chloride, though samples are found in commerce with much more," 
AрÁтнy (Mitth. Zool. Stat. Neapel, xii, 1897, p. 722) formerly employed the aurum chloratum flavum, but now prefers the fuscum.

\section{A. Pre-ımpregnation.}

362. The State of the Tissues to be Impregnated.-The once classical rule, that for researches on nerve-endings the tissues should be taken perfectly fresh, seems not to be valid for all cases. For Drasch (Sitzb. Akad. Wiss. Wien, 1881, p. 171, and 1884, p. 516 ; and Abhand. math.-phys. Cl. K. Sach. Ges. Wiss., xiv, No. 5, 1887 ; Zeit. wiss. Mik., iv, 1887, p. 492) finds that better results are obtained with tissues that have been allowed to lie after death for twelve, twenty-four, or even forty-eight hours in a cool place.

363. Cohnherm's Method (Virchow's Arch., Bd. xxxviii, pp. 346349 ; Stricker's Handb., p. 1100).-Fresh pieces of cornea (or other tissue) are put into 0.5 per cent. solution of chloride of gold until thoroughly yellow, and then exposed to the light in water acidulated with acetic acid until the gold is thoroughly reduced, which happens in the course of a few days at latest. They are then mounted in acidulated glycerin.

Results very uncertain and anything but permanent.

364. Löwit's Method (Sitzgsber. Akad. Wien, Bd. lxxi, 1875, p. 1).-The following directions are from Fischer's paper on the corpuscles of Meissner (Arch. mik. Anat., xii, 1875, p. 366).

Small pieces of fresh skin are put into dilute formic acid (1 volume of water to 1 of the acid of $1.12 \mathrm{sp}$. gr.), and remain there until the epidermis peels off. They then are put for fifteen minutes into gold chloride solution ( $1 \frac{1}{2}$ to 1 per cent.), then for twenty-four hours into dilute formic acid ( 1 part of the acid to $1-3$ of water), and then for twenty-four hours into undiluted formic acid. (Both of these stages are gone through in the dark.) Sections are then made and mounted in dammar or glycerin. Successful preparations show the nerves alone stained.

365. RanvieR's Formic Acid Method (Quart. Journ. Mic. Sci. [N.S.], $1 \times x x, 1880$, p. 456). - The tissues are placed in a mixture of chloride of gold and formic acid (4 parts of 1 per cent. gold chloride to 1 part of formic acid) which has been boiled and allowed to cool (RANVIER's Traité, p. 826). They remain in this until thoroughly impregnated (muscle twenty minutes, epidermis two to four hours); reduction is affected either by daylight in acidulated water, or in the dark in dilute formic acid ( 1 part of the acid to 4 parts of water).

366. RanvieR's Lemon-juice Method (Traité, p. 813).-RANVIER finds that of all acids lemon juice is the least hurtful to nerve- 
endings. He therefore soaks pieces of tissue in fresh lemon juice until they become transparent (five or ten minutes in the case of muscle). They are then rapidly washed in water, brought for about twenty minutes into 1 per cent. gold chloride solution, washed again in water, and brought into a mixture of 50 c.c. of distilled water and 2 drops of acetic acid. They are exposed to the light for twenty-four to forty-eight hours. The preparations thus obtained are good for immediate study, but are not permanent, the reduction of the gold being incomplete. In order to obtain perfectly reduced, and therefore permanent, preparations, the reduction should be done in the dark in a few cubic centimetres of dilute formic acid ( 1 part acid to 4 of water), which takes about twenty-four hours.

36\%. Viallane's Osmic Acid Method (Hist. et Dév. des Insectes, 1883, p. 42).-The tissues are treated with osmic acid (1 per cent. solution) until they begin to turn brown, then with 25 per cent. formic acid for ten minutes; they are then put into solution of chloride of gold of 1 : 5000 (or even much weaker) for twenty-four hours in the dark, then reduced in the light in 25 per cent. formic acid. I find this an excellent method.

Kerschner (Arch. mik. Anat., lxxi, 1908, p. 522) puts till brown into a mixture of 10 parts 5 per cent. formic acid with 1 part 2 per cent. osmic acid, washes, puts for two to six hours into 1 per cent. gold chloride in the dark, washes, puts for twelve hours into 25 per cent. formic acid in the dark and then for twenty-four in the light, and mounts in 50 per cent. glycerin with 1 per cent. of formol.

368. Other Methods.-The numerous other methods that have been proposed differ from the foregoing partly in respect of the solutions used for impregnation, but chiefly in respect of details imagined for the purpose of facilitating the reduction of the gold.

Thus BAstian employed a solution of gold chloride of a strength of 1 to 2000 , acidulated with $\mathrm{HCl}$ ( 1 drop to 75 c.c.), and reduced in a mixture of equal parts of formic acid and water kept warm.

Hénocque (Arch. de l'Anat. et de la Physiol., 1870, p. 111) impregnates in a 0.5 per cent. solution of gold chloride, washes in water for twelve to twenty-four hours, and reduces in a nearly saturated solution of tartaric acid at a temperature of $40^{\circ}$ to $50^{\circ} \mathrm{C}$. Reduction is effected very rapidly, sometimes in a quarter of an hour.

Hoyer (Arch. mik. Anat., ix, 1873, p. 222) says that the double chloride of gold and potassium has many advantages over the simple gold chloride. He impregnates in solutions of 0.5 per cent. strength, and reduces in water containing 1 or 2 drops of a pyrogallic acid 
developing solution, such as is used in photography, or in a warm concentrated solution of tartaric acid, at the temperature of an incubating stove.

I have myself used the double chloride of gold and sodium with good results.

Ciaccio (Journ. de Microgr., vii, 1883, p. 38) prefers the double chloride of gold and cadmium.

Flechsig (Die Leitungsbahnen in Gehirn, 1876; Arch. Anat. u. Phys., 1884, p. 453) reduces in a 10 per cent. solution of caustic soda.

NESTEROFFsKy treats impregnated preparations with a drop of ammonium sulphide, and finishes the reduction in glycerin (quoted from GIERKE's Färberei z. mik. Zwecken).

Bонм reduces in Pritchard's solution-amyl alcohol, 1 ; formic acid, 1 ; water, 98.

ManFREdi (Arch. per le Sci. med., v, No. 15) puts fresh tissues into gold chloride, 1 per cent., for half an hour ; then oxalic acid, 0.5 per cent., in which they are warmed in a water-bath to $36^{\circ}$. Mount in glycerin. Sunny weather is necessary.

BocCardi (Lavori Instit. Fisiol. Napoli, 1886, i, p. 27 ; Journ. Roy. Mic. Soc., 1888, p. 155) recommends oxalic acid of 0.1 per cent. or of 0.25 to 0.3 per cent., or a mixture of 5 c.c. pure formic acid, 1 c.c. of 1 per cent. oxalic acid, and 25 c.c. of water, reducing in the dark not longer than two to four hours.

Kolossow (Zeit. wiss. Mik., v, 1888, p. 52) impregnates for two or three hours in a 1 per cent. solution of gold chloride acidulated with 1 per cent. of $\mathrm{HCl}$, and reduces for two or three days in the dark in a 0.01 per cent. to 0.02 per cent. solution of chromic acid.

GEBERG (Intern. Monatsschr., x, 1893, p. 205) states that previous treatment of tissues for twenty-four hours with lime-water (ARNSTEIN's method) greatly helps the reduction.

Bernheim (Arch. Anat. Phys., Phys. Abth., 1892, Supp., p. 29) adds to LöwIT's dilute formic acid a piece of sulphite of sodium. (must be fresh and smell strongly of sulphurous acid).

Dr. Lindsay Johnson writes to me that besides the "sunning" of the impregnating solution recommended above ( $\$ 349$ ), the gold should be carefully acidulated with a neutral acetate or formate, or acetic or formic acid, at least twenty-four hours before using; and then afterwards the tissue must be washed until no reaction occurs to test-paper.

AрÁtHY (Mikrotechnik, p. 173; Mitth. Zool. Stat. Neapel, xii, 1897, pp. 718 - 728) lays stress on the necessity of having the objects thoroughly penetrated by light from all sides during the process of 
reduction. Objects, therefore, should always be so thin that light can readily stream through them. He impregnates for a few hours in 1 per cent. gold chloride ( $\$ 361$ ) in the dark, then brings the objects without washing out with water, the gold solution being just superficially mopped up with blotting-paper, into 1 per cent. formic acid. They are to be set up in this, in a tube or otherwise, so that the light may come through them from all sides, and exposed to diffused daylight in summer, or direct sunlight in winter, for six to eight hours without a break. They must not be moved about more than can be helped in the acid. If the acid becomes brown it may be changed for fresh. The temperature of the acid should not be allowed to rise over $20^{\circ} \mathrm{C}$., whence direct sunlight is to be avoided during the summer. He mounts in glycerin or his syrup (§343). He finds such preparations absolutely permanent.

\section{Post-Impregnation.}

369. Gerlach's Method (Stricker's Handb., 1872, p. 678) : Spinal cord is hardened for fifteen to twenty days in a 1 to 2 per cent. solution of bichromate of ammonia. Thin sections are made and thrown into a solution of 1 part of double chloride of gold and potassium to 10,000 parts water, which is very slightly acidulated with $\mathrm{HCl}$, and after ten to twelve hours are washed in hydrochloric acid of 1 to $2: 3000$ strength, then brought for ten minutes into a mixture of 1 part $\mathrm{HCl}$ to 1000 parts of 60 per cent. alcohol, then dehydrated and mounted in balsam.

See further, for Nerve Centres, under "Nervous System."

3\%. Golgr (Mem. Accad. Torino [2], xxxii, 1880, p. 382) puts tissues previously hardened in 2 per cent. solution of bichromate of potash for ten to twenty minutes into 1 per cent. solution of arsenic acid, then into $\frac{1}{2}$ per cent. solution of chloride of gold and potassium for half an hour, washes in water, and reduces in sunlight in 1 per cent. arsenic acid solution, which is changed for fresh as fast as it becomes brown. Mount in glycerin. Sunny weather is necessary.

3\%1. Apáthy's Method (Zeit. wiss. Mik., x, 1893, p. 349 ; Mitth. Zool. Stat. Neapel, xii, 1897, p. 729): The material to be used must. have been fixed either in sublimate or in a mixture of equal parts of saturated solution of sublimate in 0.5 per cent. salt solution and 1 per cent. osmic acid (this more particularly for Vertebrates). The material should be imbedded as quickly as possible, either in paraffin or in celloidin. Sections are made and fixed on slides, and after the usual treatment with iodine, etc., are either put into distilled 
water for from two to six hours, or are rinsed in water, treated for one minute with 1 per cent. formic acid, and again well washed with water.

They are then put for twenty-four hours, or at least overnight, into the gold-bath, which is preferably 1 per cent. gold chloride (see $\S 361$ ), but may be weaker, down to $0 \cdot 1$ per cent., after which they are just rinsed with water or superficially dried with blottingpaper. The slides are then set up on end in a sloping position, the sections looking downwards, so that precipitates may not fall on them, in glass tubes filled with 1 per cent. formic acid. The tubes are then exposed to light until the gold is reduced, as directed in $\S 368$ sub fin.

I seem to have found it advantageous to reduce in weak solution of formaldehyde, either with or without formic acid.

Szütz (Zeit. wiss. Mik., xxix, 1912, p. 292) reduces as AрÁtнy for one day, then rinses and puts back for the night into the gold, then for the next day again into the formic acid.

372. Impregnation of Marine Animals.-For some reason the tissues of marine animals do not readily impregnate with gold in the fresh state. It is said by FoL that impregnation succeeds better with spirit specimens.

373. Preservation of Impregnated Preparations.-Preparations may be mounted either in balsam or in acidulated glycerin ( 1 per cent. formic acid).

Theoretically they ought to be permanent if the reduction of the metal has been completely effected, but they are very liable to go wrong through after-blackening. RANviER states that this can be avoided by putting them for a few days into alcohol, which he says possesses the property of stopping the reduction of the gold.

Blackened preparations may be bleached with cyanide or ferricyanide of potassium. REDDING employs a weak solution of ferricyanide, Cyвulsky a 0.5 per cent. solution of cyanide.

Preparations may be double-stained with the usual stains (safranin being very much to be recommended), but nuclei will only take the second stain in the case of negative impregnation.

\section{Other Metallic Stains.}

394 Osmic Acid and Pyrogallol.-This method was first published by me in 1887 (La Cellule, iv, p. 110). It consists in putting tissues that have been treated with osmic acid into a weak solution of 
pyrogallol, in which they quickly turn greenish black, sometimes much too much so.

Hermann (Arch. mik. Anat., xxxvii, 4, 1891, p. 570) put platinoaceto-osmic material hardened in alcohol for twelve to eighteen hours into raw pyroligneous acid. This acid ought (Ergebnisse der Anat., ii, 1893, p. 28) to be as raw as possible, and to be of a dark brown colour and evil-smelling. (The stain obtained in this way is not due to a mere reduction of the osmic acid, but also to coloration by the brown pyroligneous acid; for HERManN has obtained the same stain with sublimate material, or alcohol material (op. cit., i, 1891 [1892], p. 7).

I find this gives much better results than the pure osmic acid process, but not the best possible. I now proceed as follows :

Hermann or Flemming material is brought in bulk, directly after fixing, into a weak aqueous solution of pyrogallol. The tissues may remain in it for twenty-four hours, but for small objects an hour or less is sufficient. An alcoholic solution of pyrogallol may be taken if desired. RAwITz (Lehrbuch, p. 60) takes 20 per cent. aqueous sol. of tannin.

There is thus obtained a black stain, which is at the same time a plasma stain and a nuclear stain, chromatin being so far stained that it is not necessary to have recourse afterwards to a special chromatin stain. With Invertebrates it sometimes gives very elegant differentiations of nervous tissue. It is a very easy method, and if pyrogallol be used a very safe one (with pyroligneous acid not so safe).

If it be desired to add a chromatin stain, I greatly recommend safranin (stain very strongly, twenty-four hours at least, and start the extraction with acid alcohol).

This method has been attributed to von MaEhrenthal. See also under "Nervous System" modifications of this method by Azoular and Heller and GUMPertz; also one by Kolossow (Zeit. wiss. Mik., ix, 1892, p. 38, and ix, 1893, p. 316).

375. Perchloride of Iron.-This reagent, introduced by PolaILlon (Journ. de l'Anat., iii, 1866, p. 43), sometimes gives useful results, especially in the study of peripheral nerve-ganglia, in which it stains the nervous tissue alone, the connective tissue remaining colourless.

The HogGans proceed as follows (Journ. Quekett Club, 1876; Journ. Roy. Mic. Soc., ii, 1879, p. 358):-The tissue (having been first fixed with silver nitrate, which is somewhat reduced by a short exposure to diffused light) is dehydrated in alcohol, and treated for a few minutes with 2 per cent. solution of perchloride of iron in spirit; then with a 2 per cent. solution of pyrogallic acid in spirit, and in a few minutes more, according to the depth of tint required, may be washed in water and mounted in glycerin. 
Fol fixes in perchloride $(\S 80)$ and treats for twenty-four hours with alcohol containing a trace of gallic acid.

Polatllon (loc. cit.) reduces in tannic acid.

The method is not applicable to chromic objects.

Golodetz and UnNA (Monats. prakt. Derm., xlviii, 1909, p. 153) put sections of skin for five minutes into fresh mixture of 1 per cent. perchloride of iron and 1 per cent. sol. of ferricyanide of potassium. See also UnNa and Golodetz, ibid., xlix, 1909, p. 97.

Roosevelt (Med. Rec., ii, 1887, p. 84 ; Journ. Roy. Mic. Soc., 1888, p. 157) employs a stain composed of 20 drops of saturated solution of iron sulphate, 30 grms. water, and 15 to 20 drops pyrogallic acid.

376. Palladium Chloride (see Schulze, § 77). Prussian Blue (see LEBER, Arch. Ophthalm., xiv, p. 300 ; RaNvier, Traité, p. 108). Cupric Sulphate (see LeBER, ibid.). Lead Chromate (see LEBER, ibid.). Sulphides (see LandoIs, Centralb. med. Wiss., 1885, No. 55; and GIERKE, in Zeit. wiss. Mik., i, 1884, p. 497). Molybdate of Ammonia (MERKel ; KRAUSE) (see GiERKE, ibid., i, 1884, p. 96). Oxychloride of Ruthenium (Nicolis and Cantacuzène) (see Ann. Inst. Pasteur, vii, 1893, p. 331). Ruthenium Red (Ruthenium Sesquichloride) (EIsEN, Zeit.wiss. Mik., xiv, 1897, p. 200 ; in my hands totally useless). Oxide of Manganese (Golodetz and UnNa, Monats. prakt. Derm., xlviii, 1909, p. 151). 


\section{CHAPTER XVIII.}

\section{OTHER STAINS AND COMBINATIONS.}

37\%. Kernschwarz (Platner, Zeit. wiss. Mik., iv, 1887, p. 350).A black liquid on sale by Grübler \& Hollborn. Mayer (Grundzüge, LEE \& MAYER, 1st ed., p. 202) finds that it contains iron, combined with some gallic acid. I use it as follows :

Sections (I have not tried material in bulk) are fixed on slides and treated with Kernschwarz until the required depth of stain is obtained, which will be from a few minutes to twenty-four hours, according to the material.

There is obtained a black or neutral-tint stain, which is either a pure chromatin stain, or at the same time a plasma stain. If overstaining should have occurred, the stain is easily differentiated by means of any weak acid, either in water or alcohol. Platner took alkalies, preferably carbonate of lithia, for differentiation.

It may be well, if a good plasma stain has been obtained, to afterstain for twenty-four hours with safranin, followed by differentiation in either neutral or acid alcohol, and clove oil. The stain is perfectly permanent in balsam, and is stated to be a good one for preparations that it is desired to photograph.

I greatly recommend this stain, which is safe and easy. The combination with safranin gives a better chromatin stain than safranin alone.

378. Brazilin, the colouring matter of Brazilian redwood or Pernambuco wood, has been recommended by EISEN (Zeit. wiss. Mik., xiv, 1897, p. 198) and Hickson (Nature, lxii, 1900, p. 589, and Quart. Journ. Mic. Sci., 1901, p. 469). MAYER (Grundzïge, p. 203) finds that, in alum solution, it gives a stain similar to that of hæmatein, but much weaker.

Iron-Brazilin (Hickson, Quart. Journ. Micr. Sci., xliv, 1901, p. 470 ) is better. Sections are mordanted for one to three hours in 1 per cent. sol. of iron alum in alcohol of 70 per cent. (made by dissolving $1 \mathrm{grm}$. of the salt in 23 c.c. of water, warm, and adding 77 c.c. of 90 per cent. alcohol after cooling), rinsed with alcohol, and put for three to sixteen hours into 0.5 per cent. sol. of Brazilin in alcohol of 70 per cent.

379. Orchella (Orseille), see WedL (Arch. path. Anat., lxxiv, p. 143) and FoL (Lehrb., p. 192), and early editions of this work. 
380. Orcein (Israke, Virchow's Archiv, cv, 1886, p. 169 ; and Prakticum der path. Hist., 2 Aufl., Berlin, 1893, p. 72) is a dye obtained from the lichen, Lecanora parella, and is not to be confused with orcin, another derivative of the same lichen. It is said to unite in itself the staining properties of the basic and acid stains, and also the combination of two contrast colours. Israel stains sections in a solution containing 2 grms. of orcein, 2 grms. of glacial acetic acid, and 100 c.c. of distilled water, washes in distilled water, and passes rapidly through absolute alcohol to thick cedar oil, in which the preparations remain definitely mounted. Nuclei blue, protoplasm red.

See also "Connective Tissues" in Part II, and Laurent, Zeit. wiss. Mik., xiii, 1896, p. 302 ; RuzIčKA, ibid., xiv, 1898, p. 455 ; and WoLfF, ibid., xix, 1903, p. 488.

381. Purpurin, see Ranvier's Traité technique, p. 280 ; Duval's Précis de Technique histologique, p. 221 ; and GEenacher's formula in Arch. mik. Anat., xvi, 1879, p. 470. A very weak stain.

382. Indigo.-Indigo is employed in histology in the form of solutions of so-called indigo carmine, or sulphindigotate of soda or potash. The simple aqueous solution gives a diffuse stain, but is of use when employed in conjunction with carmine. See below.

Thiersch's Oxalic Acid Indigo-Carmine (see Arch. mik. Anat., i, 1865, p. 150).

383. Other Vegetal Dyes.-See early editions. Those recommended by Claudius (Zeit. wiss. Mik., xvii, 1900, p. 52) are superfluous.

\section{Carmine Combinations.}

384. Seiler's Carmine followed by Indigo-Carmine (Am. Quart. Mic. Journ., i, 1879, p. 220). - Stain in borax-carmine, wash out with HCl alcohol, wash out the acid, and after-stain in an extremely dilute alcoholic solution of indigo-carmine ( 2 drops of saturated aqueous solution added to an ounce of alcohol and filtered).

I find this method gives good results with sections, but not if it be attempted to stain in bulk.

385. Merkel's Carmine and Indigo-Carmine in One Stain (MERKEL, Unters. anat. Anst. Rostock, 1874 ; Month. Mic. Journ., 1877, pp. 242 and 317).

Also Norris and Shakespeare, Amer. Journ. Med. Sci., January, 1877 ; Merkel, Mon. Mic. Journ., 1877, p. 242 ; MARsh, Section Cutting, p. 85 ; BayerL, Arch. Mik. Anat., xxiii, 1885, pp. 36, 37 ; Macallum, Trans. Canad. Instit., ii, 1892, p. 222 ; Journ. Roy. Mic. Soc., v, 1892, p. 698 .

386. MAYER's Carmalum (or Hæmalum) and Indigo-Carmine in One Stain.-MaYer (Mitth. Zool. Stat. Neapel, xii, 1896, p: 320) obtains very good results by taking a solution of $0 \cdot 1 \mathrm{grm}$. of indigocarmine in 50 c.c. of distilled water, or 5 per cent. alum solution, and combining it with from 4 to 20 volumes of carmalum or hæmalum.

38\%. Carmine and Picro-Indigo-Carmine (RAMON y CAJaL, Rev. 
de Cienc. med., 1895 ; CalleJA, Rev.trim. Microgr., ii, 1897, p. 101 ; Zeit. wiss. Mik., xv, 1899, p. 323).-For use after a carmine stain, RAMón takes a solution of $0.25 \mathrm{grm}$. of indigo-carmine in $100 \mathrm{grms}$. saturated aqueous solution of picric acid. Stain (sections) for five to ten minutes, wash in weak acetic acid, then in water, then remove the excess of picric acid with absolute alcohol, clear and mount.

RAMón also (Elementos de Histologia, 1897; quoted from La Cellule, xix, 1901, p. 212) employs the picro-indigo mixture after Magenta ; stain strongly in saturated solution of magenta, rinse in water until no more colour comes away, and pass into the indigo mixture. See also Borrex, Ann. Inst. Pasteur, 1901, p. 57, or Lee et Henneguy, Traité, p. 268.

388. Carmine and Anilin Blue (or Bleu Lumière, or Bleu de Lyon) (Duval, Précis de Technique Microscopique, 1878, p. 225).-Stain with carmine; dehydrate, and stain for a few minutes (ten minutes for a section of nerve-centres) in a solution of 10 drops of saturated solution of anilin blue in alcohol to 10 grms. of absolute alcohol. Clear with turpentine, without further treatment with alcohol, and mount in balsam.

Other authors recommend, instead of anilin blue, bleu de Lyon, dissolved in 70 per cent. alcohol acidulated with acetic acid (MAURICE and Schulgiv), or bleu lumière.

The solutions of both these colours should be extremely dilute for sublimate material, but strong for chrom-osmium material. It is possible to use them for staining in bulk.

Baumgarten (Arch. mik. Anat., xl, 1892, p. 512) stains sections (of material previously stained in borax-carmine) for twelve hours in a 0.2 per cent. solution of bleu de Lyon in absolute alcohol, and washes out for about half that time before mounting in balsam. He recommends the process for cartilage and nerve-centres.

389. Carmine and Malachite Green.-MAAS (Zeit. wiss. Zool., 1, 4, 1890 , p. 527) recommends borax-carmine followed by weak alcoholic solution of malachite green, with a final washing out with stronger alcohol.

390. Carmine and Picro-nigrosin (PIANese).-See Journ. Roy. Mic. Soc., 1892, p. 292.

391. Carmine and Picric Acid.—See § 298.

Hamatein or Hamatoxylin Combinations.

392. Hæmatoxylin and Picric Acid.-See § 298.

393. Hæmatoxylin and Eosin.-This popular combination gives results that are æsthetically beautiful, but (for most objects) is not 
so useful as many others, the eosin lacking in electivity. Objects may be stained with hæmatoxylin (either in the mass or as sections) and the sections stained for a few minutes in eosin. I think it is better to take the eosin weak, though it has been recommended (SтöHr, see Zeit. wiss. Mik., i, 1884, p. 583) to take it saturated. Either aqueous or alcoholic solutions of eosin may be used.

Hickson (Quart. Journ. Mic. Sci., 1893, p. 129) stains sections for one hour in a strong solution of eosin in 90 per cent. alcohol, washes with alcohol, and stains for twenty minutes in a weak solution of hæmatoxylin.

This method is most particularly recommendable for embryological sections, as vitellus takes the eosin stain energetically, and so stands out boldly from the other germinal layers in which the blue of the hæmatoxylin dominates.

See also List (Zeit. wiss. Mik., ii, 1885, p. 148); Busch (Verh. Berl. Phys. Gses., 1887) ; Gierke (Zeit.wiss. Mik., i, 1884, p. 505).

Sections should be very well washed before being passed from eosin into hæmatoxylin or the reverse, as eosin very easily precipitates hæmatoxylin.

For the complicated and superfluous mixtures of RENAUT and of Everard, Demoor and Massart, see Fol's Lehrbuch, p. 196, Ann. Inst. Pasteur, vii, 1893, p. 166, or early editions.

See especially ScotT's method, $§ 676$.

393a. Hæmatoxylin and Azoeosin or Biebrich Scarlet.-See under "Nucleoli," § 676.

\section{Hæmatoxylin and Congo.-See $\S 305$.}

395. Hæmatoxylin and Safranin.-RABL (Morph. Jahrb., x, 1884, p. 215) stained very lightly with very dilute DELAFIELD's hæmatoxylin for twenty-four hours, then for some hours in (PFITZneR's) safranin, and washed out with pure alcohol. The plasma stain is here given by the hæmatoxylin.

Similarly Regaud, Verh. Anat. Ges., xiv, 1900, p. 112.

Fò̀ (Festschr. Virchow, 1891, p. 481) stains in a mixture of 25 c.c. of Böhmer's hæmatoxylin, 20 of 1 per cent. solution of safranin, and 100 of water for one to three minutes.

396. Hæmatoxylin and Säurefuchsin.-Stain first with iron hæmatoxylin or hæmalum, then stain (sections) in 0.5 per cent. aqueous solution of Säurefuchsin, dehydrate and mount.

39\%. Hæmatoxylin and Säurefuchsin and Orange.-Proceed as above, using for the second stain the following mixture: Säurefuchsin, 1 grm. ; orange, 6 grms. ; rectified spirit, 60 c.c.; water, 
240 c.c. (from SQuire's Methods and Formula, p. 42). Using orange $G$ (not mentioned by SQUIRE), I have had very good results.

The method of Cavazzani (Riforma Med., Napoli, 1893, p. 604 ; Zeit. wiss. Mik., xi, 3, 1894, p. 344) is far too complicated.

398. Hæmatoxylin and Picro-Säurefuchsin (vaN Gieson, New York Med. Journ., 1889, p. 57 ; quoted from Moeller, Zeit. wiss. Mik., xv, 2, 1898, p. 172, which see for further details).-Proceed as above, using for the second stain the picro-Säurefuchsin mixture, $\S 299$. The second stain must not be too prolonged.

WEIGERT (Zeit. wiss. Mik., xxi, 1904, p. 1) stains first in his ironhæmatoxylin mixture ( $(244)$, rinses in water, and stains for a short time in his picro-Säurefuchsin ( $(299)$, rinses, dehydrates with 90 per cent. alcohol, and clears with carbolic acid-xylol mixture (§ 167). 


\section{CHAPTER XIX.}

\section{EXAMINATION AND PRESERVATION MEDIA.}

399. Introductory. - I comprehend under this heading all the media in which an object may be examined to advantage.

All preservative media may be used for mounting, though the only media that will afford an absolutely sure preservation of soft tissues are the resinous ones.

400. Refractive Indices of Examination Media.-An examination medium should be of such a refractive index as to afford a due degree of visibility of colourless (unstained) elements. The visibility of these is inversely as their transparency when penetrated by the medium. It is directly proportional to the difference between the refractive indices of the object and of the medium in which it is mounted. The greatest transparency is obtained when the refraction of the medium is the same as that of the tissue elements. Media having a lower index than that of the tissues give diminished transparency, but greater visibility. Media having a higher index than that of the tissues give great transparency, but diminished visibility of (unstained) details. Now the index of refraction of most tissue elements, after fixation and dehydration, is occasionally higher than that of Canada balsam: so that media of the greatest clearing power (i.e. giving the greatest transparency) must be looked for amongst reagents having an index superior to that of balsam, whilst for enhanced visibility of detail we must employ less refractive media, such as castor oil, glycerin, or water.

The following short list, extracted from BeHRENs' Tabellen zum Gebrauch bei mikroskopischen Arbeiten, Braunschweig, 1892, p. 42 , and other sources, may be useful as a guide to the optical effects of various media. The figures give the approximate indices of refraction. They should be accepted with some caution, on account of the variability of samples. The figures given for balsam refer evidently to the resin in the solid state and not to the solutions used for mounting, which are certainly much lower, according to the lower index of the solvent. 


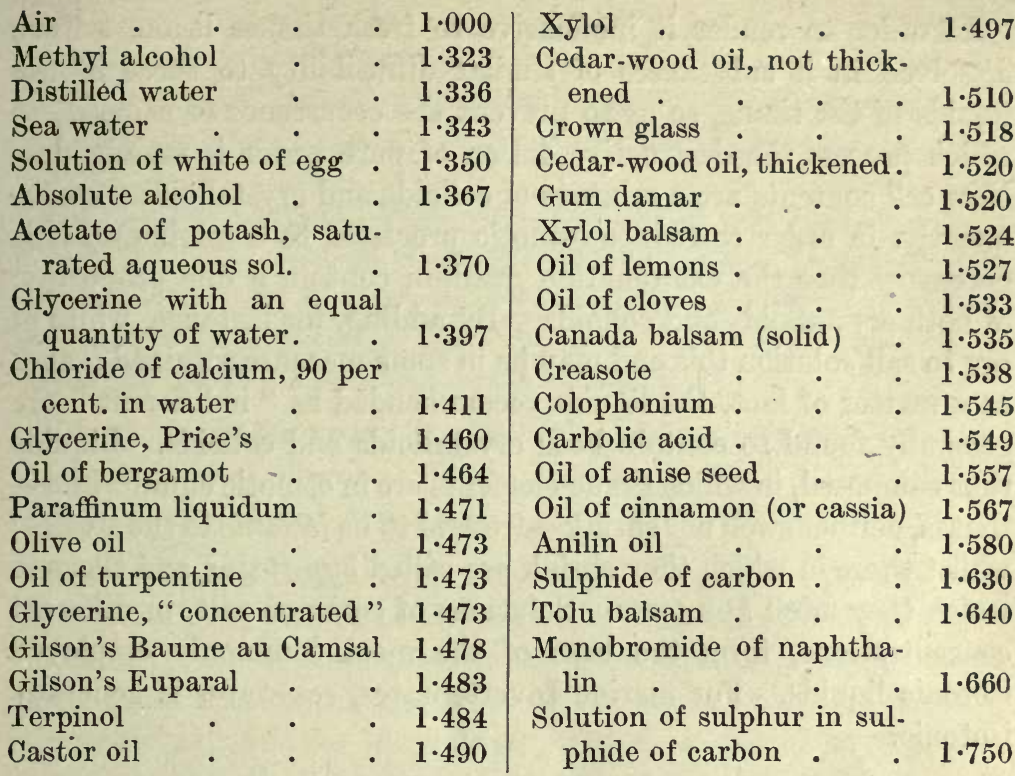

It will be seen that cedar oil has nearly the index of crown glass (this is true of the oil in the thick state to which it is brought by exposure to the air-not of the new, thin oil, which is less highly refractive); it therefore clears to about the same extent as Canada balsam. Clove oil has a much higher index, and therefore clears more than balsam; cinnamon oil higher still. Turpentine and bergamot oil have much lower indices, and therefore clear less.

\section{Watery Media.}

401. Isotonic and "Indifferent" Liquids.-The old distinction of "Indifferent" liquids, and those which have some action on tissues, appears to be misleading more than helpful ; for no medium is without action on tissues except the plasma with which they are surrounded during the life of the organism; and this plasma itself is only "indifferent" whilst all is in situ; as soon as a portion of tissue is dissected out and transferred to a slide in a portion of plasma the conditions become artificial.

Water may be employed for the examination of structures that have been well fixed; but this is by no means applicable to the examination of fresh tissues. It is very far from being an "indifferent" liquid; many tissue elements are greatly changed by it (nerve-end structures, for instance), and some are totally destroyed by its action if prolonged (for instance, red-blood corpuscles). 
In order to render it inoffensive to fresh tissues it must have dissolved in it substances of similar diffusibility to those of the liquids of the tissue, so as to prevent the occurrence of osmosis, to which process the destructive action of pure water is mainly due. Now cell contents are a mixture of colloids and crystalloids ; consequently, in order to reduce osmotic processes to a minimum, it is necessary that the examination medium contain a due proportion of both crystalloids and colloids. By adding, for instance, white of egg to salt solution this end may be in some measure attained; and, as a matter of fact, the liquids recommended as "indifferent" are generally found to contain both crystalloids and colloids. Liquids thus composed, in which tissue-elements are in osmotic equilibriumthat is, neither swell nor shrink-are said to be isotonic to the tissues; whilst those in which they shrink are called hypertonic, and those in which they swell hypotonic. Solutions of common salt, in different concentrations, form the base of the most commonly employed isotonic liquids. For marine Invertebrates, sea-water is generally isotonic.

402. Salt Solution ("normal salt solution," "physiological salt solution"). -0.75 per cent. sodium chloride in water. CARNOY recommends the addition of a trace of osmic acid.

RINGER's solution, much used in physiology, consists of sodium chloride 0.8 parts, calcium chloride 0.02 , potassium chloride 0.02 , sodium bicarbonate 0.02 and water 100 (with or without 0.1 dextrose).

According to Locke (Boston Med.-Surg. Journ., 1896, p. 514) there should be added to salt solution (which to be isotonic should contain, according to HAMBURGer, 0.9 to 1 per cent. of salt) 0.01 per cent. chloride of potassium, and 0.02 per cent. chloride of calcium, in order to obtain an "indifferent" liquid.

Malassez (C. R. Soc. Biol., iii, 1896, pp. 504 and 511) takes for erythrocytes about 1 per cent. sodium chloride.

Dekнuyzen (Onderz. Phys. Lab. Leiden., 1900, p. 149) takes for blood of Rana $0 \cdot 8$ per cent.

For Selachians, Muskens (Tijd. Nederb. Dierk. Ver., 1894, p. 314) finds $2 \frac{1}{4}$ per cent. right; and Rodin (Comptes Rend., 1900, p. 1009) 1.5 to 2.6 per cent., according to the species.

Engelmann (Deutsch. med. Wochenschr., xxix, 1903, p. 64) finds that 0.9 per cent. is isotonic with human blood-serum, and 0.64 per cent. for red blood corpuscles of the frog.

Kronecker's "Artificial Serum" (from Vogt et Yung, Traité 
d'Anat. Comp. Prat., p. 473) consists of common salt 6 parts, caustic soda $0 \cdot 06$, distilled water 1000 .

Böнм und OpPeL (Taschenbuch, 3 Aufl., p. 19) take carbonate of soda instead of caustic soda.

403. Pictet's Liquid (Mitth. Zool. Stat. Neapel, x, 1891, p. 89).5 to 10 per cent. solution of chloride of manganese. These proportions are for marine animals, and for terrestrial animals will generally be found much too high. For these from 1 to 3 per cent. will be nearer the mark. I find this liquid excellent.

404. Aqueous Humour, Simple White of Egg.-Require no preparation beyond filtering. They may be iodised if desired (see next §), or mixed with salt solution.

405. Iodised Serum.-Max Schultze. (Virchow's Archiv., xxx, 1864, p. 263). I take the following from Ranvier (Traité, p. 76).

The only serum that gives really good results is the amniotic liquid of mammals. Flakes of iodine are added to it, and the flask frequently agitated for some days. The flask should have a wide bottom, so that the serum may form only a shallow layer in it.

Another method is as follows: Serum is mixed with a large proportion of tincture of iodine; the precipitate that forms is removed by filtration, and there remains a strong solution of iodine in serum. This should be kept in stock, and a little of it added every two or three days to the serum that is intended for use. In general for maceration purposes a serum of a pale brown colour should be employed.

406. Artificial Iodised Serum (Frey, Das Mikroskop, 6 Aufl, 1877 , p. 75 ).-Distilled water 270 grms., white of egg 30, sodium chloride 2.5. Mix, filter, and add tincture of iodine.

407. Migula's Glycerised Blood-serum (see the paper in Zeit.f. wiss. Mik., vii, 2, 1890, p. 172).

408. Chloride of Calcium (Harting, Das Mikroskop, 2 Aufl., p. 297). -The aqueous solution, either saturated or diluted with 4 to 8 parts of water, has a low refractive index and does not dry up.

409. Acetate of Potash (Max Schultze, Arch. mil. Anat., vii, 1872, p. 180).-A nearly saturated solution in water. The index of refraction is lower than that of glycerin.

410. Syrup.-A good strength is equal parts of loaf sugar and water. Dissolve by boiling. To preserve it from mould, chloral hydrate may be dissolved in it ( 1 to 5 per cent.) - I have used as much as 7 per cent., and found no disadvantage - or carbolic acid (1 per cent.). 
It may be used as a mounting medium, but there is always risk of the sugar crystallising out.

Fabre-Domergue (Bull. Soc. Philomath, ix, 1899, p. 115) dissolves 200 parts of sugar in 400 of water, and adds 1 part of formaldehyde, and camphor to saturation.

411. Chloral Hydrate. 5 per cent. in water (Ladowsky, Arch. $f$. mik. Anat., 1876, p. 359).

Or, 2.5 per cent. in water (BraDy, British Copepods).

Or, 1 per cent. in water (Munson, Journ. Roy. Mic. Soc., 1881, p. 847).

\section{Mercurial Liquids.}

(I give these as examination media only, not as permanent mounting media Media containing sublimate always end by making tissues granular.)

412. Gilson's Fluid (CARnoy's Biologie Cellulaire, p. 94). Alcohol of 60 per cent. . . . . 60 c.c. Water Glycerin 30 , Acetic acid (15 parts of the glacial to 85 of

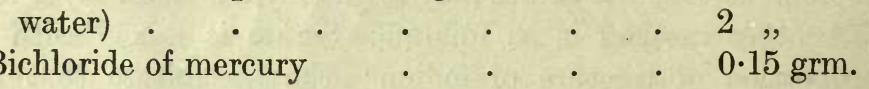

413. GAGE's Albumen Fluid (Zeit.f. wiss. Mik., 1886, p. 223).

White of egg

Water

Corrosive sublimate

Salt

$$
\begin{gathered}
15 \text { c.c. } \\
\text {. } 200 \text {, } \\
\text {. } 0.5 \text { grm. } \\
\text {. } 4 \text { grms. }
\end{gathered}
$$

Mix, agitate, filter, and preserve in a cool place. Recommended for the study of red blood-corpuscles and ciliated cells.

414. PacinI's Fluids (Journ. de Mik., iv, 1880 ; Journ. Roy. Mic. Soc., [N.S.] ii, 1882, p. 702, and early editions of this work).-Antiquated and superfluous. They consist essentially of corrosive sublimate of from $\frac{1}{2}$ to $\frac{1}{3}$ per cent. strength, with the addition of a little salt or acetic acid.

415. GодDвт's Fluids (Micro. Dict., art. "Preservation," or early editions of this work).-Quite unsuited for histological purposes.

\section{Other Fluids.}

416. Chloride and Acetate of Copper (RIPART et Petit's fluid, see $\S 90)$.

417. Tannin (CARNoy, Biol. Cellulaire, p. 95).-Water 100 grms., powdered tannin $0.40 \mathrm{grm}$., as an examination medium only.

418. Wickersheimer's Fluid (Zool. Anz., 1879, p. 670).-Worthless for histological purposes. 
419. Medium of Farrants (Beale, How to Work, etc., p. 58).Picked gum arabic 4 ozs., water 4, glycerin 2. See also the Micrographic Dictionary, and A. F. Stanley Kent, in Journ. Roy. Mic. Soc., 1890, p. 820.

420. Gum and Glycerin Medium (Langerhans, Zool. Anzeig., ii, 1879, p. 575).

Gummi arab.

$\circ . \quad \cdot \quad \cdot 5 \cdot 0$

to which after twelve hours are added-

Glycerini . . . . . $\quad 5 \cdot 0$

Sol. aquosa acid. corbal. $(5 \cdot 100)$. . . $10 \cdot 0$

421. Allen's Gum and Glycerin.-Prof. F. J. Allen (in litt.). Solution of gum arabic of the consistency of glycerin, strained, and $\frac{1}{8}$ volume of glycerin and $\frac{1}{20}$ of formol gradually incorporated. Sets hard.

422. HOYER's Gum with Chloral Hydrate or Acetate of Potash (Biol. Centralb., ii, 1882, pp. 23, 24).-A high 60 c.c. glass with a wide neck is filled two-thirds full with gum arabic (in pieces), and then either a solution of chloral (of several per cent.) containing 5-10 per cent. of glycerin is added or officinal solution of acetate of potash or ammonia. Filter after solution. The solution with chloral is for carmine or hæmatoxylin objects-that with acetate for anilin objects.

423. CoLE's Gum and Syrup Medium. See 183.

424. АрÁтнy's Gum and Syrup Medium (see § 343).-This medium sets very hard and may also be used for ringing glycerin mounts.

425. FAbre-Domergue's Glucose Medium ( La Nature, No. 823, 9 Mars, 1889, supp.).-Glucose syrup diluted to $25^{\circ}$ of the areometer (sp. gr. $1 \cdot 1968$ ) 1000 parts, methyl alcohol 200, glycerin 100, camphor to saturation. The glucose is to be dissolved in warm water, and the other ingredients added. The mixture, which is always acid, must be neutralised by the addition of a little potash or soda. It is said to preserve without change almost all animal pigments, but the mounts do not keep indefinitely.

426. Brun's Glucose Medium (from Fabre-Domergue's Premiers Principes du Microscope, 1889, p. 123).-Distilled water 140 parts, camphorated spirit 10, glucose 40 , glycerin 10 . Mix the water, glucose, and glycerin, then add the spirit, and filter. Hennegur informs me that this liquid preserves the colour of preparations stained with anilin dyes, methyl green included.

427. Levulose is recommended by Behrens, Kossel u. SchiefFERDECKER (Das Mikroskop, etc., 1889). It is uncrystallisable, and preserves well carmine and coal-tar stains (hæmatoxylin stains fade 
somewhat in it). The index of refraction is somewhat higher than that of glycerin. Objects may be brought into it out of water.

428. Amann's Lactophenol (from Langeron, C. R. Soc. Biol., lviii, 1905, p. 750).-Carbolic acid, 20 ; lactic acid, 20 ; glycerin, 40 ; water, 20. For Nematodes, Acarids, etc. Add gradually drop by drop to the water containing the organisms. Not for mounting. Mount in glycerin jelly.

\section{Glycerin Media.}

429. Glycerin.-Glycerin diluted with water is frequently employed as an examination and mounting medium. Dilution with water is sometimes advisable on account of the increased visibility that it gives to many structures. But for efficacious preservation undiluted glycerin, the strongest that can be procured, should be used (see BEALE, How to work, etc.).

For closing glycerin mounts, the edges of the cover should first (after having been cleansed as far as possible from superfluous glycerin) be painted with a layer of glycerin jelly; as soon as this is set a coat of any of the usual cements may be applied. See next chapter.

Glycerin dissolves carbonate of lime, and is therefore to be rejected in the preparation of calcareous structures that it is wished to preserve.

430. Extra-refractive Glycerin.-The already high index of refraction of glycerin (Price's glycerin, $n=1 \cdot 46$ ) may be raised by dissolving suitable substances in it. Thus the refractive index of a solution of chloride of cadmium $\left(\mathrm{CdCl}_{2}\right)$ in glycerin may be 1.504 ; that of a saturated solution of sulphocarbolate of zine in glycerin may be 1.501 ; that of a saturated solution of SCHERING's chloral hydrate (in crusts) in glycerin is 1.510 ; that of iodate of zine in glycerin may be brought up to 1.56. For further details see previous editions, or Journ. Roy. Mic. Soc., ii, 1879, p. 346 ; iii, 1880, p. 1051 ; (N.S.), i, 1881, pp. 943 and 366.

431. Glycerin and Alcohol Mixtures.-These afford one of the best means of bringing delicate objects gradually from weak into strong glycerin. The object is mounted in a drop of the liquid, and left for a few hours or days, the mount not being closed. By the evaporation of the alcohol the liquid gradually increases in density, and after some time the mount may be closed, or the object brought into pure glycerin or glycerin jelly.

1. Calberla's Liquid (Zeit. wiss. Zool., xxx, 1878, p. 442).Glycerin 1 part, alcohol 2, water 3.

2. I strongly recommend the following for very delicate objects :Glycerin 1 part, alcohol 1, water 2 . 
3. Hantsch's Liquid.-Glycerin 1 part, alcohol 3, water 2.

4. Jäger's Liquid (Vogt and Yung's Traité d'Anat. Comp. Prat., p. 16).-Glycerin 1 part, alcohol 1, sea water 10 .

\section{Glycerin Jellies.}

432. Glycerin Jellies have a higher index than pure glycerin, and set hard enough to make luting unnecessary, though it is well to varnish the mount. To use them, you melt a small portion on a slide, introduce the object (previously soaked in water or glycerin), and cover. They seem very plausible, but for delicate work I do not recommend them, and should advise instead either pure glycerin or euparal.

433. Lawrence's Glycerin Jelly (Davies, Preparation and Mounting of Microscopic Objects, p. 84). - Soak some gelatin for two or three hours in cold water, pour off the superfluous water, and heat until melted. To each fluid ounce of the gelatin, whilst it is fluid but cool, he adds a fluid drachm of the white of an egg. Boil until the albumen coagulates and the gelatin is quite clear, and to each ounce of the solution add 6 drachms of a mixture composed of 1 part of glycerin to 2 parts of camphor water.

434. BrandT's Glycerin Jelly (Zeit. wiss. Mik., ii, 1880, p. 69).Melted gelatin 1 part, glycerin $1 \frac{1}{2}$ parts. The gelatin to be soaked in water and melted as above. After incorporating the glycerin, filter through spun glass pressed into the lower part of a funnel. He describes a simple arrangement for keeping the funnel warm during the filtering (see early editions). Some drops of carbolic acid should be added.

435. KAISER's Glycerin Jelly has been given $\S 155$.

436. SquiRE's Glycerin Jelly (SQUIRE's Methods and Formula, etc., p. 84).-Soak 100 grms. of French gelatin in chloroform water, drain when soft, and dissolve with heat in 750 grms. of glycerin. Add 400 grms. of chloroform water with which has been incorporated about 50 grms. of fresh egg-albumen; mix thoroughly, and heat to boiling-point for about five minutes. Make up the total weight to 1550 grms. with chloroform water. Filter in a warm chamber.

43\%. Heidenhain (Zeit. wiss. Mik., xx, 1905, p. 328) takes of gelatin 9 parts, glycerin 7 , and water 42 , and to the filtrate adds drop by drop 14 parts of absolute alcohol.

438. Fischer (ibid., xxix, 1912, p. 65) takes 5 grms. of borax dissolved in 240 c.c. of water and adds 25 c.c. of glycerin. To this 
he adds 40 grms. of gelatin, dissolves with heat, and continues to heat gently until the solution has somewhat thickened. This remains fluid at ordinary temperatures.

439. GiLson's Chloral Hydrate Jelly (communicated by GiLson).1 vol. of gelatin, melted secundum artem, and 1 vol. of Price's glycerin. Mix, and add crystals of chloral hydrate until the volume has increased by one-half; warm till dissolved. This gives a very highly refractive medium.

GEoffroy, Journ. de Botan., 1893, p. 55 (see Zeit. wiss. Mik., ix, 1893 , p. 476), dissolves, by the aid of as little heat as possible, 3 to 4 grms. of gelatin in 100 c.c. of 10 per cent. aqueous solution of chloral hydrate.

\section{High Refractive Liquids.}

440. Stephenson's Biniodide of Mercury and Iodide of Potassium (Journ. Roy. Mic. Soc. [N.S.], ii, 1882, p. 167).-A solution prepared by adding the two salts to water until each is in excess; the liquid will then be found to have a refractive index of 1.68. (If [AMANN, Zeit. wiss. Mik., xiii, 1896, p. 21] glycerin be taken instead of water, it rises to 1.78 or 1.80 . BeHRENs [Tabellen, 1898, p. 71] takes biniodide 65 parts, iodide 50, and water 25. $n=1 \cdot 71$.) Any lower index can be obtained by suitable dilution with water. This fluid is very dense, its specific gravity being 3.02 . It is highly antiseptic.

For marine animals a weak solution is probably well adapted, as about a 1 per cent. solution ( 5 minims to the ounce) will give the specific gravity of sea water.

Covers should be sealed with white wax, and the mounts finished with two or three coatings of gold size and one of shellac.

I have experimented both with strong and with weak solutions. They are not adapted, I find, for the purposes of a permanent mounting medium, for the preparations are ruined by a precipitate which forms in the fluid. But as a temporary examination medium I have occasionally found this solution valuable. Its optical properties are wonderful ; it allows of the examination of watery tissues, without any dehydration, in a medium of refractive index surpassing that of any known resinous medium.

See further details in early editions.

441. Monobromide of Naphthalin.-See Journ. Roy. Mic. Soc., 1880, p. 1043 (ABBe and VAN HeUrCK), and Zool. Anz., 1882, p. 555 (MAX FLESOH). 


\section{Resinous Media.}

442. Resins and Balsams.-Resins and balsams consist of a vitreous or amorphous substance held in solution by an essential oil. By distillation or drying in the air they lose the essential oil and pass into the solid state. It is these solidified resins that should be employed for microscopical purposes ; for the raw resins always contain a certain proportion of water, which makes it difficult to obtain a clear solution with the usual menstrua, is injurious to the optical properties of the medium and to the preservation of stains. All solutions should therefore be made by heating gently the balsam or resin in a stove until it becomes brittle when cold, and then dissolving in an appropriate menstruum.

Solutions made with volatile menstrua, such as xylol and chloroform, set rapidly, but become rapidly brittle. Solutions made with non-volatile media, such as turpentine, set much less rapidly, and pass much less rapidly into the brittle state.

Turpentine media preserve the index of visibility of the preparations much longer than do media made with more volatile menstrua. Preparations made with these often become so transparent in course of time that much fine detail is often lost. (Such mounts may, however, be revivified without removing the cover by putting them for a day or two into a tube of benzol; the benzol penetrates the balsam, and brings it down to a lower refractive index.)

For a permanent mounting medium of somewhat low index I unhesitatingly recommend Euparal. For cases in which a still lower index is desired, Gilson's camsal balsam. Turpentine colophonium is a safe and excellent medium, but is injurious to alumhæmatein stains. For these, and in general where a strongly clearing medium is desired, xylol balsam is about the most recommendable, though it is not perfectly safe, the mounts sometimes developing granules. Seiler's alcohol balsam is a fine medium, and perfectly stable. Oil of cedar is sometimes useful, it keeps perfectly, and with time it thickens sufficiently to hold the cover in place ; or if desired, preparations may be luted with BeLL's eement.

443. Canada Balsam.-Prepare with the solid balsam as described last §. The usual menstrua are xylol, benzol, chloroform, and turpentine. Turpentine has the advantages pointed out last $\S$, but the defect that it does not always give a homogeneous solution with Canada balsam, as it does with colophonium. For most purposes the xylol solution is the best. If time be an object, a benzol solution should be preferred, as it sets much quicker than the xylol solution. 
The chloroform solutions become very brown with age, and are injurious to stains made with tar dyes. Benzol is good when chemically pure and free from water.

SAHLI (Zeit. wiss. Mik., ii, 1885, p. 5) dissolves in cedar oil.

AрÁтHy (Fauna Flora Golf. Neapel, xxii, 1909, p. 18) takes balsam 2 parts, cedar oil (immersion) 1, and chloroform 1.

Samples of balsam that are acid are frequently met with, and are injurious to some stains.

443a. How to make Neutral Balsam.-Take balsam crystals dry. Grind up in mortar and add an equal part of sodium bicarbonate : mix thoroughly. Transfer to a bottle and add enough xylol or benzole to make a quite liquid solution. Allow this to stand for three or four days, shaking occasionally. Filter, and place filtrate on a thermostat to evaporate down to the right consistency. S. G. ScotT (Journ. Path., xvi), recommends keeping a piece of marble in the balsam bottle, and either placing the latter in a tin box, or painting it black outside in order to protect the balsam from the light. See Colucci (Giorn. Ass. Med. Natural Napoli, vii, 1897, p. 172).

444. Seiler's Alcohol Balsam (Proc. Amer. Soc. Mic., 1881, pp. 60-2; Journ. Roy. Mic. Soc. [N.S.], ii, 1882, pp. 126-7).Dissolve solid balsam in warm absolute alcohol, and filter through absorbent cotton. Objects may be mounted in it direct from absolute alcohol. I find it for most purposes admirable. It is one of the most stable solutions known to me. Care should be taken not to breathe on it, as this may cause cloudiness.

445. Damar (Gum Damar, or Dammar, or d'Ammar). - The menstrua are the same as for balsam. I find xylol the best. For directions for preparing solutions, by various authors, see early editions. After ample experience I am convinced that not one of these solutions can be depended on for permanent preservation. Sooner or later, sometimes after a few weeks or days, or it may be only after months or years, granules make their appearance in the mounts.

446. Colophonium.-A solution of pale colophonium in oil of turpentine keeps well and gives very good definitions. The solution should not be too thick, as it thickens with age.

This medium dries very slowly (so that ample time is afforded for arranging objects in it). In the winter a slide will take about a month before it will be hard enough to be safe with oil-immersion lenses; whereas an alcohol-balsam mount will be dry enough in a couple of days. It injures alum-hæmatein stains ; as it sometimes develops clouds of globules it is not to be depended upon. 
Dr. S. G. ScotT of Oxford used both damar and colophonium instead of balsam, and very few of his preparations left after his decease are good. A large number have become granular.

ReHM (Zeit. wiss. Mik., ix, 1893, p. 387) dissolves 1 part colophonium in 10 of benzin. Solutions in chloroform or xylol are also used by some, see NissL in Encycl. mik. Techn., ii, p. 274.

447. Venice Turpentine (Vosseler, Zeit. wiss. Mik., vi, 1889, pp. 292 et seq.).-Commercial Venice turpentine is mixed in a tall cylinder glass with an equal volume of 96 per cent. alcohol, allowed to stand in a warm place for three or four weeks, and decanted. Preparations may be mounted in this medium direct from absolute alcohol. Celloidin sections can be mounted direct from 96 per cent. Stains keep well, according to VOSSELER, but MAYER finds hæmalum stains fade in it.

SuChanneK (ibid., vii, 1896, p. 463) prepares it with equal parts of Venice turpentine and neutral absolute alcohol.

448. Thickened Oil of Turpentine has been used as a mounting medium by some workers. To prepare it, pour some oil into a plate, cover it lightly so as to protect it from dust without excluding the air, and leave it until it has attained a syrupy consistency.

449. Gilson's Sandarac Media (La. Cellule, xxiii, 1906, p. 427 : the formulæ have not been published, on account of the extreme difficulty of preparation, but the products are on sale by Grübler \& Hollborn, even if not listed). There are three of these. They are all of them solutions of gum Sandarac in "Camsal" and other solvents ("Camsal" is a liquid formed by the mutual solution of the two solids salol and camphor).

(1) Camsal balsam (baume au camsal), propylic alcohol formula; a mixture of sandarac, camsal, and propylic alcohol, $n=0 \cdot 478$.

(2) Camsal balsam, isobutylic alcohol formula, $n=1 \cdot 485$. ephane $=(3)$ Euparal, * a mixture of camsal, sandarac, eucalyptol, and paraldehyde, $n=1 \cdot 483$. There are two sorts of this, the colourless and the green ("euparal vert"), the latter containing a salt of copper, which intensifies hoematoxylin stains.

Objects may be prepared for mounting in camsal balsam by a bath of propylic or isobutylic alcohol ; and for euparal by a bath of the special solvent (supplied by Grübler \& Hollborn under the name of " essence d'euparal "). But this is not necessary. Objects may always be mounted direct from absolute alcohol, and even at a pinch from alcohol of 70 per cent. I myself generally prefer alcohol of 95 per cent. (absolute is dangerously volatile for sections). In

* Now manufactured by Flatters \& Garnett (\$11) according to Gilson's original formula. 
difficult cases you may pass through a mixture of the medium and the solvent.

These media work very kindly, and do not dry too rapidly. They are not oxidant, and preserve delicate stains (perfectly, so far as I know). The mounts seem to keep perfectly, without scaling: all of mine, the oldest being eight years old, have kept without the slightest deterioration in any respect.

The primary intention of these media is to spare delicate objects the usual treatment with absolute alcohol and essential oils. But they have another useful property-their low index of refraction. I find that that of euparal is just right for most delicate cytological researches, giving just the desired increase of visibility to unstained elements. Thus I frequently find that unstained spindles which are totally invisible in balsam become strongly visible in the most minute details in euparal. The camsal balsam, $n=1 \cdot 478$, I have also sometimes found valuable, but its index is a little too low for most things, and I generally prefer euparal, which I find I am now using almost as much as balsam. I consider that all the media which have been recommended on the score of a slightly lower index than balsam, such as damar, colophonium, Venice turpentine, castor-oil, are now superseded by these media.

450. Sandarac (LavdowsKy, form Ref. Handbook Med. Sci., Supp., p. 438).-Gum sandarac 30 grs., absolute alcohol 50 c.c. Not trustworthy, the mounts scale badly.

451. Photographic Negative Varnish (for mounting large sections without cover-glasses).-See WeIGERT, Zeit. wiss. Mik., iv, 1887, p. 209.

452. Castor Oil.-See Grenacher, Abhandl. naturf. Ges. Halle-a.-S., Bd. xvi ; Zeit. wiss. Mik., 1885, p. 244. I have not had good results with it.

453. Terpinol. $-n=1 \cdot 484$. See $\S 131$.

454. Parolein (a pure form of paraffinum liquidum) is recommended by Coles (Lancet, 1911, p. 878) as being quite neutral and preserving certain coal tar stains. Ring mounts with Apáthy's gum syrup, § 343. Its index is 1.471 , which I find too low for most things.

455. Cedar Oil.-See § 442, sub fin.

456. Gum Thus, dissolved in xylol, is recommended by EISEN, Zeit. wiss. Mik., xiv, 1897, p. 201.

457. Styrax and Liquidambar.-See Journ. Roy. Mic. Soc., 1883, p. 741 ; ibid., 1884, pp. $318,475,655$, and 827 ; and the places there quoted. Also Bull. Soc. Belge de Mic., 1884, p. 178; and For, Lehrb., p. 141. These are very highly refractive media, therefore seldom useful in histology. 


\section{CHAPTER XX.}

\section{CEMENTS AND VARNISHES.}

458. Introduction.-Two, or at most three, of the media given below will certainly be found sufficient for all useful purposes. For many years I have used only one cement (BELL's). I recommend this both as a cement and varnish ; gold size may be found useful for turning cells; and MrLueR's caoutchouc cement may be kept for occasions on which the utmost solidity is required. Marine glue is only necessary for making glass cells.

For the operations of mounting in fluids, and of making cells and ringing, see CARPEnTER's The Microscope.

CARPEnter lays great stress on the principle that the cements or varnishes used for fluid mounts should always be such as contain no mixture of solid particles, for those that do always become porous after a certain lapse of time. All fluid mounts should have the edges of the cover carefully dried and be ringed with glycerin jelly before applying a cement; by this means all danger of running in is done away with. See $\S \S 460$ and 461 . But no method yet devised will make a glycerine mount absolutely permanent.

See also Aubert, The Microscope, xi, 1891, 150, and Journ. Roy. Mic. Soc., 1891, p. 692 ; Beck, The Microscope, xi,. 1891, pp. 338, 368, and Journ. Roy. Mic. Soc., 1892, p. 293 ; Behrens' Tabellen zum Gebrauch bei mikroskopischen Arbeiten (Bruhn, Braunschweig, 1892) ; Rousselet, Journ. Quek. Mic. Club, vii, 1898, p. 93 ; and as to the comparative tenacity of divers cements, BeHrens, Zeit. wiss. Mik., ii, 1885, p. 54, and Aubert Amer. Mon. Mic. Journ., 1885, p. 227 ; Journ. Roy. Mic. Soc., 1886, p. 173.-AuBERT places MILLER's caoutchouc cement at the head of the list, LovetT's cement coming halfway down, and zinc white cement at the bottom, with less than one-quarter the tenacity of the caoutchouc cement.

459. Paraffin.-Temporary mounts may be closed with paraffin, or white wax, by applying it with a bent wire, as described $\S 471$, and be made more or less permanent by varnishing.

460. Gelatin Cement (Marsh's Section-cutting, 2nd ed., p. 104).Take half an ounce of NELson's opaque gelatin, soak well in water, 
melt in the usual way, stir in 3 drops of creasote. It is used warm.

When the ring of gelatin has become quite set and dry, it may be painted over with a solution of bichromate of potash made by dissolving $10 \mathrm{grs}$. of the salt in an ounce of water. This should be done in daylight, in order to render the gelatin insoluble. The cover may then be finished with BELL's cement. This process is particularly adapted for glycerin mounts.

461. The Paper Cell Method.-By means of two punches I cut out rings of paper of about a millimetre in breadth, and of about a millimetre smaller in diameter than the cover-glass. Moisten the paper ring with mounting fluid, and centre it on the slide. Fill the cell thus formed with mounting fluid; arrange the object in it; put the cover on; fill the annular space between the paper and the margin of the cover with glycerin jelly (a turn-table may be useful for this) ; and as soon as the gelatin has set turn a ring of gold-size on it, and when that is quite dry, varnish with BELL's cement.

For greater safety, the gelatin may be treated with bichromate, according to MARSH's plan, last §.

462. Rousselet's Method for Aqueous Mounts (op. cit., § 458).Close the mount with a ring of a mixture of 2 parts of a solution of damar in benzol and 1 part gold-size. When dry, put on three or four thin coats of pure gold-size at intervals of twenty-four hours, and finish with a ring of WARD's brown cement.

463. Miller's Caoutchouc Cement.-Composition unknown. May be obtained from the opticians. A very tenacious and quickly drying cement. It may be diluted by a mixture of equal parts of chloroform and strong alcohol (see Rousselet, Journ. Quek. Club, v, ii, 1895, p. 8).

464. Asphalt Varnish (Bitume de Judée).-Unquestionably one of the best of these media, either as a cement or a varnish, provided it be procured of good quality. It can be procured from the opticians.

465. Brunswick Black. -See early editions, or BEALE, How to Work, etc., p. 49.

466. Gold-Size.-Best obtained from the opticians. It is soluble in oil of turpentine. A good cement, when of good quality, and very useful for turning cells.

46\%. Turpentine, Venice Turpentine (Csokor, Arch. mik. Anat., xxi, 1882, p. 353 ; Parker, Amer. Mon. Mik. Journ., ii, 1881, 
pp. $229-30)$.-Venice turpentine, or common resinous turpentine, evaporated by heat until brittle on cooling. It is used for closing glycerin mounts in the following manner: Square covers are used, and superfluous glycerin is cleaned away from the edges in the usual way. The cement is then put on with a piece of wire vent at right angles ; the short arm of the wire should be just the length of the side of the cover-glass. The wire is heated in a spirit lamp, plunged into the cement, some of which adheres to it, and then brought down flat upon the slide at the margin of the cover. The turpentine distributes itself evenly along the side of the cover, and hardens immediately, so that the slide may be cleaned as soon as the four sides are finished. It is claimed for this cement that it is perfectly secure and never runs in. It sets hard in a few seconds.

468. ApÁthy's Cement for Glycerin Mounts (Zeit. wiss. Mik., vi, 1889 , p. 171).-Equal parts of hard $\left(60^{\circ} \mathrm{C}\right.$. melting-point) paraffin and Canada balsam. Heat together in a porcelain capsule until the mass takes on a golden tint and no longer emits vapours of turpentine. Used by warming and applying with a glass rod or brass spatula. One application is enough. Does not run in, and never cracks.

469. Canada Balsam, or Damar.-Cells are sometimes made with these. They are elegant, but in my experience are not reliable for permanent mounts.

470. Tolu Balsam Cement (CARnoy's Biol. Cell., p. 129).-Tolu balsam, 2 parts, Canada balsam 1, saturated solution of shellac in chloroform, 2 parts. Add enough chloroform to bring the mixture to a syrupy consistence. Carnoy finds this cement superior to all others.

4\%1. For the cements of Ward, Bell and Clarke and for Krönig's Colophonium and Wax, Marine Glue, Amber and Copal, and Sealing Wax Varnish, see last edition. 


\section{PAR'T II.}

\section{SPECIAL METHODS AND EXAMPLES.}

\section{CHAPTER XXI.}

INJECTION-GELATIN MASSES (WARM).

472. Introduction.-Injection masses are composed of a coloured substance called the colouring mass, and of a substance with which that is combined called the vehicle.

For instructions as to the operation of injecting, and the necessary apparatus, see the Micrographic Dictionary, Rutherforn's and SCHÄFER's Practical Histology, the treatises of RoBIN and Ranvier, Beale's How to Work with the Microscope, the Lehrbuch der vergleichenden Mikroscopischen Anatomie of FoL, and (for apparatus especially) the article in the Encycl. d. mik. Technik. For injections for the study of the angiology of Vertebrates the practice of Robin and Ranvier may safely be followed. For injections of Invertebrates (and indeed, for vertebrates if it is desired to demonstrate the minute structure of environing tissues at the same time as the distribution of vessels) masses not containing gelatin are, I think, generally preferable to gelatin masses; and I would recommend as particularly convenient the Prussian blue glycerin masses of BEALE. Glycerin masses have the great advantage that they are used cold.

All formulæ which only give opaque masses, or are only suitable for coarse injections for naked eye study, have been suppressed.

In $\S 592$ is a section on injection of embryos.

473. Vaso-dilators. - In order that an injection may run freely it is necessary that the vessels of the subject be in a relaxed state. To this end the older anatomists used to wait until rigor mortis had passed off before injecting. But it is evidently preferable in the interest of the proper preservation of the tissues to inject before rigor mortis has set in. Unfortunately, when this is done, it is found that most injection masses-glycerin masses especially- 
stimulate the contraction of the vessels, so that frequently it is very difficult to get the injection in. In these cases it may be advisable to use a vaso-dilator. The animal may be anæsthetised with a mixture of ether and nitrite of amyl, and finally killed with pure nitrite. Or, after killing by nitrite, a little nitrite of amyl in salt solution may be injected before the injection mass is thrown in. In any case it is advisable to add a little nitrite to the mass just before using. The relaxing power is very great (see OviatT and SARgEnt, in St. Louis Med. Journ., 1886, p. 207 ; and Journ. Roy. Mic. Soc., 1887, p. 341).

BAYLISS (in personal communication) suggests for prevention of coagulation, to wash out in citrate of soda (4 per cent.) instead of - $75 \mathrm{NaCl}$, or to add $\frac{1}{2}$ per cent. oxalate of calcium to $\cdot 75$ per cent. $\mathrm{NaCl}$. To relax arterial walls, add sodium nitrite 1 in 500 to the washing out fluid.

Or, morphia may be added to the injection mass, or 1 per cent. of lactic acid. Mozejкo (Zeit. wiss. Mik., xvi, 1909, p. 545) prefers a saturated solution of neutral Peptonum siccum, which has the advantage of hindering coagulation. For warm-blooded animals the mass should be warmed to body-temperature; and in all cases masses that tend to dehydrate tissues should be avoided if possible.

\section{RoBin's Masses.}

474. RoBIN's Gelatin Vehicle (Traité, p. 30).-One part of gelatin soaked and melted in $7,8,9$, or even 10 parts of water, on a waterbath.

This vehicle, like all gelatin masses, is liable to be attacked by mould if kept long; camphor and carbolic acid do not suffice to preserve it. Chloral hydrate 2 per cent. is said to do so.

475. RoвIN's Glycerin-Gelatin Vehicle (Traité, p. 32).-Dissolve in a water-bath 50 grms. of gelatin in 300 grms. of water, in which has been dissolved some arsenious acid ; add of glycerin 150 grms., and of carbolic acid a few drops. Unlike the pure gelatin vehicles, . this mass does keep indefinitely.

Frankl (Zeit. f. wiss. Zool., lxiii, 1897, p. 28) prepares a similar vehicle, and adds to it a little solution of corrosive sublimate and a crystal of thymol.

476. RobIN's Carmine Colouring Mass (Traité, p. 33).--Rub up 3 grms. of carmine with a little water and enough ammonia to dissolve it. Add 50 grms. of glycerin and filter. 
Take 50 grms. of glycerin with 5 grms. of acetic acid, and add it by degrees to the carmine-glycerin, until a slightly acid reaction is obtained (as tested by very sensitive blue test-paper, moistened and held over the mixture).

One part of this mixture is to be added to 3 or 4 parts of the vehicles given above.

477. Robin's Ferrocyanide of Copper Colouring Mass (ibid., p. 34).Take -

(1) Ferrocyanide of potassium (concentrated solution) . . . . . 20 c.c. Glycerin . . . . . . 50 ,

(2) Sulphate of copper (concentrated solution). 35 ", Glycerin . . . . 50 ,

Mix (1) and (2) slowly, with agitation; at the moment of injecting combine with 3 volumes of vehicle.

4\%8. Robin's Prussian Blue Colouring Mass (ibid., p. 35, and 2nd ed., p. 1013).

Take-

(A) Ferrocyanide of potassium* (sol. sat.) . . 90 c.c. Glycerin

(B) Liquid perchloride of iron at $30^{\circ}$ Baumé . . 3 , Glycerin . . . . . . 50 ,

Mix slowly and combine the mixture with 3 parts of vehicle. It is well to add a few drops of $\mathrm{HCl}$.

\section{Carmine-gelatin Masses.}

479. RANVIER's Carmine-Gelatin Mass (Traité technique, p. 116).Take 5 grms. Paris gelatin, soak until quite swollen and soft, wash, drain and melt it in the water it has absorbed over a water-bath. When melted add slowly, and with continual agitation, $2 \frac{1}{2}$ grms. of carmine rubbed up with a little water, and just enough ammonia, added drop by drop, to dissolve the carmine into a transparent solution.

The mixture is now neutralised by adding cautiously, drop by drop, with continual agitation, a solution of 1 part of glacial acetic acid in 2 parts of water. (When the mass is near neutrality, dilute the acetic acid still further.) The instant of saturation is determined by the smell of the solution, which gradually changes from ammoniacal to sour. As soon as the sour smell is perceived the liquid must be examined under the microscope. If it contains a granular precipitate of carmine, too much acid has been added, and it must be thrown away.

* Erratum "Sulphocyanide" in 1st ed. of RoBIn's Traité. 
The mass, having been perfectly neutralised, is strained through new flannel.

480. How to Neutralise a Carmine Mass (Ville, Gaz. hebd. d. Sci. méd. de. Montpellier, Fév., 1882; may be had separately from Delahaye et Lecrosnier, Paris).-VILLe points out that when carmine is treated with ammonia a certain proportion of the ammonia combines with the carmine and the rest remains in excess. It is this excess that it is required to neutralise precisely, not the whole of the ammonia employed.

To neutralise the acidity of commercial gelatin, it should be washed for an hour or so in running water.

As to the neutralisation of the colouring mass, VILLE is of opinion that the sour smell cannot be safely relied on in practice, and prefers to employ dichroic litmus paper (litmus paper sensitised so as to be capable of being used equally for the demonstration of acids and bases). For directions for preparing this see loc. cit. or previous editions.

481. Hoyer's Carmine-Gelatin Mass (Biol. Centralb., 1882, p. 21).-Take a concentrated gelatin solution and add to it the needful quantity of neutral carmine staining solution (loc. cit., p. 17). Digest in a water-bath until the dark violet-red colour begins to pass into a bright red tint. Then add 5 to 10 per cent. by volume of glycerin, and at least 2 per cent. by weight of chloral, in a concentrated solution, and strain.

482. Fol's Carmine-Gelatin Mass (Lehrb., p. 13). This can be kept in the dry state for an indefinite length of time.

Gelatin in sheets is cut into strips which are macerated for two days in carmine solution (prepared by diluting one volume of strong ammonia with three of water and adding carmine to saturation, and filtering after a day or two). The strips are then rinsed and put for a few hours into water acidulated with acetic acid, then washed on a sieve for several hours in running water, dried on parchment paper, or on a net, and preserved for future use. To get the mass ready for use, the strips are soaked for an hour in water and melted on a water-bath in 10 to 20 parts of water.

For another process, which is said to give somewhat better results, but is more complicated, see loc. cit., or Zeit. wiss. Zool., $\mathrm{xxxviii,} \mathrm{p.} \mathrm{492,}$ or previous editions.

483. KRaUSE's Carmine-Gelatin Mass (Zeit. wiss. Mik., xxvi, 1909, p. 1). -100 grms. gelatin soaked in water, put for two to three days into a solution of $15 \mathrm{grms}$. carmine in 2 litres of water with $100 \mathrm{grms}$. of borax, 
washed, treated for a short time with hydrochloric acid of 2 per cent., washed, melted and preserved with eamphor.

484. Other Carmine-Gelatin Masses.-Thierscir's, see Arch. mik. Anat., 1865, p. 148. Gerlach's, see Ranvier, Traité, p. 118, Carter's, see Beale, p. 113. Davies, see his Prep. and Mounting of Mic. Objects, p. 138.

\section{Blue Gelatin Masses.}

485. Ranvier's Prussian Blue Gelatin Mass (Traité, p. 119).Make a concentrated solution of sulphate of peroxide of iron in distilled water, and pour it gradually into a concentrated solution of yellow prussiate of potash. There is produced a precipitate of insoluble Prussian blue. Wash this on a felt strainer, underneath which is arranged a paper filter in a glass funnel, for some days, until the liquid begins to run off blue from the second filter. The Prussian blue has now become soluble. The strainer is turned inside out and agitated in distilled water; the Prussian blue will dissolve if the quantity of water be sufficient.

The solution may now be injected just as it is, or it may be kept in bottles till wanted, or evaporated in a stove, and the solid residuum put away in bottle.

For injections, if a simple aqueous solution be taken, it should be saturated. Such a mass never transudes though the walls of vessels. Or it may be combined with one-fourth of glycerin, or with one twenty-fifth of gelatin soaked for an hour in water and melted over a water-bath in the water it has absorbed. The gelatin is to be poured gradually into the Prussian blue, on the water-bath, stirring continually until the curdy precipitate that forms at first has disappeared. Filter through new flannel and keep at $40^{\circ} \mathrm{C}$. until injected.

486. BRÜCKE's Soluble Berlin Blue (Arch. mik. Anat., 1865, p. 87).Make a solution of ferrocyanide of potassium containing $217 \mathrm{grms}$. of the salt to 1 litre of water, and one of 1 part commercial chloride of iron in 10 parts water. Take equal volumes of each, and add to each of them twice its volume of a cold saturated solution of sulphate of soda. Pour the chloride solution into the ferrocyanide solution, stirring continually. Wash the precipitate on a filter until soluble, dry it, press between blotting paper in a press, break the mass in pieces, and dry in the air.

The concentrated solution of the colouring matter is to be gelatinised with just so much gelatin that the mass forms a jelly when cold. For another method, see previous editions.

487. Other Blue Gelatin Masses.-Hoyer's, Arch. mik. Anat., 1876, p. 649 ; GUIGNeT's, Journ. de Microgr., 1889, p. 94 ; Journ. Roy. Mic. 
Soc., 1889, p. 463 ; ThIersch's, Arch. mik. Anat., i, 1865, p. 148 ; 'Fol's, Zeit. wiss. Zool., xxxviii, 1883, p. 494 ; and previous editions.

\section{Other Colours.}

488. Hoyer's Silver Nitrate Yellow Gelatin Mass (Biol. Centralbl., ii, 1882, pp. 19, 22). - A concentrated solution of gelatin is mixed with an equal volume of a 4 per cent. solution of nitrate of silver and warmed. To this is added a very small quantity of an aqueous solution of pyrogallic acid, which reduces the silver in a few seconds ; chloral and glycerin are added as directed $\S 489$.

This mass is yellow in the capillaries and brown in the larger vessels.

489. Other Colours.-Hoyer's Green (Biol. Centralb., ii, 1882, p. 19). Made by mixing a blue mass and a yellow mass. ThIERsch's Green (Arch. mik. Anat., 1865, p. 149). Robin's Scheele's Green (Robin, Traité, p. 37). Harting's White (see Frey, Le Microscope, p. 190). Frey's White (ibid.). Teichmann's White (ibid., p. 191). Fol's Brown (Zeit. wiss. Zool., xxxviii, 1883, p. 494). MilleR's Purple (see Amer. Mon. Mic. Journ., 1888, p. 50 ; Journ. Roy. Mic. Soc., 1888, p. 518). Fol's Lead Chromate (Lehrb., p. 15). RoBIN's Cadmium (his Traité, p. 36). Thiensch's Lead Chromate (Arch. mik. Anat., 1865, p. 149). HoYer's Lead Chromate (ibid., 1867, p. 136); or, for any of these, see early editions.

490. RaNviER's Gelatin Mass for Impregnation (Traité, p. 123).Concentrated solution of gelatin, 2, 3, or 4 parts ; 1 per cent. nitrate of silver solution, 1 part.

Nedvilue (Ann. Sci. Nat., xiii, 1901, p. 36) takes a solution of 10 grms. of soaked gelatin in 100 c.c. of 1 per cent. solution of nitrate of silver.

491. Friedenthal's Hardening Mass (Centralb. Phys., xiii, 1899, p. 267). - A 10 per cent. solution of gelatin, combined with a colouring mass, and with 1 volume of 4 per cent. formol, serves for injecting vessels and hardening the tissues at the same time. 


\section{CHAPTER XXIl.}

INJECTIONS-OTHER MASSES (COLD).

492. Fol's Metagelatin Vehicle (Lehrb., p. 17).-If a slight proportion of ammonia be added to a solution of gelatin, and the solution be heated for several hours, the solution passes into the state of metagelatin, that is, a state in which it no longer coagulates on cooling and can be injected without warming. Colouring masses may be added to this vehicle, which may also be thinned by the addition of weak alcohol. After injection the preparations are thrown into strong alcohol or chromic acid, which sets the mass.

According to the Encycl. mik. Technik., metagelatin is usually prepared by warming with concentrated acetic or oxalic acid. It may be neutralised afterwards with carbonate of lime.

493. TANDLER's Gold Gelatin Mass (Zeit. wiss. Mik., xviii, 1901, p. 22).-Five grms. of gelatin are soaked in 100 e.c. of water, warmed and melted, and combined with Berlin blue. Then 5 to 6 grms. of iodide of potassium are slowly incorporated. The mass generally remains liquid enough for injection down to a temperature of $17^{\circ} \mathrm{C}$, but if it should coagulate a little more iodide should be added. After injection you may fix with 5 per cent. formol. The specimens will bear decalcification with hydrochloric or sulphurous acid.

Pearl (Journ. Appl. Micr., v, 1902, p. 1736) takes 8 to 10 per cent. of the iodide.

MAYER (Grundzüge LeE and MAYer, 1910, p. 250) takes simply 10 grms. gelatin, 10 grms. hydrate of chloral and 100 c.c. water.

MozeJкo (Zeit. wiss. Mik., xxvii, 1910, p. 374) finds that 10 per cent. (or more) of sodium salicylate will retard the setting of gelatin for hours at normal temperatures.

Any of these masses may be made to set in the tissues by means of weak formol.

\section{Glycerin Masses.}

494. Beale's Carmine Glycerin Mass (How to Work, etc., p. 95).Five grains of carmine are dissolved in a little water with about 5 drops of ammonia, and added to half an ounce of glycerin. Then add half an ounce of glycerin with 8 or 10 drops of acetic or hydrochloric acid, gradually, with agitation. Test with blue litmus 
paper, and if necessary add more acid till the reaction is decidedly acid. Then add half an ounce of glycerin, two drachms of alcohol, and six drachms of water. I have found this useful, but not so good as the two following.

495. Beale's Prussian Blue (How to Work, etc., p. 93).

Common glycerin

Spirits of wine.

Ferrocyanide of potassium . . . 12 grains.

Tincture of perchloride of iron . . 1 drachm.

Water

4 ounces.

Dissolve the ferrocyanide in one ounce of the water and glycerin, and add the tincture of iron to another ounce. These solutions should be mixed together very gradually, and well shaken in a bottle, the iron being added to the solution of the ferrocyanide of potassium. Next the spirit and the rest of water are to be added very gradually, the mixture being constantly shaken.

Injected specimens should be preserved in acidulated glycerin (e.g., with 1 per cent. acetic acid), otherwise the colour may fade.

496. Beale's Acid Prussian Blue (ibid., p. 296).

Price's glycerin .

Tinct. of sesquichloride of iron

Ferrocyanide of potassium .

Strong hydrochloric acid

Water
- 2 fluid ounces.

- 10 drops.

- 3 grains.

3 drops.

1 ounce.

Proceed as before, dissolving the ferrocyanide in one half of the glycerin, the iron in the other, and adding the latter drop by drop to the former. Finally add the water and HCl. Two drachms of alcohol may be added to the whole if desired.

I find this excellent.

497. Ranvier's Prussian Blue Glycerin Mass (Traité, p. 120).-The Prussian blue fluid, $§ 494$, mixed with one fourth of glycerin.

498. Thома's Indigo-Carmine (Arch. Anat. Phys., Anat. Abth., 1899 , p. 270).-Dissolve 0.15 grm. sulphindigotate of soda in 50 c.c. water, filter, add 40 c.c. glycerin and gradually, with agitation, 10 c.c. of a filtered 10 per cent. solution of sodium chloride in water. If desired, 3 c.c. of a 1 per cent. solution of morphia may be added to dilate arteries. A fine precipitate is formed, which is injected with the mass.

499. Gamboge Glycerin (HaRting, Das Mikroskop, 1866, 2, Theil, p. 124).-Gamboge rubbed up with water and added to 
glycerin; or a saturated alcoholic solution of gamboge added to a mixture of equal parts of glycerin and water. Any excess of alcohol may be got rid of by allowing the mass to stand for twenty-four hours.

500. Other Colours. - Any of the colouring masses, $\S \S 485$ to 498 , or other suitable colouring masses, combined with glycerin, either dilute or pure.

\section{Purely Aqueous Masses: (See § 592.)}

501. RanvieR's Prussian Blue Aqueous Mass (Traité, p. 120).- The soluble Prussian blue, § 494, injected without any vehicle. It does not extravasate.

502. MülleR's Berlin Blue (Arch. mik. Anat., 1865, p. 150).Precipitate a concentrated solution of Berlin blue by means of $\frac{1}{2}$ to 1 volume of 90 per cent. alcohol. The precipitate is very finely divided; and the fluid may be injected at once.

503. Mayer's Berlin Blue (Mitth. Zool. Stat. Neapel, 1888, p. 307).-A solution of 10 c.c. of tincture of perchloride of iron in 500 c.c. of water is added to a solution of 20 gr. of yellow prussiate of potash in 500 c.c. of water, allowed to stand for twelve hours, decanted, the deposit washed with distilled water on a filter until the washings come through dark blue (one to two days), and the blue dissolved in about a litre of water. It is well to add a little acetic acid and to put up the objects in an acid liquid.

504. Emery's Aqueous Carmine (ibid., 1881, p. 21). - To a 10 per cent. ammoniacal solution of carmine is added acetic acid, with continual stirring, until the colour of the solution changes to blood-red. The supernatant clear solution is injected cold without further preparation. The injected organs are thrown at once into strong alcohol to fix the carmine. For injection of fishes.

505. TAGuchi's Indian Ink (Arch. mik. Anat., 1888, p. 565).Chinese or (better) Japanese ink well rubbed up on a hone until a fluid is obtained that does not run when dropped on thin blottingpaper, nor form a grey ring round the drop. Inject until the preparation appears quite black, and throw it into some hardening liquid (not pure water).

Della Rosa (Ver. Anat. Ges., 1900, p. 141) recommends the liquid Chinese ink sold in the shops.

Partially Aqueous Masses.

506. Joseph's White-of-Egg (Ber. naturw. Sect. Schles. Ges., 1879, pp. 36-40; Journ. Roy. Mic. Soc., ii, 1882, p. 274).—“ Fil- 
tered white-of-egg, diluted with 1 to 5 per cent. of carmine solution. ... This mass remains liquid when cold, coagulates in dilute nitric acid, chromic or osmic acid, and remains transparent in the vessels." For invertebrates.

Grosser (Zeit. wiss. Mik., xvii, 1900, p. 178) rubs up Indian ink with white-of-egg; Hoffmann (Zeit. Morph. Anthrop., iii, 1901, p. 240) with blood-serum; so also Hamburger, Zeit. wiss. Mik., xxv, 1908, p. 1 (2 vols. of the ink-"Perltusche" - to 3 of serum).

50\%. BJeloussow's Gum Arabic Mass (Arch. Anat. Phys., 1885, p. 379).-Make a syrupy solution of gum arabic and a saturated solution of borax in water. Mix the solutions in such proportions as to have in the mixture 1 part of borax to 2 of gum arabic. Rub up the transparent, almost insoluble mass with distilled water, added little by little, then force it through a fine-grained cloth. Repeat these operations until there is obtained a mass that is free from clots. It should then coagulate in the presence of alcohol, undergoing at the same time a dilatation to twice its original volume. The vehicle thus prepared may be combined with any colouring mass except cadmium and cobalt.

After injection the preparation is thrown into alcohol, and the mass sets immediately, swelling up as above described, and consequently showing vessels largely distended.

Cold-blooded animals may be injected whilst alive with this mass. It does not flow out of cut vessels. Injections keep well in alcohol. If it be desired to remove the mass from any part of a preparation, this is easily done with dilute acetic acid.

508. Milk has been recently recommended by Fischer (Centralb. allg. Path., xiii, 1902, p. 277 ; Zeit. wiss. Mik., xx, 1903, p. 224). It runs well, does not extravasate, and can be used for auto-injection of the living subject.

After injection it should be coagulated by putting the organs for at least twenty-four hours into a mixture of 75 parts of formol, 15 of acetic acid, and 1,000 of water (pure formol will not do). They are then sectioned, and the sections stained with Sudan III or Scharlach $R$, which stain the milk. They cannot be mounted in balsam.

\section{Celloidin and other Masses.}

509. SchiefFerdeCKer's Celloidin Masses (Arch. Anxt. Phys., 1882 [Anat. Abth.], p. 201). (For Corrosion preparations.)-See previous editions; HochstetTER's Modification of SCHIEFFERDECKER's Mass (Anat. Anz., 1886, p. 51) ; BuDGE's Asphaltum Mass (Arch. mil. Anat., 
xiv, 1877, p. 70), or early editions; HOYER's Shellac Mass (Arch. mik. Anat., 1876, p. 645). For this and that of BELlarminow (Anat. Anz., 1888 , p. 605), see early editions; HOYER's Oil-colour Masses (Internat. Monatsschr. Anat., 1887, p. 341); Severeanu's, Verh. Anat. Ges., 21 vers, 1906, p. 275 ; Panscir's Starch Mass (Arch. Anat. Entw., 1877, p. $480 ; 1880$,pp. 232,$371 ; 1881$, p. $76 ; 1882$, p. $60 ; 1883$, p. 265 ; and a modification of the same by GAGE, Amer. Mon. Mic. Journ., 1888, p. 195); Teichmann's Linseed-Oil Masses (S. B. Math. Kl. Krakau - Akad., vii, pp. 108, 158 ; Journ. Roy. Mic. Soc., 1882, pp. 125 and 716, and 1895, p. 704); Fuint's Celluloid (Amer. Journ. Anat., i, 1902, p. 270) ; Huber's (ibid., vi, 1907, p. 393); Krassuskaja's Photoxylin (Anat., Heft. 2, xiii, 1904, p. 521).

510. Natural Injections (RoBIN, Traité, p. 6).-To preserve these throw the organs into a liquid composed of 10 parts of tincture of perchloride of iron and 100 parts of water.

Retterer and Zenker use solution of Müller, see Journ. Anat. Phys., 1894, p. 336, and Arch. Path. Anat., 1894, p. 147.

511. Starch Masses. See "Guides for Vertebrate Dissection," Kingsley, New York, 1907. 


\title{
CHAPTER XXIII.
}

\author{
MACERATION, DIGESTION, AND CORROSION.
}

\section{Maceration.}

512. Methods of Dissociation.-It is sometimes necessary, in order to obtain a complete knowledge of the forms of the elements of a tissue, that the elements be artificially separated from their place in the tissue and separately studied after they have been isolated both from neighbouring elements and from any interstitial cement-substances that may be present in the tissue. Simple teasing with needles is often insufficient, as the cement-substances are frequently tougher than the elements themselves, so that the latter are torn and destroyed in the process. In this case recourse must be had to maceration, by which is meant prolonged soaking (generally for days rather than hours) in media which have the property of dissolving, or at least softening, the cement substances or the elements of the tissue that it is not wished to study, whilst preserving the forms of those it is desired to isolate. . When this softening has been effected, the isolation is completed by teasing, or by agitation with liquid in a test-tube, or by the method of tapping, which last gives in many cases (many epithelia, for instance) results which could not be attained in any other way. The macerated tissue is placed on a slide and covered with a thin glass cover supported at the corners on four little feet made of pellets of soft wax. By tapping the cover with a needle it is now gradually pressed down, whilst at the same time the cells of the tissue are segregated by the repeated shocks. When the segregation has proceeded far enough, mounting medium may be added and the mount closed.

A good material for making wax feet is obtained (Vosseler, Zeit. wiss. Mik., vii, 1891, p. 461) by melting white wax and stirring into it one-half to two-thirds of Venice turpentine.

The most desirable macerating media are those which, whilst dissolving intercellular substances, do not attack the cells themselves. Those which contain colloids have been found to give the best results in this respect. Iodised serum is an example.

513. Iodised Serum (Chap. XIX.).-The manner of employing it for maceration is as follows: A piece of tissue smaller than a pea 
must be taken, and placed in 4 or 5 c.c. of weakly iodised serum in a well-closed vessel. After one day's soaking the maceration is generally sufficient, and the preparation may be completed by teasing or pressing out, as indicated last $\S$; if not, the soaking must be continued, fresh iodine being added as often as the serum becomes pale by the absorption of the iodine by the tissues. By taking this precaution the maceration may be prolonged for several weeks.

This method is intended to be applied to the preparation of fresh tissues, the iodine playing the part of a fixing agent with regard to protoplasm, which it slightly hardens.

514. Iodide of Potassium (Anvold, Arch. mik. Anat., lii, 1898, pp. 135 and 763 ). -10 c.c. of 10 per cent. aqueous sol. of potassic iodide with 5 to 10 drops of a similar solution, containing also 5 per cent. of iodine.

515. Alcohol.-RANVIER employs one-third alcohol (1 part of 90 per cent. alcohol to 2 parts of water). Epithelia will macerate well in this in twenty-four hours. It macerates more rapidly than iodised serum.

Other strengths of alcohol may be used, either stronger (equal parts of alcohol and water) or weaker ( $\frac{1}{4}$ alcohol, for isolation of the nerve-fibres of the retina, for instance-THIN).

516. Salt Solution.-Ten per cent. solution of sodium chloride is a valuable macerating medium. Weaker strengths, down to 0.6 per cent., are also used.

51\%. Moleschott and Piso Borme's Sodium Chloride and Alcohol (MoleschotT's Untersuchungen zur Naturlehre, xi, pp. 99-107; RaNvier, Traité, p. 242).-Ten per cent. solution of sodium chloride, 5 volumes; absolute alcohol, 1 volume.

For vibratile epithelium RANVIER finds the mixture inferior to one-third alcohol.

518. Sodium Chloride and Formaldehyde.-GAGE recommends the addition of 2 parts of formalin to 1,000 parts of normal salt solution (quoted from Fish, Proc. Amer. Mic. Soc., xvii, 1895, p. 328).

519. Caustic Potash, Caustic Soda.-These solutions should be employed strong, 35 to 50 per cent. (МоLeschotт); so employed they do not greatly alter the forms of cells, whilst weak solutions destroy all the elements. (Weak solutions may, however, be employed for dissociating the cells of epidermis, hairs, and nails.) The strong solutions may be employed by simply treating the tissues with them on the slide. To make permanent preparations, the alkali should be neutralised by adding acetic acid, which forms 
with caustic potash acetate of potash, which constitutes a mounting medium (see Behrens, Kossel, and SchiefFerdecker, Das Mikroskop, i, 1889, p. 156). See also GAGE, Proc. Amer. Soc. of Microscopists, 1889, p. 35.

520. Baryta-water, Lime-water (FoL, Lehrb., p. 110).-Baryta-water will macerate nerve, muscle, and connective tissue in a few hours, lime. water in a few days.

521. Sulphocyanides of Ammonium and Potassium (StirLing, Journ. Anat. and Phys., xvii, 1883, p. 208).-10 per cent. solution of either of these salts, for epithelium. Macerate small pieces for twenty-four to forty-eight hours.

Soulier (Travaux de l'Inst. Zool. de Montpellier, Nouv. Sér., 2, 1891, p. 171) has found that STIRLING's solution greatly deteriorates cellular elements, but that good results are obtained by combining it with a fixing agent. The best results were obtained with a 2 per cent. solution of sulphocyanide combined with liquid of RIPART and PETIT; good ones, by combining liquid of RIPART and PETIT with artificial serum of KRONECKER instead of sulphocyanide, or with pepsin, eau de Javelle, 10 per cent. sulphate of soda, or $\mathbf{1 . 5}$ per cent. solution of caustic soda ; also by combining solutions of chloride of sodium, or solutions of caustic potash or soda, with any of the usual fixing agents.

522. Landors's Solution (Arch. mikr. Anat., 1885, p. 445). Saturated sol. of neutral chromate of ammonia

Saturated sol. of phosphate of potash

Saturated sol. of sulphate of soda

Distilled water.

5 parts.
$5 \quad 5 "$
$5 \quad$
$5 \quad 100 "$

Small pieces of tissue are macerated for one to three, or even four to five days, in the liquid, then brought for twenty-four hours into ammonia carmine diluted with 1 volume of the macerating liquid.

GIERKE particularly recommends this liquid for all sorts of macerations, but especially for the central nervous system, for which he finds it superior to all other agents. It is also recommended for the same purpose by Nansen (v. Zeit. wiss. Milk., v, 1888, p. 242).

\section{Bichromate of Potash. $-0 \cdot 2$ per cent.}

Eisig (Fauna u. Flora Golf. Neapel, 16 Monog., 1887, p. 297) macerates Capitellidæ in 0.5 to 1 per cent. solution for months or years, a little thymol being added against mould.

Müller's Solution, diluted to same strength, or combined with saliva, has also been used.

BRock (for nervous system of Mollusca, Intern. Monatssch. Anat., 
i, 1884, p. 349) takes equal parts of 10 per cent. solution of bichromate of potash and visceral fluid of the animal.

524. Permanganate of Potash is recommended, either alone or combined with alum, as the best dissociating agent for the fibres of the cornea (RoLLETT, Stricker's Handbuch, p. 1108). I have found it, for some objects, very energetic.

525. Chromic Acid.-Generally employed of a strength of about 0.02 per cent. Specially useful for nerve tissues and smooth muscle. Twenty-four hours' maceration will suffice for nerve tissue. About 10 c.c. of the solution should be taken for a cube of 5 millimetres of the tissue (RANVIER).

526. Osmic and Acetic Acid (the Hertwigs, Das Nervensystem $u$. die Sinnesorgane der Medusen, Leipzig, 1878, and Jen. Zeitschr., xiii, 1879 , p. 457).

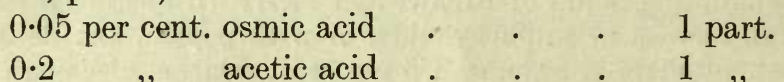

Medusœ are to be treated with this mixture for two or three minutes, according to size, and then washed in repeated changes of 0.1 per cent. acetic acid until all traces of free osmic acid are removed; they then remain for a day in $0 \cdot 1$ per cent. acetic acid, are washed in water, stained in BEALE's carmine, and preserved in glycerin.

For Actinice the osmic acid is taken weaker, 0.04 per cent.; both the solutions are made with sea water; and the washing out is done with 0.2 per cent. acetic acid. If the maceration is complete, stain with picro-carmine; if not, with BEALE's carmine.

527. MöвIUs's Media (Morph. Jahrb., xii, 1887, p. 174).

1. One part of sea water with 4 to 6 parts of $0 \cdot 4$ per cent. solution of bichromate of potash.

2. $0 \cdot 25$ per cent. chromic acid, $0 \cdot 1$ per cent. osmic acid, $0 \cdot 1$ per cent. acetic acid, dissolved in sea water. For Lamellibranchiata. Macerate for several days.

528. Nitric Acid.-Most useful for the maceration of muscle. The strength used is 20 per cent. After twenty-four hours' maceration in this, isolated muscle-fibres may generally be obtained by shaking the tissue with water in a test-tube. Preparations may afterwards be washed with water and put up in strong solution of alum, in which they may be preserved for a long time (Hopkins, Proc. Amer. Soc. of Microscopists, 1890, p. 165).

Maceration is greatly aided by heat, and at a temperature of $40^{\circ}$ to $50^{\circ} \mathrm{C}$. may be sufficiently complete in an hour (GAGE). 
A mixture of equal parts of nitric acid, glycerin, and water is recommended by MARCACCI (Arch. Ital. Biol., iv, 1883, p. 293) for smooth muscle.

529. Nitric Acid and Chlorate of Potash (KüHnE, Ueber die peripherischen Endorgane, etc., 1862; Ranvier, Traité, p. 79).Chlorate of potash is mixed, in a watch-glass, with four times its volume of nitric acid. A piece of muscle is buried in the mixture for half an hour, and then agitated with water in a test-tube, by which means it entirely breaks up into isolated fibres.

530. Nitric and Acetic Acid (Аpáthy, Zeit. wiss. Mik., x, 1898, p. 49). -3 volumes glacial acetic acid, 3 of nitric acid, and 20 each of water, glycerin, and absolute alcohol. Macerate leeches for twenty-four hours, and bring them into 70 per cent. alcohol, in which they swell; then after twenty-four hours, 50 per cent. glycerin, changed till the acid is removed.

531. Hydrochloric Acid.-Königstein (Sitzb. Akad. Wien, lxxi, 1875) takes (for gold-impregnated cornex) equal parts of the concentrated acid, glycerin, and water ; Freud (ibid., lxxviii, 1879, p. 102, for nerveimpregnations), 10 parts of acid, 7 of water, 3 of glycerin ; and SCHUBERG and Schröder (Zeit. wiss. Zool., lxxvi, 1904, p. 516) take (for fresh muscles of Hirudinea) hydrochloric acid of 5 per cent.

532. Béla Haller's Mixture (Morphol. Jahrb., xi, p. 321).-One part glacial acetic acid, 1 part glycerin, 2 parts water. For the central nervous system of Mollusca a maceration of thirty to forty minutes may be sufficient.

533. Sulphuric Acid (Ranvier, Traité, p. 78).-Macerate for twenty-four hours in 30 grms. of water, to which are added 4 to 5 drops of concentrated sulphuric acid. Agitate. For nasal mucosa, crystalline, retina, etc.

ODEnIUs found very dilute sulphuric acid to be the best reagent for the study of nerve endings in tactile hairs. He macerated hairfollicles for from eight to fourteen days in a solution of from 3 to 4 grs. of "English sulphuric acid" to the ounce of water.

Hot concentrated sulphuric acid serves to dissociate horny epidermic structures (horn, hair, nails).

534. Oxalic Acid.-Maceration for many days in concentrated solution of oxalic acid has been found useful in the study of nerveendings.

535. SchiefferdeckeR's Methyl Mixture (for the retina) (Arch. mik. Anat., xxviii, 1886, p. 305).-Ten parts of glycerin, 1 part of 
methyl alcohol, and 20 parts of distilled water. Macerate for several days (perfectly fresh tissue).

536. GaGe's Picric Alcohol (Proc. Amer. Soc. of Microscopists, 1890 , p. 120).-Ninety-five per cent. alcohol, 250 parts ; water, 750 ; picric acid, 1. Recommended especially for epithelia and muscle. A few hours suffice.

53\%. Chloral Hydrate.-In not too strong solution, from 2 to 5 per cent. for instance, chloral hydrate is a mild macerating agent that admirably preserves delicate elements. Lavdowsky (Arch. mik. Anat., 1876, p. 359) recommends it greatly for salivary glands, Hickson (Quart. Journ. Mic. Sci., 1885, p. 244) for the retina of Arthropods.

538. Lysol (Reinke, Anat. Anz., viii, 1892, p. 582).-Ten per cent. solution in distilled water or in water with alcohol and glycerin. Spermatozoa of the rat or cortical cells of hairs are said to be resolved into fibrils in a few minutes, epithelial cells of salamandra to be dissociated instantaneously.

\section{Digestion.}

539. Digestion is maceration in organic juices, which by dissolving out some of the constituents of tissues earlier than others serves to isolate those which resist. The chief liquids employed are gastric juice (or pepsin) and pancreatic juice (pancreatin or trypsin).

Pepsin is best employed in acidified solution, pancreatin in alkaline.

The most favourable temperature for digestion is about $40^{\circ} \mathrm{C}$.

Pepsin digests albuminoids, collagen substance and mucin more or less readily, elastin more slowly. Nuclein is either not dissolved or very slowly. Keratin, neurokeratin, chitin, fat and carbohydrates are not attacked.

Pancreatin (trypsin) digests albuminoids, nuclein, mucin, and elastic tissue; whilst collagen substance, reticular tissue, chitin, horny substances, fat and carbohydrates are not attacked.

Tissues for digestion should be fresh, or fixed with alcohol, not with chromic acid or other salts of the heavy metals.

540. Pepsin (Beale's, Archives of Medicine, i, 1858, pp. 296 316).-The mucus expressed from the stomach glands of the pig is rapidly dried on glass plates, powdered, and kept in stoppered bottles. Eight-tenths of a grain will dissolve $100 \mathrm{grs}$. of coagulated white of egg. 
To prepare the digestion fluid, the powder is dissolved in distilled water, and the solution filtered. Or the powder may be dissolved in glycerin. The tissues to be digested may be kept for some hours in the liquid at a temperature of $100^{\circ} \mathrm{F}$. $\left(37^{\circ} \mathrm{C}\right.$.).

BRÜCKE's (from CARNOY's Biologie cellulaire, p. 94).

Glycerinated extract of pig's stomach . 1 volume.

$0 \cdot 2$ per cent. solution of $\mathrm{HCl} \quad$. . 3 volumes.

Thymol, a few crystals.

Bickfalvi's (Centrabl. med. Wiss., 1883, p. 838).-One grm. of dried stomachal mucosa is mixed with 20 c.c. of 0.5 per cent. hydrochloric acid, and put into an incubator for three or four hours, then filtered. Macerate for not more than half an hour to an hour.

Kuskow's (Arch. mik. Anat., xxx, p. 32).-One part of pepsin dissolved in 200 parts of 3 per cent. solution of oxalic acid. The solution should be freshly prepared, and the objects (sections of hardened Ligamentum Nuchæ) remain in it at the ordinary temperature for ten to forty minutes.

541. Pancreatin.-SchIEFFERdecker's (Zeit. wiss. Mik., iii, 1886, p. 483).-A saturated solution of the "Pankreatinum siccum," prepared by Dr. Witte, Rostock, is made in distilled water, cold, and filtered. Pieces of tissue (epidermis) are macerated in it for three to four hours at about body temperature.

KÜHNE's (Unters. a. d. Phys. Inst. Univ. Heidelberg, i, 2, 1877, p. 219). -Very complicated.

See also GEDOELST, La Cellute, iii, 1887, p. 117, and v, 1889, p. 126 ; MaAS, Festschr. Kupffer, 1899; p. 211, and HoEHL, Arch. Anat. Phys., Anat. Abth., 1897, p. 136 ( $\frac{1}{5}$ to $\frac{2}{5}$ per cent. solution of Mall's or Merck's pancreatin, with $0 \cdot 3$ per cent. of oarbonate of soda ; for demonstrating adenoid tissue in paraffin sections).

\section{Corrosion.}

542. Corrosion is the operation of destroying the soft parts that surround hard parts that it is desired to study-in short, a means of cleansing hard parts for microscopic study. It has been applied to the removal of surrounding tissue from injected vessels or cavities. For this, see Altmann's Method (Arch. mik. Anat., 1879, p. 471, or previous editions); also REJSEK (Bibliogr. Anat., iv, 1897, p. 229); BrüHL (Anat. Anz., xiv, 1898, p. 418); Denker (Anat. Hefte., 1900, p. 300); Тнома and Fromherz (Arch. Entwickelungsmech, vii, 1898, p. 678); Peabody (Z. Bull., Boston, 1897, p. 164). The following sections relate chiefly to the cleansing of native hard parts. 
543. Caustic Potash, Caustic Soda, Nitric Acid.-Boiling, or long soaking in a strong solution of either of these is an efficient means of removing soft parts from skeletal structures (appendages of Arthropods, spicula of sponges, etc.).

544. Eau de Javelle (Hypochlorite of Potash) (Nolu, Zool. Anzeig., cxxii, 1882, p. 528).-A piece of sponge, or similar object, is brought on to a slide and treated with a few drops of eau de Javelle, in which it remains until all soft parts are dissolved. (With thin pieces this happens in twenty to thirty minutes.) The preparation is then cautiously treated with acetic acid, which removes all precipitates that may have formed, dehydrated, and mounted in balsam.

The process is applicable to calcareous structures.

545. Eau de Labarraque (Hypochlorite of Soda) may be used in the same way as eau de Javelle. Looss (Zool. Anzeig., 1885, p. 333) finds that either of these solutions will completely dissolve chitin in a short time with the aid of heat. For this purpose the commercial solution should be taken concentrated and boiling.

If solutions diluted with 4 to 6 volumes of water be taken, and chitinous structures be macerated in them for twenty-four hours or more, according to size, the chitin is not dissolved, but becomes transparent, soft and permeable to staining fluids, aqueous as well as alcoholic. The most delicate structures, such as nerve-endings, are stated not to be injured by the treatment. The method is applicable to Nematodes and their ova, and also to the removal of the albumen from ova of Amphibia, ett. 


\section{CHAPTER XXIV.}

DECALCIFICATION, DESILICIFICATION, AND BLEACHING.

\section{Decalcification.}

546. Decalcification.-In order to obtain the best results, it is important to employ only material that has been duly fixed and hardened, and it is well not to put too much confidence in reagents that are said to have the property of hardening and decalcifying fresh material at the same time.

It is generally well also to employ fluids that contain substances having a shrinking action on tissues, so as to neutralise the swelling frequently brought about by the decalcifying acids. Large quantities of liquid should be employed.

After decalcification the excess of acid should be carefully removed by washing, not in water, which favours swelling, but in some liquid that has rather a shrinking action, e.g., alum solution. Lastly, the tissues should be neutralised by treatment with carbonate of lime, or a salt of lithium or sodium or the like.

Rousseau (Zeit. wiss. Mik., xiv, 1897, p. 207) imbeds fixed material in celloidin, brings it into 85 per cent. alcohol, decalcifies in a very acid mixture ( 15 to 40 per cent. of nitric acid in alcohol) washes out the acid in alcohol containing precipitated carbonate of lime, then cuts sections. This for Porifera, corals, Echinoderms, etc. Tissues are said to be well preserved.

This process has been applied to the study of the temporal bone of Mammals by Stein (Anat. Anz., xvii, 1900, p. 318).

Similarly BöDECKER (Zeit. wiss. Mik., xii, p. 190 ; xxv, p. 21 ; xxvi, p. 206 ; and xxviii, p. 158), in a complicated way, adding the acid ( 6 to 10 per cent.) to the thin celloidin solution taken for imbedding.

54\%. Decalcification of Bone.-I take the following from Busch : Arch. mik. Anat., xiv, 1877, p. 481 ; see also HaUG, in Zeit. wiss. Mik., viii, 1891, p. 1; and Schaffer, ibid., xix, 1903, pp. 308 and 441, and his paper in the Encycl. mik. Technik.

The most widely used, though not the best, agent for decalcification is hydrochloric acid. Its action is rapid, even when very 
dilute, but causes serious swelling of the tissues. To remedy this, chromic acid or alcohol may be added to it. Or a 3 per cent. solution of the acid may be taken and have dissolved in it 10 to 15 per cent. of common salt. Or (WALDEYER) to a $\frac{1}{1000}$ per cent. solution of chloride of palladium may be added $\frac{x}{10}$ of its volume of $\mathrm{HCl}$.

Chromic acid is also much used, but has a very weak decalcifying action and a strong shrinking action on tissues. For this reason it should never be used in solutions of more than 1 per cent. strength, and for delicate structures much lower strengths must be taken.

Phosphoric acid has been recommended for young bones.

Acetic, lactic and pyroligneous acids have considerable decalcifying power, but cause great swelling. Picric acid has a very slow action, and is only suitable for very small structures.

548. Nitric Acid (Busch, loc. cit.).-To all other agents BuscH prefers nitric acid, which causes no swelling and acts most efficaciously.

One volume of chemically pure nitric acid of sp. gr. $1 \cdot 25$ is diluted with 10 volumes water. It may be used of this strength for very large and tough bones; for young bones it may be diluted down to 1 per cent.

Fresh bones are first laid for three days in 95 per cent. alcohol; they are then placed in the nitric acid, which is changed daily, for eight or ten days. They must be removed as soon as the decalcification is complete, or else they will become stained yellow. When removed they are washed for one or two hours in running water and placed in 95 per cent. alcohol. This is changed after a few days for fresh alcohol.

Young and foetal bones may be placed in the first instance in a mixture containing 1 per cent. bichromate of potash and $\frac{1}{10}$ per cent. chromic acid, and decalcified with nitric acid of 1 to 2 per cent., to which may be added a small quantity of chromic acid ( $\frac{1}{10}$ per cent.) or bichromate of potash (1 per cent.). By putting them afterwards into alcohol a green stain is obtained.

549. Nitric Acid (Schaffer, Zeit. wiss. Mik., xix, 1903, p. 460).SchAFFER also finds nitric acid the best reagent. It should be taken pure ; the addition of formol, alcohol, or the like, slows the reaction. The best strength is from 3 to 5 per cent. Objects must not be washed out directly with water, and washing in salt solution, alcohol, phloroglucin, or formol is not sufficient to prevent swelling. Alum in 5 per cent. solution is good, but not necessary. Material should 
be well fixed and imbedded in celloidin ( $\$ 546)$; harden in alcohol ; remove the alcohol with water; put for twelve to twenty-four hours (large specimens longer) inta nitric acid of 3 to 5 per cent., then into a 5 per cent. solution of sulphate of lithium or sodium, to be changed once in the course of twelve to twenty-four hours; running water, forty-eight hours ; alcohol.

550. Nitric Acid and Alcohol.-Three per cent. of nitric acid in 70 per cent. alcohol. MAYER has long used 5 per cent. acid in 90 per cent. alcohol. Soak specimens for several days or weeks. Pure nitric acid, even if weak, readily exercises a gelatinising action on bone; whilst the addition of alcohol (or of alum) counteracts this action (Fish, Ref. Handb. Med. Sci., Supp., p. 425).

Thома (Zeit. wiss. Mik., viii, 2, 1891, p. 191) takes 5 volumes of 95 per cent. alcohol and 1 volume pure concentrated nitric acid. Leave bones in this mixture, changing the liquid every two or three days, until thoroughly decalcified, which should happen, even with large bones, in two or three weeks. Wash out until every trace of acid is removed (i.e., for some days after no acid reaction is obtained with litmus paper) in 95 per cent. alcohol containing an excess of precipitated carbonate of lime. This may take eight to fourteen days, after which the tissues will stain well and may be treated as desired.

551. Nitric Acid and Formol.-ScHRIDde (Homatol. Techn., Jena, 1910 , p. 21) decalcifies material fixed in formol or formolMüller in a mixture of 1 part of formol, 1 of nitric acid, and 9 of water.

552. Nitric Acid and Alum (GAGE, quoted from FisH, § 550 \%.-A saturated aqueous solution of alum is diluted with an equal volume of water, and to each 100 c.c. of the dilute solution is added 5 c.c. of strong nitric acid. Change every two or three days, until the decalcification is complete. For teeth this is said to be, perhaps, a better decalcifier than the alcohol mixture.

553. Sulphurous Acid (Ziegler, Festschr.f. Kupffer, 1899, p. 51). -A saturated solution in water. Wash out for twenty-four hours. Acts rapidly and preserves well. Best used after fixation with formol.

554. Hydrochloric Acid (see $\S 547$ ). - RANVIER says that it may be taken of 50 per cent. strength, and then has a very rapid action. To counteract the swelling action of the acid, sodium chloride may be added (von EBNER), see. HAUG's paper quoted § 547. He takes either 100 e.c. 
cold saturated solution of sodium chloride in water, 100 c.c. water, and 4 c.c. hydrochloric acid. Preparations to be placed in this, and 1 to 2 c.c. hydrochloric acid added daily until they are soft. Or, 2.5 parts of hydrochloric acid, 500 of alcohol, 100 of water, and $2 \cdot 5$ of sodium chloride.

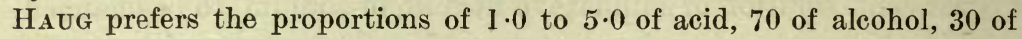
water, and 0.5 of salt.

555. Hydrochloric Acid and Chromic Acid (BAYERL, Arch. mik. Anat., 1885, p. 35).-Equal parts of 3 per cent. chromic acid and 1 per cent. hydrochloric acid. For ossifying cartilage. HAUG recommends equal parts of 1 per cent. hydrochloric acid and 1 per cent. chromic acid (loc. cit.).

556. Hydrochloric Acid and Glycerin.-Glycerin, 95; hydrochloric acid, 5 (SqurRE's Methods and Formula, p. 12).

55\%. Trichloracetic Acid.-PaRTsch (Verh. Ges. D. Naturf. Aertze, 1895, 2 Theil, 2 Hälfte, p. 26) uses a 5 per cent. aqueous solution, and Neuberger (Centralb. Phys., xi, 1897, p. 494) a 4 per cent. one. Action energetic, preservation said to be excellent.

558. Picric Acid should be taken saturated and changed frequently. Its action is weak, but it gives good results with small objects.

Picro-nitric or Picro-hydrochloric Acid.-Action very rapid.

559. Phosphoric Acid. - 10 to 15 per cent. (HAUG, loc. cit. in $\$ 547$ ). Somewhat slow, staining not good. According to SCHAFLER, $\$ 549$, it produces swelling.

560. Lactic Acid. - 10 per cent. or more. Fairly rapid, preserves well, and may be recommended (HAUG, loc. cit.).

561. Chromic Acid is employed in strengths of from $0 \cdot 1$ per cent. to 2 per cent. (but see $\$ 547$ ), the maceration lasting two or three weeks (in the case of bone). It is better to take the acid weak at first, and increase the strength gradually. Action excessively slow.

562. Chromic and Nitric Acid.-Seiler (For, Lehrb., p. 112) takes 70 volumes of 1 per cent. chromic acid, 3 of nitric acid, and 200 of water. The action is still excessively slow, frequently requiring months to be complete.

563. Chromo-aceto-osmic Acid (Van ver Stricht, Arch. Biol., ix, 1889, p. 29 ; and Schaffer, Zeit. wiss. Mik., x, 1893, p. 179).Objects to be left in it for months, the liquid being changed at first every two days, afterwards less frequently. Structure well preserved.

564. Arsenic Acid. -4 per cent. aqueous solution, used at a temperature of $30^{\circ}$ to $40^{\circ} \mathrm{C}$. (SQUIRE's Methods and Formulae, etc., p. 11).

565. Phloroglucin with Acids (Andeer, Centralb. med. Wiss., xii, xxxiii, pp. 193, 579; Intern. Monatsschr., i, p. 350 ; HAUG, Zeit. wiss. 
Mik., viii, 1891, p. 8 ; Ferreri, ibid., ix, 1892, p. 236 ; Bull. R. Acad. Med. di Roma, 1892, p. 67). - This is said to be the most rapid method of any. Phloroglucin by itself is not a solvent of lime salts ; its function in the mixture given below is so to protect the organic elements of tissues against the action of the mineral acid that this can be used in a much more concentrated form than would be otherwise advisable.

ANDEER takes a saturated solution in warm water, and adds to it 5 to 50 per cent. of hydrochloric acid. Wash out in running water.

Other acids than hydrochloric may, of course, be taken. 'See HAUG, Zeit. wiss. Mik., viii, 1891, p. 8, and Ferreri, Bull. Acad. Med. Roma, 1892, p. 67 , or (for both) fifth edition.

\section{Desilicification.}

566. Hydrofluoric Acid (Mayer, Zool. Anz., 1881, p. 593).-The objects are brought in alcohol into a glass vessel coated internally with paraffin. Hydrofluoric acid is then added drop by drop (taking great care to avoid the fumes, which attack mucous membranes with great energy). Small pieces of siliceous sponges will be completely desilicified in a few hours, or at most a day. The tissues do not suffer.

For sponges I find that this dangerous method can be avoided. If well imbedded, sections may be made from them without previous removal of the spicula, which appear to break off sharp before the knife.

Rousseau imbeds the objects in celloidin, as described $\$ 549$, then brings the block, in a covered caoutchouc dish, for a day or two into a mixture of 50 e.c. alcohol and 20 to 30 drops of hydrofluoric acid, and washes out the acid with alcohol containing carkonate of lithia in powder.

\section{Bleaching.}

56\%. MayeR's Chlorine Method (Mitth. Zool. Stat. Neapel, ii, 1881, p. 8).- Put into a glass tube a few crystals of chlorate of potash, add 2 or 3 drops of hydrochloric acid, and as soon as the green colour of the evolving chlorine has begun to show itself, add a few cubic centimetres of alcohol of 50 to 70 per cent. Now put the objects (which must have previously been soaked in alcohol of 70 to 90 per cent.) into the tube. They float at first, but eventually sink. They will be found bleached in from a quarter of an hour to one or two days, without the tissues having suffered. Only in obstinate cases should the liquid be warmed or more acid taken. Sections on slides may be bleached in this way. Instead of hydrochloric acid nitric acid may be taken, in which case the active agent evolved is oxygen instead of chlorine.

This method serves both for removing natural pigments, such as those of the skin or of the eyes of Arthropods, and also for bleaching 
material that has been blackened by osmic acid, and, according to renewed experiments of MAYER's, is to be preferred to the peroxide of hydrogen method.

For bleaching chitin of insects, not alcohol but water should be added to the chlorate and acid (MAYer), Arch. Anat. Phys., 1874, p. 321).

See also MAYER in Zeit. wiss. Mik., xxiv, 1907, p. 353 (paraffin sections exposed to the vapour of chlorine water).

Grynfeltt and Mestrezat (C. R. Soc. Biol., lxi, 1906, p. 87) add 2 c.c. of 20 per cent. solution of chloric acid $\left(\mathrm{HClO}_{3}\right)$ to 15 c.c. of alcohol and put sections (of retina) into it for several hours at $42^{\circ} \mathrm{C}$.

568. Eau de Labarraque. Eau de Javelle (see $\S \S 544,545$ ). - These are bleaching agents. For the manner of preparing a similar solution see early editions, or Journ. de Microgr., 1887, p. 154, or Journ. Roy. Mic. Soc., 1887, p. 518. Of course, the method cannot be used for bleaching soft parts which it is desired to preserve.

569. Peroxide of Hydrogen (Oxygenated Water) (Poucher's method, M. Duval, Précis, etc., p. 234).-Macerate in glycerin, to which has been added a little oxygenated water ( $\$ 35), 5$ to 6 drops to a watch-glass of glycerin. Solger (Centralbl. med. Wiss., xxi, 1883, p. 177) takes a 3 per cent. solution of peroxide. Fürst (Morph. Arb. Schwalbe, vi, 1896, p. 529) points out that after a time it macerates.

The method serves both for removing pigments and for bleaching osmic and chromic material.

570. Peroxide of Sodium (Carazzi, Zool. Anz., 444, 1894, p. 135).See previous editions.

571. Peroxide of Magnesium (MAYER, Grundzüge, p. 290).-Use as chlorine, §567. A slow but delicate method.

5\%2. Sulphurous Acid.-Prof. GiLson writes me that he finds alcoholic solution of sulphurous anhydride $\left(\mathrm{SO}_{2}\right)$ very convenient for the rapid decoloration of bichromate objects. A few drops suffice. MönCKeBerg and Bethe (Arch. mik. Anat., liv, 1899, p. 135) obtain the acid by adding to 10 c.c. of a 2 per cent. solution of bisulphate of sodium 2 to 4 drops of concentrated hydrochloric acid. Objects are put into the freshly prepared solution for six to twelve hours.

573. Permanganate of Potash.-Alfieri (Monitore Zool. Ital., viii, 1897, p. 57) bleaches celloidin sections of the choroid, etc., for eight to twenty-four hours in a 1:2000 solution of permanganate of potash, then washes them out for a few hours in a solution of oxalic acid of $1: 300$ strength, or weaker. 
574. GrenaCher's Mixture for Eyes of Arthropods and other Animals (Abh. nat. Ges. Halle-a.-S., xvi ; Zeit. wiss. Mik., 1885, p. 244).

Glycerin

80 per cent. alcohol . . . . 2 parts.

Mix and add 2 to 3 per cent. of hydrochloric acid.

Pigments (i.e. those in question) dissolve in this fluid, and so doing form a stain which suffices in twelve to twenty-four hours for staining the nuclei of the preparation.

575. Nitric Acid.-Parker (Bull. Mus. Comp. Zool., Cambridge, U.S.A., 1889, p. 173) treats sections (of eyes of scorpions) fixed to the slide with SCHÄLLIBAUM's medium, for about a minute with a solution of up to 50 per cent. of nitric acid in alcohol, or, still better, with a 35 per cent. solution of a mixture of equal parts of nitric and hydrochloric acid in alcohol. To make the solution, the acid should be poured slowly into the alcohol (not vice versâ), and the mixture kept cool.

JANDER (Zeit. wiss. Mik., xv, 1898, p. 163) takes for removal of pigments SEILER's chromo-nitric acid ( $\$ 562)$; twelve to forty-eight hours is enough for small objects.

See also under "Arthropoda.",

576. Caustic Soda.-RAwitz (Leitfaden, p. 29) dissolves the pigment of the mantle of Lamellibranchia by means of 3 to 9 drops of officinal caustic soda solution added to 15 to 20 c.e. of 96 per cent. alcohol. 


\section{CHAPTER XXV.}

\section{EMBRYOLOGICAL METHODS.*}

57\%. New Advances. - In nearly every case the newest advances in ordinary embryological technique are constituted by the improvements in fixation reported in the new sections on Cytology ( $\$ 681$ ). It would be a great mistake for observers to consider that fixation and staining methods, such as those of Champy-Kull, Kopsch, or Flemming-without-acetic acid, and iron hæmatoxylin, are of no concern to embryologists. For instance, amphibian embryos, such as those of Triton (Molge) prepared by Champy-Kull's method are extremely beautiful and instructive for study, for not only does one procure cytological perfection, but also a staining which is polychromatic. For the study of invertebrate embryology, the mitochondrial methods open up a new field for research.

The reliability of many of the new neurological methods (see $\S \S 865$ to 921 ) has been brought to a state which should now induce embryologists to devote even rare material for preparation, and to use the neurological methods much more widely than at present is the case.

In $\S 768$ is a special treatment of the study of fats and lipoids, which can readily be used for embryological studies. In $\S 646$ is a section on "Glycogen," and in $\S 650$ one on "Iron and Copper." In $\S \S 1035-1045$ is a Chapter on the "Tissue Culture" methods. In various parts of the book further notes on intravital staining have been inserted.

578. Artificial Fecundation.-This practice, which affords the readiest means of obtaining the early stages of development of many animals, may be very easily carried out in the case of the amphibia

* The sections in this chapter treating of Mammalia, Aves, and Pisces, closely follow the Traité des Méthodes Techniques, Isee et Hennegur, and are due almost entirely to HENNEGUY. The corresponding parts of the Grundzüge, LEE and MAYER, are taken from this work, and therefore also due to Hennegur, which Dr. Lee regrets to observe has not always been understood, though duly pointed out in the Preface to the first edition of the Grundzüge. This foot-note does not apply to any new material introduced into the present edition (J. B. G.). 
Anura, Teleostea, Cyclostomata, Echinodermata, and many Vermes and Colenterata.

In the case of the Amphibia, both the female and the male should be laid open, and the ova should be extracted from the uterus and placed in a watch-glass or dissecting dish, and treated with water in which the testes, or, better, the vasa differentia, of the male have been teased.

Females of Teleostea are easily spawned by manipulating the belly with a gentle pressure ; and the milt may be obtained from the males in the same way. (It may occasionally be necessary, as in the case of the Stickleback, to kill the male, and dissect out the testes and tease them.) The spermatozoa of fish, especially those of the Salmonidæ, lose their vitality very rapidly in water; it is, therefore, advisable to add the milt immediately to the spawned ova, then add a little water, and after a few minutes put the whole into a suitable hatching apparatus with running water.

Artificial fecundation of Invertebrates is easily performed in a similar way. For methods of artificial Parthenogenesis see HARveY, Biol. Bull. Wood's Hole, 1910, p. 269.

579. Superficial Examination. - The development of some animals, particularly some invertebrates, may be to a certain extent followed - by observations of the living ova under the microscope. This may usefully be done in the case of various Teleosteans, such as the Stickleback, the Perch, Macropodus, and several pelagic forms, and with Chironomus, Asellus aquaticus, Ascidians, Planorbis, many Colenterata, etc.

Some ova of insecta and Arachnida which are completely opaque under normal conditions become transparent if they are placed in a drop of oil ; if care be taken to let their surface be simply impregnated with the oil, the normal course of development is not interfered with (BALBIANI).

580. Fixation.-Osmic acid, employed either alone or in combination with other reagents, is an excellent fixing agent for small embryos, but not at all a good one for large ones. It causes cellular elements to shrink somewhat, and therefore brings out very clearly the slits that separate germinal layers, and any channels or other cavities that may be in course of formation.

In virtue of its property of blackening fatty matters, myelin amongst them, it is of service in the study of the development of the nervous system.

Chromic acid is indispensable for the study of the external forms 
of embryos ; it brings out elevations and depressions clearly, and preserves admirably the mutual relations of the parts; but it does not always preserve the forms of cells faithfully, and is a hindrance to staining in bulk.

Picric liquids have an action which is the opposite of that of osmic acid; they cause cellular elements to swell somewhat, and thus have a tendency to obliterate spaces that may exist in the tissues. But notwithstanding this defect, the picric compounds, and especially Kleinenberg's picro-sulphuric acid, are amongst the best of embryological fixing agents.

Schridde (Zeit. wiss. Mik., xxvii, 1910, p. 362) finds Orth's "Formol-Müller" in general the best fixative. Fix for not more than twenty-four hours, and pass through graded alcohols (twenty minutes in each) into absolute (one to two hours), cedar oil, xylol, and paraffin.

RABL (Zeit. wiss. Mik., xi, 1894, p. 165) recommends for embryos of Vertebrates, and also for other objects, his platinic sublimate, $\S 76$. This serves for a large number of blastoderms and young embryos (Pisces, Amphibia, Aves, Mammalia). Advanced embryos of Teleostea ought to be fixed in the warmed mixture, in order to avoid rupture of the muscles and shrinkage of the chorda.

Some of his best results were obtained by a not too prolonged fixation in a mixture of

Platinic chloride, 1 per cent. solution . 1 volume.

Picric acid, saturated aqueous . . 2 volumes.

Distilled water . . . . . 7 ,

RABL's picro-sublimate mixture has been given $§ 70$. I recommended especially for somewhat advanced embryos, such as embryo chicks from the third or fourth day, and other embryos of a similar size.

Boveri (Verh. Phys. Med. Ges. Würzburg, xxxix, 1895, p. 4), in order to imbed and cut together numbers of ova of Echinoderms, wraps them in pieces of sloughed epidermis of Cryptobranchus (of course, other Urodela will do). Sовотта (Arch. mik. Anat., 1, 1897, p. 31) takes pieces of amnios of Mammalia.

SANzo (Zeit. wiss. Mik., xxi, 1904, p. 449) describes an automatic apparatus for fixing material at definite stages.

581. Peter's Double-stain for Yolk and Tissue, see $\S 224$.

582. Removal of Albumen.-The thick layers of albumen that surround many ova are a serious obstacle to the penetration of reagents. CHILD (Arch. Entwickelungsmech., ix, 1900, p. 587) gives the following as of very general applicability. After fixation 
(in any way except with chromic acid) the ova are brought through graduated alcohols up to that of 80 per cent., in which they are hardened. They are then brought down again through successive alcohols into water acidified lightly with any acid (except chromic acid), and the albumen is found to become transparent and dissolve.

583. Reconstruction of Embryos from Sections. - To facilitate the study of series of sections, recourse may be had to graphic or plastic reconstruction of the objects.

In simple cases it may be sufficient to adopt the plan described by SchafFer (Zeit. wiss. Mik., vii, 1890, p. 342). Careful outlines of the sections to be constructed are drawn on tracing paper with the aid of the camera lucida, superposed, and held up against the light for examination by transparence. Vosmaer (Anat. Anz., xvi, 1899, p. 269) draws on plates of celluloid, and sets them up in a rack for examination. KeRR (Quart. Journ. Mic. Sci., xlv, 1902 , p. 1) draws on plates of ground glass which he afterwards superposes and makes transparent by oil of cloves run in between them. Pensa (Zeit. wiss. Mikr., xxvii, 1910, p. 48) takes sheets of lithographic gelatin. WooDworth (Zeit. wiss. Mik.; xiv, 1897, p. 15) proceeds as follows : (1) Draw an axial line of the length of the object multiplied by the magnification employed. (2) Measure with a micrometer the greatest diameter of each section. (3) Plot these diameters down transversely on the axial line at distances corresponding to the thickness of the sections multiplied by the magnification. (4) Join the extremities of these diameters; this will give you an outline of the object. (5) Measure off on each section the nearest and farthest limits (from the margin) of the organs to be filled in, and plot them down on the transverse lines (3), and join the points as before, i.e. from section to section; this will give you the outlines of the organs.

This process is best applicable to reconstruction from transverse sections, but it can be applied to reconstruction from sections in any plane if the object can be provided with a plane of definition at right angles to the plane of section. This may be established by cutting off one end of the object, or the like (see also Orientation, $\S \S 142,161)$.

To make a simple plastic reconstruction, camera drawings (or photographs) of the sections (all made at the same magnification) are pasted on pieces of cardboard of a thickness equal to that of the sections multiplied by the magnification employed. Then the parts of the drawings representing the cavities of the objects are 
cut out with a knife or fretsaw, cutting through the cardboard ; and the pieces of fretwork thus obtained are pasted together.

Many useful modifications of this method have been devised. Cardboard is rather hard to cut, and not conveniently got of the required thickness. Professor Arthur Thompson, of Oxford, uses numbers of sheets of blotting paper to the required thickness, soaked in beeswax; this makes a very tough substance, and the models, when made, can be handled without chance of injury; other workers use beeswax plates alone, drawing the outline with some sharp instrument and cutting out with a hot knife.

Mr. Pittock, of the Zoological Laboratory, University College, London, uses a modification of K. Peter's method (vide infra). Rather thin paper is used for drawing the outline of the object. In this laboratory (Professor J. P. Hill), special rolls of paper are used, so that the diagram of each of hundreds of sections may be safely rolled up in order till wanted. A large flat stone is used for the manufacture of the wax plates, with two brass gauges of the required thickness placed at a distance which will accommodate in between them the square of paper with the drawing. Instead of treating the paper with turpentine, according to Mr. Pittock's method the drawing is rapidly floated over the surface of a dish of water, drawing side down, then laid upon the stone, between the metal gauge and the superfluous moisture smoothed off with a sheet of blotting paper. The melted wax is poured on to the paper, and a heated metal roller passing over the metal gauge leaves just the required amount of wax on the paper. The latter easily peels off the surface of the stone.

For more elaborate processes of plastic reconstruction (very complicated and seldom necessary) see BorN, "Die Plattenmodellirmethode," in Arch. mik. Anat., 1883, p. 591, and Zeit. wiss. Mik., v, 1888, p. 433 ; STRASSER, ibid., iii, 1886, p. 179, and iv, pp. 168 and 330 ; KASTSCHENKo, ibid., iv, 1887, pp. 235-6 and 353, and v, 1888, p. 173 ; SCHAPER, ibid., xiii, 1897, p. 446 ; AleXANDER, ibid., p. 334, and xv, 1899, p. 446 ; Peter, ibid., xxii, 1906, p. 530; Born and Peter, ibid., xv, 1, p. 31 ; and Verh. Anat. Ges., xiii, 1899, p. 134; Johnston, Anat. Anz., xvi, 1899, p. 261 ; Fol, Lehrb., p. 35 or previous editions; Broman, Anat. Hefte, xi, 1899, p. 557 ; Peter. "Die Methoden d. Rekonstruction" (Fischer, Jena, 1906); Schönemann, Anat. Hefte, xviii, 1901, p. 117 ; GAGE, Anat. Record, i, 1907, p. 167 ; Neumayfr, Festschr. f. Kupffer, 1899, p. 459 ; Mark, Proc. Amer. Acad. Sci., xlii, 1907, p. 629 (electric wax-cutter for cutting out plates).

Hill (Bull. Johns Hopkins Hosp., xvii, 1906, p. 114) finds that embryos of mammalia taken from 95 per cent. alcohol and put into caustic potash of $I$ per cent. become so transparent that they can be studied without cutting and reconstructing. 


\section{Mammalia.*}

584. Times for Early Development.-The entry of the sperm into the egg of the mouse takes place from six to ten hours after copulation (Вовотта, Arch. mikr. Anat. Bd., 45). The pronuclei stage of fertilisation is found from eighteen to twenty-two hours, two-cell stage twenty-six hours, four-cell, fifty hours, eight-cell, sixty hours after copulation : the egg remains in the tube about eighty hours. J. A. Long and E. L. Mark (Contrib. Zool. Lab. Museum, Harvard, Carneg. Inst. Wash., No. 142, 1911) find in the mouse that ovarian eggs within fifteen or sixteen hours after parturition have formed the first maturation spindle. Fertilised eggs are obtained from animals killed between twenty-three and thirty-one hours post partum. The time required for the spermatozoa, after introduction into the uterus (either artificially or by coitus) to reach the eggs in the first part of the oviduct varies from four to seven hours in mice inseminated about the same number of hours post partum. To obtain free eggs for study, Mark and Long kill mice fourteen to seventeen hours after parturition, the ova being found in a fold of the oviduct.

In the rat the eggs are found in the oviduct about 18.7 hours and ovulation occurs in less than eighteen hours post partum.

In the rabbit the pronuclei stage of fertilisation occurs about fourteen hours, in the guinea-pig, twenty-two to twenty-four hours after copulation (КовоттA). The rabbit's egg, like that of the guinea-pig, remains about eighty hours, the dog's egg eight to ten days in the tube (RöтніG, Embryol. Technik).

Condition of Ovary as Index to Pregnancy.-On opening the body cavity of a mammal, first of all examine the ovary. By so doing one can estimate roughly the time that has elapsed since the discharge of the ovum or ova. Prominent stigmata or areas with a blood-shot centre indicate recent ovulation, while a smooth surface of yellowish appearance indicates a corpus luteum, which means that some time has elapsed since ovulation.

585. Isolation of the Eggs and Early Stages.- The tubæ and uterus or uteri are dissected out and treated in one of two ways : either the isolated tuba after straightening is washed out from the funnel opening with warm salt solution, or with some fixative like formalin or weak osmic acid, or on the other hand the whole length of the tube is laid open and spread out with a scalpel or sharp scissors and needles, and the eggs are looked for under a dissecting microscope.

* Revised by J. B. G. 
If the method of washing out is adopted, it is best to use a good rubber bulb attached to a glass tube which has been drawn out finely enough to pass into the oviducal opening. Kölliker used Müller solution or weak osmic acid for injection, collecting the fluid in a series of watch glasses ; J. P. Hill uses solid crystal dishes, which can easily be examined under a stereoscopic binocular microscope. As a fluid for washing out Hill's picronitric osmic (vide infra), weak formalin, or weak osmic acid are probably as good as anything. The success of this injection method depends on the amount of mucous in the tuba and on the condition of the folds in its mucosa; if the eggs are not found after the injection, the walls of the tube may be opened up with scissors and the lining scraped away with a small scalpel; the mucus thus procured may be diluted with a little indifferent fluid and examined on a slide under the microscope. Both operations of injection or of opening the tuba may succeed with comparatively large animals like the rabbit and dog. It is practically impossible to slit open the tuba of the cat.

In cases where the subject is small, as, for instance, the mouse, it is necessary to preserve the whole oviduct and use a fixative sufficiently penetrative to act quickly. Even with the guinea-pig the lumen of the tube is so small that it is difficult to remove the ova; we consider that attempts to press out the contents of the tubes are dangerous. In such cases it seems better to cut the tube into lengths with a razor and to fix whole (vide infra). ВischоFF in his study on the guinea-pig (Giesson, 1852), and BALlowitz (Arch. Anat.Physiol, 1883) both resorted to the method of squeezing out the contents of the tubes.

When found the ova are picked up with the point of a cataract needle or a scalpel, on a piece of black paper cut to a point, or with a pipette, and either examined fresh in the peritoneal fluid or blood serum of the animal, or in Kronecker's or other artificial serum media, or better fixed immediately.

In the case of a large animal such as the rabbit, the same doe may be made to serve for two observations, at some hours' or days' interval. A longitudinal incision of 8 to 10 centimetres' length is made on the median or a lateral line of the abdomen ; an assistant keeps the intestines in their place; a ligature is placed at the base of one of the uterine cornua, beneath the neck, and a second ligature around the mesometrium and mesovarium. The ovary, the tuba, and the cornu of that side are then detached with scissors. The abdomen is then closed by means of a few sutures passing through the muscle-layers and the skin. The animals support the operation perfectly well, and the development of the ova of the opposite side is not in the least interfered with. When it 
is desired to study these the animal may be killed, or may be subjected to a secondary laparotomy if it be desired to preserve it for ulterior observations. This method, however, cannot be carried out in this country owing to the Vivisection Acts.

This procedure was also adopted by Hartmann in his study on Didelphys (vide infra).

586. Fixation of the Isolated Ova.-These can be fixed in a chromeformalin fluid of some kind: Müller-formol, Helly, Zenker-withoutacetic acid and formol are indicated. Eggs may be left in one of these fluids overnight, then washed in distilled water and transferred either to 1 per cent. $\mathrm{OsO}_{4}$, or to some chrome-osmic fluid, this to preserve the fat. The chrome fixation will form insoluble compounds with lipoids, but less so with fats of the type of olein. It seems likely that the fixation technique of Champy-Kull, of Schridde and of Murray (see $\S 689$ ) will be of great value.

For a study of the Golgi elements the methods of Cajal and Da Fano and of Mann-Kopsch are worthy of trial, but rather more difficult to work than chrome-osmic or chrome-formol techniques. Where there may be a difficulty of penetration chrome-formol fluids will be found better than chrome-osmium. A perusal of the sections on Mitochondria and Golgi apparatus will provide suggestions for the treatment of the early stages in mammalian development. Van Beneden (Arch. de Biol., 1880, p. 149) brings the living ovum into a drop of 1 per cent. $\mathrm{OsO}_{4}$ on a slide, and thence into a solution of Müller. After an hour the liquid is changed, and the whole is put into a moist chamber, where it remains for two or three days. It is then treated with glycerine of gradually increasing strength, and at last mounted in pure glycerine acidified with formic acid. I am inclined to believe that the Champy-Kull or Regaud fixation (the latter with a post-osmication) would be much superior to the above method, that is, for sectioning.

Many authors have used picro-nitric, picro-sulphuric, picroformol with or without corrosive, chromic-acetic acid, Flemming and Hermann, and so on, but one cannot help thinking that the more modern and logical fixation methods will be better. This seems borne out by the late work of Lams (Arch. de Biol., t., xxiii), and Levi (Arch.f. Zellf., xiv).

J. P. Hill (Quart. Jour. Micr. Soc., 1910) gives the formula of a "Marsupial mixture" for fixation of ova and blastocysts of Marsupials. This fluid is made by adding to 96 c.c. of Mayer's picronitric, 2 c.c. of 1 per cent. $\mathrm{OsO}_{4}$. Two c.c. of glacial acetic acid may be added, but the picric acid is sufficiently penetrative without the addition of acetic acid. 
J. A. Long (Contrib. Zool. Lab. Museum Compar. Zool. Harvard, 1912) describes an ingenious constant temperature box for working with fresh egg of mammalia. A circulation slide is also described in detail. So far J. A. Long has succeeded in keeping mice eggs alive and under observation for only twelve hours.

J. A. Long and E. L. MARK (op. cit.) use a modified Zenker for their study on mouse eggs. They fix for from twenty to sixty minutes. (A) 4 per cent. bichromate of potash. (B) 4 per cent. (aq. sol.) sublimate and 20 per cent. acetic acid. For use, mix equal portions of $\mathbf{A}$ and $B$. Wash out in warm water for twelve to fourteen hours, 70 per cent. alcohol and iodine twelve to fourteen hours, quickly dehydrate, clear in xylol and embed in paraffin. Mark and Long's fixative appears to me (on paper at least) to be far too acid. It may be indicated for chromosome work.

58\%. Subsequent Treatment of Ova.-After fixation the eggs or blastocysts should be brought into 30 per cent. alcohol and slowly upgraded to 90 per cent. alcohol : at this stage they may be stuck on pieces of liver or brain by Minchin's albumen method; the egg is placed on the liver and albumen is gently pipetted over it. The alcohol coagulates the albumen, and enables the object to be handled more easily. Another method used by J. P. HiLl (Quart. Jour. Micr. Science, 1910) is to bring the ova into alcohol absolute and then into equal parts of alcohol absolute and ether. Then take a hand-cut section of liver or brain (which has been stored in absolute) place 1 drop of 0.5 per cent. solution of photoxylin (or celloidin) in equal parts of absolute alcohol and ether; then transfer the egg on a flat camel hair brush to this drop, and harden the object in 15 per cent. chloroform in 90 per cent. alcohol. Transfer to equal parts of absolute alcohol, xylol and chloroform. Then equal parts of chloroform and xylol, and embed in paraffin wax.

The process of sticking the eggs to the hard cut liver or brain section should be carried out under a dissecting microscope.

588. Uterine Eggs.-During the fourth, fifth, and sixth days after copulation the ova of the rabbit are free in the uterine cornua ; they are easily visible to the naked eye, and may be extracted by the same manipulations as those of the tubes. After the sixth day they are at rest in the uterus, but have not yet contracted adhesions with the mucosa, so that they can still be extracted whole. At this stage the parts of the cornua where the ova are lodged are easily distinguishable by their peculiar aspect, the ova forming eminences of the size of a pea. The cornua should be cut up transversely into as many segments as there are eminences, care being taken to have the ova in the centre of the segments. You then fix each segment 
by means of two pins on the bottom of a dissecting dish, with the mesometrial surface downwards and the ovular eminence upwards. The dissecting-dish is then filled up with serum or liquid of MüLLER, or 0.1 per cent. solution of osmic acid, Bouin's fluid, Hill's fluid, Helly's fluid or 10 per cent. formol. See sections on "Cytology," $\S \S 673$ to 696 . With a small scalpel a longitudinal incision is made on the surface of the ovular eminence, not passing deeper than the muscular layer; the underlying uterine mucosa is then gently dilacerated with two pairs of small forceps, and the ovum set free in the liquid.

From the moment the ova have become adherent to the uterine mucosa they can no longer be extracted whole. The embryo being always situated on the mesometrial surface, the ovular eminence is opened by a crucial incision, and the strip of mucosa to which the embryo remains adherent is fixed with pins on the bottom of the dish. Ed. v. Beneden (see Arch. de Biol., v, fasc. iii, 1885, p. 378) has been able by operating in this way in serum of Kronecker, and keeping the whole at blood temperature, to observe the circulation of the embryo for hours together. (If this be desired to be done, the crucial incision should not be too extended, so as to leave the terminal sinus intact.)

Retterer (C. R. Soc. de Biol., 1887, p. 99) advises that for ova of the seventh day the segment of uterus containing them be opened on the mesometrial surface, for at that date no adhesion has yet been contracted with that side. By running in liquid of Kleinenberg by means of a pipette between the ovum and the free surface of the uterus, the ovum may be got away in the shape of a closed vesicle.

C. G. Hartmann (Jour. Morph., 1916), in his study of the development of the opossum, used Carnoy's, Bouin's, Fleming's and Hill's fluids. He found Hill's "Marsupial mixture" a perfect fixing fluid for marsupial eggs. J. P. Hill now recommends leaving out the acetic acid for delicate objects.

589. Blastoderms and Later Embryos.-The routine methods of embryology apply here in general. Great care must be exercised to avoid rough treatment caused by upgrading the object too quickly. The same remark applies even more particularly to clearing, which to get the best result should be done very gradually.

In order to bring out the outlines of blastoderm cells the living ovum may be brought into $\frac{1}{3}$ per cent. solution of nitrate of silver. After remaining there for half a minute to two minutes, according to the age of the vesicle, it is brought into pure water and exposed to the light. The preparations thus obtained are 
instructive, but blacken rapidly, and cannot be permanently preserved.

The blastodermic vesicle can be opened with fine needles, and the blastoderm washed, stained, or impregnated with gold, and mounted in glycerin or balsam.

For embryonic areas and more advanced embryos, refer to "Cytology," §§ 673-696. KöLLIKER recommends putting the ovum into 0.5 per cent. solution of osmic acid until it has taken on a somewhat dark tint, which happens in about an hour, and then treating it with successive alcohols for several hours. If the ovum be adherent to the uterine mucosa the portion of the membrane to which it is fixed should be left, stretched out with pins, in 0.1 per cent. solution of osmic acid for from four to six hours. The blastodermic vesicle can then easily be removed, and further treated as before. For sections KöLLIKER fixes with osmic acid. v. BenEden treats the ova for twenty-four hours with 1 per cent. solution of chromic acid, then washes well, and brings them through successive alcohols. Chromic acid has the advantage of hardening thoroughly the vesicle, and maintaining at the same time the epiblast cells perfectly adherent to the zona pellucida. v. BENEDEN also recommends the liquid of Kleinenberg. Hennegur writes that he frequently employs it for embryonic areas and embryos of various ages, always with excellent results. Fol's modification of the liquid of Flemming, and Ranvier and Vignal's osmic acid and alcohol mixture $(\S 36)$ also give excellent results. For staining, HENNEGUY recommends borax-carmine, or Delafield's hæmatoxylin for small embryos; for large ones he found that his acetic acid alum-carmine was the only reagent that would give a good stain in the mass.

For sections imbed in paraffin, or double imbed.

590. On the Fixation of Whole Tubes.-This may be done in Carnoy, Bouin or Helly. For rapidity of fixation, and faithfulness of preservation of cell aggregates Carnoy's fluid or preferably Sansom's modification of Carnoy are to be recommended. Chromeformalin mixtures penetrate less readily, but often give fine results. Bouin's fluid I have found capricious. On the whole I think that warm Helly or Müller-formol as a preliminary fixation are to be recommended for small tubes. Regaud's or Schridde's methods should give efficient fixation ( $\$ \$ 684-689$ ). Many workers have used the picric mixtures like picro-sulphuric and nitric, and Kleinenberg's picric acid. Flemming's fluid has also been used.

In -later stages of development some workers open the uterus 
under fixative, or ligature one end of the organ and inject some fixing medium.

Corrosive formol mixtures have been much used for this purpose.

Neutral formalin of from 3 to 10 per cent. strength is often used for preserving later stages, after the uterus has been opened out. The advantage of this procedure from the cytological point of view is that any methods such as those of Regaud, BensleyCowdry, Sjövall, or formol-silver nitrate neurological techniques may subsequently be used. The chrome-picric or alcoholic acetic formol mixtures are not so suitable if one has cytological study in view.

591. On Clearing Mammalian Material.-This is an important matter, because delicate embryos are easily shrunken up, or even not properly dealcoholised, by injudicious methods. J.P. Hill clears in two stages. Dehydrated embryos are brought into cedar wood oil in which they are left overnight. The cedar wood oil is subsequently washed out in benzole for several hours according to size of object. Paraffin parings are then added to the benzole, contained preferably in a tube, and the latter is then left overnight uncovered on the top of the bath, and subsequently put into pure wax. This method insures a gentle dealcoholisation, and an efficient imbedding. Neither cedar wood oil nor benzole cause the tissue to become brittle as happens often when one uses xylol or chloroform (see $\S \S 120-135)$.

Imbedding.-For embryological work of a critical character, especially with post-blastoderm stages, double-imbedding in celloidin and wax is generally indispensable. It is only necessary to

- contrast serial sections of chick blastoderms prepared by this method, with those obtained by wax imbedding alone to become convinced of the inability of the latter method to do complete justice to the details of the structure and relations of the embryonic tissues (Wilson and Hill, Phil. Trans. Roy. Soc., 1907).

See also J. P. Hrll (Anat. Anz., Bd. xviii, 1900 ; Quart. Journ. Micr. Science, Ivi, 1910); Hartmann (Jour. Morph., xxvii, 1916). The latter recommends punching a hole in the side of larger blastoderms to facilitate penetration of dehydrating and clearing fluids. Weysse, Proc. Amer. Acad. Arts and Sci., 1894, p. 285 (blastodermic vesicle of Sus scrofa); Зовотта, Arch. mik. Anat., xlv, 1895, p. 15 (ovum of the Mouse; fixation in Flemming's weak mixture, sections stained with BendA's iron hæmatoxylin), and Anat. Hefte, 1 Abth., viii, 1897, p. 476 (Rabbit ; fixation with liquid of F'lemming or picro-sublimate with 2 per cent. acetic acid); BONNET, ibid., ix, 1897, p. 426 (Dog; fixation in sublimate); SelenkA, Stud. Entw. d. Thière, Wiesbaden, 1883, p. 5, and 
1887, p. 107 (picro-sulphuric acid for the mouse, and picric acid with $\frac{1}{10}$ per cent. of chromic acid for Didelphys); KeIBeL, Morph. Arb., ii, 1893, p. 11 (Sus scrofa); NedmaYer, Festschr. f. Kupffer, 1899, p. 458 (embryos of the sheep best fixed in Carnoy's acetic acid, alcohol, and chloroform, § 85); Winiwarter, Arch. Biol., xvii, 1900, p. 39 (mixture of 50 parts saturated sublimate in salt solution, 50 parts alcohol, 20 of 1 per cent. platinum chloride, and 5 of acetic acid); SPEE, Encycl. mik. Techn., 1910, p. 353 (cornua of Cavia fixed for twelve to twenty-four hours in sublimate, and put into 0.5 per cent. osmic acid till light brown, then into iodine alcohol, in which the osmium is reduced); WIDAKowICH, Zeit. wiss. Zool., xciv, 1909, p. 243 (Mus rattus, fixation in Zenker's mixture, or 2 parts of alcohol of 80 per cent. with 1 of formol; also instructions for dissection).

592. Injection and Clearing of Larger Embryos. - A considerable amount of useful work has lately been carried out on embryonic blood and lymph vessels, and on the cerebro-spinal cavities, by micro-injection apparatus. A suitable injection medium is blown or forced into the vessels of an embryo, the latter is fixed and then dehydrated, and cleared by the Spalteholz method (Über das Durchsichtigmachen von menschlichen und tierschen Präparaten, und seine theoretischen Bedingungen, Leipzic, S. Herzel, 1911 ; 2 Aufl., 1914).

In an early stage in the formation of embryonic vessels and cavities the walls are thin and often ill-marked, and care must be taken not to burst through boundaries by excessive pressure. Very fine metal needles or, better, finely drawn out glass cannulæ are. used for injecting the specimens ; the tube leading to the cannula is filled with the injection medium, which, by means of a rubber tube leading to the operator's mouth, is blown carefully into the perforated vessel or cavity. Or, one may use a rubber bulb either . worked by hand, or placed on the floor and compressed by the foot. See E. M. Gregory, Anat. Record, xi, 1917.

The injection media most commonly used are india-ink, a saturated solution of Prussian blue, an aqueous suspension of lamp black, or silver nitrate ( 5 per cent.). The Prussian blue and india-ink give about equal results, the blue clearing better, the ink being more opaque. The ink flows the better. Silver nitrate preparations are very beautiful and easy to analyse, but its caustic action prevents the finer vessels from filling. Lamp black tends to precipitate in fine flakes (Cunningham, vide infra). Evans (vide infra), for cerebro-spinal spaces of pig embryos, injected potassium ferrocyanide, 0.5 grms., iron ammonium citrate, 0.5 grm., aq. dest., 100 c.c., and afterwards immersed the embryo for one to ten minutes in a 10 per cent. formaldehyde solution containing 1 per cent. $\mathrm{HCl}$. 
The embryo was then fixed in Bouin's fluid, but the Prussian blue faded after about a year.

SABIN (vide infra) and CunNingham, after india-ink injection, fix in Carnoy's fluid, place in 80 per cent. alcohol, dehydrate in graded alcohols, clear thoroughly, first in benzine (or benzol), and then in oil of wintergreen (Spalteholz). Embryos cleared by Spalteholz's method may later be embedded from oil of wintergreen by transferring to half wax, half oil of wintergreen, and then pure wax. Tissues left in oil of wintergreen do not go brittle even after a year or two (Sabin).

For areas of osteoblastic activity, see $\S 780$, and cartilaginous skeletons, $\S 779$.

See also R. S. Cunningham (Contrib. Carng. Inst. Wash., 1916, No. 12) ; L. H. Weed (ibid., No. 14, 1917) ; F. SABIN (Johns Hopkins Hosp. Report Monographs, N.S., No. 5, Baltimore, 1913); Contrib. to Embryol. Carneg. Inst. Wash., No. 7, 1915); P. G. SHIPley and C. C. MACKLIN (Anat. Record, x, 1915-16).

\section{Aves.}

593. Superficial Examination.-Instructions on this head are given in Foster and BALFouR's Elements of Embryology. The following is of more recent publication.

If it be desired to observe a living embryo by transmitted light, the egg should be opened under salt solution, as described below. A little of the white is then removed through the window, the egg is lifted out of the liquid, and a ring of gummed paper is placed on the yolk so as to surround the embryonic area. As soon as the paper adheres to the vitelline membrane, which will be in a few minutes, a circular incision is made in the blastoderm outside the paper ring. The egg is put back into the salt solution, and the paper ring removed, carrying with it the vitelline membrane and the blastoderm, which may then be brought into a watch-glass or on to a slide and examined under the microscope (DUVAL).

Gerlach's Window Method (Nature, 1886, p. 497).--Remove with scissors the shell from the small end of the egg; take out a little white by means of a pipette; the blastoderm will become placed underneath the window just made, and the white that has been taken out may be replaced on it. Paint the margins of the window with gum mucilage, and build up on the gum a little circular wall of cotton wool; place on it a small watch-glass (or circular cover-glass), and ring it with gum. When the gum is dry the cover is further fixed in its place by means of collodion and amber varnish, and the egg is put back in its normal 
position in the incubator. The progress of the development may be followed up to the fifth day through the window.

A description of further developments of this method, with figures of special apparatus, will be found in Anat. Anz., ii, 1887, pp. 583, 609.

See also Paton, Journ. Exper. Zool., xi, 1911, p. 469 (cultivation of the embryo in vitro).

594. Preparation.-D During the first twenty-four hours of incubation, it is extremely difficult to separate the blastoderm from the yolk, and they should be fixed and hardened together.* In later stages, when the embryo is conspicuous, the blastoderm can easily be separated from the yolk, which is very advantageous. To open the egg, lay it on its side and break the shell at the broad end by means of a sharp rap; then carefully remove the shell bit by bit by breaking it away with forceps, working away from the broad end until the blastoderm is exposed. The egg should be opened in salt solution, then lifted up a little, so as to have the blastoderm above the surface of the liquid; the blastoderm is then treated with some fixing solution dropped on it from a pipette ( 1 per cent. solution of osmic acid, or Ranvier and Vignal's osmic acid and alcohol mixture, iodised serum, solution of Kleinenberg, 10 per cent. nitric acid, etc.). By keeping the upper end of the pipette closed, and the lower end in contact with the liquid on the blastoderm, the blastoderm may be kept well immersed for a few minutes, and should then be found to be sufficiently fixed to be excised. (Of course, if you prefer it, you can open the egg in a bath of any fixing liquid [10 per cent. nitric acid being convenient for this purpose] of such a depth as to cover the yolk; and having exposed the blastoderm, leave it till fixed [fifteen to twenty minutes]; but I think the procedure above described will generally be found more convenient.)

The egg is put back into the salt solution, and a circular incision made round the embryonic area. The blastoderm may then be floated out and got into a watch-glass, in which it may be examined, or may be brought into a hardening liquid.

Before putting it into the hardening fluid, the portion of vitelline membrane that covers the blastoderm should be removed with forceps and shaking.

* Andrews (Zeit. wiss. Mik., xxi, 1904, p. 177) separates the blastoderm at this stage by injecting picro-sulphuric acid (not any rapidly acting fixative) firstly, between the blastoderm and the vitelline membrane, so as to separate the two above, and then between the blastoderm and the yolk, so as to free the blastoderm below and float it up. This done, the membrane may be incised and the blastoderm removed. The, injection is best done with a pipette having a fine point bent upwards, 
Fixation in 10 per cent. nitric acid has the advantage of greatly facilitating the separation of the blastoderm. The acid should be allowed to act for ten minutes, after which it is well to bring the preparation into 2 per cent. solution of alum ( $c f$. Hofmann, Zeit. wiss. Mik., x, 1893, p. 485). Mitrophanow (Anat. Hefte, xii, 1899, p. 200) fixes with nitric acid of 3 per cent.; Suschkin (Nouv. Mém. Soc. Nat. Moscow, xvi, 1899, p. 34) with sublimate; Fischel (Morph. Jahrb., xxiv, 1896, p. 371) with Rabl's platino-sublimate, $\S 76$ (embryos of the duck); Patterson (Biol. Bull. Wood's Hole, xiii, 1907, p. 252) with picro-sulphuric acid containing 8 per cent. of acetic acid, for an hour (ova of Columba); Hoskins (Kansas Univ. Sci. Bull., iv, 1907, p. 176), after removing shell, for five to fifteen minutes in a mixture of 3 parts of 10 per cent. formol with 1 of 10 per cent. nitric acid, and then excises the embryo.

In order to counteract the turning up of the edges of the blastoderm that generally happens during the process of hardening, it is well to get the blastoderm spread out on the convex surface of a watch-glass, and leave it so during the hardening.

For hardening HenNegur prefers the osmic acid and alcohol mixture of Ranvier and Vignal, or Flemming's mixture followed by successive alcohols.

Stain and imbed by the usual methods.

Up to about the fiftieth hour embryos may be mounted entire in glycerin or balsam.

595. M. Duval's Orientation Method (Ann. Sc. Nat., 1884, p. 3).In the early stages of the development of the ova of Aves, before the appearance of the primitive streak, it is difficult to obtain a correct orientation of the hardened cicatricula, so as to be able to make sections in any desired direction. Duval, starting from the fact that during incubation the embryo is almost always found to be lying on the yolk in such a position that the big end of the egg is to the left and the little end to the right of it, marks the position of the blastoderm in the following way.

With a strip of paper 5 millimetres wide and 50 millimetres long you construct a sort of triangular bottomless box. You lay this on the yolk, enclosing the cicatricula in such a position that the base of the triangle corresponds to what will be the anterior region of the embryo, and its apex to the posterior region ; that is to say, if the big end of the egg is to your left, the apex of the triangle will point towards you. You now, by means of a pipette, fill the paper triangle with $0 \cdot 3$ per cent. solution of osmic acid. As soon as the preparation begins to darken you put the whole egg into weak 
chromic acid, remove the white, and put the rest into clean chromic acid solution for several days. After hardening you will find on the surface of the yolk a black triangular area, which encloses the cicatricula and marks its position; you cut out this area with scissors and a scalpel, and complete the hardening with chromic acid and alcohol.

See also the method of Hirota, Journ. Roy. Mic. Soc., 1895, p. 118.

596. KIoNkA's Orientation Method (Anat. Hefte, 1 Abth., iii, 1894 , p. 414).- Open the egg under salt solution, free it from the shell and albumen, and mark the poles by sticking into it, at about a centimetre from the blastoderm, two hedgehog spines, the one at the obtuse end being marked with a red thread. Put the whole for ten minutes into water at $90^{\circ} \mathrm{C}$., then bring into 70 per cent. alcohol, and after twenty-four hours cut out the blastoderm and a little yolk round it in the shape of an isosceles triangle, whose base marks the anterior end of the blastoderm. Paraffin sections stained with borax-carmine, washed out with acid alcohol containing 1 drop of concentrated solution of Orange $\mathrm{G}$ for each 5 c.c., which stains the yolk.

597. Vialleton's Method (Anat. Anz., vii, 1892, p. 624).-Egg opened in salt solution, blastoderm excised and removed to a glass plate, then treated with 1 per cent. nitrate of silver solution, washed with water, and put into 70 per cent. alcohol for six to twelve hours in the dark. Borax-carmine, alcohol, damar.

598. Chick and Reptile Blastoderms.-Gerhardt (Anat. Anz., $\mathrm{xx})$ uses :-

Chromic acid 1 per cent. • • . . 150 c.c. Sat. corr. subl. . . . . . . 150 , Aq. dest. - . . . . . . . 135 ,

Acetic acid . . . . . . $15 \%$

Formalin . . . . . . 150 ,

Leave in twenty-four hours. Wash twenty-four hours in running water, upgrade from 70 per cent. alcohol, 90 per cent. with iodine, pure 90 per cent., etc. Recommended by Prof. J. P. Hill.

Reptilia.

599. General Directions. - The methods described above for birds are applicable to reptiles. During the early stages the blastoderm should be hardened in situ on the yolk; later the embryo can be isolated, and treated separately.

BöHM and OPPEL (Taschenbuch, 1900, p. 186) remove the shell 
under salt solution, fix in sublimate with 20 per cent. acetic acid, or in Lo BIANco's chromo-sublimate ( $\$ 72)$, then remove the blastoderm and bring it into alcohol.

600. Special Cases.-MItsukuri (Journ. Coll. Sc. Japan, vi, 1894, p. 229) fixes embryos of tortoises chiefly with picro-sulphuric acid. To study the blastoderm he removes the whole of the shell and as much as possible of the albumen, marks the place where the blastoderm lies with a hair, brings the whole, with the blastoderm uppermost, into the fixative, and after a few hours cuts out the blastoderm and further hardens it by itself. Young embryos generally adhere to the shell and can, therefore, be fixed in a piece of it made to serve as a watch-glass, then after half-an-hour can be removed from it and further hardened alone. If the embryonal membranes have been formed, the shell may be scraped away at some spot and there treated with picro-sulphuric acid until a small hole is formed; then by working away from this spot, by means of scraping and dropping acid on to it, the whole of the shell may be removed.

Will (Zool. Jahrb., Abth. Morph., vi, 1892, p. 8) opens ova of Platydactylus in the fixative (chiefly chromic acid, or chromoaceto-osmic acid with very little osmic acid) and hardens the embryos on the yolk; so also for Cistudo and Lacerta (1893 and 1895). MehneRt (Anat. Anz., xi, 1895, p. 257) does not approve of these methods ; for his own see Morph. Arb. Schwalbe, i, 1891, p. 370.

GerhardT (Anat. Anz., xx, 1901, p. 244) fixes ova of Tropido. notus for twenty-four hours in Nowak's mixture, 112.

Ballowitz (Entwickl. d. Kreuzotter, 1903, p. 19) first fixes seg. ments of the uterus, each containing an ovum, for one or two hours, then tears them open with forceps, isolates the ova, and puts them into fresh fixative, and thence into alcohol of 40 per cent.

Nicolas (Arch. Anat. Mic., 1900, p. 457) finds the best fixative for ova of the slow-worm, as for other large ova, is 'Bouin's picro. formol (§ 110).

See also Perényi, § 48, and Zool. Anz., 1888, pp. 139 and 196, and other methods in early editions.

\section{Amphibia.}

601. Preliminary.- In order to prepare ova for section-cutting, it is essential to begin by removing their thick coats of albumen. This may be done by putting them for two or three days into 1 per cent. solution of chromic acid, and shaking well; but ova thus treated are very brittle, and do not afford good sections. A better 
method is that described by WhITMAN (Amer. Natural., xxii, 1888, p. 857), and by Blochmann (Zool. Anz., 1889, p. 269). Whitman puts the fixed eggs into a 10 per cent. solution of sodium hypochlorite diluted with 5 to 6 volumes of water, and leaves them there till they can be shaken free, which happens (for Necturus) in a few minutes. Blochmann takes eau de Javelle (potassium hypochlorite), and dilutes it with 3 to 4 volumes of water, and agitates the eggs previously fixed with solution of Flemming, for fifteen to thirty minutes in it.

Lebrun (La Cellule, xix, 1902, p. 316) advises fixing ova of Anura for not less than one and a half hours in liquid of Gilson, $\S 69$. The outer envelopes are then hard, and may be easily incised and the ovum extracted by pressing on the pole opposite to the incision. The operation should not be delayed until after hardening in alcohol. Similarly (ibid., xx, 1902, p. 12) for Urodela.

Guyer (Amer. Nat., xli, 1907, p. 400) finds it suffice to roll the ova (either fresh or fixed, but before bringing into alcohol) on blotting paper.

602. Imbedding.-A great difficulty with the ova of Amphibia lies in their becoming extremely brittle on imbedding in paraffin. Carnoy and Lebrun (La Cellule, xii, 1897, p. 212) fix ovaries or ovarian ova for fifteen minutes to three-quarters of an hour (but see last §) in Gilson's mercuro-nitric fluid, § 69, and preserve them in 80 per cent. alcohol. To imbed, they are brought for a quarter of an hour into 95 per cent. alcohol, five minutes in absolute alcohol, then into a mixture of alcohol and chloroform in equal parts, and as soon as they sink in that they are put into pure chloroform. Paraffin is added to the chloroform, enough to about double the volume of the whole, and the whole is put for about three hours into a stove at $35^{\circ} \mathrm{C}$. Lastly, the ova are put for not more than five minutes into a bath of pure paraffin at $52^{\circ} \mathrm{C}$.

Later (ibid., xix, 1902, p. 317) LEBRUN explains that it is important not to dehydrate completely with absolute alcohol ; the ova should be left in alcohol of 96 per cent. until chloroform can be added without the mixture becoming turbid, and a second bath of clean paraffin should be added.

See also Morgan, Devel. of the Frog's Egg, New York, 1897, p. 171.

603. Siredon. - The ova are easier to prepare than those of the Anura, because the yolk is separated from the albuminous layer by a wide space filled with a liquid that is not coagulated by reagents. Put the eggs for a few hours into picro-sulphuric acid, then pierce 
the inner chorion with fine scissors or needles, and gently press out the ovum. Harden in alcohol.

Frck (Zeit. wiss. Zool., lvi, 1893, p. 529) uses a mixture of 250 parts of 1 per cent. chromic acid, 1 of acetic acid, and 750 of water.

604. Triton (Scotт and Osborn, Quart. Journ. Mic. Soc., 1879, p. 449). - The albumen is here present in the form of several concentric coats, which are very delicate. Incise each of them separately with fine scissors, turn out the ovum, and fix it in solution of Kleinenberg.

HerTwig (Jen. Zeit. Naturw., 1881-2, p. 291) puts the eggs into a mixture of equal parts of 2 per cent. acetic acid and 0.5 per cent. chromic acid. After ten hours he incises the membranes, opening one end of the inner chorion, and turns out the embryos and brings them into successive alcohols.

Mrchaelis (Arch. mik. Anat., xlviii, 1896, p. 528) fixes ova, with their envelopes, in a mixture of concentrated sublimate solution and concentrated picric acid, 20 parts each, glacial acetic acid 1, and water 40 , but removes the envelopes before bringing into alcohol.

605. Salamandra (RABL, Morphol. Jahrb., xii, 2, 1886, p. 252).For his more recent methods see $\S 580$.

Grönross (Anat. Anz., xiv, 1898, p. 461) fixes the ova with a mixture of 50 parts each of saturated sublimate and 0.5 per cent. chromic acid with 1 part of acetic acid.

606. Rana.-I have found that the following mixture often gives very good results for the eggs of Rana temporaria; it dissolves away the albumen coat, preserves yolk and mitochondria, and leaves the eggs soft enough to cut in paraffin with a rotary microtome:-

Bichromate of potash of 2 per cent. $\quad$ - 100 c.c. Chromic acid of 1 per cent. . . . . 100 , Nitric acid .

Use at least 40 c.c. to twenty or thirty eggs for fifteen to twentyfour hours. Slightly shake, and the albumen coats fall off if not already dissolved. Wash out for about one hour in running water and then upgrade from 70 per cent. alcohol (one half-hour), 90 per cent. (one hour), to absolute alcohol, two changes of one hour each. Clear in benzol for fifteen minutes. Add chips of wax and place in thermostat for half an hour. Transfer to pure wax for one-half (to three-quarters) of an hour. Avoid unnecessary heat. 
The eggs thus treated can often be cut $6 \mu$ on a rotary microtome provided with a sharp knife. The method is indicated where large numbers of stages of the early development of the frog are required for junior class purposes. The main objection to the method is that the chromic acid attacks pigment. Fertilisation and segmentation stages and general cytology are often extremely good; stain in any way (J. B. G.).

O. Hertwig (Jen. Zeit. Naturw., xvi, 1883, p. 249).-The ova are thrown into nearly boiling water $\left(90^{\circ}\right.$ to $96^{\circ} \mathrm{C}$.) for five or ten minutes. The albuminous envelope of the ovum is then cut open, and the ovum extracted under water. The ova are then brought into 0.5 per cent. chromic acid for not more than twelve hours, or into alcohol of 70,80 , and 90 per cent. Chromic acid makes ova brittle and attacks the pigment, whilst alcohol preserves it, which is frequently important for the study of the germinal layers.

Morgan (Amer. Nat., xxv, 1891, p. 759, and Devel. of the Frog's $E g g, 1897$, p. 171) has the following. During the periods in which it is difficult or impossible to remove the inner jelly-membrane the eggs can be freed as follows : Each egg is cut out with scissors from the general jelly-mass, and put for from one to twelve hours into saturated solution of picric acid in 70 per cent. alcohol containing 2 per cent. of sulphuric acid. Wash in several changes of alcohol of 70 per cent. About the second day in this the inner membrane begins to swell, and on the third or fourth day may be pierced by a needle, and the egg removed and placed in 80 per cent. alcohol. See also Whitman, Meth. of Research, p. 156.

Schultze (Arch. mik. Anat., lv, 1899, p. 174) removes with scissors the outer layers of albumen, and puts the ova for five minutes in 2 per cent. formol warmed to $75^{\circ}$ or $80^{\circ} \mathrm{C}$. The membrane left on the ova then rises up sufficiently to allow the ova to be got out with needles.

See also Born (ibid., xliii, 1894, p. 1).

KING (Journ. Morph., xvii, 1901, p. 295, and xix, 1908, p. 370) fixes (for a few minutes) the spawn (of $B u f o$ ) in sublimate (saturated with 5 per cent. of acetic acid), or in Flemming, Zenker, or Hermann, brings into alcohol, first of 50 and then 80 per cent., and removes the jelly after a few days.

Bles (Trans. Roy. Soc. Edinburgh, xli, 1905, p. 792) takes for ova formol of 10 per cent., but for embryos and larvæ the mixture given $§ 109$.

BouIN takes for larvæ of Rana the formol-sublimate mixture $\S 112$. 
607. Sulphate of Copper Liquid (FoL, Lehrbuch, p. 106, after ReMAK and Goette); for hardening ova of Amphibia :

2 per cent. solution of sulphate of copper. 50 c.c.

Aleohol of 25 per cent. . . . . 50 , Rectified wood vinegar . $\quad$. . 35 drops.

\section{Pisces.}

608. Teleostea in General.- The ova of many of the bony fishes can be studied by transmitted light in the living state; but those of the Salmonidæ must be hardened and removed from their envelopes for the study of the external forms of the embryo.

To this end they may be put for a few minutes into water containing 1 to 2 per cent. of acetic acid, and thence into 1 per cent. chromic acid. After three days the capsule of the ovum may be opened at the side opposite to the embryo, and be removed with fine forceps. The ovum is put for twenty-four hours into distilled water, and then into successive alcohols. Embryos thus prepared show no deformation, but the vitellus rapidly becomes excessively hard and brittle, so as greatly to interfere with sectioncutting.

The following processes give good results as regards sectioncutting.

Put the ova for a few minutes into 1 per cent. osmic acid; as soon as they have taken on a light brown colour bring them into Müller's solution. Open them therein with fine scissors-the vitellus, which immediately coagulates on contact with air, dissolves, on the contrary, in Müller's solution-and the germ and cortical layer can be extracted from the capsule of the ovum. They should be left in clean Müller's solution for a few days, then washed with water for twenty-four hours, and brought through. successive alcohols.

Another method (Henneguy) is as follows: The ova are fixed in solution of Kleinenberg containing 10 per cent. of acetic acid. After ten minutes they are opened in water containing 10 per cent. of acetic acid, which dissolves the vitellus. The embryos are put for a few hours into pure solution of Kleinenberg, and are then brought through alcohol of gradually increasing strength.

Child (quoted from Sumner, Mem. New York Acad. Sci., ii, 1900 , p. 78) fixes for about a minute in sublimate with 10 per cent. of acetic acid, and brings into formalin of 10 per cent., which is said to give a good fixation of the embryo without the yolk becoming hard. 
609. Kollmann's Fixative (Kollmann, Arch. Anat. Phys., 1885, p. 296).

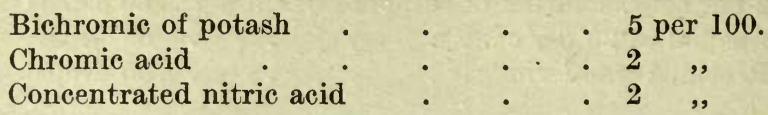

For ova of Teleostea. Fix for twelve hours, wash with water for twelve hours, then remove the chorion, and put the ova into 70 per cent. alcohol.

610. RaBL's Method, see $\S 587$; for KowalewskY's see Zeit. wiss. Zool., xliii, 1886, p. 434, or Third Edition.

611. Salmonidæ.-HenneguY's methods have been given, $\S 609$.

Kopsch (Arch. mik. Anat., li, 1897, p. 184), on the suggestion of VIRCHOw, fixes embryos for five or ten minutes in a mixture of 1 part of chromic acid to 50 of glacial acetic acid and 450 of water, then removes into chromic acid of $1: 500$, and as soon as may be removes the capsule and yolk under salt solution, and completes the hardening in the chromic acid or the saturated sublimate solution.

Similarly, Behrens (Anat. Hefte, x, 1898, p. 233). He opens the ova in the salt solution from the antipolar side, and frees the embryo from the yolk that remains by blowing the latter away with a fine-pointed glass tube.

Similarly also Sовотта (ibid., 1902, p. 579).

Gudger (Proc. U.S. Nation. Mus., xxix, 1906, p. 448) fixes blastoderms in fresh liquid of Perényi, which does not make the yolk too hard; later stages in WORCESTER's liquid (9 parts of saturated solution of sublimate in formol of 10 per cent. and 1 part of acetic acid), for half an hour to an hour, and brings gradually into alcohol of 70 per cent.

Bouin (C. R. Soc. Biol., lv, 1903, p. 1691) fixes for thirty-six to forty-eight hours in picro-formol.

RABL-RÜCKHARD's Method (Arch. Anat. Entw., 1882, p. 118).-Fix in 10 per cent. nitric acid for fifteen minutes. Remove the membranes to avoid deformation of the embryos, and put the ova back into the acid for an hour. Wash out in 1 to 2 per cent. solution of alum for an hour and harden in alcohol.

Modification of this method by Goronowitsci (see Morph. Jahrb., x, 1884, p. 381).

612. Selachia.-BEARd (Anat. Anz., xviii, 1900, p. 556) has found that the best fixatives for embryos of Raja are Rabl's picroplatinic mixture, $§ 587$, and sublimate.

Living embryos can be observed by scraping the shell thin with a knife (Kastschenko, Anat. Anz., iii, 1888, p. 445, and His, Arch. 
Anat. Phys., Anat. Abth., 1897, p. 3). See also Braus, Morph. Jahrb., xxxv, 1906, p. 250.

613. Amphioxus.--овотта (Arch. mik. Anat., 1, 1897, p. 20) fixes for twenty-four hours in liquid of Flemming; HATscheK ( $A r b$. Zool. Inst. Wien., iv, 1881) in picro-sulphuric acid. Impregnation takes place in the evening, and segmentation is completed during the night.

Legros (Grundzüge, LeE and Mayer, 1910, p. 288) fixes ova and embryos in equal parts of formol and Flemming. Sublimate is not good ; Rabl's mixtures are better. Larvæ and young animals ought first to be anæsthetised with cocain in sea-water. After fixation they should remain only for as short a time as possible in alcohol.

Cerfontaine (Arch. Biol., xxii, 1906, p. 287) fixes with Flemming or Hermann. For study of ova in toto he orients them on a slide in clove-oil-collodion which he sets with chloroform, and adds balsam. For sectioning, he orients in the same way on a layer of paraffin spread on a cover glass and imbeds the whole in paraffin.

614. Pelagic Fish Ova.-Whitman (Amer. Natural., xvii, 1883, pp. 1204-5; and Methods of Research, etc., p. 152). -Fix by treatment first for five to ten minutes with a mixture of equal parts of sea-water and $\frac{1}{2}$ per cent. osmic acid solution, and then for one or two days with a solution (due to Eisig) of equal parts of $\mathbf{0 . 2 5}$ per cent. platinum chloride and 1 per cent. chromic acid. Prick the membrane before transferring to alcohol. See also Agassiz and Whitman, in Proc. Amer. Acad. Arts and Sciences, xx, 1884 ; and Collinge, Ann. and Mag. Nat. Hist., x, 1892, p. 228.

RaFfaede (Mitth. Zool. Stat. Neapel, xii, 1895, p. 169) fixes chiefly with liquid of Hermann (1 to 2 days), or with a mixture of Mingazzini (absolute alcohol 1, acetic acid 1, saturated sublimate solution in water 2).

Heinke and Ehrenbaum (Wiss. Meeresunt. Komm. Wiss. Unt. D. Meere, iii, Heligoland, 1900, pp. 205 and 213) prefer formol with 39 volumes of sea-water.

\section{Tunicata.}

615. Ova.-Davidorf (Mitth. Zool. Stat. Neapel, ix, 1, 1889, p. 118) fixes the ova of Distaplia with a mixture of 3 parts of saturated solution of corrosive sublimate and 1 of glacial acetic acid for from half an hour to an hour; or with a mixture of 3 parts of saturated solution of picric acid and 1 of glacial acetic acid for three to four hours; then 70 per cent. alcohol.

CASTLE (Bull. Mus. Harvard Coll., xxvii, 1896, p. 213) advises for ova of Ciona liquid of Perényi for twenty minutes, followed by 
70 per cent. alcohol for twenty-four hours, and for the larvæ picronitric acid.

616. Test-Cells of Ascidians (Morgan, Journ. of Morphol., iv. 1890, p. 195).-Tease fresh ovaries in very weak osmic acid, wash in distilled water, treat for half an hour with 1 per cent. silver nitrate, wash for half an hour in 2 per cent. acetic acid and reduce in sunlight. Imbed in paraffin. By this process the limits of the follicle cells are demonstrated.

61\%. Buds.-Pizon (Ann. Sc. Nat., xix, 1893, p. 5) studies the gemmation of the composite Ascidians either on entire corms, which he first bleaches with peroxide of hydrogen and then stains, or by making sections, after anæsthetising the colonies with cocain of $1: 1000$, fixing in glacial acetic acid or picro-sulphuric or liquid of Flemming, and staining in toto with borax carmine or alum carmine, or with a strong solution of methylen blue in alcohol of 90 or 100 per cent. (after BERNARD, ibid., ix, 1890, p. 97).

RitTer (Journ. of Morph., xii, 1896, p. 150) recommends for fixing Perophora and Goodsiria picro-sulphuric acid.

\section{Bryozoa.}

618. Statoblasts.-Braki (Bibl. Zool., Chun and Leuckart, $6 \mathrm{Heft}, 1890$, p. 95) fixes statoblasts of Cristatella with hot concentrated solution of sublimate for ten minutes, brings them into water and there incises them with a razor, and after half an hour passes them gradually into alcohol. He stains with picro-carmine.

\section{Mollusca.}

619. Cephalopoda (Ussow, Arch. de Biol., ii, 1881, p. 582).Segmenting ova are placed in 2 per cent. solution of chromic acid for two minutes, and then in distilled water, to which a little acetic acid (1 drop to a watch-glassful) has been added, for two minutes. If an incision be now made into the egg-membrane, the yolk flows away and the blastoderm remains; if any yolk still clings to it, it may be removed by pouring away the water and adding more.

Watasé (Journ. of Morphol., iv, 1891, p. 249) kills the ova in the macerating mixture of the Hertwigs ( $\$ 534$ ), and as soon as the blastoderm turns white and opaque removes it under dilute glycerin. Treatment with liquid of Perényi is recommended for surface views.

Vialleton (Ann. Sc. Nat., vi, 1887, p. 168) brings ovarian ova of Sepia into a freshly prepared mixture of picro-sulphuric acid and 2 per cent. solution of bichromate of potash in equal parts, 
and after one or two minutes incises them in the equator, fixes for an hour and a half in picro-sulphuric acid the halves that contain the formative vitellus, separates this from the nutritive vitellus with a spatula, spreads it out, and hardens it in alcohol of 70 to 90 per cent. He fixes entire ova in liquid of Flemming or osmic acid.

Korschelt (Festchrift Leuckart, Leipzig, 1892, p. 348) fixes advanced embryos of Loligo in liquid of Flemming, sublimate, picro-sulphuric acid, or 0.2 per cent. chromic acid. This last is specially good for young embryos if it is washed out with many changes of picric acid.

Faussek (Mitth. Zool. Stat. Neapel, xiv, 1900, p. 83) recommends picro-nitric acid. Fix in this, harden in alcohol, bring the ova, still in their albumen, into hæmalum, stain for twenty-four hours, wash in 1 per cent. alum solution for twenty-four hours, when the albumen will be found softened so that the ova can easily be extracted.

620. Gastropoda (Henneguy).-Ova of Helix may be fixed for from four to six hours in Mayer's picro-nitric acid. The carbonate of lime that encrusts the external membrane is thus dissolved, and the albuminous coat of the egg is coagulated. The egg is opened with needles, the albumen comes away in bits, and the embryo can be removed.

Henchman (Bull. Mus. Comp. Zool., Harvard, xx, 1890, p. 171) fixes ova of Limax with $0 \cdot 33$ per cent. chromic acid, or with liquid of Perényi. It is best to remove only the outer envelope before putting into the chromic acid, the inner membrane being removed after two or three minutes therein. Where Perényi is used the membranes must be removed first, as the albumen will else coagulate in such a way as to prevent the removal of the embryos.

Meisenheimer (Zeit. wiss. Z Zool., lxii, 1896, p. 417) dissects out the embryos of Limax and fixes them with picro-sulphuric acid or concentrated sublimate. Advanced embryos are first got into extension by means of 2 per cent. cocaine, or are rapidly killed with hot sublimate.

Schmidt (Entw. Pulmonaten, Dorpat, 1891, p. 4) fixes the ova in toto with concentrated sublimate, and dissects them out afterwards.

Similarly Koford (Bull. Mus. Harvard Coll., xxvii, 1895, p. 35). Or, preferably, the ova are put into salt solution, the shell removed, the albumen removed with a pipette full of salt solution, which dissolves it; the ova are then fixed for one minute in Fol's modification of liquid of Flemming, and brought direct into Orth's picro-lithumcarmine. See also Linville, ibid., 1900 , p. 215 , who adopts this 
method of shelling, but prefers fixing in acetic-acid sublimate, or liquid of Perényi.

Heyder (Zeit. wiss. Zool., xciii, 1909, p. 92), before imbedding embryos of Arion that have been fixed with sublimate, treats them for an hour or two with carbonate of soda of $\frac{x}{10}$ to $\frac{1}{15}$ per cent., which makes the stomach and intestine less brittle.

GatenBy (Quart. Journ. Micr. Science, 1919), for Limncea stagnalis ova, used Flemming's strong fluid without acetic acid, Champy's fluid for two days to a week, and Kopsch's method.

HoLmes (Journ. of Morph., 1900, p. 371) teases the egg-capsules of Planorbis in nitrate of silver of $\frac{3}{4}$ per cent., exposes to sunlight until the cell-limits come out, rinses with $0 \cdot 2$ per cent. hyposulphite of soda, puts. for a few minutes into picric acid, and then through alcohol into balsam.

See also WASHBURN, Amer. Anat., xxviii, 1894, p. 528 (liquid of Flemming, or $\mathbf{0 . 3}$ per cent. chromic acid, or 1 per cent. osmic acid, followed by liquid of Merkel).

CoNkLin (Journ. of Morph., xiii, 1897, p. 7) fixes ova of Crepidula for fifteen to thirty minutes in piero-sulphuric acid, and stains with dilute acidified hæmatoxylin of Delafield.

Kostanecki and Wierzejski (Arch. mik. Anat., xlvii, 1896, p. 313) fix the spawn of Physa fontinalis either in $1 \frac{1}{2}$ to 2 per cent. nitric acid, or in "sublimate and 3 per cent. nitric acid in the proportion of $2: 1$," and bring through successive alcohols. They imbed entire ova in paraffin, but isolated embryos in celloidin.

621. Chiton, see MetcalF, Stud. Biol. Lab. Johns Hopkins Univ., v, 1893, p. 251. (Ova with young embryos put for twenty to forty-five seconds into eau de Labarraque, then into water, in which the chorion swells and can easily be removed.)

622. Lamellibranchiata.-STAUfFacher (Jena Zeit., xxviii, 1893, p. 196) fixes embryos of Cyclas in sublimate, stains with hæmalum, and cuts in paraffin.

Lillie (Journ. of Morph., x, 1895, p. 7) fixes ova of Unio for ten to twenty minutes in liquid of Perényi, and preserves them in 70 per cent. alcohol, or advanced embryos with liquid of Merkel or sublimate, larvæ with 0.05 to 0.1 per cent. osmic acid, preserving them in glycerin. Glochidia may be cut with the shell in paraffin of $58^{\circ}$ melting-point; they may be anæsthetised with chloral hydrate before fixing.

Arthropoda.

623. Fixation of Ova.-In many cases the ova of Arthropods are best fixed by heat ( $(13)$. This may be followed either by alcohol or some watery hardening agent. If it be desired to avoid heating, picro-nitric acid may be tried. 
624. Removal of Membranes. - It may often be advisable not to attempt to remove them, but to soften them with eau de Javelle or eau de Labarraque. See $\S 545$.

Morgan (Amer. Natural., xxii, 1888, p. 357) recommends (for the ova of Periplaneta) eau de Labarraque diluted with 5 to 8 volumes of water, and slightly warmed. This will soften the chitin membranes sufficiently in thirty to sixty minutes, if employed before fixing. Fixed ova take longer. The fluid must, of course, not be allowed to penetrate into the interior of the ovum.

625. Henking's Methods (Zeit. wiss. Mik., viii, 1891, p. 156).HENKING generally kills ova by plunging them into hot water, or by pouring hot water on to them in a watch-glass, and then removing into 70 per cent. alcohol.

He thinks that eau de Javelle for softening membranes is best avoided. They should either be dissected away or left in situ, and cut with the rest of the egg, according to the nature of the case. To avoid brittleness of the yolk proceed as follows: After fixing and treating with alcohol, prick the chorion and stain with boraxcarmine. Put the stained ova for twelve hours into a mixture containing 20 c.c. of 70 per cent. alcohol, 1 drop of concentrated hydrochloric acid, and a knife pointful of pepsin (it is not necessary that all the pepsin should be dissolved). The ova may then be treated with alcohol, oil of bergamot, and paraffin, and (with some exceptions, amongst which is Bombyx mori) will be found to cut without crumbling.

626. Diptera (Henking, Zeit. wiss. Zool., xlvi, 1888, p. 289).Ova still contained within the fly may be fixed by plunging the insect for some time into boiling water, then dissecting out and bringing them into 70 per cent. alcohol. Laid eggs may have boiling water poured over them, or be put into solution of Flemming in a test-tube which is plunged into boiling water until the eggs begin to darken (about a minute). Cold solution of Flemming easily causes a certain vacuolisation of the contents of the ova. Open the ova at the larger end, stain with borax-carmine for fifteen to thirty hours, and cut in paraffin.

Bruel (Zool. Jahrb., Abth. Morph., x, 1897, p. 569) fixes larvæ and pupæ in absolute alcohol heated to $70^{\circ}$ to $75^{\circ} \mathrm{C}$., and containing a "little" sublimate. See also VAN REES, ibid., iii, 1888, p. 10.

Bengtsson (Handl. Fysiogr. Scallsk Lund., viii, 1897) finds hot alcoholic solution of sublimate (Frenzel's, $§ 69$ ) the best fixative for 
larvæ of Phalacrocera. He could not succeed in softening the chitin with eau de Javelle.

PÉRez (Arch. Zool. expér., (4), v, 1910, p. 11) fixes pupæ in Bouin's picro-formal, or Marchoux's mixture, for twenty-four hours.

62\%. Lepidoptera (BoвRETzky, Zeit. wiss. Zool., 1879, p. 198). -Ova are slightly warmed in water and put for sixteen to twenty hours in 0.5 per cent. chromic acid. The membranes can then be removed.

628. Hymenoptera.-CARRIÈre and Bürger (Nova Acta Acad. Leop. Car., lxix, 1897, p. 273) kill ova of Chalicodoma by warming in water to $60^{\circ} \mathrm{C}$, and fix in aqueous picric acid, or alcohol of 70 per cent.

Petrunkewitsch (Zool. Jahrb., Abth. Morph., xiv, 1901, p. 576) fixes for twenty-four hours in his sublimate mixture, and passes into alcohol of 70 per cent. with iodine.

629. Orthoptera (PatTen, Quart. Journ. Mic. Sci., 1884, p. 549).The ova or larvæ (of Blattida) are placed in cold water, which is gradually raised to $80^{\circ} \mathrm{C}$. You leave off heating as soon as the ova have become hard and white. Pass very gradually through successive alcohols, beginning with 20 per cent.

WHEELER (Journ. of Morph., iii, 1889, p. 292) dissects out ovarian ova in salt solution and fixes in liquid of Perényi (fifteen minutes), then treats with alcohol, and stains with borax-carmine. Laid eggs may be killed by Patten's method. After heating, the two lips of the crista of the capsule may be separated with fine forceps and pieces of the walls torn away, and the eggs pushed out of the compartments formed by their choria and hardened as desired. Good results are also obtained by heating to $80^{\circ} \mathrm{C}$. for ten minutes in liquid of Kleinenberg, and preserving in 70 per cent. alcohol. This causes the envelopes to dilate and stand off from the surface of the egg, so that they can easily be dissected away.

Heymons (Zeit. wiss. Zool., liii, 1892, p. 434), for young embryos, incises the cocoon at the end by which it adheres in the body of the mother, brings it for two minutes into water heated to $90^{\circ} \mathrm{C}$., and opens in Flemming, in which the embryo is dissected out.

Morgan (Amer. Natural., xxii, 1888, p. 357) puts ova of Periplaneta for thirty minutes or an hour into eau de Javelle diluted with 4 to 8 volumes of water and slightly warmed, which softens the capsules.

630. Coleoptera.-Hirschler (Zeit. wiss. Zool., xcii, 1909, p. 628) fixes ova of Donacia (after incising the chorion) for two to three 
hours in equal parts of sublimate of 6 per cent. and nitric acid of 3 per cent.

Gatenby (Quart. Journ. Mic. Sci., 1917) for Donacia uses Petrunkewitsch or picro-nitric. In the latter case the chorion must be incised.

Saling (Dissert. Marburg, 1906, p. 10) fixes ova of Tenebrio for about two minutes in a hot mixture of 40 parts of alcohol of 96 per cent., 4 of nitric acid, and 50 of saturated aqueous sublimate; or for three minutes in a hot mixture of 1 part of formol with 3 of water.

KaraWaiew (Biol. Centralb., xix, 1899, p. 124) kills larvæ of Anobium in hot water, freezes them with ether spray, cuts away a lateral strip, lets them thaw, and puts for twenty-four hours into picro-sulphuric acid.

631. Phalangida.-The ova of Phalangium opilio possess a chorion covered with yellow corpuscles that render them opaque. BALBIANI puts them into water with a few drops of caustic potash, and raises to boiling point. The ova are then laid on filter paper, and the chorion removed by rubbing with a camel's hair brush, the vitelline membrane remaining intact, so that the embryo can be studied through it.

Henking's method (Zeit.wiss. Zool., xlv, 1886, p. 86).-Fix with boiling water or Flemming. Preserve the ova in 90 per cent. alcohol. To open the chorion, bring them back into 70 per cent. alcohol, which causes them to swell up so that the chorion can easily be pierced with needles, and the ovum turned out.

632. Araneida.-Kishinouye (Journ. Coll. Sci. Imp. Univ. Japan, iv, 1891, p. 55 ; Zeit. wiss. Mik., ix, 1892, p. 215) fixes in water warmed to $70^{\circ}$ or $80^{\circ} \mathrm{C}$., puts into 70 per cent. alcohol, and after twenty-four hours therein pierces the membranes and passes through stronger alcohol.

See also Locy, Bull. Mus. Comp. Zool. Harvard, xii, 3, 1886. Fix by hot water. The liquid of Perényi may also be used; it has the advantage of not making ihe yolk so granular.

Montgomery (Journ. Morph., xx, 1909, p. 628) fixes ova of Theridium for one or two hours in Carnoy \& Lebrun's mixture.

LAMBERT (ibid., p. 420) fixes ova of Epeira in picro-sulphuric acid warmed to $70^{\circ}$ or $80^{\circ} \mathrm{C}$.

Purcell (Quart. Journ. Micr. Sci., liv, 1909, p. 7) fixes ova of Atta in boiling saturated sol. of sublimate in alcohol of 70 per cent.

Hamburger (Zeit. wiss. Zool., xcvi, 1910, p. 3) fixes ova of Argyroneta in Gilson's mixture. 
633. Limulus.-Kingsley (Journ. Morph., vii, 1892, p. 38) kills ova by heating in sea-water to $70^{\circ}$ or $75^{\circ} \mathrm{C}$. and brings into alcohol of 30 to 70 per cent. Similarly Kishinouye, Journ. Coll. Sci. Japan, v, 1893, p. 56.

634. Decapoda.-Reichenbach (Abh. Senckenberg Ges. Frankfurt, xiv, 1886, p. 2) fixes ova of Astacus in water gradually warmed to $60^{\circ}$ or $70^{\circ} \mathrm{C}$. (if the chorion should burst, that is no evil), hardens for twenty-four hours in 1 to 2 per cent. bichromate of potash or 0.5 per cent. chromic acid, washes out for the same time in running water, and brings into alcohol. Remove the chorion, and remove the embryo from the yolk with a sharp knife.

Herrick (Bull. U.S. Fish, Comm., xv, 1896, p. 226) kills the ova in hot water, shells and fixes in picro-sulphuric acid.

For Homarus, see WAIte, Bull. Mus. Comp. Zool., xxxv, 1899, p. 155 .

635. Amphipoda.-Della VAlle (Fauna u. Flora Golf. Neapel xx, Monog., 1893, p. 170) puts ova of Orchestia by means of a pipette into boiling, cold-saturated sublimate solution, removes them instantly into sea-water, and thence into weak alcohol. If the chorion does not burst of itself it must be pricked with a needle.

636. Cladocera.-HAEKer (Zellen. u. Befruchtungslehre, 1899, p. 60) fixes females of Sida with winter eggs in a hot mixture of 100 c.c. alcohol of 70 per cent. with 1 to 2 c.c. saturated sol. of sublimate. See also SAmter, Zeit. wiss. Zool., lxviii, 1900, p. 176.

63\%. Copepoda.-Krueger (Arch. Zellforsch., vi, 1911, p. 173) fixes ovaries of Harpactida in Zenker's mixture with 10 per cent. of formol added, No other liquids give good results.

\section{Vermes.}

638. Rotatoria.-Jennings (Bull. Mus. Harvard Coll., xxx, 1896, p. 101) finds the best fixative for pregnant females is the strong liquid of Flemming, but the ova must then be bleached with chlorate of potash ( $\$ 575)$.

Lenssen (La Cellule, xiv, 1898, p. 428) fixes ova of Hydatina with sublimate for twenty seconds.

639. Turbellaria.-GARDINER (Journ. of Morph., xi, 1895, p. 158) finds the best fixative for ova of Polychoerus is a mixture of equal parts of absolute alcohol and glacial acetic acid.

Bresslau (Zeit. wiss. Zool., lxxvi, 1904, p. 219) fixes Mesosto- 
midæ with summer-eggs in Tellyesniczky's mixture (either cold or warmed to $60^{\circ}$ or $70^{\circ} \mathrm{C}$.) for ten to twelve hours, and washes out for the same time. He incises winter-ova at one pole, fixes and brings into alcohol of 95 per cent., then makes an incision at the other pole, and imbeds in paraffin through cedar oil. In the paraffin, slices of the shell may be removed with a scalpel, and the ova reimbedded when sufficiently shelled.

VAN DER STRICHT (Arch. Biol., xv, 1898, p. 370) finds that ova of Thysanozoon will only cut well when they have been not more than two minutes in absolute alcohol followed by chloroform and paraffin as used by Carnoy and Lebrun, $\S 602$.

See also, for Polyclads, Francotte, Arch. Zool. Expér., vi, 1898, p. 196 ; and, for fresh-water Planaria, Irsnma, Zeit. wiss. Zool., xl, 1884, p. 359 .

640. Cestoda (v. Beneden, Arch. Biol., ii, 1881, p. 187).-Ova of Tania in which a chitinous membrane has formed around the embryo are impervious to reagents. They may be put on a slide with a drop of some liquid and covered. Then, by withdrawing the liquid by means of blotting-paper, the cover may be made to gradually press on them so as to burst the membranes, and the embryo may then be treated with the usual reagents.

Haswelu (Quart. Journ. Micr. Sci., liv, 1909, p. 417) fixes ova of Temnocephala in " sublimate alcohol," brings them into 90 per cent. alcohol with iodine added, and thence gradually back into water, softens the shells in weak sodium hypochlorite, washes and imbeds.

641. Trematoda.-CoE (Zool. Jahrb., Abth. Morph., ix, 1896, pp. 563, 566), for the special study of the excretory system of the Miracidia of Distomum, kills with osmic acid, rinses with distilled water, and puts for a couple of days into $\frac{1}{4}$ per cent. solution of silver nitrate.

Egg-capsules may be softened with 5 per cent. caustic potash and then burst open (Heckert, Bibl. Zool., iv, 1889).

642. Nematoda.-The ova of Ascaris megalocephala, a classical object of study, are one of the most impervious things in the animal kingdom. Years ago Fol related to me that he had had ova segmenting right through absolute alcohol into balsam. BAtallloN (Arch. Entwickelungsmech., 1901, p. 149) has had ova showing living embryos after having been for six months in liquid of Flemming, and found them to remain alive for months after drying for twentyfour hours at $35^{\circ} \mathrm{C}$., and mounting in balsam, and for weeks in acids or alkalies. 
Doubtless the best fixative yet made known for ova furnished with their capsules will be found to be that of CARNOY and LEBRUN, $\S 86$ (La Cellule, xiii, 1897, p. 68). After fixation the ova are carefully brought into 80 per cent. alcohol, in which they are preserved. Imbedding should be carefully done as recommended for the ova of Amphibia ( $(602)$, but they ought not to remain in the pure paraffin for more than a minute to a minute and a half. But these authors prefer the celloidin method. At least six weeks' soaking in the different strengths of celloidin will be necessary to ensure penetration. They stain with iron hæmatoxylin.

Zur Strassen (Arch. Entwickelungsmech., iii, 1896, p. 29) fixes for twenty-four hours in a mixture of 4 parts 96 per cent. alcohol and 1 part acetic acid, brings into pure alcohol, stains with hydrochloric acid carmine, and brings gradually into glycerin.

Similarly ZoJA (Arch. mik. Anat., xlvii, 1896, p. 218) and ERLANGER (ibid., xlix, 1897, p. 309). Zoja stained with Bismarck brown and examined in dilute glycerin; Erlanger made paraffin sections and stained with iron hæmatoxylin.

Kostanecki and Siedlecki (ibid., xlviii, 1896, p. 184) employed concentrated sublimate solution, or 3 per cent. nitric acid or mixtures of these two, for ovarian ova.

Van Beneden and Neyt (Bull. Acad. Belg., 1887, p. 214) took equal parts of alcohol and acetic acid. BovenI (Jena Zeit., xxi, 1887, p. 423) fixed in his picro-acetic acid, $\$ 95$ - a clearly inadequate method. GuLICK (Arch. Zellforsch., vi, 1911) has "fixed" ova of Heterakis for twenty-two hours in one-third saturated picric acid with 3 per cent. of glacial acetic acid, and had them develop in alcohol of 70 per cent. to stages representing a normal development of several weeks.

BoRING (Arch. Zellforsch., iv, 1909, p. 121) spreads ova of Ascaris on a layer of Mayer's albumen on a slide, sets the albumen with a drop of formol, fixes with 4 parts of alcohol to 1 of acetic acid, stains in alcoholic hydrochloric acid carmine, and mounts in glycerin.

Artom (Zeit. wiss. Mik., xxv, 1908, p. 5) freezes segments of the uteri of Ascaris in salt water, and cuts them with the freezing microtome into disks $30 \mu$ thick, and fixes these with divers liquids.

Cerfontaine (ibid., xxix, 1912, p. 305) brings fixed ova from alcohol into absolute alcohol with 1 per cent. of clove oil, evaporates this down to one-tenth, puts into absolute alcohol with 5 per cent. of clove oil, evaporates again down to one-tenth, then into the same 
with 5 per cent. of collodion added, evaporates almost entirely away, and passes through cedar oil into paraffin.

For methods for the Mitochondria and Golgi apparatus it is necessary to treat uteri as does Artom (above explained), and then fix in the proper fluid.

Echinodermata, Coelenterata, and Porifera. See the chapter on " Zoological Methods." 


\section{CHAPTER XXVI.}

\section{CYTOLOGICAL METHODS.}

643. Study of Living Cells.-In the young larvæ of Amphibia, both Anura and Urodela, the gills and caudal " fin," and sometimes other regions, may be studied in the living state.

The larvæ may be fixed in a suitable cell, or wrapped in moist blotting-paper, or may be curarised; or the tail may be excised. (It is preferable to cut through the larva close in front of the hind limbs.)

In the living animal the epithelial cells and nuclei (in the state of repose) are so transparent as to be hardly visible in the natural state. They may, however, be brought out by curarising the larva; or, still better, by placing the curarised larva for half an hour in 1 per cent. chloride of sodium solution. Normal larvæ may be used for the study of the active state of the nucleus, but much time is saved by using curare.

Curare.-Dissolve 1 part of curare in 100 parts water, and add 100 parts of glycerin. Of this mixture add from 5 to 10 drops (according to the size of the larva), or even more for large larvæ, to a watch-glassful of water. From half to one hour of immersion is necessary for curarisation. The larvæ need not be left in the solution until they become quite motionless; as soon as their movements have become slow they may be taken out and placed on a slide, wrapped in blotting-paper. If they be replaced in water they return to the normal state in eight or ten hours, and may be re-curarised several times.

Other Narcotics.-Three per cent. alcohol or 3 per cent. ether, or infusion of tobacco, may be used in a similar way. These reagents cause no obstruction to the processes of cell-division.

Indifferent Media.-One per cent. salt solution, iodised serum, syrup, cold water $\left(+1^{\circ} \mathrm{C}\right.$.), and warm water $\left(35^{\circ}-40^{\circ} \mathrm{C}\right.$.). The tail may be excised from the living animal and studied for a long time in these media (Peremeschko, Arch. mik. Anat., xvi, 1879, p. 437).

For the processes of staining living cells see $\S 208$. 
644. Study of Fresh and Lightly Fixed Cells.-So-called "indifferent" liquids must not be believed to be without action on nuclei. Iodised serum, salt solution, serum, aqueous humour, lymph, better deserve the name of weak hardening agents. Between these and such energetic hardening agents as Flemming's mixture come such light fixing agents as picric acid or very dilute acetic acid. These it is whose employment is indicated for the study of fresh isolated cells.

A typical example of this kind of work is as follows: Tease out a piece of living tissue in a drop of acidulated solution of methyl green $(0.75$ per cent. of acetic acid). This is a delicate fixing agent, killing cells instantly without change of form. Complete the fixation by exposing the preparation for a quarter of an hour to vapour of osmium, and add a drop of solution of Ripart and Petit and a cover.

Or you may fix the preparation, after teasing, with vapour of osmium for half a minute to two minutes, then add a drop of methyl green, and after five minutes wash out with 1 per cent. acetic acid, and add solution of Ripart and Petit and cover.

Or you may kill and fix the cells by teasing in solution of Ripart and Petit (to which you may add a trace of osmic acid if you like), and afterwards stain with methyl green.

I have found Pictet's chloride of manganese ( $\$ 403$ ) useful as an examination medium. A little solution of dahlia may be added to it.

Henking (Zeit. wiss. Mik., viii, 1891, p. 156) recommends a liquid composed of-

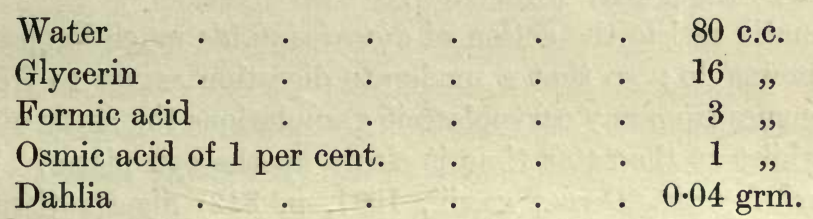

Other fixing agents, such as picric acid or weak sublimate solution, may of course be used. Other stains, too, such as Bismarck brown, and of course other examination media than solution of Ripart may be employed. But, for general purposes, the methyl-green-osmiumand-Ripart's-medium method gives such good results, and is so very convenient, that it may be called a classical method for the study of fresh cells.

645. Some Microchemical Reactions.-Methyl green is a test for chromatin, in so far as (with fresh cells) it colours nothing but the chromatin in the nucleus, see $§ 276$. It is, however, not a perfect 
test, for the intensity of the coloration it produces varies greatly in different nuclei, and may in certain nuclei be extremely weak, or (apparently) even altogether wanting. In these cases other tests must be applied in order to establish with certainty the presence or absence of that element.

Chromatin is distinguished from albuminoids by not being soluble, as these are, in water and in weak mineral acids, such as $0 \cdot 1$ per cent. hydrochloric acid. It is easily soluble in concentrated mineral acids, in alkalies, even when very dilute, and in some alkaline salts, such as carbonate of potash and biphosphate of soda. In the presence of 10 per cent. solution of sodium chloride it swells up into a gelatinous mass, or even, as frequently happens, dissolves entirely (CARnoy, Biol. Cell., pp. 208-9). It is only partially digestible (when in situ in the nucleus) in the usual laboratory digestion fluids.

The solvents of chromatin that are the most useful in practice are 1 per cent. caustic potash, fuming hydrochloric acid, or cyanide of potassium, or carbonate of potash. These last generally give better results than dilute alkalies. They may be employed in solutions of 40 to 50 per cent. strength. If it be desired to remove all the chromatin from a nucleus the reaction must be prolonged, sometimes to as much as two or three days, especially if the operation be conducted on a slide and under a cover-glass, which is the safer plan.

These operations must be performed on fresh cells, for hardening agents render chromatin almost insoluble in ammonia, potash, or sodic phosphate, etc. Hydrochloric acid, however, still swells and dissolves it, though with difficulty.

Chromatin resists the action of digestive fluids much longer than the albumins do; so that a moderate digestion serves to free the chromosomes from any caryoplasmic granulations that may obscure them, whilst at the same time it clears up the cytoplasm. UNNA (Monatschr. prakt. Derm., xxxiii, 1901, p. 342) digests tissues in solutions of sodium chloride, to remove the granoplasm. See also $§ \S 652,664$ and 668 .

\section{Glycogen.}

646. Glycogen is a carbohydrate which occurs in many cells, both glandular and genital : it is found in both inter- and intracellular positions, in the form of small areas of flocculent appearance. For its study in a tissue or organ two methods should be used:(1) An iodine technique; and (2) that of Best's carmine. The specificity of the latter method has been questioned, and both techniques must be used for comparison (see p. 338). 
64\%. Iodine Method.-Fix tissue in Carnoy, or alcohol absolute, 4 parts, acetic acid glacial, 1 part; or in absolute alcohol; or in alcoholic fixatives not containing alcohol lower than a strength of 90 per cent. It is better if the tissue is cut small. Fix for one hour, then transfer for twenty-four hours or longer, in two changes of absolute alcohol; then xylol and paraffin wax. Fix sections to slide with a mixture of 50 per cent. alcohol with a few drops of glycerin and albumen, using the alcohol as you would water; drain the slides dry. Remove wax in xylol, bring to 70 per cent. alcohol. Stain sections in Ehrlich's hæmatoxylin for five or ten minutes. Blue in tap water substitute, $\S 669$. Pass to a 2 per cent. solution of potassium iodide saturated in iodine (a Lugol solution); leave five minutes; pour away, wipe around slide, and dehydrate in absolute alcohol saturated in iodine. Clear in oleum origanum cretici for about ten minutes. Mount in origanum balsam. Such preparations should keep for years without fading much. I have some slides of human placenta which after six years still show the glycogen.

648. Best's Carmine Stain.-Material is fixed as for the iodine method and may be imbedded in celloidin. If paraffin sections are used the slide must be placed in 1 per cent. celloidin overnight, drained and allowed to dry partly, and then plunged into chloroform and absolute alcohol (equal parts), then treated as for celloidin sections. Transfer through alcohols 90 per cent. and 70 per cent. to water. Stain in Ehrlich's or iron hæmatoxylin as usual, but differentiate in acid alcohol. Then proceed to BEST's carmine stain (Zeit. f. mikros., Bd. xxiii). Make up this stock carmine solution :-

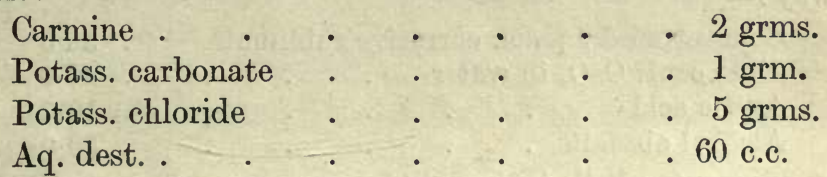

Boil gently for a few minutes; cool.

Add strong liq. ammon. 20 c.c. Keep this solution in a wellstoppered bottle in a cupboard. It may go bad in a month during summer.

Wash sections in distilled water after staining in hæmatoxylin. Stain in following solution :-

Stock carmine solution

Liq. ammon. fort.

Methyl alcohol (pure)

2 parts.

3 ",

3 ,

for five minutes. 
Differentiate in-

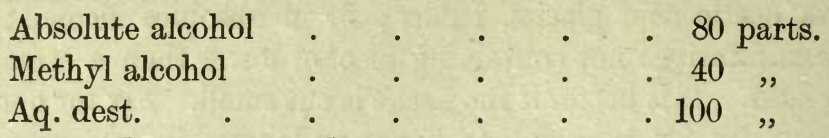

till no more red comes out (three to five minutes).

Wash in 80 per cent. alcohol, absolute and clove oil, xylol and xylol balsam; nuclei and cytoplasm, blue, glycogen red.

It is a good plan when working on glycogen to prepare triplicate slides, one for iodine stain, one for Best; the other slide is brought down to water and spat upon and set aside : the glycogen is dissolved by the diastase of the saliva, the latter is washed off in water and the slide stained as usual for Best's carmine. Comparison between the first slide and this one will assist in properly identifying glycogen; (procedure of Dr. B. R. G. Russeld, Imperial Cancer Research Bureau).

One generally succeeds at first trial with such material as the liver of a rabbit, but with invertebrate materials, especially from paraffin sections, even though soaked in 1 per cent. celloidin, the results are often disappointing. This can be overcome by practice and by slight modification in the time used for differentiation. For delicate material it seems best to work with celloidin sections.

649. Zieglwallner's Alcoholic Flemming for Glycogen and Fat.Neither the iodine nor Best's carmine method preserves fat as well as glycogen. Zieglwallner has worked out the following method for preserving both fat and glycogen. Fix small pieces of tissue in this mixture for twenty-four to forty-eight hours :-

1 per cent. chromic acid in 80 per cent. alcohol $\quad 15 \cdot 0$

2 per cent. $\mathrm{OsO}_{4}$ in water $\quad$. . : $4 \cdot 0$

Acetic acid . $. \quad . \quad . \quad .10$

In 100 c.c. of this mixture there would be 50 per cent. alcohol.

If a corrosive sublimate fixation is necessary use this mixture in the same way:-

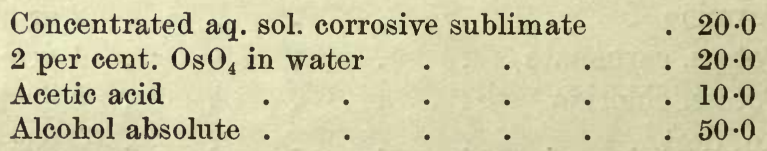

In washing out, a little iodine will be necessary. Transfer the pieces of tissue to 70 per cent., then upgrade and imbed in celloidin.

In order to preserve the brownish black colour of the osmic stain of fat, which soon disappears when the sections are brought to balsam, one may convert the reduced osmic into its sulphide by adding a small quantity of $\mathrm{Na}_{2} \mathrm{~S}$ to the 70 per cent. alcohol which replaces the fixative. Imbed in celloidin or wax : stain as by the iodine, or better in Best's carmine method, from celloidin. Dr. J. A. Murray informs me that it is generally necessary to stain sections first in warm iron alum, then warm hæmatoxylin, and then to differentiate in the cold with acid alcohol. Afterwards proceed to Best's carmine.

Paul Buchner (Praktikum der Zellenlehre I., Berlin, 1915) fixes 
overnight in a freshly-made mixture of equal parts of absolute alcohol and strong Flemming. Wash out for two days in 50 per cent. alcohol, imbed in celloidin, stain in Best's carmine.

See also Creighton, The Formative Property of Glycogen, London, 1896 ; GaGe, Trans. Amer. Micr. Soc., xxviii, 1908, p. 203 ; Kato, Arch. Ges. Phys., cxxvii, 1909, p. 125 ; Busch, Arch. Intern. Phys., iii, 1905 , p. 51 ; MaYer, Zeit. wiss. Mikr., xxvi, 1909, p. 513 ; ARnold, Sitzb. Heidelberg. Acad. Wiss., 1909, p. 1, 1910, p. 3, and 1911, 14 Abh.; Arch. path. Anat., exciii, 1908, p. 175 ; Arch. mik. Anat., lxxiii, 1909, p. 265 ; lxxvii, 1911, p. 346 ; Beitr. path. Anat., li, 1911, p. 439; Fraenkel, Virchow's Arch., 1911, p. 197; Neubert, Beitr. path. Anat., xlv, 1909, p. 38 ; ERHARD, Arch. Zellforsch., viii, 1912, pp. 447 and 507; Ehrlich and Lazarus, Die Anaemie, 1898, p. 30 ; Pekelharing, Petrus Camper, Deel I, 1901, p. 231 ; Driessen, Zeit. wiss. Mik., xxii, 1905, p. 422 ; Fischer, Anat. Anz., xxvi, 1905, p. 399 ; Fiessinger, C. R. Soc. Biol., lxvi, 1909, p. 183 ; Neukirch, Arch. paith. Anat., ce, 1910, p. 82.

650. Some Microchemical Tests.-Iron.-Organic compounds of iron, which are not ionisable into ferric and ferrous ions, and in which the iron cannot be detected by the ordinary reagents, are much more frequently present in animal and vegetable tissues than was previously believed to be the case. In addition to the albuminate compounds, there exist iron compounds giving ferric and ferrous ions, detectable with the ordinary reagents, and which, for convenience, may be designated Inorganic Iron Compounds.

The nature of many of the compounds of iron found in placentas, blood-organs, the liver, etc., is obscure; many of them appear to be formed as degeneration or excretion products, from the breaking down of hæmoglobin. See below.

Most of our knowledge of the methods for the detection of iron in tissues and cells is due to A. B. Macallum (Quart. Journ. Micr. Science, xxxviii, 1895; Journ. Physiology, 1897 ; Ergebn. d. Physiol. Wiesbaden, 1908). Macallum has shown that, to detect organic iron, one must convert it into inorganic. This can be done by allowing sulphuric or nitric acid alcohol to act upon sections, or a piece of tissue, for from one to twenty-four hours at $35^{\circ} \mathrm{C}$., according to the strength of acid and the size of the object. When masked iron is liberated in the tissues by acid alcohol, most of it is in the form of ferric salts, particularly when the oxidising nitric acid is used, and a small part occasionally of ferrous compounds. Inorganic iron compounds in tissues are usually ferric, more rarely ferrous salts.

The commonest tests for iron in tissues are the Prussian blue reaction, and Macallum's hæmatoxylin. The latter test should never 
be used alone, because its complete specificity is somewhat doubtful.

It is hardly necessary to point out that proper precautions should be taken to avoid contamination of the tissue by vessels or chemicals which may contain iron compounds. Glass needles should be used instead of steel, and the water used should have been distilled from a clean glass retort.

The tissues should either be fixed in redistilled formalin (10 per cent.), chemically pure ethyl alcohol or pure 90 to 95 per cent. alcohol, or in redistilled methylated spirit. Bouin's fluid, Flemming, and such mixtures should not be used, as such a practice is almost certain to introduce error. Material should be fixed or hardened for several days in strong alcohol. Sections are made either freehand with a bright rust-free razor wetted with absolute alcohol, or by the paraffin method with a dry rust-free knife.

Macallum's Hamatoxylin Methoj.-As an indicator Macallum uses a 0.5 per cent. solution of absolutely "pure hæmatoxylin" made up in perfectly pure aqua dest. The solution should look brownish yellow, but when alkalies or alkaline earths are added, the colour becomes violet or red. When such a pure hæmatoxylin is brought into contact with a salt of iron, the yellow colour becomes blue-black, or bluish-black; with organic iron compounds the hoematoxylin is unaffected. Such compounds must be unmasked by sulphuric or nitric acid alcohol as above mentioned.

When the compounds of iron to be investigated are found in tissues, the latter are well hardened in alcohol (purified, vide supra), sections prepared and washed in aq. dest., or the tissue simply teased out, and then the hæmatoxylin solution is added. Those parts which go blue-black or blue-violet contain inorganic iron ; the remainder of the preparation may go quite dark yellowish brown, especially nuclei, and the presence of iron may thus be obscured. To remove this excess the preparation is treated in a mixture of equal parts of absolute alcohol and ether, but not for longer than one hour. The unaffected hæmatoxylin is extracted, the blue-black compound remains. Clear in oil of cloves, mount in balsam. Such preparations are permanent.

This reaction of inorganic compounds of iron with hæmatoxylin seems to be one of oxidation (MAYer, Mitth. Zool. Stat. Neapel., x, p. 170).

Extraordinarily small traces of inorganic iron are thus demonstrated. The method is more sensitive than that of Prussian blue or ammonium sulphide. 
Organic Iron Compounds.- These will not give the iron reactions unless the complex iron compound has been broken up, that is, the iron "unmasked" by some reagent: acid alcohol is used for this. Sulphuric acid alcohol (4 per cent. in 95 per cent. alcohol) and nitric acid alcohol (3 per cent. in 95 per cent. alcohol) are better than the hydrochloric acid alcohol (Bunge's fluid). Sulphuric acid alcohol acts very slowly especially on bulk tissues, and even Protozoa take twenty-four hours at $35^{\circ} \mathrm{C}$. before their masked iron is revealed. Nitric acid alcohol acts more quickly and extracts very little of the iron it liberates (which is a danger with Bunge's fluid); the process is completed in about thirty-six hours.

Sections are treated with acid alcohol, 90 per cent. alcohol, and aq. dest., and then Macallum's hæmatoxylin is added; the sections are washed in aq. dest., stained in safranin, as described in next section, dehydrated and mounted in balsam.

Prussian Blue Reactions on Organic Compounds.-Sections after being treated in the acid alcohol (nitric or sulphuric) are washed in pure 90 per cent. alcohol and then in aq. dest. They are placed not longer than five minutes in the following solution : aq. potassic ferrocyanide 1.5 per cent., and hydrochloric acid 0.5 per cent. in aq. solution equal parts, freshly made. Again washed carefully in aq. dest. stained in eosin or safranin, dehydrated in alcohol, cleared in oil of cedar and mounted in benzole balsam. The safranin or eosin are used in 1 per cent. strength in 30 per cent. alcohol, for three minutes for eosin, and for one halfhour for safranin, and differentiated in 90 per cent. alcohol.

Ferric and Ferrous Salts both occur in inorganic iron compounds. Ferrous salts may be distinguished from ferric by the fact that only the latter give an immediate reaction with ferrocyanide of potassium, while the former react with ferricyanide of potassium. Fix material in alcohol of about 90 per cent. for several days.

Reaction for Ferric Salts.-Wash sections in aq. dest., transfer to 2 per cent. aq. sol. ferrocyanide of potassium for from three to fifteen minutes. Bring to acid alcohol (1 c.c. in 70 per cent. alcohol) for about ten minutes. The Prussian blue reaction takes place. Wash in pure 70 per cent. alcohol, dehydrate clear and mount in benzole balsam.

Counter-stain if desired in eosin or safranin (op. cit.).

Ferrous Salts.-As above, substituting ferricyanide of potassium instead of ferrocyanide. 
Simultaneous detection of both categories of salts may be made by using a solution of equal parts of ferricyanide and ferrocyanide.

Blood, AND Iron SALTs.-In $\S 789$ is given Okajima's method for elective staining of hæmatids (hæmoglobin). Degeneration products of homoglobin are homosiderin and melanin (of malaria). Hæmosiderin is found in the liver in pernicious anæmia, and also in large extravasations of blood. It is said that hæmosiderin will, but melanin will not, give the Prussian blue and other iron reactions ; both pigments survive dehydration and a clearing oil. Another pigment derived from blood is hoematoidin or bilirubin, which contains no iron and does not give the iron reactions.

Hocmoglobin itself is not unmasked by acid alcohol and will not give the iron reactions, but stains bright red with eosin from Mann's methyl-blue eosin mixture, and orange in Okajima's alizarin stain, $\S 789$. Most pigments are destroyed by concentrated sulphuric acid, which will not affect any carbon granules which may have been fed to the cells experimentally.

See also TrRmann, Goerbersdorfer Veroeffentl., ii, 1898, p. 111 ; SchneIDER, Mitth. Zool. Stat. Neapel, xii, 1895, p. 208 ; CarNoy and Lebrun, La Cellule, xii, 1897, p. 275 ; Sumita, Arch. path. Anat., cc, 1910, p. 230 ; Zaleski, Zeit. Phys. Chemie, xiv, 1890 ; Wassiermann, Anat. Hefte, xlii, 1910, p. 283, JoNes, Biochem. Jour. 1920.

Copper.-R. Boyce and W. A. Herdman, in their paper on the Green Leucocytosis in Oysters (Proc. Roy. Soc.; lxii, 1897-98), have given directions for the application of the well-known potassic ferrocyanide test of chemists, to sections of tissues in which copper is to be detected. These authors fix with proper precautions (vide supra, under "Iron ") in absolute alcohol, imbed in pure paraffin and cut sections. Care must be taken to avoid acid solutions, such as commercial turpentine or old xylol. Sections are brought from absolute alcohol to distilled water, placed in a 1.5 per cent. solution of freshly prepared potassic ferrocyanide or, preferably, in equal parts of the same ferrocyanide solution, and a 0.5 per cent. $\mathrm{HCl}$ solution, and parts where copper is present go a reddish colour. Sections are then washed in aq. dest., dehydrated in absolute alcohol, cleared in cedar-wood oil and mounted in Canada balsam.

See also Macallum, Journ. Phys. Cambridge, xxii, 1897, p. 92 ; MARFORI, Arch. Ital. Biol., xxx, 1898, p. 186.

For Zinc see Mendel and Bradley, Amer. Journ. Phys., xiv, 1905, p. 320 .

For Lime salts see Grandis and Mainini, Arch. Ital. Biol., xxxiv, 1900, p. 75 ; SCHAFFer, Zeit. wiss. Zool., lxxxix, 1908, p. 13 ; LeUtert, Encycl. mikr. Technik, ii, p. 588; Stoeltzner, Arch. path. Anat., 
clxxx, 1905, p. 363 ; Macallum, Ergeb. Phys. Wiesbaden, vii, 1908, p. 612 .

For Potassium see Macallum, Journ. Phys. Cambridge, xxxii, 1905, p. 95 ; Ergeb. Phys. Wiesbaden, vii, 1908, p. 600.

For Guanin see Gracomo, Zeit. wiss. Mik., xxvii, 1910, p. 257.

Concerning the microchemistry of the cell in general, see further fourth edition; also Carnoy and Lebrun, La Cellule, xii, 2, 1897, p. 194 ; Zrmmermann, Die Morphologie u. Physiologie des Pflanzlichen Zellkernes, Jena, 1896 (treats also of the animal cell) ; HAEcken, Praxis $u$. Theorie der Zellenund Befruchtungslehre, Jena ; Prenant, Journ. Anat. Phys., xlvi, 1910 , p. 343.

651. Cytological Fixing Agents.-A fixing agent that is good for one element of a cell is not necessarily good for all others. As regards the nucleus, all fixatives should be acid; for if not they will not satisfactorily preserve either chromatin or nucleoli. For instance, bichromate of potash, if not rendered acid, fixes chromosomes and nucleoli in a distended state so that clear images of them are not obtained. Acids contract them somewhat, and so give them sharper outlines. The fixatives mostly employed for nuclei are liquid of Flemming and liquid of Hermann. There is a slight difference between them. Liquid of Hermann, owing to the platinum chloride, causes chromatin to shrink more than liquid of Flemming does, and for this reason is supposed to give clearer images of chromosomes, especially of their splitting. I find that it generally makes them shrink too much, and that it is not at all good for spindles.

For many, if not most objects, I prefer to these two reagents BouIn's picro-formol, which gives a highly faithful preservation and a more penetrating and equable fixation.

For spindles I recommend Flemming (picro-formol does not give quite such bold images).

Some of the finest chromosomes I have seen have been fixed with Lindsay Johnson's mixture ( $\$ 44$ ), and liquid of Tellyesniczky has given me others nearly if not quite as good.

As regards the cytoplasm.-Cytoplasm is made up of two elements : a fibrillar element - the spongioplasm or mitome; and a more or less granular liquid that bathes it-the hyaloplasm or enchylema. It does not follow that a reagent that will fix one of these will also fix the other. Nor is it always desirable that both should be equally fixed.

If you fix both, you will have a full fixation; but in that case the granules of the hyaloplasm (be they vital, or be they only " precipitation forms," see $\S 29$ ), and the secretions or other enclosures that may be present in it, may so mask the fibrils of the spongio- 
plasm as to interfere with the observation of it. So that if the latter is the principal object of study, a thin fixation, one in which the spongioplasm is entirely preserved, but the hyaloplasm only partly, may be the better.

The spongioplasm is the easier to fix of the two, and the majority of acid fixatives will preserve it more or less. The best images I have obtained are those given by liquid of Flemming or Hermann in cells in which the action of the reagent has been moderate, i.e. insufficient to thoroughly fix the hyaloplasm at the same time. Nearly, if not quite, as good, is Bouin's picro-formol, which has the great advantage of being very favourable for plasma-staining. I have also had very good results with vom Rath's picro-osmic and picro-platinosmic mixtures, and with acid sublimate.

Hyaloplasm is not nearly so easy to fix, and there are only two reagents in common use that readily give a really full fixation of it ; these are osmic acid and bichromate of potash.

Osmic acid acts as a fixative of hyaloplasm in liquid of Flemming or Hermann, but only gives a full fixation in the outer layers of the material ; and in these it easily happens that many or most of the cells are ruined by over-fixation. See $\S 35$.

This defect may be to a certain degree corrected by taking the osmic acid weaker than is usual. Thus by successively reducing the proportion of this ingredient in liquid of Hermann, I have found that it can be brought down to one-eighth of the prescribed amount without loss of the distinctive characters of the fixation.

The defect of want of penetration seems to be incurable. See $\S \S 35$ and 42 . Substitution of more highly penetrating reagents such as picric acid, for the chromic acid or platinum chloride does not help in the least; you only get the osmic fixation outside, no whit deeper than before, and a picro-acetic fixation, instead of a chromo- or platino-acetic one, in the deeper layers, that is all. In view of these defects of osmic mixtures, it may often be advisable, where hyaloplasm, or its enclosures, is the chief object of study, to have recourse to bichromate of potash. The formula that has given me the finest fixations is that of LiNDSAY JoHnson, but it has the drawback that there is risk of osmication in the outer layers. In this respect liquid of Tellyesniczky, $\S 52$, is to be preferred.

Corrosive sublimate gives a fairly full fixation; but I believe it frequently produces serious artifacts, HeIdenHaIN's "Lanthanin" being one of them. Heidenhain's solution, $\S 64$, containing as it does some 11 per cent. of sublimate, without the addition of any acid to neutralise its shrinking action, seems to me to be an inad- 
missibly coarse reagent. I have, however, obtained with liquid of Carnoy-Lebrun, $\S 86$, some most excellent fixations of cytoplasm. The aqueous solutions of sublimate are frequently used in preference to liquid of Flemming on account of the facilities they afford for the employment of certain stains; but to that end I prefer Bouin's picro-formol.

652. Chromosomes; Chromatin Stains.*-For fresh tissues, see $\S 645$.

With hardly an exception modern work on chromosomes in the germ-cell cycle is carried out by use of such fixations as strong Flemming (§ 41), Bouin's picro-formol-acetic ( 110$)$, Carnoy (§ 85), or an alcoholic nitric corrosive acetic of the Gilson or Petrunkewitsch type. Stains now used much are iron hæmatoxylin of Heidenhain or Benda, thionin, safranin, well ripened Delafield, and Mayer's acid hæmalum. Gentian violet is used by many. For the study of the chromosomes, the desideratum seems to be some fixative which will penetrate evenly and rapidly, which will strip out of the cell, fats and lipoids, and which will allow the subsequent use of some dense, preferably black or dark blue stain.

Some English workers have found Flemming's strong formula, without acetic acid to give beautiful results for chromosomes (e.g. L. HoGBEN, Proc. Roy. Soc., B., xci, 1920). In nearly all my slides fixed for the cytoplasmic inclusions (\$ 673) good chromosome plates are found, but I consider that fixatives which contain lipoid solvents are indicated for chromosome work: the preparations are thereby "stripped" and cleaner, and difficult nuclei are better interpreted when superfluous materials are removed. Sister Monica Taylor (Quart. Jour. Mior. Sci., 1915) stxins first in thionin, mounts and studies the sections; then, if they prove to be worth it, removes the coverslip by soaking in xylol, and restains in iron alum hæmatoxylin. Red stains are not indicated because of the eye-strain they cause.

Iron alum hoematoxylin is especially recommended. Dr. Lee has sent me the following note on the use of this stain :- "Some cytologists have given up iron hæmatoxylin because they have found it to clog the chromosomes; but this will not occur if the following precautions are observed. Mordant sections ( 7 to $8 \mu$ ) for not more than two and a half minutes in iron alum of 2 to 3 per cent.; wash for at least a quarter of an hour ; stain in a 0.5 per cent. solution of ripened hæmatoxylin until the sections appear dark grey, but not black (about twenty-five minutes if the solution is fresh, and not more than four if it has already had several slides passed through

* By J. B. G. 
it); differentiate in the iron alum solution for at least two minutes after the chromosomes, examined in water, appear to have been sufficiently extracted, for chromosomes always appear paler in water than after they have been got into balsam." Personally I have never found iron hæmatoxylin to clog chromosomes, and can only assume that something was wrong with the fixation, or a bad specimen of stain was used.

Hot or Cold Fixation? - Some workers advocate the use of hot fixatives, others believe that the best results are obtained by keeping the capsule or vial of fixative on ice while the material is being fixed. Possibly cold, not freezing, fixatives are indicated for invertebrates and cold-blooded animals, and fixatives at body heat for warmblooded. This, however, must be left. to the discretion of the worker, who may find that either very cold or hot fixatives may improve his preparations in an unexpected manner. EzRA ALLEN recommends using his modified chromic Bouin and urea at $38^{\circ} \mathrm{C}$., while he believes that Flemming should be used in an ice-box (Anat. Record x, 1915-16). Cowdry (Contrib. Carnegie Inst. Wash., viii, 1918, recommends the use of Regaud's formol-bichromate on ice. See also $§ 31$.

653. Dissection of Animals for Chromosome Work.--Some observers have claimed that dissecting out gonads of invertebrates, in a dish of "tap-water," gives clearer chromosome figures than when one uses Ringer or such salt solutions. If possible, avoid dissecting out in any fluid. Fleas and lice, and such small insects, are to be treated as follows: cut off the end of the abdomen, hold the insect down on a glass slide by its head with the aid of a mounted needle, and with another needle press the viscera out with a stripping motion from the head backwards. Immediately transfer the viscera to a fixative (Doncaster, Quart. Jour. Micr. Sci., 1920). For bigger insects one may open the body cavity after having cut off their heads, and pipette fixative over the viscera before separating away the gonads. Then transfer to a capsule of the fixative. Read also directions in $\S \S 12$ and 676 .

654. Fusion of Chromosomes caused by Fixation. - It should be noted that unsuitable or inferior fixation may cause such artifacts as fusion of chromosomes which, intra vitam, may have merely been closely paired. With Diptera it has been found that bodies of mosquitoes, etc., should not be thrown whole into a fixer, but either finely teased or the gonads should be carefully dissected out. This obtains a more rapid and therefore a more efficient fixation (HANCE, Jour. Morph., 1917; Metz, Jour. Exp. Zool., 1916). 
In the same way more fluid chromosomes such as those of mammals may be caused to run together and so introduce error. This probably explains the discordance in the accounts given by different workers for such material as that of man.

655. Note on Fixatives for Chromosome Work.-For work on vertebrate tissues there is little doubt that Bouin's picro-formolacetic, or one of its modifications, is the best mixture to use. For invertebrates in general such Bouin fixatives are also very satisfactory, but Flemming's strong formula should always be tried. Corrosive acetic acid I hold to be a rough and unreliable fixative, and if a corrosive fixation is desired, it is better to use Gilson or Petrunkewitsch-these penetrate more rapidly and give a more delicate fixation. I have seen some excellent chromosome plates in mammals got by using Sansom's Carnoy modification $(\S 86)$. It should be noted carefully that many workers use chromosome fixatives for a short time only ; for instance, Flemming may be used on insect gonads for half to one hour only, followed by a washing out under tap, and then upgrading from 30 per cent. alcohol (from Professor Leonard Doncaster, in literis).

656. Urea and Chromosome Fixation.-It has been claimed by some American workers (McClung, Ezra Allen, R. T. Hance, etc.) that the addition of from 0.5 to 2 per cent. urea crystals to fixatives of the Flemming or Bouin type assists in penetration and gives sharper pictures of the chromosomes. The idea of using urea is, I understand, due to Professor McClung.

It is out of the province of this book to discuss whether a solution of urea in such complicated fluids as Bouin or Flemming, has the same peculiar penetrative properties as in water, or even to question whether, after the addition of the crystals to the Bouin or Flemming, disintegration of the urea does not take place. We are prepared to accept the statements of McClung, Allen and Hance, and to recommend a trial of the method. Waro Nakahara (Jour. Morph., 1919), working on Perla, did not find that the addition of urea crystals to his fixative made any appreciable difference.

65\%. Fixation of Mammalian Chromosomes.-The material must be absolutely fresh ; even half an hour's delay is fatal; prepare your fixatives and capsules, knives, etc., before you kill the animal. Apparently chromosomes of mammals will clump together about ten minutes after death. HANCE (Anat. Record, xii, 1917) gives the following method:-(1) Obtain fresh specimens of tissue from as many different animals as possible, so as to be sure of obtaining one or more in a "cycle of cell division." (2) Place small or finely 
teased pieces of fresh tissue immediately into cold Flemming's solution (on ice) plus about 0.5 per cent. urea crystals. Flemming's solution kept on ice registers about $4^{\circ}$ to $5^{\circ} \mathrm{C}$. Leave in cold solution for twenty-four hours or longer. (3) If this fixation fails, try the following: Allow small pieces of fresh tissue to remain in the air for from ten to twenty minutes after removal from the animal before placing them in the cold Flemming.* Then fix as before. (4) Wash in water about twenty-four hours. (5) Dehydrate by very gradual steps. (6) Clear from 95 per cent. alcohol in cedar oil followed by xylol. Embed in paraffin.

658. Ezra Allen's Chromic Bouin and Urea.-Used for work on the spermatogenesis of rat, etc., and generally indicated for mammals.

Picric acid, sat. sol.

Formol (pure) .

Glacial acetic

To freshly-made mixture raised to temperature of $38^{\circ} \mathrm{C}$. add and dissolve, first, 1.5 grms. of chromic acid crystals, and then 2 grms. of urea crystals. Kill animal by decapitation, remove testis immediately, snip into small pieces, fix at $38^{\circ}$ or $40^{\circ} \mathrm{C}$. for from one to two hours. Fixative replaced by "drop method " with 70 per cent. alcohol, picric acid washed out by addition to the alcohol of a sat. sol. of lithium carbonate, a few drops at a time; the alcohol is replaced by anilin oil (freshly distilled), this by synthetic oil of wintergreen, and this by paraffin of $52^{\circ}$ melting point. The paraffin is slowly added till the tissue is in a bath of high paraffin concentration. It is then passed through several changes of pure paraffin to remove oil (Ezra Allen, Jour. Morph., 1918; Anat. Record, $\mathrm{x}, 1916)$.

659. Amphibian Chromosomes.-Charles Parmenter (Jour. Morph., 1919) uses Ezra Allen's chromic Bouin with success for amblystoma. Ordinary Bouin with urea crystals, Hermann, and Flemming were also good.

660. Modified Bouin and Urea for Insects.-Miss E. Eleanor CARothers for orthopterous chromosomes uses the following fluid which has been developed in the Zoology Laboratory, Pennsylvania. It is a modification of Bouin's formula :-

Picric acid, sat. sol. aq.

Formalin (strong) 75 c.c. 15,

* This, of course, is directly contrary to all the rules of fixing technique, but it might work satisfactorily. 
Glacial acetic acid

Urea crystals . . . . . . $\frac{1}{2} \mathrm{grm}$.

Use for twenty-four hours. Stain in iron hæmatoxylin or Flemming's tricolour mixture (Jour. Morph., xxviii., 1916-17).

661. Precautions in Dehydrating and Clearing.-It is well known that too rapid dehydration or clearing will cause shrinkage and distortion. Material to be used for chromosome study should be dehydrated gradually, either by a syphon or some drop method (§3). For clearing xylol is not good, as it causes much shrinkage. The least shrinkage occurs with some vegetable oil, like bergamot, origanum, cedar wood, or cassia (cinnamic aldehyde), which, if it will not mix with paraffin, should be washed out subsequently in some paraffin solvent, like benzole or xylol. Ezra Allen (Anat. Record, x, 1915-16), following Suchannek (§ 134), uses distilled aniline oil as a substitute for the higher alcohols. One gradually brings brain and genital or such tissue to 75 per cent. alcohol, and then adds distilled anilin oil by the drop method, shaking frequently, or using some system for agitating the fluids, or by some diffusion apparatus (§3). When nearly pure anilin oil has replaced the alcohol, one transfers to pure oil until the tissue is cleared. From anilin oil one embeds as follows : warm the oil and tissue slightly, adding every ten minutes a few drops of melted paraffin, mixing thoroughly with a pipette ; continue till the mixture has 85 to 90 per cent. paraffin. Transfer to melted paraffin. If bergamot oil has been used for largish objects, at least four changes of pure paraffin must be made, one half-hour to each, and the fifth bath for at least one hour. In most cases gradual clearing in cedar-wood oil will give satisfactory results. See also $\S 5$.

662. Mounting Sections between Coverslips.-AGAR (Quart. Jour. Micr. Sci., 1911) has devised a method for mounting preparations between coverslips in order that they can be observed on both sides. Use one larger coverslip as if it were a slide. Carriers may be made by stamping out a square in stiff cardboard, or thin metal. See also, C. CÉpÈde, C. R. Soc. Biol., clv, 1913.

663. Smear Preparations of Gonads.-In some cases smear preparations of testes especially may provide useful evidence in a research on chromosomes. One may be fortunate enough to find nuclei at the prophase or metaphase of mitosis, with all the chromosomes spread out so as to be counted with ease. In many cases, to study early stages in synapsis for which very rapid penetration is essential, smear preparations are a sine qua non. 
Remove the testes; if it is large take a fragment by a pair of forceps and quickly smear along the length of a dry slide several times, so as to cover as much of the middle part of the slide as possible. If the cells are likely to stick, fix immediately by pouring on some Flemming, Bouin, or Petrunkewitsch. Set aside for a few minutes, wash off in water, upgrade from 30 per cent. alcohol, and leave overnight in 90 per cent. alcohol. Bring back to water, stain in iron hæmatoxylin, thionin, or gentian violet, etc. See also Goodrich's iodine-Bouin method (under "Protozoa"). Note that smears may be fixed in steam, acetic, osmic, formalin vapour, or "Lucidol" ( $\S \S 107,783)$, or stained and fixed simultaneously in Leishmann, acetic Bismarck brown (§277), aceto-carmine (§221), or such mixtures. Smears of very liquid testes, like those of Lepidoptera, are liable to be washed away if fixative is added too soon. It is probably best to kill the cells in some toxic vapour, then allow them to dry a little, and then fix in a liquid.

Foot and Strobell (Arch. f. Zellf., Bd. xii, 1914) recommend the following :-Place testes in drop of acidulated (acetic) "water" on end of slide, and with fine needle (No. IX.) cut from it the area at stage required (previously ascertained by examination of sections). Push this area by point of needle to middle of slide and break up the tissue by gently tapping with the needle (never roughly spread as recommended by some workers). This should be done under dissecting microscope.

See also Kernschwarz, and "Iron Carmine," § 222. For BATAILLon and KofHLER's borax-methylen-blue see Comptes Rendus, exvii, 1893, p. 521.

664. The So-called Microchemical "Tests" for Chromatin.*Among these the "digestion tests" have met with some favour. It is well-known that examination of the partly digested tissue from the gut of animals which eat fresh cells (e.g. parasitic hymenoptera) shows that the nucleus of the cell resists digestion for the longest time. On the other hand, there are a whole series of enzymes which are specially concerned in the hydrolysis of nucleic acid, each acting on some particular substrate ; these enzymes are grouped together as "nucleases." The results of tests carried out with proteolytic enzymes on the nature of unidentified cell granules must not be depended upon too much. Extracts of such organs as the spleen and pancreas are known to contain more than one kind of enzyme,

* By "chromatin" I mean that material which forms the substance of the chromosomes. The question of chromatin, nucleoli, and basophil granules is one of the most difficult in Cytology. (See GATENB Y, Quart. Jour. Micr. Sci., vol. 64, 1920, Science Progress, January, 1921, R. LUDFORD, Jour. Roy. Micr. Soc., 1921, and recent numbers of the Quarterly Journal of Microscopical Science.) 
and the zoologist especially is advised to get the help of an expert on enzymes before attempting to interpret any experiments he may have made with such intra-cellular enzymes. Because a certain protease will not dissolve away a given cell granule, while a "nuclease" may do so, does not by itself provide good evidence for the conclusion that the granule in question is "chromatin," or derived from the chromosomes.

Van Herwerden (Arch. f. Zellf., x, 1913) for instance, using the extract of spleen containing "nuclease," finds that the granules identified as "chromatin" emission by Schaxel are dissolved away. In all probability, however, such granules are phospholipin and not chromatinic, and were dissolved away by some lipolytic enzyme in the solution used by Van Herwerden.

Successful experimentation on this side of cytology calls for two desiderata: Firstly, an intimate knowledge of the nature of the enzyme solution to be used, and of what effects the latter has on various definitely identified categories of cell substances; second, a thorough study of the origin and microchemical reactions of the body to be treated by the enzyme solution. See Vernon, IntraCellular Enzymes, London, John Murray, 1908; Bayliss, The Nature of Enzyme Action, Longmans, Green \& Co., 1920 ; Euleir, General Chemistry of the Enzymes, Pope's Translation; BAyliss, General Principles of Physiology, 1919.

665. Method of Using Enzyme Solutions. -These should be used on fresh cells. It is best to begin by identifying cell or nuclear bodies or granules in properly fixed and stained preparations; after this one should familiarise oneself with the appearance of such granules or bodies in the fresh cells, with and without intra-vital staining.

The enzyme or digesting fluids may be added to fresh cells, a coverslip provided, and the preparation observed at intervals under a high power.

Another method is to immerse pieces of fresh tissue in the digestive fluid for suitable times, and then fix the tissue in some mixture by previous trials indicated for the purpose ; controls should be made by soaking other pieces of tissue for a similar time in some of the same solution inactivated by heat, or without the added enzyme.

As indicated above, such tests are of doubtful value as regards the identification of "chromatin"; the solutions used should be tried carefully on cells whose eytology is thoroughly known by the observer. The use of proprietary "pepsins" and such-like solutions whose origin and method of manufacture are unknown to the worker, is not likely to lead to the best results. See also C. Beckwith, Jour. Morph., xxv, 1914, and Jörgensen, Arch. f. Zellf., x, 1913, and § 645.

666. Chromophility.-Some workers indiscriminately call all basophil chromatic material chromatinic, which is unjustifiable: 
the chromatic " blushes" which are sometimes found surrounding the nuclei of eggs and other cells are sometimes believed to be chromatinic, but the evidence for this is very slender. Chromatin, moreover, may sometimes stain oxyphil, and it is now a well-known fact that the nature of the fixation and the method of staining are important factors in deciding whether the bodies in question will stain in the basic or the acid dye. Under "Nucleoli" are given a number of staining and fixing methods which may be tried. See also Chapter XI, § 211.

\section{6\%. "Vital" Staining of the Nucleus.-A. M. Przesmycky} (C. R. Soc. Biol., lxxviii, 1915) uses neutral red. The living nucleus is said to have a greater affinity for neutral red than protoplasm, as it stains more strongly and decolourises more slowly. This is not my experience with weak neutral red. Chambers (Science, 1912) uses janus green, which shows both chromosomes and spindle fibres. Certain physiologists doubt whether the living resting nucleus can be stained; some observations on this will be found in Chapter XI., § 207. Reference may be made to the paper of P. G. Shipley (Amer. Journ. Physiol., xlix, 1919).

It has been asserted by some observers that the nucleus may also be stained during the life of the cell by means of Bismarck brown, Congo red, methylen blue, Nile blue, and safranin. But Bolles Lee believes that it is by no means clear from the statements of these writers that the coloration observed by them is localised in the chromatin of the nucleus. It would rather appear to be a diffuse coloration brought about by mechanical and momentary retention of the dye in the nucleus-which is a very different thing from a true nuclear stain. And in some of the cases reported it is by no means certain that the coloured nuclei were really in the living state. In any case there is no dye known which is a specific intravital test for chromatin. It seems probable that the most "specific" test for chromatin known to cytology is acidulated methyl green, for which, see $\S \S 278$ and 645 .

668. Nucleoli.-The word is nowadays generally used to mean any large rounded stainable body in the nucleus. The plasmosome is " acidophilous" in so far as, in fixed material, it selects the acid dye or dyes from mixtures such as the Ehrlich-Biondi, which stains it reddish or orange. So far as known true plasmosomes of unfixed cells do not stain green with the acid methyl green, but while this may hold for the plastin nucleoli of somatic cells in general, there are to be found in eggs complicated nucleoli which have not properly 
been investigated. Even if part of these nucleoli did stain green in acidulated methyl green, it would be unsafe to interpret such material as chromatin, especially in view of the fact that methyl green will stain silk and mucin, and the secretions of many gland cells ; plasmosomes often seem to be related in some way to gland secretion.

Most of the work on plasmosomes or true nucleoli has been carried out on fixed material, and it cannot be too strongly emphasised that by such means it is not possible properly to discriminate between what is true chromatin, and what is not true chromatin. There are no perfectly specific chromatin dyes known, the nucleoli in general may be made to stain differently according as to whether they may have been fixed in acid or non-acid fixatives (this applies especially to acetic acid), or in osmicated or non-osmicated mixtures, while true chromatin may itself stain " acidophile," as, for instance, in the head of the sperm during spermateleosis, and in the egg during oögenesis.

These remarks refer also to the use of so-called specific dyes for discriminating between and identifying the various cytoplasmic inclusions. See especially $\S 5707$ et seq.

The case of the so-called karyosome or chromatin nucleolus is even less satisfactory; one often meets with " solid" intra-nuclear bodies which stain basophil with many so-called nuclear dyes, but which are only doubtfully to be regarded as chromatin. In this connection, see Bayliss ( $\$ 204$ ) on specificity of dyes.

Safranin and light green, and iron hæmatoxylin, following strong Flemming's fluid, are classic stains for nucleoli, but neither of these methods is to be regarded as providing any useful evidence as to the micro-chemical nature of the bodies they tinge. They simply stain most deeply, solid bodies.

The relationship between nucleoli and the chromatin network is quite unknown in general, but CAJAL and CARLETON (for references see Quart. Jour. Micr. Sci, lxiv, 1920) have shown by formalin and silver nitrate methods that both basophil and oxyphil nucleoli may contain an argentophile core. Carleton has followed the core through mitosis, and finds that it keeps its individuality; the definitive nucleolus of the "resting" nucleus is possibly derived after every mitosis from the core or nucleolinus. I have found nucleolini in the nucleoli of the gut cells of Saccocirrus, and in follicle-cells of the ovary of insects; nucleolini are known in many kinds of vertebrate cells.

For the study of nucleoli the following methods are advised :(1) Fixation by a corrosive sublimate, both acidified by acetic acid, and alone. (2) Fixation by hot water or steam.

(3) Fixation by a 
variety of mixtures such as Carnoy, Bouin and Zenker with or without acetic acid, etc. Staining in Ehrlich-Biondi, Ehrlich's hæmatoxylin and azoeosin or Biebrich scarlet (by Scott's method, described below), by Pappenheim's pyronin and methyl green, by Auerbach's fuchsin and methyl green, Zimmermann's fuchsin iodine green, and by Mann's methyl-blue eosin. The double and triple simultaneous stains are valuable.

Auerbach's stain consists of equal parts of 1 per cent. methyl green, and 1 per cent. acid fuchsin; Pappenheim's stain (§ 292) consists of methyl green and pyronin, a red basic stain instead of the acid fuchsin of the Auerbach. Another stain which is very valuable is the triple stain of Ehrlich, but it is less easy to work than Auerbach or Pappenheim. In all work on nuclei and nucleoli, Mann's methyl-blue eosin will be found especially helpful, because the eosin-staining from this mixture is generally more restricted and intense than when one stains in some basic dye followed by eosin, or vice versâ. Very beautiful results are occasionally procured by using Unna's polychrome methylene blue ( $\$ 337$ ).

Recourse should be made to the methods for the mitochondria, particularly those such as Champy-Kull and Bensley-Cowdry (compare with Auerbach preparations), for many nucleoli are compound bodies almost certainly containing lipoids or fats.

The formalin silver nitrate techniques of Cajal or Da Fano should be tried, and wherever possible tests on fresh cells should be carried out (e.g. digestion, methyl green, etc., etc.).

It seems indicated that further observations carried out on nucleoli of live cells in tissue cultures will provide new facts, especially with regard to the part played by these bodies during mitosis. See sections on "Tissue Culture."

669. S. G. ScotT's Standard Hæmatoxylin and Biebrich Scarlet for Chromophility (Jour. Path. and Bact., xvi, 1912).-Fix tissue in sublimate formalin, Zenker without acetic, Helly's Zenker-formalin, Müller, or formalin. All strongly acid fixatives must be avoided, for the Ehrlich's hæmatoxylin will not then stain anything but nuclei, and these only faintly. Paraffin sections are made and fixed to slide. After removal of paraffin with xylol, and this with alcohol, sections of material fixed in sublimate solutions are treated with iodine for three or four minutes $(0 \cdot 2$ per cent. iodine in 80 per cent. alcohol). Rinse off excess iodine with a little alcohol and remove all iodine from tissue with a 0.25 per cent. solution of $\mathrm{Na}_{2} \mathrm{~S}_{2} \mathrm{O}_{3}$ in 50 per cent. alcohol, not in water as recommended by HeIDENHAIN (Arch.f. d. ges. Phys., Bonn, 1902, Bd. xc, § 115). This is a most 
important part of the method, as sublimate and iodine both act detrimentally to staining. After washing in $\mathrm{Na}_{2} \mathrm{~S}_{2} \mathrm{O}_{3}$, wash off in a stream of aq. dest. ; roll the water off the slide with 1 or 2 drops of alcohol (90 per cent.), wipe the slide dry around the sections, and pour on 3 to 4 drops of Ehrlich's acid hæmatoxylin to every section. Leave exposed on bench ten minutes. Remove the hæmatoxylin by rolling it off with drops of alcohol (90 per cent.) from a drop-bottle ; do not wash off the stain with water as this lowers the viscosity of the solution and allows a diffuse staining.

Wash away alcohol with a stream of aq. dest. till every trace of stain is removed from the slide.

Blue the hæmatoxylin lake and remove acid from combination with the proteins of the section by dropping on the latter 8 to 10 drops (or more for a number of sections) of the following tap water substitute :-

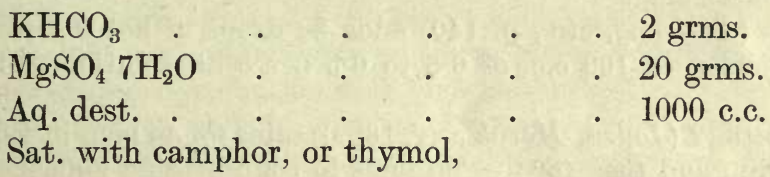

After three to five minutes wash off the alkaline solution thoroughly in aq. dest. Wipe around sections to remove superfluous water, and add a 1 in 2000 solution of azoeosin (not eosin) or Biebrich scarlet dissolved in this medium :-

Glycerol.
Methyl or 96 per cent. ethyl alcohol
Dist. water

Use azoeosin (Bayer) for bichromated material and Biebrich scarlet for non-bichromated material. Leave in the acid dye for ten to thirty minutes, rinse off in aq. dest., rapidly dehydrate in 90 per cent. and absolute alcohol; xylol, and xylol balsam.

In my hands weak watery solutions of good Eosin have not given such satisfactory results. Scott believes that sulphonated monazo colours, such as orange $G$ and Bordeaux $R$, are useless. Eosin is much less precise than Biebrich scarlet and azoeosin. The special points about this method are :-(1) Getting rid of all corrosive and iodine. (2) Staining by placing hæmatoxylin on slide where the solution can evaporate, and thus arrive at a state of viscosity which automatically prevents overstaining. (3) Washing off stain in alcohol, not water, thus preventing diffuseness. (4) A definitely alkaline tap-water substitute. (5) A precise counter-stain.

Histologists and cytologists carrying out work on chromophility are recommended to use this method as a standard for either basophility or oxyphility. Note, however, that granules which stain basophil by this 
method are not necessarily ehromatinic. Occasionally this method seems to overstain genital cells, but this gives the correct degree of chromophility of such cells in comparison with the cells of other tissues. After having used the method critically, I have come to the conclusion that it is more precise than the usual procedure which introduces differentiation in an acid solution. Scott worked out this method mainly with sections of mammalian red bone marrow.

669a. OBSt (Zeit. wiss. Zool., lxvi, 1899) fixes in sublimate, stains in borax carmine, and then stains the sections for three hours in very dilute aqueous methyl green or solid green. Nucleoli blue, chromatin red.

Buchner (Arch. Zellforsch., iii, 1909, p. 337) has found this useful for distinguishing the accessory chromosome in testis cells of Orthopteranormal chromosomes red, accessory and chromatin nucleoli blue-violet.

ZimmermanN (Zeit. wiss. Mik., 1896, p. 463) stains for ten minutes in a fresh mixture of 9 parts 0.1 per cent. aqueous iodine green with 1 part concentrated aqueous solution of fuchsin, and differentiates in absolute alcohol with 1 per cent. of acetic acid and 0.1 per cent. of iodine. Nucleoli red, chromatin blue.

Fischer (Fixirung, etc., p. 140) adds 30 drops of hot $0 \cdot 1$ per cent. fuchsin solution to 100 e.c. of 0.3 to 0.5 per cent. solution of methyl green.

MONTGOMERY (Journ. Morph., xv, 1899) stains for an hour in Ehrlich's hæmatoxylin, and then for five minutes in concentrated aqueous eosin, or first with concentrated aqueous methylen blue, and then with concentrated alcoholic solution of Brazilin.

For a series of papers on staining nucleoli, and illustrated by profuse coloured plates, see Max Jörgensen's memoirs in the Arch. f. Zellf., x, 1913. Another paper worth consulting is CHAMPY's magnificent work on the male germ-cells of amphibia in the Arch. de Zool. Expér., 1913.

See also Reddingius, Virchow's Arch., elxii, 1900, p. 206. For nucleoli of ova, List, Mitth. Zool. Stat. Neapel, xii, 1896, p. 480; of nerve-cells, Ruzicka, Zeit. wiss. Mik., xiv, 1898, p. 453, and Levi, Riv. Pat. Nerv. Ment. Firenze, iii, 1898, p. 289.

6\%0. Plasma Stains.-Dr. Bolles Lee states that he has been unable to discover a single thoroughly satisfactory one. Almost all of them colour too readily the enchylema or hyaloplasm at the same time as the spongioplasm. And, on the other hand, there are many important elements of cells which cannot be got to stain sufficiently. We consider Säurefuchsin and Biebrich scarlet the most generally recommendable, especially after iron hæmatoxylin. See also Bordeaux R.

Flemming's orange method has been much used. It is very capricious and unreliable. Ehrlich-Biondi mixture is a celebrated plasma stain. 
The Iron-Hamatein Lakes of Benda and M. Heidenhain give good plasma stains, according to the degree of extraction, and would be inferior to none were it not that they stain in the same tone as the chromatin. See also Ehrlich's tri-acid, and his acidophilous mixture, also gold chloride, Apáthy's process, § 371, and Kernschwarz.

Imperfectly stained plasma structures can often be well brought out by mounting in Euparal instead of balsam.

6\%1. Centrosomes.-These can be stained by some "acid" anilin dyes, better by a "neutral " dye (e.g. Flemming's orange method, or the Ehrlich-Biondi-Heidenhain stain). But by far the best stain is iron-hoematoxylin.

It is said by Heidenhain that the stain is obtained in a sharper form by combining the hæmatoxylin stain with a foregoing stain with Bordeaux $R$. He directs (Arch. mil. Anat., xlii, 1894, p. 665) that the sections (sublimate sections were used by him) are to be stained for twenty-four hours or more in "a weak" solution of Bordeaux, until they have attained such an intensity of colour as that " they would just be fit for microscopic examination with high powers" ( $l$. c., p. 440, note), and that they be then brought into the ferric alum. After mordanting and staining, the hæmatoxylin is to be extracted in the iron alum until the chromatin has become entirely or almost entirely colourless. Instead of Bordeaux, "anilin blue " may be used in the same way.

The images of these objects given by iron-hæmatoxylin require to be interpreted with special care. Globular or even elongated objects, such as chromosomes, do not always yield up their stain simultaneously and equally throughout their whole depth, but lose it suddenly and entirely in their outer layers, whilst retaining it in its full strength in their deeper layers. It seems that certain erroneous observations that have been published have been due to this deception.

672. Cell Granules.-For the study of the conspicuous "granules," undoubtedly metabolic products, occurring in certain gland-cells and blood- and lymph-corpuscles, and in certain elements belonging to the group of connective tissues, see the sections on "Connective Tissues," "Mitochondria" and "Fat." The most generally employed stains are the mixtures of EHRLICH.

Intra-vitam staining is useful here. See $\S 208$. See also ArNold, Anat. Anz., xxi, 1902, p. 417.

Benda (Verh. phys. Ges. Berlin, 1899-1900, Nr. 1-4, and Verh. Anat. Ges., xv, 1901, p. 172) gives the following method for demonstrating secretion-granules and distinguishing them from other granules: Harden for twenty-four hours in 10 per cent. formalin, 
then for one day in 0.25 per cent. chromic acid, one in 0.33 per cent. and two to three in 0.5 per cent., wash one day in water, dehydrate and make paraffin sections. Then stain with one of Ehrlich's mixtures, according as the granulations are basophilous, acidophilous, or neutrophilous. The methylen-blue and eosin process of Michaelis is recommended.

See also Mallory, §§ 271 and 272 ; Mann, § 328.

\section{Mitochondria,* Golgi Apparatus, $\dagger$ Yolk, Fat, and other} Cytoplasmic Inclusions. ${ }_{-}$-The mitochondria and Golgi apparatus never clearly appear in stained sections prepared by such methods as fixation in corrosive acid, Gilson, Bouin, Carnoy or Flemmingwith-acetic acid, and staining in Ehrlich's hæmatoxylin and eosin, toluidin-blue and eosin, paracarmine and borax carmine. Though the mitochondria and Golgi apparatus are properly fixed by formalin, Müller, Flemming-without-acetic acid, Champy, Altmann, etc., they will rarely appear visible in stained sections which have been prepared in Ehrlich's or Delafield's hæmatoxylin or carmine stains, or in fact in any of the current laboratory stains used for general zoological purposes. The mitochondria and Golgi apparatus may appear visible in sections fixed in formalin, Müller, etc., and stained in Altmann's acid, fuchsin-picric acid, iron-hæmatoxylin, Benda's alizarin and crystal-violet, etc. The Golgi apparatus rarely becomes visible after any of the above methods, and to study it one must use more specialised methods; to study the Golgi apparatus and the mitochondria by routine zoological laboratory technique is not possible, simply because these methods will not demonstrate the bodies in question. Nearly all of the older fixing mixtures contain either alcohol, chloroform, or acetic acid, but the last few years of cytological research have shown that the picture given by a fixing mixture containing them is incorrect and inadequate, and one cannot fail to be surprised at the improvement produced when these reagents are omitted. Nearly all the modern research on the cytoplasm has to be carried out by observers using chrome- or platinumosmium fixatives, followed by iron-alum hæmatoxylin, Benda's crystal violet, or Altmann's acid fuchsin; or by the important Kopsch and Mann-Kopsch, and Sjövall osmium tetroxide methods ; or by the useful methods of Cajal, Golgi or Da Fano's modification

* Chondriosomes, chondriokonts, plastochondria, "chromidia," bio. blasts, chondriome, chondriomites, etc., etc.

† Nebenkern batonettes, idiozome rods, "Golgi-Kopsch apparat," apparato interno reticolare, dictyosomes, Binnennetz, etc.

‡ By J. B. G. 
of Cajal, which consist of silver nitrate impregnation following formalin fixation. Intra-vitam methods, such as janus green, neutral red, or dahlia violet are also used extensively. The mitochondria are extremely fuchsinophile, and after chrome-osmium fixation stain strongly in iron-alum hæmatoxylin. The Golgi apparatus of somatic cells and of ovarian cells rarely stains by these methods (Altmann or Heidenhain) unmodified, although the Golgi apparatus of the male germ cells nearly always stains in fuchsin or hæmatoxylin after chrome-osmium or formalin fixation. Besides observing the Golgi apparatus and mitochondria, certain workers have turned their attention to the study of fats and yolk (vitellus) in cells.

In $\S 768$ is a special article on fats and lipoids, and on methods for their study; on the following pages are set forth various techniques for the investigation of definite cell organs known to be partly lipoid in nature. The application of all these methods to embryological study opens the way to a valuable field for research. In $\S 768$ will be found definitions of the terms "fat," "lipoid," and "lipin." Fats or lipoids form a special part of almost all cell-organs, as seems to be indicated by fixing tests, and so far as we know such substances are always intimately associated with protoplasm. Many of the lipoids appear to be able to form with certain metallic salts or oxides such as $\mathrm{CrO}_{3}, \mathrm{~K}_{2} \mathrm{Cr}_{2} \mathrm{O}_{7}, \mathrm{PtCl}_{4}, \mathrm{OsO}_{4}$, etc., compounds insoluble or only slowly soluble in alcohol or such clearing oils as xylol, benzole, or chloroform ; this is one of the several reactions which take place when a cell is fixed in such a fluid as that of Flemming (without acetic acid), Champy, or Altmann, and subsequently dehydrated and cleared. See $\$ \S 29,30$ and 31.

674. Choice of Method.*-We have given below a number of methods for lipoid granules, mitochondria, and other cell inclusions, and not all are suitable for every piece of work. It is very rare to find that one single method will produce the same good result in both vertebrate and invertebrate tissues. In the same way, methods which act satisfactorily with amphibia will often give disappointing results with mammalia. Osmic-chrome fixation will nearly always be found excellent for all classes of invertebrata ; Flemming-withoutacetic acid and Champy-Kull can be highly recommended. For amphibia the addition of some $\mathrm{K}_{2} \mathrm{Cr}_{2} \mathrm{O}_{7}$ to the Flemming is necessary before a correct fixation of the mitochondria is obtained; thus

* The beginner is recommended to master such techniques as those of Bouin's fluid and borax carmine, or Zenker and Ehrlich's hæmatoxylin before trying these methods. 
Champy's fluid was invented for amphibia and gives very satisfactory results ( $\$ 43$ ). For mammalian tissues a preliminary fixation in osmic acid fixatives is not generally indicated; the tissues of mammals are far more "fatty" than those of invertebrata or amphibia, and one finds that the $\mathrm{OsO}_{4}$ becomes reduced very rapidly and penetration is very poor. For mammalian tissues formalin-chrome (Regaud, Bensley-Cowdry), formalin-corrosive or formalin alone are indicated as a preliminary treatment at the least. Formalin does not destroy lipoids, and by subsequently placing small pieces of chrome-formalin fixed tissues in osmic acid (post-osmication), a fixation of lipoids and fats is obtained (Schridde); the same result may be got by fixing tissues in chrome salts and then transferring to osmic acid. It should be noted, however, that previous fixation in a chrome salt prevents the blackening of the Golgi apparatus; the formalin-osmic acid method (Sjövall) is indicated especially for mammalian tissues, when an impregnation of the Golgi apparatus is required by means of an osmic method, but the formalin silver nitrate methods of Golgi, Cajal and Da Fano are always clearly indicated for work on the Golgi apparatus of mammalian tissues. So far as possible intra vitam and fresh smear preparations should be used, as these nearly always give valuable results.

\section{6\%5. Specificity of Techniques for Cytoplasmic Inclusions, Fats,} and Lipoids.-As a rule the lipoid granules, vacuoles, and cell organs containing fats or lipoids are formed not of one pure substance, but of a mixture of several. Consequently it is necessary to proceed with caution in claiming a specificity for the techniques for various lipoid substances : properly used, however, microchemical methods may give valuable evidence as to the precise chemical nature of any special body: microchemical methods, which depend for their application on the use of complicated fixing and staining methods are to be used cautiously. For example, Benda (\$683) and AltmannBensley methods ( $\S \S 680$ and 686 ), will stain granules other than mitochondria, while the Cajal formalin uranium and silver nitrate technique impregnates bodies apart from the Golgi apparatus. In all these cases, however, the number of such exceptions to a specificity is small, and suitable differentiation between two doubtful bodies can be made by some other method. See Bayliss on "Specificity" of staining methods, $§ 211$.

676. On Killing Animals for Cytological Purposes.-So far as possible avoid narcotics of any sort. Either cut off the heads of invertebrates, or, if delicate like some worms, drop them whole in 
the fixative; kill vertebrates by a blow on the head, or by pithing. If for a study of brain, bleed, or anæsthetise in coal gas, less preferably chloroform or ether. Insects can be killed with cyanide or xylol.

Hints on removing Tissues and Cutting.-Avoid pinching the material with forceps, as this will introduce artifacts; it is preferable to remove tissue without recourse to dissection under tap-water or salt solution; for Kopsch techniques, quickly remove blood or lymph, etc., from surface of material with aq. dest. before placing in fixer; for cutting tissue the best instrument is a new safety razor blade stuck in a special holder made for the purpose, or in a split penholder, or held by artery forceps. When working on arthropods, it is best to dissect the organ from the animal, instead of preserving the whole body; surrounding fat, etc., should be removed. See also $\S \S 12$ and 653.

69\%. Chrome-osmium Techniques.-Potassium bichromate or chromium trioxide, used in watery solutions will not oxidise true fats (see $\S 768$ ) in such a way that full vacuoles of the latter will appear in finished sections prepared by routine methods; but combinations of such salts with osmium tetroxide provide fixatives which will preserve almost all cell elements in finished sections. The basis of all chrome-osmium techniques consists in a preliminary fixation of small pieces of tissue, small embryos or eggs, in such a fluid as Benda, Champy, Flemming-without-acetic acid, or Altmann, for from at least twelve hours to a week. The osmic reaction is then, in some methods (Kull, Benda), "set" or strengthened by the reducing effect of pyroligneous acid; following this treatment is a further "chroming" in 3 per cent. bichromate of potash, and, finally, a thorough wash out under the tap. Material treated in this way is generally perfectly preserved, and fit for selective staining. Arranged below are chrome-osmium techniques of progressive intensity and difficulty.

678. Modified "Flemmings" for Cell Inclusions.-BENDA : 15 c.c. chromic acid 1 per cent., 4 c.c. osmic acid 2 per cent., 3 to 6 drops of acetic acid. Meves: 15 c.c. of chromic of 0.5 to 1 per cent., containing 1 per cent. sodium chloride, with 3 to 4 c.c. of 2 per cent. osmic acid, and 3 to 4 drops of acetic (Encycl. mik. Techn., 1910). Gatenby: Strong Flemming without acetic acid; and same solution diluted by one-half or one-third (Quart. Journ. Micr. Sci., 1919). The presence of a small quantity of acetic acid is always liable to introduce distortion, but less so among vertebrates 
than among invertebrates. See also "Champy's Fluid” (§ 43) and "Altmann's Fluid” (§ 42), and $\S 41$.

\section{Flemming's Strong Fluid without Acetic Acid, and Iron} Hæmatoxylin.-Small organs freshly dissected out in normal saline, or parts of organs cut with safety razor blade, not more than 5 millimetres in diameter, are placed in about 15 c.c. of one of the abovementioned fixing fluids, preferably Gatenby's modification. A glass-covered capsule or vial is the best vessel to use, and the material is left for at least twenty hours, and not longer than one week. We find about twenty-four hours gives a satisfactory fixation of most tissues. After fixation the liquid is poured away, and the material is washed for at least two hours, and not necessarily longer than five, in running tap-water. It is then passed through up-graded alcohols, beginning at 30 per cent., giving the material at least three hours in the strengths 30,50 and 70 per cent., and overnight in 90 per cent. The pieces of tissue are dehydrated two or three hours in two changes of absolute alcohol, and then transferred to a mixture of half absolute alcohol and half xylol for a quarter of an hour ; then pure xylol, and embedded in wax. Sections are cut from 4 to $8 \mu$, but I generally find $6 \mu$ to be convenient. Leave eight to ten hours in iron alum, twelve to twenty hours in hæmatoxylin. This method gives a delicate and precise stain of the mitochondria (and Golgi apparatus or nebenkern batonettes of male germ-cells only), fat is black, while yolk is generally greenish brown. Especially recommended for germ-cells, and histology of Invertebrata, but with vertebrate tissues, and especially mammalian material (not embryos), it often gives atrocious results; for such material, Helly, Zenker, or Regaud's methods are indicated (Gatenby, Quart. Journ. Micr. Sci., 1919).

Note that Fleming-without-acetic acid is not a suitable fixative for after-staining in Altmann's acid fuchsin. For this the material must be washed in distilled water for a short time after fixation, and then transferred to 3 per cent. bichromate of potash for three days; or the more elaborate mordantage as for Champy-Kull may be used ( $\$ 681)$.

680. Altmann's Acid Fuchsin and Picric Acid (Die Elementarorganismen, Leipzig, 1890).-Fix twenty-four hours in mixture of equal parts of 5 per eent. bichromate of potash and 2 per cent. osmic acid. Embed in paraffin, stain sections on slide for one minute over flame, with a solution of 20 grms. (sic) of acid fuchsin in 100 c.c. of aniline oil-water ( $\$ 286)$. Cool, and wash out in a saturated alcoholic solution of picric acid diluted with 2 volumes of water, 
heat being used as before to aid differentiation; blot, dip into 90 per cent. or absolute alcohol, xylol, balsam. This method only stains granules which can be seen intra vitam; properly used it never produces artifacts, and Fischer's critique is quite wrong (Fixirung Faerbung u. Bau des Protoplasmas). Altmann's original method has been superseded more or less by the following method of Champy-Kull. (Both Dr. J. A. Murray and I find that the 20 grms. of acid fuchsin will not dissolve in 100 c.c. of aniline oilwater; only about 5 to 7 grms. will dissolve, and this quantity will make a perfectly efficient solution.)

681. Champy-Kull's Acid Fuchsin, Toluidin Blue and Aurantia (Kull, Anat. Anz., Bd. xlv, 1913).-The following method, while being generally useful, will be found very convenient for work on Invertebrata. It gives results intermediate between those of Benda and Altmann, but is shorter and undoubtedly better than the method of Benda. It will be found very useful for embryological research, and probably also for protozoology. Fix in Champy $(\S 43)$ (we find Flemming-without-acetic acid will do, too) for twentyfour hours. Pieces to be fixed must be small. After fixation wash half an hour in aq. dest., and then transfer to a mixture of 1 part acid acet. pyrolignosum rect., and 2 parts 1 per cent. chromic acid, for twenty hours. Wash half an hour in aq. dest., and transfer to a 3 per cent. solution of potassium bichromate for three days. Wash under tap for twenty-four hours; pass through up-graded alcohols to xylol; embed in paraffin wax (or celloidin method, if desired). Section 4 or $5 \mu$. Proceed as follows:-(1) Stain in Altmann's acid fuchsin aniline oil mixture (5 to 10 grms. of acid fuchsin in 100 c.c. of aniline oil-water), and heat till steaming. (2) Set slide aside to cool for six minutes (this is important), pour off, and wash quickly in aq. dest. (3) Counter-stain in either a 0.5 per cent. solution of toluidin blue or a saturated solution of thionin in aq. dest. for one to two minutes. Wash in aq. dest. In some cases the time in the blue stain must be shortened. Transfer to a 0.5 per cent. solution of aurantia in 70 per cent. alcohol for from twenty to forty seconds, watching extraction of fuchsin stain under microscope. Differentiate the blue stain in 96 per cent. alcohol, then absolute, xylol, and balsam. The chromatin is generally blue, mitochondria (and occasionally Golgi apparatus) are red, and the ground cytoplasm is golden-yellowish to green. This modification of Altmann's method is a most brilliant three-colour stain which is highly recommended. We have found that it is useful for histological as well as cytological purposes; sections of Annelids, or of 
flat-worms, for instance, prepared by Champy-Kull show beautiful colour graduations in their different tissues. The preparations begin to fade after a year.

After Champy-Kull fixation you can: $(a)$ stain in iron hæmatoxylin (long method, $\S 242$ ), (b) stain as for Benda ( $\$ 683),(c)$ mount unstained for examination of osmicated granulations, $(d)$ stain in safranin and light green ( $\$ 286)$. For a chart illustrating ChampyKull technique, see below.

Maxımow (C. R. Soc. Biol., Paris, lxxix, p. 462) fixes in Champy, washes slightly in water, transfers to mixture of 1 per cent. chromic acid 1 part, glacial acetic 2 parts, for twenty-four hours. Wash again for half-an-hour, place for three days in 3 per cent. $\mathrm{K}_{2} \mathrm{Cr}_{2} \mathrm{O}_{7}$. Wash in running water. Stain sections as above.

\section{Champy-Kull Fixation.}

\begin{tabular}{|c|c|c|c|c|c|}
\hline $\begin{array}{c}\text { Subsequent } \\
\text { method. }\end{array}$ & $\begin{array}{l}\text { Mito- } \\
\text { chondria. }\end{array}$ & Fat. & Yolk. & Golgi apparatus. & Nucleus. \\
\hline Mount unstained . & Yellowish. & $\begin{array}{l}\text { Black (extract- } \\
\text { able in tur- } \\
\text { pentine). }\end{array}$ & $\begin{array}{l}\text { Yellow } \\
\text { to black. }\end{array}$ & $\begin{array}{l}\text { Yellowish or does not } \\
\text { show. }\end{array}$ & Yellowish. \\
\hline Champy-Kull stain . & $\begin{array}{l}\text { Red or } \\
\text { pink. }\end{array}$ & Black. & $\begin{array}{l}\text { Yellow } \\
\text { to black. }\end{array}$ & $\begin{array}{l}\text { Generaliy will not } \\
\text { show in somatic } \\
\text { cells or ovaries, red } \\
\text { in male germ cells. }\end{array}$ & $\begin{array}{l}\text { Chromatin blue to } \\
\text { greenish (nucleolus } \\
\text { red). }\end{array}$ \\
\hline Iron haematoxylin & Black. & $\begin{array}{l}\text { B l a c k, pro- } \\
\text { vided it has } \\
\text { not been ex- } \\
\text { tract } \theta \text { d in } \\
\text { turpentine. }\end{array}$ & $\begin{array}{l}\text { Same as } \\
\text { above. }\end{array}$ & $\begin{array}{l}\text { Same as above, but } \\
\text { black in male germ } \\
\text { cells. }\end{array}$ & $\begin{array}{l}\text { Chromatin grey to } \\
\text { b la c k (nucleolus } \\
\text { black). }\end{array}$ \\
\hline $\begin{array}{l}\text { Benda stain (alizarin } \\
\text { and crystal violet) }\end{array}$ & Vlolet. & Same as above. & $\begin{array}{l}\text { Same as } \\
\text { above. }\end{array}$ & $\begin{array}{l}\text { Same as above, but } \\
\text { violet in male germ } \\
\text { cells. }\end{array}$ & $\begin{array}{l}\text { Chromatin brown or } \\
\text { yellowish (nucleolus } \\
\text { violet). }\end{array}$ \\
\hline
\end{tabular}

683. BENDA's Alizarin Method (Ergebnisse der Anat., xii, 1902 (1903), p. 752, and other places) is as follows:-Harden for eight days in strong liquid of Flemming, the acetic acid therein being reduced to 3 drops (or as for Champy-Kull or Regaud). Wash for an hour in water and put for twenty-four hours into a mixture of equal parts of pyroligneous acid and 1 per cent. chromic acid, then for twenty-four hours into bichromate of potash of 2 per cent., wash for twenty-four hours and embed in paraffin. Sections on the slide are mordanted for twenty-four hours with 4 per cent. solution of ferric alum or diluted liq. ferri sulfur. oxydat., then rinsed with water and put for twenty-four hours into an amber-yellow aqueous solution of Kahlbaum's sulfalizarinate of soda, prepared by dropping 1 c.c. of saturated alcoholic solution thereof into 80 to 100 c.c. of water. Rinse in water, flood the slides with the solution of crystal 
violet ( $\$ 330$ ) diluted with an equal volume of water, and warm till vapour is given off. Rinse, differentiate one or two minutes in 30 per cent. acetic acid (till the nuclei come out reddish), wash in running water for five to ten minutes, dry with blotting-paper, dip into absolute alcohol, pass through bergamot oil into xylol and balsam. Mitochondria violet, chromatin and " archoplasm " brownred, certain secretion granules pale violet, centrosomes red violet.

Instead of the staining solution prescribed above (which may be kept in stock) you may take (Encycl., ii, p. 198) a freshly prepared mixture of equal parts of anilin water and saturated alcoholic solution of crystal violet-and this is to be preferred.

Some worker's prefer to harden as BENDA, but to stain with iron hæmatoxylin instead of by the alizarin process ; the special hardening rendering the hæmatoxylin stain sufficiently specific.

ARNold (Arch. Zellf., viii, 1912, p. 256) stains first with iron hæmatoxylin, differentiates, stains for twenty to thirty minutes with saturated aqueous solution of thionin, passes up to absolute alcohol, stains for two minutes with Orange $\mathrm{G}$ dissolved in clove oil, and passes through xylol into balsam. Chromatin blue, chondriosomes black.

684. Formalin-Chrome Techniques.*-The methods of Regaud, Bensley-Cowdry, Schridde, Murray, etc., are of importance on account of their suitability for vertebrate, and especially mammalian tissues. The tissues are fixed either in neutral formalin or in formalin-chrome mixtures, washed, and then mordanted in 3 per cent. $\mathrm{K}_{2} \mathrm{Cr}_{2} \mathrm{O}_{7}$. As with Champy-Kull, it is possible to stain after such fixation by a variety of methods:-iron hæmatoxylin, acid fuchsin, alizarin and crystal violet, safranin, etc. The Regaud and Bensley-Cowdry methods do not preserve neutral fat in the finished sections, but by post-osmication, as for Schridde ( $\$ 687)$, or Murray ( $\$ 689)$, this can be done.

685. REGAUD's Formol-Bichromate and Iron Hæmatoxylin (Arch. d'Anat. micr., t. xi, 1910).-Fix in a mixture of 3 per cent. potassium bichromate 80 .volumes, commercial formalin 20 volumes, for four days, changing every day. Mordant in potassium bichromate for seven days, changing every second day. Wash in running water twenty-four hours, dehydrate (twenty-four hours each strength),

* Note that formalin-chrome mixtures consist of a reducer and an oxidiser, and will not keep. Such solutions should always be made up just before use. Zenker's fluid, too, keeps better without the acetic acid, which, if baing used, should be added just before the material is put in the fixative. 
clear and embed in paraffin. Pass sections on slide down to water ; 5 per cent. iron alum at $35^{\circ} \mathrm{C}$. for twenty-four hours; rinse in aq. dest., not tap-water. Stain twenty-four hours in this solution :$1 \mathrm{grm}$. of pure crystals of hæmatoxylin in 10 c.c. of absolute alcohol, added to 10 c.c. of glycerine and 80 c.c. of aq. dest. Differentiate in 5 per cent. iron alum, watching process under microscope. The main point is to avoid washing out the mordant too much when the slides are being transferred from the iron alum to the hæmatoxylin. Permanent stain, very good for vertebrate tissues. See also CowDRY, Amer. Journ. Anat., xix, p. 441. I find ordinary iron hæmatoxylin is quite good after Regaud fixation.

686. Bensley-Cowdry Acid Fuchsin and Methyl Green Stain (Cowdry, Contrib. Carnegie Inst. Wash., viii, 1918).--Fix as for Regaud, either by immersion or injection; formalin should be neutralised in magnesium carbonate, and, if possible, the fixation should be done in an ice-box, but this is not necessary. Pass sections down to aq. dest. through toluol (or xylol), absolute alcohol, etc., thirty seconds in each; transfer to 1 per cent. potassium permanganate for thirty seconds, but time must be determined experimentally; then 5 per cent. oxalic acid for thirty seconds. (Note.The permanganate and oxalic acid may generally be omitted.) Then rinse in several changes of aq. dest. for about one minute (incomplete washing prevents staining in acid fuchsin). Stain in Altmann's fuchsin ( $(680)$ as follows : dry around sections with duster, add stain, warm over spirit lamp until fumes come off ; cool for six minutes; wipe around sections with duster, rinse off in aq. dest., so that the only remaining stain is in the sections (or a precipitate forms with the methyl green); pipette a little 1 per cent. methyl green over the sections for about five seconds at first, modify time as experimentally found convenient; drain off excess, plunge into 95 per cent. alcohol for a second or two. Rinse in absolute alcohol, clear in toluol, mount in balsam. Difficulties are that the methyl green may remove the fuchsin (due to incomplete chrome mordanting during fixation), or the fuchsin may have overstained (due to too much mordanting). Sometimes, if the methyl green is too weak, it is better to omit the 95 per cent. alcohol, dehydrating in absolute. The difficulties of this modification of Altmann's stain are easily overcome; I have used it for a senior histology class, and with success. Like the Champy-Kull method, this stain is not so permanent as Iron Hæmatoxylin. See also Bensley, Amer. Journ. Anat., xii, p. 308 ; Duesberg, ibid., xxi, p. 469. 
68\%. SCHRIDDE's Method for Mitochondria, modified (Ergeb. Anat. u. E. Merk. Bonnet, xx, 1911).-Fix in this mixture : formol (1 part), Müller (9 parts), for two days; then place in Müller, two to four days; then 2 per cent. $\mathrm{OsO}_{4}$, for two days. Wash overnight, dehydrate, clear in xylol, cut paraffin sections $5 \mu$. Stain as follows : iron alum hot for a quarter of an hour, then hæmatoxylin hot, a quarter of an hour. Differentiate in alum in the cold. This has the advantage over pure formol-chrome techniques in that the introduction of the $\mathrm{OsO}_{4}$ preserves fat; recommended by Duesberg. With this mordanting it should be possible to stain either as for Altmann, Bensley-Cowdry, Champy-Kull, or Benda.

Levi, G. (Areh. f. Zellf., Bd. xi), ovary of mammals.

10 e.c. $2 \cdot 5$ per cent. $\mathrm{K}_{2} \mathrm{Cr}_{2} \mathrm{O}_{7}$.

10 , . 5 per cent. sublimate containing 2 c.c. of formol.

$2,, \quad$. 2 per cent. $\mathrm{OsO}_{4}$.

Leave for three or four days. Wash out well in running water. Stain in Regaud, Benda, ete.

688. A. H. DRew's Formol-Chrome-Hæmatoxylin Method (Journ. R. Micr. Soc., 1920). - This method is used for demonstrating rodlike bodies in the cytoplasm of plant cells. These rods are supposed to be the homologue of the Golgi apparatus of animal cells. The method will undoubtedly be useful for studying animal tissues. Fix plant root tips, etc., for twenty-four hours in a mixture of formol, 20 c.c.; cobalt nitrate, 2 grms. ; sodium chloride, $0 \cdot 8$ grm. ; water to 100 c.c. (preferably at temperature of $37^{\circ} \mathrm{C}$.). Soak fixed tissues in gum-syrup for at least an hour, and cut sections on freezing microtome. Wash in water, and fix on gelatin-coated slides with formalin. See $\S \S 172$ and 182 . Rinse in water to remove excess formalin, mordant at $50^{\circ}$ to $55^{\circ} \mathrm{C}$. in chromic acid 4 per cent., osmic acid 2 per cent., equal parts, on slide for varying periodsfifteen minutes to one hour, or longer. Rinse in water and stain with iron alum 3 per cent. for fifteen minutes, followed by $\frac{1}{2}$ per cent. hæmatoxylin for fifteen minutes, at $50^{\circ} \mathrm{C}$. Differentiate in the cold in iron alum till the nuclei show pale brown. Transfer to 2 per cent. pyridin for two minutes, dehydrate, and mount in xylolbalsam.

In specimens chromed for short periods the mitochondria alone are visible, while in those chromed for a longer time the mitochondria stain less well, while gradually the long Golgi elements appear in the best chromed cells. In animal cells, too, Drew finds short chroming shows the mitochondria, while it requires longer treatment in the chrome to demonstrate the Golgi apparatus. This is my own experience with the Golgi elements or "nebenkern batonettes" of Mollusca. 
689. J. A. Murray's Chrome-Osmic Method for Mitochondria and Bacteria of Mammalian Tissue.-Fix tissue in formol-salt or formolMüller overnight. Thin slices are then placed in Müller's fluid for from two to seven days, and then transferred to 2 per cent. $\mathrm{OsO}_{4}$ for two days more. Wash overnight in running water, dehydrate, embed in paraffin. Sections to be not more than $5 \mu$ thick, fixed on slide, and stained in $3 \frac{1}{2}$ per cent. iron alum at $50^{\circ} \mathrm{C}$. for fifteen minutes, followed by $\frac{1}{2}$ per cent. aqueous hæmatoxylin in same way and for same time. Sections should now be jet black. If such sections be decolourised in the ordinary way in iron alum, both mitochondria and bacteria (if present) will retain the stain, and nuclei are decolourised.

If such sections are decolourised in 0.5 per cent. $\mathrm{HCl}$ in 70 per cent. alcohol, the mitochondria give up the lake and the bacteria remain deeply stained. At the same time the details of the nuclei are sharply stained. Wash sections for twenty minutes in tap-water, counterstain in Van Gieson, mount in balsam (Report Imp. Cancer Research Bureau, 1919).

690. Double-Staining in Hæmatoxylin and Acid Fuchsin.-It is well known that different cell elements have varying powers of resisting decolourisation or differentiation after iron alum or such hæmatoxylin stains. Thus in a hermaphrodite gonad or during fertilisation it is sometimes noticed that the mitochondria of the egg hold the hæmatin lake much faster than those of the sprrm or spermatogenesis stages. It is possible in certain cases to make use of this fact for studying differentially cell granules, etc.

Fix tissue by some prolonged mordanting method, such as that of Champy-Kull, or Regaud. Wash out well in running water and prepare thin paraffin sections. Stain by some intense hæmatoxylin method, such as that of Benda or Heidenhain; differentiate the cell element which you wish to $b_{3}$ stained subsequently a red colour, till it looks pale greyish under the microscope: wash well in water, and counterstain in Altmann's acid fuchsin. Extract the fuchsin to the right stage in 95 p r cent. alcohol, quickiy dehydrate and clear in xylol; mount in balsam. If necassary, aftrr staining in acid fuchsin, you may apply the picric acid of Altmann's method $(\S 680)$, but this necessitates underdifferentiation in the iron alum.

I have found that after staining in the acid fuchsin you may differentiate partly in aurantia as for the Kull method ( $\$ 681$ ).

A method to be tried only by experienced cytologists. The difficulty is to differentiate the hæmatoxylin just to the right stage, and to avoid washing away the acid fuchsin (GATEnby, Journ. Roy. Micr. Soc., 1919 ; Haxs Hend; Arch. f. mikr. Anat., Bd. lxxxix).

691. On Post-Chroming and Post-Osmication in General.-By soaking tissues in $\mathrm{K}_{2} \mathrm{Cr}_{2} \mathrm{O}_{7}$, with or without $\mathrm{CrO}_{3}$, one produces 
stainab'e compounds of cell proteins and lipoids which are not easily dissolved out by alcohol and a clearing oil. One may fix in almost any mixture not containing alcohol; chloroform and acetic acid. Wash the tissue in aq. dest. for a short time (say half an hour or less), cut into small pieces, and transfer to 3 per cent. potassium bichromate for several days, and then to 1 or 2 per cent. $\mathrm{OsO}_{4}$ for a day. Wash under tap overnight and stain in Heidenhain or an Altmann. Thus tissues or embryos fixed in special formol-chrome, corrosive formol, chrome-corrosive, and other mixtures which one has found most suitable for one's purpose, may be post-chromed, or post-osmicated as well. Schridde's and Murray's methods (above) include both post-chroming and post-osmication.

692. Kopsch's Osmium Tetroxide Method (Sitzungberg. $d . k$. preuss. Akad. d. Wiss. zu Berlin).-Osmium tetroxide solution will fix both fats and lipoids, and proteid substances. As has been mentioned above, the various cell inclusions, such as mitochondria, Golgi apparatus, yolk and fat, are nearly älways mixtures of different quantities of several definite substances, and consequently will reduce the osmic solution in varying degrees of intensity. Kopsch methods are somewhat capricious, but one gets results unequalled by other methods ; for chrome-osmium, or chrome-formol, followed by iron hæmatoxylin, or Altmann, generally will not demonstrate the Golgi apparatus (except in male germ-cells), while the Kopsch methods preserve and demonstrate Golgi apparatus, mitochondria, yolk, fat and chromatin structures, and occasionally neurofibrils of embryos.

For this method dissect out organs, and cut tissue into small pieces; dip these quickly into aq. dest. to remove blood or cell detritus from surface, and then transfer to a small glass-stoppered or glass-covered capsule of 2 per cent. $\mathrm{OsO}_{4}$. Leave in a darkened cupboard for two weeks (fourteen days) at room temperature. Wash in running water for several hours, dehydrate, embed in hard wax; section about $3 \mu$. Mount unstained, or stain chromatin in safranin or methyl-blue eosin. Unsaturated fats black, others yellowish, Golgi apparatus, and sometimes mitochondria, black.

This method succeeds for mollusc and many invertebrate and vertebrate tissues, but the following Mann-Kopsch method is generally superior. Note that a trace of chromic acid, potassium bichromate, or platinum chloride, in the $\mathrm{OsO}_{4}$ solution will inhibit the blackening of the Golgi apparatus, but not of fat. See also $\S 776$. 
693. The Mann-Kopsch Method (Weigl, Bull. Acad. Scien. Cracovie, 1912; Hirschler, Arch. mikr. Anat. 89; Gatenby, Journ. R. Micr. Soc., 1919). For a study of cell structure, and in general cytology, the Mann-Kopsch method gives invaluable results. It is an alternative to the formalin-silver nitrate techniques of Golgi, Cajal or Da Fano, but in addition preserves fatty substances.

The Mann-Kopsch technique in itself is easy to work, but the subsequent steps in staining are often extremely difficult. The ordinary Kopsch technique may cause extreme shrinkage, and is not generally so specific. First fix in Mann's osmo-sublimate fluid (§ 71) for from one quarter-hour to two or three hours or more. Pieces to be fixed must be small (not exceeding a centimetre in diameter) and should only be left in the osmo-sublimate long enough to complete the penetration of the fluid. For an insect ovary, or small invertebrate, one half-hour is sufficient; for solid tissues like nerve, longer is necessary. These times must be ascertained experimentally. After fixation the pieces are washed in two changes of distilled water for half an hour or less, according to the size of the tissue and its accessibility to the water. The pieces are transferred to a glass-stoppered bottle containing just enough 2 per cent. $\mathrm{OsO}_{4}$ in aq. dest. to cover them. Then they are left in a cupboard at room temperature, for at least ten days, and preferably two weeks. Every few days the bottle should be examined to see whether the $\mathrm{OsO}_{4}$ is evaporating, or whether it has completely disintegrated. Should either have happened the pieces should be washed quickly in aq. dest., and new $\mathrm{OsO}_{4}$ solution added. It should be noted, however, that the osmic solution nearly always becomes slightly dark, but not until it has gone black or no longer smells of $\mathrm{OsO}_{4}$ should new liquid be added. When the right period has elapsed the objects are taken out of the osmic, and preferably washed for several hours in running water before transference to 50 per cent. alcohol. They are upgraded and embedded in hard paraffin. Sections to be cut from 3 to $6 \mu$. They are stuck on the slide with albumen and water in the usual way and dried overnight. One of the slides is taken, the wax removed in xylol, and it is mounted in xyol balsam. Examination of this slide will enable one to ascertain to what extent the process has acted successfully. In completely successful preparations the Golgi apparatus, yolk and fat alone are blackened, while nuclear organs, mitochondria and cytoplasm are stained in shades of yellow and greenish brown. Having studied this untreated slide, and noted the extent of the 
blackening effect of the $\mathrm{OsO}_{4}$, one may then proceed to make experiments. Several alternative methods may be tried :-

(a) The blackening may be extracted step by step in turpentine, and the appearance of the cell granules studied at intervals.

(b) If the mitochondria are not stained black by the $\mathrm{OsO}_{4}$, one may proceed directly to Altmann's method (but preferably after cautious treatment in $\cdot \mathbf{1 2 5}$ per cent. permanganate of potash).

(c) The nuclear structures may be stained in safranin, crystal violet, or acid fuchsin. The sections are brought down to distilled water and transferred to watery solutions of the dye. A few minutes generally suffice to stain the nuclei.

We find that in successtul Mann-Kopsch preparations especially of Invertebrata, the mitochondria do not generally become black, but are either unstained or go yellowish. In many, but not all, cases it will be found that where the mitochondria do become black after $\mathrm{OsO}_{4}$, the colour is more readily extracted from them than from the Golgi elements, so that a distinction can nearly always be made by the Mann-Kopsch method itself, without recourse to other methods which will generally stain mitochondria and not Golgi apparatus (Regaud, Flemming, as described in $\$ 679$ ). Among the most useful differentiation or extraction methods after Mann-Kopsch, turpentine is probably the best. The wax is removed from the sections on the slide by means of xylol, and the slide is transferred to a jar of turpentine. After about half a minute the section is examined under a $\frac{1}{6}$ th.inch objective, and the effect of the turpentine is noted; one sometimes finds that the black colour in fat globules and yolk spheres is extracted before a quarter of an hour has elapsed, while the Golgi apparatus retains its black condition. In most cases it is therefore possible to distinguish between yolk and fat on the one hand, and the Golgi apparatus on the other.

694. Mann-Kopsch-Altmann Combination (GATEnby, Journ. Roy. Micr. Soc., 1921).- -If examination of the first Mann-Kopsch section showed that the Golgi apparatus was blackened, and the mitochondria were either not stained or only straw or light-brown coloured, one may proceed directly to the Altmann stain. Should the examination show that the mitochondria as well as the Golgi apparatus have become blackened, the sections must be extracted in turpentine in an endeavour to remove the blackening from the mitochondria. If the latter treatment does not succeed properly the only course is to make new Mann-Kopsch preparations, allowing less time in the $\mathrm{OsO}_{4}$, say seven or eight days instead of the two weeks.

When one has succeeded in procuring sections in which the Golgi apparatus alone is blackened, it is possible to stain in Altmann's aniline acid fuchsin and picric acid method, so that the mitochondria (and nucleoli) become red, the Golgi apparatus is black and the ground cytoplasm yellowish. The Mann-Kopsch sections are 
brought down to distilled water, and cautiously treated in a 5 to 125 per cent. solution of potassium permanganate, in order to recover the staining properties of the tissue. A very short time suffices. Wash in water; then stain ( $(680)$.

An important part of the technique is to ascertain the optimum length of time to leave the tissue in the Mann's fluid. Examples are as follows :-

(1) Saccocirrus (entire) overnight in Mann.

(2) Cavia testis about five hours.

(3) Chick embryos about one quarter-hour.

The Mann-Kopsch technique can be used in combination with the Kull staining method ( $\S 681)$. We find that the cells are rather liable to overstain in the toluidin blue, which must be left on for a very short time.

Explanation.-Mann's osmo-sublimate fixes the cells successfully, bacause the $\mathrm{HgCl}_{2}$ aids penetration and the $\mathrm{OsO}_{4}$ is not so strong as to cause shrinkage. Thus, before the tissue is transferred to the Kopsch fluid $\left(\mathrm{Os}_{4}\right.$ of 2 per cent.), a complete fixation has taken place and the distorting effect of the strong $\mathrm{OsO}_{4}$ is avoided. Left for two weeks, the lipoid materials which partly form the substance of the Golgi apparatus, the unsaturated fats, and the special lipoids of the mitochondria, are all able to reduce the $\mathrm{OsO}_{4}$ in varying dogrees. The subsequent treatment of the sections by turpentine (oxidiser) introduces a further differentiation, and so the various inclusions can be distinguished. The acid fuchsin stains presumably the lipoid content of the mitochondria. See also $\S 768$.

695. Osmic Vapour Method (W. Cramer, Imp. Cancer Research Fund Report, 1919).-Choose a small glass-stoppered bottle, and place a piece of wide glass tube open at both ends, in the bottom. Arrange a piece of gauze over the top of the inner tube. Add some 2 per cent. $\mathrm{OsO}_{4}$ to the outer vessel. Objects to be fixed by the osmic vapour are placed on the gauze. All the surrounding (fatty) tissue should be removed from the organ or material to be treated; if too dry the outside of the material should be slightly wetted.

The bottle, with object suspended in the $\mathrm{OsO}_{4}$ vapour, is kept at temperature of $40^{\circ} \mathrm{C}$. for one and a half hours. Removed from bottle, the tissue is placed in 50 per cent. alcohol and upgraded and embedded in paraffin. Cut sections, mount in balsam without staining. Such wax sections may be treated in 10 per cent. hydrogen peroxide in 80 per cent. alcohol for two hours, after which they may be stained in ordinary methods (e.g. iron hæmatoxy๋lin).

GATfenby (Quart. Journ. Micr. Sci., 1920) suggests two modifications. (a) Fix as above for one and a half hours, and then transfer to 2 per 
cent. $\mathrm{OsO}_{4}$ in water at $37^{\circ} \mathrm{C}$. for several days as for $\mathrm{Kopsch}$. Then wash in water for several hours, dehydrate, embed and sectiou. Mount unstained, or eautiously treat in permanganate of potash or hydrogen peroxide and then stain in acid fuchsin (Altmann) or iron hæmatoxylin.

(b) Tissues may also be fixed as above, and then transferred to Altmann's or Champy's fluid, and subsequently stained in Altmann's fuchsin and pieric acid.

Cramer fixes wet films for about three minutes. We think that a subsequent treatment of films as in above two paragraphs should be useful. The main point to note is that substances in a tissue which might b3 dissolved out or altered by the water added to the $\mathrm{OsO}_{4}$ crystals are fixed in situ, and without the danger of alteration. This method should be of value to histologists and cytologists.

696. SJövall's Formol Osmic Acid Method (Anat. Hefte., Bd. xxx, 1906).

Material fixed in formalin, but without chrome salts or platinum chloride, may ba used for Sjövall's technique (Anat. Hefte., Bd. xxx). Fix pieces of tissue or small embryos in neutral formalin ( 5 to 20 per cent. neutralised with magnesium carbonate) for two days. Cut into smaller pieces and wash in several changes of aq. dest.

Transfer to 2 per cent. $\mathrm{OsO}_{4}$ solution for from two to fourteen days at room temperature, as for Kopsch. Wash well in water, dehydrate clear and embed. Cut sections $3 \mu$, it necessary decolourise in peroxide $(\S 695)$ and mount unstained in balsam.

This technique is eapricious, much more so than Kopseh, and depends firstly on a suitable fixation in formalin, and secondly on the right time in $\mathrm{OsO}_{4}$ : it is convenient to cut the tissue into several pieces, which are removed from the $\mathrm{OsO}_{4}$ at different intervals. At times Sjövall demonstrates mitochondria and not Golgi elements, and in all probability it is not of such value as Mann-Kopsch. We consider that the method is of use to the skilled eytologist, although it may not bs applicable for general purposes.

Note.-Corrosive-formalin $(\S 112)$ and osmicated picric ( $(100)$ may also be used as preliminary fixers before a Kopsch or Sjövall osmication. We cannot recommend the latter, as the picric acid probably introduces maceration; the former is good.

697. Champy's Iodide of Osmium Method (Journ. de l'Anat. et Phys., xlix, 1913). - Champy finds that osmium iodide prepared as below has the power of blackening fat and eertain other cytoplasmic inclusions whose identity seems doubtful, but which he ealls for the time being eatalyosomes or lyosomes.

Just bafore use prepare the following solution :-

Osmic acid 2 par cent.

Iodide of sodium 3 per cent.

1 part.

1 ",

It produces a golden yellow colour. Place relatively large pieces of tissue ( 5 to 6 millimetres) in the solution. If one takes very small pieces as for the Kopsch techniques, one gets notbing but the osmic reaction while the iodine reaction, which takes place deeper in, is masked. Leave tissue in for at least twenty-four hours, and use a good deal of the liquid 
for a few pieces of tissue. Upgrade in alcohol, pass through toluol, embed in wax. The bodies reducing the osmium iodide go black, on a pale grey background. One may subsequently stain in Altmann (mitochondria red, lyosomes black) or in iron hæmatoxylin. It is possible to distinguish between lyosomes and fat, by fixing some of the same tissue in Flemming and comparing the two preparations; or by comparing the central part of the osmium iodide preparation with the periphery where the fixation is due to the $\mathrm{OsO}_{4}$ exclusively.

I have tried this method, at present with disappointing results.

\section{Marine or Fresh Water Organisms and Techniques for the} Cell Inclusions.-- If you are going to use a formalin-chrome technique, kill the animals by adding neutral formalin to the water; if an osmic technique is to follow, kill with $\mathrm{OsO}_{4}$; wash slightly in aq. dest. in both cases and then transfer to the special fixative. See also under "Protozoa" and "Plankton."

699. The Centrifuge and Polariscope Microscope in Oögenesis Studies, etc.-In examining large differentiated cells, such as ovarian or nerve, most valuable help can be obtained by use of a powerful centrifuge. Tissues or small gonads or whole invertebrates are placed in a tube and centrifuged at high speeds (circa 3,000 revolutions) for from a quarter of an hour to one hour. The centrifuged tissues or animals are immediately divided out among several capsules and fixed by several methods which have previously been found to show the various cytoplasmic inclusions : the specifically stained layers can then be examined. We are of the opinion that no study of the inclusions during cell differentiation is complete without recourse to this method. See also Gatenby, Quart. Journ. Micr. Sci., 1920; Lillie, Biol. Bull., 1908-9.

Fauré-Fremiet (C.R. Soc. Biol., Paris, lxxxiii) attaches a small platform to the centrifuge, so that preparations on the slide, under coverslip, can be centrifuged and examined from time to time.

The polariscope microscope has proved very useful, not only for studying the musculature of small animals, but also for discriminating between various fatty and lipoid materials. In working on accumulations of masses of metaplastic substances in embryos, or in eggs and other differentiated cells, polarised light is often most helpful. The subject is dealt with in $\S 768$ by Cramer.

700. Vital Staining of the Mitochondria.-There is probably no specific intra vitam stain for the mitochondria alone, most of the so-called specific stains will tinge other bodies. E. V. Cowdry has summarised the various methods used for this purpose (Contrib. to Embryol. Carneg. Inst., Washington, viii, 1918).

Four stains (Janus green B, Janus blue, Janus black I, and diethylsafranin) will give an intensely positive reaction on the mitochondria of freshly drawn human lymphocytes. All these are Hoechst proprietary preparations;' I find the Janus green of 
Grübler is much less satisfactory. Nile blue B, Janus green G, methylen blue med., pyronin, Bismarck brown, and methyl violet $5 \mathrm{~B}$, will all tinge the mitochondria, but faintly. Cowdry claims that in dilution 1:500,000 Janus green is specific, and will not stain granules other than mitochondria: it seems certain at any rate that exceptions to its specificity for mitochondria alone are rare. Cowdry (loc. cit.), in his admirable discussion on the Janus colours (pp. 86-93), states that the specificity of these dyes is due to a diethylsafranin group. Janus black consists partly of Janus green and another substance ; so also Janus blue and grey. See also "Bayliss," $\$ 207$. For a treatment of the Benzidine dyes, see $§ 790$.

\%01. Mitochondria of Blood and Cell Smears (CowdRy, Internat. Monat. f. Anat. u. Phys., Bd. xxxi).-Janus green B, $1: 10,000$ in 0.85 per cent. $\mathrm{NaCl}$. Place a drop on a series of six or more slides : add a small amount of freshly drawn blood (etc.), and then apply coverslip. Do not attempt to mix the cells and the Janus green. The mitochondria stain rapidly, and the preparation, after ringing with vaseline, will last hours.

902. Injection of Janus Green B (Bensley, Amer. Journ. Anat., xiii, 1911).-Kill the animal (we believe coal gas seems preferable for this), inject 1:10,000 Janus green in salt solution, through left ventricle or aorta by gravity pressure ; in order to obtain a good penetration clamp up the return flow momentarily. After ten minutes' perfusion small pieces of the gland (pancreas in this case) may be removed and examined microscopically for mitochondria. When the desired depth of staining has been reached the entire gland is placed in salt solution pending further study.

\%03. Neutral Red.-Use as above for Janus green ( $\$ 700)$ in $1: 2,000$ to $1: 10,000$ solution (see $\$ \S 308,790$, and 804 ).

r04. Toxic and other Examining Media for Mitochondria of Blood, Protozoa, and Fresh Cells.-The mitochondria can be examined successfully in toxic solutions such as weak $\mathrm{OsO}_{4}$, with or without an added dye.

Meves' Victoria Green.-Add a "small" quantity of Victoria green (malachite green) to a 4 per cent. iodic acid solution. Place a drop on a slide with the cells to be examined (Anat. Anz., Bd. xxvi).

Osmicated solution of Ripart and Petit, ef. $\$ 90$, recommended to leave out the acetic acid of the original formula.

Dahlia saturated solution in 0.75 per cent. $\mathrm{NaCl}$.- Stains mito- 
chondria and Golgi apparatus (nebenkern) of male cells distinctly violet. Both methyl violet $5 \mathrm{~B}$ (Grübler) and methyl blue in aqueous solutions will stain the mitochondria. Bismarck brown gives, a less clear picture. See under these dyes in Part I.

705. Cajal, Golgi, and Da Fano Silver Nitrate Methods for the Golgi Apparatus.-For vertebrate tissue Cajal or Golgi, and for invertebrate tissue Cajal and Da Fano methods are a great aid to research on the cell. Every cytologist is recommended to master either Cajal's or Da Fano's method. These methods will be found in the neurology section $(\$ \S 844,849)$.

706. Differentiation between Cell Inclusions.-It is frequently somewhat difficult to distinguish between the various categories of cell inclusions. In this section we have provided a series of tables intended to act as a tentative guide to the interpretation of the various images got by representative cytological techniques. These tables are based on work carried out on animals of most orders, but it would be injudicious for the researcher to depend upon them implicitly, because many exceptions are met with, and the personal factor is to be taken into consideration. The use of such tables, if made with several methods and in conjunction with a careful study of the origin and morphology of any doubtful cell body, will, however, provide reliable evidence for identification. Another warning must be given-never try to ascertain the nature of granules in developing eggs without first studying the oögenesis of the animal in question. Eggs after spawning or laying are difficult objects to study by these methods, and even the most experienced worker is unable to give a valuable interpretation until he has worked at the oögenesis. It should also be remembered that there are periods in the development of the cell during which the mitochondria are often able to resist becoming dissolved in lipoid solvents, these periods are in the early spermatogonium in some animals, and during the last stages of spermatogenesis (spermateleosis) in all animals, and sometimes in large oöcytes. See also the work of Regaud, Arch. d'Anat. micr., xi.

Nota Bene.-With regard to the oil used for clearing and imbedding, it should be pointed out that all these tables are based on preparations cleared and imbedded in xylol, which occasionally tends to extract lightly osmicated fat. Vegetable oils like cedar wood oil seem to be less active in this way. I have not found that chrome-osmicated fat, or "Kopsched" fat, is extracted either by xylol or xylol-balsam. See also $§ 768$. 
70\%. Differentiation between Mitochondria and Fat (Olein, Stearin and Palmitin Mixtures especially).

\begin{tabular}{|c|c|c|c|c|c|}
\hline $\begin{array}{l}\text { Method } \\
\text { employed. }\end{array}$ & $\begin{array}{l}\text { Fresh tissue stained } \\
\text { in Herxheimer's scarlet } \\
\text { red or such alcoholic } \\
\text { fluids. }\end{array}$ & $\begin{array}{l}\text { Fixed in Flem- } \\
\text { ming with } \\
\text { acetic acid } \\
\text { examined in } \\
\text { unstained sec- } \\
\text { tions on slide. }\end{array}$ & $\begin{array}{l}\text { Kopsch or Mann- } \\
\text { Kopsch. }\end{array}$ & $\begin{array}{l}\text { Regand or for- } \\
\text { malin fixation, } \\
\text { iron hæma- } \\
\text { toxylin. }\end{array}$ & $\begin{array}{l}\text { Fresh tissue } \\
\text { stained in } \\
\text { Janus green } \\
1: 10,000 .\end{array}$ \\
\hline $\begin{array}{l}\text { Mitochon- } \\
\quad \text { dria. }\end{array}$ & $\begin{array}{l}\text { Will not stain bright- } \\
\text { ly, generally dis- } \\
\text { solved away. }\end{array}$ & $\begin{array}{l}\text { Do not show, } \\
\text { generaily dis- } \\
\text { solved away, } \\
\text { except in cer- } \\
\text { tain cases } \\
\text { wh h r e the } \\
\text { mitochondria } \\
\text { are more re- } \\
\text { sistant to } \\
\text { acetlc acld. }\end{array}$ & $\begin{array}{l}\text { Yellow or black : if } \\
\text { black, colour often } \\
\text { difficult to extract } \\
\text { in turpentine ; if } \\
\text { y ellow, c a n b e } \\
\text { stained in acid fuch- } \\
\text { sin of Altmann. }\end{array}$ & Black. & Green. \\
\hline $\begin{array}{c}\text { Fat (see } \S \\
768) .\end{array}$ & $\begin{array}{l}\text { Stains brightly. (It } \\
\text { should be noted here } \\
\text { that while Herx- } \\
\text { heimer's scarlet red } \\
\text { will not stain mito- } \\
\text { chondria it may pos- } \\
\text { sibly stain lipoids } \\
\text { other than true fat.) }\end{array}$ & $\begin{array}{c}\text { Black } \\
768)\end{array}$ & $\begin{array}{l}\text { Black : colour easily } \\
\text { extracted after a } \\
\text { few hours in turpen- } \\
\text { tlne. }\end{array}$ & $\begin{array}{l}\text { Not stained, as } \\
\text { it has been } \\
\text { dissoived out } \\
\text { by the clear- } \\
\text { ing reagent } \\
\text { (xy lol or } \\
\text { chloroform, } \\
\text { not vegetable } \\
\text { oils). }\end{array}$ & $\begin{array}{l}\text { Does not } \\
\text { stain. }\end{array}$ \\
\hline
\end{tabular}

\section{Differentiation between Golgi Apparatus and Mitochondria.*}

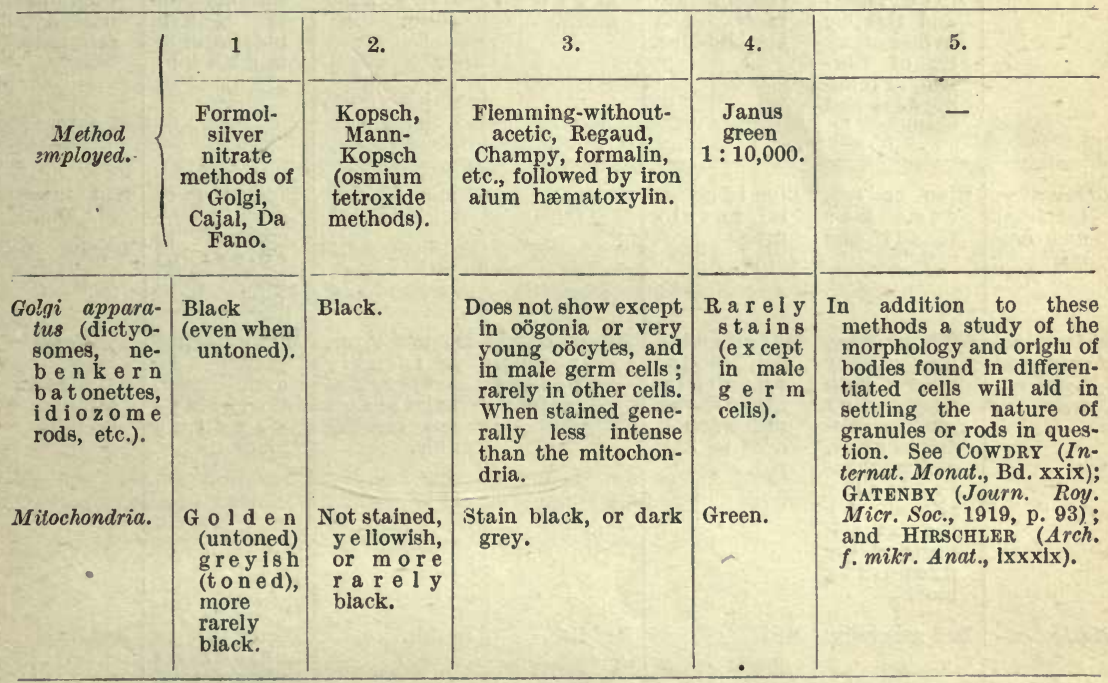

709. Mitochondria, Golgi Apparatus, Chromatin, True Chromidia, and Nucleoli.-In $\S 710$ is a table giving the main fixing and staining reactions of these bodies. It will be found to hold for a discrimination between chromatin of the nucleus and mitochondria and Golgi apparatus, especially in somatic cells; but the chief diff- 
culties arise in the study of oögenesis and gland secretion, where one meets with profound changes in the chromophility of the nucleus, and with complicated nucleoli, whose real nature is as yet undetermined ; moreover, at certain stages in the differentiation of a cell, true chromatin may stain oxyphil. Such exceptions must be taken into consideration; always ascertain carefully the origin and behaviour of a doubtful body and remember that the safest tests for chromatin lie not in staining, but in the behaviour of the doubtful material during mitosis and in the sexual or reproductive phenomena of the cell. See also Gatenby, Journ. R. Mic. Soc., 1919 and 1920.

910. Chromatin and the Inclusions.

\begin{tabular}{|c|c|c|c|c|c|c|}
\hline & 1. & 2. & 3. & 4. & 5. & 6. \\
\hline $\begin{array}{l}\text { Technique } \\
\text { employed. }\end{array}$ & $\begin{array}{l}\text { Fixation in al- } \\
\text { cohol acetic, } \\
\text { Carnoy (a t } \\
\text { le a s t on e } \\
\text { hour); Pet- } \\
\text { r u nkewitsch } \\
\text { or G il o on } \\
\text { (o e r r nlght) } \\
\text { and staining } \\
\text { in hæmatoxy- } \\
\text { lin of Ehr- } \\
\text { lich, or tolui- } \\
\text { dln-blue and } \\
\text { eosin. }\end{array}$ & $\begin{array}{l}\text { If e } t h \text { o d of } \\
\mathrm{Ch} \mathrm{a} \mathrm{m} \mathrm{p} \mathrm{y} \mathrm{-} \\
\text { Kull, } i . e \text {, fix- } \\
\text { a t i o n in } \\
\text { chrome os- } \\
\text { mium, stain- } \\
\text { ing in acid } \\
\text { fuchsin au- } \\
\text { rantia and } \\
\text { toluidin blue. }\end{array}$ & $\begin{array}{l}\text { A lt m a n u- } \\
\text { B e n s ley, } \\
\text { chrome-os- } \\
\text { mium fixa- } \\
\text { tion, stain- } \\
\text { ing in acid } \\
\text { fuchsin and } \\
\text { me t h y l } \\
\text { green. }\end{array}$ & $\begin{array}{l}\text { Chrom e-os- } \\
\text { mium fixa- } \\
\text { tion and } \\
\text { staining in } \\
\text { Ehrlich's } \\
\text { hrmatoxylin } \\
\text { or toluidin } \\
\text { blue and } \\
\text { cosin. }\end{array}$ & $\begin{array}{l}\text { Cajal's for- } \\
\text { mol uranium } \\
\text { nitrate and } \\
\text { silver method } \\
\text { for Golgi ap- } \\
\text { paratus coun- } \\
\text { terstained in } \\
\text { safranin (or } \\
\text { in methyl- } \\
\text { blue eosin or } \\
\text { m e t h y l- } \\
\text { green). }\end{array}$ & $\begin{array}{l}\text { Mann - Kopsch, } \\
\text { i.e., fixation } \\
\text { in corrosive- } \\
\text { osmic, after- } \\
\text { treatment in } \\
\text { OsO }_{4} \text { of } 2 \% \\
\text { for } 14 \text { days, } \\
\text { then stain in a } \\
\text { basic dye like } \\
\text { safranin. }\end{array}$ \\
\hline $\begin{array}{l}\text { Chromatin } \\
\text { (nucleus) } \\
\text { and chro- } \\
\text { midia. }\end{array}$ & $\begin{array}{l}\text { Blue or red, } \\
\text { et tc. } \mathrm{See} \\
\$ 821.1 \text { and } \\
666 .\end{array}$ & $\begin{array}{l}\text { Blue to green- } \\
\text { ish, r a r e l y } \\
\text { red. }\end{array}$ & Green. & Bluish. & $\begin{array}{l}\text { Blue, red or } \\
\text { green, a c- } \\
\text { cording to } \\
\text { colour of o r } \\
\text { basic stain } \\
\text { used. }\end{array}$ & $\begin{array}{l}\text { Red in safra- } \\
\text { nin, etc. }\end{array}$ \\
\hline $\begin{array}{l}\text { Mitochon- } \\
\text { dria and } \\
\text { Golgi ap- } \\
\text { paratus. }\end{array}$ & $\begin{array}{l}\text { Will not show, } \\
\text { because they } \\
\text { h a v e been } \\
\text { nearly or } \\
\text { q u it e dis- } \\
\text { solved away, } \\
\text { and morpho- } \\
\text { log i c all y } \\
\text { altered b y } \\
\text { the fat sol- } \\
\text { vents of the } \\
\text { prep paring } \\
\text { media. }\end{array}$ & $\begin{array}{l}\text { M i t ochondria } \\
\text { red, G o lg i } \\
\text { a p pa r a tus } \\
\text { rarely shows, } \\
\text { but when it } \\
\text { does so it is } \\
\text { red. }\end{array}$ & $\begin{array}{l}\text { Mitochondria } \\
\text { red, Golgi } \\
\text { a p p a ratus } \\
\text { also as for } 2 .\end{array}$ & $\begin{array}{l}\text { Do not show, } \\
\text { or fa in t ly } \\
\text { oxyphil, gra- } \\
\text { nules as such } \\
\text { not identif- } \\
\text { able. }\end{array}$ & $\begin{array}{l}\text { M i tochondria } \\
\text { golden to o } \\
\text { dark brown, } \\
\text { G olg I a p. } \\
\text { p a r a t u s } \\
\text { black. }\end{array}$ & $\begin{array}{l}\text { Yellowish to } \\
\text { black, will } \\
\text { not stain in } \\
\text { safranin. }\end{array}$ \\
\hline Nucleolus. & Red generally. & Red. & Reddish. & Bluish. & $\begin{array}{l}\mathrm{Reddish} \text { or } \\
\text { colour of } \\
\text { plasma stain. }\end{array}$ & Reddish. \\
\hline
\end{tabular}

See also remarks on nucleoli in $\$ \$ 664$ and 668 .

y11. Cytoplasmic Inclusions in Gametogenesis.-In the table below is a summary of the fixing and staining reactions of the inclusions during oögenesis and spermatogenesis. In the male germ cells the Golgi apparatus (nebenkern batonettes) show through- 
out; those of the egg can generally only be demonstrated by methods 1 and 2. The fat-methods of Daddi, Herxheimer Martinotti, and Lorrain Smith should be tried as well, and reference made to tables given below. If yolk granules contain olein or such unsaturated fat they will stain in $\mathrm{OsO}_{4}$ like fat, but by slow decolourisation as in paragraph 2 (with turpentine), their proteid basis will be noted, and they will generally be demonstrated in methods 1 and 4, while fat vacuoles disappear completely. See also the special sections on "Fat," §§ 768 to 772.

r12. Inclusions in Gametogenesis.

\begin{tabular}{|c|c|c|c|c|c|c|}
\hline & 1. & 2. & 3. & 4. & 5. & 6. \\
\hline Method. & $\begin{array}{c}\text { Cajal or Da Fano } \\
\text {. }\end{array}$ & Kopsch series. & $\begin{array}{c}\text { Chrome-osmiuin } \\
\text { and iron hæma- } \\
\text { toxylin (or Alt- } \\
\text { mann). }\end{array}$ & $\begin{array}{l}\text { Bouln and } \\
\text { corrosive } \\
\text { acetic } \\
\text { and Ehr- } \\
\text { lich's } \\
\text { hæm. }\end{array}$ & $\begin{array}{l}\text { Champy- } \\
\text { Kull. }\end{array}$ & Benda. \\
\hline $\begin{array}{l}\text { Mitochon- } \\
\text { dria. }\end{array}$ & $\begin{array}{l}\text { Either do not } \\
\text { show or greyish } \\
\text { or g o l d e n } \\
\text { brown, accord- } \\
\text { I n g a t o } \\
\text { whether sec- } \\
\text { tions have been } \\
\text { toned. }\end{array}$ & $\begin{array}{l}\text { Often will not } \\
\text { show, or faintly } \\
\text { yellowish, more } \\
\text { rarely black or } \\
\text { brown, but can } \\
\text { often be decolour- } \\
\text { ised rapidly in } \\
\text { turps. }\end{array}$ & Black (or red). & $\begin{array}{l}\text { Do not } \\
\text { show. }\end{array}$ & Red. & Violet. \\
\hline $\begin{array}{c}\text { Golgi ap- } \\
\text { paratus. }\end{array}$ & Black. & $\begin{array}{l}\text { Black, and resists } \\
\text { decolourisation in } \\
\text { turps longer than } \\
\text { mitochondria, fat } \\
\text { or yolk. }\end{array}$ & $\begin{array}{l}\text { Rarely shows, } \\
\text { when it does, } \\
\text { black (or red), } \\
\text { or not stained. }\end{array}$ & $\begin{array}{c}\text { Does not } \\
\text { show. }\end{array}$ & $\begin{array}{l}\text { Rarely } \\
\text { shows, If } \\
\text { so, red. }\end{array}$ & $\begin{array}{l}\text { Rarely } \\
\text { shows, if } \\
\text { so, violet. }\end{array}$ \\
\hline $\begin{array}{l}\text { Yolk gra- } \\
\text { nules. }\end{array}$ & $\begin{array}{l}\text { Either will not } \\
\text { show, greyish } \\
\text { or g o l d e n } \\
\text { brown, accord- } \\
\text { ing as to toning. }\end{array}$ & $\begin{array}{l}\text { Yellowish, or black } \\
\text { easily decolour- } \\
\text { ised In turps. }\end{array}$ & $\begin{array}{l}\text { May or may not } \\
\text { go black (or } \\
\text { unstained), very } \\
\text { rarely red. }\end{array}$ & $\begin{array}{l}\text { Not } \\
\text { stalned or } \\
\text { yellowish. }\end{array}$ & $\begin{array}{l}\text { Yellowish } \\
\text { or black. }\end{array}$ & $\begin{array}{l}\text { Yellowish } \\
\text { or black. }\end{array}$ \\
\hline $\begin{array}{l}\text { Fat vacu- } \\
\text { oles. }\end{array}$ & Do not show. & $\begin{array}{l}\text { Black, easily de- } \\
\text { colo uris ed in } \\
\text { turps. }\end{array}$ & $\begin{array}{l}\text { Black in un- } \\
\text { sta ined pre- } \\
\text { paration. }\end{array}$ & $\begin{array}{l}\text { Not } \\
\text { stained, } \\
\text { washed } \\
\text { away. }\end{array}$ & Black. & Black. \\
\hline $\begin{array}{l}\text { Chromatin } \\
\text { granules. }\end{array}$ & $\begin{array}{l}\text { Do not show, but } \\
\text { may subse- } \\
\text { qu e } \mathrm{t} \text { l y be } \\
\text { stained in a } \\
\text { basic colour, } \\
\text { like m e th y } \\
\text { green or safra- } \\
\text { nln. }\end{array}$ & $\begin{array}{l}\text { Yellowish, wlll sub- } \\
\text { sequently st a in } \\
\text { In hæmatoxylin } \\
\text { or safranln. }\end{array}$ & $\begin{array}{l}\text { Black to grey } \\
\text { (or re d dis h } \\
\text { purple). }\end{array}$ & $\begin{array}{l}\text { Bluish to } \\
\text { purple. }\end{array}$ & Blue. & $\begin{array}{l}\text { Brownish } \\
\text { yellow. }\end{array}$ \\
\hline Nucleoli. & $\begin{array}{l}\text { Yellowish may } \\
\text { take s u b s e- } \\
\text { quent stain. }\end{array}$ & Yellowish. & $\begin{array}{l}\text { Black or dark } \\
\text { grey. }\end{array}$ & $\begin{array}{l}\text { Reddish, } \\
\text { or reddish } \\
\text { purple. }\end{array}$ & Red. & $\begin{array}{c}\text { Violet } \\
\text { or brown. }\end{array}$ \\
\hline
\end{tabular}

713. Plan for Cytological Research. - It is best to work at some animal - which is procurable in sufficient numbers, as frequently your first preparations are disappointing. If a vertebrate, begin with Regaud, and stain in iron alum hæmatoxylin; if an invertebrate try Flemming without acetic acid, and iron hæmatoxylin; the former method will 
give mitochondria and yolk; zymogen but not fat; the latter will give all these. Champy-Kull and Bensley-Cowdry or Altmann methods are next worth trying. Mann-Kopsch and Sjövall methods may present difficulties, but again one might succeed at the first trial : if invertebrate tissue, the Mann-Kopsch method will be best, if vertebrate, the Cajal or Da Fano formalin-silver nitrate methods are most indicated for a study of the Golgi apparatus.

If your material is limited to a small amount, the Champy-Kull (or Bensley-Cowdry) and the Mann-Kopsch methods are recommended: successful preparations by both these methods will enable you to make an almost complete study (sometimes of the chromosomes and) of the cytoplasmic inclusions of every kind-excepting glycogen.* Recommended in the third place is a formalin-silver nitrate method-Cajal or Da Fano. See also Gatenby, Quart. Journ. Micr. Science, lxiv, 1920, p. 296, and E. V. Cownry, Contrib. Embryology, Carneg. Inst., Washington, viii, p. 59.

Addendum: SAguchi (Amer. Jour. Anat., Nov. 15th, 1920) finds that the Golgi apparatus of acinus cells of the frog pancreas is brought into evidence by Cajal's uranic silver nitrate method, and also by the Weigl, Kopsch, and Sjövall methods, but that in islet cells, the former method exhibits some other sort of apparatus. See also SAGUCHI, ibid., vol. $26,1920$.

* Shun Ichi Ono (Anat., Anthrop. Ass. of China, 1920) finds that osmicated mitochondrial fixatives preserve glycogen, which can be stained in Best's carmine and iron hæmatoxylin, the mitochondria (grey black) and the glycogen (reddish) showing side by side. 


\section{CHAPTER XXVII.}

\section{TEGUMENTARY ORGANS.}

714. Epithelium.-Both for surface views and for sections good results are obtained by the nitrate of silver method, the methylen blue method, the perchloride of iron and pyrogallol method of the Hoggans, $\S 375$, the osmic acid and pyrogallol process, $\S 374$, and by iron-hoematoxylin.

For the purpose of separating the epidermis from the corium, Loewy (Arch. mik. Anat., xxxvii, 1891, p. 159) recommends macerating for twenty-four to forty-eight hours, at a temperature of about $40^{\circ} \mathrm{C}$., in 6 per cent. pyroligneous acid. Acetic acid of $\frac{1}{3}$ per cent. (Philippson) is also good. Minot (Amer. Nat., xx, 1886 , p. 575) macerates embryos for several days in 0.6 per cent. salt solution, Mrtrophanow (Zeit. wiss. Mik., v, 1888, p. 573) for a quarter of an hour in 3 per cent. nitric acid, then one hour in one-third alcohol, and, if need be, twenty-four in stronger alcohol.

Mayer (Lotos, 2, xii, 1892) exposes the cornea or membrana nictitans of Rana, Bufo, and Mus for half a minute to the vapour of acetic acid, and then puts it into 0.5 per cent. salt solution.

For ciliated epithelium see the methods of Engelmann under "Mollusca."

715. Intercellular Bridges (and Canals), Prickle Cells.-See IDE, in La Cellule, iv, 1888, p. 409, and v, 1889, p. 321 ; also Kolossow, Arch. mik. Anat., lii, 1898,-p. 1. Kolossow used an osmic-acidtannin stain, § 374 .

See also Flemming, Anat. Hefte, 1 Abth, vi, 1895, p. 1.

Besides maceration, impregnation may be useful ; Mrtrophanow (Arch. Anat. Phys., Phys. Abth., 1884, p. 191) has used gold chloride.

Unna (Monatsschr. prakt. Derm., xxxvii, 1903, p. 1) has described a highly complicated process with Wasserblau and orcein, see Zeit. wiss. Mik., xxi, 1904, p. 68.

716. Plasma-fibrils of Epithelium.-Kromayer's process (Arch. mik. Anat., xxxix, 1892, p. 141) is as follows: Sections are stained for five minutes in a mixture of equal volumes of anilin -water 
(§ 286) and concentrated aqueous solution of methyl violet 6 B. They are well washed in water and treated with solution of iodine in iodide of potassium until they become blue-black (one to thirty seconds). They are again washed with water, dried with blottingpaper, and treated with a mixture of 1 volume of anilin to 2 volumes of xylol until sufficiently differentiated, when they are brought into pure xylol. Very thin sections will require more xylol in proportion to the anilin, viz. $1: 3$ or $1: 4$; thicker ones may require more anilin, viz., $3: 5$ or $3: 3$. Gentian or Krystallviolett will do instead of methyl violet, but not quite so well. See also EHrmanN and Jadassohn, Arch. Dermatol. u. Syphilis, 1892, 1, p. 303 ; Zeit. wiss. Mik., ix, 1893, p. 356 ; Herxheimer, Arch. mik. Anat., liii, 1899 , p. 510 ; and Rosenstadt, ibid., lxxv, 1910, p. 659 (takes the differentiating mixture much weaker in anilin).

UnNa (Monatsschr. prakt. Derm., xix, 1894, p. 1 and pp. 277 et seq.; Zeit. wiss. Mik., xii, 1, 1895, pp. 61, 63) has given a whole series of methods, from which the following are some extracts.

(1) Wasserblau-OrCein.-Stain sections for ten minutes in a neutral aqueous 1 per cent. solution of Wasserblau, rinse and stain for five or ten minutes in a neutral alcoholic 1 per cent. solution of Grübler's orcein. Dehydrate, clear, and mount in balsam. This may be varied as follows:

(a) Ten minutes in the Wasserblau and thirty minutes or more in the orcein.

(b) Take for the second stain an acid solution of orcein.

(c) Stain for only one minute in the Wasserblau, but for thirty or more in the neutral orcein.

(2) Stain for half an hour or more in a strong solution of hæmalum, rinse, stain for half a minute in a saturated aqueous solution of picric acid, and dehydrate for thirty seconds in alcohol containing 0.5 per cent. of picric acid.

(3) Hæmalum for two hours, neutral orcein as above for ten to twenty minutes.

More recently UNNA advocates the process mentioned last section.

See also Ranvier, Arch. Anat. Mikr., iii, 1899, p. 1.

71\%. Keratohyalin. - The keratohyalin granules of the cells of the stratum granulosum are soluble in mineral acids, and can be digested in pepsin. They can be stained with picro-carmine, alum hæmatoxylin, van Gieson's mixture, or Unna's Wasserblau-orcein, last §. FIck (Centralb. allg. Path., xiii, 1902, p. 987 ; Zeit. wiss. Mik., xx, 1903, p. 222) stains sections of alcohol material for three 
to four minutes in concentrated aqueous solution of Kresylechtviolett, differentiates in alcohol, clears in xylol, and mounts in balsam.

See also Unna, Monatsschr. prakt. Derm., xx, 1895, p. 69 ; the article "Haut" in the Encycl. mik. Technik.; and UNNA and GolodeTz, Monatsschr. prakt. Derm., xlix, 1909, p. 95 ; LAfFont, Bibl. Anat., 1909, p. 209.

For Triohohyalin, see Gavazzenr, Monatsschr. prakt. Derm., xlvii, 1908, p. 229.

718. Eleidin.-To demonstrate the stratum granulosum and the eleidin granules Ranvier (Arch. Anat. Micr., iii, 1899, p. 1) hardens with alcohol, stains with picro-carmine, and treats with lime-water. The cells swell and show up the granules, which do not change. See loc. cit., other methods for the study of skin.

BuzzI (see Encycl. mik. Technik., article "Haut.") stains sections for a few minutes in a watch-glassful of water with 2 to 3 drops of 1 per cent. Congo red. Similarly Weidenreich, Arch. mik. Anat., lvii, 1901, p. 583. Other authors recommend nigrosin, or Wasserblau, or orcein.

See also Joseph, "Dermatohist. Technik," Berlin, 1905, and Dreuw, Med. Klinik, Berlin, 1907, Nos. 27 and 28.

For Cholesterin see GoLODETZ and UNNA, Monatsschr. prakt. Derm., xlvii, 1908, p. 1.

719. Horn, Hair, and Nails.-The elements of hairs and nails may be isolated by prolonged maceration in 40 per cent. potash solution, or by heating with concentrated sulphuric acid. See also von Nathusius, Zool. Anz., xv, 1892, p. 395.

Horny tissues stain well in safranin or gentian violet (REINKE, Arch. f. mik. Anat., xxx, 1887, p. 183 ; ERnst, ibid., xlvii, 1896, p. 669 ; RABL, ibid., xlviii, 1896, p. 489).

UNNA (op. cit. last section, p. 598) stains the tyrosin-bearing keratin in sections of skin for a few seconds or minutes in a mixture of 5 parts of Millon's reagent, 5 of water, and 1 of glycerin, treats shortly with nitric acid of 25 per cent., and mounts in balsam.

720. Skin-nerves and Nerve-endings.-Impregnate with gold chloride. See Chapter XVII., especially $\S 365$.

721. Tactile Corpuscles. - See $\S \S 364-366$.-Gold methods are indicated. See also Ranvier, Traité, p. 919 ; Langerhans, Arch. mik. Anat., 1873, p. 730 ; Kultschizky, ibid., 1884, p. 358; and SuIRnow, Intern. Monatsschr. f. Anat., efc., x, 1893, p. 241, who recommends, besides the gold method of Löwit, the rapid bichromate of silver method of Golgi. 
122. Corpuscles of Herbst and Corpuscles of Grandry.-DoGIEI. (Arch. Anat. u. Entwickel., 1891, p. 182) has used the methylen blue method. Four per cent. solution of methylen blue, warmed to $40^{\circ} \mathrm{C}$., is injected into blood-vessels of the heads of ducks or geese ; pieces of skin are removed from the beaks, sectioned in pith, and the sections brought on to slides and moistened with aqueous or vitreous humour from the animal and left for ten to thirty minutes exposed to the air, then brought into picrate of ammonia, and treated as described § 343. GEBERG (Intern. Monatsschr. Anat., x, 1893, p. 205) made use of a method of ARnstein, according to which pieces of skin are put for twenty-four hours into lime-water, the horny layer removed, the pieces treated for five minutes with 0.25 per cent. gold chloride, reduced in water, and the precipitate that forms on them removed by putting into $0 \cdot 25$ per cent. cyanide of potassium and brushing.

NowaK (Anat. Anz., xxxvi, 1910, p. 217) takes UnNA's Orceïnwasserblau mixture (Wasserblau O.D., 1 part, orceïn 1 , acetic acid 5, glycerin 20, alcohol 50, water 100) and adds to it 1 part more of orceïn. To 10 c.c. of this he adds at the moment of using 10 c.c. of 1 per cent. solution of eosin in alcohol of 80 per cent. and 3 c.c. of 1 per cent. solution of hydroquinon. Stain for five to ten minutes. rinse, stain for ten minutes in 1 per cent. aqueous solution of safranin, wash, treat for thirty minutes with 0.5 per cent. solution of bichromate of potash, dehydrate and mount.

Similarly Dogies, Folia Neurobiol., iv, 1910, p. 218 (also employing Bielschowsky's neurofibril method).

123. Corpuscles of Meissner and of Krause (Cornea and Conjunctiva).-Dogiel (Arch. ff. mik. Anat., xxxvii, 1891, p. 602, and xliv, 1894 , p. 15) employs the methylen blue method; for details see previous editions.

See also Longworth's methods, Arch. mik. Anat., 1875, p. 655.

724. Similar Objects. - Papillæ Foliatæ of the Rabbit, HermanN, see Zeit. wiss. Mik., v, 1888, p. 524; ARNSTeIN, ibid., xiii, 1897, p. 240. Olfactive Organs of Vertebrates, DoGIEL, Arch. mil. Anat., 1887, p. 74. Organs of a "Sixth Sense " in Amphibia, Mitrophanow, Zeit. wiss. Mik., v, 1888, p. 513 (details as to staining with "Wasserblau," for which see also Biol. Centralb., vii, 1887, p. 175). Nerve-endings in Tongue of Frog, Fajerstain, Arch. de Zool. expér. et gén., vii, 1889, p. 705. Tongue of Rabbit, von LeNHosseK, Zeit. wiss. Mik., xi, 1894, p. 377 (Ramón y Cajal's double Golgi method).

925. Cornea.-There are three chief methods--the methylen blue, the silver, and the gold method. 
For the methylen blue method see particularly $\$ 345$.

Negative images of the corneal cells are easily obtained by the dry silver method (KLEIN). The conjunctival epithelium should be removed by brushing from a living cornea, and the corneal surface well rubbed with a piece of lunar caustic. After half an hour the cornea may be detached and examined in distilled water.

In order to obtain positive images of the fixed cells the simplest plan (RANVIER) is to macerate a cornea that has been prepared as above for two or three days in distilled water. There takes place a secondary impregnation.

The same result may be obtained by cauterising the cornea of a living animal as above, but allowing it to remain on the living animal for two or three days before dissecting it out, or by treating a negatively impregnated cornea with weak salt solution or weak solution of hydrochloric acid (His).

But the best positive images are those furnished by gold chloride. RANVIER prefers his lemon-juice method. It is important that the cornea should not remain too long in the gold solution, or the nerves alone will be well impregnated.

Zawarsin (Arch. mik. Anat., lxxiv, 1909, p. 116) removes the membrane of Descemet for study in the following manner. A cornea, fixed in sublimate, is dissected out and put for some hours into a mixture of alcohol and ether. Then collodion of 4 per cent. is poured on to the inner surface, and after some time a layer of collodion with the membrane attached can be peeled off, and the collodion removed from the tissue by a mixture of alcohol and ether.

See also Rollett, in Stricker's Handb., pp. 1102, 1115, or previous editions; TARTuferi, Anat. Anz., v, 1890, p. 524, or previous editions; Craccio, Arch. ital. Biol., iii, p. 75 ; and Renault, C. R. Acad. Sc., 1880, p. 137.

"26. Crystalline.-GerhardT (Zeit. wiss. Mik., xiii, 1896, p. 306) hardens the lens for one or two days in 4 to 10 per cent. formalin; it is then easily dissociated with needles into its fibres.

RABL (Zeit. wiss. Zool., lxv, 1898, p. 272) fixes the enucleated eye for half an hour in his platinum chloride or picro-sublimate, $§ \$ 75$ and 70 , divides it at the equator, and puts the anterior half back for twenty-four hours into the fixative.

For Maceration you may use sulphuric acid, $\S 533$.

See also Robinski, Zur Kenntniss d. Augentinse, Berlin, 1883. 


\section{CHAPTER XXVIII.}

\section{MUSCLE AND TENDON (NERVE-ENDINGS).}

\section{Striated Muscle.}

72\%. Muscle-cells. - For these and allied subjects see, inter alia, Behrens, Kossel, und SchiefFerdecker, Das Mikroskop, etc., vol. ii, pp. 154-161; and Schäfer, Proc. Roy. Soc., xlix, 1891, p. 280.

Iron hæmatoxylin gives very fine images of striped muscle, and so does Mallory's phospho-tungstic.

For dissociation methods see $\$ \S 512-545$.

To isolate the sarcolemma Solger (Zeit. wiss. Mik, vi, 1889, p. 189) teases fresh muscle in saturated solution of ammonium carbonate.

728. Nerve-endings - the Methylen Blue Method.-For BIEDERMANN's procedure for the muscles of Astacus see $\S 342$ (see also Zeit. wiss. Mik., vi, 1889 , p. 65). After impregnating as there directed the carapace should be opened, and the muscles exposed to the air in a roomy moist chamber for from two to six hours.

For Hydrophilus piceus, BIEDERMANN proceeded by injecting 0.5 c.c. of methylen blue solution between the ultimate and penultimate abdominal rings, in the ventral furrow, and keeping the animals alive in water for three to four hours, then opened the thorax by two lateral incisions, and removed the muscles of the first pair of legs and exposed them to the air for three or four hours in a moist chamber, and finally examined in salt solution.

Gerlach (Sitzb. Akad. Wiss. München, 1889, ii, p. 125) injected frogs, either through the abdominal vein or through the aorta, with 4 to 5 c.c. of a 1 : 400 solution in 1 per cent. salt solution, and examined pieces of muscle in serum of the animal, afterwards fixing with picrate of ammonia and mounting in glycerin jelly.

The procedure of DoGIEL has been given, $\S 342$.

929. Nerve-endings - the Gold Method.-Fischer (Arch. mik. Anat., 1876, p. 365) used the method of Lowiт.

Biedermand (last section) recommends for Astacus a similar 
procedure, the preliminary treatment with formic acid being omitted, and the muscles being put for a couple of days into glycerin after reduction in the acid.

RANvier (Traité, p. 813) finds that for the study of the motor terminations of Vertebrates the best method is his lemon-juice process ( $\$ 366)$.

See also the methods of APÁTHY, $\S \S 368,371$.

7330. Nerve-endings - the Silver Method.-Ranvier employs it as follows (ibid., p. 810); Portions of muscle (gastro-cnemius of frog) having been very carefully teased out in fresh serum, are treated for ten or twenty seconds with nitrate of silver solution of 2 to 3 per 1000, and exposed to bright light (direct sunlight is best) in distilled water. As soon as they have become black or brown they are brought into 1 per cent. acetic acid, where they remain until they have swelled up to their normal dimensions. They are then examined in a mixture of equal parts of glycerin and water.

This process gives negative images, the muscular substance being stained brown, and the nervous arborescence unstained. The gold process gives positive images, the nervous structures being stained dark violet.

731. Nerve-endings - the Bichromate of Silver Method.-The rapid method of GoLGI has been used by RAMóN y CAJAL for the terminations of nerves and tracheæ in the muscles of insects. See Zeit. wiss. Mik., vii, 1890, p. 332, or fourth edition. A modification is used by Wunderer, Arch. mik. Anat., lxxi, 1908, p. 523.

732. Muscle-spindles.-See Cilimbaris, Arch. mik. Anat., lxxv, 1910 , p. 692. Principally intra vitam methylen blue, by injection through the internal carotid. For elastic fibres, Weigert's resorcinfuchsin, followed by 1 per cent. orcein acidified with $\mathrm{HCl}$.

\section{Electric Organs.}

733. Electric Organs.-RANvIER (Traité, Chap. xviii), finds that osmic acid is the only reagent that will fix properly the terminal arborisations on the lamellæ. He injects a little 2 per cent. solution under the surface of the organ, removes a small portion of it after a few minutes, and puts it into a quantity of the same solution for twenty-four hours. The electric plates may then be teased out and examined in water, and will show the stag's horn ramifications ; and the dissepiments between the columns will show the bouquets 
of Wagner. The terminal arborescence may be impregnated with silver. A portion of the surface of the organ is rubbed with lunar caustic until it appears opaque, then removed and the plates teased out in water. This gives negative images.

Or, electric plates, isolated by teasing after twenty-four hours in osmic acid as above, and kept for some days in one-third alcohol, are washed and placed on a slide with their ventral surface uppermost. They are then treated with a few drops of 0.5 per cent. solution of chloride of gold and potassium, and those which become violet are washed and mounted in glycerin. This gives positive images.

These may also be obtained by putting material fixed by osmic acid into 2 per cent. solution of bichromate of ammonia for a few weeks, then teasing, staining with alum hæmatoxylin, and mounting in damar.

Torpedo.-BALlowitz (Arch. mik. Anat., xlii, 1893, p. 460) gets the best results by the rapid Golgi impregnation.

An electric column, with about $\frac{1}{2}$ to 1 centimetre of tissue round it, is dissected out, and put for three to four days into the osmium bichromate mixture; then for one to three days into $\frac{3}{4}$ per cent. silver, cut without imbedding and mounted in xylol balsam. Impregnates all the important elements. See further, on the whole subject, Ballowitz, Encycl. mik. Techn., 1910, p. 298.

Cavalí́ (Bibl. Anat., xiii, 1904, p. 214) takes material fixed with osmic acid of 2 per cent. and impregnates it with gold by the method of NABIAS, and mounts in glycerin.

Raja.-Iwanzoff (Bull. Soc. Nat. Moscou, ix, 1895, p. 74) fixes the organ in the tail of Raja with liquid of Flemming, stains with hæmacalcium and eosin, and makes paraffin sections.

Ballowitz (Anat. Hefte, 1 Abth., vii, 1897, p. 285) finds the method of Golgi excellent for this organ. He also makes sections after fixing in saturated solution of sublimate (in sea-water), or in liquid of Flemming, and examines them in water. Methylen blue may be used, intra vitam. Gold is little good.

Gymnotus.-BALLowiTz (Encycl. mik. Technik., p. 303) fixes with Flemming, and makes sections. He also commends impregnation with gold chloride, but not the Golgi method.

Malapterurus.-BALLOWITz (ibid., p. 202) fixes with picro-sublimate, with Flemming, or with various mixtures of bichromate, sublimate, and formol, and uses gold chloride and Golgi impregnations. He macerates in liquid of Miiller or saturated aqueous solution of picric acid. 


\section{Tendon.}

734. Tendons.-Retterer (C. R. Soc. Biol., x, 1898, p. 580) fixes in equal parts of saturated solutions of sublimate and picric acid, puts for one to three days into saturated picric acid with 2 to 3 per cent. of sodium chloride, to remove the mucin, and imbeds in paraffin.

735. Union of Muscle and Tendon.-For this see ReTterer and Lelièvre, C. R. Soc. Biol., 1911, No. 12 (orceïn for twenty-four hours, followed by iron hæmatoxylin); and Schultze (Verh. phys. med. Ges. Würzburg, 1911, p. 33) (treats for a day or two with a mixture of equal parts of 2 per cent. bichromate of potash and alcohol, in the dark, then for two days with 0.5 per cent. solution of hæmatoxylin in alcohol of 70 per cent., then with Van Gieson's picro-säurefuchsin).

936. Corpuscles of Golgi (RAnvier, Traité, p. 929).-Take the tendon of the anterior and superior insertion of the gemini muscles of the rabbit. Treat it by the formic acid and gold method ( $\$ 365)$, and after reduction scrape with a scalpel, in order to remove the muscle-fibres that mask the musculo-tendinous organs.

MARCHI's methods for the tendons of the motores bulbi oculi (Archivio per le Scienze Mediche, v, No. 15).- The enucleated eyes, together with their muscles, were put for not less than three days into 2 per cent. bichromate of potash. The muscles and tendons were then carefully dissected out; stained with gold chloride and osmic acid (Golgr's method), and by the method of MaNFred, $\S 368$. Mount in glycerin. The methods only succeed completely during fine, sunny weather.

Ruffint (Atti R. Acc. Lincei Roma Rend. [5], i, 1892, p. 442) recommends the method of Fischer.

Ciaccio (Mem. R. Acc. Sci. Bologna [4], t. x, 1890, p. 301) puts tendons of Amphibia into 0.1 per cent. hydrochloric acid or 0.2 per cent. acetic acid until transparent; then for five minutes into a mixture of $0 \cdot 1$ per cent. gold chloride and 0.1 per cent. potassium chloride; then back into the acetic acid, for a day in the dark, and for two or three hours more in the sunlight. When they have become somewhat violet they are put for a day into 0.1 per cent. osmic acid, and finally mounted in glycerin acidulated with 0.5 per cent. of acetic or formic acid.

Dogrel (Arch. mik. Anat., lxvii, 1906, p. 638) stretches tendons of eye-muscles on cardboard with hedgehog spines, puts for four 
or five days into nitrate of silver of 1 to 2 per cent., reduces for a day in pyrogallic acid with formol, and imbeds in celloidin.

\section{Smooth Muscle.}

193\%. Tests for Smooth Muscle.-Picro-säurefuchsin, § 299, stains muscle yellow, connective tissue red.

Picro-nigrosin, § 325 , stains muscle yellowish, connective tissue blue.

UnNA (Encycl. mik. Technik, article "Kollagen") stains for twenty-four hours in orceïn 1 part, Wasserblau $0 \cdot 25$, alcohol 60 , glycerin 10 , water 30 , which gives muscle in a mixed tone, collagen blue, elastin reddish. See also a complicated process with methylen blue in Monctssch. prakt. Dermatol., xix, 1894, p. 533, and another with orceïn, hæmatein, säurefuchsin and picric acid.

Retterer (C. R. Soc. Biol., 1887, p. 645) fixes in 10 volumes of alcohol with one of formic acid, washes well and stains in alum carmine. Muscle red, connective tissue unstained.

938. General Structure.-WERNER (Hist. d. glatten Musculatur, Dorpat, 1894, p. 22) fixes stretched intestine or bladder in Flemming, washes well and stains in Heidenhain's chrome hæmatoxylin, § 265. For demonstrating intercellular spaces, fresh intestine is put for twenty-four hours into oil, at $37^{\circ} \mathrm{C}$., then for twelve hours into Flemming, and for four to six into chromo-acetic acid.

739. Isolation of Fibres.-GAGE's methods, see $\S \S 518,528$, and 536.

Moвius, muscle of Cardium, see $\S 527$.

Ballowitz, muscle of Cephalopoda, see Arch. mik. Anat., xxxix, 1892 , p. 291.

Schultz (Arch. Anat. Phys., Phys. Abth., 1895-6, p. 521) puts muscle of Vertebrates for twenty-four hours into 10 per cent. nitric acid, rinses with water, and brings pieces for six to eight days (in the dark at first) into a mixture of equal parts of $\frac{1}{20}$ per cent. osmic acid and $\frac{1}{5}$ per cent. acetic acid, teases and mounts in glycerin.

For smooth muscle of Vermes, see AрÁtнy, Zeit.f. wiss. Mik., x, 1893 , pp. 36,319 , and $\S 530$, ante.

740. Iris.-Dogiel (Arch. mik. Anat., 1886, p. 403) puts the anterior half of an enucleated eye for some days into a mixture of two parts one-third alcohol and one part 0.5 per cent. acetic acid. The iris can then be isolated, and split from the edge into an interior and posterior plate, and these stained according to the usual methods. 
See also KoganeI, Arch. mik. Anat., 1885, p. 1 ; Canfield, ibid., 1886, p. 121 ; and Dostorewsky, ibid., p. 91.

\%41. Bladder of Frog, Innervation of (WoLFF, Arch.f. mik. Anat., 1881, p. 362). - A frog is killed and a solution of gold chloride of $1: 20,000$ injected into the bladder through the anus. (If the injection flows out on removal of the syringe, tie the frog's thighs together.) Now open the frog, ligature the intestine above the bladder, and cut away the abdomen so as to have in one piece bladder, rectum and hind legs. Put this into gold solution of $1: 2000$ for four hours; the bladder is then excised, slit open, and pinned (with hedgehog spines) on to a cork (outside downwards). Place it under running water until all the epithelium is washed away. Use a camel's-hair brush if necessary. Put for twenty-four hours into gold solution of $1: 6000$. Wash in pure water, and put away in the dark " for some time" in acidulated water, and finally reduce in fresh water in daylight.

RANvier (Traité, p. 854) recommends his two gold processes, the liquids being injected as above.

GrǗNSTEIN (Arch. mik. Anat., 1899, p. 1) injects. 1 per cent. methylen blue in normal salt solution through the vena abdominalis, and after twenty to thirty minutes excises the bladder and exposes to the air. Fix the stain with picrate of ammonia and mount in glycerin with the same (§ 343$)$. 


\title{
CHAPTER XXIX.
}

\author{
CONNECTIVE TISSUES.
}

\section{Connective Tissue.}

742. General Stains for Connective Tissue.-Connective tissue, elastic tissue, and smooth muscle are all normally acidophilous. Collagen, the distinctive element of connective tissue, absolutely requires "acid" dyes for the production of a permanent stain, whilst elastic tissue and muscle will also fix " basic" dyes. Collagen has a special affinity for Säurefuchsin and Wasserblau. Elastin has a strong affinity for acid orcein, whilst muscle has no special affinity for either, but stains energetically with picric acid.

Picro-säurefuchsin is much used and very convenient as a general differentiating stain, but not to be recommended for cytological detail. See Schaffer, Zeit. wiss. Zool., lxxx, 1905, p. 176.

E. and T. SAvini recommend Benda's Picro-Säurefuchsin, § 299.

EHRLICH-BIond mixture gives connective tissue red, but smooth muscle redder still.

UNNA's Wasserblau-orcein for distinguishing connective tissue and muscle has been given, $\S 676 . \rho^{2}$ It works after all fixatives. Stain long, and dehydrate preferably with acid alcohol.

Freeborn (Amer. Mon. Mic. Journ., 1888, p. 231) recommends (for sections) picro-nigrosin, made by mixing 5 c.c. of 1 per cent. aqueous solution of nigrosin, with 45 c.c. of aqueous solution of picric acid. Stain for three to five minutes, wash with water, and mount in balsam. Connective tissue blue, nuclei blackish, the rest yellowish.

RAḾ́N Y CAJAL's picro-indigo-carmine gives connective-tissue fibres dark blue, with red nuclei.

S. MAyer (Sitzb. k. Akad. Wiss., lxxxv, 1882, p. 69) recommends for staining fresh tissue Violet $\mathrm{B}, \S 330$. Elastic fibres and smooth muscle also stain, but of different tints.

Dubreuil (C. R. Ass. Anat., vi Sess., 1904, p. 62) uses a mixture of 23 volumes 1 per cent. picric acid and 2 volumes 1 per cent. methyl blue-with a foregoing stain with carmalum or safranin.

For RanvieR's method of artificial œdemata for the study of areolar tissue, see his Traité, p. 329. 
743. UnNA's Orcein Method.-(Encycl. mik. Technik, 1910, p. 250). Sections are stained for ten minutes in Grübler's polychrome methylen blue. They are then washed with water, mopped up, and brought for fifteen minutes into a neutral 1 per cent. solution of orcein in absolute alcohol, rinsed in pure alcohol, cleared in bergamot oil, and mounted. Collagenous ground-substance dark red, muscle bluish, elastic fibres sometimes dark red. Material may be fixed in almost any way except with nitric or picric acid, formol, or liquids of Müller and Hermann.

"744. UnNA's Methylen-blue + Säurefuchsin (UnNa, in Encycl. mik. Technik, 1910, p. 247). Stain for two to five minutes in polychrome methylen blue solution (Grübler). Wash and stain for ten to fifteen minutes in " $(0.5$ per cent.) Säurefuchsin + (33 per cent.) tannin-mixture (Grübler)." Water, alcohol, essence, balsam. Collagen, protoplasm, and muscle red, nuclei and keratin blue. On Flemming material, ełastin blue. Liquids of Hermann and Erlicki, formol and copper fixatives incompatible.

745. UnNa's Safranin + Wasserblau (ibid.). Ten minutes in 1 per cent. safranin. Wash. Ten to fifteen minutes in " 1 per cent.) Wasserblau + (33 per cent.) tannin mixture." Wash. Stains in opposite colours to the last. Formol and liquid of Hermann contra-indicated for fixing.

746. Flemming's Orange Method is said to give a very sharp differen. tiation of developing fibrils.

747. MaLLORY (Zeit. wiss. Mik., xviii, 1901, p. 175) stains sections of sublimate or Zenker material for a few minutes in Säurefuchsin of $0 \cdot 1$ per cent. mordants for a few minutes in 1 per cent. phosphomolybdic acid and stains for two to twenty minutes in anilin blue 0.5 grms., Orange G. 2, oxalic acid 2, and water 100. His phosphotungstic hæmatoxylin stains connective tissue sharply, but does not differentiate it sufficiently from elastio tissue and muscle.

748. For the complicated procedure of HoRNowskI see ibid., xxvi, 1909, p. 138.

749. For DelaMare's mixture or orcein, hæmatoxylin, Sãurefuchsin and picric acid see Verh. Anat. Ges., xix, 1905, p. 227.

750. MASson (O. R. Soc. Biol., lxx, 1911, p. 573), stains first in hæmalum, then in eosin, and then for a. few minutes in 1 per cent. solu . tion of saffron in tap water (made by boiling). Connective tissue, bone, and cartilage, yellow.

751. Benecke's stain for fibrils (Verh. Anat. Ges., vii, 1893, p. 165) is essentially that of KROMAYER, $\$ 716$. 
752. Bielschowsky's Silver Methon (post, under "Neurofibrils") has been used for connective-tissue fibrils. SNessarew (Anat. Anz., xxxvi, 1910, p. 401) employs it as follows : Tissue is hardened in neutral formol and sectioned with a freezing microtome. The sections are put for at least four days into iron alum of 2.5 to 10 per cent., changed daily. They are then silvered for thirty-six to forty-eight hours in nitrate of silver of 10 per cent., then treated with the oxide bath and reduced in formol of 20 per cent. Collagen fibres grey, but fine connective networks black, nerve fibres unstained or only weakly stained.

See also MAREsch, Zeit. wiss. Mik., xxiii, 1906, p. 356 ; STudNICKa, ibid., p. 416 ; Zimmermann, ibid., xxv, 1908, p. 10 ; Levi, Monit. zool. Ital., 1908, p. 290 ; HeINRICH, Arch. mik. Anat., lxxiv, 1909, p. 786 (dentine): Insabato, Arch. Ital. Anat. Emb., viii, 1909, p. 375 (silvers Flemming material); Athanasiu and Dragoru, C. R. Acad. Sci., cli, 1910, p. 551 (Ramón y Cajal's silver process, with alcohol fixation).

\section{Elastic Tissue.}

753. Elastic Tissue, Generalities.-Elastic fibres have a great affinity for osmium, staining with much more rapidity than most other tissue elements. They are not changed by caustic soda or potash. They are normally acidophilous, but are easily rendered artificially basophilous by means of chromic acid or other mordants, and then stain with great energy with basic dyes. Hence a group of stains of which those of Lustgarten and Martinotti are types. They have a natural affinity for orcein, whence stains of the TaenzerUnna type.

For a review of the older methods of BaLzer, UnNa, Lustgarten, and Herxheimer, see the paper by G. Martinottr, in Zeit. wiss. Mik., iv, 1887, p. 31 ; also Encycl. mik. Technik., art. "Elastin."

\section{Victoria Blue (LUSTGARTEN). See § 289.}

755. Safranin (G. MartinotTi, loc. cit., § 753).-Fix in a chromic liquid, wash, stain for forty-eight hours in strong (5 per cent. Pfitzner's) solution of safranin, wash, dehydrate, clear, and mount in balsam. Elastic fibres black.

The staining will be performed quicker if it be done at the temperature of an incubating stove (GRIESBACH, ibid., iv, 1887, p. 442). See also FerRIa (ibid., v, 1888, p. 342).

See also Mibelli, Mon. Zool. Italiano, 1, p. 17, or Zeit. wiss. Mile., vii, 1890 , p. 225 (the report in Journ. Roy. Mic. Soc., 1890, p. 803, is vitiated by a misprint). Other basic dyes have been recommended. 
756. Kresofuchsin (RöтнIG, see § 289).

75\%. Orcein.-This method is due to TAEnzer, and as modified by UNNA is known as the TAENZER-UNNA method, see third edition, or Monatssch. prakt. Dermatol., xii, 1891, p. 394.

UnNA's Modified Orcein Method (Monatssch. prakt. Dermatol., xix, 1894, p. 397 ; Zeit. wiss. Mik., xii, 1895, p. 240).-Grübler's orcein 1 part, hydrochloric acid 1 part, absolute alcohol 100 parts. Stain sections for thirty to sixty minutes, or for ten to fifteen at $30^{\circ} \mathrm{C}$., rinse in alcohol, clear, and mount. Elastin dark brown, collagen light brown.

See also Merk. Sitz. Akad. Wiss. Wion., cviii, 1899, p. 335 ; Pranter, ibid., xix, 1903, p. 361 (he takes 2 per cent. of nitric acid instead of the hydrochloric, and stains six to twenty-four hours) ; WoLfF, ibid., p. 488; the article "Elastin" in Encycl. mik. Technik.; and E. and T. SAvini, Zeit. wiss. Mik., xxvi, 1909, p. 34.

758. Weigert's Resorcin-Fuchsin Method (Centralb. allg. Path., ix, 1898, p. 290).- One per cent. of basic fuchsin and 2 per cent. of resorcin (or of carbolic acid) are dissolved in water. Two hundred cubic centimetres of the solution are raised to boiling-point in a capsule, and 25 c.c. of Liquor ferri sesquichlorati P. G. are added, and the whole is boiled, with stirring, for two to five minutes more. A precipitate is formed. After cooling the liquid is filtered, and the precipitate which remains on the filter is brought back into the capsule, and there boiled with 200 c.c. of 94 per cent. alcohol. Allow to cool, filter, make up the filtrate to 200 c.c. with alcohol, and add 4 c.c. of hydrochloric acid.

Wolfrum (Zeit. wiss. Mik., xxv, 1908, p. 219) adds 10 to 15 per cent. of acetone to the mixture.

Stain sections (of material fixed in any way) for twenty minutes to an hour, wash with alcohol, clear with xylol (not with an essence). Elastic fibres dark blue on a light ground.

Mrnervini (Zeit. wiss. Mik., xviii, 1901, p. 161) gives a variant with safranin instead of fuchsin.

See also Pranter, ibid., xix, 1903, p. 361 ; B. Fischer, Virchow's Arch., clxx, 1902, p. 285, or Zeit. wiss. Mik., xx, 1903, p. 40 (ehemistry of the dyes obtained by these processes, which he calls "Fuchselin," "Safranelin," etc.); Hart, Centralb. allg. Path., xix, 1908, p. 1; and Cilimbaris, Arch. mik. Anat., lxxv, 1910, p. 708.

759. Hæmatoxylin Methods.-HaRris (Zeit. wiss. Mik., xviii, 1902, p. 290) makes an "Elasthæmatein" as follows: Hæmatoxylin 0.2 grms., aluminium chloride 0.1 grms., alcohol of 50 per cent. 100 c.c., boil and add mercuric oxide 0.6 grms., filter and add 1 drop of $\mathrm{HCl}$. Keep for 
some weeks. Stain tor five or ten minutes, put into alcohol with 1 per cent. of nitric acid for one minute, then pure alcohol.

See also De Witt, Anat. Rec., i, 1897, p. 74 ; Duerck, Arch. Path. Anat., clxxxix, 1907, p. 62 ; Verhoeff, Journ. Amer. Med. Assoc., 1908, No. 11.?

MALLORY's phosphotungstic hæmatoxylin is good, but not specific.

For a hæmatoxylin and eosin stain for connective tissues see KRÜGER (Zeit.f. w. Mikr., xxxi, or Journ. R. Mier. Soc., 1914).

760. Other Methods for Elastic Tissue.-For the elastic tissue of the skin see Passarge and Krösing, Derm. Stud., xviii, 1894.

See also for staining and dissociation AGaвaBow, Arch. mik. Anat., l, 1897, p. 566 et seq.

For C. Martinotri's silver impregnation see Zeit. wiss. Mik., v, 1888, p. 521, or Arch. Ital. Biol., xi, 1889, p. 257.

Schumacher (Arch. mik. Anat., lv, 1899, p. 151) has had good results (for the spleen) with picro-nigrosin, §681.

See also $\S 733$.

761. Drew-Murray van Gieson-Nile Blue Method for Connective Tissues (and Bacteria).-Fix in formol-salt solution. Prepare paraffin (or frozen) sections. Stain one to three minutes in van Gieson's picric acid-acid fuchsin solution. Wash in aq. dest.; treat in 2 per cent. Nile blue sulphate solution in aq. dest. for from two to twenty-four hours. Wash in changes of aq. dest. till the latter is tinted pale blue. Stain again in van Gieson one to five minutes. Wash in aq. dest. till wash-water is pale yellow. Dehydrate rapidly with absolute alcohol from drop bottle. Clear quickly in xylol (not more than a minute). Differentiate in clove oil from five minutes to several hours (the longer period is for frozen sections). Wash in xylol-Canada balsam.

In successful preparations nuclear chromatin a saturated transparent blue, mast cell granules nearly black, collagen red, keratin and erythrocytes orange yellow (if bacteria are present they stain blue). (Report of Imper. Cancer Research Fund, 1919.)

\section{Plasma Cells.}

762. Plasma Cells and "Mastzellen"; Generalities.-Plasma cells, of which "Mastzellen" are a sub-species, are cells found in or along with connective tissue, and distinguished by their hypertrophied and very granular cytoplasm and poorly staining nucleus. The granules are highly basophilous, much more so than the nuclei ; they stain with special energy with basic anilins, and mostly metachromatically. They do not, however, stain with pure methyl green. The nuclei either do not stain at all or not in the normal way, except with pure methyl green. 
According to UnNA in Encycl. mik. Technik., 1910, ii, p. 411, material.should be fixed in chemically pure absolute alcohol and sectioned in celloidin. Care should be taken to avoid contamination of the liquids by tannin ; corks, and supports for imbedding, should be soaked for some hours before use in 2 per cent. carbonate of soda

763. Mastzellen (Nordmann, Beitr. z. Kenntniss d. Mastzellen, Inaugural diss., Helmstedt, 1884). - A concentrated solution of vesuvin containing 4 to 5 per cent. of hydrochloric acis. Stain for a few minutes, and dehydrate with absolute alcohol.

764. Plasma Cells, UnNA's Later Methods (UnNA, in Encycl. mik. Technik., 1910, ii, p. 411).

\section{A.-For Large Plasma Cells.}

(1) Ten minutes in Grübler's polychrome methylen blue solution, wash and drain. Fifteen minutes in 1 per cent. orcein solution (Grübler), without acid; absolute alcohol, so long as methylen blue comes away abundantly; berganot oil, balsam.

(2) Methylen blue as above, two minutes. Wash well. Then two minutes in glycerin-ether mixture* (Grübler) diluted with 4 volumes of water. Wash thoroughly (two to five minutes); absolute alcohol, bergamot oil, balsam.

(3) Modification of a method of Pappenheim (Virchow's Arch., clxiv, 1901, p. 111). Ten minutes in the warm, $20^{\circ}$ to $40^{\circ} \mathrm{C}$., in Grübler's carbol-pyronin-methyl-green mixture. Cool rapidly, by plunging the recipient containing the tissues into cold water. Remove the tissues with a platinum wire and rinse. Absolute alcohol, bergamot oil, balsam.

Stropeni (Zeit. wiss. Mik., xxix, 1913, p. 302) takes acridin red instead of the pyronin. This will work after various fixatives.

\section{B.-For Small Plasma Cells.}

(4) As No. 2, supra, but only half a minute in the glycerin-ether.

(5) After removal of the celloidin from the sections with alcohol and ether, five minutes in polychrome methylen blue, wash, dry with blotting-paper, dehydrate (about a minute) in a mixture of 2 parts alcohol to 3 of xylol, then one minute in xylol; then five to ten minutes in alum-anilin (prepared by allowing anilin to stand

* Glycerin ether $\mathrm{C}_{6} \mathrm{H}_{10} \mathrm{O}_{3}$, is a glycerin anhydride. It is a differentiating agent for basic dyes. The glycerin-ether mixture in question contains alcohol and glycerin, and ean be obtained from Grübler. 
over a layer of powdered alum a couple of fingers deep); xylol, balsam.

(6) As No. 3, supra, after a foregoing stain of two minutes in polychrome methylen blue.

See also EHRLICH in Virchow's Arch., clxxv, 1904, p. 198.

765. EHRLICH's Original Method for Mastzellen (Arch. mik. Anat., xii, 1876, p. 263).- Stain for at least twelve hours in-
Absolute alcohol
50 c.c.
Water
Acid. acet. glacial
100 ,
$12 \frac{1}{2}$

- to which has been added enough dahlia to give an almost saturated solution. Wash out with alcohol, and mount in resinified turpentine.

See also Scmiefferdecker and KosseL's Gewebelehre, p. 329.

966. Mastzellen, UnNA's Latest Methods (Encycl. mik. Techn., 1910, ii, p. 72).-(1) Stain three hours to overnight in polychrome methylen blue with a knife-pointful of alum to a watch-glass of the stain, rinse ; alcohol, oil, balsam. (2) Stain in polychrome methylen blue quarter of an hour, rinse, then ten minutes in glycerin-ether, $\S 702$, wash thoroughly, alcohol, oil, balsam.

These methods give a specific metachromatic stain of Mastzellen on a light ground. See also loc. cit., two other methods demonstrating plasma cells at the same time.

767. Other Methods for Plasma Cells and Mastzellen.-See, inter alios (in previous editions) PAPPENHEIM, Virchow's Arch., clxvi, 1901, p. 427 ; Bergonzini, Anat. Anz., 1891, p. 596 ; Schridde, Anat. Hefte, xxviii, 1905, p. 698 ; Maximow, Arch. mik. Anat., lxvii, 1906, p. 686 ; SchafFER, Centralb. Phys., xxi, 1907, p. 261 (fixation in absolute alcohol or 2 parts alcohol to 1 of formol, staining for half an hour in methylen blue, thionin or toluidin blue, in 70 per cent. alcohol with 1 per cent. of $\mathrm{HCl}$ ) ; Ranvier, C. R. Acad. Sci., 1890, p. 165 (his "Clasmatocytes": fix with osmium, stain with aqueous methyl violet $5 \mathrm{~B}$ ).

\section{Fatty Substances.*}

"68. Fatty Substances.-The general term "fatty substances" is used here to denote the true fats and the lipoids.

TRUE FATs are esters of the alcohol, glycerol, with the higher fatty acids, chiefly palmitic acid, stearic acid and oleic acid. The fatty acids may be "saturated " as, for instance, palmitic and stearic acid, or they may be " unsaturated" as, for instance, oleic acid.

* By Dr. W. Cramer, Imper. Cancer Research Bureau, and partly by J. B. G. 
LiPoIDs. This term was used originally to denote substances having solubilities similar to those of the true fats, i.e., substances which may be present, together with the true fats in alcohol, ether and chloroform extracts of tissues. This definition is, however, too crude, and the term as now used is restricted to certain chemically well-defined groups of substances, which in fact constitute the bulk of such extracts, namely, cholesterol and its esters, phosphatides, cerebrosides and phosphorised cerebrosides. The phosphorised cerebrosides are "compound lipoids." Their molecule is very large and consists of a chemical combination of several groups of simple lipoids, e.g., cerebrosides and a phosphatide in protagon. The existence of such compound lipoids is denied by some authors, who look upon them merely as mixtures of cerebrosides and phosphatides. These groups of substances, although differing in their chemical constitution from each other and from true fats, frequently occur together in the tissues, and may, therefore, be presumed to have a similar physiological significance. They also resemble each other and true fats in their general staining reactions, but some of them exhibit characteristic differences in this respect. Some authors use the term "lipoid" as including the true fats. Others, morphologists in particular, use the term "fat" so as to include lipoids. It seems advisable, however, to separate the true fats from lipoids, as is done here, since the two groups of substances fulfil different physiological functions. The term "LiPIN" has been proposed by some authors to denote certain groups of lipoids. The advantage of this nomenclature is not obvious, and since almost every author who has used this term has given it a new definition it will not be used here.

For a detailed account of the chemistry of these substances the reader is referred to the larger text-books and monographs, such as Hammarsten's Text-book of Physiological Chemistry, ABDerHALDEN's Biochemisches Handlexicon, Vol. iii, MaClean's monograph on Lecithin and the Allied Substances. The table on p. 358 gives only a few elementary data concerning the chemical constitution of these substances and their solubilities, which are of importance in connection with their staining reactions.

In the following, the term "true fats" will always be applied to mixtures of unsaturated and saturated fats, since in the tissues these substances always occur together. The table shows that all fatty substances occurring in the tissues contain somewhere in their molecule a double linkage (marked thus ii in the table overpage), and are therefore unsaturated compounds. This fact is of importance, 
Table of Chemical Constitution of Fatty Substances.

\begin{tabular}{|c|c|c|c|c|}
\hline Group. & Examples. & $\begin{array}{c}\text { Characteristic central } \\
\text { groups. }\end{array}$ & Additional groups. & Solubilities. \\
\hline True fats. & $\begin{array}{l}\text { Palmitin. } \\
\text { Stearin. } \\
\text { Olein. }\end{array}$ & Glycerine and fatty acids. & None. & $\begin{array}{l}\text { Readily soluble in ace- } \\
\text { tone, ether, chloro- } \\
\text { form and benzene. }\end{array}$ \\
\hline $\begin{array}{l}\text { Simple } \\
\quad \text { lipoids. } \\
\text { Sterines. }\end{array}$ & $\begin{array}{l}\text { (A.) Cholesterin } \\
\text { (Cholesterol). } \\
\text { (B.) Cholesteri- } \\
\text { nesters. }\end{array}$ & $\begin{array}{l}\text { (A.) Cholesterin free. } \\
\text { ii } \\
\text { (B.) Cholesterin and fatty } \\
\text { li }\end{array}$ & None. & $\begin{array}{l}\text { (A.) Same as true fats. } \\
\text { (B.) Not readily soluble } \\
\text { in alcohol and ace- } \\
\text { ton, soluble in ether, } \\
\text { chloroform and ben- } \\
\text { zene. }\end{array}$ \\
\hline $\begin{array}{l}\text { Phospha- } \\
\text { tides } \\
\text { (phos- } \\
\text { phorised } \\
\text { fats). }\end{array}$ & $\begin{array}{l}\text { Lecithin. } \\
\text { Kephalin. }\end{array}$ & $\begin{array}{l}\text { Glycerine, phosphoric acid, } \\
\text { and fatty acids. } \\
\qquad \mathrm{li}\end{array}$ & $\begin{array}{l}\text { A nitrogenous base, } \\
\text { e.g., cholin in the } \\
\text { case of lecithin. }\end{array}$ & $\begin{array}{l}\text { Not soluble in acetone, } \\
\text { readily soluble in } \\
\text { alcohol, ether, chloro- } \\
\text { form and benzene. } \\
\text { Kephalin, when pure, } \\
\text { is insoluble in alcohol. }\end{array}$ \\
\hline $\begin{array}{l}\text { Cerebro- } \\
\text { sides. }\end{array}$ & $\begin{array}{l}\text { Cerebron } \\
\text { (Cerebrin, } \\
\text { Phrenosin). } \\
\text { Homocerebrin } \\
\text { (Kerasin). }\end{array}$ & Galactose and a fatty acld. & $\begin{array}{l}\text { The nitrogenous base } \\
\text { sphingosin. }\end{array}$ & $\begin{array}{l}\text { Insoluble in fatty sol- } \\
\text { vents in the cold ex- } \\
\text { cept pyridine. Soluble } \\
\text { in hot alcohol, ben- } \\
\text { zene, chloroform. }\end{array}$ \\
\hline $\begin{array}{l}\text { Compound } \\
\text { lipoids. } \\
\text { Phospho- } \\
\text { rised } \\
\text { cerebro- } \\
\text { sides. }\end{array}$ & Protagon. & $\begin{array}{l}\text { A chemical comblnation } \\
\text { of the two cerebrosides, } \\
\text { cerebrin and homocere- } \\
\text { brin (vide supra), and a } \\
\text { substance sphingomye- } \\
\text { lin, allied to the phos- } \\
\text { phatides (s e e nex t } \\
\text { column). }\end{array}$ & $\begin{array}{l}\text { Sphingomyelin, which } \\
\text { contains phosphoric } \\
\text { acid, a fatty acid, } \\
\text { sphingosin and cholin } \\
\text { ii ii }\end{array}$ & Same as cerebrosides. \\
\hline
\end{tabular}

as on it depends most of the characteristic staining reactions for fatty substances.

The characteristic staining and other reactions for fatty substances fall into five groups:-

(1) Staining with Sudan III., or Scharlach R.

(2) Blackening with osmic acid, either with the acid itself or mixed with bichromate solution.

(3) Staining with hæmatoxylin after mordanting with bichromate.

(4) Their behaviour in polarised light.

(5) Staining with Nile blue.

The rationale of these methods will now be considered.

(1) Scharlach R, Sudan III.-Mixtures of the true fats, as they occur in the tissues, are readily stained by these dyes. Most lipoids, when pure, do not take this stain at all, or only slightly, except cholesterinesters, which take the stains, although not as readily as the true fats. The staining is probably a purely physical process and depends on the solution of the stain in the fatty material. Such solution occurs only when the fatty material is fluid, and this condition is fulfilled in the tissues where mixtures of the true fats 
are always present together, as, for instance, in the cells of adipose tissue, or in cells showing fatty degeneration. Mixtures of the true fats and lipoids which constitute, for instance, many of the fat globules of the adrenal cortex also take these stains. It is to be noted, however, that Scharlach $\mathrm{R}$ and Sudan III. are applied in alcoholic solution, and in the process of staining with these dyes some of the fatty substances tend to be dissolved out. The true fats are apparently not so readily dissolved as some substances, which show a double refraction, presumably cholesterin-fatty acid mixtures.

The method of Herxheimer, in which Scharlach $\mathrm{R}$ is used in strongly alkaline solution, has been recommended on the ground that it is a more "energetic" fat stain for fatty substances in the sense that it stains many cell inclusions which are not stained by Scharlach or Sudan alone. The chemistry of the reaction has not been worked out. It depends probably on the saponifying action of the alkali, which liberates some fatty acids and then produces mixtures of fatty acids and lipoids, which are more readily stained by Scharlach. The range of staining by this method is, therefore, probably as wide as that of osmic acid alone, and will be found to comprise most fatty substances, but for the purpose of differentiating between the different groups of fatty substances has lost the advantage of restricted staining, which the ordinary staining with Sudan III. and Scharlach $R$ alone possesses.

(2) Osmic Acid Methods.-The true fats and the lipoids are all blackened by osmic acid. This blackening indicates a reduction of osmic acid to a lower oxide. It is due to the fact that all these fatty substances have a double linkage (marked ii in table on p. 358) in their molecule and are, therefore, more or less easily oxidised. But the various groups of substances differ in the readiness with which they are oxidised, and consequently in the rapidity with which they are blackened by osmic acid and the depth of the blackening produced. The true fats are blackened most rapidly and most deeply, the phosphatides, lecithin and kephalin, come next in order, while cholesterin, the cerebrosides and phospho-cerebrosides are least susceptible to the oxidising action of osmic acid. In fact, these substances when solid are not blackened at all, but are so when dissolved in an appropriate solvent, such as chloroform. In the myelin sheath of peripheral nerves they are present in the form of a colloidal solution, since it can be shown that they contribute to the blackening of the sheath by osmic acid.

This difference in the reducing power of the various fatty substances can be accentuated by using osmic acid together with bichro- 
mate solutions, as in the Marchi method. The bichromate, which is itself an oxidising agent, acts in the various double linkages, and prevents the osmic acid from being reduced except by the substances having the strongest reducing power; these are the true fats and mixtures of cholesterin and unsaturated fatty acids.

Since the latter show double refraction in polarised light (see below), while the true fats do not, it is possible to differentiate these two groups of substances.

The behaviour of mixtures of cholesterin and fatty acids is paradoxical, since cholesterin alone and fatty acids alone do not blacken with osmic acid after bichromate. The same paradoxical behaviour is exhibited by these cholesterin fatty acid mixtures in their staining reaction with hæmatoxylin after mordanting with bichromate (see next paragraph). One must assume that cholesterin is present in these mixtures in a special physical state, in which it exhibits a greater reducing power.

The myelin sheath of normal nerves does not contain true fats and gives, accordingly, no blackening with bichromate-osmic acid treatment. In the early stages of degeneration globules of true fat are formed, which stain black with bichromate-osmic acid and then give the positive Marchi reaction. With other tissues rich in lipoids, such as the adrenal cortex, no such clear distinction can be obtained because the lipoids are present therein, not only together with true fats, but also mixed with them, so that a globule of fatty material frequently contains both groups of substances.

(3) Staining with Hamatoxylin after Mordanting with Bichromate.-This method, which was introduced originally by Weigert for the staining of the medullary sheath of nerves, is applicable to all fatty substances. Like the preceding method, it also depends on the presence of a double linkage in the molecule, as pointed out by Thorpe. Substances having a double linkage are oxidised by the bichromate solution and, in this process of oxidation, a chromium compound is formed which is insoluble in fat solvents and which at one stage of the oxidation has the property of forming a dark blue lake with hæmatoxylin. If oxidation be continued, however, hæmatoxylin will again cease to stain. The rate of oxidation with bichromate varies, as might be expected, with the concentration of the solution used and with the temperature at which it is carried out. Working under similar conditions, it is found again that the different fatty substances vary in the readiness with which they are oxidised by bichromate and, consequently, reach the stage of staining with hæmatoxylin after different periods 
of mordanting with bichromate. The different lipoids, when pure, differ greatly in the ease with which the stainable chromium compounds is formed; thus the cerebrosides and protagon stain after a short mordanting; the unsaturated true fats require a slightly longer time, while lecithin, and especially cholesterin, are very resistant and require prolonged mordanting. But mixtures of cholesterin and the various fatty substances show quite a different behaviour and reach the stainable stage very rapidly. This appears to be due to the fact that such mixtures form colloidal solutions in a peculiar physical condition (fluid crystals). It will be clear that a histochemical identification of the various lipoids by means of this method is not possible. Its value lies in the possibility of demonstrating, first, the presence of fatty substances by a method which gives good histological details, and secondly, by comparison with normal tissues, the occurrence of chemical changes in these substances under pathological conditions.

As introduced by Weigert, the method stains the lipoids of the normal myelin sheath. By prolonging the bichromating the degenerating nerve fibres may be made to stain in the early stage of degeneration, or the fat droplets in fatty organs may be stained. The same principle underlies Altmann's method for the staining of mitochondria, which are by some believed to consist of a central core of protein covered by an envelope of fatty material. The original method consists in mordanting with a bichromate solution and staining with acid fuchsin, just as Weigert originally used acid fuchsin for the staining of the myelin sheath. In the staining of mitochondria, the acid fuchsin can again be replaced by hæmatoxylin (Heidenhain's hæmatoxylin).

(4) Behaviour in Polarised Light.-The true fats and the fatty acids are isotropic, i.e., show no double refraction in polarised light, so that with crossed nicols the field appears dark. The lipoids are anisotropic. In fresh teased preparations they can be seen with crossed nicols as luminous droplets with a varying degree of brilliancy. The double refraction disappears on gentle heating to about $60^{\circ}$ and reappears on cooling. In formol fixed frozen sections the anisotropic lipoids appear chiefly in the form of needles and as droplets. Heating and cooling produces the effect mentioned above.

The behaviour in polarised light is, therefore, an easy and important means of differentiating the isotropic true fats from lipoids. (For a detailed description of the technique of the polarisation microscope, see Ambronn, Anleitung zur Benutzung des Polarisations Mikroskopes bei Histologischen Untersuchungen; ADAMI, 
The Myelin and Potential Fluid Crystalline Bodies of the Organism, 1906 ; Kaiserling and Ogler, "Uber das Auftreten von Myelin in Zellen, etc.," Virchow's Archiv., clxvii.).

(5) Nile Blue.-When a solution of Nile blue is boiled with sulphuric acid the solution contains, in addition to the original basic oxazine dye, which unites with fatty acids to form a blue compound, a red oxazone dye, not basic in character, but soluble in liquid fat and giving it a red colour. The stain, therefore, enables us to distinguish neutral fat and fatty acid. If a given globule contains neutral fat and no fatty acid, it will be coloured red; if it contains fatty acid only, it will be coloured blue; but if it contains both neutral fat and fatty acid, it will be coloured a tint between blue and red, depending on the proportion of neutral fat and fatty acid. The principal histochemical group reactions are summarised in the following table:-

Table of some important Histochemical Group Reaclions.

\begin{tabular}{|c|c|c|c|c|c|c|c|}
\hline & \multirow{2}{*}{ Sudan. } & \multirow{2}{*}{$\begin{array}{c}\text { Double } \\
\text { refrac- } \\
\text { tion. }\end{array}$} & \multirow{2}{*}{$\begin{array}{l}\text { Osmic } \\
\text { acid. }\end{array}$} & \multirow{2}{*}{$\begin{array}{c}\text { Osmic } \\
\text { after bi- } \\
\text { chromate. }\end{array}$} & \multicolumn{3}{|c|}{ Solubility in cold } \\
\hline & & & & & Acetone. & Alcohol. & $\begin{array}{l}\text { Chloro- } \\
\text { form. }\end{array}$ \\
\hline True fats & +++ & - & $++t$ & + & + & + & + \\
\hline $\begin{array}{l}\text { Cholesterin } \\
\text { Cholesterinesters } \\
\text { Phosphatides } \\
\text { Cerebrosides } \\
\text { Phospho-cerebrosides }\end{array}$ & $\begin{array}{l} \pm \\
\overline{-}\end{array}$ & $\begin{array}{l}+ \\
+ \\
+ \\
+ \\
+\end{array}$ & $\begin{array}{c}+ \\
+ \\
+ \\
+\end{array}$ & $\begin{array}{l}\bar{z} \\
\bar{z}\end{array}$ & $\begin{array}{l} \pm \\
\pm\end{array}$ & $\frac{ \pm}{ \pm}$ & $\begin{array}{l}+ \\
+ \\
+ \\
+\end{array}$ \\
\hline $\begin{array}{c}\text { Cholesterin - fatty } \\
\text { acid mixtures. }\end{array}$ & + & + & ++ & + & + & + & + \\
\hline
\end{tabular}

Although different groups of fatty substances differ in their behaviour towards different staining methods, it is, nevertheless, necessary to proceed with caution in interpreting the results obtained when these methods are applied to tissues. In the tissues the various groups of fatty substances are mixed, so that one globule may contain two or three different substances. The various substances may then form a special kind of colloidal solution, which modifies their physical state and alters their staining reaction completely. The behaviour of mixtures of cholesterin and fatty acids is an example and has been referred to above. Or one substance encloses another substance belonging to a different group. In such a case the staining reactions of the globule would be those of the material which constitutes the envelope. In the adrenal cortex, for instance, 
many globules contain a core of lipoids surrounded by true fats. In such a case examination in polarised light is helpful. Differentiation by means of different solvents is also possible within limits. Again, the size of the globules is, for obvious reasons, an important factor in the methods which involve mordanting with bichromate. But even if due consideration is given to these reservations, the application of these staining reactions has yielded important results and has profoundly modified our conceptions of the part taken by the fatty substances as constituents of protoplasm. In the first place, it is important to realise that no one single method is a specific staining method, either for all fatty substances or for any one group of them. Thus, Sudan III. or Scharlach R. do not stain all fatty substances, as a glance at the table of the group reactions shows. They stain intensely the true fats, and less intensely cholesterinesters and cholesterin-fatty acid mixtures. But many lipoids are not stained by these dyes, and an examination in polarised light is necessary to detect their presence. Osmic acid alone has the widest range as a reagent for fatty substances, and stains all the different groups. With some substances, however, such as cholesterin or cerebrosides, it may give negative results if they are not present in a state of colloidal solution in the cytoplasm. But since osmic acid stains also substances which are not fatty in nature, e.g., adrenalin in the cells of the adrenal medulla, it cannot be looked upon as a specific stain. In the case mentioned, a differentiation can still be effected by immersing the section in turpentine, which dissolves the fatty substances, even after osmication. The method giving the highest degree of specificity is the use of osmic acid after bichromate. This will stain only the true fats or cholesterin-fatty acid mixtures. Or, expressed in terms of everyday histological technique, the presence of black cell globules in material fixed either in bichromate-osmic mixtures, such as Altmann's, Champy's or Flemming's fixatives, or first in bichromate fixatives, such as Muller's or Zenker's fluids, and post-osmicated (\$ 691) indicates the presence either of true fats or of cholesterin-fatty acid mixtures. These two can then be differentiated by examination in polarised light. It is perhaps equally important to be able to draw the opposite conclusion, when other facts have indicated the presence of fatty substances. The absence of blackening of the globules under the conditions just mentioned definitely excludes the presence of true fats. Thus, true fats can be proved to be absent from the myelin sheath of normal nerves. The significance of the staining of fatty substances after mordanting with bichromate has already been 
referred to as revealing the essential similarity of Weigert's method for the myelin sheath of nerves and Altmann's method for mitochondria. The staining reactions of the Golgi apparatus-blackening after prolonged action of osmic acid, staining with hæmatoxylin after prolonged mordanting with bichromate-suggest that it is made up of fatty substances, and, further, that these substances are not readily oxidised. They thus correspond in their behaviour to lipoids such as cholesterin.

From what has been said, it is clear that for the study of fatty substances several methods must be applied to the tissues.

In so far as the selection of a fixative is concerned, it follows that fixatives containing alcohol or chloroform must be avoided. A fixative such as Carnoy, or such mixtures containing strong alcohol or chloroform, are themselves fat solvents, as well as lipoid solvents, and they dissolve away all fat-vacuoles and shrink up cell organs which may be formed partly of other "fatty" substances, e.g., mitochondria. Other fixatives, such as picric acid or corrosive sublimate, while in themselves not fat or lipoid solvents, are unable to prevent the fat from being dissolved away in any subsequent dehydration and clearing of the tissue. Very few reagents are known which are able to form with fat substances insoluble or scarcely soluble in alcohol and a clearing medium, like xylol; osmium tetroxide and, to a less extent, potassium bichromate, are thus valuable reagents to the histologist. Various oils act differently towards these fat $\mathrm{OsO}_{2}$ compounds ; xylol and chloroform do not easily disintegrate them, but an oxidising oil like turpentine will quickly do so. The "compounds". of $\mathrm{OsO}_{4}$ with fats and lipoids are ill understood, but Partington and Huntingford have recently shown (see $\$ 772$ ) that the reduced black substance is a hydrated form of $\mathrm{OsO}_{2}$.

Martinotti (see $\$ 772$ ) has recently introduced a new method which may prove of great importance. According to this observer the orange yellow dye chrysoidin (phenyl-azo-m-phenylene-diamide), when applied to fatty tissue and then treated with an oxidising agent, such as bichromate of potash, has the power of preventing the fat globules from being dissolved away in alcohol, benzol and xylol. In preparations the fat looks a brown orange colour.

A complete histochemical investigation of fatty cell inclusions comprises, thus, the following methods :-

(1) In fresh preparations :

(a) Examination in polarised light.

(b) Staining with Sudan III. or Scharlach R. 
(c) Staining with osmic acid in solution or as vapour.

(2) In frozen sections, material fixed in formol :

(a) Examination in polarised light.

(b) Staining with Sudan III. or Scharlach R.

(c) Staining with Nile blue.

(d) Repeat $(b)$ and $(c)$ after immersing sections in cold acetone or cold alcohol for a few minutes.

(3) In paraffin sections :

(a) Fixation in osmic acid.

(b) Fixation in bichromate osmic acid mixtures or fixation in bichromate solutions and post-osmication.

(c) Prolonged fixation in bichromate and staining with Sudan III. (Ciaccio, Bell).

(d) Staining with hæmatoxylin (Weigert, Lorrain, Smith, Dietrich).

(e) Treatment with chrysoidin and subsequent fixation in bichromate (Martinotti).

Such a plan of investigation refers especially to vertebrate materials, but with certain small modifications is applicable to the study of fatty substances in developing eggs, embryos, and to the tissues of invertebrates. Paragraphs 1 and 3 are both applicable to invertebrate tissues and embryos, while in the case of paragraph 2 the tests can be used after the whole embryos or eggs have been fixed in neutralised and suitably diluted formalin. It should be remembered that in all animal cells (and possibly plant cells, too) there exist two categories of cytoplasmic inclusions, Golgi apparatus and mitochondria, which are partly formed of lipoid materials, and which we now know may produce fats, or may metamorphose into fats. In nearly all eggs where fat granules are present, examination has revealed the fact that such "yolk" is derived either from Golgi elements or mitochondria (GATENBY and WoodGER, Journ. Roy. Micr. Soc., 1920), and in certain cells of vertebrate tissues we know that mitochondria may change into fat (MurRAY, Scientific Report Cancer Research Fund, 1919).

Reference should be made to the sections on "Mitochondria," "Golgi Apparatus," "Fat," and "Yolk" (§§ 673-713), and especially to the tables in $\S \S 702,708,710$ and 712 , where some attempt has been made to illustrate the behaviour of the various inclusions after the application of certain well-known techniques. It is always necessary to ascertain exactly the condition and behaviour of the mitochondria and Golgi apparatus in tissues or cells being investigated for fatty and lipoid substances, particularly 
in view of any inter-relationship which may exist between the former and the latter. See also BeLL (Journ. Med. Research, xxiv, 1911, p. 539 ; Journ. of Pathol. and Bact. xix, 1914, p. 105. Ciaccio (Centralblatt f. allg. Pathol. and Path. Anatomie, xx, 1909, p. 771; Arch. f. Zellforschung, v, 1910, p. 235. Cramer, Feiss and Bullock (Proceed. Phys. Soc., 1913; Journ. of Physiology, xlvi, p. 51. International Congress of Medicine, London, 1913. Section of Pathology). Dietrich (Erg. d. Allg. Pathologie und Patholog. Anat., xiii, 1909, pt. 2, p. 283 ; Deutsche Patholog. Gesellsch., xiv, 1910, p. 263). Kawamura (Die Cholesterinester verfettung. Jena. Gustav Fischer. 1911). SMith and MaIr (Journ. Pathol. and Bact., xiii, 1909, p. 14 ; Skand. Arch. f. Physiol., xxv, 1911, p. 247).

769. Fixing and Staining. - The choice of the fixative depends on the question whether the material is to be examined in frozen sections or in paraffin sections. In any case all fixatives containing acetone, alcohol, chloroform or other fat solvents are excluded. For paraffin sections the material may be fixed in osmic acid alone ( 1 per cent. in solution, or 2 per cent. if fixed in vapour), or in osmic acid mixed with bichromate solution (see fixatives of Flemming, Altmann, Champy). Or it may be fixed in formol bichromate and treated subsequently with a bichromate-osmic mixture (see methods of Schridde and Marchi). As stated in the general part (see p. 363) the different methods give different results with the various groups of fatty substances. For all these methods very small pieces of tissue must be used. For the effects of alcohol on the blackening of certain fatty substances by osmic acid, see HANDWERCK, Zeit. wiss. Mik., xv, 1898, p. 177 ; Mulon, ibid., xxii, 1905, p.138; Golodetz, ibid., xxviii, 1911, p. 213 ; and Chem. Rev. Fett u. Harzindustrie, xvii, 1910, p. 70 ; Loisel, C. R. Soc. Biol., 1903, p. 826.

Another method consists in fixing and mordanting with strong bichromate solution and subsequent staining with Sudan (see below, Bell's method, also Ciaccio) or with hæmatoxylin (methods of Weigert for nervous system; also Lorrain Smith, Dietrich).

For examination in frozen section the tissue may be fixed in formol saline or formol-bichromate, or the stain may be applied directly to the fresh tissue after teasing. For fine cytological work, the formol should be neutralised by shaking with solid calcium carbonate. Hays Bullard (Amer. Journ. Anat., xix, 1916) recommends neutralisation and distillation method of Gustav ManN (Physiological Histology, Oxford, 1902): neutralise commercial 
solution with sodium or lithium carbonate, and freshly distil. A 20 per cent. solution is then prepared and rendered isotonic: $.75 \mathrm{gms}$. of $\mathrm{NaCl}$ to 100 c.c. of fluid. With short fixation (thirty-five minutes to five hours), the quantity of fat usually does not differ from that seen in fresh tissue (Bullard). Cut on freezing microtome, stain by one of the methods given below, preferably Herxheimer's alkaline scarlet red. As control use also fresh tissue.

For quinolein blue, see $\S 322$.

DADDI (Arch. Ital. Biol., xxvi, 1896, p. 413) stains fat in tissues by treating for five to ten minutes with concentrated alcoholic solution of Sudan III. washing for the same time with alcohol, mopping up with blotting paper, and mounting in glycerin.

Similarly RIEDER, see Zeit. wiss. Mik., xv, 1898, p. 211.

The alcohol for making the stain should be of 70 per cent., according to most authors, though SATA (Beitr. path. Anat., xxviii, 1900 , p. 461 ; Zeit. wiss. Mik., xviii, 1901, p. 67) employs 96 per cent. Rosenthal (ibid., xix, p. 469 ; Verh. path..Ges., September, 1899, p. 440) insists that the washing-out be done with alcohol of exactly 50 per cent.

Michaelis (Virchow's Arch., clxiv, 1901, p. 263) recommends Scharlach R (syn. "Fettponceau"). Stain for fifteen to thirty minutes in a saturated solution in 70 per cent. alcohol, and mount in glycerin or levulose.

Other authors also commend this stain. Herxhermer (Deutsche med. Wochenschr., xxvii, 1901, p. 607 ; Zeit. wiss. Mik., xix, 1902, p. 66) makes a solution of 70 parts of absolute alcohol, 10 of water. 20 of 10 per cent. caustic soda, and Scharlach $R$ to saturation. This makes a stronger solution, and stains in a couple of minutes. Wash out with alcohol of 70 per cent.

With either solution the staining must be done in a covered vessel or the stain will precipitate.

Similarly Bfll, Amer. Journ. Anat., ix, 1909, p. 401, and Anat. Rec., iv, 1910, p. 199.

Herxhermer also (Centralb. allg. Path., xiv, 1903, p. 841 ; Zeit. wiss. Mik., xxi, 1904, p. 57) recommends a saturated solution of the dye in a mixture of equal parts of acetone and 70 per cent. alcohol.

He also (Deutsche med. Wochenschr., xxvii, 1909, p. 607 ; Zeit. wiss. Mik., xix, 1902, p. 67) has had very. fine results by staining for 20 minutes in a saturated solution of Indophenol in $70 \%$ alcohol.

Mollison (Zeit. wiss. Zool., lxxvii, 1904, p. 529) has had good results by staining gelatin sections for a few minutes in strong 
extract of Alkanna in 96 per cent. alcohol, and mounting in glycerin or syrup.

Lorrain SMith (Journ. Path. 'Bact., xii, 1907, p. 1) finds that Nile blue stains fatty acids blue and neutral fats reddish.

Similarly EisenBerg (Virchow's Arch., cxcix, 1910, p. 502), who recommends aqueous solution of Nilblau BB.

BENDA (ibid., clxi, 1900, p. 194) finds that free fatty acids can be detected by Weigert's neuroglia mordant. See also Berner, ibid., clxxxvii, 1907, p. 360, and Fischler, Zeit. wiss. Mik., xxii, 1905, p. 263.

Oкалгмa (ibid., xxix, 1912, p. 67) extracts red capsicum berries for some days with alcohol, and evaporates down to one fifth. This stains only fatty bodies : amongst them, myelin.

See also Kingsbury, Anat. Rec., v, 1911, p. 313.

770. Removal of Fatty Substances.-If not treated with osmic acid or mordanted with strong bichromate, alcohol, ether, chloroform, pyridine, xylol, will readily dissolve fatty substances. Osmicated fats and lipoids are more resistant, especially if osmic acid and bichromate have been combined. It can then be removed in a few hours by alcoholic hydrogen peroxide ( 10 per cent. $\mathrm{H}_{2} \mathrm{O}_{2}$ in 80 per cent. alcohol) or in twenty-four hours by oil of turpentine. Ether, creosote, xylol, clove oil and chloroform will also remove osmicated fats and lipoids if allowed to act sufficiently long.

See also Flemming in Zeit. wiss. Mikr., 1889, pp. 39, 178.

771. Differentiation between Fats and various Lipoids.-Fix in formol and prepare frozen sections. Stain some with Sudan or Scharlach (see above $\S 769$ ), others with osmic acid. Leave some unstainer. Globules which stain with Sudan or Scharlach and osmic acid, and which in unstained sections show no double refraction, can be identified with certainty as true fats. This may be confirmed in paraffin sections by fixing in bichromate and subsequent treatment with osmic acid as in the methods of Schridde and Marchi. These globules should then reduce osmic acid. But the presence of double refraction must not be taken as excluding the presence of true fats since the globules may be a mixture of true fats and double refracting lipoids. The deduction that true fats are absent can be made when tissue containing fatty material as indicated by blackening with osmic acid fails to give this blackening after previous treatment with bichromate, as for instance in normal peripheral nerve.

The histochemical differentiation between true fats and lipoids is much more difficult when these substances are mixed in one and the same globule than when one cell contains several globules of which some are composed entirely of true fats while others contain lipoids. In the latter case methods may be-applied which depend on differences in the solubility in various solvents.

Deflandre (Journ. Anat, Phys., 1904, p. 80) fixes in formol of 4 per 
cent. and brings into acetone, in which fat is dissolved, but not lecithin, which can then be stained by osmium.

See also Ciaccio, Arch. Zellforsch, v, 1910, p. 235 ; and Fisciller, Zeit. wiss. Mik., xxii, 1905, p. 262 ; LoISEL, C. R. Soc. Biol., lv, 1903, p. 703 .

BeLl with a modification of Dietrich's and CIACCIo's methods (Journ. Path. and Bact., xix) claimed to be able to distinguish between fat drops mainly of triolein and those that principally contain " lipoids." The former appear in annular shape, the latter are quite solid. In the former case the centre of the droplet is not chromated, and therefore dissolves out in xylol used for imbedding. Fix at $45^{\circ} \mathrm{C}$. to $50^{\circ} \mathrm{C}$. in 10 per cent. aqueous $\mathrm{K}_{2} \mathrm{Cr}_{2} \mathrm{O}_{7}, 100$ c.c., acidified by 5 c.c. of acetic. Wash, dehydrate and imbed in paraffin. Fasten 3 to $5 \mu$ sections to slide with albumen water. Treat sections in xylol, absolute alcohol, and transfer to freshly prepared solution of Sudan III in 80 per cent. alcohol for ten minutes. Rinse off excess stain in 50 per cent. alcohol; transfer to water to stop action of alcohol. Counterstain in Delafield's hæmatoxylin, wash in water, differentiate in acid alcohol, wash and mount in glycerin gum-arabic.

772. Mounting Fat.-After treatment with osmic acid sections can generally be mounted in balsam without special precautions. Many lipoids, however, fade even after osmication, if kept for a long time in balsam. In some cases it may suffice to avoid absolute alcohol and essences as much as possible, and mount direct in alcohol balsam or euparal, or clear with cedar oil, which has little solvent action. For very delicate fats it may be necessary to avoid alcohol of more than 70 per cent., or avoid it altogether, and mount in glycerin or levulose.

Chrysoidin (L. Martinotti, Zeit. Physiol. Chem., xci, 1914) fixes tissue in 10 per cent. formol, sections on a freezing microtome, washes in aq. dest., and immerses for five to ten minutes up to several hours in a 1 per cent. aqueous solution of chrysoidin. Wash not longer than one minute in aq. dest., and treat in 10 per cent. $\mathrm{K}_{2} \mathrm{Cr}_{2} \mathrm{O}_{7}$ or $\mathrm{CrO}_{3}$ for one minute, wash, dehydrate in benzol and xylol, and mount in neutral balsam.

\section{Bone. ${ }^{*} \dagger$}

v99. Bone, Non-decalcified.-RANvier (Traité, p. 297) has the following :

Bones should be plunged into water, without being allowed to dry, as soon as the surrounding soft parts have been removed, and should be divided into lengths with a saw whilst wet. The

* For a detailed review of the whole subject, see the paper of SciraFFER in Zeit. wiss. Mik., x, 1893, p. 167, or the article "Knochen und Zähne" in Encycl. mik. Technik.

$\dagger$ This section has been revised by J. Thornton Carter, Esq., F.R.M.S., of the Zoological Department, University College, London; 
medulla should then be driven out from the central canal by means of a jet of water; spongy bones should be treated as follows:

An epiphysis having been removed, together with a small portion of the diaphysis, a piece of caoutchouc tubing is fixed by a ligature on to the cut end of the diaphysis, and the free end of the piece of tubing adapted to a tap through which water flows under pressure ; they are then put to macerate for several months, the liquid being changed from time to time. As soon as all the soft parts are perfectly destroyed, the bones may be left to dry.

Thin sections may then be cut with a saw and prepared by rubbing down with pumice-stone. Compact pumice-stone should be taken and cut in the direction of its fibres. The surface should be moistened with water and the section of bone rubbed down on it with the fingers. When both sides of the sections have been rubbed smooth in this way, another pumice-stone may be taken, the section placed between the two, and the rubbing continued. As soon as the section is thin enough to be almost transparent it is polished by rubbing with water (with the fingers) on a Turkey hone or lithographic stone. Spongy bone should be soaked in gum and dried before rubbing down (but see VoN KocH's copal process and Ehrenbaum's colophonium process).

Schaffer (Zeit. wiss. Mik., x, 1893, p. 171) grinds and polishes on stones of graduated fineness.

For the process of WEIL for bones and teeth see $\S 180$.

RösE (Anat. Anz., vii, 1892, pp. 512-519) follows Koch's process. He penetrates first with a mixture of cedar oil and xylol, then with pure xylol, and imbeds in solution of Damar in chloroform or xylol. The method can bз combined with Golgi's impregnation.

FANZ (Anat. Record, xiv, 1918, p. 493) employs sand or carborundum paper of different grades of eoarseness for grinding, using the back or smooth side of a piece of sandpaper for polishing the section. He recommends shellac in preference to balsam for attaching the section to the glass slip.

White (Journ. Roy. Mic. Soc., 1891, p. 307) recommends the following: Sections of osseous or dental tissue should be cut or ground down moderately thin, and soaked in ether for twentyfour hours or more. They should then be put for two or three days into a thin solution of fuchsin in collodion, then into spirit to harden the collodion. After this they are ground down to the requisite thinness between two plates of old ground glass, with water and pumice powder, and mounted, surface dry, in stiff balsam or styrax, care being taken to use as little heat as possible. Lacunæ, canaliculi, and dentinal tubuli are found infiltrated by the coloured collodion. 
Hanazawa (Dental Cosmos, lix, 1917, pp. 125 et seq.) gives a number of methods for staining ground and decalcified sections of dentine to demonstrate its minute structure.

Matschinsky (Arch. mil. Anat., xxxix, 1892, p. 151, and xlvi, 1895, p. 290), after grinding, impregnates with nitrate of silver.

For similar method of Ruprecir, see Zeit. wiss. Mik., xiii, 1896, p. 21, wherein see also quoted (p. 23) a method of Zirmmermans.

Csokor (Verh. anat. Ges., 1892, p. 270) describes a saw which will cut fresh bone to $120 \mu$; and ARndT (Zeit. wiss. Mik., xviii, 1901, p. 146) a double saw which will also give very thin sections.

r94. Mounting. - To show lacunæ and canaliculi injected with air, take a section, or piece of very thin flat bone, quite dry. Place on a slide a small lump of solid balsam, and apply just enough heat to melt it. Do the same with a cover glass, place the bone in the balsam, cover, and cool rapidly.

When thin ground sections of enamel are mounted in Canada balsam it is found often that they appear almost structureless. To demonstrate the enamel pattern of such sections they may be etched by immersion in 6 per cent. of hydrochloric acid in 70 per cent. alcohol, or in a weak aqueous solution of picric acid, and mounted in Camsal balsam or Euparal, media which, on account of their low index of refraction, will be found to disclose the structure of the enamel more easily.

\section{ry5. Sections of Bones or Teeth showing the Soft Parts.-A} developing tooth with its epithelial enamel-organ, its mesodermal dentinal papilla, and its layers of partially calcified enamel and dentine, is made up of very delicate structures of different consistency and so peculiarly liable to unequal shrinkage, with consequent distortion during the period of fixation and in the subsequent processes passed through in the preparation of sections. Further, post-mortem changes in the ameloblasts occur within a very few minutes after death leading to a less precise behaviour to stains than is found in the case of cells which are fixed immediately after death.

For the examination of developing teeth in situ, jaws may be fixed in corrosive-formalin-acetic mixture, in Bouin's picro-formol, in Zenker's mixture or Helly's modification thereof, or in Sansom's modification of Carnoy's mixture ( $(86)$.

For the study of the micro-anatomy of the enamel-organ and the dentinal papilla, a young pup or a kitten, two or three days old, is killed, preferably by a blow on the head. The jaws are removed and the bone of the under-surface of the mandible pared away by a sharp scalpel until the bases of the tooth-germs are almost exposed. 
The muco-periosteum is grasped with a pair of forceps and stripped from the bone, when the tooth-germs will come away attached thereto.

Sansom's modification of Carnoy's mixture, employed at bloodheat, is particularly effective when the tooth-germs have been exposed in the manner outlined above, fixation therein being complete in from five to ten minutes. They are then passed through successive baths of alcohol of 30 per cent. and 50 per cent., each for fifteen minutes; 70 per cent., to which is added tincture of iodine, for four hours ; 90 per cent. for thirty minutes ; and into two changes of absolute alcohol, each for fifteen minutes or longer.

The tooth-germs are then transferred to a mixture of equal parts of absolute alcohol and carbon disulphide for one hour, two changes of pure carbon disulphide, each of fifteen minutes, then for thirty minutes into carbon disulphide saturated with paraffin at $30^{\circ} \mathrm{C}$., transferred to carbon disulphide saturated with paraffin at $42^{\circ} \mathrm{C}$. for a like period, and finally into two baths of paraffin, in each half an hour. Imbed for cutting in pure paraffin.

By the employment of this method the amount of shrinkage in the tissues is extremely slight and the dentine does not become hardened, so that the tooth-germs of the incisors may be cut without decalcification. In the case of the canine and molar tooth-germs a short period of decalcification may be necessary, and for this purpose a rapid and delicate method lies in the employment of ZEIGLER's method (Festschr. f. Kupffer, 1899, p. 51), in which, by the use of a 5 per cent. solution of sulphurous acid, the insoluble tricalcium phosphate is changed into the readily soluble monocalcium phosphate.

To demonstrate eytological detail no stain equals iron-hæmatoxylin followed by a counter-stain of picric-lichtgrun or of Rubin S in picrate of ammonia.

It cannot be too strongly emphasised that the precision of staining methods depends on the rapidity with which fixation of the tissues is effected after death. Refer to $\S 31$.

For large jaws imbedding in celloidin, or, when serial sections are required, double imbedding in celloidin, parlodion or photoxylin and paraffin is recommended ( $\S 171)$.

Mummery (Phil. Trans. B., ceviii, 1917, p. 258) deprecates the employment of paraffin for imbedding the tooth-germs of fishes, considering the heat employed to be very injurious to the delicate enamel organs, and advocates the use of the freezing method in obtaining sections. See carbon disulphide method above.

Nealey (Amer. Mon. Mic. Journ., 1884, p. 142 ; Journ. Roy. Mic. 
Soc., 1885, p. 348) says that perfectly fresh portions of bone or teeth may be ground with emery on a dentist's lathe, and good sections, with the soft parts in situ, obtained in half an hour.

Hopewell-Smiti (Journ. Brit. Dent. Ass., xi, 1890, p. 310 ; Journ. Roy. Mic. Soc., 1890, p. 529) says that for preparing sections of teeth showing odontoblasts in situ the best plan is to take embryonic tissues. A lower jaw of an embryonic kitten or pup may be taken, and hardened in solution of Müller followed by alcohol, then cut with a freezing microtome.

WEIr. (loc. cit., § 180) fixes pieces of fresh teeth in sublimate, stains with borax-carmine, brings them through alcohol into chloroform and chloroform-balsam, and after hardening this by heat proceeds to grind as usual ( $\$ 177)$.

See also Röse, § 773 .

1995 A. - For the study of the vessels in teeth, LepKowsKy (Anat. Hefte, viii, 1897, p. 568) injects with Berlin blue, hardens the teeth with a piece of the jaw for one or two days in 50 per cent. formol, decalcifies in 10 per cent. nitric acid (eight to fourteen days, change frequently) and makes celloidin sections.

For decalcification of teeth, see also $§ 546$ (Rousseau, BöDECKER and Fleis.chmans). Bödecker finds Rousseau's process not applicable to human teeth : the acid must be added to the fuid celloidin.

For the study of the lymphatics in the dental pulp, DEwEY and Noyes (Dental Cosmos, lix, 1917, pp. 436-44) first inject the blood vessels with carmin-gelatin. Then 2 grms. of Prussian blue (oil colour in tubes) is stirred with 3 grms. of turpentine oil in a glass mortar for five minutes; 15 grms. of sulphuric ether is added, and this fluid filtered through flannel or chamois skin. After the injection of this fluid the head is placed for twenty-four hours or longer in 20 per cent. formalin, and then the injected teeth are carefully removed and the pulps examined. Later it was found that more constant results were obtained when the injection of the blood vessels followed that of the Prussian blue. Prussian blue injected directly into the pulps and trypan blue or lithium carmine injected intravenously or intraperitoneally were also employed. See $\$ \S 780$ and 790 .

Wellings (Proc. Sixth Internat. Dent. Cong., pp. 47 et seq.) demonstrated intra-vitam staining of dental and adjacent tissues by means of trypan blue $(\S 780)$.

Mummery (Phil. Trans. B., ccii, 1912), for the fixation of the nerve-tissue of the dental pulp, finds formalin to be preferable to all other fixing agents, employing 10 parts of the 40 per cent. commercial formalin to 90 parts of water.

Decalcification is effected by means of 33.3 per cent. formic acid. After thorough washing he leaves for twenty-four hours in a strong 
solution of dextrin (which he finds preferable to gum arabic), and sections are cut on the freezing microtome, by the employment of which he is able usually to obtain thinner sections than when paraffin is used for imbedding.

The sections are stained either by means of iron and tannin, iron-hæmatoxylin (Benda), Congo red, Ranvier's modification of Löwet's gold chloride process, or by Cajal's method, where :-

(1) Small pieces of the decalcified tooth, not more than 4 millimetres thick, are placed in 50 c.c. of rectified spirit, to which 3 or 4 drops of ammonia may be added, and kept in this solution for from four to six hours.

(2) Transfer to absolute alcohol for twenty-four hours.

(3) Rinse with distilled water.

(4) Place in a large quantity of 1.5 per cent. solution of silver nitrate, and keep in warm incubator at about $35^{\circ} \mathrm{C}$. for five or six days.

(5) Rinse in distilled water for a few seconds.

(6) Place in the following solution for twenty-four hours :-

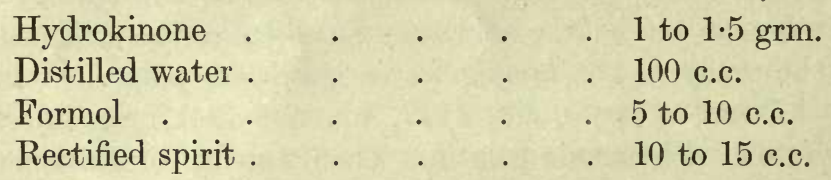

(7) Wash in water for some minutes.

(8) Cut sections, and mount.

The presence of nerve-end cells in the dental pulp was demonstrated by Mummery (Phil. Trans. B., ccix, 1920), by means of a modification of the gold method of Beckwith.

Teeth, immediately after extraction, are placed in a solution of formol and water or of formol and normal salt solution, preferably 4 per cent. of formol. This is, after a few days, changed to a 10 per cent. solution, and the teeth kept in this for at least a fortnight.

Decalcification is effected by means of a $33 \cdot 3$ per cent. solution of formic acid in distilled water, to which 5 per cent. of formol may be added. (Mummery states that neither he nor Dependorf has ever procured good nerve preparations of teeth which have been decalcified in the mineral acids.)

Wash in running water for twenty-four hours, then for a few minutes in distilled water.

The pieces are taken from the distilled water and suspended by threads in a large quantity of a weak solution of gold chloride $(1$ in 5,000). Each piece should be suspended in at least 100 c.c. 
of the solution, in which it is left in the dark for from four days to one week, according to its size. On removal from the gold solution it is washed for a few minutes only in distilled water. Reduction is effected by placing the pieces in a 20 per cent. solution of caustic soda for four minutes, then rinsing in water and placing in a 10 per cent. solution of potassium carbonate for from half an hour to an hour. This is then drained off, and the pieces are placed in a 10 per cent. solution of potassium iodide for a short time-usually five to ten minutes. As soon as seen to darken, the pieces are removed from this solution to water, placed in gum for twelve hours, and sections cut on the freezing microtome.

After dehydration the sections are mounted in camsal (propylic) balsam.

776. Vivante (Intern. Monasschr. Anxt. u. Phys., ix, 1892, p. 398) impregnates portions of frontal bone of four to six months calves, which are not more than 3 to 4 millimetres thick, by Golgi's rapid bichromate and silver process. After impregnation the specimens should be decalcified in von Ebner's mixture ( $\$ 562$ ), well washed with water, and brought into solution of carbonats of soda, and finally imbedded in paraffin. For his quinolein blue method see fourth edition.

For UNDERWOOD's gold process for teeth, and for that of LEPKOWSKI, see third edition, or Anat. Anz., 1892, p. 294.

LaAw (Proc. Roy. Soc. Med., i, 1908, p. 45) studies nerve-endings in teeth of mammals by treating paraffin sections of decalcified tissue with BETHE's molybdenum toluidin blue (details in Journ. Roy. Micr. Soc., 1908, p. 518).

VAN DER Stricht (Carnegie Instit. Embryol. Contrib., No. 21) fixes the isolated cochlea in a 5 per cent. aqueous solution of trichloracetic acid, or in Bouin's or Zenker's fluid, and stains, before imbedding, in borax-carmine. The sections are afterwards stained in iron-hæmatoxylin, Congo red and light green. He obtained the best results with the membrana tectoria by making one or two openings in the bony wall of the fresh cochlea and exposing the piece for fifteen minutes to the vapours from an aqueous solution of osmic acid or by submerging it in a 1 per cent. solution of the same for one hour. Afterwards fixation was completed by immersion in trichloracetic acid, Bouin's fluid or 'Zenker's fluid, and the series of sections therefrom stained as above. By this method some of the turns of the cochlea give very good preparations of the structure of the membrana tectoria. The mitochondria are also visible within osteoblasts, osteoclasts, connective-tissue cells, all epithelial cells, and the sensorial elements.

Mitochondria in odontoblasts and osteoblasts may be demonstrated by fixation in Flemming-without-acetic followed by staining 
in iron-hæmatoxylin ( $\S 679)$, and the Golgi apparatus in these cells is well shown by the employment of Golgi's method, Cajal's method,

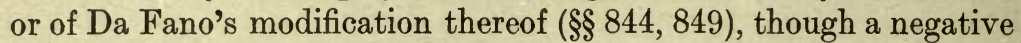
image of this cell-element is clearly shown when the tissues are fixed in Sansom's modification of Carnoy's mixture.

ry\%. Bone, Decalcified (Flemming, Zeit. wiss. Mik., 1886, p. 47).Sections of decalcified bone are soaked in water, dehydrated with alcohol under pressure, dried under pressure and mounted in hard balsam melted on the slide. They show the lacunar system injected with air as in non-decalcified sections.

7y/8. Stains for Cartilage and Decalcified Bone.-See hereon SchaFfER in Zeit. wiss. Mik., v, 1888, p. 1; and Encycl. mik. Technik., art. "Knochen."

KöLLIKER (Zeit. wiss. Zool., xliv, 1886, p. 662) treats sections of decalcified bone with concentrated acetic acid until they become transparent, and then puts for one quarter to one minute into a concentrated solution of indigo-carmine, washes and mounts in - glycerin or balsam. The fibres of Sharpey appear red, the remaining bone substance blue.

SchaFFER (Zeit. wiss. Mik., v, 1888, p. 17) employed at one time a safranin method modified from Bouma (Centralb. med. Wiss., 1883, p. 866), for which see previous editions. He now (Encycl. mik. Tech., 1910, i, p. 762) stains sections for twenty-four hours in a bath of 20 c.c. of water with 1 drop of 1 per cent. solution of safranin (or thionin) and (apparently) mounts in balsam. The safranin stain will keep if the material is cartilage which has been fixed in picrosublimate; otherwise it must be fixed with ammonium molybdate of 5 per cent. before dehydrating.

Schmorl (Centralb. allg. Path., x, 1899, p. 745) stains in a mixture of 2 c.c. concentrated solution of thionin in alcohol of 50 per cent. and 10 c.c. of water for ten minutes, rinses and puts into saturated aqueous picric acid for thirty to sixty seconds. Rinse and pass through graded alcohols into origanum oil or carbol-xylol and balsam. Matrix yellow, cells red, fat-cells violet. He also describes a more complicated method with thionin and phosphotungstic or phosphomolybdic acid.

Mols (Gentralb. Physiol., xiii, 1899, p. 225) stains embryonic cartilage for six to twenty-four hours in orcein $0.5 \mathrm{gr}$., alcohol 40 , water 20 , hydrochloric acid 20 drops, and mounts in balsam. Matrix blue, nuclei red.

Kallius (Anat. Hefte, xxx, 1905, p. 9) stains first with borax carmine or alum-carmine, then (sections) for ten minutes in satu- 
rated solution of thionin, and washes out with alcohol of 70 per cent. Said to be specific for embryonic cartilage.

Vastarini-Cresi (Att. Accad. med.-chir. Napoli, 1907, p. 4) stains sections of embryonic cartilage with borax carmine, then with muchæmatein (alcoholic solution without acid), and then with Orange G. in alcohol.

BAYERL's method for ossifying cartilage (Arch. mik. Anat., 1885, p. 35) :-Portions of ossified cartilage-are decalcified as directed, $\S 555$, cut in paraffin, stained in Merkel's carmine and indigocarmine mixture, and mounted in balsam.

MAYer (Grundzüge, LEE and MAYER, 1910, p. 393) prefers to all these resorcin fuchsin, $\S 758$, the precipitate being freed from iron chloride by washing before dissolving in the alcohol.

Aqueous solution of benzoazurin has been commended as a stain for ossifying cartilage by ZschокKe, see Zeit. wiss. Mik., x, 1893, p. 381.

A process of BAUMGarten's has been given, $\S 388$.

Moerner (Skandinavisches Arch. Physiol, i, 1889, p. 216 ; Zeit. wiss. Mik., vi, 1889, p. 508) gives several stains for tracheal cartilage, chiefly as microchemical tests, for which see third edition.

See also a critique of these methods by Wolters in Arch. mik. Anat., xxxvii, 1891, p. 492 ; and on the whole subject of cartilage see ScHIEFFERDECKER's Gewebelehre, p. 331.

FusARI (Arch. Ital. Biol., xxv, 1896, p. 200) makes sections of fresh cartilage, puts them for twenty-four hours into 1 per cent. nitrate of silver, washes, dehydrates, and exposes to the light in balsam.

See also Disse, Anat. Anz., xxxv, 1909, p. 318, a stain for dentine (hæmalum followed by a mixture of Säurerubin and Orange G); and Retterer and Lelievre, C. R. Soc. Biol., lxx, 1911, p. 630.

\section{Skeletons of Embryos.}

9y9. Cartilaginous Skeletons of embryos (Van Wruhe, Proc. $K$. Akad. Wetensch. Amsterdam, 1902, p. 47) may be studied by staining embryos for a week in a solution of $0.25 \mathrm{grm}$. methylen blue in 100 c.c. of 70 per cent. alcohol with 1 per cent. of hydrochloric acid. Wash out in alcohol with 1 per cent. of hydrochloric acid until no more colour comes away (about a week) and mount in balsam. The cartilage remains blue, all the other tissues being colourless.

Similarly, Lundvall (Anat. Anz., xxv, 1904, p. 219, and xl, 1912, p. 639), using toluidin blue. Thionin blue also may be used.

Similarly also BAKAY (Verh. A nat. Ges., 1902, p. 248), with Bismarck brown (the embryos having been previously treated with nitric acid of 3 per cent.).

For fish embryos, Professor E. S. Goodrich, of Oxford, informs me that thionin is excellent.

For the Spalteholz method of clearing such preparations see $§ 592$. 
780. Demonstration of Centres of Osteoblastic Activity by Trypan Blue (P. G. Shipley and C. C. Macklin, Anat. Record, x, 1915-16). -If an azo dye like trypan blue be administered to a very young animal, the bones are stained quickly and very intensely with vital colour. The dye is injected in a 1 per cent. solution into the peritoneal cavity (less preferably subcutaneously). The animal is killed forty-eight hours after staining, and the tissues are fixed by 10 per cent. neutral formalin.injected through blood vessels, followed by immersion in 10 per cent. formalin for twenty-four to forty-eight hours. Bones are washed thoroughly, hardened in ascending grades of alcohol, after which the soft parts are dissected away. Clear in benzol and then in oil of wintergreen. Study with dissecting microscope. Refer also to J. Thornton Carter, $\S 775 \mathrm{~A}$.

780A. Potash Method for Osteoblastic Centres (Schultze, Grundriss d. Entwickl. d. Menschens, 1897, and F. P. MaLL, Amer. Journ. Anat., v, No. 4, 1905-6).

Embryos of mammals after fixation in alcohol may be cleared, for the study of the ossification centres, by means of weak potash. For alcohol specimens Mall considers that Schultze's solution is too strong, and uses instead a 1 per cent. $\mathrm{KOH}$ solution for a few hours. With weak solutions the tissues of the smaller embryos remain firm, and, in the end, the specimen is transparent, with the bones held in place. After treatment with the potash, the embryo is placed in the following solution for days, or even months :-

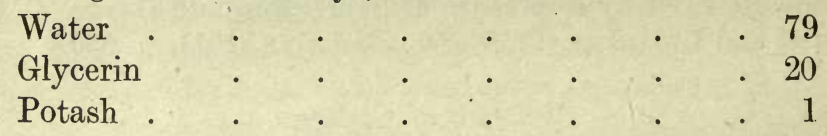

From time to time the embryo may be returned to a 3 per cent. solution of potash for a number of hours to hasten the clearing process; then returned to the glycerin solution, which helps to hold the parts together. . When properly cleared, upgrade gradually to pure glycerin, in which they may remain.

MaLL (op. cit.) clears formalin embryos in 10 per cent. potash for about a month or longer. Formalin renders the connective tissues very tough, and this strong $\mathrm{KOH}$ solution is necessary. Refer also to $\S 592$. 


\title{
CHAPTER XXX.
}

\author{
BLOOD AND GLANDS.
}

Blood.

781. Fixing and Preserving Methods.-The school of Ehrlich used to fix by heat. A film of blood was spread on a cover-glass and allowed to dry in the air, and then fixed by passing the cover a few times, three to ten or twenty, through a flame, or by laying it face downwards on a hot plate kept for several minutes or as much as two hours at a temperature at which water not only boils, but assumes the spheroidal state $\left(110^{\circ}\right.$ to $150^{\circ} \mathrm{C}$.). For details see Gulland, Scottish Med. Journ., April, 1899, p. 312 ; Rubinstein, Zeit. wiss. Mik., xiv, 1898, p. 456; Zielina, ibid., p. 463. But I believe they have now well-nigh abandoned this barbarous practice.

In wet methods either the blood is mixed at once, on being drawn, with some fixing and preserving medium, and studied as a fluid mount, or films are prepared and put into a fixing liquid before they have had time to dry, or after drying in the air without heat for a few seconds (at most ten to thirty).

To make a film, place a very small drop of blood on a perfectly clean slide. Bring down on to the slide the edge of another slide held over it at a slope; move this along till it touches the edge of the drop and the blood runs along the angle between the two slides. Then move the second slide away from the drop, and the blood will follow it and be drawn out into a film without being crushed. Similarly with two cover-glasses, to make a cover-glass film, which can be floated face down on to fixing or staining liquids in a watch-glass.

Some persons make films by flattening blood between two coverglasses which are afterwards separated by sliding the one over the other; but that produces injurious kneading of the cellular elements.

Most of the usual fixing agents are applicable to blood. But it is often necessary to employ only such as are favourable to certain stains. Those most recommended in this respect are alcohol, formol, sublimate (should not be too strong), osmic acid in very light fixation, or absolute methyl alcohol, which is an energetic fixative of dried films. 
Air-dried films ought to be fixed before putting into aqueous or glycerin stains, else they will wash off; but this is not necessary for alcoholic stains.

782. Fixing and Preserving in Bulk.-Most morphologists are agreed that by far the most faithful fixing agent for blood-corpuscles is osmic acid. A drop or two of blood (BIONDI recommends two drops exactly) is mixed with 5 c.c. of osmic acid solution, and allowed to remain in it for from one to twenty-four hours. As a rule the osmic acid should be strong-1 to 2 per cent. Fixed specimens may be preserved for use in acetate of potash solution (MAX Flesch, Zeit. wiss. Mik., v, 1888; p. 83).

GRIESBACH also (ibid., 1890, p. 328) combines the osmic acid with certain stains. He mentions methyl green, methyl violet, crystal violet, safranin, eosin, Säurefuchsin, rhodamin, and iodine in potassic iodide.

Rossi (ibid., vi, 1889 , p. 475) advises a mixture of equal parts of 1 per cent. osmic acid, water, and strong solution of methyl green, permanent mounts being made by means of glycerin cautiously added.

Ewald (Zeit. Biol., xxxiv, 1897, p. 257) mixes 3 to 4 drops of blood of amphibia or reptiles with 10 c.c. of a solution of 0.5 per cent. osmic acid in 0.5 per cent. salt solution (for mammals 0.6 to 0.7 per cent. salt), siphons off the supernatant liquid after twentyfour hours with his capillary siphon $(\S 3, p .4)$, and substitutes water, alum-carmine, etc., and lastly, 50 per cent. alcohol.

Weidenreich (Arch. mik. Anat., Ixxii, 1908, p. 213) lays a cover with a drop of blood on it on a layer of agar-agar ( 1 per cent. in salt solution of 0.8 per cent.), and after five minutes runs in osmic acid of 1 per cent., and after five minutes more removes the cover.

Dekhuyzen (Anat. Anz., xix, 1901, p. 536) recommends a mixture of either 3 or 9 volumes of 2 per cent. osmic acid with 1 of 6 per cent. acetic acid, containing $\frac{1}{8}$ per cent. of methylen blue, which he calls "Osmacet."

The mercurial liquids of Pacini ( $\$ 414$ ) used to be considered good. Hayem ("Du Sang," etc., Paris, 1889; see also Zeit. wiss. Mik., vi, 1889, p. 335 ) has the following formula: sublimate 0.5 , salt 1, sulphate of soda 5, and water 200 . This should be mixed with blood in the proportion of about $1: 100$. Eosin may be added to it. LöwIT's formula (Sitzb. k. Akad. Wiss. Wien, xcv, 1887, p. 144) consists of 5 c.c. cold saturated sublimate solution, 5 grms. sulphate of soda, 2 grms. salt, and 300 c.c. 'water. Mosso finds that both of these are too weak in sublimate. 
DuboscQ (Arch. Żool. Expér., vi, 1899, p. 481) uses (for blood of Chilopoda) a solution of acetic acid, copper acetate, copper chloride, osmic acid, thionin, $1 \mathrm{grm}$. each, water 400 , which, mixed with the blood, fixes and stains in about two minutes.

Formol has lately been used. Marcano (Arch. de Med. Expér., xi, 1899, p. 434) mixes fresh blood with a mixture of 100 parts of sodium sulphate of sp. gr. 1.020 and 1 of formol; or with water 85 to 100 parts, sodium chloride 1 , and formol 1 .

Kizer (Journ. Roy. Mic. Soc., 1900, p. 128) simply mixes 1 drop of blood with 3 of 2 per cent. formalin, and allows to stand for an hour.

Schridde (Hamat. Techn. Jena, 1910, p. 17) lets blood drop into a mixture of 1 part of formol, 9 of liquid of Müller, and 10 of water, fixes therein for two to four hours at $40^{\circ} \mathrm{C}$., filters, washes and brings through alcohol and chloroform into paraffin for sectioning.

783. Fixing and Preserving in Films.-MuIR (Journ. of Anat. and Phys., xxvi, 1892) makes cover-glass films and drops them into saturated sublimate solution, and after half an hour washes, dehydrates, and passes through xylol into balsam.

Gulland (Brit. Med. Journ., March 13th, 1897 ; Scottish Med. Journ., April, 1899) makes cover-glass films, and after a few seconds drops them face downwards into a solution of-

Absolute alcohol saturated with eosin . . 25 c.c.

Pure ether . . . . . 25 "

Sublimate in absolute alcohol (2 grms. to

$$
10 \text { c.c.) . . . . . } 5 \text { drops. }
$$

After three or four minutes they are washed, stained, and mounted in balsam.

For JenneR's fixing and staining and staining method, see next section.

Many recent authors fix wet films with formol. Benario (Deut. med. Wochenschr., 1895, p. 572) mixes 1 part of 10 per cent. formol with 9 of alcohol (the mixture must be freshly prepared), and plunges films into it for a minute.

Similarly GULLAND, with 1 part of formol to 9 of alcohol.

Similarly Wermel (see Zeit. wiss. Mik., xvi, 1899, p. 50), who combines various stains (methylen blue, eosin, gentian, etc.) with the formol.

Edington (Brit. Med. Journ., 1900, p. 19) exposes films for fifteen to thirty minutes to vapour of formol under a bell-jar.

ScotT (Journ. of Path. and Bacter., vii, 1900, p. 131) exposes 
films to the vapour for about five seconds and drops into absolute alcohol, and after fifteen minutes stains and mounts.

A short exposure (thirty seconds) to vapour of osmium has also been recommended.

Szecsi (Deutsch. med. Wochschr., 1913, p. 1584) has recommended Lucidol for blood smears, and smears of fæces containing protozoa and cysts. The formulæ for an acetone and a pyridin solution will be found on p. 59, $\S 107$, and also of an acetone-xylol solution for subsequent washing of the smears.

It is best to keep a sufficient quantity of the fixing solutions in staining jars. Make a smear, allow it to dry, and place it in the acetone peroxide of benzol solution for fifteen minutes; transfer to the acetone xylol solution for ten minutes in order to remove the lucidol; wash off in pure methyl alcohol ; the slide is now ready for staining. It will be found that most of the current stains used for such smears will act successfully after the lucidol fixation. Pappenheim's panoptic method ( $\$ 784$ ) is recommended.

For smears of frees a fixation of twenty minutes in the pyridinbenzol peroxide solution is used; wash as above, in acetone-xylol, or pyridin-xylol, and then in methyl-alcohol.

Possibly the substitution of pure acetone for the methyl alcohol bath might prove advantageous in some ways.

y84. Stains for Blood.-Fresh (unfixed) blood can be stained on the slide. See also $\S 1008$, et seq.

Toison (Journ. Sci. méd. de Lille, fév., 1885 ; Zeit. wiss. Mik., 1885 , p. 398) recommends that it be mixed with the following fluid :

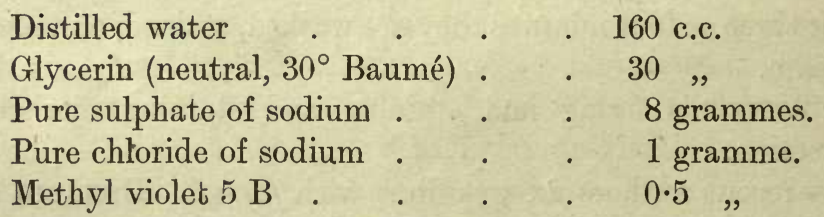

(The methyl violet is to be dissolved in the glycerin with one half of the water added to it; the two salts are to be dissolved in the other half of the water, and the two solutions are to be mixed and filtered.) This mixture stains leucocytes sharply, which facilitates enumeration.

Brzzozero and Torne (Arch. Sci. Mediche, 1880, p. 390) dilute a drop with normal salt solution containing a little methyl violet, which stains nuclei intensely, cytoplasm less intensely.

Similarly Giglio-Tos (Zeit. wiss. Mik., 1898, p. 166), diluting with saturated solution of neutral red in salt solution, which stains 
hæmoglobigenous granules in five to ten minutes. This is also recommended by EhrLich and Lazarus. See $\S 309$.

Similarly also Ross (Trans. Palh. Soc., 1907, p. 117), using polychrome methylen blue.

Levaditi (Journ. Phys. path. Gén., Paris, 1901, p. 425) allows solution of Brillantkresylblau in alcohol to dry on a slide, puts a drop of blood on the dried layer, and covers. Similarly CesarisDemel (Arch. path. Anat., 1909, p. 92), with a mixture of this dye and Sudan III ; and NAKANISHI (Centralb. Bakt., 1901, p. 98), with methylen blue BB.

Fixed films may be treated with the usual tissue stains, eosin being an important one, as it stains rose-red all parts of blood-cells that contain hæmoglobin. EHRLICH's acid hæmatoxylin, with $0.5 \mathrm{gr}$. of eosin dissolved in it, is a good general stain. Or, stain with hæmalum, and then with eosin $(0.5$ per cent. in alcohol or water).

EHRLICH's triacid ( $§ 296$ ) gives good general views, and demonstrates neutrophilous granules. His mixture for eosinophilous cells has been given ( $\$ 311)$.

Pappenheim's panoptic triacid (on sale by Grübler) is Ehrlich's triacid with methylen blue in place of the methyl green.

Chenzinski's mixture, which is good, has been given ( $\$ 313$ ). Stain for six to twenty-four hours in a stove. This gives rise to precipitates. To avoid them (Willebrand, Deutsch. med. Wochenschr., 1901, p. 57) you may make a mixture of equal parts of 0.5 per cent. solution of eosin in 70 per cent. alcohol and saturated solution of methylen blue in water, and add acetic acid of 1 per cent. drop by drop till the mixture begins to turn red, and filter before use. Or (Mrchaelis, ibid., 1899, No. 30) make (a) a mixture of 20 parts 1 per cent. aqueous methylen blue with 20 of absolute alcohol, and $(b)$ a mixture of 12 parts 1 per cent. aqueous eosin with 28 of acetone, and for staining mix equal parts of these and stain for half a minute to ten minutes.

JenNeR (Lancet, 1899, No. 6, p. 370) mixes equal parts of 1.2 to $1 \cdot 25$ per cent. water-soluble eosin (Grübler's) and 1 per cent. methylen blue, filters after twenty-four hours, washes the precipitate on the filter, dries it, and dissolves it in 200 parts of absolute methyl alcohol (the solution can be had ready made from Grübler and Hollborn). (Or, simply mix 125 c.c. of 0.5 per cent. solution of the eosin in methyl alcohol with 100 c.c. of 0.5 per cent. solution of methylen blue.) Cover-glass films are floated on to this, in which they are fixed and stained in three minutes. Wash off the stain with a little 
water (not under the tap), dry, and mount in balsam. Erythrocytes red, all nuclei blue, parasites blue, but with unstained nuclei.

The methods of MAY and GRÜNWALD are closely similar to this.

Assmann (Münch. med. Wochenschr., 1906, No. 28; “Das eosinsaure Methylenblau," Leipzig, 1908, p. 35) treats fresh films for half a minute to three minutes in a Petri dish with a few drops of Jenner's solution (from Grübler and Hollborn), then pours on 20 c.c. of distilled water with 5 drops of $\frac{1}{10}$ per cent. solution of lithium carbonate, leaves for five minutes, rinses in distilled water, dries with blotting paper, and mounts in neutral balsam.

The foregoing mixtures give a stain - seemingly due to the formation of an eosinate of methylen blue-in which the nuclei of bloodcells are blue and their plasma red to violet. It was made out by Romanowsky (St. Petersburger med. Wochenschr., 1891) that under certain conditions mixtures of these two dyes give a stain which is in some respects the inverse of this, blood-cells being stained in divers hues, according to their kinds, and any protozoan parasites that may be present showing red nuclei and blue plasma, which greatly facilitates their detection and diagnosis. This reaction appears to be due to the formation of an eosinate-not of methylen blue, but of Methylenazur (§ 377). The method, only vaguely indicated by Romanowsky, has undergone, at the hands of Ziemann, Zettnow, Nocht, Reuter, Michaelis, Ruge, Maurer, Leishman, Giemsa and others, numerous modifications which have culminated in the establishment of a process worked out by Giemsa as perhaps the most trustworthy and efficient of "Romanowsky" stains. This is as follows :

Giemsa's Azur-eosin process. You start with a mixture of eosin with methylenazur (instead of methylen blue). This mixture is very troublesome to prepare, and is best obtained ready made from Grübler and Hollborn (their "Giemsa'sche Loesung für Romanowskyfaerbung"*). Air-dried films (Deutsch. med. Wochenschr., 1907, No. 17) are fixed in alcohol or in methyl-alcohol (two to three minutes), and dried with blotting paper. They are treated for ten to fifteen minutes with a dilution of 1 drop of the stock mixture to 1 c.c. of water, washed under a tap, dried with blotting paper, and again dried in the air and mounted in balsam, or (preferably) preserved unmounted. All reagents, especially the balsam, must be strictly free from acid.

* To make this up from Grübler's powders, dissolve 3 grms. of Azur II-eosin and 8 decigrammes of Azur II in $125 \mathrm{grms}$. of glycerin and 375 of methyl-alcohol. 
Wet films (ibid., 1909, p. 1751) are treated as follows: Fix them for twelve to twenty-four hours in a mixture of 2 parts saturated aqueous solution of sublimate with 1 of absolute alcohol. Wash and treat for five to ten minutes with a mixture of 2 parts of iodide of potassium, 100 of water, and 3 of Lugol's solution. Wash, and treat for ten minutes with 0.5 per cent. solution of sodium thiosulphate. Wash, and stain as above (changing the stain for fresh after half an hour), for one to twelve hours. Then pass through mixtures of acetone with first 5, then 30 , then 50 parts per cent. of xylol into pure xylol, and mount in cedar oil. This process is applicable to sections.

Or (ibid., 1910, p. 2476) a slide is placed in a Petri dish and covered with a mixture of equal parts of methyl-alcohol and stock mixture. After half a minute this is poured off and enough distilled water poured in to cover the slide, and the whole is rocked to mix the two. After three to five minutes, wash in running water, dry, and mount in cedar oil.

By any of these processes nuclei (red) are demonstrated not only in hæmatozoa, but in many bacteria, spirochætæ, coccidia, sarcosporidia, etc.

See also, for paraffin sections, SchuberG, in Deutsch. med. Wochenschr., xxv, 1909, No. 48, or Zeit. wiss. Mik., xxvii, 1910, p. 161, who passes through acetone and xylol into balsam.

The older Romanowsky stains published by the authors mentioned above, as also Laveran's "Bleu Borrel " seem to be superseded by Giemsa's.

Leishman's Romanowsky Stain (Brit. Med. Journ., March 16th and September 21st, 1901) is as follows: To a 1 per cent. solution of Grübler's medicinal methylen blue in water add 0.5 per cent. of sodium carbonate, heat to $65^{\circ} \mathrm{C}$. for twelve hours and let stand for ten days. Then add an equal volume of $0 \cdot 1$ per cent. solution of Grübler's Eosin extra B, let stand for six to twelve hours, collect the resulting precipitate on a filter, wash it until the wash water comes off colourless, dry and powder. For staining, dissolve $0.15 \mathrm{grm}$. in 100 c.c. of pure methyl alcohol. Stain cover-glass films (airdried) for five to ten minutes; flood the film with water for one minute, and examine, or dry (without heat) and mount in xylol balsam. Nuclei in shades of red, cytoplasm bluish, parasites blue with ruby red chromatin.

RAADT (Münch. med. Wochenschr., 1911, No. 27 ; Zeit. wiss. Mik., 1912 , p. 236) obtains a Romanowsky stain of blood and parasites with JENneR's solution. Films fixed with alcohol and ether are 
first stained for five to ten minutes in solution of 1 part methylenblau med. puriss. Hoechst, 0.5 part of lithium carbonate and 100 of water, kept for at least three weeks and diluted with 10 volumes of water. Rinse with water, dry with blotting paper, flood with JENNER's solution diluted with 2 or 3 volumes of water; and stain for five to ten minutes. Wash, dry with blotting paper, and mount. See also Scotr, Folia Haem., xii, 1911.

785. Pappenneim (Anat. Anz., xlii, 1912, p. 525) recommends the following for sections of homopoietic tissues, and also of kidney, liver, hypophysis, suprarenals, lung, intestinal epithelium and central nervous system. Fix in Orth's Formol-Müller, stain sections for twenty minutes in a stove in "aqueous diluted alcoholic" solution of MAY-GRÜNWALD or JENNER diluted with 8 volumes of water; after-stain for forty minutes in the stove in "aqueous GIEMSA solution (15 c.c. of water with 10 drops of glacial acetic acid)" ; differentiate in 100 c.c. of water with 5 to 6 drops of acetic acid; wash, dry between blotting-paper ; dehydrate in mixture of equal parts of acetone and absolute alcohol, and mount in neutral balsam. The result is not a Romanowsky stain, but a pale methylen-blue-eosin stain.

See also Wright, Pub. Massachusetts Gen. Hosp., iii, 1910, p. 1, or Journ. R. Micr. Soc., 1910, p. 783.

For the special technique of eosinophilous cells see MaRtinotri in Zeit. wiss. Mikr., xxvi, 1909, p. 4 (alphabetical bibliography of eight pages).

y86. Demonstration of Blood-plates of Bizzozero (KEMP, Studies from the Biol. Lab. Johns Hopkins Univ., May, 1886, iii, No. 6 ; Nature, 1886, p. 132).-A somewhat large drop of blood is placed on a slide, and quickly washed with a small stream of normal salt solution. The blood-plates are not washed away, because they have the property of adhering to glass. They may be stained with solution of 0.02 per cent. of methyl violet or $1: 3,000$ of gentian violet, in salt solution. To make permanent preparations of them, they should first be fixed, by putting a drop of osmic acid solution on the finger before pricking it.

They may also be stained in films, especially by the Romanowsky method. According to PAPPEnheIm (Farbchemie, p. 107) Wasserblau is almost specific for them.

WRIGHT (Journ. Morph., xxi, 1910, p. 274) studies them in tissues, after fixation with formol or sublimate (not Zenker) by staining with a modified Giemsa stain, and bringing through acetone and oil of turpentine into turpentine colophonium. Details loc. cit. or Journ. Roy. Mic. Soc., 1910, p. 783.

See also Dekhuyzen, Anat. Anz., xix, 1901, p. 533 ; Корsch, 
Intern. Monatschr. Anat. Phys., xxi, 1904, p. 344, and xxiii, 1906 , p. 359 ; Deetjen, Zeit. phys. Chem., lxiii, 1909, p. 1.

78\%. Demonstration of a New Body in Red Blood Corpuscles (GoLGI, Boll. Soc. Med. Chir. Pavia, 1919, xxxi ; Homatologica (Napoli), 1920). This original communication of Golgi gives two methods of interest to workers on blood: (1) Blood-films are fixed twenty-four to forty-eight hours in equal parts of saturated solutions of mercury chloride and potassium bichromate. They are then transferred into equal parts of 2 per cent. mercury chloride and potassium bichromate, to which 5 to 10 c.c. of 1 per cent. gold chloride and 5 to 10 drops of acetic acid are added. The films are observed in glycerol, starting from the second or third day after the last treatment until the fifteenth or twentieth day; (2) drops of blood are fixed in a watch-glass by means of a fluid composed of 2 per cent. mercury chloride 60 c.c., saturated solution of picric acid 20 c.c., 1 per cent. osmic acid 10 c.c., acetic acid 5 drops, with the addition either immediately or after eight to twenty-four hours of 10 c.c. of 1 per cent. gold chloride for every 50 c.c. of fixative. Preparations are made from the sediment with some glycerol, from the second until about the tenth day after fixation.

Both methods show within the red blood corpuscles a peculiar body with a diameter of about one-third that of adult corpuscles and of about half that of foetal ones. The body occupies the central part of the erytrocytes, and particularly by means of the second process it appears to have a fine, sometimes more fibrillar, sometimes more reticular structure. Its contours, though clearly defined, are irregular, and there is no indication whatever of a limiting membrane. This "reticulo-fibrillar apparatus" is not a nucleus, as the latter remains colourless by the new methods, not only in the white, but also in the nucleated red corpuscles, in which the apparatus appears concentrically arranged round the unstained nucleus. According to Golgi this apparatus does not correspond to any of the structures already described within the red corpuscles, but it reminds one a little of the endoglobular body recently demonstrated by Petrone, by means of a lead impregnation method, and not to be confused with the well-known Petrone's bodies. Golgi is convinced that the images obtainable by his two new methods are the expression of a real structure situated within the red blood corpuscles, but he does not feel able at present to give any opinion about their significance. The new methods stain also the centrosome in the white corpuscles of the blood. 
788. WeigerT's Fibrin Stain (Fortschr. d. Med., v, 1887, No. 8, p. 228).-Sections (alcohol material) are stained in a saturated solution of gentian or methyl violet in anilin water ( $\$ 286)$. They are brought on to a slide and mopped up with blotting-paper, and a little Lugol's solution is poured on to them. After this has been allowed to act for a sufficient time they are mopped up with blottingpaper, and a drop of anilin is poured on to them. The anilin soon becomes dark, and is then changed for fresh once or twice. The anilin is then thoroughly removed by means of xylol, and a drop of balsam and a cover are added. This stain may be applied to celloidin sections without previous removal of the celloidin.

See also the modifications of this method by Kromayer ( $\$ 656)$; Benecke (\$690); UnNa (Monatssch. prakt. Dermat., xx, 1895, p. 140); WolfF (Zeit. wiss. Mik., xv, 1899, p. 310); and one of another sort by Kockel (Centralb. allg. Path., $\mathrm{x}, 1899$ ).

789. Elective Staining of Erythrocytes (K. OKaJIma, Anat. Record, $\mathrm{xi}, 1917$ ). - This stain is based on the fact that the phosphomolybdic acid lake of alizarin stains, shows a special affinity for hæmoglobin. Fix material in formol, sublimate, chrome, etc. Transfer sections on slide to aq. dest.; mordant in 10 per cent. phosphomolybdic acid solution for thirty seconds to two minutes; wash in water; stain in this mixture for twenty minutes to twenty hours :- sodium sulfalizarinate, saturated aqueous solution, 100 c.c.; and 10 per cent. phosphomolybdic acid, aq. solution, 30 c.c. (10 to 50 c.c.); wash in water; alcohols, xylol, balsam. Erythrocytes go bright yellow orange. Counter-staining may be done in Ehrlich's hæmatoxylin.

The completeness of the "specificity" of this method is open to doubt, but it gives interesting results.

990. Intravital Staining with Benzidine Dyes.-Bouffard (Ann. de l'Inst. Pasteur, xx), then Goldmann (Beit. z. Klin. Chir., lxiv, have shown that animal tissues may be "stained" intra vitam by the injection of several benzidine dyes. There are some categories of cells in the body which seem to show a special affinity for phagocytosing or, at least, ingesting in some way granules of certain of these colloid dyes. These various cells are often called pyrrhol cells (Goldmann), macrophages (Evans), histiocytes (Aschoff-Kiyono), or resting wandering cells (Tschaschin).

According to HaI. Downey (A nut. Record, xii, 1917) the process of "staining" is one of storage or ingestion, and not of true staining, and attempts to classify cells according to their reactions to these colloidal 
dyes are a failure. This author believes that blood cells behave towards these dyes just as they do to any foreign matter. Other authors do not agree. "To dismiss these cells (pyrrhol cells) as scavengers is to do them an injustice, for, however important this function may be, their service to the body is a far greater one " (P. G. SHIPLex, Amer. Journ. Physiol., xlix, 1919, p. 300).

Evans and Schulemann (Science, N.S., 1914), believe that vital staining with azo dyes is the result of "phagocytosis" of ultra-microscopic dye particles, existing in a state of fine dispersion as an hydrosol. In using the term "phagocytosis" Evans does not quite mean an engulfing by pseudopodia as with amoba. P. G. SHIPIsy (Amer. Journ. Physiol., 1919, p. 285) points out that some cells which are most active in phagocytosing bacteria and other coarse particles, take no part, under ordinary conditions, in the segregation of vital dyes in the body of the living animal.

It has been stated that the benzidine dyes are not characterised by a propensity for staining the mitochondria, as are Janus green or dahlia. The granules in cells which store ultramicroscopic particles of the benzidine dyes seem to be something apart from the mitochondria. Only occasionally the mitochondria, as such, take up a benzidine dye like trypan blue. In tissue cultures from forty to sixty hours old many of the cells are seen to contain large greyish granules which were either not present in the early stages or were not very noticeable. Such granules ("segregation granules " of Shipley, and possibly degeneration or "neutral red" granules of the Lewises, Amer. Journ. Anat., 1915), stain red in neutral red and deep purple in cresyl blue, and by using a combination of trypan red and Janus green, it can be shown, according to Shipley, that the mitochondria (green in the Janus) and the segregation granules which take up the azo red dye, are separate entities. This opinion is not shared by Tschaskin (Fol. Howm., 1914), by LEVY (R. Accad. d. Lincei, 1916), and by Maximow (Arch. Russ. d'Anat. d. Hist. et d'Embryol., 1916).

Trypan blue is also used for demonstrating areas of osteoblastic activity (Shipley and Macklin). See $§ 780$.

The Methods of using Benzidine Dyes are as follows:-Trypan blue and pyrrol-blue of 1 per cent. strength in Ringer's solution are injected subcutaneously, intraperitoneally or into the blood vessels. Whereas 1 c.c. of a 1 per cent. solution per 20 grms. of the animal's body weight injected subcutaneously has no ill-effect on the animal, no more than 0.5 c.c. of the same solution should be used for intravascular work. In the latter case coloration sets in speedily, increases up to the second day, but rapidly fades after the fourth day; in any case, quicker than when gradual absorption of the stain takes place through the lymphatic channels. It is undoubtedly safest and best for histological study to inject the staining fluids subcutaneously. Injections of 1 c.c. of a 1 per cent. solution per 20 grms. body weight may be repeated many times once a week. 
In some cases Goldmann has given fifteen consecutive injections. These remarks refer especially to small animals like the rat; for bigger animals, such as the rabbit or ape, intraperitoneal injections are preferable to subcutaneous: use the standard of 1 c.c. of a 1 per cent. solution per 20 grms. of animal's weight.

Trypan blue, isamin blue and pyrrol blue allow of fixation in 10 per cent. formalin solution (injected intravenously if possible), but it is only tissues stained in trypan blue that allow ordinary processes of histological technique, but even for trypan-blue, the fixative should contain a little formalin. Sections are cut with a freezing microtome from tissue fixed in 10 per cent. formalin not less than forty-eight hours. Stain as necessary in alum carmine or hæmalum, etc. Pappenheim's pyronin and methyl green are good for connective tissues, Ehrlich's triacid for hæmopoetic tissues (Goldmann, Proc. Roy. Soc.; lxxxv, 1912), G. B. Wislocki and H. Downey (Anat. Record, xii, 1917), after staining, fix in Zenker or formol, upgrade in alcohols, imbed in wax and section. Counterstain in hæmalum. Goldmann (loc. cit.) mentions the following "vital stains," apart from those given above: Trypan violet, benzopurpurin, diamin blue B.B., diamin black B.H., vital " neu rot," vital "neu orange," vital " neu gelb," dianil blue R (Grübler). These are used in 1 per cent. strength in salt solution. Trypan blue, trypan red, Congo red, azo blue, and benzopurpurin can be used on tissue cultures by introducing some of the dye into the culture medium (Shipley, Amer. Journ. Physiol., 1919, p. 287).

Apart from the references given above see also, Hoffman (Fol. Hom., 1914), Renault (Arch. d'Anat. Micr., 1907) ; Loele (Fol. Hocm., 1913); Batchelor (Proc. Amer. Assoc. Anat., 1914, Anat. Record, 1914).

791. Microchemical Tests for the Oxygen Place in Tissues.--Recently certain workers have claimed to be able to locate centres or regions of oxidation in the cell by means of some substances sensitive to free oxygen. Unna's method is to use a solution of rongalit white, which is a solution of the leucobase of methylen blue kept in a state of reduction by excess of rongalit, an absorption product of formaldehyde with sodium sulphite. See UnNa (Die Reduktionsorte und Sauerstofforte des tierschen Gewebes, Arch. f. Mikr. Anat., lxxviii, 1911). A. N. DruRx (Proc. Roy. Soc., 1914) has shown that Unna's claim is inadmissible, and consequently his theory of staining by oxidation and reduction is not proven. Graham (Journ. Med. Research, Boston, xxxv, 1916) claims to have demonstrated by means of $\mathrm{H}_{2} \mathrm{O}_{2}$ and naphthol, that the granules of leucocytes and myelocytes contain a peroxidase of the peroxide type. Schultze in his Oxydase Reaction uses $a$-naphthol and dimethyl-p-phenylen-diamin (Merek). Blood and marrow smears fixed in formalin vapour are treated firstly in the a-naphthol solution; pre- 
pared by melting $1 \mathrm{grm}$. of naphthol on the surface of 100 c.c. of aq. dest., and adding potassium hydrate till the naphthol dissolves. After a few minutes in this solution (cooled) the smears are transferred to a 1 per cent. solution of the dimethyl-for the same time, when a blue colour is seen to appear where the oxydases lie. Mount in glycerine jelly, but blue colour fades. Myelocytes and not lymphocytes are said to give a positive reaction.

It is doubtful how far these various colour indicators for oxygen place in cells and tissues are reliable. It has been claimed that by means of the last-mentioned method, it is possible to show that the staining (oxygen place) appears especially around the nucleus of the cell. This has not been confirmed.

\section{Glands.}

992. Mucin.-Hoyer (Arch. mik. Anat., xxxvi, 1890, p. 310) finds that the mucin of mucus cells and goblet cells stains with basic tar colours and with alum hæmatoxylin, but not with acid tar colours. He obtained his best results by means of thionin, and good ones with toluidin blue, both of these giving a metachromatic staintissues blue, mucin reddish - and also with methylen blue (which is particularly useful from its power of bringing out the merest traces of mucin), safranin, etc.

Tissues should be fixed for two to eight hours in 5 per cent. sublimate solution, and paraffin sections stained for five to fifteen minutes in a very dilute aqueous solution of the dye ( 2 drops of saturated solution to 5 c.c. of water).

Hyaline cartilage, the jelly of Wharton, and the Mastzellen of Ehrlich give the same reactions with basic dyes as mucin does.

See also Sussdorf, Deutsche. Zeit. Thiermed., xiv, pp. 345, 349 (Zeit. wiss. Mik., vi, 1889, p. 205); Bizzozero, Atti. R. Accad. di Sci. di Torino, 1889 to 1892 (reports in Zeit. wiss. Mik., vii, 1890 , p. 61 ; and ix, 1892 , p. 219) ; also UNNA, ibid., xiii, 1896, p. 42.

The safranin reaction is not obtained with all brands of the dye ; that of Bindschedler and Busch, in Bâle, gives it, whilst safranin 0 of Grübler does not. UnNA employs chiefly polychrome methylen blue.

As regards the thionin stain, see Hári, Arch. Mik. Anat., Ivviii, 1901, p. 678.

Bruno (Bull. Soc. Nat. Napoli, 1905, p. 220) fixes and stains the skin of the frog in a mixture of 100 c.c. of formol of 1.25 per cent. with 8 c.c. of 1 per cent. solution of thionin. Mucus glands red.

Kultschizky (Arch. mik. Anat., xlix, 1897, p. 8) fixes in his mixture ( $(57)$, and stains sections either in safranin with 2 per 
cent. acetic acid, or in a similar solution of neutral red (two to three days, washing out with alcohol).

MAYER (Mitt. Zool. Stat. Neapel., xii, 1896, p. 303, or last edition) gives the following two formulæ for mixtures that stain exclusively mucus.

793. MAYER's Mucicarmine (op. cit., last §). - One gramme of carmine, and $0.5 \mathrm{grm}$. of aluminium chloride with 2 c.c. of distilled water heated over a small flame for two minutes, and made up to 100 e.c. with 50 per cent. alcohol. This gives a stock solution, which is as a rule to be diluted for use tenfold with distilled or tap water.

MAYER's Muchæmatein (ibid.). - Hæmatein $0.2 \mathrm{grm}$. , aluminium chloride $0.1 \mathrm{grm}$., glycerin 40 c.c., water 60 c.c. An alcoholic solution may be made by dissolving in 100 c.c. of 70 per cent. alcohol, with or without the addition of 2 drops of nitric acid.

794. Mucicarminic Acid (RAwitz, Anat. Anz., xv, 1899, p. 439).One gramme of carminic acid, 2 of aluminium chloride, and 100 c.c. of 50 per cent. alcohol.

995. Goblet Cells. - So far as these contain mucin they give the reactions above described. See PANeth, Arch. mik. Anat., xxxi, 1888, pp. 113 et seq. ; List, ibid., xxvii, 1886, p. 481; and GUYEIsse, C. R. Soc. Biol., 1907, p. 1212.

For intestinal epithelium, especially the cells of PANETH, see also Martin, Unters. ueb. Oberflächen u. Drüsenepithel, Leipzig, 1910 ; and Kulu, Arch. mik. Anat., lxxvii, 1911, p. 541 (sections stained with alum hæmatoxylin, treated for twenty to thirty seconds with tincture of iodine, stained a few minutes with Victoria blue, then with eosin).

796. Salivary Glands.-SoLGER (Unters. z. Naturlehre d. Menschen xv, 5 and 6, pp. 2-15; Festschr. f. Gegenbaur, ii, 1896, p. 211' demonstrates the granules in serous cells and half-moons of the submaxillary gland by hardening in a 10 per cent. solution of formol, and then making sections and staining with hæmatoxylin of Delafield or of Ehrlich, the granules taking the stain.

KraUse (Arch. mik. Anat., xlv, 1895, p. 94) stains sections either with Heidenhain's iron hæmatoxylin or with Ehrlich-Biondi mixture or thionin. See also Krause, ibid., xlix, 1897, p. 709 ; and MüLler, Zeit. wiss. Zool., 1898, p. 640.

79\%. Gastric Glands.-KoLsten (Zeit. wiss. Mik., xii, 1895, p. 314) differentiates the two kinds of cells in stomach glands by over-staining with hæmatoxylin, washing out with alcohol containing 1 per cent. of $\mathrm{HCl}$, blueing with alcohol containing 1 per cent. of ammonia, and, after washing, staining for one to five minutes in a 
weak solution of Säurefuchsin. Peptic cells blue, parietal cells red. Osmic material cannot be employed.

CADE (Arch. Anat. Micr., iv, 1901, p. 4) stains material fixed with Bouin's picroformol in Victoria blue of 1 per cent.

R. and L. Monti (Rich. Lab. Anat. Roma, ix, 1902) demonstrate ducts and canaliculi of delomorphous cells by Golgi's bichromate and silver impregnation, especially with rejuvenated material (see SACERDOTTI), leaving it for five or six days in half-saturated sulphate of copper, then for twenty-four hours in the osmic-bichromate mixture. You can embed in paraffin (rapidly).

798. Intestine.-Benslex (Amer. Journ. Anat., v, 1906, p. 323) stains sections of glands of Lieberkühn in a mixture of equal parts of saturated solutions of orange $G$ and Säurerubin, and then with toluidin blue, and mounts in balsam.

999. Liver.-Braus (Denkschr. Med. Nat. Ges. Jena, v, 1896, p. 307) demonstrates the bile capillaries by the rapid method of GoLGI, hardening in a mixture of 1 part formol with 3 parts liquid of Müller or $\frac{1}{3}$ per cent. chromic acid.

Eppinger (Beitr. path. Anat., xxxi, 1902, p. 230) studies them by means of a complicated modification of WEIGERT's neuroglia stain, and Ciechanowskx (Anat. Anz., xxi., 1902, p. 426) by means of WEIGERT's myelin stain (the 1885 method).

OpPel (Anat. Anz., v, 1890, p. 144 ; vi, 1891, p. 168) puts pieces of liver or spleen (alcohol material) for twenty-four hours into a solution of neutral chromate of potash ( $\frac{1}{2}$ to 10 per cent.), then for twenty-four hours into a $\frac{3}{4}$ per cent. solution of silver nitrate, washes, dehydrates and cuts without embedding. The lattice fibres are only stained near the surface, so that tangential sections must be made.

Similarly BerkLeY, ibid., 1893, p. 772, fixing in picric acid, then in an osmium bichromate mixture, and then silvering.

See also Ranvier, Journ. de Miorogr., ix, x, 1885-6 ; Igacuschi, in Arch. path. Anat., xevii, p. 142, or Zeit. wiss. Mik., 1885, p. 243 (gold process for study of fibrous networks); KupFFER, Sitzb. Ges. f. Morph., etc., München, Juli, 1889, or Zeit. wiss. Mik., vi, 1889, p. 506 ; KraUSE (Arch. mik. Anat., xlii, 1893, p. 57); and TrMofeJew, Anat. Anz., xxxv, 1909, p. 296 (sections of frozen tissue stained with methylen blue).

800. Spleen.-For lattice fibres, see OPPEL, last $\S$.

Kultschitzky (Arch. mik. Anat., xlvi, 1895, p. 675) studies the musculature in sections (of material from liquid of Müller) stained for a day or more in a solution of lakmoid in ether and mounted in balsam. 
For elastic fibres he puts sections for half an hour or a day into a mixture of 800 parts 96 per cent. alcohol, 40 parts 1 per cent. solution of carbonate of potash, 2 parts Magdala red, and 1 part methylen blue.

For the blood vessels he puts sections of Müller material for a few minutes into a solution of 1 or 2 parts of Säurerubin in 400 parts of 3 per cent. acetic acid, washes out in 2 per cent. acetic acid, and after-stains in a similar solution of helianthin or Wasserblau until the red only remains in the erythrocytes.

See also Whiting (Trans. Roy. Soc., Edinburgh, xxxviii, 1896, p. 311); SChumacher (Arch. mit. Anat., lv, 1899, p. 151); WeIDEnreich (ibid., Iviii, 1901, p. 251).

801. Lymphatic Glands.-For lattice-fibres especially, see RoESSLE and Yoshida, Beitr. path. Anat., xlv, 1909, p. 110, or Zeit. wiss. Mik., xxvi, 1909, p. 295. Sections stained with hæmatoxylin and eosin, or Weigert's iron hæmatoxylin, or Bielschowsky's neurofibril stain as applied by Maresch, loc. cit., § 752. The sections should not remain for more than fifteen to thirty minutes in the oxide bath.

See also for the thymus some very complicated methods of SALkIND, Anat. Anz., xli, 1912, Nos. 6 and 7.

802. Kidney.-Sauer (Arch. mik. Anat., xlvi, 1895, p. 110) finds that for the renal epithelium the best fixative is Carnoy's acetic alcohol with chloroform (three to five hours, washing out with absolute alcohol). A mixture of 9 parts alcohol with 1 of nitric acid is also good, as is liquid of Perényi. He stains with iron hæmatoxylin, and after-stains in a very weak solution of Säurerubin in 90 per cent. alcohol, which stains the ciliary plateau. $\mathrm{He}$ macerates with iodised serum or one-third alcohol, staining afterwards with dahlia.

Arnold (Anat. Anz., xxi, 1902, p. 417) employs intra vitam staining methods for the study of the granules of the epithelium cells. Sections of fresh kidney are cut with a Valentin's knife, and brought into a very dilute solution of neutral red, or methylen blue, in which the granules stain in a few minutes or hours. Or saturated solutions of the dyes, or of indigo carmine, may be injected subcutaneously during life, at intervals of fifteen to twenty minutes, and after two to five injections the organ may be excised and sections made and examined (see $\S \S 208$ and 342 to 344 ).

803. Thyroid.-Benslex (Amer. Journ. Anat., xxix, 1916) uses brazilin and water blue. Fix gland in Zenker-formol. Section in paraffin and fix sections to slide with water alone, or very little 
albumen; pass through toluol, absolute alcohol, water, iodise, and place in this brazilin solution for several hours :-

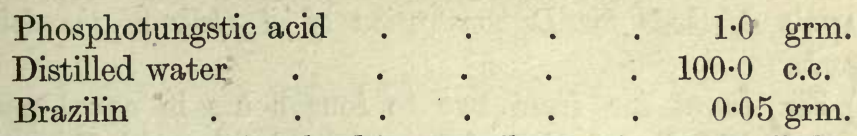

The brazilin is first dissolved in a small quantity of distilled water by the aid of heat and added to the phosphotungstic acid solution. This solution goes bad after three days. After staining in the brazilin, wash in water, and place for five minutes in this mixture :-

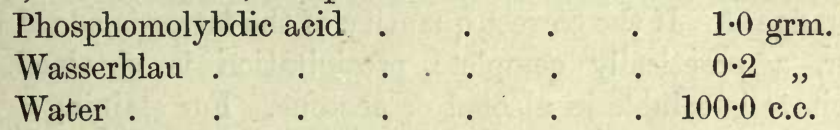

Wash rapidly in water, dehydrate in absolute alcohol, clear in toluol, and mount in balsam. Cytoplasm stains blue to lilac, nuclear chromatin deep red, contents of thyroid vacuoles sky blue, and colloid droplets of Hürthle deep blue to deep red.

804. Pancreas.-Most of the techniques given under the heading of "Mitochondria " and "Golgi apparatus," etc. (\$§ 673-712) give important results with the zymogen granules of the pancreas. The methods of Bensley-Cowdry ( $(686)$, Regaud ( $(685)$, Benda ( $(683)$, and Schridde ( $\S 687)$, all apply here. For the Golgi apparatus Cajal's formalin silver nitrate method may be used ( $\$ 847$ ).

Bensley's Neutral Red Method (Amer. Journ. Anat., xii, 1911-12). -Animal killed by bleeding; a cannula introduced into aorta and a solution of neutral red in isotonic salt solution, containing 1 in 15,000 neutral red, is injected. Immediately after the pancreas has assumed a faint rosy tint a part of the organ is removed-the islets of Langerhans stain intense yellow red, the rest faint rosy-pink. In a short time after mounting the islets remain the only stained elements, owing to bleaching in the acini. Method applicable to the counting of the islets of Langerhans.

Janus Green Method. See $\S 702$.-Islets deep blue on a red background.

Pyronin Method for Ducts.-Inject a 1 in 1,000 solution of pyronin, as above, for neutral red method. The ducts stain intensely red. Double stains may be made by injecting mixed Janus green and pyronin (BeNSLey, op. cit.).

Methylen blue, 1 in 10,000 may also be used for this purpose. After injection fix in 5 per cent. ammonium molybdate, for which see also Chapter XVI.

Grand-Moursel and Tribondeau (C. R. Soc. Biol., liii, 1901, 
p. 187) recommend for pancreas Nicolle's "thionine phéniquée," which stains the insulæ of Langerhans hardly at all, the rest strongly.

LANE's Methods for Demonstration of A Cells of the Islets of Langerhans.

(1) Fix tissue for from two to four hours in equal parts of saturated alcoholic solution of mercury chloride, and $2 \frac{1}{2}$ per cent. potassium bichromate. Wash in 50 per cent. alcohol, then upgrade and embed; $3 \mu$ sections are stained in neutral gentian, obtained by precipitation of equivalent solutions of gentian violet (crystal violet) and orange $G$. If the correct quantity of the latter is added to the former, a practieally complete precipitation is obtained. The precipitate is soluble in alcohol or acetone. For staining add the stock alcohol solution to 20 per cent. alcohol until a solution having the colour of good hæmalum is obtained. Allow to stand for twenty-four hours. Stain for twenty-four hours, blot, dehydrate in acetone, toluol, differentiate in absolute alcohol 1 part, oil of cloves 3 parts, wash in toluol, and mount in balsam.

(2) Fix in 70 per cent. alcohol, then stain in neutral gentian as above.

LANE's Methods for Demonstration of B Cells of Islets ofLangerhans.

Fix for four to twenty-four hours in :-

$$
\begin{aligned}
& \mathrm{K}_{2} \mathrm{Cr}_{2} \mathrm{O}_{7} \text {. . . . . . } 2.5 \text { grms. } \\
& \mathrm{HgCl}_{2} \text {. . . . . . } 5.0 \text {, } \\
& \text { Aq. dest. . . . . . } 100 \cdot 0 \text { c.c. }
\end{aligned}
$$

Dehydrate, clear, embed, and section; stain in neutral gentian as above.

Formalin Bichromate Method for Fixation.-This gives a very regular and reliable fixation, and is suitable where one is carrying out observations which necessitate a successful routine method. Bensley (op. cit.) uses 10 c.c. of neutral formalin to 90 c.c. of Zenker's fluid without acetic acid, for twenty-four hours. Stain in neutral gentian, acid fuchsin and toluidin blue, iron hæmatoxylin or Mallory (§ 314).

Homans (Journ. Med. Research, xxx, 1914) used Bensley's modified Altmann fixative $\left(\mathrm{OsO}_{4}\right.$ of 4 per cent., 2 c.c. ; potassium bichromate of 2.5 per cent., 8 c.c. ; glacial acetic acid, 1 drop), Lane's methods (vide supra), and ordinary hæmatoxylin and eosin.

Very pretty results are obtainable by using Mallory's polychrome methylen blue and eosin ( $\$ 314)$.

See also Babkin, Rubaschin and Ssawitsch, Arch. f. Mikr. Anat., Bd. 74 ; Helly, ibid., Bd. 67 ; Lane, Amer. Journ. Anat. vii, 1907, SAGUCHI, ibid., Vols. 26 and 28, and $\S 713$. 


\section{CHAPTER XXXI.*}

\section{NERVOUS SYSTEM-GENERAL METHODS.}

805. Introduction.-The microscopic investigation of the nervous system pursues two ends. Either it is desired to elucidate the intimate structure of nerve-cells, nerve-fibres and their supporting tissues, or to study the morphology of nerve-cells, their distribution in the grey matter, their connections with each other, and with the nerve fibres which chiefly constitute the white matter, and lastly to investigate the architectural arrangement of both nerve-cells and nerve-fibres in the various regions of the central nervous system. The processes employed in the first case form a group of cytological methods, whilst the processes used in the second instance are spoken of as the anatomical methods.

The processes used in the study of nervous tissue in peripheral organs having been described in the chapter on "Methylene Blue," "Impregnation Methods," "Tegumentary Organs," and "Muscle and Tendon," the following chapters are chiefly devoted to the description of methods for the investigation of the central nervous system.

\section{Fixation.}

806. Fixation by Injection.-Fixation, in the proper sense of the word, is of course out of the question for the human subject. But in the case of the lower animals it is possible to inject fixing fluids into their nervous centres when still in an almost living state. The practice ensures a very rapid penetration into and even distribution within the tissues of the fixing agents, and has, moreover, the capital advantage of greatly helping to prevent distortion of the nervetissues during their subsequent treatment. And as in most instances the practice does not meet with special difficulties, it should be adopted as far as possible also in the case of human subjects, but particularly for a preliminary fixation and hardening of the very soft cerebral mass of young individuals, which is particularly liable to much injury and distortion in the process of removing it from the brain case.

* Revised and in great part rewritten by Dr. C. Da Fano, King's College, University of London. 
The choice of the fluid to be injected depends upon the object in view and the subsequent treatment to which the tissues are to be submitted. In the case of animals it is a good practice to warm the fixing fluid to body-temperature before injecting it, and, whenever possible, to wash out the blood by first injecting physiological solution as suggested by Mann. The injection can be carried out through the carotids if the fixation is to be limited to the encephalon, and through the aorta if it is desired to fix the spinal cord too. The above applies to higher vertebrates and particularly to mammals ; in the case of lower vertebrates, fixation by injection has not, as a rule, the same importance, and one must have recourse to special methods.

See on this subject Golgi, op. cit., in $\S 880$; Gerota, $\S 811$; DE QUervaIn, Virchow's Arch., exxxiii, 1893, p. 481 ; ManN, Ztschr. wiss. Milkr., xi, 1894, p. 482 ; Strong, Anat. Anz., xi, 1886, p. 655 ; Journ. Comp. Neurol., xiii, 1903, p. 291 ; McFarLand, Journ. App. Mior., ii, 1899, p. 541.

\section{Hardening.}

80\%. Hardening by the Freezing Method.-This phrase has often given rise to confusion and should, therefore, be clearly understood. One can harden by freezing either fresh tissues, or material already fixed and consequently also a little hardened. In the first instance small pieces of fresh tissue, immediately after removal and without any previous treatment, are hardened on a freezing microtome. The sections are generally floated on to water, and immediately afterwards treated for a minute on the slide with a 0.25 per cent. solution of osmic acid; or otherwise treated according to the object of one's investigation. In this case the ether freezing method should be preferred, bearing in mind, however, that there is considerable difficulty in obtaining sufficiently good sections, and that the results attainable are very limited particularly since BrodmanN (Journ.Psychol. Neurol., ii, 1903-4, p. 211) has shown that formalin material can be used even for investigations by polarised light. (See also p. 361.)

The hardening by freezing of already fixed material may be also attended with some difficulty, but this will be easily overcome if pieces are relatively small, the fixing agent properly washed away, and one has, eventually, recourse to one or other of the processes described in $\S 183$. Material fixed in formalin, however, does not, as a rule, require any soaking in gum, or syrup, or the like, and is easily cut if the formalin has been washed away. In this case the $\mathrm{CO}_{\mathrm{a}}$ freezing microtome is in my opinion to be preferred. 
The hardening and section cutting by the freezing method of very large pieces require special apparatus and special methods, for which see NaGeotte, C. R. Soc. Biol., lxvii, 1909, p. 542.

808. Hardening by Reagents. - If large pieces of nervous tissue are to be hardened, it is necessary to take special precautions in order to prevent them from being deformed by their weight during the process. The spinal cord or small portions of any region of the encephalon may be cut into thin slices, laid out on cotton wool in a vessel into which the hardening fluid is poured. The specimens may also be suspended in the liquid (§ 34). Another good plan consists in adding to the hardening fluid just enough glycerine or sodium chloride to make tissues float.

If several pieces are placed in the same vessel, they should never be put on top of each other. Voluminous organs to be hardened in toto should be at least incised as deeply as possible in the less important regions. With the exception of the dura mater, the membranes are not generally removed at first, as they serve to protect the tissues. They can be removed partially or entirely later on when the hardening has made some progress. In the case of material intended for Golgi's methods it is best not to remove them at all.

The spinal cord, medulla oblongata and pons Varolii may be hardened in toto, and the preparation hung up in a cylindrical vessel with a weight attached to its lower end to prevent it from becoming distorted.

The cerebrum should have light plugs of cotton wool in the fissure of Sylvius, and as far as possible between the convolutions. If it is desired not to open the lateral ventricles, the hardening fluid may be injected into them. Unless there are special reasons to the contrary, the brain should be divided into two portions by a middle frontal section, or better into two symmetrical halves by a sagittal cut passing through the median plane of the corpus callosum.

The cerebellum should be treated in the same manner.

The action of most hardening fluids is greatly enhanced by heat. But in the judgment of most histologists this rapid hardening is not, as a rule, attended by good results, and one should have recourse to it only for particular reasons and special purposes after a tentative experiment, whenever possible, at establishing the degree of temperature at which the desired results may be obtained without otherwise injuring the delicate structure of the nervous tissues. 
On the other hand the hardening action at room temperature of certain reagents, such as solutions of chromic salts, proceeds so slowly that decomposition may set in before the fluid has had time to act effectively. For this reason voluminous preparations which are to be hardened in toto in solutions of chromic salts, and were not injected as described in $\S 806$, should be put away in a very cool place or in an ice-chest. A human cerebral hemisphere may require eight or nine months for hardening in this way.

The volume of the fluid should always be very large in proportion to that of the pieces of tissue and to their number. It should be taken in solutions as weak as is consistent with the proper preservation of the tissues. It should be frequently changed and its strength gradually increased.

MARIE's method of fixing and hardening in situ is highly recommended; for its indications and contra-indications, see SAINTON and Kattwinkel (Deutsche Arch. klin. Med., lx, 1898, p. 548) and Pfister (Neurol. Centrbl., xvii, 1898, p. 643).

809. The Reagents to be Employed.-As in the case of the fixation by injection one should bear in mind that the preservation of tissues for neuro-histological investigations greatly depends upon the purpose in view. Fixing and hardening fluids which are excellent for cytological investigations are very often unsuitable for anatomical methods. (See $\S 805$.) On the other hand, material collected and prepared for cyto-architectonic or fibro-architectonic studies can hardly be used to elucidate questions regarding the intimate structure of nerve-cells or nerve-fibres. . Alcohol, formalin* and chromic salts are most frequently used because they are generally ready at hand, and because they are useful for carrying out afterwards either a great number of methods, or certain methods, under constant conditions of hardening and staining.

810. Alcohol.- - It is generally employed in the strength of 94 to 96 , per cent., penetrates well and hardens quickly; but as it rapidly

* Wrong as it is, I find it expedient to use the term "formalin" or "formol" in the generally accepted sense, viz., as if it were a chemical reagent, while it is only a commercial denomination which ought not to have been introduced in the histological terminology. See $\S 108$. As is well known, the commercial formalin is only a 40 per cent. solution of formaldehyde; but when in this and the following chapters on the nervous system a 5,10 or 20 per cent. solution of formalin is mentioned, it is intended to mean 5, 10 or 20 parts of commercial formol, and 95, 90 or 80 parts of water, respectively, while, e.g., a 20 per cent. solution of formaldehyde is the commercial formalin diluted with half its volume of water.-C. d. F. 
absorbs water from the tissues the latter shrink considerably, whilst the alcohol loses its fixing and hardening properties through hydration. It has consequently to be changed soon for a fresh supply and used in quantities exceptionally large in proportion to the size of the pieces, which ought to be neither too small nor too large. For this reason one seldom hardens in alcohol voluminous organs, and its use has become on the whole very restricted. Alcohol, however, remains the principal fixing and hardening reagent for cytological investigations by Nissl's method (see $\S 826$ ), and for carrying out some of Ramón y Cajal's reduced silver processes (§ 827), its shrinking influence being counteracted by having recourse for the first fixation to weaker dilutions of alcohol ( 60 to 70 per cent.) to be raised gradually up to 95 or 96 per cent. within the first nine to twelve hours, and to be changed once or twice or more often in the next few days.

811. Formalin.-Since the time when it was introduced into histological technique by F. BLum (Ztschr. wiss Mikr., x, 1893, p. 314); J. Blum (Zool. Anz., xvi, 1893, p. 434); Hermann (Anat. Anz., ix, 1893, p. 112) ; Hoyer, jun. (Anat. Anz., ix, 1894, p. 236); LACH (Monit. Zool. Ital.,.v, 1895, p. 15) and many others, its use has been steadily increasing because of the many advantages it offers. As a matter of fact it penetrates more quickly than solutions of chromic salts, and even than alcohol ; it is not likely to overharden; it allows of the most various after-treatments and methods of staining, including neurofibril stains and Golgi's impregnation method.

Several writers have insisted that for nervous tissue it should not be acid, but some prefer it acid. See "Retina." For neurofibrils it should be preferably neutral. To neutralise it, it is generally sufficient to prepare its solutions with spring water, but one may shake it with magnesium or sodium or calcium carbonate. Some authors prefer to neutralise with ammonia. (See also § 108.)

The strength of the formalin solutions generally used for fixing and hardening nervous tissues varies considerably with the quality of the material in hand, but particularly with the age of the subjects. As a rule the more delicate the material and the younger the subject, the weaker should be the formalin solutions to be employed at first. Generally, however, one starts with a 3 or 5 per cent. solution in the case of very soft tissues, gradually increasing the strength up to 10 or 12 per cent. An adult human encephalon can be very well preserved in a 10 or 15 or 20 per cent. solution with two changes of 
the fluid during the first days of fixation and hardening. See further on this subject, $\S 108$.

Formalin can be associated with, or followed by, alcohol ( $\$ 109)$ or other reagents. Thus Fish (Proc. Am. Micr. Soc., xvii, 1895, p. 319) recommends :-

Water

Formalin

Sodium chloride

Zinc chloride
. 2,000 c.c.

50

100 grms.

15 ,

Brains are left in the mixture eight to ten days or longer, and then transferred into a mixture of water 2,000 c.c. and formalin 50 c.c., in which they may remain indefinitely if the jar is kept tightly stoppered.

PARker and Floyd (Anat. Anz., ix, 1896, p. 156) advise for sheep's brains a mixture of 6 volumes of 95 per cent. alcohol and 4 volumes of 2 per cent. formol. Brains may be kept in the mixture for months.

Flatau (Anat. Anz., xiii, 1897, p. 323) finds that brain increases in weight slightly in 10 per cent. formol (spinal cord somewhat more); whilst in 1 per cent. solution it may increase as much as 24 per cent.

Gerota (Int. Monatschr. Anat., xiii, 1896, p. 108) puts human brains into a 5 or 10 per cent. solution of formol, and after twentyfour hours removes the pia mater, and changes the liquid ; this is also further done every five to seven days, and in one or two weeks the hardening is complete. In the case of fœtal brains of Canis, Felis and Homo, he first injects the vascular system with a 10 to 15 per cent. solution of formol in 85 per cent. alcohol, and then brings the heads into the 5 to 10 per cent. watery solution; after one or two days he removes the brains from the skull and puts them back for fifteen to twenty days into the formol.

Kadyi (Poln. Arch. Biol. Med. Wiss., i, 1901, p. 80) takes 5 parts of formol, 100 of water, and 2 of bicarbonate of soda, for four to ten days.

Herdlicka (Proc. U. S. Nat. Mus., xxx, 1906, p. 304) takes 3 parts of formol, 25 to 45 of water, and 72 to 52 of 95 per cent. alcohol.

STRECK ER fixes small pieces for twenty-four to forty-eight hours in equal parts of 10 or 20 per cent. formol and Ehrlich-Biondi triacid mixtures, and imbeds in paraffin, thus getting a stain at the same time as a fixation. Similarly with toluidine blue fixing it with ammonium molybdate. See Ztschr. wiss. Mikr., xxviii, 1911, p. 17, and the literature discussed therein. 
812. Chromic Salts.-The most commonly used is potassium bichromate. The liquid of Erlicki has a more rapid action than other solutions of chromic salts, but it has been generally abandoned because of the alterations it very often produces.

SAHLI (Ztschr. wiss. Mikr., ii, 1885, p. 1), after investigating the action of the usual solutions, concludes that the best hardening agent for fresh tissues is pure potassium bichromate, in 3 or 4 per cent. solution, the hardening being done in a cold place. He rejects the liquid of Erlicki on account of the precipitates to which it so frequently gives rise.

OBERSTEINER is of the same opinion, and recommends pure bichromate for general hardening purposes ; whilst for the study of delicate structural details he recommends fixing in Fol's modification of Flemming's fluid ( $\$ 47$ ) for twenty-four hours, followed by washing with water and hardening in 80 per cent. alcohol.

In view of the slowness of penetration of chromic salts, it is often advisable to treat the preparations for twenty-four hours or more with 80 to 90 per cent. alcohol, or better, for a few days with a formol solution before putting them into the hardening fluid, or to add formol (say 3 per cent.) to it, in order to avoid maceration of the deeper layers of the tissues. In this case, however, the fluid must be changed after twenty-four to forty-eight hours.

Potassium bichromate should be employed at first of not more than 2 per cent. strength ; this is then gradually raised to 3 or 4 per cent. for the cord and cerebrum, and as much as 5 per cent. for the cerebellum.

Ammonium bichromate should be employed of half the strength recommended for potassium bichromate at first; it may be raised to as much as 5 per cent. for cerebellum towards the end of the hardening.

Nissl (Enzycl. Mik. Technik, ii, 1910, p. 245) uses, for rapid hardening, large quantities of Müller's fluid 100 parts, formol 3 parts, and enough glycerine to make the tissues float. If the solution is often changed, even entire brains are in a few days sufficiently hardened to be safely transferred into pure Müller's fluid, or potassium bichromate of about the same strength,

Betz's method (Arch. mik. Anat., 1873, p. 101). Brain and spinal cord are first hardened, for some days or weeks, in 70 to 80 per cent. alcohol containing enough iodine tincture to give it a light brown coloration (as soon as the alcohol becomes colourless, more iodine must be added.) They should then be definitely hardened in 3 per cent. potassium bichromate for spinal cord, medulla 
oblongata, and pons, 5 per cent. for cerebellum, and 4 per cent. for cerebrum.

The methods of Bevan Lewis (op. cit.) and Hamilton (Journ. Anat. and Physiol., 1878, p. 254) can be considered as superseded, chiefly because they are based on a fixation with methylated spirit (Bevan Lewis) or mixtures of Müller's fluid and methylated spirit (Hamilton), which cannot be used any longer for histological purposes, owing to the excess of impurities it now contains. Also the methods for encephala of Duval (Robin's Journ. de l'Anat., 1876, p. 497) and DeEcke (Journ. R. Micr. Soc., 1883, p. 449) can be considered as obsolete.

ORTH uses formol-Müller changed every few days. See $\S 113$.

Bonvrcini (Ztschr. wiss. Mikr., xxvi, 1909, p. 412) puts entire human brains into 10 per cent. formol (first injected through the carotids or into the ventricles) for six to eight days, cuts them into $1 \mathrm{~cm}$. thick slices by means of a special macrotome, and transfers them into a mixture of 4 parts of potassium bichromate and 2.5 parts of chromium sulphate in 100 of water, and keeps them therein, in the dark, for two months for cerebral hemispheres, twelve to fourteen days for medulla and pons, five or six days for spinal cord. In the case of slices of brain, the fluid must be changed every week.

RAwiTz (ibid., p. 338) puts formol material for exactly five days into alcohol with 10 per cent. tinctura iodi P. G., then for eight to ten into saturated solutions of potassium bichromate changed after the first day, and lastly into 95 per cent. alcohol for three days in the dark.

813. Other Reagents.-Osmic acid is hardly useful for specimens of more than 2 or $3 \mathrm{~mm}$. thickness.

Chromic acid is rarely used alone. Its action is rapid, but uneven, and causes shrinkage and brittleness. A very little (e.g. 3 to 5 drops of a 1 per cent. solution to every 100 c.c. of fluid), added to bichromate solutions will do no harm and quicken the hardening.

Nitric acid has been and still is employed in strengths of 10 to 12 per cent.

Neutral acetate of lead affords, according to KotLAREwSKI (Ztschr. wiss. Mikr., iv, 1887, p. 287), an excellent preservation of ganglion cells.

Corrosive sublimate solutions either alone or mixed with other reagents (see Chapter V.), have been very often used for cytological studies.

Similarly acetic alcohol.

MANN (op. cit.) for cell studies, puts small pieces for twenty-four hours into a solution of 5 parts of potassium iodide and 25 of iodine in 100 parts of water, and then into 70 per cent. alcohol.

OLMACrier ( $\$ 65$ ) recommends his mixture. KodIs (Arch. mikr. Anat., lix, 1901, p. 212) fixes tissues in a saturated solution of cyanide 
of mercury; brings them into 10 per cent. formol, and makes sections by the freezing method.

NeLrs (Bull. Ac. Sc. Belg., 1899-1900) fixes spinal ganglia for twentyfour hours in a solution of 20 grms. of copper sulphate and corrosive sublimate to saturation in a litre of 7 per cent. formol with 5 c.c. of acetic acid.

KING (Anat. Rec., iv, 1910, p. 213) after trying over twenty-five methods on brains of rats, concludes that the best is Ohlmacher's. The brain should be put into it for two to three hours, then for one into 85 per cent. alcohol, then into 70 per cent. with iodine for at least twenty. four hours, then passed through alcohols of ascending strength and alcohol-ether into 2 per cent. celloidin for two to three days, and through chloroform and benzol into paraffin. In her opinion, Bouin's is the best of the formol liquids; Tellyesnicky's is the only one of the bichromate mixtures that equals it. All sublimate mixtures fix the nuclei well, but vacuolise the cytoplasm.

See further particulars on this subject in the original papers of Trzebinski, Virchow's Arch., cvii, 1887, p. 1 ; DIOMIDofF, ibid., p. 499 ; Fisir, The Wilder Quarter-Century Book, 1893, p. 335; Donaldson, Journ. Morphol., ix, 1894, p. 123 ; MARINA, Neurol. Centrbl., xvi, 1897, p. 166 ; Trmofeew, Intern. Monatschr. Anat., xv, 1898, p. 259.

814. Nervous Centres of Reptiles, Fishes and Amphibia.-Mason (Central Nervous System of Certain Reptiles, etc. ; Whirmax's Methods, p. 196) recommends iodised alcohol, six to twelve hours; then 3 per cent. bichromate, changed once a fortnight until the hardening is sufficient (six to ten weeks).

BURCKHARdT (Das Centralnervensystem von Protopterus, Berlin, 1892 ; Ztschr. wiss. Mikr., ix, 1893, p. 347) recommends a liquid composed of 300 parts of 1 per cent. chromic acid, 10 parts of 2 per cent. osmic acid, and 10 parts of concentrated nitric acid, in which brains of Protopterus are hardened in twenty-four to forty-eight hours.

Fish (Journ. of Morphol., x, 1895, p. 234) employed for Desmog. nathus a mixture of 100 c.c. of 50 per cent. alcohol, 5 c.c. of glacial acetic acid, 5 grms. of corrosive sublimate, and $1 \mathrm{grm}$. of picric acid, fixing for twelve to twenty-four hours, and passing through the usual alcohols.

Strong (Journ. comp. Neurol., xiii, 1903, p. 296) fixes (and decalcifies at the same time) the heads of young Acanthias in a mixture of 9 parts of 5 per cent. iron alum and 1 part of formol, for about two weeks, makes paraffin sections, stains with hæmatoxylin, and differentiates in 1 or 2 per cent. iron alum.

Johnston (Morphol. Jahrb., xxxiv, 1905, p. 150) recommends for nerves of Petromyzon to make paraffin section from Zenker material, and stain them with a mixture of saturated solution of nigrosin, saturated solution of picric acid, and 1 per cent. acid fuchsin, in water mixed in proportions arrived at by trial.

\section{Sections.}

815. Imbedding is by no means always necessary, and is objected to in some cases. Indeed sections can be obtained from any part 
of the central nervous system without it, if the tissues are well hardened. Material hardened in alcohol, or in chromic solutions, or treated according to Golgi's methods may be glued on to a piece of wood or hard cork (or still better to a glass cube) by means of a rather thick solution of gum arabic. As soon as it begins to stick to the support the whole is put into 70 to 80 per cent. alcohol to harden the gum, and then fixed in the object-holder of the microtome and cut. Or one can simply make a clean cut at the bottom of the specimen, dry it with blotting paper and stick it on the support with sealing wax or paraffin of high melting point. For section cutting the knife should be wetted with alcohol or water; if the latter is used some soap may be added to it to prevent it from running into drops on the knife.

Formalin material is preferably cut by the freezing method, this being very largely used since the introduction of $\mathrm{CO}_{2}$ microtomes, by means of which many and relatively very thin sections can be rapidly obtained with great economy of time and imbedding reagents.

Imbedding in paraffin is not advised for the nervous system in general, particularly after fixation in alcohol, and bichromate solutions. One should have recourse to it only for special cytological methods, taking care not to use paraffin of too high a melting point.

Imbedding in celloidin is very largely used, and to great advantage, for many purposes.

If, notwithstanding every precaution, the celloidin has not thoroughly penetrated the tissues, good sections may still be obtained by Duval's method of collodionising the sections. The cut surface of the block is dried by blowing on it, and is covered with a thin layer of collodion laid on it with a brush. As soon as this layer has somewhat dried, which happens very rapidly, a section is cut, and the cut surface collodionised as before, and so on for each section. This process gives very good results, and may be advantageously employed even with material that has been successfully imbedded, as it gives greater consistency to brittle or otherwise delicate tissues.

The above applies to section cutting of small, medium-sized and even relatively large pieces. Also unusually large pieces, entire human hemispheres, and brains of high vertebrates can be cut into thin, and, if necessary, serial sections both without, and after imbedding either in celloidin or paraffin or by mixed methods. The processes used for the purpose do not differ essentially from those above-mentioned and fully described in Chapters VII., VIII. and 
IX., but (particularly for cyto-architectonic and fibre-architectonic studies) special apparatus and installations are needed, the description of which is outside the province of this book.

See Feist, Ztschr. wiss. Mikr., viii, 1891, p. 492 ; DEECKE, op. cit.; DeJerine, Anat. Centres Nerveux ; Strasser, Ztschr. wiss. Mikr., ix, 1892, p. 8; Brodmann, Journ. Psychol. u. Neurol., ii, 1903-4, p. 206 ; WARNKE, ibid., p. 221 ; LIFSEGANG, Ztschr. wiss. Mikr., xxvii, 1910 p. 369 ; Venderoviě, Anat. Anz., xxxix, 1911, p. 414.

\section{General Stains.}

816. Carmines.-Ammonia-carmine is good for general views. Stain very slowly in extremely dilute solutions. Bichromate material should be brought direct into the stain without passing through alcohol (see $\S 51$ ).

Picro-carmine has much the same action, but gives a better demonstration of non-nervous elements.

Bolles Lee (see 1913 ed.) prefers carmalum with formol material as giving a more delicate stain. He finds it better then paracarmine.

The best way of staining formol material with ammonia carmine, carmalum, picro-carmine and the like, consists in my opinion in cutting sections by one or the other of the freezing methods, and transferring them for a few hours either in Müller's fluid, or 0.5 per cent. chromic acid as suggested by Schwalbe (Centrbl. allg. Pathol., xii, 1901, p. 881). Sections are then washed for a longer or shorter time according to the amount of mordant one wishes to extract, proceeding afterwards to stain with one of the above-mentioned carmine solutions.

On the other hand sections of non-imbedded material fixed and hardened in one or the other of the fluids mentioned in $\$ \S 810$ to 812 may be stained not only with carmines, but also with a great variety of dyes if one so desires (see Chapter XI.). The same applies to sections of imbedded material, though the after-treatment to which it has been submitted may render more or less difficult the carrying out of certain general stains. But one should remember that in any case the results thus obtained are not very instructive, and by no means comparable with those attainable by the rational use of the special methods described in the following chapters.

For other carmine processes of staining, see Schmaus (Münch. med. Wochenschr., 1891, p. 147) ; UPSON (Neurol. Centralb., vii, 1888, p. 319); Freeborn (Journ. Roy. Mic. Soc., 1889, p. 305); Kadyi (Neurol. Centralb., xx, 1901, p. 687); ChILESotri (ibid., xix, 1902, p. 161, and Centralb. allg. Pathol., xiii, 1892, p. 191). 
817. Nigrosin and Anilin-Blue-Black.-Nigrosin has given useful results in some hands. Anilin-blue-black has been much recommended by SANKey (Lancet, 2, 1875, p. 82); Bevan Lewis (Human Brain, p. 125. and Quart. Journ. Micr. Sc., 1876, pp. 73-75); VEJAS (Arch. f. Psych. xvi, 1885, p. 200) ; Martinotti (ii, 1885, p. 478); Jelgersma (Ztschr. wiss. Mikr., 1886, p. 39) ; Schmaus (Münch. med. Wochenschr., 1891, p. 147), and others. And see also previous editions.

818. Picronigrosin.-MaRTinotTi (loc. cit., 1885, p. 478) stains for two or three hours or days in a saturated solution of nigrosin in saturated solution of picric acid in alcohol, and washes out in a mixture of 1 part of formic acid with 2 parts of alcohol.

819. KAISER (Ztschr. wiss. Mikr., vi, 1889, p. 471) stains sections of spinal cord for a few hours in a solution of 1 part of naphthylamin brown, 200 of water, and 100 of alcohol, washes with alcohol, clears with origanum oil and mounts.

820. Alizarine.-SchrötTer (Neurol. Centrbl., xxi, 1902, p. 338) stains sections for twenty-four hours in a 1 to 2 per cent. solution of sulphalizarinate of soda, differentiates for half to one minute in tap-water, dehydrates, and mounts. This is a general stain, but demonstrates Nissl bodies and other internal details.

\section{Mallory's Phosphomolybdic Acid Hæmatoxylin and Kodis'} modification; see § 271. For AuERBach's modification, see Neurol. Centrbl., xvi, 1897, p. 439.

822. Hæmatoxylin and Acid Fuchsin.-Finotri (Virchow's Arch., exliii, 1896, p. 133) stains in hæmatoxylin, counterstains for three minutes with 0.5 to 1 per cent. solution of acid fuchsin, and differentiates in 75 per cent. alcohol containing a very little caustic potash.

VAN GIESoN's hæmatoxylin and picro-fuchsin ( $\$ 398)$ may give useful general views of nerve-cells, axis-cylinders, and neuroglia.

823. Other General Stains.-ALT (Munch. med. Wochenschr., 1892, No.4) stains for a couple of hours in a solution of Congo red in absolute alcohol, and washes out with pure alcohol. This is useful for peripheral axis-cylinders and other elements.

SCarPatetTi (Neurol. Centrbl., xvi, 1897, p. 211) stains sections of formol material for five minutes in 1 per cent. hæmatoxylin, treats for five minutes with concentrated solution of neutral copper acetate, differentiates with Weigert's borax-ferricyanide, treats with concentrated solution of lithium carbonate, washes and mounts. Myelin is not stained.

Röтнig (Folia Neurobiol., ii, 1909, p. 385) fixes and stains for about four weeks in saturated solution of methylenazur $I$., in 10 per cent. formol, puts for ten to fifteen minutes into acetone, then for twelve hours into chloroform, and imbeds in paraffin. He also has a process with trichloracetate of lead and methylenazur. 
RAwITz (Ztschr. wiss. Mikr., xxvi, 1909, p. 341) has some complicated methods with Indulin, Indaminblau, and Azosäureblau, which take twenty-eight days: and (ibid., xxviii, 1911, p. 1) others with fuchsin and azofuchsin which take over thirty-six days.

ARIËNS KAPPERS (ibil., xxviii, 1911, p. 417) describes a staining method with extract of elderberries for material fixed and hardened in Müller's fluid or similar solutions. It is very simple and particularly recommended for photographic purposes; it should be carried out as follows: Stain celloidin or paraffin sections overnight in neutralised elderberries extract (obtained by fermentation at $20^{\circ}$ to $25^{\circ} \mathrm{C}$.), to which 1 per cent. carbolic acid has been added. Wash in water. Differentiate in 3 per cent. Liquor ferri sesquichlorati P.G., wash, dehydrate, and mount. 


\section{CHAPTER XXXII.*}

NERVOUS SYSTEM-SPECIAL METHODS, CHIEFLY CYTOLOGICAL.

824. Introduction.-The ordinary methods of cytology are, of course, available for nerve cells; but there are certain constituents of these cells, as well as of nerve fibres, which require, for minute study, special methods, such as the following :-

\section{A. Methods for Cells, demonstrating Tigroid Substance and other Granular Materials.}

825. Tigroid substance or bodies, chromophilic or chromatophilic substance or material or granules, Nissl's bodies or granules, etc., are all denominations for a markedly basophil substance which appears as blocks, granules or irregular patches within the cytoplasm of nerve cells under certain conditions of fixing and staining.

It is now almost universally admitted that this substance exists in the living cells as a fluid or semi-fluid " plasm rich in nutritive value," and that the blocks, granules or patches are appearances chiefly due to the coagulation of this plasm, as brought about by the fixing agents employed for their demonstration. As, however, these bodies or granules appear always the same under constant optical conditions in healthy cells fixed and stained in a constant manner, they are said to be the equivalent of such healthy cells during life. "It follows that if the cells, prepared by the same method and examined under the same conditions, show a difference from the equivalent or symbol of healthy cells, the difference is the measure of some change that has occurred during life." See Halliburton, Handbook of Physiology, London, 1920, p. 194.

This is pointed out here to make it quite clear why NISSL has always insisted that his method should be carried out according to his suggestions, and in a constant manner. At first (Neurol. Centrbl., iv, 1885 , p. 500) he used to stain sections of material fixed in alcohol with a warmed watery solution of magenta red or dahlia violet or vesuvine, and to differentiate them with alcohol. Later (Allg. Ztschr. Psych., xlviii, 1892, p. 197) he suggested floating sections on a warmed solution of methylene blue (B patent), with

* Re-written by Dr. C. Da Fano, King's College, University of London. 
subsequent differentiation with a 10 per cent. solution of anilin oil in 96 per cent. alcohol. The present form of the method was published in 1894 (Neurol. Centrbl., xiii, p. 507) ; but Nissu continued to introduce into it slight modifications, as one understands from many of his papers, to which due attention was paid when preparing the following account. It must be added here that Nissl's method has been, and still is, extremely useful for the study of nervous tissue under various physiological and pathological conditions, and that it stains, when properly carried out, not only the tigroid substance and the basophil parts of nuclei of nerve cells, but also the nuclei and certain parts of the cytoplasm of neuroglia cells and connective tissue elements normally or abnormally present in the nervous tissue.

826. Nissu's Methylene-blue Method.-Not too small pieces of fresh tissue are fixed in 96 per cent. alcohol and hardened therein for a few days. They should not be allowed to fall to the bottom of the bottle, but kept floating by means of some filter paper or cotton wool. The alcohol must be in large quantities in proportion to the number of pieces, and repeatedly changed. The pieces are cut without embedding and the sections collected in 96 per cent. alcohol, from which they are directly floated on some stain filtered into a watch glass at the moment of using it. The stain should be at least three to four months old, and shaken at the moment of filtering the quantity needed. It is prepared by carefully dissolving 1.75 grms. of Venetian soap in 1 litre of distilled water and adding to it $3.75 \mathrm{grms}$. of methylene blue (B patent). It is a good practice to vigorously shake the bottle from time to time, and to re-filter into the same bottle the amount of stain left in the watch-glass after staining one or more sections.

The watch-glass containing the stain with the section floating on it is warmed carefully over a flame until small bubbles rise to the surface. The section, which should not have fallen to the bottom of the watch-glass, is immediately transferred into a mixture of 10 parts of anilin oil and 90 parts of 96 per cent. alcohol, and as soon as no more colour is given off (it often takes only some seconds), it is lifted on to a slide, pressed with smooth filter paper, and cleared with a few drops of pure anhydrous cajeput oil. Care should be taken not to dry the section excessively with the filter paper and to pour the cajeput oil on to the section very quickly.

The cajeput oil not only clears the section, but stops the differentiation; it is, therefore, advisable to renew it after a little 
while on the section. As soon as this has become quite transparent, the cajeput oil is dried off with filter paper, the section thoroughly washed with benzol and covered with a drop of thick xylol-colophonium, rendered more fluid by passing the slide carefully over a flame, and quickly covering the section with a thin cover-glass before the colophonium sets again by cooling.

\section{2\%. Suggestions regarding the Carrying-out of Nissl's Method} (as deduced from NissL's papers; see chiefly Enz.d. mikr. Techn., ii, 1910 , pp. $252-280$, and the references therein quoted at p. 287).For the fixation of tissues alcohol should be almost exclusively used. Formalin, mixture of formalin and alcohol, sublimate and mixture of the same with alcohol or picric acid and the like, nitric acid may be occasionally employed, but no particularly good results can be expected from them. These are, however, somewhat better if tissues are placed for some time in alcohol after fixation with one or the other of the above reagents. This applies particularly to formalin material, which can be kept with advantage for many weeks, and even months, in repeatedly changed 96 per cent. alcohol. The bichromates of potassium and ammonium and mixtures containing chromic"salts, though useful for other purposes, should be entirely avoided for cytological investigations in Nissl's sense. See on this subject also BurchardT, La Cellule, xii, 1897, p. 337.

If tissues are too brittle to be sectioned without embedding, or if embedding is for any other reason desirable, one should have recourse to celloidin, paraffin being used only when unavoidably necessary or for special purposes. Pieces to be embedded in celloidin are not to be passed through alcohol-ether, but directly from absolute alcohol into thin celloidin. Embedding should, in any case, be carried out as quickly as possible.

Sections of material which was not fixed in alcohol and of embedded tissues, however fixed, stain, as a rule, very poorly by Nissl's soap-methylene-blue method; but good and even excellent results can be obtained by staining such sections with watery solutions (generally 0.5 to 1 per cent.) of toluidine blue, thionine, Unna's polychrome methylene blue, dahlia violet, vesuvine, neutral red, magenta red, Azur I, Azur II, and the like.

If one of such stains is used, it need not be warmed until bubbles come to the surface, but only until vapour arises. For the differentiation pure 96 per cent. alcohol, viz., without any addition of anilin oil, should be used. In this connection I find that very good results can be obtained from material embedded in 
celloidin if the sections are, whenever possible, freed from the celloidin before staining them, and if they are re-stained a second and a third time after having been each time completely differentiated. See Da Fano, Proc. Physiol. Soc., Journ. Physiol., liv, 1920-1.

All preparations stained by Nissl's method keep badly, but they keep a little better and may last almost unchanged even for years : (1) if the anilin-alcohol or alcohol used for the differentiation is properly washed away with pure benzol ; (2) if the xylol-colophonium for the mount is prepared with pure xylol, and of the thickness needed; (3) if sections of material embedded in celloidin are submitted to the above-mentioned succession of staining and differentiation; (4) if preparations are carefully protected from light.

828. Modifications of Nissl's Method.-ReнM (Münch. med. Wochenschr., xxxix, 1892, p. 217) floats sections for half a minute to a minute on a hot 0.1 per cent. solution of methylene blue, differentiates them in 96 per cent. alcohol and clears them with origanum oil.

LenhosséK (Fein. Bau. d. Nervens., 1895) stains sections of formol material in a concentrated aqueous solution of thionine, rinses them with water and mounts them like Nissl.

Luxenburg (Neurol. Centrbl., xviii, 1899, p. 629) stains paraffin serial sections either with Nissl's methylene blue or with thionine as Lenhossék.

Juliusburger (Neurol. Centrbl., xvi, 1897, p. 259) stains sections of material fixed in Orth's fluid and embedded in celloidin, either with Nissl's methylene blue or with warmed neutral red.

Rosin (Deutsche med. Wochenschr., xxiv, 1898, p. 615) treats sections of formol material similarly.

LenhosséK (Neurol. Centrbl., xvii, 1898, p. 577) stains paraffin or celloidin sections of spinal ganglia fixed in Carnoy's fluid with a concentrated watery solution of toluidine blue overnight, rinses with water, differentiates quickly with alcohol and clears with xylol or carbol-xylol.

Polumondwinow (Ztschr. wiss. Mikr., xvi, 1899, p. 371), uses 1 part of 1 per cent. solution of toluidine blue to 119 of distilled water and 1 of sodium carbonate.

Van Gehuchten and Nelis (La Cellule, xiv, 1898, p. 374) recommend fixing spinal ganglia in Gilson's mixture.

VAN Gehuchten (see 1913 ed.) uses paraffin sections mounted on slides by the water method and stains them for five to six 
hours in Nissl's methylene blue solution in the incubator at $35^{\circ}$ to $40^{\circ} \mathrm{C}$.

Gothard (C. R. Soc. Biol., v, 1898, p. 330) stains celloidin sections for twenty-four hours in Unna's polychrome methylene blue, and differentiates them with a mixture of 5 parts of creosote, 4 of cajeput oil, 5 to 8 of xylol, and 16 of absolute alcohol. The mixture is removed with absolute alcohol and sections mounted in xylol dammar after clearing with cajeput oil.

Luithlen and SoRgo (Neurol. Centrbl., xvii, 1898, p. 640) differentiate in Unna's glycerin-ether mixture, remove this with absolute alcohol, and clear with origanum oil.

Similarly LennHoFF (ibid., 1910, p. 20) ; or, polychrome methylene blue two minutes, distilled water quickly, carbol-pyronin-methyl green twenty minutes; distilled water quickly, absolute alcohol, oil, balsam.

LoRD (Journ. Ment. Sc., xliv, 1898, p. 693) makes sections from frozen fresh tissues, treats them for a few seconds with a mixture of equal parts of 6 per cent. formaldehyde and saturated solution of picric acid, then rinses them with distilled water and stains them in 5 per cent. solution of methylene blue B pat.

829. Bielschowsky and Plien's Cresyl Violet Method (Neurol. Centrbl., xix, 1900, p. 1141).-Celloidin or paraffin sections of material fixed either in alcohol or formalin, or sections made by the freezing method from formalin material, are stained for twenty-four hours in a very diluted solution of cresyl violet R.R., prepared by adding 6 to 8 drops of a concentrated aqueous solution to every 50 c.c. of distilled water. After a quick wash in distilled water sections are brought through the ascending series of alcohols, cajeput oil, and xylol, into balsam. The preparations are said to keep better than those stained with thionine or toluidine blue.

830. Picrocarmine has been successfully used by Messner (Journ. Psychol. Neurol., xviii, 1912, p. 204, and xx, 1913, p. 256). Sections of alcohol material, embedded in celloidin or not, are washed in water and then stained for five minutes in a warmed diluted solution of Ranvier's picrocarmine. After a quick wash, they are differentiated in 3 per cent. hydrochloric acid, dehydrated and mounted as usual. In the case of the spina] cord, medulla oblongata and pons the method succeeds also if material was fixed in formalin.

831. Other Methods for Tigroid Substance and Basophil Granules in General.-See Goldscheider U. Flatau, Norm. u. path. Anat. $d$. Nervenz, Berlin, 1898, or Ztschr. wiss. Mikr., xvi, 1899, p. 102, and 
NissL's remarks thereon, Deutsche Ztschr. Nervenheilk, xiii, 1899, p. 348 ; Ilberg, Neurol. Centrbl., xv, 1896, p. 831 ; Cox, Anat. Hefte, x, 1898, p. 75 ; Int. Monatschr. Anat., xv, 1898, p. 241 ; AUERBACH, Monatschr. Psych. Neurol., iv, 1898, p. 31 ; MYers, Anat. Rec., ii, 1908, p. 434 ; Savini, E. U. Tr., Centrbl. Bakt., I Abth., xlviii, 1909, p. 697; Mosse (argentamin stain), Arch. mikr. Anat., lix, 1902, p. 403 ; Mentz v. Krogir, Centrbl. Bakt., I Abth., Iviii, 1911, p. 95 ; Johnston, Anat. Rec., xi, 1916, p. 287.

832. HeLd's Methylene Blue and Erythrosin Method (Arch. Anat. Phys., Anat. Abth., 1895, p. 399 ; 1897, pp. 226-233, 273-385, Supplementband).-Material may be fixed in alcohol, but preferably either in picro-sulphuric acid, or in van Gehuchten's mixture of alcohol, chloroform and acetic acid, or 1 per cent. corrosive sublimate in 40 per cent. acetone. Tissues should be carefully embedded in paraffin and sections stuck to slides by the water method. They are stained with the aid of gentle heat for one to two minutes in a solution of 1 grm. erythrosin in 150 of distilled water acidulated with 2 drops of glacial acetic acid. After washing with water the slides are transferred into a mixture of equal parts of Nissl's methylene blue solution and 5 per cent. acetone, warming until all odour of the latter has disappeared. Differentiation is carried out after cooling by means of a $0 \cdot 1$ per cent. solution of alum until sections are reddish. Rinse in distilled water, dehydrate as rapidly as possible in absolute alcohol, wash in xylol and mount in balsam.

BocCARdi (Monit. Zool. Ital., x, 1899, p. 141) uses a mixture of erythrosin $0 \cdot 1$ grm., toluidine blue $0 \cdot 2$ grm., and water 100 c.c., and differentiates in 0.5 per cent. alum solution.

By means of Held's method, besides the tigroid substance, other granules-viz., Held's neurosomes-become stained. It may, therefore, be considered as a typical example of double staining of nervecells. Other double stains demonstrating basophil, acidophil and other granules have been repeatedly proposed and may be easily obtained by the combination of an acid and a basophil dye. One generally uses watery solutions, e.g., of acid fuchsin, and methylene or toluidine blue, and one stains first with the acid dye and then with the basophil one, differentiation being carried out with alcohol. One may also have recourse to EHRLICH's triacid (\$ 296) as originally proposed by Rosin (Neurol. Centrbl., xii, 1893, p. 803), or to one or other of the methods used for staining blood films ( $\$ 784$ ), such as Pappenheim's panoptic triacid stain, Jenner's mixture, Leishman's Romanowsky stain, Pappenheim's method as described in $\S 785$, and so on.

See on this subject CowDry, Int. Monatschr. Anat., xxix, 1913, 
p. 673, and for pathological specimens ALzHEIMER's methods 5, 6 and 9 in Histol. u. Histopath. Arb. über d. Grosshirnr, iii, 1910, pp. 406-412, which may be useful for the study of nerve-cells though originally proposed for the investigation of neuroglia.

B. Methods for Cells and Fibres, demonstrating Neurofibrils.

833. Neurofibrils ; General Characters.-Nerve cells and the fibres into which they are prolonged contain, in addition to the chromatic constituents shown by the methods already dealt with, a characteristic so-called achromatic element, consisting chiefly of very fine and fairly refractive fibrils which can only be seen with great difficulty in the unstained state, but may be fixed with osmic acid and made out in thin sections of medullated nerve fibres observed in diluted glycerin or water, and may be to a certain extent isolated by maceration. For their demonstration, however, one or the other of the methods chronologically described in the following paragraphs must be employed. They are all regarded as giving true stains of neurofibrils.

For the method of KuPFER (Sitzb. math. Kl. Alkad. Wiss. München, xiii, 1884) see former edition.

834. Apathy's Methods.-The gold method ("Nachvergoldung") has been given in $\S 371$. The stain is very sharp, but good results are obtained only in certain invertebrates, and even in these with considerable difficulty.

The hocmateine method (Mitth. Zool. Stat. Neapel, xii, 1897, p. 712) has the same advantages and disadvantages, and has been little used since the discovery of the Cajal and Bielschowsky processes. Material may be fixed with corrosive sublimate, Zenker's fluid, picro-sulphuric acid, or any other mixture which is not inimical to staining with alum hæmatoxylin, and should be preserved in 90 per cent. alcohol. Portions, no more than $\frac{1}{2} \mathrm{~cm}$. thick, are stained for at least fortyeight hours in hæmateine I.A. (§ 259 ), and then washed for up to twenty-four hours in absolutely pure distilled water, or preferably suspended therein. Before the stain has become washed out of the neurofibrils entirely, it is fixed by putting the preparations for three to five hours into spring water, after which they are put back for not more than two hours into distilled water, dehydrated as rapidly as possible by hanging them up in absolute alcohol, and embedded in paraffin or celloidin, after clearing with chloroform, and carefully protecting them from light whilst in chloroform or celloidin. The sections are mounted either in a resin or in neutral glycerin. 
835. Bethe's Molybdenum-Toluidine Blue Method (Ztschr. wiss. Mikr., xvii, 1900, p. 13).-Pieces of the central nervous system of vertebrates are fixed for twenty-four hours in 3 to $7 \cdot 5$ per cent. nitric acid, and then brought directly into 96 per cent. alcohol for a day or longer. They are afterwards put for twelve to twenty-four hours in a mixture of 1 part of ammonia (of sp. gr. 0.95) with 3 of distilled water and 8 of 96 per cent. alcohol ; for six to twelve hours into pure alcohol ; for twenty-four hours into a mixture of 1 part of concentrated hydrochloric acid, 3 of distilled water, and 8 to 12 of alcohol; for ten to twelve hours into pure alcohol; for two to six hours into water. They are now mordanted with 4 per cent. ammonium molybdate, washed again, dehydrated and embedded in paraffin. The sections, 8 to $10 \mu$ thick, are seriated on slides by means of egg albumen, but without water, then passed through xylol and alcohol and "differentiated," viz., covered with water poured on the sections so as to form over them a layer 1.5 to $2 \mathrm{~mm}$. deep, and put into an incubator at 55 to $60^{\circ} \mathrm{C}$. for ten minutes. They are then rinsed with water, covered with a $1: 3000$ solution of toluidine blue, stoved for another ten minutes, rinsed with water, and lastly treated with 96 per cent. alcohol till no more colour comes away. After dehydration with absolute alcohol they are mounted in the usual way.

The method is also applicable to invertebrates for which other fixing agents besides nitric acid are admissible, and the impregnation with ammonium molybdate may be done on the sections instead of previously on the uncut tissues.

For LUGARo's modification see Riv. pat. nerv. ment., x, 1905, p. 265.

836. Donaggio's Methods (Riv. Sper. Freniatr., xxx, 1904, p. 397, and xxxii, 1906, p. 394).-There are five methods of Donaggio. By the first two, pieces are stained in bulk before embedding, but results are not so good as by the other three, the most important of which is-

Method III.-Good for spinal cord, pons, medulla oblongata, spinal and sympathetic ganglia. Thin slices of tissues are fixed for five to six days in pure pyridine changed at least once, and then treated with repeatedly changed distilled water until the pyridine has been entirely eliminated. The surfaces of pieces are smoothed by means of a sharp razor, and the pieces brought for twenty-four hours into 4 per cent. ammonium molybdate to which 4 drops of hydrochloric acid have been added. After a quick wash, they are rapidly dehydrated in 95 per cent. and absolute alcohols, and em- 
bedded in paraffin. The sections, which must be rather thin ( 3 to $6 \mu$ ), are brought through xylol, absolute and 95 per cent. alcohols into distilled water and here washed.

This is the crucial point of the method because, by washing, ammonium molybdate becomes extracted from the sections, and the success of the subsequent staining depends almost entirely on carrying out the extraction up to the right point. I find that the only way of ensuring this consists in proceeding by trials, which must be repeated for every series of sections. Once the right amount of washing has been decided upon, one can proceed to stain even many slides at the same time by means of a $1: 10,000$ solution of thionine, to be freshly prepared every time from a less diluted stock solution.

The staining is a "progressive" one, and must be controlled under the microscope. It generally takes about twenty minutes to obtain it, at the end of which time the grey substance has a redpurple tone whilst the white substance appears bluish. If the staining is right the preparations can be quickly washed, dehydrated and mounted. But if the neurofibrils are not quite sharply stained, the preparations can be "differentiated" for another fifteen to twenty minutes in the ammonium molybdate solution used for mordanting the pieces, or for ten seconds in a diluted solution (1:10 to 1:20) of "pink salt" (C. Erba, Milano). Preparations last only a few months, but are sometimes of great interest. See DA Fano, Ziegler's Beiträge, xliv, 1908, p. 495.

Method $I V$, which is particularly useful for the demonstration of neurofibrils in the cells of the cortex cerebri and cerebelli, differs from Method III only in regard to a preliminary fixation of pieces for twenty-four hours in a mixture of pyridine nitrate $10 \mathrm{grms}$, and pyridine, 100 c.c. ; they are then transferred for another thirty-six hours into pure pyridine, proceeding as in Method III.

Method $V$ may be used for the demonstration of both Nissl's substance and neurofibrils. Pieces are fixed in a saturated solution of corrosive sublimate ; after a day they are treated for twenty-four hours with distilled water to which a few drops of iodine tincture have been added, then for two to three hours with pure distilled water; and lastly passed for forty-eight hours into pure pyridine, this being changed at least once. The rest as in Method III.

Paravicini (Boll. Mus. Z. Anat. Comp. Torino, xx, 1905, p. 1) fixes and mordants in the dark, and differentiates after staining with extremely weak hydrochloric acid.

TOMASELli (Ztschr. wiss. Mikr., xxiii, 1906, p. 421) fixes spinal ganglia for six to seven hours in absolute alcohol 100 c.c. with four to five drops of ammonia, and then transfers them for two days into pure 
pyridine to be repeatedly changed, the vessel with the pieces being kept at $36^{\circ}$ to $37^{\circ} \mathrm{C}$. After washing for two to three hours in running tap water, he continues as in Donaggio's method III.

For the criticism of JäDERHolm, see.Arch. mikr. Anat., lxvii, 1906, p. 108 ; and for that of Montanari, Ztschr. wiss. Mikr., xxviii, 1911, p. 22.

83\%. Ramón y CaJAL's Methods. Introductory.-It has been said by some authors that Cajal's methods were originally only modifications of the photographic process of Simarro. The criticism is unjust because even the first formula of Cajal differs so profoundly from Simarro's process as to form an entirely new method. One cannot, however, deny the existence of a certain similarity of conception between the two processes in so far as both are based on the silver-reducing power of certain photographic reagents. For this reason it has been thought expedient to briefly describe here Simarro's process, which though uncertain in its results, may still be of some value to elucidate certain histological questions.

Simarro's Process (Rev. Trim. Micr., v, 1900, p. 45) consisted in poisoning animals with subcutaneous injections of solutions of sodium or potassium bromide or iodide in order to impregnate their living nervous tissues with one or the other of these salts. As soon as the animals showed that the poisoning had reached its maximum they were killed and their central nervous system removed in the photographic dark room. Small pieces were then immersed in a solution of silver nitrate, which, by combining with the bromine or iodine with which the tissues were impregnated, gave rise to the formation of silver bromide or iodide, which is easily affected by light. Sections were then made (always in the dark room), best by means of a freezing microtome, and exposed for a little while to light. There remained only treating them with a photographic developer, such as hydroquinone, pyrogallol or the like, and fixing them with sodium hyposulphite and so on, as if they were photographic plates ; they were lastly washed, dehydrated and mounted in the usual way.

One can easily understand the many drawbacks of such a method and the reason for which it was abandoned as soon as Cajal published in 1903 his first reduced silver methods. From that time onwards, Ramón y Cajal continued improving them and adding new formulæ, which he himself summarised in a special article of his Trab. Lab. Invest. Biol., Madrid, viii, 1910, on which the following account is based. The numbering is that of Ramón y Cajal.

Formula 1a.-Small pieces of fresh tissue are directly put into 1.5 per cent. silver nitrate and kept therein for three to four and 
even to five days at a temperature of about $35^{\circ} \mathrm{C}$. In summer, with a temperature constantly over $22^{\circ} \mathrm{C}$., the stove may be dispensed with, provided the impregnation is prolonged for two to three days more. The tissues are known to be ripe for reduction when a freshly cut surface shows a brownish-yellow colour.

They are then washed for one to two minutes in distilled water and put into-

Pyrogallol or hydroquinone

Distilled water

Formalin

$$
\begin{aligned}
& 1-2 \text { grms. } \\
& 100 \text { c.c. } \\
& 5-10 \text { c.c. }
\end{aligned}
$$

The formol is not necessary but useful. One may use pyridine instead ( 1 to 3 per cent.). The addition of a small quantity of sodium sulphite $(0 \cdot 2$ to 0.5 per cent.) has been abandoned by Cajal. The stronger the pyrogallol, the greater the contrast, so that it may be useful to take, sometimes, as much as 3 per cent., but then the over-impregnation of the outer layers will be increased. Hydroquinone reduces more energetically than pyrogallol.

The pieces remain in the reducing fluid for about twenty-four hours and are then quickly washed, hardened in alcohol and embedded in paraffin or celloidin. The sections (15 to $20 \mu$ thick) are mounted in dammar after toning with a solution of gold chloride if the reaction is rather weak, without toning if the impregnation is a good one.

Faintly impregnated sections can be advantageously toned with-

Distilled water

Ammonium sulphocyanide

Sodium hyposulphite .

1 per cent. gold chloride

$$
\begin{aligned}
& \text { - } 100 \text { c.c. } \\
& \text { - } \quad 3 \text { grms. } \\
& \text { - } \quad 3 \text { " } \quad \text { a few drops. }
\end{aligned}
$$

If subsequently found to be too dark they can be bleached by Veratti's potassium permanganate and sulphuric acid mixture (see $\$ 846)$.

The sections from the outer layer are generally too dark for study, those from the innermost too pale, whilst those from the intermediate layer are good. The over-staining of the outer layer can be diminished by diluting the silver nitrate with 1 volume of water for the last twelve hours.

The method has the defect of giving an imperfect fixation of the nervous tissue and of impregnating, almost exclusively, cell bodies and dendrites. ' It is not good for ganglia and large cells of adult subjects, but excellent for small and medium-sized cells of very young subjects and early embryos.

Formula la, A.-As the last, but pieces are fixed in 3 to 6 per cent. 
silver nitrate. This formula gives better fixation, and was successfully used by Dogres (Anat. Anz., xxv, 1904, p. 558, and Arch. mikr. Anat., lxvii, 1906, p. 638) for the study of Grandry's corpuscles and other sensory nerve endings, by Kolmer (Anat. Anz., xxvi, 1905, p. 560) for the epidermis of Lumbricus, etc., and by other authors for the ganglionic chain of Hirudinea.

Formula 1a, B.-As above, but taking $0 \cdot 75$ per cent. silver nitrate and very small pieces, preferably from embryos and new-born subjects. Poor fixation, much shrinkage, but vigorous stain of the neurofibrils, nucleolar granules and the intranuclear rodlet of Roncoroni.

Formula 1a, C.-As above, but tissues are fixed in 2 per cent. silver nitrate to which one-fourth of absolute alcohol or acetone has been added. Better fixation than with pure silver nitrate. Results very similar to those obtainable by Formula la with dog, cat and rabbit, and better results with human cerebrum and cerebellum.

Formula 2a.-Fixation for twenty-four hours in 96 per cent. alcohol. Tissues not washed, but mopped with blotting paper and put into 1.5 per cent. silver nitrate for seven days at $35^{\circ} \mathrm{C}$., or six days at $40^{\circ} \mathrm{C}$. The rest as Formula la. Good impregnations of nerve centres of adults, of peripheral nerve endings, of regenerating nerves, of early embryos, and of young fishes. It impregnates medullated and many non-medullated fibres, large and medium nerve cells, the basket fibres of Purkinje's cells, etc. Results fairly constant, but sometimes showing a granular precipitate of unknown origin.

To hinder this precipitate and, at the same time, to hasten the impregnation, it is well to add to the alcohol certain substances which Cajal calls " accelerators." Such are chloral hydrate, veronal, pyridine, nicotine, ethylamine, antipyrine, and others.

Hypnotics, particularly veronal and chloral, and in a less degree pyridine and ammonia, also act as "rejuvenators," reviving the susceptibility of impregnation in tissues which have lain too long in alcohol.

Formula 2a, A.-Fixation for twenty-four to forty-eight hours in 96 per cent. alcohol with 2 per cent. of chloral hydrate. Silver bath of 1.5 per cent. for five days in the stove. The rest as usual. Veronal (same proportion) gives the same result, as do also sulphonal, trional, hedonal, etc. The results are very constant. Medullated fibres well shown.

Formula 2a, B.-Fix for twenty-four hours in 96 per cent. alcohol with 10 to 20 per cent. of pyridine; wash for some hours in pure alcohol and transfer pieces into 1.5 silver nitrate for five days. 
I understand from Cajal's pupil, Del Río Hortega, that this formula may be successfully employed for the study of peripheral nerve endings. In this case material is better fixed for twenty-four hours in pyridine to which one-third its volume of distilled water or 96 per cent. alcohol has been added. Pieces should be washed in running tap-water overnight and then transferred for six hours into pure 96 per cent. alcohol. Impregnation, reduction, embedding, etc., as above. Results are good, but pieces become extremely hard even if dehydrated very quickly, and are consequently difficult to cut. See also Formula 5a.

Formula 2a, C.-Fix for twenty-four hours in 50 c.c. of alcohol with 10 drops of nicotine. Mop up with blotting paper, without washing, and silver as usual for five days (or four at $40^{\circ}$ C.). Good results with adult tissues, especially spinal cord. Good penetration and less shrinkage than with pure alcohol.

Formula 2a, D.-Fix for twenty-four hours in allyl alcohol (the industrial product will do). Wash for some hours in several changes of water. Put for a day into 50 c.c. of alcohol with 4 drops of ammonia. Silver for four days at $35^{\circ}$ to $38^{\circ} \mathrm{C}$., and reduce as usual. Good for human tissues, especially for fibre plexuses of cerebrum and cerebellum. Instead of allyl alcohol one may take acetal or acetone. Put for six hours into acetone with 25 per cent. of water, then for twenty-four into pure acetone, wash in water, etc., as above.

Formula 3a.- Fixation in ammoniacal alcohol for twenty to fortyeight hours. The most generally useful formula is 50 c.c. of 96 per cent. alcohol with 4 to 5 drops of ammonia (of $22^{\circ}$ strength). But for cerebrum not more than 1 to 3 drops ; for cerebellum, ganglia, spinal cord and regenerating tracts, 4 drops ; for neurofibrils of the large nerve cells of the medulla oblongata and spinal cord, 9 to 10 drops. To avoid shrinkage, it is well to begin by putting the pieces for six hours into 70 per cent. alcohol, then in 85 per cent., without ammonia; then for the rest of the time into the ammoniacal alcohol. Do not wash, but mop up with blotting paper before putting into the silver. Silver for four to four and a half days (small specimens) at $40^{\circ} \mathrm{C}$, , or medium to large (3 to $4 \mathrm{~mm}$. thick) for five days at $32^{\circ}$ to $35^{\circ} \mathrm{C}$. So long as the tissues are only yellowish-white, they are not ripe for reduction ; light grey indicates ripeness ; dark grey over-ripeness. Reduce as by Formula la.

Specimens may be decalcified, after reducing and washing, in 96 per cent. alcohol to which a few drops of nitric acid have been added.

For the delicate impregnation of the neurofibrils of the large and 
medium nerve cells this formula is superior to all others. It gives good results with the majority of nerve centres, and is particularly good for non-medullated fibres, peri-cellular baskets of cerebellum, buds of Held and Auerbach in the oblongata, for human sympathetic, and for the study of regenerating nerve fibres.

Formula 3a, A.-Fix in 50 c.c. of alcohol with 10 grms. of glycerin and 6 to 10 drops of ammonia. Good for retina and non-medullated fibres, but especially for the buds of Held and Auerbach.

Formula 3a, B.-Fix in 50 c.c. of alcohol with 1.5 c.c. of a 33 per cent. alcoholic solution of ethylamine. Results the same as with ammoniacal alcohol.

Formula 4 a.-Pieces of tissue of not more than $4 \mathrm{~mm}$. in thickness are fixed for six to twelve hours in 15 per cent. formol. Wash for six or more hours in running tap-water. Put for twenty-four hours into 50 c.c. of alcohol with 5 drops of ammonia. Wipe with blotting paper, silver for five days (or four if the stove is at $38^{\circ}$ to $40^{\circ} \mathrm{C}$.). The rest as usual. Sharp impregnation of the finer fibres of nerve centres and of the terminal buds of pericellular nests. Adult tissues give better results than young ones. Energetic stain of the arborisations of the moss fibres of the cerebellum.

Formula 4a, A.-Fix in "a mixture of formol and alcohol." Wash out thoroughly with running tap-water, silver, and reduce as usual. Fixation more rapid and better; results similar to those of $3 \mathrm{a}$.

Formula 5a.- This is characterised by a preliminary fixation in pyridine as originally suggested by Held (Arch. Anat. Physiol., Anat. Abth., 1905, p. 77; Anat. Anz., xxix., 1906, p. 186). He used to fix tissues in pure pyridine, but Cajal finds that this is likely to cause much shrinkage, and he recommends fixing small pieces first for six to eight hours in a mixture of equal parts of distilled water and pyridine, then for eighteen to twenty-four hours into pure pyridine. Wash for several hours in running water, and put for a day into 90 per cent. alcohol. Wipe, and put for four to five days into 1.5 per cent. silver nitrate at $35^{\circ}$ to $38^{\circ} \mathrm{C}$, and reduce as usual. Not very good for adult organs, ibut superior to all others for the earliest phases of neurogenesis, and good for regenerative processes, as well as for peripheral nerve endings.

Formula 6a.- Put for twenty-four hours into 50 c.c. of water with 5 grms. of chloral hydrate, rinse, and put into 50 c.c. of 96 per cent. alcohol with 5 drops of ammonia (time not stated). Wipe with blotting paper; put for four to five days at $35^{\circ}$ to $38^{\circ} \mathrm{C}$. into 
1.5 per cent. silver nitrate, and reduce as usual. Results very constant, without shrinkage. Good for the fine plexuses of cerebrum, bulb and, cord, the baskets of Purkinje's cells, and moss fibres; also for motor plates and for regenerating nerves.

Formula 6a, A.-Fix for twenty-four hours in 10 per cent. chloral hydrate, wash for six, and put direct into the silver. Stove for four days. Results similar to those of Formula la. Medullated fibres well stained.

Formula 7a.-Fix for twenty-four hours in Merck's fibrolysine, wash for six, put for twenty-four into 50 c.c. of alcohol with 5 drops of ammonia. The rest as by other formulæ.

Instead of fibrolysin, lysidine may be taken.

838. Application of CAJAL's Methods to different Objects.-(1) For the study of the evolution of neuroblasts and nerve fibres in very early embryos it is necessary to avoid fixing with formol, or alcohol with an accelerator, or ammoniacal liquids. The best formulæ are 2a and 5a which are applicable to all vertebrates, but preferably to embryos of birds and fishes.

(2) For late embryos and foetus of mammals. Besides the above formulæ, 3a, 6a and alcohol with an accelerator. Best subjects, embryos of chick from the fifth day, and of rabbit from the tenth to the twelfth day; or new-born birds, with ammoniacal alcohol, or 5 a.

(3) For sympathetic ganglia. Formula 3a, or pure alcohol, or 4a and 5a. Best with man. Dog, cat, and rabbit give mostly weak reactions. The visceral ganglia are the most difficult.

(4) Sensory ganglia. Formula 2a.or 3a. Easy.

(5) Cerebellum. For Purkinje cells, 1a or 3a. For the baskets, climbing fibres, and medium and small dendrites, $2 a$ or its variants. For terminal rosettes and collaterals of moss fibres and for the plexuses of the granular layer, $4 \mathrm{a}$ or sometimes 5a or 6a. For the stellate cells of the molecular layer, $2 \mathrm{a}$ and $3 \mathrm{a}$. The best subject for the latter is the dog.

(6) Cerebrum. In general, the same formulæ as for the cerebellum, especially la for pyramids of young dogs and cats (of eight to twenty days). In Formula 3 a the proportion of ammonia should be diminished. For fine plexuses, $4 \mathrm{a}, 5 \mathrm{a}$, and $6 \mathrm{a}$.

(7) Spinal cord and bulb. All the formulæ are applicable. For neurofibrils of motor cells the best subject is the dog of four to fifteen days, with Formula 3a, with a large dose of ammonia (10 drops) ; also the alcoholic fixatives with an accelerator. For medullated 
fibres, large and small, 2a or 6a. For buds of Held and Auerbach and for fine plexuses, $4 \mathrm{a}, 3 \mathrm{a}, \mathrm{A}$, or $5 \mathrm{a}$.

(8) Ganglia of invertebrates. For the medicinal leech (not for other leeches), la, A. For Hocmopis, Aulostomum, Pontobdella and Glossiphonia, 2a or, better, $3 \mathrm{a}$, with not more than 2 to 5 drops of ammonia and 3 per cent. silver nitrate, stoving three or three and a half days. For further details see SANCHEz, Trab. Lab. Invest. Biol., Madrid, vii, 1909 , pp. $42-47$.

Lumbricus is generally refractory to Cajal's methods. Boule (Le Nevraxe, x, 1908, p. 15) obtained good impregnations by acidifying the fixatives. He takes : $(a) 25$ per cent. formol with 5 per cent. of acetic acid; or $(b)$ the same with 0.5 per cent. of ammonia; or (c) 100 c.c. of alcohol, 25 c.c. of formol, 5 c.c. of acetic acid, and 0.5 c.c. of ammonia. For the impregnation he uses 3 per cent. silver nitrate with 15 per cent. of alcohol, and reduces in the usual hydroquinone-formol solution, with the addition of 15 c.c. of alcohol. These results are confirmed by KowaLski (La Cellule, xxv, 1909; p. 292, and by Schutz (Anat. Anz., xlii, 1912, p. 262). Kowalski gets impregnations also by simply starving worms for several days, or exposing them to cold ( $-5^{\circ} \mathrm{C}$.) for a quarter of an hour.

(9) Regenerating nerve tissue. For nerves operated a month or more previously, Formula $2 \mathrm{a}$ or $3 \mathrm{a}$, with not more than 3 drops of ammonia, will stain equally the old and the new fibres ; for nerves operated not more than two to ten days previously, Formulæ 3a with 4 to 6 drops of ammonia, 5a with pyridine, and $4 \mathrm{a}$, also sometimes $6 \mathrm{a}$; for regeneration in cord, cerebrum, and cerebellum, $3 \mathrm{a}$ with 3 drops of ammonia, or 5a, or pure alcohol.

839. Modifications of RAMON Y CAJAL's Methods.-DA FANO (Ziegler's Beitr., xliv, 1908, p. 495) recommends using solutions of silver nitrate and hydroquinone in 1: 10,000 gelatin in order to obtain a deeper and sharper impregnation.

KATO (Folia neurobiol. ii, 1908) fixes in 10 to 15 per cent. formol, and silvers for one to five days at $35^{\circ} \mathrm{C}$. in 5 per cent. argentamin to which 3 per cent. of silver nitrate has been added in such a way as to have an impregnating fluid with a little argentamin in excess; or argentamin eight to ten parts, with 3 per cent. potassium bichromate thirty parts and distilled water 100 parts. For the reduction he uses 10 per cent. formol with 1 per cent. hydroquinone.

Pusateri (see Amato, Virchow's Arch., clxxxv, 1908, p. 547) fixes for three to six days at $35^{\circ}$ to $38^{\circ} \mathrm{C}$. in a mixture of 45 c.c. of tachinl (10 per cent. silver fluoride) and 155 of distilled water.

Besta (Riv. pat. nerv. ment. Firenze, xv, 1910, p. 333) fixes for fortyeight hours in alcohol with 5 per cent. nitric acid, neutralised in alcohol with ammonia. 
Liesegang (Kolloidchemie, Beihefte, iii, 1911, H. 7; Ztschr. wiss. Mikr., xxviii, 1912, p. 369) makes sections of formol material by the freezing process, and silvers them until yellow. He then adds to the silver bath an equal volume of 50 per cent. solution of gum arabic and the same amount of saturated solution of hydroquinone. After one or two minutes the sections are brought into 10 per cent. solution of sodium hyposulphite, washed and mounted. Results said to be the same as by the usual process.

Ascoli (Boll. Soc. med. chir., Pavia, 1911, p. 177) recommends for the sympathetic nervous system of Hirudinea the following: The animals cut open at the back are stretched on a piece of cork and fixed in a solution prepared by dissolving over a flame $5 \mathrm{gr}$. of pulverised crystals of silver nitrate in 100 c.c. of 95 per cent. alcohol. After a fow minutes the animals may be detached from the cork and put back in the same fixative for twenty-four to forty-eight hours in an incubating stove. They are then transferred for another twenty-four to forty-eight hours into a 10 per cent. watery solution of silver nitrate, to be kept also in the incubator. After a quick wash they are reduced for five to eight hours in Amidol-Hauff 0.5 gr., sodium sulphite cryst. 10 grms., distilled water 100 c.c., and lastly passed into glycerin. Preparations are made by teasing, the thinner ones being toned and counterstained as usual. For mounting he prefers Apáthy's syrup.

Ranson (Anat. Anz., xlvi, 1914, p. 522) has the following for the demonstration of 'non-medullated nerve fibres in cranial and peripheral nerves: Fix in absolute alcohol containing 1 per cent. of strong ammonia for forty-eight hours; rinse in distilled water, put in pyridine for twenty-four hours, wash in many changes of distilled water for twenty-four hours, place in 2 per cent. silver nitrate at $35^{\circ} \mathrm{C}$. in the dark for three days, rinse in water, and place for one day in a 4 per cent. solution of pyrogallic acid in 5 per cent. formalin.

As suggested by HABER and GuILD (Anat. Rec., vii, 1913, p. 253) the results can be improved by a preliminary injection of 95 per cent. alcohol, containing 1 per cent. of ammonia, through the arteries till tissues are thoroughly saturated, after which they are dissected out and placed in a similar ammoniated alcohol solution for from two to three days. Huber and Guild have found this method of use for the study of cranial nerves of small animals and embryos, since the entire heads can, after fixation, be decalcified by means of 7 per cent. nitric acid, brought through 80,90 , and 95 per cent. alcohols, each containing 1 per cent. of ammonia, and finally treated as above.

840. Bielschowsky's Methods. Introductory.-It is well known that, if ammonia be poured into a solution of silver nitrate, a precipitate is formed which is redissolved by the addition of some more ammonia. If an alkaline solution of formaldehyde be slowly added to this easily reducible di-ammoniacal silver nitrate $\left(\mathrm{N}\left(\mathrm{NH}_{4}\right) \mathrm{AgH}_{2} \mathrm{NO}_{3}\right)$, metallic silver is immediately precipitated and deposited on the walls of the test tube. Both FAJERsTAJN (Neurol. Centrbl., xx, 1901, p. 98) and Bielschowsky (ibid., xxi, 1902, p. 579) 
thought of taking advantage of this reaction for histological purposes with the object of finding out a silver impregnation of the nervous tissue similar to that which characterises Golgi's method. The results of their attempts were different: Fajerstajn was able to obtain only a difficult method for staining axis-cylinders which is now superseded; Bielschowsky also published, at first, a complicated silver method for impregnating axis-cylinders very similar to that of Fajerstajn, but, through successive modifications of his first process, was led to the discovery of a new method, which is as important as Cajal's reduced silver methods from an histological point of view, but is of still greater advantage than the latter for histopathological investigations. Moreover, Bielschowsky's method is applicable to any formol material, even if very old. BAYON (Die Untersuchungsmeth, etc.) succeeded with four-year-old material, and I with brains which had been left in formalin for more than eleven years.

There are at present three Bielschowsky methods : one for sections, one for peripheral nerve-fibres and axis-cylinders, and one for pieces. It seems better to describe them separately in the following account which is based on the original papers of Bielschowsky, as well as on some personal experience I gained through a visit paid to him when in Berlin.

Bielschowsky's Method for Sections (Journ. Psychol. Neurol., iii, 1904, p. 169 ; and xii, 1909, p. 135).-Pieces from central nervous organs, fixed in 15 to 20 per cent. formalin, are washed for some hours in running tap-water and then cut by means of a $\mathrm{CO}_{2}$ freezing microtome. The sections are collected in distilled water, thoroughly washed therein and passed in a 2 or 3 per cent. solution of silver nitrate where they are left for twenty-four hours in a dark place, and at room temperature. The sections can also be passed first into pure pyridine for twenty-four to forty-eight hours, washed in many changes of distilled water until the pyridine has been completely eliminated and then transferred into 2 to 3 per cent. silver nitrate as above.

The pyridine bath is optional and has the advantage of ensuring a sharper stain of axis-cylinders whilst neuroglia, which is more or less coloured when the pyridine bath is dispensed with, remains unstained. Also connective tissue and nuclei are generally very faintly stained after the pyridine treatment. Intracellular neurofibrils, however, are not always so well shown as by the direct passage of sections into the silver nitrate solution.

Before proceeding further, one should prepare the Bielschowsky 
ammoniacal silver nitrate-and-oxide bath as follows:-Pour 5 c.c. of a 20 per cent. solution of silver nitrate into a measuring cylinder and add to it first 5 drops of a 40 per cent. solution of $\mathrm{NaOH}$, and then ammonia, drop by drop, until the brown precipitate formed disappears; dilute to 25 c.c. with distilled water, and filter through paper washed with the same water.

For staining take sections one by one from the silver nitrate bath, quickly wash them in distilled water and transfer them into the ammoniacal silver bath. Here they remain for about ten minutes when they become yellowish-brown and should be, once more, quickly washed in distilled water and placed in 20 per cent. formalin prepared with spring water. The reduction takes place immediately, and if one works with a number of sections it is advisable to retransfer them into a fresh bath of 20 per cent. formalin.

At the end of half an hour and even less, the reduction can be considered as accomplished and sections can be washed in distilled water and toned with a diluted $(0 \cdot 2$ per cent.) solution of gold chloride. This may be slightly acidified with acetic acid if one wishes to obtain a faintly purple background, or neutralised with a few drops of a diluted solution of sodium or lithium carbonate if one prefers greyish-white backgrounds. Instead of gold chloride one can use a slightly acid solution of chloroplatinic acid. After toning there remains only the washing once more of sections in distilled water, and the passing of them for a few minutes in a 5 per cent. solution of sodium hyposulphite, or any diluted fixing bath for photographic plates. Wash again, dehydrate in alcohols of increasing strength up to 95 per cent., clear in carbol-xylol, and mount in balsam.

For other details about the toning and fixing of sections see the original papers of BreLschowsky (op. cit. and Journ. Psychol. Neurol., iv, $1904-5$, p. 227), as well as WoLfF (Biol. Centrbl., xxv, 1905, p. 683), and DA Fano (Proc. Physiol. Soc. Journ. Physiol., liii, 1920).

Bielschowsky states that this method is also suitable for sections of celloidin or paraffin blocks of formol material, but he does not recommend the practice, and I have no experience of it.

Bielschowsky's Method for Peripheral Nerve-fibres (Journ. Psychol. Neurol., iv, 1904 -5, p. 227).-This method can be applied to the study of spinal and sympathetic ganglia, peripheral nerveendings, and end-organs in normal conditions, but its chief applications belong to the domain of histopathology. According to my experience good results are rarely obtained, and the method requires important modifications to become as useful as the above and following ones. 
The staining is carried out on sections of formol material in the same way as described above. There is only this difference that the staining in the ammoniacal silver bath is carried on a few minutes longer, viz., until the sections have taken a decidedly brown colour, after which they are washed in 10 c.c. of distilled water acidified with 5 drops of acetic acid, when they acquire (sometimes in a few seconds) a yellowish tinge. They should then be immediately transferred into the usual 20 per cent. solution of formalin. For the toning a neutral gold bath is necessary: sections should be left therein until red-violet. In the finished preparations axis-cylinders are black, myelin red-violet, connective tissue violet or blue-violet. The washing in acidified water and the prolonged toning both answer for the purpose of creating a sharp contrast between nerve fibres and connective tissue fibres, which might otherwise become stained almost as black as the axis-cylinders.

Bielschowsky has also a method for central nerve-fibres. Sections made by freezing from formol material are placed for twenty-four hours or longer in a 4 per cent. solution of copper sulphate or Weigert's mordant for neuroglia stain ( $\$ 910)$. After washing they are placed for a few seconds in the usual ammoniacal silver bath and then washed, reduced, toned and fixed as above. The preparations are similar to those obtainable by the methods of Fajerstajn, Strahüber and Kaplan.

Bielschowsky's Method for Pieces (op. cit.)._-Good for peripheral nerve-endings and embryonic material, and also for small specimens of adult subjects. This method has been described by Bielschowsky in various ways, probably because of the difficulty of giving fixed rules in a case in which the greatest freedom had to be left to histo. logists to adapt the method to the quality of their material and the purpose of their investigations. In what follows two forms of the method are described: one without and one with pyridine treatment of pieces.

A. Method for Pieces without Pyridine Treatment.-Thin slices or small pieces of formol material are washed for some hours, first in running tap-water and afterwards in distilled water. They are then placed in a 2 per cent. solution of silver nitrate for from one to eight days in the dark. The use of an incubator at $35^{\circ}$ to $37^{\circ} \mathrm{C}$. is optional. After a wash in several changes of distilled water (to be prolonged for some minutes up to some hours according to the length of time during which pieces have been kept in the silver bath, and if in an incubator or not) they are transferred into an ammoniacal solution of silver nitrate prepared as in the method for sections, but diluted 
up to 100 c.c. They are kept therein for from an hour up to six, washed once more in distilled water, passed for twelve to twenty-four hours into the usual 20 per cent, solution of formalin. Wash, dehydrate quickly, embed, preferably in paraffin, tone sections as described above, counterstain, if necessary, mount in balsam.

B. Method for Pieces with Pyridine Treatment.-Pieces of formol material, up to $1 \mathrm{~cm}$. thick for adult tissue, and up to $5 \mathrm{~cm}$. long for embryos, is put for two, three or four days into pure pyridine, washed for some hours in several changes of distilled water and put for three to five days into 3 per cent. silver nitrate at $36^{\circ} \mathrm{C}$. Wash in distilled water and transfer into the diluted ammoniacal silver bath as above, but leaving pieces therein for twenty-four hours. Wash for about two hours in several changes of distilled water, reduce in 20 per cent. formalin. The rest as above.

841. Modifications of BIELSCHOWSKY's Methods.-FavoRSKY (Journ. Psychol. Neurol., vi, 1906, p. 260) uses 10 per cent. silver nitrate for the first silver bath instead of 2 or 3 per cent.

Paton (Mitth. Zool. Stat. Neapel, xviii, 1907, p. 576) fixes fish embryos in 4 per cent. formaldehyde neutralised with carbonate of magnesia. For the first silver bath he uses 0.75 to 1 per cent. silver nitrate and keeps material therein four days in summer, five to seven in cooler weather. To make the ammoniacal silver nitrate-andoxide bath he takes 20 c.c. of 0.75 to 1 per cent. silver nitrate, adds to it 4 drops of 40 per cent. caustic soda and then ammonia drop by drop in the usual way. The embryosare first washed in distilled water, then kept for five to fifteen minutes in 10 c.c. of water acidified with 5 drops of acetic acid, washed once more in pure water, and transferred for twelve hours into a reducing fluid consisting of 1 per cent. hydroquinone 20 c.c., neutralised formalin, 2 c.c. After embedding in paraffin, the sections are toned as usual and counterstained with 1 per cent. eosin in absolute alcohol.

Sснüтz (Neurol. Centrbl., xxvii, 1908, p. 909) finds that the times given by Bielschowsky are too short and washes sections for twentyfour hours after the 2 per cent. silver nitrate bath, leaves them thirty to forty minutes in the ammoniacal silver bath, and twenty-four hours in the 20 per cent. formalin. For toning he puts them for ten minutes into 10 c.c. of water with 2 drops of acetic acid, then for thirty to forty-five minutes into 10 c.c. of water with 3 drops of a 1 per cent. gold chloride solution (until blackish-grey).

BoEke (Anat. Anz., xxxv, 1910, p. 193) has obtained excellent results by the use of Bielschowsky's method for pieces when applied 
to the study of peripheral nerve endings. He fixes in 10 per cent. formalin prepared with 60 per cent. alcohol, changes the fluid two or three times, and then either leaves material therein until wanted or keeps it in 70 to 80 per cent. alcohol. For staining, pieces are brought into 10 to 12 per cent. formalin, and left in it until they are quite free from alcohol. The rest as by Bielschowsky's method for pieces, with or without pyridin treatment. Boeke finds that the method succeeds also after other kinds of fixation.

Schlemmer (Ztschr. wiss Mikr., xxvii, 1910, p. 22) makes the ammoniacal silver nitrate-and-oxide bath by adding to any silver nitrate solution, 40 per cent. caustic soda, drop by drop, until no more precipitate is formed. He then washes the precipitate by repeated decantation until the wash water no longer gives an alkaline reaction, takes it up with the smallest possible quantity of ammonia, and filters through glass wool. This concentrated solution keeps for many days unaltered, and should be diluted ten times its volume before using it.

Dex Río-Hortega (Trab. Lab. Invest. Biol., Madrid, xiv, 1916, p. 181) has made known a similar method used in those laboratories for preparing the ammoniacal silver nitrate bath. Forty drops of 40 per cent. caustic soda are added to 30 c.c. of 10 per cent. silver nitrate, and the precipitate washed ten to twelve times by means of about a litre of distilled water. Fifty cubic centimetres of water are then added to it, and ammonia, drop by drop, until the precipitate is dissolved. The solution, brought finally to 150 c.c. and filtered into a dark brown bottle, keeps well for many months. I find that the ammoniacal silver bath thus prepared can be further diluted with one, two, up to five times its volume of water, and usefully employed for Bielschowsky's method for pieces, particularly for the study of peripheral nerve endings.

AGDuHR (Ztschr. wiss. Mikr., xxxiv, 1917, pp. 1-99), who has exhaustively investigated almost all questions relating to the results obtainable by Bielschowsky's method for pieces, has come to the conclusion that material is best fixed in neutral or slightly acid 20 per cent. formaldehyde (50 per cent. formalin). Pieces should then be washed in distilled water for many days until the wash-water is free from substances reducible by an ammoniacal silver nitrate solution used as test. For the first silver bath he uses 3 per cent. silver nitrate, and for the second a solution obtained by adding to 10 c.c. of 10 per cent. silver nitrate, first 20 drops of 25 per cent. $\mathrm{NaOH}$, then from 200 up to 600 c.c. of distilled water, and lastly ammonia enough to dissolve the precipitate. For the reduction he 
uses again 20 per cent. formaldehyde. To avoid an excessive impregnation of the connective tissue he also finds it useful to. wash pieces in acidified distilled water (see the Bielschowsky method for peripheral nerve-fibres), but he uses as much as five times the amount suggested by Bielschowsky.

842. DA FANO's Modifications.-An important point of this series of modifications of Bielschowsky's method for sections is the use of distilled water, re-distilled on potassium permanganate, with the object of ensuring elimination of any trace of organic matter from the ordinary distilled water and of avoiding the formation of precipitates.

Da Fano's first modification (Mod. 1) (Atti. Soc. Lomb. Sc. Med. Biol., Milano, iii, 1914) was meant for the study of recticular tissue of spleen, lymph glands, and other organs, and is to be carried out as follows :-(1) Fix small pieces of fresh tissue in 10 to 20 per cent. formalin or in Kayserling's first fluid (forty-eight hours at least), or in Orth's fluid (twenty-four to forty-eight hours). (2) Wash pieces in running tap-water for twenty-four to thirty hours, and then in distilled water for another twenty-four hours. (3) Wash sections made by the freezing method in re-distilled water (twenty-four hours), and then place them in filtered 2 per cent. silver nitrate (prepared with redistilled water) in a Petri dish, taking care that they do not touch each other. Here they are kept in the dark and at room temperature from six hours to three days. (4) Treat sections for twenty to thirty minutes with Bielschowsky's ammoniacal silver nitrate solution prepared with only 2 drops of 40 per cent. caustic soda and diluted with redistilled water to 40 to 70 c.c. (5) Reduce, tone, counterstain, and mount as by Bielschowsky's method for sections.

Mod. 2 (Proc. Physiol. Soc. Journ. Physiol., lii, 1919) consists in an application to nervous tissues of Mod. 1. The use of redistilled water and the mode of preparing the ammoniacal silver bath are the same, but Da Fano lays stress on the following points :(1) Nervous tissue must be fixed in 10 up to 20 per cent. formalin for at least three weeks, better still for two months. Attempts to obtain a rapid fixation with 10 to 20 per cent. formalin at $37^{\circ} \mathrm{C}$. gave bad results. (2) Sections of nervous tissues may be placed, after washing in redistilled water, in anhydrous pyridine (six to twelve hours), then repeatedly washed and left overnight in redistilled water, to get rid of all pyridine. This treatment appears to render neurofibrils a little thinner and, consequently, a little 
sharper, but increases the length and cost of the method, and may cause precipitates to form, especially where much myelin is present. (3) It is possible to keep sections, which cannot be stained immediately, for some days or even a fortnight, in redistilled water to which a few drops of formalin have been added. Thorough washing with redistilled water is then imperative before they are transferred into the 2 per cent. silver nitrate solution. (4) Sections of nervous tissues must not remain in the 2 per cent. silver nitrate more than forty-eight hours, or precipitates may form. The longer their stay there, the longer must be the washing before staining; this, however, must not, as a rule, exceed five minutes. (5) The volume to which the ammoniacal silver nitrate is diluted should be 35 to 45 c.c., and the sections remain in it fifteen to twenty minutes. The subsequent washing before transferring the sections into 20 per cent. formalin should not occupy more than ten to fifteen seconds, and their stay in the final formalin solution (especially for cerebral cortex) should not exceed two to three hours.

The other eight Da Fano modifications (Proc. Physiol. Soc., Journ. Physiol., liii, 1919-20) were all proposed for the study of cortex cerebelli, and are characterised by a special treatment of the sections (cut by the freezing method) with various reagents before transferring them into the 2 per cent. silver nitrate solution, nothing having been changed, however, in regard to the long fixation of material in formalin and the use of redistilled water. They may be summarised as follows:-

Mod. 3. Place sections, after washing in redistilled water, in 2 to 3 per cent. silver nitrate at $36^{\circ}$ to $37^{\circ} \mathrm{C}$. for about twenty-four hours; wash quickly; stain in ammoniacal silver nitrate solution diluted to 40 c.c. for thirty minutes. Wash, reduce, tone, and mount as usual.

Mod. 4. Place sections in 50 per cent. pyridine for six to eighteen hours; wash in redistilled water for twenty-four to forty-eight hours ; 2 per cent. silver nitrate at $37^{\circ} \mathrm{C}$. for twenty-four hours, etc., as in Mod. 3.

Mod. 5. Place sections in pure pyridine for four to twelve hours. Wash in redistilled water overnight. Transfer sections into 20 per cent. formalin prepared with redistilled water for about twenty-four hours. Wash again in redistilled water overnight; 2 per cent. silver nitrate at $37^{\circ} \mathrm{C}$., etc., as before.

Mod. 6. Sections are treated first with 20 per cent. formalin, and then with pure pyridine, in the reverse order of Mod. 5.

Mods. 7 and 8. The same as Mods. 5 and 6, but replacing the 
pyridine with a mixture of 3 parts of methyl-alcohol and 2 parts of water.

Mod. 9. Place sections in a mixture of equal parts of 20 per cent. formalin and methyl alcohol for twenty-four hours; wash in redistilled water for six to twenty-four hours; 2 per cent. silver nitrate at $37^{\circ} \mathrm{C}$. for twenty-four hours, etc., as before.

Mod. 10. Place sections into 20 per cent. formalin for twentyfour hours, transfer them, without washing, into a mixture of equal spart of 20 per cent. formalin and methyl alcohol, etc., as in Mod. 9.

Mod. 3 is particularly suitable for human material of young individuals: Mod. 4 for adult subjects. Mods. 5 and 6 are useful for the study of neurofibrils in the various elements of the cortex cerebelli and for the staining of the granules. Mods. 7, 8 and 9 are to be preferred for the demonstration of pericellular baskets and nervous processes. Mod. 10 gives very complete stainings, and is the most certain of all ; preparations are, however, fairly dark, and, therefore, more suitable for general view.

Perdrau (Journ. Pathol. Bact., xxiv, 1921) has worked out a modification which appears to be particularly suitable for the study of the connective tissue in nervous organs. He washes pieces and sections as in Da Fano's modifications, but without having recourse to redistilled water. He then places sections for about ten minutes in 0.25 per cent. potassium permanganate, washes, and treats them as by Pal's modification of Weigert's myelin stain (see § 857). After another wash in distilled water, he transfers sections into 2 per cent. silver nitrate, and continues as in Da Fano's Mod. 1. Nerve cells, nerve fibres, neuroglia, etc., unstained; connective tissue and elastic fibres stained in various shades of purple-grey to black.

843. Neurofibrils; Other Methods.-Cox's Method for fibrils of spinal ganglion cells; see Ztschr. wiss. Mikr. xiii, 1896, p. 498, and Anat. Hefte, x, 1898, p. 98.

S. MeYeR's Berlin blue, see Anat. Anz., xx, 1902, p. 535.

LUGARo's collargol (colloidal silver) method, see Monit. Zool. Ital., $\mathrm{xv}, .1904, \mathrm{p} .353$.

Joris' colloidal gold method has not been received with favour; see Bull. R. Acad. Med. Belg., xviii (S. iv), 1904, p. 293.

Sand (C.R. Ass. Anat. Bruxelles, 1910 ; Bibliogr. Anat. Supp., 1910 , p. 128, or Ztschr. wiss. Mikr., xxviii, 1911, p. 500) gives the following as entirely certain for man, dog, cat, and rabbit. Specimens of not more than $5 \mathrm{~mm}$. in thickness are fixed for forty-eight hours in a freshly prepared mixture of 90 parts of acetone and 10 of nitric acid, to be changed for fresh after half an hour, and once 
again within twenty-four hours. Wash out for at least six hours in pure acetone, changed two or three times. Make paraffin sections and bring them through xylol and acetone into distilled water; silver for three days at about $37^{\circ} \mathrm{C}$. in 20 per cent. solution of silver nitrate. Put for ten minutes into a mixture (at least three days old) of 1000 parts of water, 10 of sodium acetate, 5 of gallic acid, and 3 of tannin (to be changed if it becomes turbid). Mount at once or tone until grey (five minutes) in 80 parts of water with 17 of 2 per cent. ammonium sulphocyanide and 3 of 2 per cent. gold chloride; fix for a few seconds in 5 per cent. sodium hyposulphite. Neurofibrils grey-violet, shown in cells, dendrites, and axons. Terminal buds of Held also clearly shown, and nothing else stained. One may counterstain in any way, even by Weigert's or Benda's methods for neuroglia stain.

The methylene blue intra vitam method is important, and may be usefully employed for the study of neurofibrils. See the processes of Apathy, Dogiel, and Bethe in Chapter XVI.

\section{Methods for the Demonstration of Golgi's Internal Apparatus.}

844. Introduction.-The discovery of the "apparato reticolare interno" was made by Golgi in 1898 by means of his rapid process (see Chapter XXXIV). Soon afterwards he had recourse to a mixture due to Veratti (see next paragraph), and Negri, Pensa, and others of Golgi's pupils found that the internal apparatus is not a peculiarity of nerve cells. In 1902 Kopsch showed that the apparatus can be stained by a simple immersion of nervous tissues (spinal ganglia) into 2 per cent. osmic acid for eight to ten days. Since then the apparatus was shown to exist in almost every kind of cells, and new processes proposed for its demonstration in nervous and other tissues by Sjövall, Golgi, Ramon y Cajal, Gatenby, Da Fano.

As the methods of Kopsch and Sjövall, the Mann-Kopsch method, and Gatenby's Mann-Kopsch-Altmann combination have already been fully discussed in Chapter XXVI, and particularly described in $\$ \S 692-694$ and 696 , there remain to be described here only those methods which are particularly suitable for the study of the internal apparatus in nervous tissues, with exception of Golgi's rapid process, for which see Chapter XXXIV, § 882 .

845. Golgi-Veratit's Method (see Golgi, Anat. Anz. Verh. Anat, Ges., xiv, 1900, p. 174).--Small pieces are hardened for a time 
varying from a few hours to ten days or longer in Veratti's mixture, consisting of-

5 per cent. potassium bichromate

30 parts.

$0 \cdot 1$ per cent. chloroplatinic acid

1 per cent. osmic acid

15 to 30

From time to time pieces are put in one or other of Golgi's rejuvenating fluids (as described in $\S 888$ ), and thence into 0.8 to 1 per cent. silver nitrate. Sections are cut and mounted as by Golgi's bichromate and nitrate of silver method (see $\S 882$ ).

846. Golar's Arsenious Acid and Silver Nitrate Method (Arch. Ital. Biol., xlix, 1908, p. 272).-Small pieces of quite fresh tissues are fixed for three, six, eight or twelve hours in equal parts of 20 per cent. formalin, saturated solution of arsenious acid, and 96 per cent. alcohol. After a quick wash with distilled water, they are passed for some hours (or days) into 1 per cent. silver nitrate, and then treated with a reducing fluid, usually Cajal's hydroquinone mixture (hydroquinone 20 grms., sodium sulphite 5 grms., formalin 50 c.c., water 1000 c.c.). Wash quickly, dehydrate, and embed either in celloidin or paraffin. The sections are toned with equal parts of 1 per cent. gold chloride and a mixture consisting of water 1000 c.c., with 30 grms. each of sodium hyposulphite and ammonium sulphocyanide, and then rapidly bleached by the following method, due to Veratis :- Wash the toned sections in distilled water and transfer them for one, two or three minutes into potassium permanganate 0.5 grm., distilled water 1000 c.c., sulphuric acid 1 c.c. ; wash again ; transfer into 1 per cent. oxalic acid until the yellowish colour imparted to the sections by the potassium permanganate has disappeared; wash thoroughly in repeatedly changed distilled water ; counterstain, dehydrate, and mount as usual.

84\%. RAMÓN Y ('AJAL's Uranium Nitrate and Silver Nitrate Method (Trab. Lab. Invest. Biol., Madrid, xii, 1914, p. 127).--(1) Small pieces of quite fresh tissues are fixed for ten to fourteen hours in a mixture of neutralised formalin 15 c.c., distilled water 85 c.c., uranium nitrate $1 \mathrm{grm}$. Instead of uranium nitrate, uranium acetate, as suggested by Del Río-Hortega, may be sometimes used. Should a very fine reaction be desirable, the following formula may be employed:- Uranium nitrate 1 grm., ethyl or methyl alcohol 30 c.c., distilled water 80 c.c., neutralised formalin 15 to 20 c.c. (2) After a quick wash in distilled water, pieces are transferred into 1.5 per cent. silver nitrate and kept therein for thirty-six to fortyeight hours at room temperature. If the pieces are only a few and 
small, 1 per cent. silver nitrate will be sufficient. (3) Wash quickly and reduce for eight to twenty-four hours in hydroquinone 1 to 2 grms., formalin 15 c.c., distilled water 100 c.c., sodium sulphite 0.5 grm. (4) Wash quickly, embed in paraffin or celloidin, or make sections by the freezing method. (5) Tone and counterstain sections if desirable. Dehydrate and mount as usual.

Best results are obtained from vertebrates, preferably kittens and young rabbits. The method may be applied to human material, if available in a sufficiently fresh condition. From invertebrates results are not so good, and rather uncertain, so that Cajal advises a simple fixation in formalin or formalin-acetone, followed by impregnation with silver nitrate, as by his reduced silver methods for neurofibrils.

848. Da Fano's Cobalt Nitrate Modification (Proc. Physiol. Soc., Journ. Physiol., liii, 1920 ; Journ. R. Micr. Soc., 1920, p. 157).Small pieces of quite fresh tissues are fixed for six to eight hours at room temperature in cobalt nitrate 1 grm., distilled water 100 c.c., formalin 15 c.c. The solution can be prepared beforehand, and keeps unaltered for months. The formalin need not be neutralised unless strongly acid or containing free sulphuric acid, in which case it is necessary to neutralise it by one of the usual methods. For the fixation of embryonic organs and in all cases in which a shrinkage of delicate tissues is to be feared, the quantity of the formalin may be reduced to 10,8 , or 6 c.c. for every 100 c.c. of distilled water. The time of fixation should be shortened to three to four hours or even less in the case of very small pieces, such as spinal ganglia of mice and rats, the pituitary body of the same animals, etc. Pieces of spinal cord, cerebrum, cerebellum of adult animals give better results if fixed for about eight to ten hours. The fixation may be prolonged in special cases to twelve to twenty hours, but should not exceed twenty-four hours. The fixation in an incubator at a temperature varying between $25^{\circ}$ and $37^{\circ} \mathrm{C}$. has been attempted with success in the case of tissues of adult subjects, but it leads to a staining of both the internal apparatus and intracellular formations, which, according to their morphology, are to be considered as mitochondria.

For the impregnation, Da Fano quickly washes the pieces in distilled water, makes their surfaces smooth if necessary, and then places them into 1.5 per cent. silver nitrate in the dark for twentyfour to forty-eight hours at room temperature. For very small fragments, 1 per cent. silver nitrate may be used, whilst for pieces of 
spinal cord of adult subjects, 2 per cent. should be preferred. For the reduction he uses Cajal's hydroquinone-formalin mixture, taking care in further recutting the pieces, before transferring them into the reducing fluid, so that their thickness should not exceed $2 \mathrm{~mm}$. He dehydrates and embeds pieces, preferably in paraffin, or he makes sections by the freezing method. He usually tones these by means of $0 \cdot 2$ per cent. gold chloride, fixes with 5 per cent. sodium hyposulphite, counterstains and mounts as usual.

The method gives good results also with material from lower vertebrates and invertebrates.*

849. Other Methods and Modifications.-Besta (Anat. Anz.: xxxvi, 1910, p. 477) fixes for two days in 20 parts of formol with 2 of acetic aldehyde and 80 of water, washes for twenty-four hours in distilled water changed seven or eight times, and puts for two days in 4 per cent. solution of ammonium molybdate, makes paraffin sections, stains in a 1:1000 solution of thionin, differentiates in 3 parts of creosote to 1 of absolute alcohol, and passes through pure creosote and xylol into neutral balsam. Recommended for Purkinje cells and spinal ganglia of young animals.

Suchanow (Neurol. Centrbl., xxi, 1902, p. 777) has obtained good results by the use of Golgi-Veratti mixture, keeping pieces of spinal cord and spinal ganglia for twenty to thirty days in the mixture and for two to three days in the rejuvenating fluid.

LEGENDRE (Anat. Anz., xxxvi, 1910, p. 209) omits the toning and bleaching by Golgi's arsenious acid method, and embeds in paraffin.

Similarly Collin et LuCren, Bibliogr. Anat. Supp., 1909, p. 238.

SavaGnone (Pathologica, i, 1909) silvers pieces fixed in Golgi's arsenious acid mixture with 30 c.c. of tachiol (10 per cent. silver fluoride) in 100 of water.

Carleton (Journ. R. Micr. Soc., 1919, p. 321) reduces pieces treated according to Cajal's uranium nitrate method for only two hours in the usual hydroquinone mixture.

- Penfield (Brain, xliii, 1920) has successfully employed Cajal's uranium nitrate method for his experimental investigations on the alterations of Golgi's apparatus in nerve cells of spinal cord and spinal ganglia of young cats. He adds 20 c.c. (instead of 15) to Cajal's fixing fluid and as much as 1.5 grms. of sodium sulphite to the hydroquinone-formalin solution. He finds it imperative to dehydrate pieces very quickly before embedding them in paraffin. In order to obtain perfect fixation of the spinal cord he sometimes performs a laminectomy in the lower lumbar region of the anæsthe-

* Da Fano's method has been used by me with great success for the study of gametogenesis of many invertebrata (J. B. G.). 
tised animal, passes a needle in the subarachnoid space, and allows the fixative to flow in " under a gravity pressure of $75 \mathrm{~cm}$." The heart stops about a minute after the beginning of the injection, which is continued for twenty hours. At the end of this time the cord is removed, pieces cut and dropped directly into the silver bath.

For counterstaining Penfield finds it particularly useful to immerse untoned sections into a diluted solution of Unna's polychromemethylene blue for one to four hours, this being followed by passage through alcohols of increasing strength and differentiation in absolute alcohol. By this method, also Holmgren's, trophospongium is sometimes stained. But for the study of the relationship between the latter and Golgi's apparatus, Penfield (in litteris) prefers to make drawings of the apparatus from certain selected cells, subsequently removing the coverslip and bringing the slides through graded alcohols into 5 per cent. iron alum for twelve to twenty-four hours. This removes all silver from the cells as well as the counterstain, and at the same time mordants the tissues for further staining by Heidenhain's iron-hæmatoxylin method. If the proper amount of differentiation has been secured of the particular cells already drawn, the trophospongium will be found stained with great detail.

\section{Addendum.}

Holmaren's Method for Trophospongium.-Fix small pieces or ganglia in trichlorolactic acid for twenty-four hours. Dehydrate and embed in paraffin as usual. Stain thin sections for twenty-four hours in Weigert's resorcin fuchsin solution for elastic fibres (see $\$ 758$ ) recently prepared and a little diluted. Dehydrate and mount as usual.

\section{Methods for the Demonstration of the Sustaining Apparatus of Medullary Sheaths, Neurokeratin, etc.}

850. Methods demonstrating Funnels and Spiral Filaments.Golgi (see Rezzonico, Arch. p. l. Sc. Med., iv, 1880, p. 78; GolgI, Opera Omnia I, p. 163) puts small pieces of spinal cord in 2 per cent. potassium bichromate for eight to fifteen days in summer, or a month in winter. After a quick wash he transfers them into 0.50 to 0.75 per cent. silver nitrate for two or three days in summer, or eight, ten or more in winter. The pieces are then washed in 95 per cent. alcohol, dehydrated in absolute alcohol, cleared in oil of turpentine and teased therein. The preparations, mounted in dammar, must be exposed to sunlight for eight to ten days; or to diffused daylight for twenty to forty days, 
For peripheral nerve fibres, Golgr (Op. Omnia I, p. 162) has proposed two methods. Of these the first is a modification of his rapid process (see $\S 882$ ), and should be carried out as follows :Tracts of peripheral nerves are cut with care not to stretch them, and put in a mixture of 10 parts of 2 per cent. potassium bichromate and 2 of 1 per cent. osmic acid. After about one hour the tract or tracts of nerves are sufficiently hardened to be further recut in pieces of about $\frac{1}{2} \mathrm{~cm}$. in length, which are put back in the same mixture. After another three hours, and successively at intervals of three hours during twenty-four hours, pieces are transferred into 0.5 per cent. silver nitrate where they may remain for any time, but no less than eight hours. Preparations are made and mounted as above.

The other method is a modification of that used for central nerve fibres, the only difference consisting in keeping the pieces in the bichromate for a much shorter period, i.e., for from four hours to at most two days, and in transferring specimens into the silver bath at intervals of about three hours. After twelve to twenty-four hours preparations can be made as described above.

The preparations made by the first method show the spiral filaments very clearly, but do not keep well. The preparations. made by the second method do not show the spiral filaments so completely, but are more useful for the demonstration of the funnels and last longer.

Cattani (Arch. Ital. Biol., vii, 1886, p. 345) either fixes in Flemming's fluid and teases and mounts in glycerine, or puts pieces into Golgi's bichromate and osmic acid mixture, dehydrates and passes into oil of turpentine to be changed until it remains colourless. The turpentine dissolves the myelin and leaves funnels and spiral filaments visible. Cattani also has a modified Golgi method, now superseded.

Sala (Verh. Anat. Ges. Anat. Anz., 1900, p. 176) employs the Golgi-Veratti method for the intracellular network (see $\S 845$ ).

See also concerning these methods, Mondino, Arch. p. l. Sc. Med., viii, p. 45 .

GALLI (Ztschr. wiss. Mikr., iii, 1886, p. 467) hardens peripheral nerves for eighteen to twenty days into Müller's fluid, cuts out pieces 5 to $6 \mathrm{~mm}$. long, and keeps these in Müller's fluid diluted with 2 parts of water for another two days. He then stains for fifteen to twenty minutes in aqueous solution of China blue, washes out in alcohol, clears in essence of turpentine, and mounts in damar.

RAMóN y CAJAL has successfully employed some modifications of 
his reduced silver and uranium nitrate methods, for which see Trab. Lab. Invest. Biol., Madrid, x, 1912, p. 221.

851. Methods demonstrating Neurokeratin Network.-PlatneR (Ztschr. wiss. Mikr., vi, 1889, p. 186) fixes for several days in a mixture of 1 part of Liq. Ferri Perchlor. (Ph. G., ed. 2) and 3 to 4 parts of water or alcohol, washes out well in water and stains for several days or weeks in a concentrated solution of "Echtgrün" (dinitroresorcin) in 75 per cent. alcohol. See also BEER, Jahrb. Psychiatrie, ii, 1893.

Cox (Anat. Hefte, i, 1898, p. 102, note) fixes nerves in 2 per cent. osmic acid (rabbit) or 1 per cent. (frog), washes, dehydrates, clears with bergamot oil, and mounts in balsam. The bergamot oil dissolves out the myelin, and leaves the neurokeratin visible. It may be necessary to leave the nerves for forty-eight hours in the oil.

Corning (Anat. Anz., xvii, 1900, p. 309) studies the neurokeratin network in the sciatic of the frog by means of sections of sublimate material strongly stained with iron hæmatoxylin.

KaPLAN (Arch. Psychiatr., xxxv, 1902, p. 825) stains sections with acid fuchsin and differentiates them by Pal's method.

Gedoelst (La Cellule, v, 1889, p. 136) has the following: (a) A nerve is treated with liquid of Perenyi, either pure or with addition of a trace of osmic acid, and examined in glycerin. By this treatment the myelin loses its excessive refractivity and the neurokeratin network comes out clearly. (b) Silver nitrate. Good images, but uncertain. (c) Treatment with a mixture of 1 per cent. osmic acid and absolute alcohol. The network comes out black. 


\title{
CHAPTER XXXIII.*
}

\author{
MYELIN STAINS.
}

852. Iron Hæmatoxylin.-According to A. Bolles Lee (see 1913 Ed.) the simplest way of staining myelin is to make paraffin sections of formol material and stain them with iron hæmatoxylin exactly as for central corpuscles (say, twelve to fourteen hours in the mordant, six in the hæmatoxylin, and a few minutes for the differentiation). Sections best not over $15 \mu$. One may counterstain the cells with carmalum, but not for more than half an hour, or the hæmatoxylin will be attacked. The stain is not so æsthetic as Weigert's, but quite as sharp. Axis cylinders are not shown.

Similarly Regaud (C. R. Acad. Sc., cxlviii, 1909, p. 861), but adding a chrome mordantage either concurrently with the formol fixation, or subsequently. Also Nageotte (C. R. Soc. Biol., lxvii, 1909 , p. 542), with sections of formol material by the freezing method; Houser (Journ. Comp. Neurol., x, 1901, p. 65), and Brookover (ibid., xx, 1910, No. 2) ; Spielmeyer (Neurol. Centrbl., xxix, 1910, p. 348); and his Technik d. mikrosl. Untersuch. d. Nervensystems, 1911, p. 87 , with sections of 25 to $35 \mu$ by the freezing method; Loyez (C. R. Soc. Biol., lxix, 1910, p. 511), who differentiates first lightly, till the grey matter begins to appear, in the iron alum, then washes, and differentiates further in Weigert's borax ferricyanide; GILBERT (Ztsch. wiss. Mikr., xxviii, 1911, p. 279), who mordants with iron alum, stains with molybdic acid hoematoxylin, and differentiates with the borax ferricyanide; STOELTzNer (ibid., xxiii, 1906, p. 329), who mordants celloidin sections of formol material for five minutes in Liq. ferri sesquichlorati, stains in 0.5 per cent. hæmatoxylin, and differentiates in the mordant or in borax ferricyanide; and KodIS (Arch. mik. Anat., lix, 1902, p. 211), who fixes for one or two days in saturated solution of mercury cyanide, hardens in 10 per cent. formol, and stains sections, made by the freezing method, with Heidenhain's iron hæmatoxylin.

853. WEIGERT's Methods.-There have been in all three methods of WEIGERT:- the 1884 method, the 1885 method, and the 1891 method.

* Revised by Dr. C. Da Fano, King's College, University of London. 
The 1884 method (Fortschr.d. Med., ii, 1884, pp. 120, $190 ;$ Ztschr. wiss. Mikr., i, 1884, pp. 290, 564), which depends on the formation of a chrome lake of hæmatoxylin, may be considered as superseded. Not so the two others, which depend on the formation of a copper lake in addition to the chrome lake.

854. WeIGERT's 1885 Method (Fortschr.d. Med., iii, 1885, p. 236 ; Ztschr. wiss. Mikr., 1885, pp. 399, 484 ; Ergebn. Anat., vi, 1896 (1897), p. 10).-The tissues are hardened in potassium bichromate. WEIGERT takes (Ergebn., p. 10) a 5 per cent. solution, and if time is an object hardens in a stove. (Other bichromate mixtures will do, e.g., Müller's, Kultschitzky's, Zenker's ; Erlicki's is not to be recommended.) The tissues are "ripe" for staining when the hardening has been carried to a certain point. They are first yellow, without differentiation of the grey matter from the white; these are unripe. Later they show the grey matter light brown, the white matter dark brown; and these are ripe.

More recently (Ergebn., p. 14) he added to the bichromate solution 2 per cent. of chrome alum or of chromium fluoride, which hastens the hardening, so that small specimens become brown and ripe in four to five days, without stoving.

After hardening, tissues are generally embedded in celloidin and the blocks hardened in the usual way. They are then put for one or two days, in an incubating stove, into a saturated solution of neutral copper acetate diluted with 1 volume of water. By this treatment the tissues become green and the celloidin bluish-green. They may then be kept, till wanted for sectioning, in 80 per cent. alcohol.

Sections are made, well washed in water, and brought into a stain composed of :-

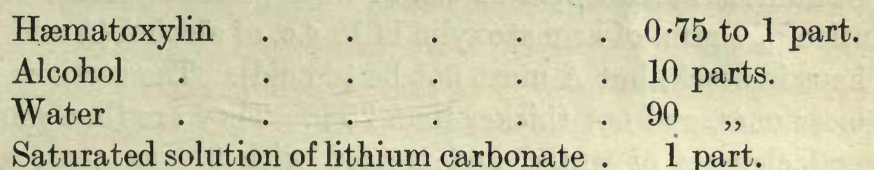

They remain there, for spinal cord, two hours; for medullary layers of brain, two hours; for cortical layers, twenty-four hours.

They are then again well washed with water, and brought into a decolorising solution composed of :-

Borax

Ferricyanide of potassium

Water

$2 \cdot 0$ parts.

$2 \cdot 5 \quad$

$200 \cdot 0$,

They remain there until complete differentiation (half an hour to 
several hours), and are then well washed with water (running, or changed several times), dehydrated, and mounted in balsam. They may be previously counterstained, if desired, with alum-carmine.

The method is applicable to the study of peripheral nerves as well as to nerve centres, and also the study of lymphatic glands, skin (see SchIEFFERDECKer, Anat. Anz., ii, 1887, p. 680), bile capillaries, and other objects.

The process is applicable to tissues that have been hardened in alcohol or in any other way, provided that they be put into a solution of a chromic salt until they become brown before mordanting them in the copper solution.

It is not necessary that the mordanting be done in bulk. Max FlesCH (Ztschr. wiss. Mikr., iii, 1886, p. 50) prefers (following LICHTHEIM) to make the sections first, and to mordant them separately. - Vassale (Riv. sperim. Freniatr., xv, 1889, p. 102) first stains the sections in 1 per cent. hæmatoxylin for three to five minutes, then puts them for three to five minutes into saturated solution of copper acetate, and differentiates as Weigert.

855. WeIGERT's 1891 Method (Deutsche med. Wochenschr., xvii, 1891 , p. 1184). - The material is hardened in bichromate and embedded in celloidin (see last §). It is then (according to the latest form of the process (Enzycl. mik. Technik., 1903, p. 942)), put for twenty-four hours in a stove into a solution of $2 \frac{1}{2}$ parts of chromium fluoride, 5 of copper acetate, and 5 of acetic acid in 100 of water.*

Sections are then made and stained for from four to twentyfour hours at room temperature in a freshly prepared mixture of 9 volumes of (A), a mixture of 7 c.c. of saturated aqueous solution of lithium carbonate with 93 c.c. of water, and 1 volume of (B), a solution of $1 \mathrm{grm}$. of hæmatoxylin in 10 c.c. of alcohol ( $\mathrm{A}$ and $\mathrm{B}$ may be kept in stock, but A must not be too old). The sections should be loose ones, and not thicker than $25 \mu$. They are then washed in several changes of water, and treated with 90 per cent. alcohol, followed by carbol-xylol, or by a mixture of 2 parts of anilin oil with 1 of xylol, then pure xylol and xylol balsam (not chloroform balsam).

It was, however, found that preparations thus made, without differentiation, did not keep well, and WEIGERT (Ergebn. Anat., iii, 1894, p. 21) reverted to the practice of differentiating with the borax-ferricyanide mixture.

* Instead of the chromium fluoride one may use chrome alum, as Weigert did at one time, and as some still do. But then one must boil, as directed for Weigert's Neuroglia stain. 
Later still (Enzycl. mik. Technik., 1903, p. 942) he employed a stain composed of equal parts of (A), a mixture of 4 c.c. of the officinal Liquor ferri sesquichlorati P. G. with 96 of water, and (B), a mixture of 10 c.c. of a 10 per cent. alcoholic solution of hæmatoxylin with 90 of 96 per cent. alcohol. The two (A and B) must be mixed immediately before use, and the sections should remain in the stain overnight or longer, then rinsed and differentiated as usual. This has the advantage of demonstrating very fine fibres, and of giving a colourless back ground.

For difficult objects the differentiating liquid may be diluted with water, and gives better results than dilute acetic or hydrochloric acids or the like, which were formerly recommended.

By means of Weigert's methods only the myelin sheaths of normal nerve-fibres are stained, whilst those of degenerated tracts are of a paler colour and, if the degeneration is sufficiently old, they may even be stainless. See also $\$ 870$.

856. Formol Material (Ergebn. Anat., vi, 1896, p. 14) may be employed if mordanted till brown (four or five days) in 5 per cent. solution of potassium bichromate with 2 per cent. of chromium fluoride.

I understand from Dr. Perdrau that this method is the most satisfactory of all for routine work, and relatively small pieces; but particularly for the histopathological investigation of parts of the human spinal cord, medulla oblongata, pons, and midbrain. He generally cuts from material fixed in formalin, for no less than ten days, slices $\frac{1}{3}$ to $\frac{1}{2} \mathrm{~cm}$. thick, and places them direct in the mordant (potassium bichromate 5 grms., chromium fluoride, 2.5 grms., water, 100 c.c.) for five to six days. Relatively large pieces may be left in the mordant four or five days longer. After a thorough wash in running tap water, he dehydrates and embeds in celloidin. The sections are stained overnight in Kultschitzky's hæmatoxylin ( $\$ 859$ ) several months old, washed in water, and placed in a bowl of distilled water to which about 2 c.c. of a saturated solution of lithium carbonate have been added. They are stirred about several times and transferred into a fresh bath of the same solution if necessary, until the celloidin is all but colourless. He lastly differentiates, as by Pal's method ( $\$ 857$ ), washes, and counterstains either in alum carmine for ordinary work or in an alcoholic solution of eosin if the preparations are to be photographed.

P. Mexer (Neurol. Centrbl., xxviii, 1909, p. 353) embeds formalin material in celloidin and cuts before putting into Weigert's copper fluid. 
For Sheldon's modification, which is also based on a formalin fixation, see Folia Neurobiol, viii, 1914, p. 1.

\section{Modifications of Weigert's Method.}

85\%. PAL's Method (Wien. med. Jahrb., N.F. i, 1886, p. 619; Ztschr. wiss. Mikr., iv, 1887, p. 92 ; Med. Jahrb., N.F. ii, 1887, p. 589).-One proceeds as in WEIGERT's process, but omitting the copper bath. After staining in the hæmatoxylin solution the sections are washed in water (if they are not stained of a deep blue a trace of lithium carbonate must be added to the water). They are then brought for twenty to thirty seconds into 0.25 per cent. solution of potassium permanganate, rinsed in water, and brought into a decolorising solution composed of :-

Oxalic acid

Potassium sulphite $\left(\mathrm{SO}_{3} \mathrm{~K}_{2}\right)$

Dist. water
$1.0 \mathrm{grm}$.

1.0

$200 \cdot 0$ c.c.

In a few seconds the grey substance of the sections is decolorised, the white matter remaining blue. If the differentiation is not complete the whole process can be repeated a second time, and so on. The sections should now be well washed out, and may be counterstained with Magdala red or eosin, or (better) with picrocarmine or acetic acid carmine.

Pal's process gives brilliant results, the ground of the preparations being totally colourless. WEIGERT (Ergebn. Anat., vi, 1896, p. 21) considered it superior to his own for thick sections, but not so safe for very fine fibres.

Marcus stains by the Pal method sections of material hardened in formalin.

GUDDEN (Neurol. Oentrbl., xvi, 1897, p. 24) makes celloidin sections of material hardened in 5 to 10 per cent. formol followed by alcohol, treats them for ten hours with 0.55 per cent. chromic acid, rinses with water, and treats with 80 per cent. alcohol; then stains by the method of Pal, adding to the hæmatoxylin a few drops of dilute nitric acid (MINNICH).

TSCHERN YSCHEW and KARUSIN (Ztschr. wiss. Mikr., xiii, 1896, p. 354) stain for twenty-four hours in KULTSCHITZKY's hæmatoxylin.

Pavlow (ibid., xxi, 1904, p. 14) uses the permanganate twice as strong as Pal.

KozOWSKY (Neurol. Centrbl., xxiii, 1904, p. 1041) stains as Weigert, and differentiates the sections first with 1 per cent. permanganate, till the grey matter comes out brown, and finishes the differentiation with Liq. ferri sesquichlorati.

Pötrer (Ztschr.wiss. Mikr., xxvii, 1910, p. 238) stains as Weigert, last $\S$, and differentiates first in 0.25 per cent. permanganate, then in borax ferrieyanide. 
858. KaISER (Neurol. Centrbl., xii, 1893, pp. 364) hardens first in Müller's fluid, then for eight days in Marchi's fluid (\$ 870), mordants sections for five minutes with sesquichloride of iron (1 part to one of water and 3 of 70 per cent. alcohol), stains and differentiates with Pal's liquid. For details see early editions.

Bolton (Journ. Anat. \& Phys., xxxii, 1898, p. 247) makes sections of formalin material, and mordants them for a few minutes in 1 per cent. osmic acid, or for a few hours in iron-alum or ammonium molybdate, stains in KULTSCHITZKY's hæmatoxylin (next §), and differentiates by Pal's process.

Similarly WynN, ibid., xxxiv, 1900, p. 381.

LAASLETT (Lancet, 1898, p. 321) mordants in Marchi's fluid (1 week), makes sections, stains by KuLTscHITzKY's method, and differentiates by PAL's.

859. Kultschitzky's Method (Anat. Anz., iv, 1889, p. 223 ; and v, 1890 , p. 519).- Specimens are hardened for one or two months in ERLICKI's fluid, imbedded in celloidin or photoxylin, and cut. Sections are stained for from one to three hours, or as much as twenty-four, in a stain made by adding $1 \mathrm{grm}$. of hæmatoxylin dissolved in a little alcohol to 100 c.c. of 2 per cent. acetic acid. They are washed out in saturated solution of lithium or sodium carbonate. Differentiation is not necessary, but by adding to the lithium carbonate solution 10 per cent. of a 1 per cent. solution of potassium red prussiate, and decolorising therein for two or three hours or more, a sharper stain is obtained. After this the sections are well washed in water and mounted in balsam. Myelin dark blue.

Wolters (Ztschr. wiss. Mikr., vii, 1890, p. 466) proceeds as Kultschitzky, except that he stains at $45^{\circ} \mathrm{C}$. for twenty-four hours, after which the sections are dipped in Müller's fluid, and differentiated by Pal's method.

Similarly KAES (Neurol. Centrbl., x, 1891, p. 456). Myelin dark blue, cells yellow-brown.

860. Mrtrophanow (Ztschr. wiss. Mikr., xiii, 1896, p. 470) mordants photoxylin sections for at least twenty-four hours at $40^{\circ} \mathrm{C}$. in a mixture of equal parts of saturated aqueous solution of copper acetate and 90 per cent. alcohol, stains for ten minutes in KuLTSCHITzKx's hæmatoxylin, and differentiates with Weigert's ferricyanide fluid.

861. BerkLey's Rapid Method (Neurol. Centrbl., xi, 1892, p. 270). -Slices of tissue of not more than $2 \frac{1}{2} \mathrm{~mm}$. in thickness are hardened for twenty-four to thirty hours in FLEmming's fluid, at a temperature of $25^{\circ} \mathrm{C}$., then in absolute alcohol, then imbedded in celloidin and cut. After washing in water the sections are put overnight into a saturated solution of copper acetate (or simply warmed therein to $35^{\circ}$ to $40^{\circ} \mathrm{C}$. for half an hour). They are then 
washed, and stained for fifteen to twenty minutes in a lithium carbonate hæmatoxylin similar to Weigert's, warmed to $40^{\circ} \mathrm{C}$., allowed to cool, and differentiated for one to three minutes in Weigert's ferricyanide liquid, which may be diluted if desired with one third of water.

862. HrLl (Brain, xix, 1896, p. 1 ; Phil. Trans., 184, в, 1894, p. 399) stains well-washed Müller material in bulk in alum carmine, cuts and mordants sections for twenty-four hours in half-saturated solution of copper acetate, stains and differentiates as Weigert, taking the differentiating fluid only half as strong.

863. BENDA's Rapid Method (Berlin klin. Wochenschr., xl, 1903, p. 748). Sections of formol material by the freezing process (alcohol being avoided) are stained (without any mordanting) for twenty-four hours in Boehmer's hæmatoxylin, differentiated with Weigert's ferricyanide, and mounted in balsam. Only recommended for peripheral nerves, or for preliminary examination of the central nervous system.

Similarly, NaGeotte, C.R. Soc. Biol., ii, 1908, p. 408, staining with hæmalum.

Similarly the Enzycl. mik. Technik., 1910, ii, p. 239, with fresh material cut by the freezing process, and the sections mounted in lævulose (as alcohol somewhat extracts the stain).

864. Streeter (Arch. mik. Anat., lxii, 1903, p. 734) stains small nerve-centres in bulk (after mordanting in Weigert's bichromate and fluoride mixture, $\$ 845$ ) with. Weigert's hæmatoxylin (four to six days), washes for a couple of days in 70 per cent. alcohol, makes paraffin sections, and differentiates them by the method of Weigert or Pal.

865. Besta's Ammonio-Chloride of Tin Methods (Riv. Sperim. Freniatr., xxxi, 1905, p. 569).--Pieces of peripheral nerves are fixed for one to three days in 100 c.c. of water with 25 of formol, and 4 grms. of Merk's ammonio-chloride of tin, and then dehydrated and embedded as usual. The sections may be stained in different ways : (a) For twenty-four hours in Mallory's phosphomolybdic-carbolicacid hæmatoxylin with subsequent differentiation in Lugol's solution; (b) for thirty to sixty minutes in a very diluted solution of Delafield's hæmatoxylin and then for a minute in Held's acetic solution of erythrosin; (c) for five to ten minutes in erythrosin, and then for two hours in a mixture of equal parts of 1 per cent. hæmatoxylin and 4 per cent. ammonium molybdate with 3 drops of acetic acid to every 50 c.c. of the mixture.

866. Gallein.-ARONson (Centrbl. med. Wiss., xxviii, 1890, p. 577) stains sections of material, hardened in liquid of Erlicki or Müller and mor- 
danted with copper acetate, for twelve to twenty-four hours in a solution of 3 to 4 e.c. of Gallein in 100 c.c. of water with 20 of alcohol and three drops of a concentrated solution of sodium carbonate. Sections are differentiated by the method of Weigert, or Pal. Nerve-fibres red. A second stain with methylene blue may follow (best after differentiating with potassium permanganate). Similarly SCHRöTter (Centrabl. allg. Path., xiii, 1902, p. 299).

867. Schrötter (Neurol. Centrbl., xxi, 1902, p. 338) also stains sections for two to three hours in a 5 per cent. solution of sodium sulphalizarinate, to which a few drops of 5 per cent. oxalic acid (enough to give an orange tint) are added, then differentiates until no more colour comes away in sodium carbonate solution of $\frac{3}{1000}$ strength, and mounts in balsam. Myelin red, on a colourless ground.

868. Toluidine Blue and Methylene Blue.-Harris (Philadelphia Med. Journ., i, 1898, p. 897) stains sections (of material hardened as for Weigert's stain) for several hours in a 1 per cent. solution of toluidine blue in I per cent. borax solution, and differentiates in saturated aqueous solution of tannic acid. Similarly, but with methylene blue, in a complicated way Fraenkel (Neurol. Centrbl., xxii, 1903, p. 766).

Bing and Ellermann (Arch. Anat. Phys., Phys. Abth., 1901, p. 260) harden in 9 parts of acetone to 1 of formol, cut without imbedding, stain for five to ten minutes in saturated methylene blue solution, and put for one or two into saturated solution of picric acid.

869. Other Modifications or Similar Methods.-FLECHSIG, Arch. Anat. Phys., Phys. Abth., 1889, p. 537; Breglia, Ztschr. wiss. Mikr., vii, 1890, p. 236; Rossi, ibid., vi, 1889, p. 182; MERCIER, ibid., vii, 1891, p. 480 ; HaUG, ibid., p. 153 ; WALSEM, ibid., xi, 1894, p. 236 ; ROBERTson, Brit. Med. Journ., 1897 (1), p. 651.

Strong (Journ. Comp. Neur., xiii, 1903, p. 291) finds copper bichromate (of 2 to 3 per cent.) the best mordant; and that the mordanting is best done before bringing into celloidin. After staining, he treats for half a minute with $0 \cdot 25$ per cent. osmic acid and differentiates as Pal.

K. Kocr (Berl. Klin. Wochenschr., li, 1914, p. 422) makes sections by the freezing method of formalin material imbedded in gelatine, and after staining with Weigert's iron hæmatoxylin, differentiates by Pal's method, and mounts in glycerin jelly.

870. MarChI's Method (for Degenerate Nerves) (Riv. sperim. Fren., xii, 1886, p. 50).-Small pieces of nervous tissue are hardened for a week in Müller's solution, and then put for a few days into a mixture of 2 parts of Müller's solution and 1 part of 1 per cent. osmic acid. Sections are cut, best without imbedding, and mounted in balsam. The myelin sheaths of normal nerve fibres take a yellowish-brown colour, those of degenerated fibres a black one.

This process, therefore, gives positive images of the degenerated elements, Weigert's process only giving negative ones.

For a critical review of this method and its modifications, see 
Weigert (Ergebn. Anat., vii, 1897 (1898), pp. 1-8); Matuszewski (Arch. path. Anat., clxxix, 1905, p. 12); De Lange (Le Nevraxe, x, 1908, p. 83) ; and Lewy (Fol. Neurobiol., ii, 1909, p. 471).

Frnotwi (Virchow's Arch., cxliii, 1896, p. 133) makes sections of material that has been in Müller's fluid for not more than a few weeks or months, and puts them for four to ten hours (in the dark) into a freshly prepared mixture of 1 or 2 parts of 1 per cent. osmic acid, and 1 part of a concentrated solution of picric acid in one-third alcohol. For peripheral nerves; myelin (normal), black.

ORR (Journ. Path. and Bact., vi, 1900, p. 387) treats small pieces of fresh tissue with a mixture of 8 c.c. of 2 per cent. osmic acid, and 2 c.c. of 1 per cent. acetic acid, which increases the penetration. Should the mixture be darkened at the end of twenty-four hours, then it ought to be renewed. After forty-eight hours, the pieces are placed in 10 per cent. formalin for three days to complete reduction and hardening.

Vassale (Arch. Ital. Biol., xxvii, 1897, p. 131) takes 75 c.c. of Müller's solution, 25 c.c. of 1 per cent. osmic acid, and 20 drops of nitric acid.

NissL (Encycl. mik. Technik., ii, p. 248), holding that alcohol attacks the myelin, cuts without imbedding, and hurries sections through alcohol and bergamot oil into balsam.

RaMon Y CaJal (Trab. lab. Biol. Madrid, ii, 1903, p. 93) has a complicated method of treating Marchi material.

Busch (Neurol. Centralb.; xvii, 1898, p. 476) puts formol material for five to seven days into a solution of 1 part osmic acid, 3 of iodate of sodium, and 300 of water. Same stain as Marchi's, but more penetrating and sharper.

See also Venderovič (Anat. Anz., xxxix, 1911, p. 414) who cuts slices of formol material $0.5 \mathrm{~cm}$. thick, and treats these, with Marchi's fluid, thus getting increased depth of reaction.

SteEnsland (Anat. Rec., viii, 1914, p. 123) recommends clearing sections of Marchi material with oleum origani cretici, and mounting in chloroform-balsam.

Osmic Acid (ExNer, Sitzb. Akad. Wiss. Wien, 1xxxiii, 1881, Abth. 3, p. 151; BeVAN LewIS, The Human Brain, 1882, p. 105).-A portion of brain, not exceeding a cubic centimetre in size, is placed in 1 per cent. osmic acid, and after five to ten days is cut (best without imbedding). The sections are treated with caustic ammonia (20 drops to 50 c.c. of water), which clears up the general mass of the brain substance, leaving the medullated fibres black. The preparations are not permanent, unless (RANVIER, Traité, 1 ed., p. 1086) they are fixed for a quarter of an hour in osmic acid vapour. 
871. Azoulay's Osmic Acid Methods (Anat. Anz., x, 1894, p. 25).(A) Sections of Müller material are put for five to fifteen minutes into a solution of $1: 500$ or 1 to 1,000 of oxmic acid, rinsed with water, and put for two to five minutes into a 5 or 10 per cent. solution of tannin, warming them therein over a flame till vapour arises, or in a stove at $50^{\circ}$ to $55^{\circ} \mathrm{C}$. Wash for five minutes in water, counterstain with carmine or eosin, and mount in balsam. If the sections are too thick it will be necessary to differentiate by Pal's process, or with eau de Javelle diluted with 50 volumes of water. (B) Material that has been in an osmic mixture (fluids of Flemming, or Marchi, or Golgi). Sections as before, then tannin bath, warming for three to ten minutes, the rest as before.

872. Heller and Gumpertz (Ztschr.wiss. Mikr., xii, 1895, p. 385) give for peripheral nerves, and Heller (ibid., xv, 1898, p. 495) for central nervous system, the following method. Sections of Müller material are put into 1 per cent. osmic acid (twenty-four hours at $37^{\circ} \mathrm{C}$. for peripheral nerves ; ten to thirty minutes, at room temperature, for central nerve fibres). They are treated with pyrogallic acid (a photographic developer will do) till the nerve fibres are black, then with a violet-coloured solution of potassium permanganate till the sections become brown, then with 2 per cent. oxalic acid till they become yellow-green. Wash out well between each operation.

Similarly, TelJatnik (Neurol. Centrbl., xvi, 1897, p. 521); Robertson (Brit. Med. Journ., 1897 (1), p. 651), the material being previously mordanted with Weigert's chrome alum-copper fluid for neuroglia; and ORR, Journ. Path. and Bact., vi, 1900, p. 387. See also Rossolimo and Busch, Ztschr. wiss. Mikr., xiv, 1897, p. 55.

WitTmaAcK (Arch. Ohrenheilk., lxi, 1904, p. 18) mordants till green (temporal bones) in 90 parts of Müller's fluid with 10 of formol and 3 to 5 of acetic acid, decalcifies with nitric acid and formol, treats sections (paraffin or celloidin) for a few minutes with 2 per cent. osmic acid, and reduces in 5 per cent. pyrogallol.

873. Iron.-Allerhand (Neurol. Centrbl., xvi, 1897, p. 727) puts sections of Müller material for fifteen minutes into warm 50 per cent. solution of Liquor ferri sesquichlorati, then for an hour or two into 20 per cent. tannin solution (old and brown). They are then differentiated by PAL's method, using, however, the liquids twice as strong.

An iron-alum process is described by Strong in Journ. Comp. Neurol., xiii, 1903, p. 291.

874. Silver Nitrate--Vestarini-Cresi (Att. Accad. Med. Chir. Napoli, 1, 1896) hardens in formol, cuts thick sections, washes them with 
40 per cent. alcohol, puts them in the dark into 1 per cent. solution of silver nitrate in 40 to 70 per cent. alcohol, then washes thoroughly.

Similarly, Mosse (Arch. mik. Anat., lix, 1902, p. 401), impregnating bichromic material with 1 per cent. solution of argentamin, and reducing in 10 per cent. pyrogallic acid, and differentiating by the method of PAL.

\section{Myelin-and-axis-cylinder Stains.}

875. Methylene Blue.-SAHLI (Ztschr.wiss. Mikr., ii, 1885, p. 1) stains sections of tissue hardened in bichromate for several hours, in concentrated aqueous solution of methylene blue, rinses with water, and stains for five minutes in saturated aqueous solution of acid fuchsin. If now the sections are rinsed first with water, then for a few seconds in a $1: 1,000$ alcoholic solution of caustic potash, and lastly brought into a large quantity of water, the stain becomes differentiated, axis-cylinders being shown coloured red and the myelin sheaths blue.

- Or (ibid., p. 49), the sections are stained for a few minutes or hours in :-

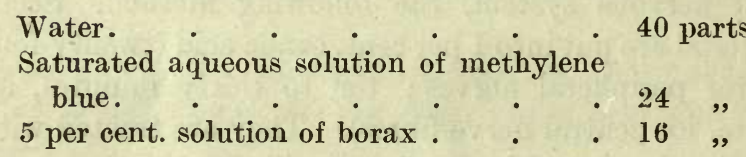

then washed either in water or alcohol until the grey matter is distinctly differentiated from the white substance, cleared with cedar wood oil, and mounted in balsam. Preparations similar to those obtainable by Weigert's method.

876. Acid Fuchsin.-Finotti (Virchow's Archiv., cxliii, 1896, p. 133) stains strongly in Delafield's hæmatoxylin, then for a few seconds in concentrated solution of picric acid, then in 0.5 per cent. acid fuchsin, and treats lastly with alkaline alcohol.

OHLMACHER (Journ. Exper. Med., ii, 1897, p. 675) stains sections for one minute with gentian violet in anilin-water, then for a few seconds in a 0.5 per cent. solution of acid fuchsin in saturated solution of picric acid diluted with 1 volume of water, and differentiates with alcohol and clove oil.

KaPLAN (Arch. Psychiatr., xxxv, 1902, p. 825) mordants for months in Müller, stains sections for a day or more in $\frac{1}{3}$ per cent. aqueous solution of acid fuchsin, rinses in water acidulated with $\mathrm{HCl}$, and differentiates by the method of Pal.

877. Safranine-AdamkIEwicz (Sitzb. Akad. Wiss. Wien. Math. Naturw. $K l$., lxxxix, 1884, Abth. 3, p. 245) stains sections of Müller material in concentrated solution of safranine, differentiates in alcohol and clove oil, brings back again into water, washes in water acidified with acetic acid, and stains in methylene blue. Myelin red, nuclei violet.

Similarly, Ciaglinski (Ztschr. wiss. Mikr., viii, 1891, p. 19) and STROEBE (ibid., x, 1893, p. 384), the former employing safranine followed by anilin blue, whilst the latter first stains with anilin blue, then differentiates with alcohol containing a very little caustic potash, and counterstains with safranine. 
878. Congo Red.-Nissu (Ztschr. wiss. Mikr., iii, 1886, p. 398) stains for three days in Congo red ( 5 parts to 400 of water) and differentiates in alcohol with 3 per cent. of nitric acid.

Other Methods. RöTHIG's Vital-Scharlach VIII Counterstain (Neurol. Centrbl., xxxiii, 1914, p. 219, and xxxiv, 1915, p. 265).Sections stained and differentiated by Weigert-Pal's method are kept for twenty-four hours at room temperature in a counterstaining fluid consisting of 90 c.c. of distilled water and 10 to 20 c.c. of a solution of Vital-Scharlach VIII, saturated at room temperature. They are then washed in distilled water for fifteen minutes and differentiated in 70 per cent. alcohol for from one or two hours up to twenty-four, when the celloidin will be found to be colourless. After another wash in 96 per cent. alcohol, sections are mounted as usual. Nerve cells and their processes, as well as axis-cylinders red, the latter being visible within the deep blue myelin sheaths.

The method does not succeed if the sections were previously treated with an osmic acid solution. Vital-Scharlach VIII may also be used as a general stain, in which case the finished preparations are similar to those obtainable by the usual carmine stains.

Paladino's palladium chloride methods; see Rendic $R$. Accad. Scienze, Napoli, iv, 1891; p. 14; Arch. Ital. Biol., xvii, 1892, p. 145, and xix, 1893, p. 26.

For WoLTER's vanadium chloride process, see next chapter.

Zosin's magenta red method; see Neurol. Centrlb., xxi, 1902, p. 207.

Perusini's remarks and methods for the study of the white substance of the spinal cord : see Journ. Psychol. Neurol., xix, 1912, p. 61. 
P.M. table even twenty-four to forty-eight hours after death. It should be divided into pieces of not more than $1 \mathrm{~cm}$. or $1 \frac{1}{2} \mathrm{~cm}$. in size.

The most difficult point of the method consists in finding out the exact degree of hardening after which the material can be successfully submitted to the further treatment. In summer good results may be obtained after fifteen to twenty days of hardening, and the material may continue to be in a state suitable for the silver impregnation up to thirty, forty or fifty days. In cold weather good results can seldom be obtained under a month; when this is the $\cdot$ case, the material may continue to give good impregnations for two, three or even four months. The only way to make sure is to pass, at intervals, trial portions of the tissue into the silver nitrate solution-in summer frequently, in winter every eight or ten days - and observe whether and when the reaction has been obtained.

It is a good practice to inject the organs (see $\S 806$ ) with the hardening fluid, generally 2.5 per cent. potassium bichromate, to which, according to Golgi, 5 to 6 per cent. of gelatine may be added, in which case, however, the fluid must be injected after warming it to body temperature. Stoving at a temperature of $20^{\circ}$ to $25^{\circ} \mathrm{C}$. is useful for abridging the hardening, but there is a risk of overhardening; and Golgi thinks that the results are never quite so delicate as after hardening at room temperature.

(b) Impregnation.-As soon as the pieces of tissue have attained the proper degree of hardening, they are brought into a large quantity of silver nitrate solution, the usual strength of which is 0.75 per cent., but 0.50 per cent. may be used for material which has not been quite enough hardened, and 1 per cent. for material that has been slightly over-hardened.

The moment the pieces are put into the silver bath an abundant precipitate is formed. This, of course, weakens the bath pro tanto. It is, therefore, advisable first to wash them well in a weaker silver solution until, on being put into a fresh quantity of it, no further precipitate is formed. Used solutions will do for this purpose. The final silver bath needs, generally, no further attention; but it should be changed for a fresh one if it becomes yellowish, as it sometimes does, particularly in the case of tissues which have taken up a great deal of bichromate.

It is not necessary to keep the material in the dark during the impregnation; in winter it is well to keep it in a warmed room. The time generally necessary for the impregnation is from twenty- 
four to forty-eight hours; but tissues may remain in the bath without hurt for days, weeks or months.

(c) Preservation.-As soon as a trial has shown that a sufficiently satisfactory impregnation has been obtained, the pieces are brought into 80 to 90 per cent. alcohol. The alcohol is changed two, three or more times, until it remains transparent, even after specimens have been two or three days in it ; for, in view of good preservation, it is necessary that the excess of silver nitrate should be washed out from them thoroughly.

Sections are now made (see $\S 892$ ). These are to be washed thoroughly in three or four changes of absolute alcohol and cleared, first in creosote, in which they should remain only a few minutes, then in oil of turpentine, in which they are usually left for three to fifteen minutes, though they may be kept in it even for some days without being spoiled. They are then mounted in thick xyloldamar (rather than in balsam), without coverslip. Preparations mounted with coverslips in the usual way always go bad sooner or later, whilst those mounted without a cover keep well for years, especially if they are protected from dust and light.

Instead of creosote and oil of turpentine, fluid cedar-wood oil is now used in Golgi's laboratory for clearing the sections, which are then mounted, without cover, in thick cedar-wood oil. But care must be taken to leave the sections in fluid cedar-wood oil no longer than one hour or so, as otherwise they become brittle and difficult to mount. To make sure of complete dehydration and that no curling of the sections should take place in the fluid cedar-wood oil, they are quickly passed through liquid absolute guaiacol, the whole procedure being carried out as follows : A small quantity of absolute guaiacol is poured in a watch-glass and some fluid cedar-wood oil in two other small glass dishes. Two or three sections are carried from the absolute alcohol into the guaiacol by means of a perforated spatula, which is to be used for all the other passages, and cleaned at every passage. After a few seconds the sections are transferred into the first dish of fluid cedar-wood oil and there left for the time necessary to pass another two or three sations from the absolute alcohol into the guaiacol. The first batch of sections is now transferred into the second dish of cedar-wood oil, the second batch into the first cedar-wood oil and a fresh batch into guaiacol, and so on until all sections are collected in the second dish of cedar-wood oil.

For mounting the sections are lifted, one by one, by means of the same small spatula, and arranged in the order and number one may 
P.M. table even twenty-four to forty-eight hours after death. It should be divided into pieces of not more than $1 \mathrm{~cm}$. or $1 \frac{1}{2} \mathrm{~cm}$. in size.

The most difficult point of the method consists in finding out the exact degree of hardening after which the material can be successfully submitted to the further treatment. In summer good results may be obtained after fifteen to twenty days of hardening, and the material may continue to be in a state suitable for the silver impregnation up to thirty, forty or fifty days. In cold weather good results can seldom be obtained under a month; when this is the -case, the material may continue to give good impregnations for two, three or even four months. The only way to make sure is to pass, at intervals, trial portions of the tissue into the silver nitrate solution-in summer frequently, in winter every eight or ten days - and observe whether and when the reaction has been obtained.

It is a good practice to inject the organs (see $\S 806$ ) with the hardening fluid, generally 2.5 per cent. potassium bichromate, to which, according to Golgi, 5 to 6 per cent. of gelatine may be added, in which case, however, the fluid must be injected after warming it to body temperature. Stoving at a temperature of $20^{\circ}$ to $25^{\circ} \mathrm{C}$. is useful for abridging the hardening, but there is a risk of overhardening; and Golgi thinks that the results are never quite so delicate as after hardening at room temperature.

(b) Impregnation.-As soon as the pieces of tissue have attained the proper degree of hardening, they are brought into a large quantity of silver nitrate solution, the usual strength of which is 0.75 per cent., but 0.50 per cent. may be used for material which has not been quite enough hardened, and 1 per cent. for material that has been slightly over-hardened.

The moment the pieces are put into the silver bath an abundant precipitate is formed. This, of course, weakens the bath pro tanto. It is, therefore, advisable first to wash them well in a weaker silver solution until, on being put into a fresh quantity of it, no further precipitate is formed. Used solutions will do for this purpose. The final silver bath needs, generally, no further attention; but it should be changed for a fresh one if it becomes yellowish, as it sometimes does, particularly in the case of tissues which have taken up a great deal of bichromate.

It is not necessary to keep the material in the dark during the impregnation; in winter it is well to keep it in a warmed room. The time generally necessary for the impregnation is from twenty- 
four to forty-eight hours; but tissues may remain in the bath without hurt for days, weeks or months.

(c) Preservation.-As soon as a trial has shown that a sufficiently satisfactory impregnation has been obtained, the pieces are brought into 80 to 90 per cent. alcohol. The alcohol is changed two, three or more times, until it remains transparent, even after specimens have been two or three days in it ; for, in view of good preservation, it is necessary that the excess of silver nitrate should be washed out from them thoroughly.

Sections are now made (see $\S 892$ ). These are to be washed thoroughly in three or four changes of absolute alcohol and cleared, first in creosote, in which they should remain only a few minutes, then in oil of turpentine, in which they are usually left for three to fifteen minutes, though they may be kept in it even for some days without being spoiled. They are then mounted in thick xyloldamar (rather than in balsam), without coverslip. Preparations mounted with coverslips in the usual way always go bad sooner or later, whilst those mounted without a cover keep well for years, especially if they are protected from dust and light.

Instead of creosote and oil of turpentine, fluid cedar-wood oil is now used in Golgi's laboratory for clearing the sections, which are then mounted, without cover, in thick cedar-wood oil. But care must be taken to leave the sections in fluid cedar-wood oil no longer than one hour or so, as otherwise they become brittle and difficult to mount. To make sure of complete dehydration and that no curling of the sections should take place in the fluid cedar-wood oil, they are quickly passed through liquid absolute guaiacol, the whole procedure being carried out as follows : A small quantity of absolute guaiacol is poured in a watch-glass and some fluid cedar-wood oil in two other small glass dishes. Two or three sections are carried from the absolute alcohol into the guaiacol by means of a perforated spatula, which is to be used for all the other passages, and cleaned at every passage. After a few seconds the sections are transferred into the first dish of fluid cedar-wood oil and there left for the time necessary to pass another two or three sections from the absolute alcohol into the guaiacol. The first batch of sections is now transferred into the second dish of cedar-wood oil, the second batch into the first cedar-wood oil and a fresh batch into guaiacol, and so on until all sections are collected in the second dish of cedar-wood oil.

For mounting the sections are lifted, one by one, by means of the same small spatula, and arranged in the order and number one may 
wish, either on ordinary slides, or on coverslips if the Golgi hollowedout wooden slides are preferred for definite preservation. The excess of cedar-wood oil carried with the spatula is removed by covering the sections, after having definitely arranged them on the slides, first with a sheath of cigarette paper and then with a folded piece of filter paper, to be held by the left hand while the right is passed over it so as to press down the sections and absorb the oil. The whole manœuvre may be repeated a second time, and then a drop of thick cedar-wood oil put on each section. On the next day the oil which may have run from the sections is cleaned from the edges of the slides and a fresh drop of the thick cedar-wood oil put on the sections, to be protected from dust and light at least until the oil has become quite dry.

Preparations mounted in this way last for years unaltered; in fact, I have some which were made in Golgi's laboratory over fifteen years ago and I find that they have kept without change. I have no experience of the use of creosote or of the mixture, originally proposed by Andriezen, of equal parts of pyridine and xylol instead of the guaiacol, but they should equally well serve the purpose.

As a general rule one makes sections of 20 to $40 \mu$; thicker sections of 50 to $60 \mu$, or more, show more than thin ones but do not seem to keep so well.

The order in which the elements of nervous tissues impregnate is generally-first, axis-cylinders, then nerve cells, and lastly, neuroglia cells.

882. Golgr's Bichromate and Nitrate of Silver Method. RAPID Prockss. - Small pieces of very fresh tissues are hardened in a mixture of 2 to 2.5 per cent. potassium bichromate 8 parts, and 1 per cent. osmic acid 2 parts. Or, if a very quick hardening is desirable, 2 parts of 3 per cent. bichromate to one of 1 per cent. osmic acid. In Golgi's laboratory mixtures of 3 parts of 3 per cent. bichromate and 1 of 1 per cent. osmic acid are now generally used. The tissues begin to be in a state suitable for the silver impregnation from the second or third day; in the next following days they are in a still more favourable state, but this soon declines, and is generally quite lost by the tenth or twelfth day.

The silver impregnation is conducted exactly in the same way as in the slow process, and sections are prepared and mounted in the same manner, but they should not be left in alcohol for more than an hour or so before mounting. 
There is this difference, that the impregnated material cannot be preserved for any length of time in alcohol and must not remain in it for more than one or two days. But it may be kept in the silver solution until wanted for sectioning. According to v. Gehuchten ( La Cellule, vi, 1890, p. 405) pieces may be kept with advantage for many days, weeks and months in the silver nitrate solution. An abundant impregnation was found by him after many days up to six months where almost none had been seen after twenty-four to forty-eight hours only. But the material must be kept in the dark.

As to the proper duration of the hardening process in different cases, it must be pointed out that definite rules can hardly be given, while investigators can easily find out the right moment for successfully transferring the pieces into the silver bath by means of attempts made in accordance with the purpose in view and the quality of the material with which they are working. However, the following points should be borne in mind :-

Spinal cord of chick from the sixth to the tenth day of incubationtwelve to forty-eight hours in the hardening mixture (up to the fifth day the embryos may be treated whole, later the vertebral column should be dissected out and cut into two or three segments; it need not be opened). The spinal column of newborn rats and mice should be treated in the same way, and remain in the mixture for twenty-four hours for spinal ganglia, or for two to six days for the cord itself. The encephalon of these subjects may be treated in just the same way, without being dissected out.

v. LENHOSSEK (op. eit.) recommends for human foetal cord two to three days for neuroglia, three to five for nerve-cells, and five to seven for nerve-fibres and collaterals.

Cerebellum of new-born subjects three to five days in the hardening mixture.

Cerebral cortex of young subjects two to three days (mice), or as long as five (rabbit, eat); cortex of adults, eight to fifteen days. The most favourable region of the brain is the Ammon's horn, especially in the rabbit.

Retina-twenty-four to forty-eight hours in the mixture, then " double " impregnation ( $(923)$.

Sympathetic.-SALA, L. (Mon. Zool. Ital., iii, 1892) found the inferior cervical ganglion particularly suitable for staining by Golgi's rapid process. He proceeds thus: osmium bichromate mixture, three days; quick wash in distilled water; silver bath, two to three days ; further wash in distilled water and passage into the same osmiumbichromate mixture for about four days; a third impregnation can be resorted to, in which case pieces should remain in the hardening fluid for five to seven days.

Spinal cord of larvee of Amphibia.-The entire larvæ (best 2 to $2.5 \mathrm{~cm}$. 
long) should be put for two to five days into the hardening mixture, and for one to two into silver nitrate.

Epidermis of Lumbricus. - Three to six days in the mixture, and two in the silver, or double impregnation if necessary.

Nervous system of Helix (glia-cells). The above mixture for eight to ten days, then silver of 0.75 to 1 per cent.

As a general rule, the younger the subject the shorter should be the hardening. If it has been too short, sections will have a brownish-red opaque aspect, with precipitates, and irregular impregnation of cells and fibres. If it has been too long, the ground will be yellow, without precipitates, but with no impregnated elements, or hardly any.

This process has the advantage of great rapidity, and of sureness and delicacy of results, and it is the one that has found most favour with other workers. But for the methodical study of any given part of the nervous system GoLGI himself prefers the following:-

888. GolGI's Bichromate and Nitrate of Silver Method. MIXED Process.-Fresh pieces of tissues are put for periods varying from two to twenty-five or thirty days into the usual bichromate solution $(\S 881$ ). Every two or three or four days some of them are passed into the osmio-bichromate mixture of the rapid process, hardened therein for from three or four to eight or ten days, and finally impregnated with silver nitrate and subsequently treated exactly as by the rapid process.

The reasons for which Golgi prefers this process are : The certainty of obtaining samples of the reaction in many stages of intensity, if a sufficient number of pieces of tissues have been used for the purpose. The advantage of having at one's disposal a considerable time-some twenty-five days-during which the tissues are in a suitable state for taking the silver. The possibility of greatly hastening the process whenever desired by simply bringing all the pieces over at once into the osmic mixture. Lastly, a still greater delicacy of results, particularly noticeable in the staining of axons and their collaterals.

884. Theory of Impregnation.- - It was once held that the reaction depends on the formation in the tissues of a precipitate of some salt of silver. And Kallius has put forward the suggestion that this precipitate may consist of a protein-silver-chromate combination. But this seems to B. Lee incorrect (see 1913 Ed.). In agreement with v. Lenhossék, he finds that the colouration is not due to a visible precipitate, but is a true stain accompanied, particularly in unsuccessful impregnations, by precipitates which not only do not 
help the stain, but are injurious to it. It has been maintained that the stain is merely superficial, and the method has been called an " incrustation method." But it is easy to realise that it generally extends throughout the whole thickness of the impregnated elements, though in special cases or by slight modifications of the original method, the stain may be limited to certain constituents of the nerve-cell body, such as Golgi's pericellular investment and intracellular network.

The chemical nature of the stain has not as yet been discovered.

A critical review of the Golgi method by WeIGERT may be found in Ergebn.d. Anat., v, 1895, p. 7. See also Hill (Brain, xix, 1896, p. 1), and KALliUs (op. cit.).

\section{Morlifications of Golgi's Bichromate and Silver Nitrate Method concerning the Impregnation of Tissues.}

885. Instead of potassium bichromate, ammonium bichromate has been recommended by GoLGI and sodium bichromate by KaLLIUs. Both these salts appear to penetrate more quickly into the tissues than potassium bichromate. According to Strong (N.Y. Acad. Sc. Proc. xiii, 1894) lithium bichromate hardens more rapidly than potassium bichromate. The influence on the reaction of the bichromates of ammonium, sodium, calcium, magnesium, rubidium, lithium, zinc and copper, has been investigated by L. SalA (see Kallius, op. cit., i, p. 564), but he came to the conclusion that they do not offer any particular advantage, with the exception of calcium bichromate, this last to be preferred for the staining of the tangential fibres of the cerebral cortex.

Ramon y CaJal (Ztschr. wiss. Mikr., vii, 1890, p. 332) gives 3 per cent. as the strength of the bichromate in the mixture for the rapid process, but in numerous other places has given it as 3.5 per cent. This latter strength has been adopted by many workers for the rapid process, and the mixture containing this proportion of bichromate is generally known as the RAMON y CAJAL mixture.

886. Ramon y CaJaL's Double-Impregnation Process (La Cellule, vii, 1891, p. 130). - Sometimes the usual rapid method fails to give a good impregnation. This, however, may frequently be obtained by putting the tissues back for a day or two into the osmiumbichromate mixture used for the first hardening, or into a fresh but weaker one containing 2 parts of 1 per cent. osmic acid and 20 parts of 3 per cent. potassium bichromate. Tissues are then washed quickly with distilled water or with a weak solution of silver nitrate, 
and put for a second time into the silver bath, where they should remain from thirty-six to forty-eight hours. It is important to find out the proper duration of the first hardening. If it has been too long (four days) or too short (one day) the second impregnation will not succeed. In this case a third impregnation may be resorted to, the objects being again treated with the weak osmium-bichromate mixture and then again with the silver nitrate solution. I find that this modification, which is the most important that has hitherto been made, gives excellent results if one proceeds by tests, viz., re-transferring into the weak osmium-bichromate mixture those pieces in which the reaction has been found to have succeeded to some extent.

887. Kolossow's Modification (see Zuschtschenco, Arch. Mikr. Anat., xlix, 1897). - Tissues are hardened for one to seven days in 3 to 5 per cent. potassium bichromate containing 0.25 per cent. of osmic acid. They are then washed quickly in distilled water, dried with filter paper and transferred for two to three days into a bath of 2 to 3 per cent. silver nitrate to which 0.25 to 0.5 per cent. of osmic acid has been added. This is a good modification for sympathetic ganglia.

888. Golgi's Processes for the Rejuvenation of Over-hardened Tissues.-Tissues which have been too long in the osmium-bichromate mixture will no longer take on the silver impregnation. They can, however, be made to impregnate by one or the other of Golgi's so-called processes of rejuvenation. These can be carried out in various ways given here with sufficient detail, as they may be of great use not only for rejuvenating ordinary pieces of central nervous system, but also, and particularly, for the staining of nerve-endings in glandular and other tissues, internal apparatus, spiral filaments of peripheral nerve-fibres, etc.

Golgi at first suggested washing the over-hardened pieces in a half-saturated solution of copper acetate until they no longer give a precipitate, afterwards putting them back again for five or six days into the osmium-bichromate mixture, and subsequently transferring them into the silver nitrate solution.

Later he advised leaving tissues in 3 to 4 per cent. copper sulphate or 1 to 2 per cent. arsenic acid. After one, two and three days some pieces are brought back into the osmium-bichromate mixture in which they had been hardened, or into a weaker one, proceeding further as in the rapid process, viz., as if the pieces had been freshly fixed in the osmium-bichromate mixture.

More recently Golgi appears to have preferred mixtures of equal parts of 2 or 3 or 4 per cent. copper sulphate or acetate and 4 to 5 per 
cent. potassium bichromate, filtering them if copper acetate was used, and treating the pieces as stated above. As a rule these copper acetate and potassium bichromate mixtures ought to be tried first and in preference to others. As with other points of Golgi's methods, so also in this case, one must proceed by tentative experiments, according to the purpose of one's investigation and the quality of the material in hand, but chiefly according to the length of time during which the tissues have been left in the osmio-bichromate solution.

See on this subject SACERDotTr, Intern. Monatschr. Anat., xi, 1894, p. 326 ; GolGI, Cinquant. Soc. Biol., 1899, p. 514, and Opera Omnia II, 1903, p. 677 ; Fusari, Tratt. Elem. Istol. Tecn. Istol., Torino, 1909 ; Sala G., Anat. Anz., xviii, 1900, p. 176 ; Gemmelli, Anat. Anz., 1913, p. 444.

889. Formaldehyde Modifications of GoLGI's Bichromate and Nitrate of Silver Method.-Many investigators have found that formaldehyde can take the place of the osmic acid in the osmio-bichromate mixture of the rapid process. This has certain advantages: A cheap reagent is employed instead of the expensive osmic acid. Pieces much larger than by Golgi's original process may be used. The stage of hardening favourable for a good impregnation lasts longer, i.e., formalin-bichromate mixtures do not over-harden. Moreover, the formaldehyde modifications can be usefully resorted to for impregnating nervous tissues of adult or young subjects, as well as for material which after repeated attempts has been found impervious to the osmic mixtures. However, it should be remembered that many investigators have failed to obtain good results by the formaldehyde methods and that they are unsuitable for embryonic specimens.

Hoyer, Jun. (Anat. Anz., ix, 1894, p. 236) was the first to point out that material fixed in formalin could be used for carrying out Golgi's method.

LACHI (Monit. Zool. Ital., v, 1895, p. 15) used, at first, to harden tissues for five to nine days in equal parts of 20 per cent. formalin and 6 per cent. potassium bichromate. Afterwards (Anat. Anz., $\mathrm{x}, 1895$, p. 790$)$ he adopted the mixture proposed by his pupil Dell'Isola (Boll. Acc. Med. Genova, 1895, No. 2) of equal parts of 10 per cent. formalin and 10 per cent. potassium bichromate, with the addition of 1 part of 1 per cent. osmic acid to every 10 of the mixture, this last formula being particularly suitable for quick work, as forty-eight hours afterwards pieces can be already transferred into the silver bath.

Strong (Anat. Anz., x, 1895, p. 494) suggested fixing pieces of 
brain of adult specimens in mixtures of 100 volumes of 3.5 per cent. potassium bichromate and from $2 \frac{1}{2}$ to 5 volumes of formalin. One or more pieces are, during several days, daily transferred into 1 per cent. silver nitrate. Or the tissues are left for one to two days in the above formalin-bichromate mixture and then passed into a fresh one consisting of 2 volumes of 5 per cent. potassium bichromate and 1 volume of formalin; after another twelve to twenty-four hours all the pieces are transferred into the silver bath.

DuRIG (ibid., p. 659) obtained good results by fixing $\frac{1}{2} \mathrm{~cm}$. thick pieces in 3 per cent. bichromate containing 4 to 6 per cent. of formalin, and hardening therein for three days. After silvering for two days, the pieces are brought back into the fixing mixture and one proceeds as in Ramon y Cajal's double impregnation process.

Frsh (Proc. Amer. Micr. Soc., xvii, 1895, p. 319) uses 2 c.c. of formalin for every 100 c.c. of 3 per cent. potassium bichromate, and leaves tissues three days in this fluid, and another three days in 0.75 per cent. silver nitrate; or, with advantage, Müller's fluid, 100 c.c. ; 10 per cent. formalin, 2 c.c. ; 1 per cent. osmic acid, 1 c.c.; silvering as above.

Kopsch (Anat. Anz., xi, 1896, p. 727) uses 4 parts of 3.5 per cent. potassium bichromate and one of formalin ; after twenty-four hours he transfers all pieces to pure 3.5 per cent. bichromate for at least two days (retina) or three to six (central organs). He finds that by this means, precipitates are almost entirely avoided. B. Lee (1913 ed.) confirms this, but points out that the method gives a too abundant impregnation of capillaries.

Gerota (Intern. Monatsch. Anat., xiii, 1896, p. 108) first hardens brains for a week or two in 5 to 10 per cent. formalin, then puts small pieces for three to five days into 4 per cent. bichromate, and lastly transfers these into the silver bath, where they are left with advantage for ten to twenty days.

Bolton (Lancet, 1898 (1), p. 218 ; Journ. R. Micr. Soc., 1898, p. 244) has obtained good results from brains of cats and half-grown kittens placed whole in 5 per cent. formalin and from human brains hardened whole in formalin of the same strength for two to twelve months. Small pieces are then cut out, and placed into 1 per cent. ammonium bichromate and left therein for from a few hours up to five days, some being transferred at intervals into 1 per cent. silver nitrate.

Schreiber (Anat. Anz., xiv, 1898, p. 275) obtained good results from appendages of Crustacea impervious to the osmic mixture, with 5 parts of 2.5 per cent. potassium bichromate to 1 of 4 per cent. 
formalin, or 1 part of 2.5 per cent. bichromate to 2 of 5 per cent. formalin, the specimens remaining for one day in the first mixture and for two in the second.

Similarly Dubosq (Arch. de Zool. exp., vi, 1898-90).

Smirnow (Arch. mikr. Anat., lii, 1898, p. 201) fixes the cerebellum of a freshly-killed animal in 5 per cent. potassium bichromate 4 parts, and formalin 1 part, for one to eight weeks. He then divides the organ in two halves and places them into pure 3.5 per cent. bichromate, where they are left for another two to five weeks. Of the two halves one may be used for Weigert's myelin stain; the other is divided in pieces 1 to $2 \mathrm{~cm}$. thick, and these put for one to one and a half weeks into a mixture of 5 per cent. potassium bichromate, 5 parts, and 2 per cent. osmic acid, 1 part. Pieces are then transferred, at first into a weak silver bath and then into the usual 1 per cent. silver nitrate solution. The method gives good results also in human brains of adult subjects.

Odier (La Rachicocainisation, Genève, 1903, p. 27) takes 100 c.c. of Müller's fluid with 2 c.c. of undiluted formalin and 1 c.c. of 1 per cent. osmic acid. The mixture should be kept in the dark and made up at the instant of using it. Odier finds that formalin-bichromate mixtures generally afford a more abundant impregnation with fewer precipitates than the osmio-bichromic ones.

Brookover (Journ. comp. neurol., xx, 1910, p. 49) finds useful for adult specimens a preliminary fixation in 4 per cent. " formaldehyde," neutralised with lithium carbonate or ammonia, before carrying out Golgi's rapid process.

890. Other Modifications.-VASsale and Donaggio (Monit. Zool. Ital., vi, 1895, p. 82) harden pieces of at most $1 \mathrm{~cm}$. in thickness for fifteen to twenty days in a mixture of 5 parts of acetic aldehyde and 100 of 3 to 4 per cent. potassium bichromate, changing the fluid as soon as it has become dark. The rest as Golgi.

RAMON Y CAJAL (Rev. Trim. Histol., No. 2, 1888, note) found that the addition of a very little formic acid to the silver bath facilita ted reduction. According to van Gehuchten ( $L a$ Cellule, vii, 1891, p. 83) 1 drop of the acid should be added to every 100 c.c. of the silver nitrate solution. But the practice is now generally abandoned.

MARTINOTTI (Rif. med., 1887 ; Ztschr. wiss. Mikr., v, 1888, p. 88) pointed out that Golgi's method can be successfully carried out on relatively large pieces by using unusually large quantities of silver nitrate solution with 5 per cent. glycerin added to it, and by keeping this for thirty days at a temperature of $25^{\circ} \mathrm{C}$. to impregnate nerve-cells, and of $35^{\circ}$ to $40^{\circ} \mathrm{C}$. to stain the neuroglia.

ANDRIEZEN (Brit. Med. Journ., i, 1894, p. 909) found useful for human brain to suspend thin slices of 2 to $4 \mathrm{~mm}$. in diameter in 95 c.c. 
of 2 per cent. potassium bichromate to which after ten to fifteen minutes 5 c.c. of 1 per cent. osmic acid are added. The mixture is kept in the dark and after twenty-four hours changed for a fresh one made up with 90 c.c. of $2 \frac{1}{2}$ per cent. bichromate and 10 c.c. of 1 per cent. osmic acid. After another two days the mixture is changed over again for one made according to the proportions given by Golgi ( 3 per cent. potassium bichromate, 80 c.c.; 1 per cent. osmic acid, 20 c.c.). Pieces are transferred into the silver bath after three and a half days (for nerve cells and neuroglia) up to six days. They are washed for five to fifteen minutes in $\frac{3}{4}$ per cent. silver nitrate, and then put into a solution of silver nitrate of the same strength, but to which 1 drop of formic acid to every 100 or 120 c.c. of solution has been added. The whole is kept in an incubator at $25^{\circ}$ to $27^{\circ} \mathrm{C}$. for about three days, changing the silver bath after the first twenty-four hours. The same author advised, for the impregnation of neuroglia (Intern. Monatschr. Anat. x, 1893, p. 533), adding 1 drop of a saturated solution of chromic acid and 1 drop of formic acid to the first hardening bath.

BERKELEY (Johns Hopkins Hosp. Rep., vi, 1897, p. 1) hardens tissues in Müller's fluid until they are of sufficient consistency to admit of fairly thin sections (about two weeks at room temperature). The portions of the brain selected are cut into slices $3 \mathrm{~mm}$. thick and immersed for about three days in a mixture of 3 per cent. potassium bichromate, 100 parts, and 1 per cent. osmic acid 30 parts. For the impregnation, tissues are removed from the hardening fluid, dried a little with filter paper, washed in a weak solution of silver nitrate, and put for no less than two to three days into a freshly prepared solution of 2 drops of 10 per cent. phosphomolybdic acid and 60 c.c. of 1 per cent. silver nitrate, which in winter should be kept at a temperature of about $26^{\circ} \mathrm{C}$.

HILL (op. cit. $\S 884$ ) uses, instead of silver nitrate, a $\frac{3}{4}$ per cent. solution of silver nitrite, with $0 \cdot 1$ per cent. formic acid added.

GUDDEN (Neurol. Centrbl., xx, 1901, p. 151) ùses the lactate of silver (sold as " actol "), and finds it more penetrating.

891. Avoidance of Precipitates. - Golgi's method frequently gives rise to the formation at the surface of the pieces of irregular and sometimes voluminous precipitates, which destroy the clearness of preparations. To minimise this, SEHRWALD (Ztschr. wiss. Mikr., vi, 1889 , p. 456 ) pours 10 per cent. gelatin, which is just liquid, into a paper box, embeds the tissues in it with the aid of a little heat, and brings them therein into the silver bath; or the tissues are coated with gelatin by dipping and cooling several times. After the impregnation is completed the gelatin is removed, before cutting, by means of warm water saturated with silver chromate. MANN (Physiol. Histol., 1902, p. 276) finds that the method gives good results provided the gelatin is not rendered insoluble by the action of light. To prevent this he proceeds thus :-Either in the photographic dark room or in the evening, by artificial light, tissues, tied loosely to a thread, are immersed three times into liquefied 
10 per cent. gelatin, and, as soon as this has set, they are put into the silver bath, keeping the latter in some dark place. It appears that surrounding a tissue with gelatin makes the impregnation slower, and for this reason Mann allows a day longer for the silver bath.

MaRtinotTi (op. cit.) covers pieces with a layer of a pap of filter paper and distilled water.

Athias wraps tissues in wafer papers.

RAMON Y CAJAL covers them with a layer of congealed blood, which need not be removed before cutting, or with celloidin or peritoneal membrane. See "Retina."

\section{Modifications concerning the Preservation of the Preparations.}

892. Cutting.-As pointed out in $\S 880$, one of the chief qualities of Golgi's method consists in allowing one to follow nerve-cell processes for a great distance. Evidently this cannot be done with very thin sections; and as sufficiently thin ones can be obtained without embedding, the general practice is simply to wash the pieces taken from the silver-bath with distilled water, fix them with gum to a cork or wooden cube, put the whole into alcohol for a little while to harden the gum, and cut by means of a sliding microtome without embedding.

But quick embedding, particularly in celloidin, is quite possible, and should be resorted to for material either brittle or otherwise difficult to cut. Pieces of tissue as small as possible are brought in the course of about two hours through the ascending series of alcohols into absolute alcohol ; after having changed this a couple of times, pieces are transferred for another one or two hours in thin celloidin, then coated with thick celloidin, and by means of this fixed to a wooden cube, the celloidin being a little hardened by means of chloroform vapour, as usual. The whole is left for a little while in 70 per cent. alcohol, and sections made in the usual way. If these operations are started in the morning, when going into the laboratory, pieces are ready for cutting at about 2 p.m., sufficient time remaining for the further treatment of the sections according to the directions given above ( $\$ 881$ ). Care should be taken, of course, not to transfer the sections into absolute alcohol if it is not considered safe to dissolve the celloidin. In this case dehydration can be carried out as usual up to 98 per cent. alcohol, and the sections transferred into fluid absolute guaiacol and cedar-wood oil as already described in $\S 881$. 
Embedding in paraffin is also possible, but results are usually rather poor, and one should have recourse to it only for special objects, such as muscles (see Veratri, Mem. R. Inst. Lomb. Sc., xix, 1902, p. 87). In any case tissues should be passed quickly through the lower grades of alcohol, and remain only a few hours in 95 per cent. and absolute alcohols. They should be cleared with cedarwood oil, as xylol and similar reagents may be injurious to the silver impregnation. One should transfer pieces directly into paraffin of as low a melting point as possible. According to Brookover (op. cit.), cedar-wood oil should be used over and over again, as it becomes saturated with silver nitrate.

893. Mounting.-As pointed out in $\S 881$, Golgi preparations do not keep well if mounted under a cover-glass in the usual way. How and why this happens it is very difficult to say. Though an elaborate discussion between SEHrwald (Ztschr. wiss. Mikr., vi, 1889, p. 443), Samassa (ibid., vii, 1890, p. 26), and Fish (ibid., viii, 1891, p. 168) has furnished the net practical result that watery fluids should be avoided as much as possible during the aftertreatment, it is not clear why preparations should deteriorate, when mounted under a cover-glass in thick cedar-wood oil or neutral balsam ; while ManN (op. cit., p. 277) states, on the other hand, that sections keep well if mounted under a cover-glass in Price's No. 1 pure neutral glycerin.

For these reasons the general practice is to mount sections without a cover, either on ordinary slides or on cover-glasses to be inverted for study over the aperture of a hollowed-out wooden slide.

If mounting under a cover is desirable, this should either be raised free of contact with the slide by means of wax feet or the like, or the balsam of the mount should be rendered perfectly anhydrous by careful heating it on the slide with the section in it, until it immediately sets hard on cooling, when a slightly-warmed cover can be applied.

- This last method is also recommended by Huber (Anat. Anz., vii, 1892 , p. 587). B. LEE (see previous editions) advises keeping the preparations uncovered until the sections have become quite dry and the balsam, applied from time to time in thin layers, quite hard, and then to cover them with a warmed cover-glass, this being slightly pressed down on the sections.

Various processes have been devised for mounting Golgi's preparations at once under a cover, but none of them give really satisfactory results. One should have recourse to them either for special objects, or if counterstaining with carmine or hæmatoxylin, or by Weigert-Pal's 
method, or the like, is particularly desirable. In this case one of the following methods may be employed :-

Greppin (Arch. Anat. u. Entwick., Anat. Abth., Supp., 1889, p. 55) treats sections for thirty to forty seconds (until whitish) with 10 per cent. hydrobromic acid, washes them in several changes of water, dehydrates, clears with clove oil and exposes them for ten to fifteen minutes to sunlight.

OBREGIA (Virchow's Arch., exxii, 1890, p. 387) transfers sections into a mixture of absolute alcohol, 10 c.c., and 1 per cent. gold chloride, 10 drops, to be previously exposed to diffuse daylight for half an hour. Sections are then passed into it and put in a dark place. After fifteen to thirty minutes they are washed successively in 50 per cent. alcohol, distilled water, 10 per cent. sodium hyposulphite (five to ten minutes), and repeatedly changed distilled water. They may be then counterstained, dehydrated and mounted in balsam under a cover.

Kallius (Anat. Hefte., ii, 1893, p. 271) uses 230 c.c. of distilled water and 20 c.c. of commercial hydroquinone solution (hydroquinone 5 grms., sodium sulphite 40 grms., potassium carbonate 75 grms., distilled water 250 c.c.). The solution is further diluted before using with one-third to one-half its volume of absolute alcohol and the sections (freed from unreduced silver by washing them in many changes of alcohol) left in it for several minutes. Here they become dark-grey to black, and are then transferred for ten to fifteen minutes into 70 per cent. alcohol, for five minutes into 20 per cent. sodium hyposulphite, and for twenty-four hours into a large quantity of distilled water. Counterstain, dehydrate, clear and mount as usual.

Eberth and RUNGe (Arch. mikr. Anat., xlvi, 1896, p. 370) have successfully used a process similar to that of Greppin. They convert the silver impregnation into silver chloride by keeping sections in chlorine water for fifteen to twenty minutes, and they then reduce the white silver chloride, either through exhibition to sunlight just before mounting, or by means of Kallius' process.

BolTon (op. cit.) has obtained good results with Kallius' process applied to his formol-bichromate modification.

CURRERI (Anat. Anz., xxxii, 1908, p. 432), after fixing by Kallius' method, tones in $0.7 \mathrm{grm}$. of gold chloride, $3 \mathrm{grm}$. of sodium acetate and 100 c.c. of water.

Zimmermann's process (Arch. mikr. Anat., lii, 1898, p. 554). Paraffin sections of formol-Golgi material are brought from alcohol into a large quantity of a mixture of 1 part of physiologieal salt solution and 2 parts of 96 per cent. alcohol. They are kept in motion therein for ten to fifteen minutes, after which they are brought into 75 to 96 per cent. alcohol in a bright light until they have become dark (about half a day); or sections are left for half to one hour in 100 c.c. of absolute alcohol to which a few drops of ammonium hydrosulphide have been added. In the first case the silver deposit becomes converted into silver chloride, in the second into silver sulphide. Later (Arch. mikr. Anat., lxxviii, 1911, p. 199) he reduces for several hours in 20 c.c. of saturated solution of sodium carbonate (made up with 50 per cent. alcohol) to which $0.5 \mathrm{grm}$. of adurol are added. These processes are useful for 
studying the inter-relationship between gland-ducts and gland-cells (stomach, liver) if the silver chloride sections are afterwards stained with thionin or toluidine blue or safranin, the sulphide sections with Delafield's hæmatoxylin, and the adurol ones with hæmalum or alum cochineal.

For toning, fixing and counterstaining sections of tissues treated by the sublimate method and the like, see next paragraph.

\section{The Sublimate Method.}

894. Golgr's Bichromate and Sublimate Method (Arch. Sc. Med., iii, 1878 ; Rend. R. Inst. Lomb. Sc. (2), xii, 1879, p. 205 and (2), xxiv, 1891; Arch. Ital. Biol., op. cit., §880; Rif. Med., 1891; Opera Omnia, I, p. 143, and II, pp. 505 and 607).-For hardening, use either a solution of potassium bichromate progressively raised from 1 to 3 per cent., or Müller's fluid. It is best to take small pieces of tissue, large quantities of hardening fluid, and change the latter frequently. But the reaction can be obtained with much larger pieces, even entire hemispheres. In this case the brain should at first be treated with "repeated" injections of the fixing agent, or this should be injected from the carotid or the aorta. Pieces, particularly if small, begin to be ready for the subsequent treatment eight to ten days afterwards, but it is advisable to wait until the twentieth or thirtieth day of immersion, this being not injurious if prolonged for several months; it is, on the contrary, to be recommended if the pieces are uncommonly large.

When it is thought that the tissues have been hardened enough, they are passed directly from the bichromate into 0.5 to 1 per cent. mercury bichloride. One generally prefers weak solutions $(0.5$ per cent.) if pieces have been left in the fixing fluid for a relatively short period, having recourse to the stronger ones ( 1 per cent.) for materials which have been hardened for many weeks or months. The sublimate solution must be changed at first every day, and later as often as it becomes yellowish. At the end of the reaction pieces will be found decolourised and almost with the aspect of fresh tissue. To obtain a good reaction, about ten days of immersion in the mercury bichloride are necessary if pieces are small, longer periods, and even months, being required for large pieces and entire hemispheres. Particularly fine results were obtained by Golgi from brains which had been kept in 1 per cent. sublimate for as long as two years.

The reaction may be said to have begun by the time tissues are nearly decolourised. From that time onwards sections may be made and mounted if successful. 
Embedding is not necessary, but in many cases desirable. It can be easily carried out by washing pieces in many changes of alcohol of ascending strengths and embedding them in celloidin. Sections, however made, must be repeatedly washed with distilled water, otherwise they will soon be spoilt by the formation of opaque granules and needle-like crystals which very much hinder proper observation. After dehydrating, sections can be passed through creosote and turpentine and mounted, preferably without a coverglass, in dammar or balsam.

It is, however, preferable to treat sections by the following fixing-and-toning process which was suggested by Golgi for transforming the whitish mercury impregnation (to which the reaction is due) into a full-black stain, much more suitable for observation under high power. Moreover, the process helps in preventing the formation of opaque precipitates, and allows of mounting in the usual way without any danger of spoiling the specimens.

One proceeds thus:- Sections of pieces embedded in celloidin are thoroughly washed in many changes of water, and then transferred for a few minutes into a photographic fixing and toning bath to be prepared at the moment of using, as follows :-

\section{Solution A.}

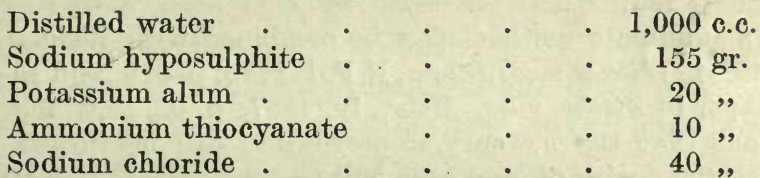

Allow to stand for eight days and then filter.

Gold chloride

Distilled water

Solution B.

For use take 50 c.c. of sol. A, 7 c.c. of sol. B, and 40 c.c. of old combined bath.

From the fixing and toning bath sections are transferred into distilled water and again thoroughly washed; they are then slightly counterstained with an acid solution of carmine diluted with some alcohol, dehydrated, cleared, and mounted in the usual way.

The elements stained by the method are:-(1) Nerve cells with all their processes and ramifications. (2) Nuclei, which is not the case with the silver process. (3) Neuroglia cells. But the reaction in this case is far less precise and complete than that obtained by the silver method. (4) Blood vessels, and particularly their muscle fibre-cells. 
The method gives particularly good results with cerebral cortex and Ammon's horn, very poor ones with the cerebellum and spinal cord. It is superior to the silver method in so far that the reaction can always be obtained with certainty in a certain time; that the preparation can be preserved by the usual methods; that large pieces of tissue can be impregnated. Moreover, it is cheaper and may give a more abundant and finer impregnation than even the rapid process.

\section{Modifications of GoLGI's Bichromate and Sublimate Method.--} MoNdiNo (Ztsch. wiss. Mikr., ii, 1885, p. 157) has obtained good results from even whole human brain treated according to Golgi's original method.

Flatad (Arch. mikr. Anat., xlv, 1895, p. 158) fixes whole human brain in 3 to 4 per cent. potassium bichromate. After two or three months slices $\frac{1}{2} \mathrm{~cm}$. thick and 1 to $2 \mathrm{~cm}$. wide are brought into 0.1 per cent, mercury bichloride to be changed every two to three days for the first three weeks or so: Pieces are ripe for cutting after nine to twelve months, at which time they are washed and embedded in celloidin. Sections are passed through alcohols, cleared in carbolxylol and mounted in balsam.

PAL (Erratim "Tal ") (Ztschr. wiss. Mikr., iv, 1887, p. 497) converts the whitish mercury impregnation into a black one by treating sections with 1 per cent. sodium sulphide. They may then be counterstained with Magdala red.

GoLGI's sublimate method may be combined with Weigert's myelin stain (see PAL, Wiener med.Jahrb., N.F. 1, 1886, p. 619, and the abstract of this paper in Ztschr. wiss. Mikr., iv, 1887, p. 92, in which EDINGER pointed out that the mercury impregnation can be turned black by treating sections with diluted ammonia).

Flechsig (Arch. Anat. Phys., Physiol. Abth., 1889, p. 537) has published a rather complicated combination of Brama's Guinea red-wood process for medullated nerve-fibres and Golgi's sublimate method, as slightly modified by Held.

896. Cox's Process (Arch. mikr. Anat., xxxvii, 1891, p. 16).-This is the most important of all modifications of Golgi's bichromate and sublimate method. Cox found that the sublimate and bichromate can be used together, and that potassium chromate can be usefully added to the mixture in order to reduce the normally acid reaction of the bichromate, as otherwise axis-cylinders are not impregnated. He used a fluid consisting of 20 parts of 5 per cent. potassium bichromate, 20 parts of 5 per cent. corrosive sublimate, 16 parts of 5 per cent. potassium chromate, and 30 to 40 parts of distilled water. To prepare it, the bichromate and sublimate are mixed together, the chromate diluted with the water and added to the mixture.

One generally uses small pieces of tissues, but also relatively large 
ones can be employed, and whole brains of small animals, particularly if some of the fluid has been previously injected through the carotid or aorta. The duration of the impregnation is from two to three months, but material can be left in the mixture for much longer, certainly without danger and, very likely, with advantage.

MaNN (op. cit.) recommends warming the mixture to the temperature of the incubator and diluting it to one-half the strength advocated by Cox, particularly for material of adult subjects. Portions of the brain measuring $1 \mathrm{~cm}$. in thickness or entire brains of young animals are placed by him on cotton-wool in this solution and left in the incubator for twenty-four hours, when the solution is changed. After a second change on the third day the vessel (which should contain the mixture in proportion of $30: 1$ of the brain) is sealed with vaseline and left in the incubator for at least a month, but preferably for two. I find this way of carrying out the GolgiCox method very good, but, after incubating for a month or so, I prefer keeping the vessel at room temperature, and cutting after another two or three months or longer.

There is considerable difficulty in making and preserving sections which ought to be made either by free hand or by means of a freezing microtome after slight preliminary washing of the pieces with water, and impregnating them with 20 per cent. dextrin for one to three days as suggested by Mann.

To convert the white mercury impregnation into a black one, Cox suggested treating the sections for an hour or two with 5 per cent. sodium carbonate, but 5 to 10 per cent. ammonia is now generally used. They are then thoroughly washed in distilled water, carefully dehydrated, cleared by one of the usual ways, and mounted, without a cover, either in thick xylol balsam or in the original medium suggested by Cox and composed of:-Gum sandarac 75 grms., camphor 15 , oil of turpentine 30 , oil of lavender $22 \cdot 5$, alcohol 75 , castor oil 5 to 10 drops. For examination, add a drop of castor oil, and cover.

89\%. Methods for rendering Golgi-Cox Preparations more permanent.--Various authors (see SANDERs, 1898, in litt. to A. B. Lee, Vade-Mecum, 1913 ed., p. 433 ; Bremer, Anat. Rec., iv, 1910, p. 263) have proposed washing tissues treated according to Cox's process in many changes of alcohol, and embedding them in celloidin - this chiefly with the object of overcoming the difficulty of cutting brittle pieces by means of the freezing microtome, and also of rendering preparations more permanent by removing the excess of corrosive 
sublimate not utilised by the reaction, and which still permeates the tissues. As a matter of fact sections of pieces thus treated are very easily cut and can be transferred from one to another fluid without danger of injuring them. Moreover, they can be counterstained, and the impregnation keeps sufficiently well, particularly if sections are mounted without a cover-glass. But in such preparations, sometimes quickly, sometimes slowly, opaque granules and minute needle-like crystals become almost always developed.

To avoid this I recently proposed (see DA FANo, Proc. Physiol. Soc. Journ. Physiol., liv, 1921) to treat sections much in the same way as by the so-called process of toning and fixing Bielschowsky preparations and the like. (See DA Fano, ibid., liii, 1920.) I proceed thus:- Pieces which, by a trial section, have been found well impregnated, are washed for some hours in distilled water and then brought, through many changes of alcohol of ascending strengths, into absolute alcohol, and then embedded in celloidin in the usual way. The celloidin blocks are hardened in 70 per cent. alcohol, where they can be safely left for many days and weeks. Sections of the desired thickness are collected in 60 per cent. alcohol, transferred into distilled water and here thoroughly washed. They are then treated for five to ten minutes with 5 per cent. ammonia and washed over again in two or three changes of distilled water. At this point toning is carried out by means of a slightly acidified $0 \cdot 2$ per cent. gold chloride solution, in which sections are left for ten or fifteen or twenty minutes, according to their thickness. After a quick washing in distilled water they are passed for three to five minutes in 5 per cent. sodium hyposulphite and washed once more in distilled water. From this they are transferred successively into 30,50 , and 70 per cent. alcohols, to each of which 1 drop of saturated iodine tincture to every 5 c.c. of alcohol has been added. Sections remain in each alcohol ten to fifteen minutes and are lastly transferred into pure 70 per cent. alcohol.

At this point the process is ended, and one can proceed to mount the sections in the usual way, or re-transfer them into distilled water, counterstain them lightly with a carmine solution, dehydrate with alcohols of ascending strength up to 95 per cent., pass them through two changes of carbol-xylol and mount them under a thin coverglass in xylol-colophonium or balsam. If desirable and safe, the celloidin can be removed before definite mounting by passing sections through absolute alcohol, and alcohol-ether if necessary.

The process is simpler than the rather complicated platinum substitutions of Robertson and Macdonald (Journ. Ment. Sc., 
xlvii, 1901, p. 327) and is so quickly and easily carried out that many sections can be manipulated at the same time.

\section{Processes similar to Golgi's Methods or suitable for the same Purposes.}

898. Zienen's Gold and Sublimate Method (Neurol. Centrbl., x, 1891, p. 65). - Small pieces of fresh tissues are put into a large quantity of a mixture of equal parts of 1 per cent. corrosive sublimate and 1 per cent. gold chloride, and left therein for at least three weeks, preferably for several months up to five, by which time they will have become of a metallic red-brown colour. They are then gummed to a cork or wooden cube and cut without embedding. Sections are treated either with LUGoL's solution diluted with 4 volumes of water, or with diluted tincture of iodine, until duly differentiated, then washed, dehydrated, and mounted in balsam. Both medullated and non-medullated nerve-fibres, as well as nerve-cells and neuroglia cells are stained.

899. KrohnthaL's Lead Sulphide Impregnation (Neurol. Centrbl. xviii, 1889 ; Ztschr. wiss. Mikr., xvi, 1899, p. 235).-Pure formic acid is slowly added to a saturated solution of lead acetate till white crystals of lead formiate are abundantly formed. The mother liquid is filtered off, and the crystals are dissolved to saturation in distilled water. Equal volumes of this saturated solution of lead formiate and 10 per cent. formalin form the fixing fluid in which pieces of brain or spinal cord are left for five days. Tissues are then directly brought into a mixture of equal parts of 10 per cent. formalin and sulphuretted hydrogen. After a few minutes the first discoloured portion of this mixture is poured off and replaced with fresh solution, in which pieces remain for another five days. They are then gradually dehydrated and embedded in celloidin. Sections are cleared in carbol-xylol $(1: 1)$ and mounted in balsam under a cover. Nerve-cells and nerve-fibres are extensively impregnated.

Corning (Anat. Anz., xvii, 1900, p. 108) hardens the tissues in 10 per cent. formalin and then brings them into the lead formiate, which he buys from Merk. He prefers to cut without embedding.

900. Wolter's Chloride of Vanadium Process (Ztschr. wiss. Mikr., vii, 1891, p. 471).-Central or peripheral nervous tissues are fixed in Kultschitzky's solution, followed by alcohol as described in $\S 55$. Celloidin sections, 5 to $10 \mu$ thick, are mordanted for twenty-four hours in a mixture of 2 parts of 10 per cent. vanadium chloride and 8 parts of 8 per cent. aluminium acetate. They are then washed 
for ten minutes in water, stained for twenty-four hours in an incubator in Kultschitzky's haematoxylin, and differentiated in 80 per cent. alcohol acidified with 0.5 per cent. of hydrochloric acid until slightly blue-red. The acid is then removed by washing with pure alcohol, and the sections dehydrated, cleared with origanum oil, and mounted in balsam. Axis-cylinders, nerve-cells and glia cells are stained, the myelin being coloured only when the differentiation in the acid alcohol has been insufficient.

901. Azoulay's Ammonium Vanadate Process (Bull. Soc. Anat., Paris, lxix, 1894, 5th S., p. 924).-Wash in water thin sections of material fixed in a bichromate solution and embedded in celloidin. Lift a section on a slide and pour on it a few drops of 0.5 per cent. ammonium vanadate, wait a moment, pour off the stain, wash with a little distilled water and pour on the section a few drops of 2.5 per cent. tannin. After a few minutes pour off the tannin solution, wash, and start all over again, and so on until axis-cylinder and nerve-cells are stained dark green. Wash quickly, dehydrate and mount. These preparations photograph well.

902. Fajerstajn's Hæmatoxylin (Poln. Arch. Biol. Med. Wiss., i, 1901, p. 189).-Make sections, by means of the freezing microtome, of material fixed for two to seven days in 5 to 10 per cent. formalin. Transfer them into 0.25 to 0.5 per cent. chromic acid, and after twenty-four hours wash them well, and put them to stain for another twenty-four hours in 1 per cent. aqueous solution of hæmatoxylin Differentiate by Pal's method.

903. Nabias' Method (C. R. Soc. Biol., lvi, 1904, p. 426).-Sections of material fixed in alcohol-corrosive sublimate or any other fixing agent easily allowing the penetration of iodine are treated until yellow with Lugol's solution (Gram's formula). They are then quickly washed, and treated for a few minutes with 1 per cent. gold chloride, quickly washed once more, and reduced in 1 per cent. watery solution of anilin oil or resorcin. Dehydrate and mount in balsam.

904. Lennhoff's Processes (Neurol. Centrbl., xxix, 1910, p. 20).(1) Polychrome-methylene blue and potassium sulphocyanide method for axis-cylinders: Fixation not stated. Stain sections in polychrome methylene blue for two to five minutes, wash them in distilled water and transfer them for half to twenty-four hours into potassium sulphocyanide (strength not stated). Wash, dehydrate, clear, and mount in balsam. (2) Polychrome-methylene blue and potassium forricyanide method for axis-cylinders and nerve cells. Sections of material fixed in alcohol are treated as above, using potassium ferricyanide instead of 
the sulphocyanide. (3) Iron method. Sections are kept for thirty seconds in 2 c.c. of a 15 per cent. solution of tannin to which 3 drops of a 5 per cent. solution of oxalic acid have been added. Rinse them first in distilled water and then for a few seconds in 1 per cent. solution of iron chloride until no further blackening occurs. Wash, dehydrate and mount in balsam. Axis-cylinders black, nerve-cells grey.

APathy's Gold Method. See § 371.

Gerlach's Bichromate and Gold Process. See $\S 369$.

Ramon y CaJal's Gold Method. See Rev. trim. Micr., v, 1900, p. 95.

Upson's Gold and Iron and Ztschr. wiss. Mikr., vii, 1891, p. 474.

Magini's Zine Chloride Process. See Boll. Acc. med. Roma, 1886, or Ztschr. wiss. Mikr., v, 1888, p. 87.

Montr's Copper Process. See Rend. R. Acc. Lincei, Roma, v, 1889, p. 705 .

Strahuber's Anilin Blue Method. See Centrbl. allg. Path., xii, 1901, p. 422 .

Chilesotti's Carmin Stain. See Centrbl.allg. Path., xiii, 1902, p. 191 ; Ztschr. wiss. Mikr., xix, 1902, p. 161, and xx, 1903, p. 87.

KaPlaN's Anthracen Ink Method. See Arch. Psych., xxxv, 1902, p. 825 .

MaLLORY's Phosphomolybdic Hæmatoxylin. See § 271.

DoNAGGIo's Tin Stain. See § 273.

\section{Methylene-blue Methods not considered in Chapter XVI.}

905. S. MeyeR's Method for the Central Nervous System (Arch. mikr. Anat., xlvi, 1895, p. 282, and xlvii, 1896, p. 734).-The method consists essentially in injecting animals subcutaneously with large quantities of a solution of methylene-blue B.X., and in treating the central organs (brains) with Bethe's fixing bath. S. Meyer used, at first, a 1 per cent. solution; later, a solution of methylene-blue B.X. saturated at the body temperature of the animal to be injected (viz., about 5 to 6 per cent.). The injections are to be made at short intervals and in such a way that the animal receives the total quantity it can support in about one to two hours. A cat can support even 150 c.c.; half-grown rabbits, 30 to 50 c.c.; fully developed guinea-pigs, 30 to 50 c.c. ; new-born kittens, 15 to 25 c.c. As soon as the animal used is dead, the brain is removed, divided into two to four pieces, and these plunged in 10 per cent. ammonium molybdate to which 1 drop of $\mathrm{HCl}$ for every gram of ammonium molybdate is added. Here they remain for about twenty-four hours at $0^{\circ} \mathrm{C}$. Pieces are then washed for two hours in running tap-water, passed quickly through the ascending series of alcohols into absolute alcohol, and, lastly, embedded in paraffin in the usual way. 
906. Ramon y Cajal's Diffusion Process (Rev. Trim. Micr., i, 1896, p. 123).- The brain is exposed, and by means of a sharp razor the cortex is divided into slices about $2 \mathrm{~mm}$. thick. The slices are then covered on both sides, either with finely powdered methylene blue or impregnated with a saturated solution of the same and replaced in their natural situation. The brain is covered over again with its case for about half an hour, after which the slices are removed and fixed for a couple of hours in Bethe's ammonium molybdate solution. They are then washed and hardened for three or four hours in a mixture of 5 parts of chloroplatinic acid, 40 parts of formalin, and 60 parts of distilled water. After another quick wash and a brief treatment (? a few minutes) with a $1: 300$ alcoholic solution of chloroplatinic acid, they are dehydrated and embedded in paraffin. The sections may also be quickly treated with the same weak alcoholic solution of chloro-platinic acid, cleared with xylol or bergamot oil, and mounted in the usual way.

90\%. Catois' Method for Fishes (C. R. Ac. Sc., cxxiv, 1897, p. 204). -Small quantities (2 to 3 c.c.) of a concentrated solution of methylene blue, prepared with physiological salt solution, is injected into the branchial vessels or intramuscularly. The brain is removed after half an hour, divided into slices, and then left for another half an hour in the same concentrated solution used for injecting the animal. The slices are then fixed in the usual ammonium molybdate solution, or in Cajal's chloroplatinic acid mixture.

908. See also the valuable account of DoGIEL Methylen-blau zur Nervenfärbung in the Enzykl. mikr. Techn., 2nd ed., 1910, and the article of Gordon in Anat. Rec., iv, 1910, p. 267 ; and that of MichaILOw in Ztschr. wiss. Mikr., xxvii, 1910, p. 1, in which the literature of the subject is critically discussed. 


\title{
CHAPTER XXXV.
}

\author{
NEUROGLIA AND SENSE ORGANS.
}

\section{Neuroglia.*}

909. Introduction.-Neuroglia cells may be isolated by teasing after maceration in weak solutions of potassium bichromate or 33 per cent. alcohol, and then stained, preferably by means of dilute picrocårmine or other carmine solutions. They may be studied, also, in sections made from non-embedded material fixed in solutions of chromic salts and stained with carmine, nigrosin, orcein and so on. Sections made from either fresh material hardened by the ether freezing method and treated with a weak solution of osmic acid ( $\$ 807$ ), or from tissues hardened in potassium bichromate, can be advantageously stained with watery solutions of anilin-blue-black or nigrosin. Also, sections cut from material fixed, hardened and embedded by the usual methods may, up to a point, be employed for getting a general, though incomplete, view of the amount and arrangement of the neuroglia in a given nervous organ. Iron hæmatoxylin, particularly after fixation in corrosive sublimate or other fluids containing it, gives good results with sections of central nervous organs of lower vertebrates, chiefly of fishes.

See Golgi, Opera Omnia, i, pp. 1 and 3 to 70 ; ii, p. 61 ; Ranvier, Traité, etc.; Bevan Lewis, op. cit.; E. MÜLLeR, Arch. mikr. Anat., Iv, 1900, p. 17; Srudnicka, Anat. Hefte, xv, 1900, p. 316, and the literature quoted therein.

But the best method for the study of the morphology and relationship of ependyma cells and astrocytes has been for many years, and in a sense still is, Golgi's rapid process ( $\S 882$ ), the best material being that which has been placed for about two or three days in the osmio-bichromic mixture.

This method, however, does not allow of any tinctorial differentiation, either between neuroglia cells and nerve cells, or between neuroglia cells and neuroglia fibres. One might even say that it is unsuitable for the demonstration of the latter, the existence of which was clearly established only after the publication of

* Rewritten by Dr. C. Da Fano, King's College, University of London. 
WEIGERT's method (see next §), the first and, perhaps even now, most important of all so-called specific processes for staining neuroglia fibres.

But the Weigert method, whilst staining neuroglia fibres and nuclei of neuroglia cells intensely and, up to a point, specifically, leaves the cell-bodies of the latter entirely, unstained. It consequently led to the erroneous conclusion that the processes of neuroglia cells were one and the same thing as the neuroglia fibres shown by the new method, and that the latter were, in the adult state, only contiguous to-viz., independent of - the former.

Efforts were, therefore, made to discover new methods suitable for the study of neuroglia fibres and neuroglia cells and their reciprocal relations. Many modifications of Weigert's neuroglia stain. the methods of Benda, Mallory, Anglade and Morel, Held, Rubaschikin, Da Fano, etc., may be considered as the direct outcome of such efforts.

None of these methods, however, was sufficient to entirely solve the problems resulting from Weigert's discovery, and from the comparison between the results attainable by the new neuroglia stain and Golgi's process. Hence the publication of the methods of Ramón y Cajal, Achucarro, Del Río-Hortega, and the modern conception that the neuroglia consists essentially of cells provided with variously ramified processes (protoplasmic neuroglia), and of fibres which, though a product of differentiation of the former, remain, very likely, throughout life continuously connected with the protoplasmic bodies and processes of neuroglia cells (fibrous neuroglia).

With all that, the very meaning of the word " neuroglia" and the methods for its study are just at present the subject of fresh discussions and investigations. It is, consequently, expedient to fully describe in the following paragraphs only the principal methods in use for the demonstration of neuroglia, taking this term to mean the whole of the sustaining tissue of the central nervous organs, which is plainly not connective tissue. For minute technical details and methods almost exclusively used in histopathology, the original papers quoted in the following paragraphs should be consulted, as well as Alzheimer, Histol. u. Histopathol. Arb., iii, 1910, pp. 406 to 412 ; Nissu, Enzykl. mikr. Techn., ii, 1910, pp. 280 to 283; Bonome, Atti R. Inst. Veneto Sc. Ixvii, 1909.

910. Weigert's Neuroglia Stain (Weigert's Beitr. zur Kenntniss d. norm. mensch. Neuroglia, Frankfurt-a-Main, 1895; and the 
article "Neurogliafärbung" in Enzykl. mik. Technik, ii, 1910).-Pieces of very fresh tissue of not more than $\frac{1}{2} \mathrm{~cm}$. in thickness are put, for at least four days, into 10 per cent. formol. They are then mordanted for four or five days at $36^{\circ}$ to $37^{\circ} \mathrm{C}$. (or for at least eight days at the temperature of the laboratory) in a solution containing 5 per cent. of neutral copper acetate, 5 per cent. of acetic acid, and $2 \frac{1}{2}$ per cent. of chrome alum, in water. (Add the alum to the water, raise to boiling point, and add the acetic acid and the acetate, powdered, or, instead of chrome alum, take chromium fluoride, which obviates the necessity of boiling.) If preferred, the mordant may be dissolved in the formol solution, so that the hardening and mordanting are done at the same time.

After mordanting, the tissues are washed, dehydrated, embedded in celloidin, and cut. The sections (not too thick) are treated for ten minutes with a $\frac{1}{3}$ per cent. solution of potassium permanganate and well washed in water. They are then treated for two to four hours with a-solution of "chromogen." This is a naphthaline compound prepared by the Hoechst dye manufactory. The solution to be used is prepared as follows : 5 per cent. of "chromogen" and 5 per cent. of formic acid (of $1.20 \mathrm{sp}$. gr., about four times as strong as the officinal) are dissolved in water, and the solution carefully filtered. To 90 c.c. of the filtrate, 10 c.c. of a 10 per cent. solution of sodium sulphite are added.

After this the sections are put till the next day into a saturated (about 5 per cent.) solution of "chromogen." (According to Bolles Lee, Pal's potassium sulphite may be used instead of the "chromogen.")

They are next carefully washed and stained. This is best done on the slide. The stain is a warm-saturated solution of methyl violet in 70 to 80 per cent. alcohol (to which, after cooling and decanting, there may be added, if desired, 5 per cent. of a 5 per cent. aqueous solution of oxalic acid). The sections are treated with this for from a few seconds to one minute, and mopped up with blottingpaper, then treated for an instant with saturated solution of iodine in 5 per cent. potassium iodide. They are then differentiated till clear and light blue with a mixture of equal parts of aniline oil and xylol, washed thoroughly with pure xylol, and mounted in balsam or, preferably, in turpentine-colophonium.

Glia fibres and nuclei blue, cytoplasm stainless.

This method only gives good results with the human subject.

911. Modifications of WeIGERT's Method.-MaLLORY (Journ. Exper. Med., 1897, p. 532) fixes tissues for four days in 10 per cent. solution of 
formalin, then for four to eight in saturated solution of picric acid (or for the same time in a mixture of the two), then mordants for four to six days at $37^{\circ} \mathrm{C}$. in 5 per cent. solution of ammonium bichromate, makes sections (celloidin) and stains them in Weigert's fibrin stain.

STORCH (Virchow's Archiv., clvii, 1899, p. 127), instead of mordanting the material in bulk with the copper fluid, first makes celloidin sections and then mordants them.

BARTEL (Ztschr. wiss. Mikr., xxi, 1904, p. 18) first makes paraffin sections and treats them with all the reagents used by Weigert without removing the paraffin, until they have passed the aniline-xylol mixture which should consist of 1 part of aniline to 10 of xylol (or more), and be allowed to act for twelve to twenty-four hours.

SAND uses material fixed as for his neurofibril stain ( $\$ 843$ ), and stains it according to Weigert.

See also Aguerre, Arch. mik. Anat., lvi, 1900, p. 509 ; Krause, Abh. k. Akad. Wissench. Berlin. Anhang, 1899; Wrmmer, Centrbl. allg. Pathol u. pathol. Anat., xvii, 1906, p. 566; GaleSescu, C. R. Soc. Biol., lxv, 1908, p. 429.

Rubaschkin (Arch. mik. Anat., lxiv, 1904, p. 577) recommends injecting centres of small mammals with the fixing liquid. To make this, take 100 parts of 2.5 per cent. solution of potassium bichromate and 0.5 to 1 of copper acetate, boil, and add 2.5 to 5 of glacial acetic acid. To this (which may be kept in stock) add, just before use, 10 per cent. of formol. Inject warm, and after ten minutes dissect out and harden in the same fluid for five to seven days at $35^{\circ}$ to $40^{\circ} \mathrm{C}$. Dry superficially, put for six to twelve hours in 95 per cent. alcohol and embed in celloidin or paraffin. Stain sections on the slide for six to twelve hours in saturated aqueous solution of methyl-violet B ; treat for half a minute to a minute with Gram's iodine in iodide of potassium; differentiate in aniline or clove oil, and pass through xylol into balsam. The method gives very sharp results with small mammals.

912. BENDA's Method (Neurol. Centrbl., xix, 1900, p. 796 ; and his article "Neurogliafärbung," Enzykl. mik. Technik, ii, 1910, p. 308) is as follows:- The material is to be fixed in 90 or 93 per cent. alcohol for no less than two days. Pieces, not thicker than $\frac{1}{2} \mathrm{~cm}$. are put for twenty-four hours in officinal nitric acid 1 part, and distilled water 10 parts; for another twenty-four hours in 2 per cent. potassium bichromate; for forty-eight hours in 1 per cent. chromic acid. After washing for twenty-four hours, they are dehydrated in alcohols of ascending strength, cleared first in creosote (twenty-four hours), then in benzol (twenty-four hours), and lastly embedded slowly in paraffin, this being dissolved in benzol to saturation first at room temperature, then successively at $38^{\circ}, 42^{\circ}$ 
and $45^{\circ} \mathrm{C}$., so that pure paraffin, melting at $58^{\circ} \mathrm{C}$., is used only for the embedding proper.

The sections, stuck to slides, are mordanted for twenty-four hours in 4 per cent. iron alum or in 50 per cent. Liquor ferri sulfurici oxydati P.G., thoroughly washed, put for.two hours into an amberyellow aqueous solution of sodium sulfalizarinate as directed in $\S 683$, rinsed with tap water, and put to stain in 0.1 per cent. toluidine blue either for fifteen minutes by warming until vapour arises, or for twenty-four hours at room temperature. After rinsing in 1 per cent. acetic acid or in a very dilute solution of picric acid, the sections are dried with filter paper, passed through absolute alcohol, and differentiated for about ten minutes with creosote. They are then dried once more with filter paper, washed with xylol and mounted in balsam.

Besides this, Benda recommends hardening and making paraffin sections as above, then staining by Weigert's method ( $(910)$, but without passing the sections through the saturated solution of " chromogen," and using instead of Weigert's methyl violet solution a freshly prepared mixture of 1 volume of saturated solution of crystal violet, 1 volume of 1 per cent. acid alcohol, and 2 volumes of aniline water.

Benda also uses Heidenhain's iron hæmatoxylin to stain paraffin sections of pieces treated as described, differentiating either with 2 per cent. iron alum or with Weigert's borax-ferricyanide mixture.

913. Mallory's Hæmatoxylin Stains (Journ. Exper. Med., v, 1900 , p. 19).-Tissues to be fixed, mordanted and cut as directed under MaLLORx, $\S 911$. The sections are put for a quarter of an hour into 0.5 per cent. solution of potassium permanganate, washed and put for another quarter of an hour into 1 per cent. solution of oxalic acid, well washed and stained for twelve to twenty-four hours or more in MALLORY's phosphotungstic hoematoxylin. Wash, dehydrate in 95 per cent. alcohol, clear with origanum oil, mount in xylol-balsam. Axis cylinders and nerve cells pink, neuroglia blue. To get a more isolated stain of neuroglia, the sections. should be brought for five to twenty minutes, after staining, into a 30 per cent. alcoholic solution of iron sesquichloride. Neuroglia and fibrin blue, the rest colourless.

MALLORY's phospho-molybdic hamatoxylin may also be used for the stain, but it is less elective.

914. ANGlade and Morel's Victoria Blue Method (Rev. Neurol., ix, 1901, p. 157).- Harden in a mixture of 3 parts of liquid of Fol. 
(§ 47) with 1 of 7 per cent. sublimate solution, dehydrate with alcohol followed by acetone, make paraffin sections, and stain in saturated aqueous solution of Victoria blue heated till it steams ; rinse with Gram's fluid, differentiate with xylol 1 part, aniline 2 parts, and mount in balsam. Simple, applicable to lower animals, and gives very sharp pictures.

915. Da Fano's Methods (Ricerche Lab. Anat. Roma ed altri Lab. Biol., xii, 1906).-Method I. is a modification of MALLORY's phosphotungstic hæmatoxylin process ( $\$ 913)$. Small pieces of fresh tissue are fixed for twenty-four to forty-eight hours in a mixture of 72 volumes of pyridine and 28 of 50 per cent. nitric acid. After washing for about six hours, the pieces are dehydrated and embedded in paraffin. The sections, stuck to slides by the albumin method, are treated as by MALLORY's method, and stained with an old solution of MaLlory's phosphotungstic hæmatoxylin, but prepared without the addition of hydrogen peroxide. In order to increase the contrast between neuroglia fibres (blue-violet) and the protoplasm of neuroglia cells (pink) DA Fano dehydrates the stained sections in 95 per cent. alcohol to which a small quantity of an alcoholic solution of eosin has been added.

Method II. is a modification of BeNDA's process ( $\$ 912$ ). Very small pieces are fixed for thirty-six to seventy-two hours in a mixture of 2 volumes of the fixing fluid used for Method I. and 1 volume of 1 per cent. osmic acid. After washing for six to twelve hours, the pieces are embedded in paraffin. The sections, stuck to slides, are successively mordanted for twenty-four hours each with WEIGERT's copper acetate-chromium fluoride fluid ( $\$ 910)$, 2 per cent. chromic acid, and 2 per cent. iron alum rinsing in water before passing them from one into the other mordant. They are lastly either treated and stained as by BENDA's alizarine-toluidine blue process, or as by HeIDENHAIN's iron hæmatoxylin method.

Method III. was arrived at in an endeavour to make use of unsuccessful preparations made by CAJAL's reduced silver method. Pieces treated as by Cajal's formula la or one of its modifications ( $\$ 837$ ), or simply fixed in 2 or 3 per cent. silver nitrate at $36^{\circ}$ to $37^{\circ} \mathrm{C}$., are embedded in paraffin. The sections, stuck to slides, are bleached by Pal's differentiation method for myelin stain, and then mordanted and stained as by Method II.

916. HeLD's Method for Marginal Neuroglia (Monatschr. Psych. Neurol., xxvi, 1909 ; Ergänzungsh., p. 360).-Tissues are preferably fixed by means of a modified Zenker's fluid consisting of Müller's 
fluid 100 c.c. and sublimate 3 grms., with the addition at the moment of use of acetic acid 3 c.c., formalin 0.5 c.c. The fluid should be warmed at $35^{\circ}$ to $40^{\circ} \mathrm{C}$. and injected through the blood-vessels, the blood being first washed away by means of Ringer's solution to which 1: 1000 of amylnitrite was added. The tissues are treated in the usual way and embedded in celloidin. The sections are first treated for five minutes with a 1 per cent. solution of caustic soda in 80 per cent. alcohol and washed in distilled water, and then mordanted for a few minutes in 5 per cent. iron alum and washed once more. For staining, Held adds to some distilled water a few drops of a very old molybdic acid hæmatoxylin, enough to impart to the water a bluish-violet tone, and stains therein for twelve to twenty-four hours at $50^{\circ} \mathrm{C}$. The stain is prepared by dissolving $1 \mathrm{grm}$. of hæmatoxylin in 100 c.c. of 70 per cent. alcohol and adding an excess of molybdic acid. Differentiation is carried out by means of the same iron alum solution used for mordanting; wash well ; counterstain with v. Gieson picro-fuchsin solution; wash in 96 per cent. alcohol, dehydrate and mount as usual.

Neuroglia cells and fibres greyish-black; marginal neuroglia (membrana limitans marginalis and membrana limitans perivascularis) sharply differentiated; connective tissue pink-red.

917. Other similar Methods.-Liérmitte and Guccione (Semaine Méd., xxix, 1909, p. 205) have the following modification of ANGLADE and MOREL's method: Sections, made by the freezing method from formalin material, are collected in distilled water and then kept for two hours in a cold-saturated solution of sublimate and for two days in a mixture consisting of 3 parts of 1 per cent. osmic acid, 35 of 1 per cent. chromic acid, 7 of 2 per cent. acetic acid, 55 of distilled water. The rest as Anglade and Morel.

Similarly Merzbacker (Journ. Psychol. Neurol., xii, 1909, p. 1).

De Albertis (Pathologica, xii, 1920, p. 240) has recently proposed the following combination of the methods of Weigert, Mallory, and ANGLADE and MOREL. Sections are made by means of a freezing microtome from pieces fixed in 15 to 20 per cent. formalin for about twenty-four hours, but not longer than three days. They are transferred into a bath of 2 per cent. acetic acid in 1 per cent. chromic acid (time not stated), and then washed for some hours in repeatedly changed distilled water, oxidised for ten to fifteen minutes in $\frac{1}{2}$ per cent. potassium permanganate, washed again in distilled water, reduced for fifteen to twenty minutes in 1 per cent. oxalic acid and lastly put to stain for twelve to twenty-four hours in a saturated solution of Victoria blue. For the further treatment sections are washed in distilled water and from this lifted, one by one, by means of a thin glass or platinum spatula, this to be used to plunge each section for an instant first into concentrated Lugal's solution, then into absolute alcohol, and lastly into equal parts of xylol and aniline oil, where the differentiation is accom- 
plished in a few seconds. Sections are finally collected and washed in slightly warmed and repeatedly changed xylol, and mounted in xyloldamar without a cover.

Kultschitzky Rubin's Method (Anat. Anz., viii, 1893, p. 357) is no longer used. For the slight modification of this method of Popow, see Ztschr. wiss. Mikr., xiii, 1896, p. 358, and for that of BURCIARDT, La Cellule, xii, 1897 , p. 364 .

The method of Yamagiwa (Virchow's Arch., clx, 1900, p. 358) is also no longer used.

\section{Methods for Protoplasmic Neuroglia and Neuroglia Granules:-} Oppenheim (Neurol. Centrbl., xxvii, 1908, p. 643) mordants sections made from frozen formalin material with Weigert's copper acetatechromium fluoride mixture and then stains them with Weigert's iron hæmatoxylin prepared without hydrochloric acid. An important point of this method is that the material and the sections should not have been treated with alcohol before staining.

Eisath (Monatschr. Psych. Neurol., xx, 1906, p. 3 ; Arch. Psych. Nervenkrankh., xlviii, 1911, p. 897) fixes large pieces in a modified Orth's formol-Müller mixture consisting of water 1000 c.c., potassium bichromate 25 grms., sodium sulphate 15 grms., and formalin 150 c.c., to be added at the moment of using the mixture. After about four weeks the tissues are ready for being cut without embedding, but can be kept for many months, and even years, in 4 per cent. formalin. The sections are collected in 4 per cent. formalin, in which they may be kept until wanted. For the staining the sections are put for thirty seconds in a $0 \cdot 2$ per cent. solution of sublimate, well washed in water, and lifted on to the slide, a dilution of an old Mallory's phosphomolybdic-carbolic acid hæmatoxylin being poured on them. After a few minutes they are washed with water, differentiated with a mixture of equal parts of 40 per cent. tannic acid, 50 per cent. alcohol, and 20 per cent. pyrogallic acid in 80 per cent. alcohol. Wash in alcohol, dehydrate, clear and mount.

Fieandt (Arch. mikr. Anat., lxxvi, 1910-11, p. 125) fixes in Heidenhain's sublimate-trichloracetic mixture, and treats pieces for five to seven days with 96 per cent. alcohol, to be changed three times during the first twenty-four hours and daily in the following days. After dehydration the pieces are embedded in paraffin as directed by Prantner. The sections, 3 to $5 \mu$ thick, are stuck to slides, freed from sublimate by the usual iodine treatment, and then stained for twelve to twenty-four hours with Mallory's phosphotungstic hæmatoxylin. Dry with filter paper, differentiate for a few hours in 10 per cent. iron perchloride in absolute alcohol, blot once more with filter paper, wash, dehydrate and mount. 
Neuroglia fibres, cytoplasm of neuroglia cells, and glia granules stained in various shades of blue and greyish-blue; all other elements yellowish-grey or yellowish-brown.

Ranke (Ztschr. ges. Neurol. u. Psych., vii, 1911, p. 355) uses for similar purposes either celloidin sections of foetal tissues fixed in picric acid-alcohol or sections made by freezing from formalin (pathological) material. In the first case the sections are stuck to slides by pressing with filter paper and then pouring on them methylalcohol until all celloidin is dissolved. He next stains them for a few minutes with his acid eosin-thionin solution (see further on), washes with water, and re-stains them, with the help of gentle heat, with 5: 1000 Giemsa's "Methylenazur I"; quick differentiation with distilled water ; 96 per cent. alcohol, cajeput oil, xylol, balsam. In the case of pathological material the sections are first treated with 1 per cent. osmic acid in order to stain fatty products of degeneration, etc., then pressed on to slides and stained as above. To prepare the acid eosin-thionin mixture, mix and shake repeatedly 1000 c.c. of each $1: 1000$ watery solution of eosin W.G. and $1: 1000$ watery solution of thionin. Leave for forty-eight hours, pour out the fluid part, and wash the sediment into a paper filter with distilled water until the wash water is only a little stained. Dry what remains in the filter, and dissolve it in methyl-alcohol in the proportion of 0.3 to 0.5 per cent.

919. RAMón y CaJaL's Gold Chloride and Sublimate Method (Trab. Lab. Invest. Biol., Madrid, xi, 1913, pp. 219 and 255; xiv, 1916, p. 155). - At first Cajal used to harden pieces of quite fresh tissues in 14 per cent. formalin, but in his successive papers he recommended fixing from two to ten days in-

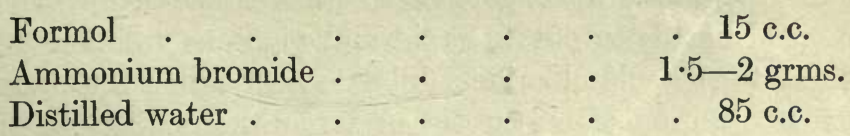

Relatively thick sections (20 to $25 \mu$ ) are made by the freezing method, and collected in distilled water to which a few drops of formalin have been added. After a quick wash, batches of four to six sections are each transferred into glass dishes of about $6 \mathrm{cms}$. in diameter, and each containing 15 c.c. of a mixture of-

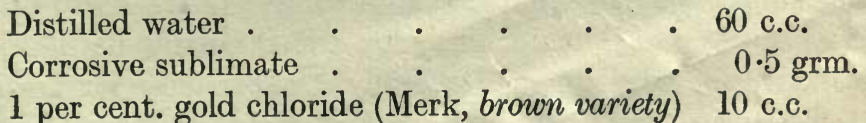

After about four hours the sections will be found to have become 
an intense purple, and can be passed, for five to ten minutes, into a fixing bath consisting of -

Concentrated solution of sodium hyposulphite 5 c.c.

Distilled water . . . . . . 70,

Alcohol . . . . . . 30 "

Concentrated solution of sodium bisulphite . 5 ",

Wash in 50 per cent. alcohol, lift sections on to slides, dry with filter paper, wash with absolute alcohol, clear with origanum oil, wash with xylol, and mount in balsam.

To ensure successful results the following points should be borne in mind:-(1) The gold chloride must be of the brown variety. Its solution remains unaltered for months if kept in the dark. (2) The solution of mercury chloride becomes very easily altered, and is best prepared when required, dissolving it with the help of some heat, and filtering before adding it to the gold chloride solution. (3) Best results are obtained by keeping the glass dishes, with the sections and the gold chloride-sublimate mixture, at a temperature of $18^{\circ}$ to $20^{\circ} \mathrm{C}$. If the reagent is freshly prepared, the reaction will be complete in about four up to six hours. At temperatures between $14^{\circ}$ and $17^{\circ} \mathrm{C}$. three or four hours more are necessary to obtain good stains. With temperatures below $14^{\circ}$ or $12^{\circ} \mathrm{C}$. it is very difficult to obtain any reaction at all. One may have recourse to temperatures above $20^{\circ} \mathrm{C}$., up to $27^{\circ}$ or $30^{\circ} \mathrm{C}$. in special cases, as Del Río-Hortega has done for the neuroglia of the pineal body. (4) More diluted gold baths may be used for economical reasons, but in this case one must have recourse either to higher temperatures or to greater lengths of time. (5) To proceed quicker, one may either double the proportion of sublimate in the formula given above or double the proportion of gold chloride and treble that of sublimate. (6) A good means to obtain rapid and vigorous reactions consists in adding to the gold chloride-sublimate bath either 2 to 3 drops of a $1: 1000$ solution of erythrosin or a minute quantity of the dry dye, enough to impart to the bath a slightly orange tone. (7) All other conditions being the same, results are greatly influenced by the length of time during which the pieces have been kept in the fixing fluid. As a rule, they begin to be ripe for cutting from the end of the third day, and they continue to be in a state favourable for obtaining good reactions for another five or six up to fifteen or twenty days. Good stains may be exceptionally obtained after two months of hardening. Generally, the capacity for taking the gold disappears first from the protoplasmic, and then from the fibrous, neuroglia. 
By means of Cajal's method two categories of neuroglia elements become stained a dark purple on a much lighter purplish background. The first category consists of neuroglia cells provided with a changing number of variously ramified protoplasmic processes, which inter-cross with those of other cells, and thus give origin to Cajal's pleurigenic plexus. These neuroglia cells prevail in the grey layers of the human cerebral cortex, and form the bulk of the protoplasmic neuroglia (\$ 909). In Cajal's preparations they appear beset with vacuoles, situated both within their cytoplasm and along their processes. The vacuoles or spaces are occupied by granules (gliosomes), which may be stained either by Cajal's uranium nitrate method ( $(847$ ) (superficial sections) or by methods generally used for the demonstration of mitochondrial formations as well as by the methods of Eisath and Fieandt. The other category of neuroglia elements shown by the gold chloride and sublimate method consists of astrocytes, viz., of neuroglia cells, also provided with a changing number of processes, but chiefly characterised by the absence of gliosomes and the presence of fibres which, though a product of differentiation of the protoplasmic portions of the astrocytes, never become entirely independent of the latter. These fibres appear to correspond to those stainable by the methods described in $\S \S 910$ to 916 . The astrocytes prevail in the white matter of the central nervous system, and form the bulk of the fibrous neuroglia (\$909). Neuroglia cells, in part protoplasmic and in part fibrous, occur chiefly at the points of transition between the grey and the white substances of central nervous organs.

The gold chloride and sublimate method leaves unstained $a$ third category of elements, the existence of which was at first recognised by Cajal by means of this negative character, but they were subsequently studied by him in superficial sections of pieces stained by his uranium nitrate method and other cytological methods. The cells belonging to the category now considered appear in uranium nitrate preparations as roundish elements, but, as a matter of fact, they also are provided with a changing number of variouslyramified protoplasmic processes (see § 919). As Cajal was not able to come to any definite conclusion in regard to their nature, he proposed to term them the "third element," i.e., a category of cells which, though non-nervous in character, do not plainly form part either of the connective tissue (blood-vessels, pial septa) or of the neuroglia, this term being, in Cajal's opinion, reserved for those elements which are genetically derived from an evolution of the ependymal epithelium. 
920. ACHúCARro's Tannin Method and DeL Rfo-Hortega's Modifications.-The methods described in this paragraph can be considered as the direct outcome of various efforts at modifying the Bielschowsky method for sections ( $(840)$ in such a way as to obtain a neuroglia stain. As a matter of fact, they all stain both neuroglia cells (astrocytes) and connective tissue elements. In other words, they are not elective, and may be used for the study of reticular tissue in non-nervous organs, as well as of other histological details in nervous and non-nervous tissues.

Perusini's modification of Bielschowsky's method (Neurol. Centrbl., xxix, 1910, p. 1256) should be first remembered. Pieces of fresh material were fixed in Weigert's formalin-copper acetatechromium fluoride mixture for neuroglia stain (\$910), cut by the freezing method, and stained as by Bielschowsky's method for sections, without pyridine treatment. Achúcarro did the same, except for silvering by Ramon y Cajal's reduced silver process.

ACHúcarro's tannin method (Bol. Soc. Espan. Biol., Madrid, 1911, p. 139) consisted in putting sections made from frozen formol material into a cold-saturated solution of tannin and warming this until vapour arose. Without waiting for the tannin to become cool again, the sections were, one by one, quickly rinsed in water and put to stain for about ten minutes into three successive glass dishes, each containing 10 c.c. of distilled water and 6 to 8 drops of Bielschowsky ammoniacal silver nitrate-and-oxide bath, prepared beforehand, as described in $\S 841$. As soon as they turned dark yellow, they were transferred into 10 per cent. formalin, and, after about ten minutes, washed, dehydrated and mounted.

The results obtained by such a method were rather uncertain and Achúcarro himself felt the necessity of modifying it in the following way, published by Des Río-Hortega (Trab. Lab. Invest. Biol., Madrid, xiv, 1916, p. 181) :-(1) Fix pieces, 2 to $3 \mathrm{~mm}$. thick, for two or three days in formalin neutralised with ammonia. (2) Make sections of $10 \mu$, and mordant them in 10 per cent. tannin until vapour arises. (3) Without waiting for the tannin to become cool, wash the sections in distilled water alkalised with a few drops of ammonia until they have again acquired their flexibility. (4) Treat them with the diluted ammoniacal silver nitrate solution as described above, but adding only 2 or 3 drops of it to every 10 c.c. of distilled water. (5) Reduce in 20 per cent. formalin, either neutralised as for fixing, or (according to Del Río-Hortega) containing an excess of ammonia, say, 6 to 8 drops to every 10 c.c. of 20 per cent. formalin. 
DeL Río-Hortega (op. cit.) found that the method could be further modified, and usefully employed for the staining not only of the neuroglia, but also of centrosomes of nerve cells and neuroglia cells, mitochondria, secretion granules, intra-epithelial fibrils, reticular tissue, collagenous fibres, etc. The modifications proposed by Del Río-Hortega for these various purposes are four in number, and known as the variants of Achucarro's method.

Modification I.- Suitable for the staining of fibrous neuroglia as well as for elastic membranes and connective tissue cells. (1) Fix tissues for no less than ten days in 10 per cent. formalin. (2) Make sections by the freezing method, and mordant them for five minutes in 3 per cent. tannin kept at a temperature of $50^{\circ}$ to $55^{\circ} \mathrm{C}$. (3) Wash them in distilled water alkalised with ammonia, and transfer them successively into three glass dishes, each containing 1 c.c. of ammoniacal silver nitrate, prepared as described in $\S 841$, and 10 c.c. of distilled water. (4) As soon as they have taken a distinct yellowish-brown colour, wash them in distilled water and reduce them in a $1: 500$ gold chloride solution kept for twenty or thirty minutes at a temperature of about $40^{\circ}$ to $45^{\circ} \mathrm{C}$. (5) Fix with 5 per cent. sodium hyposulphite, wash, dehydrate and mount as usual.

Modification II.-Good chiefly for reticular tissue and its histogenesis. Material may be fixed either in 10 per cent. formalin or Bouin's fluid, or alcohol ; if one or the other of these last two fluids has been used, it is advisable to re-transfer pieces for a few days into a formalin solution. Sections should, as a rule, be made by the freezing method, but pieces may also be embedded in celloidin, this being dissolved after cutting. The sections, however obtained, are mordanted for five minutes at $50^{\circ}$ to $55^{\circ} \mathrm{C}$. or for fifteen to thirty minutes at $40^{\circ}$ to $45^{\circ} \mathrm{C}$. in a 1 per cent. alcoholic solution of tannin. Stain as in Modification I; reduce for half a minute in 20 per cent. formalin, neutralised by shaking with chalk; wash, dehydrate and mount.

Modification III.-Particularly good for collagenous fibres, but also for neuroglia fibres. Proceed as in Modification II until the sections are placed in the staining bath; keep them therein until brown; reduce and fix as in Modification I.

Modification IV (op. cit., xv, 1918, p. 375, note).- Suitable for the demonstration of the protoplasmic neuroglia. Frozen sections of formalin material are treated for some minutes at $45^{\circ}$ to $50^{\circ} \mathrm{C}$. with a mixture of tannin, 3 grms.; ammonium bromide, 1 grm.; distilled water, 100 c.c. Wash and stain as in Modification I; reduce in 20 per cent. formalin neutralised with chalk; tone with 
$0 \cdot 2$ per cent. gold chloride; fix, wash, dehydrate and mount as usual.

921. Del Río-HortegA's Carbonate of Silver Method (Trab. Lab. Invest. Biol., Madrid, xv, 1918, and xvii, 1920 ; Bol. Soc. Esp. Biol., viii, 1918).--Pieces of quite fresh nervous tissues are fixed in Cajal's ammonium bromide-formalin mixture, and kept therein for different periods of time, according to the purposes in view. If it is desired to stain the protoplasmic neuroglia, pieces are best fixed for twenty to thirty or forty days; after this time they are for some months in a condition particularly suitable for the staining of the fibrous neuroglia. But if the time of fixation is limited to one or two days at the temperature of about $35^{\circ} \mathrm{C}$., or to two up to four days at room temperature, the tissues are in a state favourable to the impregnation of Cajal's "third element" (§ 919), which Del Río-Hortega proposes to term either microglia or mesoglia, the first of these two denominations being simply used with reference to the smallness of the elements thus named, the second implying that they do not belong to the neuroglia as this term is understood by Cajal and his pupils. See $\S 919$. For the staining one may choose one or the other of the following three processes:-

Process I, for protoplasmic and fibrous neuroglia. - Sections made by the freezing method are washed in two or three changes of distilled water and transferred into a crystallising basin containing 5 or 10 c.c. of ammoniacal silver carbonate solution, prepared as follows:-To 50 c.c. of 10 per cent. silver nitrate an equal or greater quantity of cold-saturated lithium carbonate solution is added, so as to precipitate all silver in the form of silver carbonate. The fluid part is poured off, and the precipitate first washed with 200 to 300 c.c. of distilled water, and then taken up with about 50 c.c. of diluted ammonia, by means of which it is entirely dissolved. The solution is diluted with distilled water up to a total volume of 250 c.c. and poured into a dark brown bottle, where it keeps indefinitely, if put away in some dark place.

The crystallising basin, with the ammoniacal silver carbonate and the sections placed therein, is warmed, either in an incubating stove at $45^{\circ}$ to $50^{\circ} \mathrm{C}$. or over a flame, until the sections become a greyishyellow colour. This requires only a few minutes if the sections are moved about so that they may stain uniformly. Good results may be also obtained by staining at $35^{\circ} \mathrm{C}$. for twelve to fourteen hours or at room temperature for one or two days. Without waiting for 
the silver solution to become cool, the sections are quickly washed in distilled water and then transferred, one by one, into 20 per cent. formalin neutralised with chalk. After one or two minutes, the reduction is complete, and the sections may be washed, toned, fixed and dehydrated, cleared with a mixture of carbolic acid 5 parts, xylol 45 parts, creosote 50 parts, and mounted in balsam.

Process II, for microglia.- Sections are made as above, and then treated for ten or fifteen minutes at $50^{\circ}$ or $55^{\circ} \mathrm{C}$. with the bromideformalin solution used for fixing. After washing in two or three changes of water, one continues as in Process I, but warming the ammoniacal silver carbonate solution at $50^{\circ}$ or $55^{\circ} \mathrm{C}$. until the sections are dark yellow.

Process III, also for microglia.-The pieces are warmed for ten minutes in the fluid used for fixing, and then cut by the freezing method. The sections are washed in distilled water and stained for ten to thirty minutes, either at room temperature or by careful gentle warming, with an ammoniacal silver carbonate solution, prepared by adding to 10 c.c. of 10 per cent. silver nitrate, first, 30 c.c. of 5 per cent. sodium carbonate, then ammonia, drop by drop, until the precipitated silver carbonate is dissolved, and, lastly, distilled water up to a total volume of 150 c.c. The sections are kept in the impregnating bath for from ten to thirty minutes at room temperature, but they should nevertheless remain almost colourless. For the reduction, 1 per cent. formalin is used, and sections are left therein until they have taken a greyish-yellow tinge ; wash, tone, etc., as in Process I.

The above refers to material fixed in Cajal's ammonium bromideformalin mixture; if nervous tissues are fixed in 10 per cent. formalin and sections treated as in Process I, nerve cells and axis cylinders become stained as by Bielschowsky's method. If formol sections of non-nervous tissues are treated in the same way, the reticular tissue becomes stained.

\section{Retina.*}

922. Fixation and Hardening. - Notwithstanding the Encycl. mik. Technik., 2nd ed., p. 75, I hold that osmic acid is by far the best fixing agent. The retina of small eyes is best prepared by fixing the entire unopened bulb with osmium vapours.

Besides the sources quoted in the text, see Seligmann, Die mikroskopischen Untersuchungsmethoden des Auges, Berlin, S. Karger (Karl-

* By A. Bolles Lee. 
strasse 13), 1899 ; GREEF, Anleitung zur Mikr. Untersuch. d. Auges, Berlin, Hirschwald, 3rd ed., 1910 ; and the Art. "Retina" in Encycl. mik. Technik., 2nd ed., p. 575.

Szent-Györgi (Zeit. f. wiss. Mikr., xxxi, 1914), uses the following fluid :-

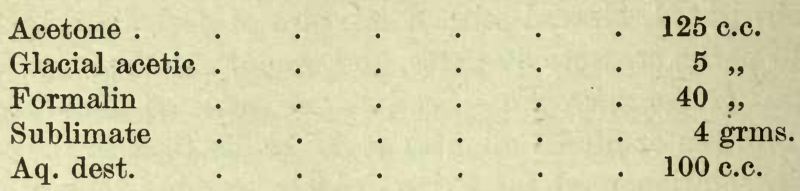

Leave whole small eyes in 100 c.c. of this mixture for two to three days, larger whole eyes six to seven days, after which one adds an additional 50 c.c. of acetone to the fixative and leaves for a further two or three days. Transfer to pure acetone for three or four days, renewing on the last day; then bring the eyes into a vessel of acetone, with a thick layer of desiccated calcium chloride at its bottom, for three or four days, renewing the $\mathrm{CaCl}$, if necessary. Transfer from the acetone into a mixture of half ether, half absolute alcohol, then proceed as for celloidin embedding.

According to RANviER (Traité, p. 954) you may fix the eye of a triton (without having previously opened the bulb - the sclerotic being very thin) by exposing it for ten minutes to vapour of osmium. Then divide it by an equatorial incision, and put the posterior pole for a few hours into one-third alcohol.

Somewhat larger eyes, such as those of the sheep and calf, may be fixed in solutions without being opened. But it is generally the better practice to make an equatorial incision, and free the posterior hemisphere before putting it into the liquid.

The older practice was to use strong solutions of pure osmic acid alone ; but most of the best recent work has been done with chromic mixtures following the osmium.

Dr. Lindsay Johnson tells me that he now gets the best results by suspending the globe over the steam of a 1 per cent. osmic acid solution raised to the temperature at which vapour is seen to be given off (but not to boiling point) for five minutes in the case of human adults, or for one to three minutes in the case of human infants, all monkeys and small mammals, as in them the sclerotics are very thin. As soon as the sclerotic is felt to be firm to the touch, it should be opened by a small nick with a razor just behind the ciliary body; or if the eye be that of an adult, the cornea and lens may be removed. The eye is then put for twelve hours into the mixture, $\S 44$; it is then washed in running water, and suspended in a large volume of 2.5 per cent. bichromate of potash for two days, then passed gradually through successive alcohols, beginning with 
20 per cent., and ending with absolute, taking five days from first to last.

Similarly Rochon-Duvigneaud (Arch. Anat. Micr., ix, 1907, p. 317).

Other hardening liquids, however, also give good results, provided that the fixation by the osmic acid has been properly performed: amongst them liquid of Flemming, and that of Müller. Formaldehyde mixtures he does not recommend.

LEBER (Münch. med. Wochenschr., xli, 1894, p. 605; Zeit. wiss. Mik., xii, 1895, p. 256) advises a solution of formol 1, water 10. After a few days hardening in this, the eyes may be cut through, it is said, without derangement of the parts. The retina lies flat, and is at least as well preserved as with solution of Müller.

See also HIPPEL (Arch. f. Ophthalm., xlv, 1898, p. 286; Zeit. wiss. Mik., xvi, 1899, p. 79), who finds that formol fixes the lens badly, the retina well, so far at least as the absence of folds from shrinkage is concerned; and Herzog (Arch. mik. Anat., lx, 1902, p. 517, and Encycl. mik. Technik., p. 75), who also approves of formol, but insists that it should be acid, and adds 3 to 5 per cent. of acetic acid.

Kolmer (Arch. Gesammte Phys., cxxix, 1909, p. 35), fixes for twelve to twenty-four hours in a mixture of 4 parts saturated solution of bichromate, 4 of formol of 10 per cent., and 1 of acetic acid.

Benda (Verh. Ges. Naturf. Atrzte, lxxi, Vers., 1900, p. 459) fixes in nitric acid of 10 per cent., and hardens in liquid of Müller, twenty-four hours in each.

ZüRn (Arch. Anat. Phys., Anat. Abth., 1902, Supp., p. 106) advises (for mammals) fixing in saturated solution of sublimate in salt solution of $0 \cdot 6$ per cent., with 1 to $1 \frac{1}{2}$ per cent. of acetic acid after removing the anterior pole and the vitreous. Wash out in alcohol of 35 per cent. made 5 per cent. stronger each day up to 50 per cent. ; then pass on to stronger and cedar oil and paraffin.

923. Staining.-For general views I recommend iron-hæmatoxylin, followed by Säurefuchsin or Picro-Säurefuchsin, or preceded by Bordeaux; or Kernschwarz, followed by safranin, or the EhrlichBiondi stain.

The Methylen-blue intra-vitam stain has given valuable results; see the methods of DoGIEL.

But the most important method is the bichromate and silver impregnation of GoLGI, first applied to this object by TARTUFERI (Intern. Monatsschr., iv, 1887, p. 421). This author employed the rapid process. So also RAḾ́N y CAJAL (La Cellule, ix, 1893, p. 121) with the double-impregnation process, $\S 886$. To avoid the formation of precipitates on the tissues, he covers the retina, before silvering, with a piece of peritoneal membrane, or a thin layer of collodion. Or, better, he rolls the retina (op. cit., p. 130). After 
removing the vitreous, the retina is cut away around the papilla with a punch or fine scalpel, and separated from the choroid. It is then rolled up (after being cut into quadrants or not), so as to form a solid block. This is painted with 2 per cent. celloidin, which is allowed to dry for a few seconds, and the whole is put into the bichromate mixture, and further treated as a solid mass of tissue.

RAMón also employs his neurofibril silver method, see Intern. Monatsschr. Anat. Phys., xxi, 1905, p. 393.

GoLGr's sublimate impregnation (Cox's form) has also been successfully employed by Krause and RAmón.

The bichromate and silver method serves for the study of the fibres of Müller and neuroglia cells, as well as neurones. Weigert's neuroglia stain does not give good results.

Lennox (Arch. f. Ophthalm., xxxii, 1886, 1; Zeit.wiss. Mik., iii, 1886, p. 408) has used Weigert's hæmatoxylin method.

Kunnt (Jen. Zeit. Naturw., Bd. xxiv, 1890, p. 177) employs Pal's modification. Similarly Schaffer (Sitzb. Akad. wiss. Wien., xcix, 1890, Abth. 3, p. 110; Zeit. wiss. Mik., viii, 1891, p. 227). These methods give a differential stain of rods and cones.

For the zonula and ciliary body see MAwas, Arch. d'A nat. micr., xii, 1910 , p. 103.

924. Dissociation.-For maceration preparations you may use weak solutions $(0.2$ to 0.5 per cent.) of osmic acid for fixation, and then macerate in 0.02 per cent. chromic acid (M. Schultze), or in iodised serum (M. Schultze), or in dilute alcohol (LANDolT), or in Müller's solution, or (RANvieR, Traité, p. 957) in pure water, for two or three days. THin (Journ. of Anat., xiii, 1879, p. 139) obtained very good results by fixing for thirty-six to forty-eight hours in one-third alcohol, or in 25 per cent. alcohol, and then staining and teasing.

SCHIEFFERDECKER macerates fresh retina for several days in the methyl mixture, $\S 543$.

KraUse (Intern. Monatsschr. Anat., i, 1884, p. 225) recommends treatment for several days with 10 per cent. chloral hydrate solution ; the rods and cones are well preserved.

\section{Inner Ear.}

925. Inner Ear, Dissection.-For the dissection of the human ear see Politzer, "Die anatomische $u$. histologische Zergliederung d. menschlichen Gehöroganes," Stuttgart (Enke), 1889 (Zeit. wiss. Mik., vii, 1890 , p. 364). Amongst the lower mammalia, the guinea-pig is a favourable subject, as here (as with some other rodents) the cochlea projects freely into the cavity of the bulla, and may be easily removed 
with a scalpel and brought into a fixing liquid, and opened therein. With fishes and amphibia also the membranous labyrinth may easily be got away.

926. Preparation. - Schwalbe (Beitr. z. Phys. ·(C. Ludwig's Festschr), 1887, p. 200).-Fix (cochlea of guinea-pig) for eight to ten hours in "Flemming," wash in water, decalcify (twenty-four hours is enough) in 1 per cent. hydrochloric acid, wash the acid out, dehydrate, and embed in paraffin.

Prenanti (Intern. Monatsschr. Anat., ix, 1892, p. 28).-Open the cochlea in solution of Flemming or of Hermann, and fix therein for four to five hours. Avoid decalcification as far as possible, but if necessary take 1 per cent. palladium chloride. Make paraffin sections.

Isolation preparations of the stria vascularis may be made by putting a cochlea for a day into 1 per cent. solution of osmic acid, then for four to five days into $0 \cdot 1$ per cent. solution; the stria may then be got away whole.

Katz (Zeit. wiss. Mik., xxv, 1908, p. 111) fixes the inner ear, opened, for one or two hours in 30 c.c. of 0.5 per cent. osmic acid with 5 drops of acetic acid, then adds 10 drops of acetic acid and 60 c.c. of chromic acid (or platinum chloride) of 0.5 per cent. and leaves it for four days therein. He then rinses, puts for twelve to twenty-four hours into pyroligneous acid or pyrogallol or tannin solution, decalcifies (not necessary for mice) in 200 parts of water with 1 of chromic acid and 4 to 10 of nitric or hydrochloric acid, and embeds in celloidin or sometimes paraffin.

Similarly WIтTMAack, see $§ 799$.

Bielschowsky and Bruehl (Arch. mik. Anat., lxxi, 1908, p. 27) fix the petrous in formol of 20 per cent., decalcify it in nitric acid of 5 per cent., wash this out, and put back for a few days into the formol, cut by the freezing method, and silver by the neurofibril method ( $\$ 840$-twenty-four hours in nitrate of 4 per cent., but only a few minutes in the oxide bath).

Similarly Mullenix (Bull. Mus. Comp. Zool. Harvard Coll., liii, 1909, p. 215).

Stein (Anat. Anz., xvii, 1900, p. 398) decalcifies in celloidin by the method of Rousseau. So also KIshi (Arch. mik. Anat., lix, 1902, p. 173).

For staining, Ravvien (Traité, p. 991) employs his gold and formic acid method.

The bichromate and silver method of GoLGi may be employed with foetal or new-born subjects. The methylen blue intra vitam method 
has given good results. For the higher vertebrates the injection method should be employed. The Encycl. mik. Technik., i, p. 511, recommends injection of 1 c.c. of 0.5 to 1 per cent. solution every five minutes through the vena femoralis until the death of the animal. The cochlea then to be got out, exposed to the air for fifteen or thirty minutes, and fixed for some hours (overnight) in 10 per cent. ammonium molybdate with a little osmic acid. Then decalcified in trichloracetic acid of 5 per cent. with a trace of platinum chloride, washing for twenty-four hours and got into paraffin.

For fishes and amphibia the immersion method will suffice.

927. Other Methods.-WALDEYER, Stricker's Handb., p. 958 (decalcification either in 0.001 per cent. palladium chloride containing 10 per cent. of $\mathrm{HCl}$, or in chromic acid of $0 \cdot 25$ to 1 per cent.).

Urban Pritcirard (Journ. Roy. Mic. Soc., xii, 1872, p. 380).-Decalcification in 1 per cent. nitric acid.

LAVDowsky (Arch. mik. Anat., xiii, 1877, p. 497). - Fresh tissues (from the cochlea) are treated with 1 per cent. solution of silver nitrate, then washed for ten minutes in water containing a few drops of 0.5 or 1 per cent. osmic acid solution, and mounted in glycerin.

Max Flescir (Arch. mik. Anat., xvi, 1879, p. 300); Tafani (Arch. Ital. de Biol., vi, 1884, p. 207); EICHLER (Abh. math-phys. Cl. Sächs. Ges. Wiss, xviii, 1892, p. 311 ; Zeit. wiss. Mik., ix, 1892, p. 380 (injection of blood-vessels of the labyrinth)); SiebenMann (Die Blutgefässe im Labyrinthe des menschlichen Ohres, Wiesbaden, Bergmann, 1894; Zeit. uiss. Mik., xi, 1894, p. 386); GRAY (Journ. Anat. Phys., Xxxvii, 1903, p. 379); Sсотт (ibid., xliii, 1909, p. 329).

928. Olfactive Nerve-endings, Tactile Corpuscles, etc.-Besides the gold method, Chapter XVII, and the methylen-blue method, Chapter XVI, the rapid bichromate and silver method of GoLGI should be employed, and for the olfactive mucosa gives the best results. See van Gehuchten, La Cellule, vi, 1890, p. 405. For intra-epidermic nerve-endings, besides the methods given in Chapter XXVII, the Golar method should be employed. According to van Gehuchten ( $L a$ Cellule, ix, 1893, p. 319) it gives much better results than gold methods. He uses the rapid process. For tactile corpuscles, etc., besides the methods given in $\$ \$ 721$ and 722 , see RAMÓN Y CAJAL's neuro-fibril methods. 


\section{CHAPTER XXXVI.}

\section{METHODS FOR INVERTEBRATES.}

\section{Tunicata.}

929. Fixation of Tunicata. - A method of Lo BIANCo * for killing simple Ascidians in an extended state has been given, $\S 25$. Some forms, such as Clavellina, Perophora, Phallusia, Molgula, Cynthia, etc., should first be narcotised by treatment for from three to twelve hours with chloral hydrate ( $1: 1000$ in sea water), then killed in a mixture containing chromic acid of 1 per cent. 10 parts, acetic acid of 50 per cent. 100 parts, and finally hardened in 1 per cent. chromic acid.

The compound Ascidians with contractile zooids may be left in clean sea water till the zooids have become fully extended, then fixed by VAN BENEDEN's acetic acid process, $\S 84$ (steel instruments being avoided for manipulating them). I strongly recommend this process.

S. Lo Bravco recommends for this group the chloral hydrate process, followed by fixation with sublimate or chromo-acetic acid.

CAULlery (Bull. Sc. France Belg., xxvii, 1895, p. 5) first stupefies the animals with cocaine (LAHILLE, a few drops of 5 per cent. solution to 30 c.c. of sea water), then fixes in liquid of Flemming or acetic acid.

Most small pelagic Tunicates are very easily fixed with osmic acid or acid sublimate solution.

I have found the acetic acid process very good for Pyrosoma. Lo BIANCO puts them for a quarter of an hour into 50 per cent. alcohol containing 5 per cent. of hydrochloric acid, then into successive alcohols, beginning with 60 per cent. He kills the hard forms of Salpa with acetic acid of 10 per cent., the semi-hard ones with 1 per cent. chromic acid containing 5 per cent. acetic acid, the soft ones with 1 per cent. chromic acid containing $\frac{1}{50}$ per cent. osmic acid, or 10 parts of 1 per cent. chromic acid, with 1 of formol and 9 of sea water, Doliolidæ with sublimate, or the above osmic mixture, or a

* References to methods of Lo Bianco in this Chapter are all to his paper in Mitth. Zool. Stat. Neapel, ix, 1890, p. 435. 
mixture of 10 parts 10 per cent. solution of sulphate of copper with 1 part concentrated sublimate solution, or the formol mixture.

\section{Molluscoida.}

930. Bryozoa.-For some methods of killing and fixing see $\$ \S 13$, 20, and 21. S. Lo Branco employs for Pedicellina and Loxosoma the chloral hydrate method, fixing with sublimate. For Flustra, Cellepora, Bugula, Zoobothrium, he employs the alcohol method of EIsig, § 18. For Cristatella see $\S \S 16,20$. See also Braun.

1 Conser (Trans. Amer. Mic. Soc., xvii, 1896, p. 310) kills the fresh water forms with cocaine, puts them for an hour into 1 per cent. chromic acid, and passes through water into alcohol, etc.

Similarly Calvet (Hist. Nat. Bryozoaires, Montpellier, 1900, p. 15), for marine forms.

ZsChiesche (Zool. Jahrb., xxviii, 1909; p. 6) fixes larvæ of Alcyonidium (settled down on a layer of celloidin or paraffin) with 8 parts of sublimate and 2 of acetic acid to 90 of sea water, for twenty-five to thirty minutes.

931. Brachiopoda.-Lo Bianco kills small animals in 70 per cent. alcohol, larger ones being first narcotised with alcohol and sea water.

Blochmann (Untersuch. fein. Bau Brachiopoden, Jena, 1892, p. 5) fixes principally with sublimate, macerates by the HeRTwigs' method, § 526, decalcifies with 1 per cent. chromic acid (for thick shells add a little hydrochloric or nitric acid), or with nitric acid in alcohol of 50 to 70 per cent., and embeds in paraffin or celloidin.

See also Ekman, Zeit. wiss. Zool., lxii, 1896, p. 172.

\section{Mollusca.}

932. Fixation.-To kill Mollusca extended for dissection make up stock solution A : 90 parts absolute alcohol, 10 parts turpentine ; 10 per cent. of A to 90 per cent. water. Leave in twelve to twentyfour hours. Lo Branco narcotises Lamellibranchs for six to ten hours or more with alcohol, $\S 18$, and then kills them.

List (Fauna Flora Golf. Neapel, xxvii, 1902, p. 292) narcotises Mytilidæ with 2 per cent. of cocaine in sea water, and (for preservation of cilia) fixes in sea water, with 10 per cent. of formol.

Lo Bianco advises that Prosobranchiata, and, amongst the Heteropoda, Atlantidæ, be narcotised with 70 per cent. alcohol, $§ 18$. For Opisthobranchiata I recommend sudden killing with liquid of Perényi, or the acetic method, §929. Aplysia may first be narcotised 
by subcutaneous injection of about 1 c.c. of a 5 to 10 per cent. solution of hydrochlorate of cocaine (Robert, Bull. Scient. de la France, etc., 1890 , p. 449 ; Zeit. wiss. mik., ix, 1892, p. 216), or (Schönlein, Zeit. Biol., xxx, 1893, p. 187) 1 c.c. of 4 per cent. solution of Pelletierin. For Lo Bianco's various methods see the original, p. 467.

For Pteropoda in general, liquid of Perényi. Creseis is a difficult form. Lo Branco advises the alcohol method, § 18. For the Gymnosomata he narcotises with $0 \cdot 1$ per cent. chloral hydrate.

For terrestrial Gastropods see $\S \S 23$ and 26. MARCHI (Arch. mik. Anat., 1867, p. 204) gets rid of the mucus of the integument of Limax, which may be an obstacle to preparation, by putting the living animal into moderately concentrated salt solution, in which it throws off its mucus and dies in a few hours.

LANG (Anat. Hefte, 1902, p. 84) puts Helix into water with enough chromic acid to make it of a Rhine-wine colour, with an air-tight cover to the vessel, and when the animals are extruded injects into them a quarter to a half of a Pravaz syringe of 1 per cent. cocaine, and after five to fifteen minutes dissects and fixes.

Heymans (Bull. Acad. Belg., xxxii, 1896, p. 578) injects ethyl bromide under the skin of Cephalopoda.

Lo Bianco uses for fixing them his chromo-acetic acid, No. 1 (§39), with a double quantity of acetic acid, for twenty-four hours.

933. Liver of Mollusca.-Enriques (Mitth. Zool. Stat. Neapel., xv, 1901, p. 289) fixes the liver of Octopus and Sepia with sublimate. For Aplysia (especially in summer) alcohol, formol, and chromic mixtures are counter-indicated, on account of the carbohydrates in the cell. Sublimate is best.

934. Nervous System of Pulmonata.-B. de Nabias (Act. Soc. Linn. Bordeaux, 1894 ; Rech. Hist. centres nerveux des Gastéropodes, 1894, p. 23) opens the animals and fixes the ganglia for one hour in a mixture of 6 parts glacial acetic acid to 100 of 90 per cent. alcohol, or for fifteen to twenty minutes in 5 per cent. sublimate with 5 per cent. acetic acid. He stains in bulk, with Renaut's hæmatoxylic eosin, or R. Heidenhain's hæmatoxylin, or a copper hæmatoxylin of Viallanes, and embeds in paraffin. He also stains by the rapid method of Golar, embedding, however, the ganglia in celloidin directly after the hardening in osmic acid and bichromate, and treating the sections with the silver (p. 458). He stains with methylen blue by treating the ganglia in situ for twelve to twentyfour hours with a 1 per cent. solution. 
Dreyer (Zeit. wiss. Zool., xcvi, 1910, p. 380) narcotises Nudibranchs with cocaine, and for studying the nerves fixes them with MAYER's picro-formol, puts for a week into a mixture of $1 \mathrm{grm}$. of iron alum with 2 c.c. of formol and 40 of water, makes sections and stains with iron hæmatoxylin.

See also, for nerve-cells, McClure, Zool. Jahrb., 1898, p. 17 (MANN's methyl blue and eosin, or BENDA's safranin and Lichtgrün), and Legendre, Arch. mic. Anat., x, 1909, p. 312.

935. Eyes of Gastropoda (Flemming, Arch. mik. Anat., 1870, p. 441).-To obtain the excision of an exserted eye, make a rapid cut at the base of the peduncle, and throw the organ into very dilute chromic acid, or 4 per cent. bichromate; after a short time it will evaginate, and remain as completely erect as if alive. Harden in 1 per cent. osmic acid, in alcohol, or in bichromate.

Sмптн (Bull. Mus. Comp. Zool. Harvard, xlviii, 1906, p. 238) macerates eyes for at least two days in 9 parts of water with 1 of weak mixture of Flemming, followed by glycerin of 10 per cent. He bleaches them (in sections) with nitric acid and chlorate of potash.

936. Eyes of Cephalopoda and Heteropoda (Grenacher, $A b h$. naturf. Ges. Halle-a.-S., Bd. xvi, 1896, p. 213).-Depigment with hydrochloric acid (in preference to nitric acid). The mixture $\S 574$ may also be used. If you stain with borax-carmine and wash out in this mixture, the pigment will be found to be removed quicker than the stain is washed out.

Lenhossék (Zeit. wiss. Zool., lviii, 1894, p. 636; Arch. mik. Anat., xlvii, 1896, p. 45) applies the method of GoLGr to the eyes of Cephalopods.

Similarly Kopsch (Anat. Anz., xi, 1895, p. 362), but using formol instead of the osmic acid.

Hesse (Zeit. wiss. Zool., lxviii, 1900, p. 418) fixes eyes of Heteropoda with 1 of formol to 4 of water, and (p. 257) bleaches those of Cephalopoda by the methods of GRENACHER and that of JANDER, $\S 575$.

See also Merton, ibid., lxxix, 1905, p. 326.

93\%. Eyes of Lamellibranchiata.-See Patten, Mitth. Zool. Stat. Neapel, vi, 1886, p. 733, and RAwrtz, Jena. Zeit. Naturw., xxii, 1888 , p. 115 , and xxiv, 1890 , p. 579 (bleaches with caustic soda); see $\S 576$. Hesse (op. cit., last $\S$, p. 380 ) employs the method of JANDER for Arca. He fixes the eye of Pecten in 10 per cent. formol for five minutes, followed by sublimate or picro-nitric acid. 
938. Shell.-Sections of non-decalcified shell are easily obtained by the usual methods of grinding, or, which is often a better plan, by the methods of v. Koch or Ehrenbaum. Moseley (Quart. Journ. Mic. Sci. (2), xxv, 1885, p. 40) decalcifies with nitric acid of 3 to 4 per cent. and then makes sections. This method serves for the study of the eyes of Chitonidz.

939. Injection of Acephala (Flemming, Arch. mik. Anat., 1878, p. 252).-To kill the animals freeze them in a salt and ice mixture, and throw them for half an hour into lukewarm water. They will be found dead, and the injection-pipe may be tied in the heart, and the entire animal filled and covered up with plaster of Paris, which serves to occlude cut vessels that it is not possible to tie. As soon as the plaster has hardened the injection may be proceeded with. See also Dewrtz, Anleit. zur Anfert. zootom. Präp., Berlin, 1886, p. 44 (Anodonta) and p. 52 (Helix).

Dakin (Liverpool Mar. Biol. Comm., xvii, 1909, p. 76) narcotises by adding alcohol and glycerin for eighteen to twenty-four hours, puts for half an hour into formol of 5 per cent., and injects from a branchial vessel.

MozeJko (Zeit. wiss. Mik., xxvi, 1909, p. 353, and 1910, p. 542) puts for half an hour into water at $40^{\circ}$ to $50^{\circ} \mathrm{C}$., removes the shell, and injects carmine by auto-injection through the heart. For occluding vessels he takes cotton-wool soaked with gelatin and plaster of Paris. He takes for a vaso-dilator a saturated solution of peptonum siccum.

940. Maceration Methods for Epithelium.-Engelmann (Pflüger's Arch., xxiii, 1880 , p. 505) macerates the intestine of Cyclas in osmic acid of 0.2 per cent. (after having warmed the animal for a short time to $45^{\circ}$ to $50^{\circ} \mathrm{C}$.), or in concentrated boracic acid solution.

Cilia.-The entire intra-cellular fibre apparatus may be isolated by teasing fresh epithelium from the intestine of a Lamellibranch (e.g., Anodonta) in either bichromate of potash of 4 per cent. or salt solution of 10 per cent. To get good views of the apparatus in situ in the body of the cell, macerate for not more than an hour in concentrated solution of boracic or salicylic acid. Very dilute osmic acid (e.g., $0 \cdot 1$ per cent.) gives also good results. The "lateral cells" of the gills are best treated with strong boracic acid solution ( 5 parts cold saturated aqueous solution to 1 part water).

Dr. Orton uses borax carmine and picro-nigrosin (in litera).

Bela Haller's Mixture, see § 532; Brock's Medium, § 523; Möвrus's Media, $\$ 527$; the second of these is much recom- 
mended by Drost (Morphol. Jahrb., xii, 1866, p. 163) for Cardium and Mya.

Patten (Mitth. Zool. Stat. Neapel, vi, 1886, p. 736) takes sulphuric acid, 40 drops to 50 grms. of water. Entire molluscs, without the shell, may be kept in it for months.

Bernard (Ann. Sci. Nat., ix, 1890, p. 191) macerates the mantle of Prosobranchs in a mixture of 1 part each of glycerin and acetic acid, 2 parts each of 90 per cent. alcohol and 0.1 per cent. chromic acid and 40 parts water, which acts in from a quarter of an hour to three hours. He also (pp. 102, 306) uses a weak solution of chloride of ruthenium, especially for nerve-tracts, mucus-cells and cilia. Alcohol material may be macerated in a mixture of 1 part glycerin, 2 of acetic acid and 40 of water.

941. Mucus Glands.-Racovitza (Arch. Zool. expér. [3], ii, 1894, p. 8) studies these in Nudibranchs (and Annelids) by killing with acetic acid, staining in toto with methyl green dissolved in liquid of Ripart and Petit, and after three to six days, when only the glands show the stain, examining in mixture of equal parts of glycerin and the liquid.

\section{Arthropoda.}

942. General Methods for Arthropoda.-As general methods for the study of chitinous structures, the methods worked out by Paul Mayer (see $\$ \S 8,96$ and 97) are excellent. It is, at all events, absolutely necessary, in the preparation of entire organisms or unopened organs, that all processes of fixation, washing and staining should be done with fluids possessing great penetrating power. Hence picric acid combinations should in general be used for fixing, and alcoholic fluids for washing and staining. Concentrated picro-sulphuric acid (or picro-nitric) is the most generally useful fixative, and 70 per cent. alcohol is the most useful strength for washing out. Alcoholic picrosulphuric acid may be indicated for fixing in some cases.

But if the animals or organs can first be properly opened, the usual methods may be employed.

942A. Mounting Small Arthropods.-Dr. A. D. Imms informs me that for mounting and clearing aphids and other small insects, etc., the following formula as used by Professor Berlese for Acarina gives good results :-

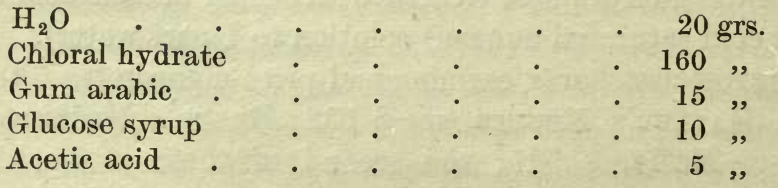


The living specimens may be placed direct on the medium on the slide, or may be killed by a short immersion in 10 per cent. acetic acid, or boiling water. If the specimens are in alcohol they should be washed in 10 per cent. acetic acid before mounting. After the cover glass is put on, gently warm the slide, then allow to cool, and leave for one or two weeks to dry and set. Ring the slide with a waterproof substance and finally ring with a layer of Canada balsam.

Personally, I often kill in 90 per cent. or absolute alcohol, leave for a few days, and mount in Euparal (see § 449).

943. Crustacea.-Some forms are very satisfactorily fixed with sublimate. Such are the Copepoda and the larvæ of Decapoda. It is sometimes indicated to use the sublimate in alcoholic solution. Some Copepoda, however (Copilia, Sapphirina), are better preserved by means of weak osmic acid, and so are the Ostracoda. In many cases the osmic acid will produce a sufficient differentiation of the tissues, so that further staining may be dispensed with; so for Copilia and Phyllosoma. The pyrogallic process ( $\$ 374$ ) may be useful. GIESBRECht takes for marine Copepods a concentrated solution of picric acid in sea water, to which a little osmic and acetic acid may be added. For fresh-water forms, Zacharias (Zool. Anz., xxii, 1899, p. 72) takes chromo-acetic acid.

Giesbrecht fixes larvæ of Stomatopoda for five to ten minutes in formol of 10 per cent. warmed to $40^{\circ}$ or $50^{\circ} \mathrm{C}$., opens them in sea water and puts for one and a half to two and a half hours into formol 1 part and sea water 5 parts, and brings into alcohol of 70 per cent. StAPPERs (La Cellule, xxv, 1909, p. 356) fixes Sympoda in GiLson's copper formol, $\S 115$, or in HoRNELL's mixture of 100 parts of 5 per cent. formol with 40 of alcohol ; and for softening the chitin puts for twelve to thirty-six hours into 3 per cent. solution of sublimate with 5 per cent. of nitric acid.

Netrovitch (Arb. z. Inst. Wien, xiii, 1900, p. 3) fixes Argulus with liquid of Tellyesniczky, $\S 52$, warmed to $50^{\circ} \mathrm{C}$.

For Fischel's intra-vitam stain of Cladocera with alizarin, etc., see $§ 207$.

944. Tracheata.-Kenyon (Tufts Coll. Stud., No. 4, 1896, p. 80) fixes Pauropoda in Carnoy's acetic alcohol and chloroform, $\S 85$, cuts them in two for staining, etc., and embeds in celloidin followed by paraffin.

Hennings (Zeit. wiss. Mik., xvii, 1900, p. 311) takes-Nitric acid 16 parts, chromic acid of 0.5 per cent. 16 parts, sublimate saturated in 60 per cent. alcohol 24 parts, picric acid saturated in water 12 , and absolute alcohol 42 , fixes for twelve to twenty-four 
hours, and washes out with iodine alcohol. He says that this mixture not only fixes, but softens chitin enough to allow of paraffin sections being made through hard parts.

Hamann (Sitz. Naturw. Freunde Berlin, 1897, p. 2) fixes small Tracheata in 10 per cent. formol and finds the chitin sufficiently soft for sections to be made.

VAn Leeuwen (Zool. Ainz., xxxii, 1907, p. 318) takes for larvæ of Hexapoda 12 parts of 1 per cent. solution of picric acid in absolute alcohol, 2 of chloroform, 2 of formol, and 1 of acetic acid.

Hollande (Arch. d'Anat. mic., xiii, 1911, p. 171) takes 12 parts of saturated solution of picric acid in formol of 40 per cent., 54 of absolute alcohol, 3 of benzene, and 1 of nitric acid, and finds that this fixes quickly enough not to make chitin too hard.

Nuttald, Cooper and Robinson (Parasitology, 1908, i, p. 163) fix for a few minutes in hot picrosulphuric acid.

945. Methods for Clearing and Softening Chitin.-The methods of Looss have been described $\S 545$, those of Hennings and HamanN last §.

Lrst (Zeit. wiss. Mik., 1886, p. 212) treats Coccidæ (after hardening) for eighteen to twenty-four hours with eau de Javelle, diluted with 4 volumes of water. After washing out they may be embedded in paraffin, and good sections obtained.

Saling (Dissert. Marburg., 1906, p. 11) boils larvæ of Tenebrio for some minutes in eau de Labarraque, the heat serving to fix the soft parts, which in successful cases are well preserved. Wash out with warm water, then alcohol.

Sazepin's method for antennæ of Chilognatha (Mém. Acad. Imp. St. Pétersb., xxxii, 1884, pp. 11, 12) consists in steeping antennæ (that have been dehydrated with alcohol) for twenty-four hours in chloroform containing a drop of fuming nitric acid (shake occasionally).

BEthe (Zool. Jahrb., viii, 1895, p. 544) puts telsons of Mysis for eight to fourteen days into 40 per cent. alcohol, to which nitric acid is gradually added, so that by the end of that time they have been brought into alcohol containing 20 per cent. of the acid. This softens the chitin, and somewhat breaks down the structure of the otolith, so that good sections through it are occasionally obtained.

Similarly Herbst, Arch. Entwickelungsmech, ix, 1899, p. 291.

See also the depigmentation processes, $\S 567$ to 576 .

945A. Double Embedding of Insects.-Dissolve 1.5 grs. desiccated celloidin chips in 50 c.c. clove oil, or, better, add the celloidin in 
an ordinary ether-alcohol solution and evaporate off the solvent in the oven overnight.

It takes many days to dissolve thoroughly, but the time may be shortened by keeping it at $90^{\circ} \mathrm{F}$. Fix objects in absolute alcohol ; bring them into clove oil, and allow this to clear the preparations, then transfer to the clove oil celloidin. The time in this must be gauged according to the size and nature of the insect; fleas, if a rupture is made in the chitinous covering, are penetrated in twentyfour hours or less. When ready to embed, dip a cover-glass in melted paraffin wax, to get a smooth surface on whicb the celloidin solution will not spread, but forms a thick drop. Place the insect on the prepared glass slip in a drop sufficient to completely cover it, and arrange in any desired position. Invert the cover-glass and float on to some chloroform; leave for half an hour or longer, according to the size of the drop. The drop of celloidin should fall away from the glass. Transfer to melted wax, and allow time for the wax thoroughly to permeate (twenty minutes is long enough for fleas and lice). The result will be a small tablet-shaped mass of spongy celloidin impregnated with wax; this can be at once embedded, or may be put away for future use. (Professor Boycott informs me that he has never left his preparations for more than two or three weeks at a time, so that he has no data as to how long the same could be kept, but they could probably be stored indefinitely.)

945B. Carbon Bisulphide Embedding of Insects, etc.-HeIdenHain many years ago recommended carbon bisulphide as a medium for embed. ding in wax. This fluid is both smelly and dangerous and should only be used in electric thermostats. The wax is dissolved in carbon bisulphide, and dehydrated insects, etc., are placed in some of the fluid which is allowed to evaporate at a gentle warmth. Subsequently the material is rapidly treated in pure wax in the thermostat. This method certainly curtails the length of time in the thermostat, and overheating is a serious matter when one is working at chitinous or brittle organisms.

946. Test for Chitin (ZANDER, Pflüger's Arch., lxvi, 1897, p. 545).Treat for a short time with a drop of freshly prepared solution of iodine in iodide of potassium and add a drop of concentrated chloride of zinc. This is then removed with water as far as possible, and the violet reaction is obtained.

See also Wester, Zool. Jahrb., Abth. Syst., xxviii, 1910, p. 531.

947. Bethe's Stain for Chitin (loc. cit., § 945).- Sections are put for three or four minutes into a freshly prepared 10 per cent. solution of anilin hydrochloride, to which has been added 1 drop of hydrochloric acid for every 10 c.c. They are then rinsed in water, and the slide is put with the sections downwards into 10 per cent. solution of bichro. 
mate of potash. The stain is at first green, but becomes blue in tap water or alcohol containing ammonia.

MAYer simply uses a solution of pyrogallol in alcohol or glycerin; and Hofmann (Zeit. uiss. Zool., Ixxxix, 1908, p. 684) puts for a day or more into raw pyroligneous acid.

Dr. ORTON writes to me that he simply uses picro-nigrosin and borax carmine.

948. Tracheæ may be studied by the Golgi bichromate and silver process. Martin (C. R. Soc. Philomath., 1893, p. 3) injects them with indigo white (through the body cavity), and puts into hot water from which the air has been expelled by boiling. Tracheæ blue.

949. Brain of Bees.-Kenyon (Journ. Comp. Neurol., vi, 1896, p. 137 ; Journ. Roy. Mic. Soc., 1897, p. 80) treats by the GolgI process (seldom successful), or hardens in a mixture of 1 part formol and 2 of 5 per cent. sulphate of copper, followed by staining in Mallory's phospho-molybdic hæmatoxylin.

JoNEscu (Jena. Zeit., xlv, 1909, p. 111) has employed the silver methods of Ramón y Cajal and Bielschowsky and Wolff.

950. Ventral Cord.-Floyd (Mark. Anniv. vol., 1904, p. 355) fixes the ganglia of Periplaneta for eighty minutes with vapour of formol, and brings into alcohol.

See also Binet, Journ. Anat, Phys., xxx, 1894, p. 469.

951. Eyes of Arthropods.-For the methods of LANKESTER and Bourne (Quart.Journ. Mic. Sci., 1883, p. 180 : Limulus) ; Hrckson (ibid., 1885, p. 243 : Musca) ; PARker (Bull. Mus. Harvard Coll., xx, 1890, p. 1 ; Zeit. wiss. Mik., viii, 1891, p. 82 : Homarus) see early editions.

Parker (Mitth. Zool. Stat. Neapel, xii, 1895, p. 1) also applies the methylen blue method to the retina and optic ganglia in Decapods, especially in Astacus. He injects 0.1 c.c. of a 0.2 per cent. solution into the ventral sinus. After twelve to fifteen hours the animals are killed, the ganglia quickly dissected out, and the stain fixed as described, $\S 343$.

For his method for eyes of Scorpions see $\S 575$.

For the methods of Purcell for the eyes of Phalangida see Zeit. wiss. Zool., lviii, 1894, p. 1 . He has the following stain. The cephalothorax is removed and brought for twenty minutes into 50 per cent. alcohol warmed to $45^{\circ}$ or $50^{\circ} \mathrm{C}$., and saturated with picric acid. The pigment dissolves in this solution and stains the nuclei and some other parts of the rhabdoms, so that no further - stain is required. 
Hennings (Zeit. wiss. Mik., xvii, 1900, p. 326) depigments sections by putting them for ten minutes (Musca) to twelve hours (Myriopoda) into a mixture of 2 parts of 80 per cent. alcohol with 1 of glycerin and 2 per cent. of nitric acid, best kept at $35^{\circ} \mathrm{C}$. The elements are well preserved.

Widmann (Zeit. wiss. Zool., xc, 1908, p. 260) makes the lens of Arachnida fit for sectioning by putting for a day or so into alcohol with 10 to 15 per cent. of nitric acid; and bleaches sections with 1 part of chlorine water to 2 of alcohol.

See also Rosenstadt, Arch. mik. Anat., xlvii, 1896, p. 478; Viallanes, Ann. Sci. Nat., xiii, 1892, p. 354 ; and Dietrich, Zeit. wiss. Zool., xcii, 1909, p. 465 (fixes in alcoholic formol, and bleaches with dilute aqua regia).

952. Injections (Arachnida and Crustacea especially).-Armé Schneider (Tablettes Zool., ii, 1892, p. 123) recommends lithographic Indian ink, the animals being narcotised with chloroform, then injected and thrown into strong alcohol. Similarly CAUSARD (Bull. Sc. France Belg., xxix, 1896, p. 16).

953. Arctiscoida (Doy k̀re, Arch. mik. Anat., 1865, p. 105).-Examination of living animals after partial asphyxiation in boiled water. See early editions.

\section{Vermes.}

954. Chætopoda: Cleansing Intestine.-KüKentHaL (Journ . Roy. Mic. Soc., 1888, p. 1044) puts Lumbricus into a glass vessel filled with bits of moistened blotting-paper. They gradually evacuate the earthy particles from the gut, and fill it instead with paper.

Vogt and Yung (Traité d'Anat. Comp. Prat., v) recommend coffee-grounds instead of paper, as they cut better after embedding.

JoEST (Arch. Entwicklungsmech., v, 1897, p. 425) simply keeps the worms for a few days in moist linen, and finds the gut empty.

Pearl (Journ. appl. Mic., iii, 1901, p. 680) injects alcohol of 6 per cent. through the gut of narcotised worms.

955. Chætopoda : Fixation.-Lumbricus may be anæsthetised by putting the animals into water with a few drops of chloroform. Perrier puts them into water in a shallow dish, sets up a watch-glass with chloroform in the corner of it, and covers the whole.

Cerfontaine (Arch. de Biol., x, 1890, p. 327) injects interstitially about 2 c.c. of a 1 : 500 solution of curare. 
JAQUET (Bib. Anat., iii, 1895, p. 32) kills Lumbricus in extension in 1 part of nitric acid to 125 of water.

Collin (Zeit. wiss. Zool., xlvi, 1888, p. 474) puts Criodrilus lacuum into a closed vessel.with a little water, and hangs up in it a strip of blotting-paper soaked in chloroform. KüKenTHAL (Die mik. Technik, 1885 ; Zeit. wiss. Mik., 1886, p. 61) puts Annelids into a glass cylinder filled with water to the height of $10 \mathrm{~cm}$., and then pours 70 per cent. alcohol to a depth of 1 to $2 \mathrm{~cm}$. on to the water. For Opheliadæ he also employs $0 \cdot 1$ per cent. of chloral hydrate in sea water.

Many marine Chætopoda may be successfully narcotised (Lo Bianco) in sea water containing 5 per cent. of alcohol, or by means of the mixture, $\S 18$.

The Polychceta sedentaria may sometimes be satisfactorily fixed by bringing them rapidly into corrosive sublimate. Cold, not hot, solutions should be taken, as heat frequently shrivels up the branchiæ. Eunice and Onuphis may be treated in the same way.

Lo Branco advises killing Chætopteridæ, Sternaspidæ, Spirographis, Protula, by putting them for half an hour into 1 per cent. chromic acid. Some of the sedentaria may be got protruded from their tubes by leaving them for some hours in $0 \cdot 1$ per cent. chloral hydrate in sea water.

For Ersig's methods for Capitellidæ see Fauna u. Flora Golf. Neapel, xvi, 1887, p. 295.

See also $§ 14$ (lemon juice), and the methods $\S \S 20$ to 26,39 and 49.

956. Blood-vessels of Annelids (KüKenthal, Zeit. wiss. Mik., 1886, p. 61). - The animals should be laid open and put for two or three hours into aqua regia (4 parts of nitric acid to 2 of hydrochloric acid). Vessels black, on a yellow ground.

Bergh (Anat. Hefte, xlv, 1900, p. 392, and xlix, 1900, p. 599) puts small Annelids for a week or more into equal parts of 1 per cent. ritric acid and 1 per cent. nitrate of silver, or into 50 parts of nitrate, 25 of formic acid, and 25 of water, dissects out the organs and exposes to light. Marine forms may be treated by HaRMER's process.

95\%. Nerves of Annelids. - Note the methylen blue method and the bichromate of silver method of Golgi (the rapid method). For the latter see v. LenHossék (Arch. mik. Anat., xxxix, p. 102).

LANGDON (Journ. Comp. Neur., x, 1900, p. 4) injects strong solution of methylen blue into the body cavity of Nereis, and puts the animal for some hours into sea water in the dark, fixes the stain by Bethe's method, and makes paraffin sections. 
See also M. Lewis, Anat. Anz., xii, 1896, p. 292 ; Atheson, ibid., xvi, 1899, p. 497 ; and the methods of Apatry $\S \S 342,368,371$, and 834.

958. Hirudinea.-For the methods of killing see those given for Lumbricus in $\S 955$, also $\S \S 20$ to 26 , and 49.

Whitman (Meth. in mic. Anat., p. 27) recommends that they be killed with sublimate.

I have obtained better results myself by narcotising with carbonic acid (§ 26), and fixing. with liquid of Flemming. I have also found that lemon juice kills them in a state of very fair extension.

AрÁtнy succeeds with alcohol of 40 per cent.

GraF (Jen. Zeit., 1893, p. 165) has obtained good results by narcotising with a decoction of tobacco.

959. Injection.-Whitman (Amer. Natural., 1886, p. 318) states that very perfect natural injections may often be obtained from leeches that have been hardened in weak chromic acid or other chromic liquid.

JACQUET (Mitth. Zool. Stat. Neapel, 1885, p. 298), for artificial injections, puts leeches into water with a very small quantity of chloroform, and allows them to remain a day or two in the water before injecting them.

960. Nervous System.- Impregnation with gold. BRISTOL (Journ. of Morph., xv, 1898, p. 17) kills in formic acid of 15 to 20 per cent., puts for twenty-five minutes into 1 per cent. gold chloride, reduces in formic acid of 1 per cent. (twelve to eighteen hours), and embeds in paraffin. See also $\S \S 342,368,371$ and 834 .

961. Nephridia.-Shearer (Quart. Journ. Micr. Sci., lv, 1910, p. 288) stains Histriobdella intra vitam with a very weak solution of Methyl blue, which allows the course of the nephridia to be made out.

962. Gephyrea.-Vogt and Yung (Anat. Comp. Prat., p. 373) direct that Siphunculus nudus be kept for some days in perfectly clean basins of sea water, changed every day, in order that the intestine of the animals may be got free from sand, and then anæsthetised with chloroform.

Ward (Bull. Mus. Comp. Zool., Cambridge, Harvard Coll., xxi, 3, p. 144) puts them into a shallow dish with sea water and pours 5 per cent. alcohol in a thin film on to the surface of the water, and as soon as they make no contractions on being stimulated removes to 50 per cent. alcohol. 
Lo Branco says killing with 0.5 per cent. chromic acid or with 0.1 per cent. chloral hydrate in sea water may be tried. Phascolosoma and Phoronis should be treated by the alcohol method, larvæ of Sipunculus with cocaine, $\S 21$.

Apel (Zeit. wiss. Zool., xlii, 1885, p. 461) puts Priapulus and Halicryptus into a vessel with sea water and heats on a water bath to $40^{\circ} \mathrm{C}$. ; or they may be thrown into boiling water, which paralyses them so that they can be quickly cut open and thrown into $\frac{1}{3}$ per cent. chromic acid or picro-sulphuric acid.

963. Rotatoria.-For quieting them for study in the living state, Weber (Arch. de Biol., viii, 4, 1888, p. 713) finds that 2 per cent. solution of hydrochlorate of cocaine gives the best results. Warm water gave him good results for large species, such as those of Hydatina and Brachionus.

HaRdy (Journ. Roy. Mic. Soc., 1889, p. 475) recommends thick syrup added drop by drop to the water. Hudson (ibid., p. 476) mentions weak solution of salicylic acid.

Volk (Jahrb. Hamburg. wiss. Anst., xviii, 1901, p. 164) quiets them in quince mucilage, $40 \mathrm{grm}$. of the seeds to 1 litre of water. Cf. $\S 1018$.

HirschFelder (Zeit. wiss. Zool., xcvi, 1910, p. 211) studies them living in neutral red of $1: 50,000$.

See also $\S \S 23,24$ and 27. Methylene blue, $\S 339$, may be found useful.

Permanent preparations may be made by the method of Rousselet (Journ. Quekett Mic. Club, v, March, 1895, p. 1): The animals are got together in a watch glass and are narcotised by adding to the water at intervals a few drops of the following mixture :

Hydrochlorate of cocaine 2 per cent. solution

Methylated spirit

Water

As soon as the cilia have ceased to beat, or are seen to be on the point of ceasing to beat, they are fixed by adding a drop of liquid of Flemming or of $\frac{1}{4}$ per cent. osmic acid. After half a minute or less the animals are taken out with a pipette, and thoroughly washed by passing them through two or three watch glasses of distilled water. They are then definitely mounted in a mixture of formol $2 \frac{1}{2}$ parts, distilled water $37 \frac{1}{2}$ parts.

Zograf (Comptes Rend., cxxiv, 1897, p. 245) narcotises as RousseLET, but without the spirit, fixes with osmic acid for two to four 
minutes, then replaces this by raw pyroligneous acid diluted with 8 to 10 volumes of water, and after five to ten minutes washes in several changes of water, and passes through successive alcohols into glycerin or balsam.

Lenssen (La Cellule, xiv, 1898, p. 428) for the embryology of Hydatina, kills with hot saturated sublimate, dehydrates, stains lightly, embeds in paraffin and stains with hæmalum.

HIRSCHFELDER (op. cit., supra) narcotises with cocaine, and fixes with Fol's picro-chromic acid.

Beauchamp (Arch. Zool. Expér., iv, 1906, p. 29) finds 1 per cent. stovaïne better than cocaine for some forms. He (ibid., x, 1909, p. 77) fixes for five to ten minutes in 4 parts of 1 per cent. osmic acid, with 1 of 6 per cent. sublimate, and 5 of 5 per cent. bichromate of potash, and 1 drop of acetic acid for each 2 c.c., and embeds in celloidin, and then through chloroform in paraffin (three to ten minutes).

See also Tozer (Journ. Roy. Micr. Soc., 1909, p. 24).

964. Acanthocephali. - SAEFFTigen (Morph. Jahrb., x, 1884, p. 120) obtained the best results by killing gradually with $0 \cdot 1$ per cent. osmic acid; the animals placed in this contract during the first hours, but stretch out again and die fully extended. Similarly with $0 \cdot 1$ per cent. chromic acid; Echinorhynci live for days in it, but eventually die fully extended.

Hamann (Jen. Zeit., xxv, 1890, p. 113) has succeeded with sublimate, and also with alcohol containing a little platinum chloride.

KAISER (Biblioth. Zool., H. vii, 1 Hälfte, 1891, p. 3) found that a saturated aqueous solution of cyanide of mercury, warmed to $45^{\circ}$ to $50^{\circ} \mathrm{C}$., and allowed to act for from fifteen to sixty minutes, and then washed out with 70 per cent. alcohol, was the best of all fixing media.

965. Nematodes. - The impermeable cuticle is a great obstacle to preparation. According to Looss (Zool. Anz., 1885, p. 318) this difficulty may be overcome in the manner described in $\$ 545$.

Wash in 1 per cent. saline (if necessary) and fix in boiling 70 per cent. alcohol; store in fresh 70 per cent. for examination. If this method is properly applied the worms will die extended and straight. For examination transfer to 70 per cent. alcohol made up with 5 per cent. glycerine. Place small bottle of this fluid, plus worms, on incubator at $60^{\circ} \mathrm{C}$, and allow to evaporate slowly for about twentyfour hours or even two days, which finally leaves the worms in viscid, almost pure, glycerine. Examine in pure glycerine, or glycerine 
jelly. For rapid examination after killing in alcohol, transfer to absolute alcohol for thirty minutes, and clear in " white " creosote. (LEIPER, in Science of the Sea, London. John Murray. 1912.)

For fixing, most recent authors recommend sublimate solutions ; chromic solutions seem to have a tendency to make the worms brittle.

But, according to Zur Strassen (Zeit. wiss. Zool., liv, p. 655), Bradynema rigidum ought to be fixed for at least twelve hours in mixture of Flemming.

Augstein (Arch. Naturg., lx, 1894, p. 255) takes for Strongylus filaria Mayer's picro-nitric acid.

Vejdovsky (Zeit. wiss. Zool., lvii, 1894, p. 645) advises for Gordius 0.5 per cent. chromic acid (twenty-four hours).

Lo Bianco employs for marine forms concentrated sublimate or picro-sulphuric acid.

Looss (Zool. Anz., xxiv, 1901, p. 309) prefers hot $\left(80^{\circ}\right.$ to $90^{\circ}$ C.) alcohol of 70 per cent.

Glaue (Zeit. wiss. Zool., xcv, 1910, p. 554) kills Ascaris in a hot mixture of 100 parts of saturated sublimate, 100 of alcohol, and 1 of acetic acid.

- Staining is frequently difficult, and sometimes alcoholic carmine $\$ 234 \mathrm{~A}$, is the only thing that will give fair results.

Braun (see Journ. Roy. Mic. Soc., 1885, p. 897) recommends that small unstained Nematodes be mounted in a mixture of 20 parts gelatin, 100 parts glycerin, 120 parts water, and 2 parts carbolic acid, which is melted at the moment of using. Canada balsam, curiously enough, is said to sometimes make Nematodes opaque.

Demonstration of living Trichino.-BARNES (Amer. Mon. mik. Journ., xiv, 1893, p. 104) digests trichinised muscle (of the size of a pea) in a mixture of $3 \mathrm{gr}$. of pepsin, $2 \mathrm{dr}$. of water, and 2 minims of hydrochloric acid, kept at body temperature for about three hours. The flesh and cysts being dissolved, the fluid is poured into a conical glass, and allowed to settle; the trichinæ are drawn off from the bottom with a pipette, got on to a slide with water and examined on a hot stage.

Graham (Arch. mik. Anat., 1, 1897, p. 216) isolates Trichinæ by macerating for one or two days in 2 per cent. acetic acid, staining with aceto-carmine, and teasing.

966. Nemertina.-My best results have always been obtained by fixing with cold saturated sublimate solution, acidified with acetic acid. The other usual fixing agents, such as the osmic and chromic mixtures, seem to act as irritants, and provoke such violent muscular contractions that the whole of the tissues are crushed out of shape by them. 
Prof. DU Plessis has suggested to me fixing with hot (almost boiling) water. I have tried it and found the animals die in extension, without vomiting their proboscides. So also Joubin, Bull. Mus. Hist. Nat., 1905, p. 326.

I have tried FoetTingen's chloral hydrate method (\$ 20). My specimens died fairly extended, but vomited their proboscides. According to Lo Branco narcotisation with a solution of $0 \cdot 1$ to $0 \cdot 2$ per cent. in sea water for six to twelve hours is useful.

Oestergren ( $\$ 18$ ) recommends his ether water.

Dendy (see Journ. Roy. Mic. Soc., 1893, p. 116) has succeeded with Geonemertes by exposing it for half a minute to the vapour of chloroform.

For staining fixed specimens in toto I have found that it is well-nigh necessary to employ alcoholic stains. Borax-carmine or Mayer's alcoholic carmine may be recommended; not so cochineal or hæmatoxylin stains; on account of the energy with which they are held by the mucin in the skin.

Sections by the paraffin method, after penetration with oil of cedar (chloroform will fail to penetrate sometimes after a lapse of weeks).

Bürger (Fauna u. Flora Golf. Neapel, xxii, 1895, p. 443) studies the nervous system, nephridia, skin, muscle and intestine by the intra vitam methylen-blue method. He injects the animals with 0.5 per cent. solution in distilled water, or 0.5 per cent. salt water, and allows them to lie for six to twelve hours or more in moist blotting-paper.

See also Montgomery (Zool. Jahrb., Abth. Morph., x, 1897, p. 6) ; and Böнмig (Zeit. wiss. Zool., lxiv, 1898, p. 484).

96\%. Cestodes. - Wash gently in 1 per cent. saline, and then fix in hot corrosive sublimate acetic (at circa $50^{\circ} \mathrm{C}$.) and allow the tapeworms to remain in the dish till the fluid becomes cold. Wash in running water for twelve hours and transfer to 70 per cent. alcohol. Stain as in general methods.

As pointed out by Vogt and Yung (Traité d'Anat. Comp. Prat., p. 204), the observation of the living animal may be of service, especially in the study of the excretory system. And, as shown by PINTNER, Tæniæ may be preserved alive for several days in common water to which a little white of egg has been added.

Tower (Zool. Jahrb.; xiii, 1899, p. 363) has kept Moniezia expansa alive for several days in a mixture of 100 c.c. of tap water, 10 grs. of white of egg, 2 of pepsin, 2 of sugar, and 5 of prepared beef ("Bovox"). Chloride of sodium, he says, should be avoided. 
LönnberG (Centralb. Bakteriol., xi, 1892, p. 89 ; Journ. Roy. Mic. Soc., 1892, p. 281) has kept Trianophorus nodulosus alive for a month in a slightly acid pepsin-peptone solution containing from 3 to 4 per cent. of nutritive matter and less than 1 per cent. of $\mathrm{NaCl}$.

For the nervous system, Tower (Zool. Anz., xix, 1896, p. 323) fixes in a picro-platin-osmic mixture (stronger than that of 0 . voM RATH, $§ 101)$ for ten hours, then treats for several hours with crude pyroligneous acid, and lastly with alcohol, and embeds in paraffin.

Zernecke (Zool. Jahrb., Abth. Anat., ix, 1895, p. 92) kills Ligula in the osmio-bichromic mixture of GoLGI $(4: 1)$, impregnates as usual, makes sections in liver, and treats them by the hydroquinon process of KaLlius. Besides the peripheral and central nervous system, muscle-fibres, parenchyma cells, and the excretory vascular system are impregnated.

He has also obtained good results by the methylen-blue method.

Blochmann (Biol. Centralb., xv, 1895, p. 14) recommends the bichromate and sublimate method of GoLGI.

See also KöHLER, Zeit. wiss. Zool., lvii, 1894, p. 386 (stretches Tæniæ round a glass plate or on cork, and fixes with 5 per cent. sublimate); LüHe, Centralb. Bakt., xxx, 1901, p. 166, and RANsom, U. S. Nation, Mus. Bull., lxix, 1909, p. 8.

968. Trematodes.--If necessary, clean by shaking up in 1 per cent. saline (parasites). Decant off dirty liquid, one-third of the tube is filled again with 1 per cent. saline, in which the worms are shaken vigorously, and an equal quantity of $\mathrm{HgCl}_{2}$ solution is added quickly, the vigorous shaking being continued for several minutes thereafter. This treatment should kill the flukes in an extended condition. Leave in the fixer as indicated (corrosive one or two days, wash in water twelve hours if 10 per cent. formalin be substituted for the $\mathrm{HgCl}_{2}$; leave about same time and store in 3 per cent. formalin).

Fischer (Zeit. wiss. Zool., 1884, p. 1).-Opisthotrema cochleare may be mounted entire in balsam. For sectioning, he recommends a mass made by dissolving 15 parts of soap in 17.5 parts of 96 per cent. alcohol. The sections should be studied in glycerin.

Lo Bianco fixes Trematodes with hot saturated sublimate.

Looss (Arch. mik. Anat., 1895, p. 7) takes for Bilharzia warm $\left(50^{\circ}\right.$ to $60^{\circ} \mathrm{C}$.) 1 per cent. sublimate in 70 per cent. alcohol.

BetTendorf (Zool. Jahrb., Abth. Morph., x, 1897, p. 308) has had good results with the rapid Golgi method only on Distoma hepaticum, and prefers methylen blue. 
Havet (La Cellule, xvii, 1900, p. 353) has also had results with the Golgi method on this form, and also with thionin (after fixing with sublimate), which demonstrates tigroid substance.

Cercarice.-Schwarze (Zeit. wiss. Zool., xliii, 1886, p. 45) found that the only fixing agent that would preserve the histological detail of these forms was cold saturated sublimate solution warmed to $35^{\circ}$ to $40^{\circ} \mathrm{C}$.

For an "indifferent" liquid, HoFmann (Zool. Jahrb., xii, 1899, p. 176) takes 1 part of white of egg in 9 of normal salt solution.

969. Turbellaria.-Braun (Zeit. wiss. Mik., iii, 1886, p. 398) gets entire animals (Rhabdocœla) on to a slide, lightly flattens out with a cover, and kills by running in a mixture of 3 parts of liquid of Lang with 1 of 1 per cent. osmic acid solution. (BöHмrg [ibid.], commenting on this, says that for some of the tissues, such as muscle and body parenchyma, nitric acid and picro-sulphuric acid are very useful.) Sections may be made by the paraffin method.

Delage (Arch. de Zool. exp., iv, 2, 1886) recommends fixation (of Rhabdocœla Acœla) by an osmium-carmine mixture, for which see loc. cit., or by concentrated solution of sulphate of iron. Liquid of Lang was not successful.

For staining, he recommends either the osmium-carmine or impregnation with gold ( $\frac{1}{3}$ formic acid, two minutes ; 1 per cent. gold chloride, ten minutes ; 2 per cent. formic acid, two or three days in the dark).

BöнміG (Zeit. wiss. Mik., iii, 1886, p. 239) has obtained instructive images with Plagiostomidæ fixed with sublimate and stained with the osmium-earmine.

Graff (Turbellaria Accela, Leipzig, 1891 ; Zeit. wiss. Mik., ix, 1892, p. 76) says that chromo-aceto-osmic acid, followed by hæmatoxylin, is good for the skin, but not for the Rhabdites, which in Acœla and Alloiocœla seem to be destroyed by swelling. The same method is also good for the parenchyma of Amphichorus cinereus, Convoluta paradoxa and C. sordida. Sublimate is good for Convoluta Roscoffensis. The nervous system may be investigated by the methods of DELAGE.

For Dendrocœla sublimate solutions, sometimes hot, appear indicated for fixing ; see the mixture of LANG, $\S 64$, also CHICHкoFF (Arch. de Biol., xii, 1892, p. 438).

Arnold (Arch. Zellforsch., iii, 1909, p. 433) kills Dendrocolum in extension (?) with strong liquid of Flemming.

Oestergren narcotises Dendrocolum with his ether-water, $\$ 18$. JAENICHEN (Zeit. wiss. Zool., lxii, 1896, p. 256) advises for Planaria, eyes especially, picro-sulphuric acid for an hour or two; 
osmic acid is not good, and liquid of Müller macerates. He stains with borax-carmine, makes sections, and puts them for ten minutes into osmic acid, then for five minutes into pyroligneous acid, on the top of the stove. He macerates the visual rods in a mixture of 1 part common salt, 1 of acetic acid, and 100 of water. He bleaches the pigment of the eyes with peroxide of hydrogen.

Wilhelmi (ibid., lxxx, 1906, p. 548) throws Triclads into almost boiling mixture of Zenker, and after ten to thirty minutes removes to water for some hours, and then passes into iodine alcohol.

\section{Echinodermata.}

9\%0. Holothurioidea.-These are difficult to fix on account of their contracting with such violence under the influence of irritating reagents as to expel their viscera through the oral or cloacal aperture.

Vogt and Yung (Anat. Comp. Prat., p. 641) say that Cucumaria Planci (C. dotiolum, Marenzeller) is free from this vice; but they recommend that it be killed with fresh water, or by slow intoxication, $\S 25$.

Synapta may be allowed to die in a mixture of equal parts of sea water and ether or chloroform (S. Lo Bianco).

Oestergren ( $\$ 18$ ) puts Synapta into his ether water, but Dendrochirota first into magnesium sulphate of 1 to 2 per cent., for some hours.

Gerould (Bull. Mus. Harvard Coll., xxix, 1896, p. 125) paralyses Caudina with sulphate of magnesia, $\S 24$, and fixes with liquid of Perenyi (or sublimate for the ovaries).

Holothurids, Dr. WEBER informs me, are admirably preserved in formaldehyde; a weak solution is sufficient.

For the staining of muscles with methylen blue, see Iwanzofr, Arch. mik. Anat., xlix, 1897, p. 103; and for the study of calcareous plates, see Woodland, Quart. Journ. Micr. Sci., xlix, 1906, p. 534 (fixation with osmic acid, staining with picro-carmine, followed by Lichtgrün).

971. Echinoidea.-I advise that they be killed by injection of some fixing liquid. For preservation, formaldehyde has proved admirable in all respects, and greatly superior to alcohol (WEBER).

Lo BIANco kills by pouring over them (mouth upwards) a mixture of 10 parts acetic acid and 1 of 1 per cent. chromic acid, and brings at once into weak alcohol. Or he makes two holes in the shell, lets the water run out and alcohol run in.

Sections of spines may be made by grinding, see $§ 177$. 
Spicula and the skeleton of pedicellariæ may be cleaned by eau de Javelle, see Döderlein (Wiss. Ergeb. Tiefsee-Exped,, v, 1906, p. 67).

972. Asteroidea.-Hamans (Beitr. Hist. Echinodermen, ii, 1885, p. 2) injects the living animal with a fixing liquid through the tip of a ray. The ambulacral feet and the branchiæ are soon distended by the fluid, and the animal is then thrown into a quantity of the same reagent.

In order to study the eyes, with the pigment preserved in situ, they should be removed by dissection, should be hardened in a mixture of equal parts of 1 per cent. osmic acid and 1 per cent. acetic acid, and sectioned in a glycerin gum mass, or some other mass that does not necessitate treatment with alcohol (which dissolves out the pigment, leaving the pigmented cells perfectly hyaline). For maceration use one-third alcohol, the aceto-osmic mixture failing to preserve the rods of the pigmented cells.

Specimens for externals only preserve in 70 per cent. alcohol or formalin. They retain their shape better if they are put for two or three minutes into fresh water before being placed in the fixer. If the internal anatomy is to be studied, cut along the length of each arm so as to allow fluid to enter, and preserve in 2 per cent. chromic acid, etc. Wash in running water, transfer to 70 per cent. alcohol ; or the specimen may be preserved in formalin spirit or 5 per cent. formalin.

Formaldehyde is not to be recommended for the cell preservation of Ásteroidea (Weber). See also Lo Branco, op. cit. (he kills Brisinga with absolute alcohol), also $\S \S 17,20$.

973. Ophiuridea should in general be killed in fresh water if it be desired to avoid rupture of the rays (De Castellarnau, $L a$ Est. Zool. du Napoles, p. 135).

Lo BiAnco kills small forms with weak alcohol, Ophiopsila with absolute alcohol, and Ophiomyxa with 0.5 per cent. chromic acid.

Russo (Richerche Lab. Anat. Roma, iv, 1895, p. 157) fixes Ophiothrix for an hour or two in 0.5 per cent. osmic acid and then decalcifies in solution of Müller for six to ten days. Or he fixes for three minutes in a mixture of 2 parts concentrated sublimate solution, 1 part 70 per cent. alcohol, and 1 part acetic acid (sp. gr. 1.06), and decalcifies in Müller or in 70 per cent. alcohol with 10 per cent. of acetic acid. He stains with paracarmine.

974. Crinoidea.-Lo Bianco (loc. cit., p. 458) fixes Antedon rosacea with 70 per cent. alcohol, $A$. phalangium with 90 per cent. 
9\%5. Larvæ of Echinodermata (from instructions written down for me by Dr. BARRoIs).-For the study of the metamorphoses of the Echinoidea and Ophiuridea it is necessary to obtain preparations that show, the calcareous skeleton preserved intact (a point of considerable importance, since this skeleton frequently affords landmarks of the greatest value), and that give clear views of the region of formation of the young Echinoderm (which is generally opaque in the living larva). They should also possess sufficient stiffness to allow of the larva being turned about in any desired way, and placed in any position under the microscope.

Pluteus larvæ should be fixed in a cold saturated solution of corrosive sublimate, for not more than two or three minutes, then washed with water, and brought into dilute Mayer's cochineal $(\S 235)$. This should be so dilute as to possess a barely perceptible tinge of colour. They should remain in it for from twelve to twentyfour hours, being carefully watched the while, and removed from it at the right moment and mounted in balsam, or, which is frequently better, in oil of cloves or cedar-wood.

Auricularia and Bipinnaria.-As above, but the earlier stages of the metamorphosis of Auricularia are better studied by fixing with osmic acid, staining with Beale's carmine, and mounting in glycerin.

Larvae of Comatula are best fixed with liquid of Lang, and stained with dilute borax-carmine. It is important (for preparations that are not destined to be sectioned) to use only dilute borax-carmine, as the strong solution produces an over-stain that cannot easily be reduced.

Narcotisation by chloral hydrate before fixing is useful, especially for the study of Pentacrinus larvæ and of the young Synapto formed from Auricularia. Without this precaution you generally get preparations of larvæ either shut up (Pentacrinus), or entirely deformed by contraction (young Synaptoe).

See also MACBRIDE on the development of Amphiura squamata, Quart. Journ. Micr. Sci., xxxiv, 1892, p. 131 (osmic acid followed by liquid of Müller and alcohol ; decalcification with nitric acid in aloohol ; staining with Mayer's paracarmine or hæmalum); and SEELIGER on the development of Antedon, Zool. Jahrb., Abth. Anat., vi, 1892, p. 161.

MacBride (Quart. Journ. Micr. Sci., xxxviii, 1896, p. 340) fixes larvæ of Asterina in osmic acid, brings into liquid of Müller for twelve to fourteen hours, imbeds in celloidin followed by paraffin (see $\S 171$ ), and stains sections with carmalum or Delafield's hæmatoxylin, best after a foregoing stain of twenty-four hours in borax carmine.

Mayer (Grundzüge, Lee and Mayer, 1910, p. 486) arranges a number of fixed and stained Plutei on a sheet of gelatin foil gummed 
to a slide with euparal, dehydrates by adding alcohol by drops, and adds euparal and a cover. See also Woodland, Quart. Journ. Micr. Sci., xlix, 1905, p. 307.

\section{Coelenterata.}

976. Thread-Cells.-Iwanzoff (Bull. Soc. Nat. Moscou, x, 1896, p. 97) advises for the Nematocysts of Actiniæ maceration by HeRTwIGS' method, $\S 534$, or better, fixation for two to five minutes with vapour of osmium followed by a short washing with sea water or distilled water.

For Medusæ he also advises Hentwigs' method, § 526, or treatment with a solution containing methyl green and gentian violet with a little osmic acid.

97\%. LitTle (Journ. App. Mic., vi, 1903, p. 2116 ; Journ. Roy. Mic. Soc., 1903, p. 237) kills Hydra in hot saturated sublimate in 70 per cent. alcohol, washes with alcohol, stains for five minutes in strong solution of methylen blue, dehydrates rapidly, clears with cedar or bergamot oil, and mounts in balsam. Nematocysts blue, the rest unstained.

9\%8. Actinida.-Anæsthetise in menthol (§ 15), which will take some twelve hours or more. For ordinary sea anemones, formalin ( 5 to 10 per cent.) followed by formalin spirit is to be preferred. For corals, such as Caryophyllia, Alcyonium or Gorgonia, anæsthetise, and then add hot corrosive sublimate or 5 per cent. formalin, followed by cold saturated corrosive sublimate. Ninety per cent. spirit, not allowed to get weaker than 70 per cent., gives good results for anatomical work (ALLEN and Browne, loc. cit.).

For other narcotisation methods see $\S 15$ to 26.

979. Fixation.-In Le Attinie, Fauna u. Flora d. Golfes v. Neapel, ANDREs says that hot corrosive sublimate often gives good results. In the case of the larger forms the solution should be injected into the gastric cavity.

Freezing sometimes gives good results. A vessel containing Actiniæ is put into a recipient containing an ice and salt freezing mixture and surrounded by cotton-wool. After freezing, the block of ice containing the animals is thawed in alcohol or some other fixing liquid.

DUERDEN (Journ. Inst. Jamaica, ii, 1898, p. 449) narcotises with magnesium sulphate, $\S 24$, and fixes with formol of 3 to 5 per cent.

980. Maceration.-For the Hertwigs' method (Jen. Zeit., 1879, p. 457) see $\$ 526$. The tissues should be left to macerate in the acetic acid for at least a day, and may then be teased in glycerin. 
List (Zeit. wiss. Mik., iv, 1887, p. 211) treats tentacles of Anthea cereus and Sagartia parasitica for ten minutes with a mixture of 100 c.c. of sea water with 30 c.c. of Flemming's strong liquid, then washes out for two or three hours in 0.2 per cent. acetic acid, and teases in dilute glycerin. Picro-carmine may be used for staining.

981. Nervous system.-This group is generally held to be refractory to the Golgi impregnation. Haver, however (La Cellule, xviii, 1901, p. 388), has obtained good results by the rapid method on young specimens of Metridium dianthus. Besides nerve-cells, there are impregnated neuro-muscular cells, gland-cells, and nematocysts. He leaves for five to eight days in the osmic mixture. He has also had good results by the intra vitam methylen blue method (this is also good for nematocysts). So also has Groselu (Arb. Zool. Inst. Univ. Wien, xvii, 1909, p. 269), adding the dye to the water with the animals till it gives a steel-blue tint.

982. Zoantharia with Calcareous Skeletơns are difficult to deal with on account of the great contractility of the polyps. Sublimate solution, which ought very often to be taken boiling, sometimes gives good results.

See also Lo Bianco, loc. cit., p. 446.

Sections.-See $\S \S 177$ and 17̣, for undecalcified specimens.

983. The Alcyonaria have also extremely contractile polyps. In a former edition I suggested for their fixation either hot sublimate solution or glacial acetic acid (\$ 84). S. Lo Bianco has since recommended essentially similar processes. GARBINI (Manuale, p. 151) drenches them with ether, and brings into strong alcohol.

Wilson (Mitth. Zool. Stat. Neapel, 1884, p. 3) kills Alcyonaria with a mixture of 1 part of strong acetic acid and 2 parts of concentrated solution of corrosive sublimate, the animals being removed as soon as dead and hardened for two or three hours in concentrated sublimate solution.

984. Zoantharia and Alcyonaria.-Braun (Zool. Anz., 1886, p. 458) inundates Alcyonium palmatum, Sympodium coralloides, Gorgonia verrucosa, Caryophyllia cyathus, and Palythoa axinelloe with a mixture of 20 to 25 c.c. of concentrated solution of sublimate in sea water with 4 to 5 drops of 1 per cent. osmic acid, and after five minutes passes into successive alcohols.

(This method also gives good results with Hydra and some Bryozoa and Rotifers.)

See also $§ 14$. 
Bujor (Arch. Zool. expér., ix, 1901, p. 50) kills Veretillum in sea water containing 10 per cent. each of formol and etherr, and after a minute passes into 2 per cent. solution of formol in sea water.

985. Hydroidea in General.-Directly the tow-net comes on board, the Plankton must be poured into a glass jar and jelly fishes at once picked out by means of a lifter or pipette, and placed in another very clean jar of sea water. Leave in this jar for half an hour to allow organisms to recover from shock. Note that the slightest trace of chemicals in the jar will prevent their expanding. The secret of successful preservation depends on keeping the animals in motion while you pour in the fixer. First stir the organisms very slowly and gently, and when all are in motion begin to pour the formalin slowly down the side of the vessel. About 10 c.c. of 10 per cent. formalin should go to 100 c.c. of sea water, but better more than this quantity. Keep stirring for at least two minutes after addition of fixer. Leave for a few hours and then transfer to 5 per cent. formalin; finally store in 10 per cent. 'To obtain medusæ in a nice state of expansion it is necessary to use an anæsthetic (see especially $\$ 15$ ). Hydrochloride of cocaine is possibly the best; use a 1 or 2 per cent. solution. Place the medusæ in a small glass vessel with just enough sea water to allow them to swim. After they have expanded add a little cocaine (3 c.c. of 1 per cent. solution for every 100 c.c. of sea water). If the medusæ at the end of ten to fifteen minutes do not contract when touched with a glass rod no more cocaine is needed ; if they are still active add more narcotiser and stir ; an over-dose will cause prolonged contraction. After anæsthetisation add the formalin and keep stirring, and continue for a minute, or longer. Do not leave specimens in solutions of cocaine longer than necessary. (ALLEN and Browne in Science of the Sea. London. John Murray. 1912.)

For further description of narcotisation methods see $\S \S 15$ et seq.

For killing by heat see $\S 13$.

Fixation.-In general polyps may be very well killed in saturated sublimate solution, in which they should be plunged for an instant merely, and be brought into alcohol. The solution should be employed cold in general for Gymnoblastea, hot for most Calyptoblastea.

Ether attentively administered gives good results with Campanularidæ. Hydra is very easily killed by a drop of osmic acid on a slide. For the methylen blue intra vitam method, see Chapter XVI; also HADŽI, Arb. Zool. Inst. Wien, xvii, 1909, p. 225.

986. Medusæ : Fixation.-For narcotisation see $\S 17$ and above. Trachymedusæ and Acalephæ may be fixed in the usual way in 
chromic or osmic mixtures. Osmic acid may be added to the sea water containing the animals, which should be removed to spring water as soon as they begin to turn brown.

987. Bigelow (Mem. Boston Soc. nat. Hist., v, 1900, p. 193) fixes the seyphistomes of Cassiopeia in Lo Bianco's mixture of 10 parts of 10 per cent. solution of cupric sulphate with 1 of saturated sublimate, and hardens them in 5 per cent. bichromate of potash.

988. Medusæ: Sections.-Paraffin and collodion are certainly not satisfactory as all-round methods for these watery organisms. The HeRTwigs (Nervensystem der Medusen, 1878, p. 5) embedded in liver with the aid of glycerin gum, and hardened the objects and the mass in alcohol.

See also JoLIET's glycerin gum method, and the gelatin methods in Chapter VIII.

989. Medusæ : Maceration.-See, especially for the study of the nervous system, $\S 526$. Doubtless in many cases the pyrogallic acid reaction, $\S 374$, would give enhanced differentiation.

990. Siphonophora.-For the cupric sulphate method of BEDOT (Arch. Sci.phys. et nat., xxi, 1889, p. 556), which is admirable for the preparation of museum specimens, but not necessary for histological work, as well as for those of Lo Bianco (op. cit., p. 454), FriedLÄNder (Biol. Centrbl., x, 1890, p. 483), and Davidoff (Anat. Anz., xi, 1896, p. 505) see previous editions. Lo Bianco fixes most forms with the mixture given in $\S 1021$.

For preserving, according to WEBER, formaldehyde is better than alcohol. Davidoff (loc. cit.) fixes in it.

991. Ctenophora : Fixation.-Never store in formalin, always in 70 per cent. alcohol. Pleurobrachia are best killed in 5 per cent. formalin in sea water. Fill large measuring jar with this fluid, drop in the animals and leave till they sink to the bottom; transfer to 5 per cent. formalin in pure water. After a week or so (not longer) transfer to very dilute alcohol, and up-grade to 70 per cent. strength. Beroë : Bring into small quantity of sea water, and when expanded add large quantity of corrosive sublimate saturated solution in sea water. When specimens become white, decant and add fresh water; wash in several changes to remove corrosive, up-grade to 70 per cent. alcohol. Bolina dissolves at once in formalin; kill in Flemming, selecting small specimens: leave half an hour, wash slightly, up-grade to 70 per cent. alcohol. (ALLEN and Browne in Science of the Sea. London. John Murray. 1912.) 
Small forms are very easily prepared by means of osmic acid. For the large forms see Lo Bianco, loc. cit., p. 457. He uses his copper sulphate mixture, $\S 987$.

Samassa makes sections by the double-embedding method. See Arch. mik. Anat., xl, 1892, p. 157.

992. Plankton, Preservation of, without Sorting (E. J. AlLEN and E. T. Browne in Science of the Sea. John Murray. 1912).--Preservation of whole catch of a tow-net is performed by stirring around the Plankton with a rod and adding a little 5 to 10 per cent. formalin. Keep on stirring for about a minute, then allow the organisms to settle to the bottom; as soon as this occurs pour off as much of the liquid as possible and transfer the Plankton to a bottle; again allow to settle and reduce the fluid to a minimum; then fill the bottle with 5 or 10 per cent. formalin. A bottle should be not more than half full of Plankton. After a few days, or on the appearance of opalescence of the fluid, change the liquid. Another method is first to fill the Plankton by pouring some saturated solution of picric acid into the jar containing the organisms, then add some formalin 5 or 10 per cent. and leave for an hour or two, occasionally stirring. Finally decant and add 5 or 10 per cent. formalin as before described; the yellow colour of the fluid can be neglected. Never use corrosive sublimate with formalin, as crystals form, which adhere to the organisms.

\section{Porifera.}

993. Spongiæ : Fixation.-The smaller forms can be fairly well fixed by the usual reagents, osmic acid being one of the best. For the larger forms absolute alcohol is apparently the best. If any watery fluid be preferred, care should at all events be taken to get the sponges into strong alcohol as soon as possible after fixation, on account of the rapidity with which maceration sets in in watery fluids. Fiedler (Zeit. wiss. Zool., xlvii, 1888, p. 87) has been using (for Spongilla), besides absolute alcohol, an alcoholic sublimate solution and the liquids of Kleinenberg and Flemming.

Staining. - To avoid maceration, I hold that alcoholic stains should be alone employed, and I recommend Mayer's tincture of cochineal, § 235. Von Lendenfeld (Zeit. wiss. Mik., xi, 1894, p. 22) uses aqueous solutions of Congo red and anilin blue for the coloration of collar-cells.

Minchin (Quart. Journ. Mic. Sci., xl, 1898, p. 569) stains spicula sheaths with Freeborn's picro-nigrosin, § 742. 
Rousseau (Ann. Soc. Belg. Mic., xxiv, 1899, p. 51) stains in nigrosin, picro-nigrosin, or indulin, or MAYER's picro-magnesia carmine.

Prof. Dendy informs me that he uses Hickson's brazilin ( $\$ 378$ ) a great deal in his work on sponges.

For intra vitam staining, see LoIseL, § 207, ante, p. 130.

For silvering, see $§ 356$.

Sectioning.-Calcereous sponges may be decalcified in alcohol, acidified with hydrochloric or nitric acid, and then embedded in the usual way. Siliceous sponges may be desilicified, $§ 566$.

For Rousseau's methods, see $\S 566$. Vosmaer and Pekelharing decalcify with a solution of picric acid in absolute alcohol (see Zeit. wiss. Mik., xv, 1899, p. 462).

See also Johnstone-Lavis and Vosmaer, $§ 179$.

Preparation of Hard Parts.-Siliceous spicules are easily cleaned by treating them on a slide with hot concentrated nitric or hydrochloric acid, or solution of potash or soda. The acids mentioned are very efficient, but may attack the silex of some delicate spicules. Potash solution is, therefore, frequently to be preferred, notwithstanding that, in my experience, it does not give such clean preparations.

According to NoLL, eau de Javelle is preferable to any of these reagents, see $\S 544$.

Embryos and Larvæ.-MAAS (Zool. Jahrb., Abth. Morph., vii, 1894, p. 334) fixes larvæ in liquid of Flemming or Hermann, one to three minutes, and stains with borax-carmine, or with gentian violet and Orange G (Flemming). He also (Zeit. wiss. Zool., lxvii, 1900, p. 218) fixes young Sycons in absolute alcohol and stains with ammonia carmine (spicules in situ).

Delage (Arch. Zool. Expér., x, 1892, p. 421) fixes larvæ of Spongilla that have settled down on cover-glasses for three minutes in absolute alcohol, stains in alcoholic carmine, and brings through alcohol into oil of bergamot, then either mounts direct in balsam, or detaches the larvæ from the cover and imbeds in paraffin (three. minutes).

Gatenby (Journ. Linnean Soc., 1920) uses methods for mitochondria, especially Champy-Kull and Kopsch.

\section{Protozoa.*}

994. Introduction.-In the special study of the protozoa the various reagents and methods of general cytology are in great part

* By Dr. A. Drew, Imperial Cancer Research Fund. 
applicable, but numerous modifications have been introduced to meet special cases. Speaking generally, the main lines of study applied to the protozoa may be summed up under the following headings :-Culture, collection, determination of life cycles, general morphology, physiology. It will perhaps be appropriate to say a few words by way of introduction under each of these headings.

995. Culture.-No general method is applicable to all cases; for many of the free living protozoa, such as infusoria, amœbæ and flagellates, a simple 1 or 2 per cent. hay infusion is suitable, which may be conveniently placed in small petri dishes; these give the additional advantage of providing a large surface. Amœbæ are in many cases best grown on a solid agar medium, to be described later. Special media have been described for the culture of many pathogenic forms, such as trypanosomes, and quite lately Sr. Monica Taylor has described a useful method for cultivating Amœbæ proteus (§ 1013).

996. Pure Mixed Cultures of Amœbæ.-When working with protozoa it is extremely desirable to have pure cultures whenever possible. In the case of amœbæ, bacteria are necessary as food, so that we must grow our amœbæ with bacteria. A culture containing one species of amœba, together with a pure culture of any particular bacterium, is spoken of as a pure mixed culture, and it is necessary to obtain such pure mixed cultures in order properly to study the biology of the amœbæ. The preparation of a pure mixed culture involves two distinct processes : (1) The separation from its fellows of a single amœba; and (2) its subsequent cultivation with a pure culture of some selected bacterium.

(1) Separation of a Single Cyst.-The easiest method of accomplishing this is by means of the following method. Take a glass capillary tube about 5 to $10 \mathrm{~cm}$. in length and $1 \mathrm{~mm}$. in diameter. Flame the centre, and draw out quickly to the fineness of a hair. Break into two equal portions and reduce each of these to a length of $5 \mathrm{~cm}$, so that one obtains two short tubes consisting of a wider portion and a very fine capillary portion. Next select a culture of the amœbœ rich in cysts, add a drop of sterile water, and rub a portion of the growth into this with a sterile platinum wire. Allow a minute portion of this emulsion to run into the capillary end of the prepared tube, and then run in sterile water till about $0.5 \mathrm{~cm}$. of the broad portion of the tube is filled. Mix the contents of the tube by vigorous rotation. Now prepare an agar film on a microscope slide, by melting one of the stock tubes of the agar and pouring 
a few drops on a slide. Allow this to set. Place on the microscope and focus the upper surface with an inch objective. Tap out on to filter paper some of the liquid in the capillary tube, and then, whilst looking through the microscope, gently touch the film with the fine end of the tube. A small volume of the suspension of cysts will run on to the jelly and will spread out in an area which is quite visible, and which occupies only a small portion of the field. If no cyst is present, or if there should be more than one, place another drop on a fresh film and repeat till a single cyst is obtained on the film. The method is simple, and with practice one can make half a dozen such cultures in an hour. Place the slide film surface downwards above water in a petri dish (this is conveniently done by resting on two corks), and cover the dish. Examine day by day, till numerous amœbæ are found, and then allow them to encyst. From this culture, prepare cultures in test tubes containing the special agar sloped as for bacteriological work. Allow these to grow for a week till covered with cysts. This may be observed with a low-powered lens through the wall of the test tube. Prepare a 3 per cent. solution of hydrochloric acid in sterile distilled water and cover the jelly film in the test tube with this solution and replace the plug. Allow this to act for twenty-four hours. Pour off the acid, and fill up with sterile water, and again pour off, using aseptic precautions throughout. With a platunim loop scrape some of the emulsion of cysts off the tube on to the surface of a fresh sterile tube of agar. Then add a loopful of a very dilute emulsion of the selected bacterium and rub gently over the tube. In the course of twenty-four to fortyeight hours the amœbæ will excyst, and multiply, and will generally be found to be in pure culture with the bacterium added. Should they not be so on bacteriological analysis, allow to encyst and again treat the cysts with the acid and proceed as already described. It will be found that the best organisms wherweith to grow amœbæ are those naturally occurring in water, one of the best being Bacillus fuorescens non-liquefaciens. When pure mixed cultures have been obtained, the stock cultures should always be kept in test tubes, as the petri dish method over water, although excellent for impure stock cultures, is very liable to allow contaminations to occur. By means of the method described, amœbæ have been obtained in pure mixed culture with the following bacteria, and with the results detailed below. 
B. pyocyaneus

B. prodigiosus

B. megatherium

B. subtilis

B. proteus vulgaris

B. coli

B. typhosus

B. phlei .

B. Rabinowitch

B. Nabarro 3 .

Pneumococcus .

Meningococcus

Staphylococcus aureus
- Excystation fairly rapid, growth good but amœbæ encyst rapidly.

Excystation fairly rapid, growth good.

Excystation fairly rapid, growth poor, amœbre encyst very soon.

Excystation extremely slow, growth very poor.

Excyst fairly rapidly, growth slow, encystment very slow.

Excyst fairly rapidly, growth fair.

99\%. Collection.-The great majority of the free living protozoa are inhabitants of either fresh or salt water, and to obtain satisfactory specimens some method of collection is essential.

There are several pieces of apparatus which are more or less essential, viz., a collecting stick with ring and net, collecting bottle and cutting knife, a good pocket magnifier (about $\times 10$ ), and small specimen bottles. The collecting stick can be obtained from all opticians, and it is usually fitted with the necessary appliances. The net is perhaps the most essential part of the apparatus. It consists of a bag of soft mull muslin fastened to a brass ring about 9 inches in diameter at one end, whilst the other carries a rimmed glass bottle about 3 inches long by 1 inch wide. The cutting knife is a strong curved hook with a sharp knife edge, which is screwed to the end of the stick, and is occasionally useful for cutting pieces of water weeds, which would otherwise be out of reach. A flat bottle, which can be obtained from most opticians, is extremely useful for examining specimens secured by the net. In a round bottle it is often extremely difficult to see minute organisms clearly when examined with the magnifier, but with the flat bottle one can usually quickly ascertain whether a sample is worth keeping for study with the microscope. The magnifier should be a good one and should be aplanatic. The most generally useful will be found to be either Watson's aplanatic loops, or one of Zeiss's hand lenses. The most generally useful powers will be either the $\times 6$ or $\times 10$, preferably both.

The various forms of pond life can be divided, for collecting purposes, into the free, and attached, and these groups will require different treatment in the methods of collection. The free swimming forms are often designated by the term "plankton," and these will be best obtained by means of the net. In order to collect material with the net it is passed through the water half a dozen times or so and then withdrawn ; the pond water runs out, but the various infusoria, rotifera, etc., are retained and are finally condensed in the bottle. They should then be emptied either into the flat bottle for preliminary examination, 
or into one of the specimen tubes to carry home. The attached forms, such as hydra, polyzoa, vorticella, are generally found on water weeds or bits of stick or submerged roots. In order to secure such organisms the weeds, etc., are severed with the cutting knife and are dragged on shore; placed in water in the flat bottle and examined. Polyzoa seem to have a preference for submerged rootlets, whilst forms such as vorticella are more frequently found on the roots and stems of duckweed.

998. Determination of Life Cycles. - The only really satisfactory method of determining life cycles is to follow a single organism continuously throughout its various changes in the manner originally used by the late Dr. Dallinger, but such observations should be controlled by examination of suitably stained specimens in the different stages, under critical illumination. In the case of many of the parasitic pro. tozoa such methods are impossible, as cultures cannot be obtained. In such cases we are only able to examine fixed and stained preparations and endeavour to piece out a life cycle from the appearances observed. This must be checked by observations of the living material wherever possible. For free living protozoa some means of keeping a drop of the culture fluid from drying is necessary, but any means adopted must permit of the continuous examination of the organisms by high-power lenses. One of the best is that used by Dallinger and Drysdale, and described in The Microscope and its Revelations, edited by DALLINGER, 8th ed., Part I, pp. 341 to 344 . For the majority of flagellates this is an excellent arrangement. Its chief drawback seems to be due to the fact that it does not permit the aeration of the culture fluid, and it is, therefore, found that organisms such as large ciliates, many amœbæ, vorticellæ, etc., soon die out from lack of oxygen. The simplest method of examining such forms is the well-known hanging drop arranged on a hollow ground slide. This, however, does not permit of critical illumination and completely upsets the corrections of the condenser. To get over this difficulty a slide with a small table ground out is used. The table is surrounded by a trench, and a ring of vaseline is painted round the outside of the trench, and a drop of the fluid containing the organisms to be studied is placed on the table. A cover-glass is then lowered on to the drop and adjusted for pressure by gently pressing on to the vaseline. It is advisable in most cases to arrange the drop so that it does not spread entirely to the edge of the table; this ensures an air supply. For amœbæ and many ciliates and flagellates the live slide described by Drew and GRIFFIN (Journal of Royal Microscopical Society, February 21st, 1917) may be used. This form consists of a glass plate cut to fit the mechanical stage of the microscope, and with a glass arm cemented along one side. A piece of linen has a hole slightly larger than the cover-glasses to be used cut in it, and this is then damped with water and laid upon the slide. A small glass vessel filled with water is attached to the arm and is put in communication with the cloth by means of a piece of linen or soft wick. The linen is thus kept moist by capillarity. A drop of the culture is placed on the slide and a cover-glass placed upon it, and adjusted so that the circular opening in the linen touches it at the margins, pressure being regulated, if necessary, 
by the insertion of cigarette paper between slide and cover-glass. So long as water remains in the vessel attached to the arm, the linen and the fluid under the cover will remain moist. For many purposes the live slide devised by Botterill and described in The Microscope and its Revelations by DALIINGER, p. 340, will be found extremely useful.

999. General Morphology.-Here great attention must be paid to modern cytologic methods. The most perfect fixing and staining technique should be used in any detailed study of the protozoa, and yet this is seldom found. Methods of fixation will necessarily differ according to whether one wishes to study nucleus, cytoplasm or such cytoplasmic inclusions as mitochondria or Golgi apparatus. Different methods are also frequently necessary according to the organisms studied. For purposes of convenience it is proposed to treat the fixing and staining of protozoa under the following headings:-Amoboe, Coccidia, Ciliata, Flagellata, Hamamoebce.

1000. Amœbæ.-For temporary purposes many amœbæ may be stained and fixed by running a drop of 1 per cent. chromic acid under the cover-glass and then running in a little alum carmine followed by water. Staining intra vitam is very conveniently carried out by means of an agar jelly. A 2 per cent. solution of agar in distilled water is made; this is cleared with egg white and filtered hot and distributed in 5 c.c. quantities in test tubes. For use a tube is melted by steeping in boiling water in a beaker and a few drops of the selected stain is added and well mixed. A little of the molten agar is then poured on to a slide and allowed to set. The amœbæ are distributed in a drop of fluid on a cover-glass, which is then inverted on the jelly. The preparation is at once examined with the microscope. Staining takes place progressively till the nucleus is tinted, when " death" occurs. One of the best stains for such a method is Unna's polychrome methylene blue. For permanent preparations one of the most satisfactory methods is that used by the writer. Slides are coated with a very thin film of molten agar. This is conveniently done by pouring a very small quantity of the agar on one end of a perfectly clean slide and spreading it out quickly into a thin film over the slide by means of a warmed glass rod. As soon as the agar has set the slides are stored in a moist chamber till required. A drop or two of the fluid culture containing the amœbæ is spread gently over the surface of the agar with either a platinum loop or a glass rod, great care being taken not to break the thin agar film. The slide is then placed film side up in a moist chamber and allowed to remain for from ten minutes to half an hour or more. In this time the amœbæ have generally 
spread themselves out on the agar and many are in states of division. The slide is quickly removed and placed in a larger tube containing wool saturated with 2 per cent. osmic acid. The vapour fixes the organisms and the slide is removed in two to five minutes, and at once carefully placed in 50 per cent. alcohol. It should remain in this for fifteen minutes and is then transferred to 70 per cent., 90 per cent. and absolute alcohol fifteen minutes in each. It is then brought down to distilled water by passage through the alcohols, and is stained by Heidenhain's iron hæmatoxylin method. The secret of success in this method is to prepare the agar coated slides with as thin a film as possible, otherwise it will peel off in the alcohol.

Amœbæ may be mixed with 1 per cent. serum water, and spread in a thin layer over slides, which are then fixed in Schaudinn's fluid, passed, as before, through the upgraded alcohols and back again to water, and stained with iron alum. Dobell's alcoholic iron hæmatin method may be used for staining the free forms of amœbæ, but is not so good for the cysts. A modification of Mann's stain by Dobell is also an excellent stain for amœbæ and cysts, and also sections of intestinal ulcers and tissues. The stain consists of Mann's methyl blue-eosin, which is made up in the usual way. Differentiation is carried out in dilute orange $\mathrm{G}$ in 70 per cent. alcohol. A simple staining in Lugol's iodine solution is also of use, especially in the routine examination of fæces.

1001. Coccidia.-These parasites stain rather badly and consequently may be examined by adding a drop or two of 1 per cent. aqueous eosin to the material containing them. The coccidia stand out as unstained bodies on a pink ground. Tissues may be fixed in the following solution :-

10 per cent. cobalt chloride in distilled water 20 c.c.

2 per cent. chromic acid in distilled water . 5,

Formic acid . . . . . . 1 drop.

Schaudinn's alcoholic sublimate is also a good fixative. Bertarelli's method consists in fixing in saturated perchloride of mercury and staining in Grenacher's hæmatoxylin and differentiating in acetic alcohol.

Borrel's method is of considerable utility.

Tissues are fixed in the following solution for twenty-four hours :-

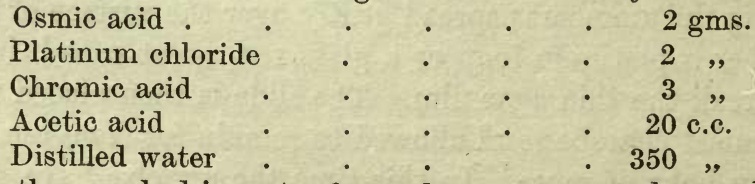

They are then washed in water for an hour or two, and passed through 
the upgraded alcohols to paraffin. Sections are stained for one hour in saturated magenta, and are placed for five to ten minutes in a saturated solution of picric acid in water, l part, and saturated aqueous indigo carmine, 2 parts. They are washed in water and are decolourised in absolute alcohol and then in clove oil, and are mounted in balsam.

1002. Ciliates.-Osmic acid fixation is amongst those best suited for the ciliates, generally being employed either as vapour or wet on the slide. Many forms may be mixed with serum water (serum 1 part, water, 20 parts), and then spread on slides and fixed either in the osmic vapour or by means of Schaudinn's solution. Bouin's fluid is also very suitable for fixation of many forms such as Opalina and Lophomonas. Tissues are best fixed in either 10 per cent. formol saline or Flemming's solution.

Staining may be by means of methyl green or any of the stains used for amœbæ. In many cases Hollande's chloro-carmine gives very satisfactory results.

1003. Flagellata.-For the majority of these forms the technique employed for the study of the trypanosomes may be used. Slides may be coated with either serum water or a very thin layer of glycerin albumen. A drop of the fluid containing the flagellates such as blood may be spread quickly over it before drying occurs, and the slide at once placed in Schaudinn's sublimate or Flemming's solution for varying periods. Slides are then treated with upgraded alcohols, the weaker ones containing a little iodine. They are then brought down to water and stained with Heidenhain's iron hæmatoxylin. Dried blood films prepared in the usual manner may be fixed in alcohol-ether or absolute methyl alcohol and stained with Giemsa's method.

1004. Hæmamœbæ.-These forms, the principal of which are the well-known malarial parasites, will be found in the blood, and hæmatological methods must be used.

Blood is spread in thin layers on slides by placing a drop at one end of the slide and touching it with the end of another. The blood will spread out in a thin layer and may then be drawn across the slide when a thin, usually one-cell, layer is obtained. Films made by streaking a drop of the blood over a slide by means of eigarette paper are also good.

Such films are best fixed either in alcohol-ether (equal parts of each) for half an hour to one hour, or pure methyl alcohol free from acetone one hour. For a single method of staining such films, probably Rees' thionin is the best. It is prepared as follows. Thionin 1.5 grms., absolute alcohol 10 c.c., 5 per cent. carbolic acid solution, 100 c.c. Dissolve the thionin in the alcohol and add the carbolic solution. It is 
best diluted for use about one in five, and films are stained for from five to thirty minutes, washed, dried and mounted in balsam or euparal. The hæmamœbæ are stained purple, nuclei blue, red cells faint blue or grey. Films may be differentially stained by means of eosin-methylene blue, Borrel's blue, or still better, by one of the Romanowsky methods.

1005. Eosin-Methylene Blue.-The film is prepared and fixed either by alcohol-ether or methyl alcohol, and is then stained for thirty seconds in a 0.5 per cent. solution of eosin, and is then washed and stained in a saturated solution of methylene blue for thirty seconds. In a successful preparation the red cells are stained pink and the nuclei of leucocytes and parasites blue.

1006. Ronald Ross's Thick Film Method.-Frequently the parasites in these cases are only present in very small numbers, and in the examination of the ordinary thin films their presence may be overlooked. To meet such cases Ross recommends about 10 to 20 cubic $\mathrm{mm}$. of the blood to be spread in a thick film on a slide, which is dried by waving gently over a flame and is then washed in water. By such treatment the hæmoglobin is dissolved out from the erythrocytes. The film may now be stained for a minute in 0.5 per cent. to 1 per cent. aqueous eosin, followed by a 1 per cent. aqueous solution of methylene blue, made alkaline by the addition of 0.5 per cent. sodium carbonate and heated. For this may, with advantage, be substituted a solution of Unna's polychrome methylene blue diluted 1 in 2 or 3 with water. Films after staining are washed, dried, and mounted in balsam. Only the leucocytes and the parasites are stained by this method.

100\%. Borrel's Blue.-This method depends upon the formation of an oxidation product of methylene blue. Silver oxide is prepared by dissolving the nitrate in distilled water and precipitating the oxide with a 10 per cent. solution of sodium hydroxide. The precipitate obtained is washed thoroughly in distilled water and a saturated solution of methylene blue is added. The mixture is well shaken and allowed to stand for about a fortnight. The supernatant fluid is then pipetted off and constitutes Borrel's blue. Films are prepared and fixed for half an hour in either alcohol, etheralcohol or absolute alcohol, and are then stained with Laveran's solution prepared as follows :-

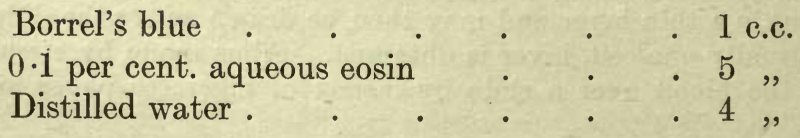

The slides are placed film side downwards in this fluid and allowed to stain for from five to fifteen minutes, and should then be washed in distilled water treated with a 5 per cent. aqueous solution of tannic acid for one to two minutes, washed and dried. Red cells are stained pink, nuclei of leucocytes purple violet; whilst the 
cytoplasm of the parasites is stained faintly blue their nuclei is reddish purple.

1008. Romanowsky Methods. - The Romanowsky methods depend essentially upon the formation of Azur and other oxidation products of methylene blue, either alone or in combination with eosin. The most generally used are Leishman's stain, Wright's stain and the well-known Giemsa's stain.

1009. Leishman's Stain.-This stain is an extremely useful one; it is best purchased, but may be prepared as described in $\S 784$, p. 385. Films are prepared in the ordinary manner, but are merely dried, not fixed. Five to 10 drops of the stain are poured on from a pipette and allowed to act for thirty seconds. An equal quantity of distilled water is then added and the diluted stain allowed to act for a further period of from five to ten minutes. The preparation is then well washed in distilled water, and is dried and mounted in balsam. Red cells are stained pale pinkish, the nuclei of the leucocytes red, parasites blue and their nuclei reddish purple. See $\$ \S 784$ et seq.

1010. Wright's Stain.-This stain is best prepared by dissolving Grubler's methylene blue, 1 grm. in 0.5 per cent. sodium bicarbonate 100 c.c. The mixture is then heated at $70^{\circ}$ to $80^{\circ} \mathrm{C}$. for an hour. Cool and add 500 c.c. of a 0.1 per cent. solution of eosin (the yellowish shade water soluble variety). The solution of eosin should be added rather slowly, with constant stirring: till the blue colour disappears and the mixture is purple; at this stage the fluid should have a metallic appearance on the surface, when a finely granular blackish precipitate is formed. This is collected and dried at $37^{\circ} \mathrm{C}$. and a 0.3 per cent. solution of this dye is then made in pure absolute methyl alcohol. When required for use it is diluted with methyl alcohol (4 stain, 1 methyl alcohol). Stain as described for Leishman's method.

1011. Wilson's Stain.-This stain also depends upon the production of polychrome derivatives of methylene blue. It gives very satisfactory results when carefully used, closely resembling those obtained in good Giemsa preparations. Two grms. of silver nitrate are dissolved in 15 c.c. of distilled water, and to the solution so obtained is added 250 c.c. of a freshly-prepared lime water. The mixture should be shaken well and the precipitate of silver oxide collected on a filter and well washed with distilled water. The precipitate is then dried in an oven at a temperature not above $70^{\circ}$ to $80^{\circ} \mathrm{C}$. The moist silver oxide prepared from $\mathrm{AgNO}_{3}$ and $\mathrm{NaOH}$ solution may be used in place of the dry product. To the oxide so obtained 2 grms. of methylene blue dissolved in 200 c.c. of 0.5 per cent. sodium bicarbonate solution is added. The mixture is then gently boiled in a porcelain dish for twenty to thirty minutes, stirring from time to time. Pour off one-third of the contents into a cylinder and then add to the remaining solution in the dish an amount of distilled water equal to that poured off. Boil the mixture in the dish again for twenty to thirty minutes. Again pour one-third of the contents of the dish into the cylinder, and boil the remainder for a 
further twenty to thirty minutes. Pour the contents of the dish into the mixture in the cylinder and make up the total volume to 200 c.c. Filter into a cylinder and add at once solution No. II., which is prepared as follows: 1 grm. water soluble eosin (yellow shade) in 200 c.c. distilled water. Mix and allow to stand for half an hour, and collect the resulting precipitate on a filter. Dry the precipitate at $60^{\circ} \mathrm{C}$. In order to prepare the stain dissolve $0.2 \mathrm{grm}$. of the dried precipitate in 50 c.c. pure acetone-free methyl alcohol. The method of staining is identical with that of Leishman and Wright.

1012. GIEMsa's Method.-In many ways this is the easiest and best of the Romanowsky stains; it is best bought ready prepared, either as a solution or the solid products of Burroughs, Wellcome \& Co. For use, films are fixed in absolute methyl alcohol, alcohol-ether or absolute alcohol. They are then placed film side down in the staining fluid, which is prepared as follows :-

Giemsa's stain

Neutral distilled water

They should be stained for about one hour in this stain, washed, dried and examined.

It is highly important that the water used should be neutral. This is best secured by boiling fresh distilled water for fifteen minutes, and then rapidly cooling and storing in a syphon vessel over soda-lime.

1013. M. TAYloR's Method for Amœba Cultures (Nature, April, 1920).-Water from such places as the drainage cuttings in birch, alder, and willow woods, or from the margins of ordinary pools and ponds, together with the filamentous algæ and the brown scum, and included diatoms overlying the dead leaves and the other decaying organic matter forming the floor of such places, is gathered in autumn or in early spring. This is allowed to stand in tap-water for some time, until a rich brown scum appears on the top. The surface water, with the scum, is poured off into another glass vessel, and wheat is added ( 1 gram to a litre of water). In February, minute amœbæ begin to make their appearance; these become fully grown in May and June, and will then divide rapidly, forming a luxuriant culture until the late autumn, when encystment of most individuals again takes place.

Once started, amœba cultures require no further attention than a supply of water to compensate for evaporation, and the addition of wheat from time to time.

Dr. J. Brontë Gatenby informs me that using Sr. Monica Taylor's method he has made successful cultures in boiled and unboiled London tap-water, in London rain-water and in spring-water. Sub-culturing is easily managed. Simply take about a pint of fresh rain-water or boiled tap-water; about eight wheat seeds are just brought to the boil in a test tube of water, the latter poured away and the seeds shaken into a flat dish containing the rain.water. The dish is then inoculated 
from an old culture and securely covered. In from two to three weeks the cultures may be going strongly. It is best to make half a dozen different cultures, placing them at different distances from an oven or thermostat. Not all of them "take." Small flagellates always seem to accompany successful cultures, but the appearance of rotifers and small annelids usually heralds the end of the amœbæ.

Sr. Monica, in a paper in press (Journ. Roy. Mic. Soc. 1921), makes a further contribution to this subject: there are periods of depression in the cultures, new cultures may not " take "immediately, the cultures are best kept in the light near a window, and to avoid extinction of a strain by means of rotifers or small worms, one can only subculture.

\section{The Growth of Paramœcium in Sterile Culture Medium (by} R. A. Peters, Phys. Proceed., 1920).-Culture of a race of paramœcia about $50 \mu$ in length, isolated from a single individual, have been obtained upon the following medium, the cultures being considered sterile for the reasons given below.

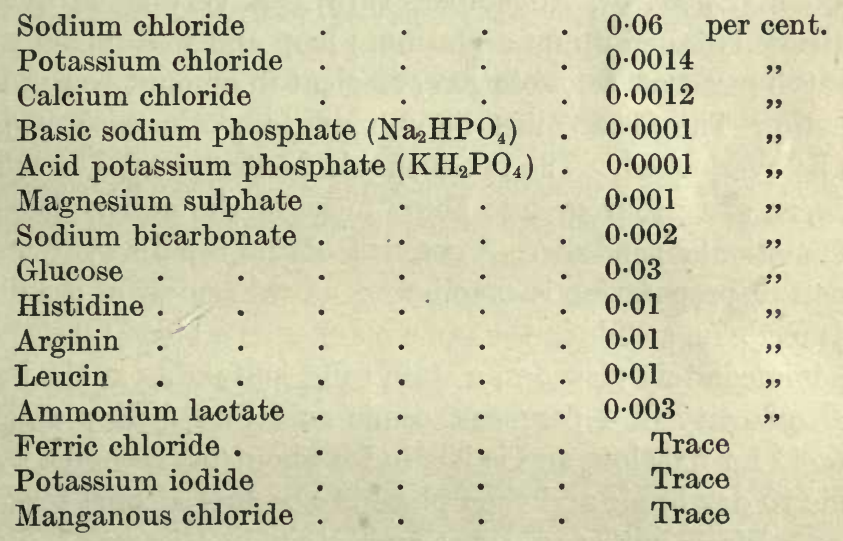

The substances are made up with glass distilled water. The constituents are autoclaved separately, and the final mixture sterilised by heating to $80^{\circ} \mathrm{C}$. on three successive days.

The organisms were cultivated first in sterile media in depression slides, experiment showing the most suitable concentration for division. The first divisions were apt to be slow. After a number of individuals have been obtained in this way, they are sub-cultured in test tubes, using all sterile precautions, and when a satisfactory test tube culture has been obtained, it can be used for culture purposes as required. A culture has now been kept going by weekly sub-culture upon this medium for three months. (Temperature from $15^{\circ}$ to $20^{\circ} \mathrm{C}$.)

From successful eultures grown in this way with the paramøcium in question, it was impossible to obtain any bacterial growth (or other 
growth) by sowing a drop of the paramœcial culture with a platinum loop, either upon (1) nutrient broth, (2) nutrient agar, (3) glucose agar (anærobic) and (4) litmus milk at room temperature or at $36^{\circ} \mathrm{C}$. It was also not possible to obtain growth upon the medium itself stiffened with a trace of agar. Examination of a growing culture under the $\frac{1}{12}$ oil immersion lens, however, showed the presence of peculiar rodshaped bodies. Thesê were about $10 \mu$ long and $2 \mu$ broad. They were motile, but appeared as a rule to be anchored at one end to the slide. They were never observed to divide. After a varying time they would cease to move in the moist drop preparation. When stained they were found to lie in rows, varying in shape from curved to straight. The absence of any sign of parasite or symbiotic organism in the paramocium and the general resemblance of these bodies to split off cilia has led to the belief that the cultures in question really contain no other organism than the paramœcium.

1015. Method for Examination of Fæces for Protozoa (H. M. Woodcock, B. M. J., November, 1915).-A very small quantity of the fæces is taken up on a platinum loop and well mixed with a drop of 0.5 per cent. salt solution sufficient in amount to run under a coverslip. The fæces must be well diluted, otherwise cysts are apt to be overlooked. The frces should be examined as freshly as possible, as after four or five hours most of the active flagellates become motionless and die. A convenient and rapid way of making a permanent preparation is as follows :-A thin smear of the diluted fæces is made on a slide in the same manner as a blood film, and the slide is immediately placed in a stain tube containing at the bottom a small quantity of 4 per cent. osmic acid plus 1 drop of glacial acetic acid for fixation, and is left in for about ten seconds. Allow the slide to dry in air and then place in absolute alcohol for fifteen minutes. Wash with tap-water and stain in Giemsa, 1 drop to 1 c.c. neutral distilled water for twenty minutes or so. Rinse with tap-water.

\section{Donaldson's Method of Detecting Protozoal Cysts in Fæces} by Means of Wet Stained Preparations (Lancet, 1917).-Donaldson recommends the use of two solutions, $\mathrm{A}$ and $\mathrm{B}$.

A. (1) Five per cent. aqueous potassium iodide saturated with iodine to which is added an equal volume of ether.

B. (1) A saturated aqueous solution of Rubin S. ; or

(2) A saturated aqueous solution of eosin ; or

(3) Stephen's scarlet writing fluid.

Equal parts of stains A and B are mixed just before use. A few loopfuls of one of the above stain combinations are placed on a clean slide, a loopful of fæces is taken and rubbed up with the stain 
to form a fairly smooth emulsion, and a clean coverslip gently lowered on to the drop. In order to get the best possible definition, it cannot be emphasised too strongly that the film so made should be spread out under the cover-glass by capillarity so as to form the thinnest layer possible, preferably a layer which is no thicker than the diameter of an Entamœba coli cyst.

The exact amount of stain to be used will soon be learned after one or two attempts, and depends upon the size of the loop employed and the size of the coverslip. It is a matter of considerable importance the way in which the film is made, especially where Rubin S., or, to a less extent, eosin has been used in the staining combination, for if the layer of fluid between the slide and coverslip be too thick the superfluous film of fluid overlying the eysts tends to render the latter less bright. In the case of eysts of the size and shape of Entamœba coli or histolytica, this makes little difference, but if one is hunting specially for the Lamblia, or, still more, Tetramitus mesnili, the colour contrast is not so marked, owing to the smaller size of the cysts and the conse quent thicker red layer of fluid overlying them. Where the worker has not acquired sufficient dexterity in making such a film, the difficulty may be got over by using Rubin S. or eosin of only half saturation in the stain combination, or by employing the scarlet writing fluid mentioned. In this way the effect of the super-imposed deeper red is to some extent obviated. In a wet preparation stained by this method there is a more or less homogeneous red background, from which the cysts stand out as brilliant yellow or greenish-yellow spheres which even the tyro cannot miss seeing.

1017. Method for the Tsetse Flies (M. Robertson, Trans. Roy. Soc., Series B, vol. 203, p. 161). - The newly hatched flies are starved for twentyfour to thirty-six hours and are then fed on the infecting monkey once, or in some cases twice. The infecting feed is the first blood ingested by the flies. After the infecting feed the cage is starved for. one or two days and thereafter fed on elean monkey's blood every second or third day. Daily feeding is not essential to the welfare of glossina, and does not appear to occur in nature. Dissections are made in a drop of physiological salt solution. The trypanosomes are studied both in the live state and in fixed and stained preparations. Preserved material is fixed while wet by dropping the coverslip film side downwards into Schaudinn's solution; the preparations are subsequently stained by Heidenhain's iron hæmatoxylin.

1018. Immobilisation. - See the narcotisation methods $\S \S 20$ to 25 . According to Schürmayer (Jen. Zeit., xxiv, 1890, p. 402), nitrate of strychnin, of 0.01 per cent. or less, gives good results with some forms, amongst which are Stentor and Carchesium. Antipyrin $(0.1$ per cent.), or cocaine of 0.01 per cent., seems only to have given good results as regards the extension of the stalk in stalked forms.

Eismond (Zool. Anz., xiii, 1890, p. 723) slows the movements of 
small organisms (small worms and Crustacea as well as Ciliata) by means of a drop of thick aqueous solution of cherry-tree gum added to the water containing them (gum arabic and the like, it is stated, will not do). The objects remain fixed in their places, with cilia actively moving, and all vital processes retaining their full activity.

Certes (Bull. Soc. Zool. France, xvi, 1891, p. 93) has found that an intra vitam stain may be obtained by adding methyl blue or "violet dahlia, No. 170 " to the gum solution.

Jensen (after Stahl; see Biol. Centralbl., xii, 1892, p. 558) makes a solution of 3 grms. of gelatin in 100 c.c. of ordinary water by the aid of heat. This makes a jelly at the normal temperature. It is slightly warmed, and a drop of it is mixed in a watch-glass with a drop of water containing the organisms.

See also Volк, ante, $\S 886$; Statkewitsch, Arch. Protistenk., v, 1904, p. 17 ; Lyon, Amer. Journ. Phys., xiv, 1905, p. 427 (neutralised gum).

1019. Staining intra vitam.-See hereon BRANDT (Verh. physiol. Ges. Berlin, 1878); Certes (Bull. Soc. Zool., 25 janv., 1881); and Henneguy (Soc. Philom., 12 fév., 1881). See also § 208.

BRANDT recommends a $1: 3000$ solution of Bismarck brown; also (Biol. Centralb., i, 1881, p. 202) "a dilute solution of hæmatoxylin."

Certes (op. cit., pp. 21, 226, 264, and Zool. Anz., iv,.1881, pp. 208, 287 ) found that living Infusoria stain in weak solutions of cyanin, Bismarck brown, dahlia, violet $5 \mathrm{~B}$, chrysoidin, nigrosin, methylen blue, malachite green, iodine green, and other tar colours, and hæmatoxylin. The solutions should be made with the liquid that constitutes the natural habitat of the organisms. They should be very weak, that is, of strengths varying between $1: 10,000$ and $1: 100,000$. For cyanin, $1: 500,000$ is strong enough.

As to the staining of the Nucleus, see Przesmycki, Biol. Centralb., vii, 1897, p. 321 ; and as to that of the Granula, the same author, Zeit. wiss. Mik., xiii, 1896, p. 478. Also Lorsel, § 208.

Examination in a coloured medium in which the organisms do not stain, but show up on a coloured background, is sometimes helpful. Certes (Bull. Soc. Zool. de France, xiii, 1888, p. 230) recommends solution of anilin black-Infusoria will live in it for weeks; FABREDomergue (Ann. de Microgr., ii, 1889, p. 545) concentrated solution of diphenylamin blue.

For the mitochondria and other granules, see FAURÉ-FREMIET (Arch. d'Anat. micr., xi, p. 457). Dahlia in salt solution, Pictet's fluid, Janus green, or crystal violet being useful. 
For mitochondria and Golgi apparatus of a sporozoon, J. HIRSCHLER (Anat. Anz., xlvii, 1914-15) used the Mann-Kopsch method, § 693.

1020. Demonstration of Cilia (Waddington, Journ. Roy. Mic. Soc., 1883, p. 185).-A drop of solution of tannin, or a trace of alcoholic solution of sulphurous acid, added to the water containing the living organisms is efficacious.

1021. Fixing and Preserving.--Protozoa may be killed by heat, by toxic vapours or by toxic liquids. Almost instantaneous fixation can sometimes be obtained by steam or by iodine (Kent) or iodine vapour (Overton) : see $\S 83$.

E. S. GOodRICH (Quart. Journ. Micr. Science, lxiv, 1919) modifies Kent's method in a way which we have found useful for amœbæ. A strong solution of iodine in potassium iodide is diluted to about the colour of sherry with normal saline for terrestrial and fresh water animals, and with sea water for marine organisms. Such a solution is run under the coverslip and followed by the definitive fixing agent, e.g., Bouin's fluid, etc., and the preparation proceeded with in the usual way. The iodine does not appear to destroy any of the cell contents.

With regard to fixation, read $\S \S 29,30, \S 655, \S 663$, and especially $\S \S 673$ to 713 . See also the important section from $\S \S 768$ to 772 , dealing with fats.

WoodCock and WrLson's Modification of Schaudinn's Fixative (Phil. Trans. Roy. Soc. B., ccvii, 1916, p. 379 ; and Univ. Calif. Pub., xvi, 1916, p. 244).-Woodcock gives saturated aqueous sublimate, 2 parts; absolute alcohol, 1 part; and acetic acid, 5 per cent. Wilson uses alcoholic sublimate +5 per cent. acetic. See also Gilson and Petrunkewitsch fluids, $§ 69$, and acetic alcohol, $\S 86$. For Schaudinn's original fixative, refer to $\S 1031$.

Lucidol or Peroxide of Benzol. See $\$ \$ 107$ and 783.

For killing by heat, see $\S 13$.

Pfitzner (Morph. Jahrb., xi, 1885, p. 454) used concentrated solution of picric acid run in under the cover.

ENTz (Zool. Anz., iv, 1881, p. 575) adds liquid of Kleinenberg to the water containing the organisms in a watch glass.

KoRschelt (ibid., v, 1882, p. 217) employs in the same way 1 per cent. osmic acid, or, for Amœbæ, 2 per cent. chromic acid.

LANSBERG (ibid., p. 336) advises the same reagents, but recommends bringing the organisms into the fixing liquid with a pipette.

For sulphurous acid, $\S 62$.

Cattaneo (Bollettino Scientifico, iii and iv; Journ. Roy. Mic. 
Soc., 1885 , p. 538) fixes for a few minutes with $\frac{1}{3}$ per cent. solution of chloride of palladium.

Brass (Zeit. wiss. Mik., 1884, p. 39) employs a mixture of 1 part each of chromic acid, platinum chloride, and acetic acid with 400 to 1,000 parts of water.

Certes (Comptes rend., lxxxviii, 1879, p. 433) fixes with 2 per cent. osmic acid, or its vapours (ten to thirty minutes). For details, see previous editions.

Longhi (Bull. Mus. Zool. Univ. Genova, 1892, No. 4) kills in 10 c.c. of 1 per cent. sulphate of eserin with 1 drop of 1 per cent. sublimate.

Scala (Rev. Mus. La Plata, xv, 1908, p. 57) fixes for five or ten minutes in a mixture of $2 \mathrm{mg}$. of atropin, 10 drops of formol, $10 \mathrm{grms}$. of glycerin and 50 c.c. of water.

See also Puschkarew, Zeit. wiss. Mik., xxviii, 1911, p. 145 (agar process for fixing and staining Amœbæ).

Fou (Lehrb., p. 102) fixes delicate marine Infusoria (Tintinnodea) with the perchloride of iron solution $(\S 80)$, added to the water containing them, and stains with gallic acid.

Lo Branco (loc. cit., p. 444) fixes Gregarinæ with picro-sulphuric acid (one hour), Vorticellæ with hot sublimate, Acinetæ with sublimate in sea water, or with osmic acid, Thalassicola with 0.5 per cent. chromic acid (one hour), Acanthometræ and Aulacanthæ with 50 per cent. alcohol or with concentrated sublimate, or by adding a little osmic acid to the water. For Sphærozoa he proceeds as BRANDT, $\S 1019$.

Zograf fixes Rhizopoda and Infusoria as Rotatoria, $\S 886$, but without narcotisation.

See also Fabre-Domergue, Ann. de Microgr., ii, 1889, p. 545, and 1890 , p. 50 ; SCHEWIAKoff, Biblioth. Zool., v, 1889, p. 5 ; Journ. Roy. Mic. Soc., 1889, pp. 832, 833 ; ZoJA, Boll. Sci. Pavia, 1892 ; Zeit. wiss. Mik., ix, 1893, p. 485 ; LaUterborn, Zeit. wiss. Zool., lix, 1895, p. 170 ; Schaudinn, ibid., p. 193 ; Baldiani, Zool. Ans., xiii, 1890, p. 133 ; KarawaIew, ibid., xviii, 1895, p. 286.

1022. Embedding of Protozoa and other Small Objects (MrNchin, Q. J. M. S., Ix, 1915, p. 508).-A thin slice of a block of amyloid liver preserved in alcohol is floated into a shallow glass vessel with a flat bottom, containing alcohol. The dish is placed on the stage of a dissecting microscope. The objects to be embedded are taken up in a pipette and placed on the slice of liver and orientated as desired.

A tiny drop of glycerine and albumen solution is taken up on the 
point of a needle and caused to touch the surface of the alcohol immediately above the small objects. The dense albumen solution falls at once through the alcohol and spreads out over the objects on the liver; at the same time the glycerine is extracted and the albumen coagulated by the alcohol, with the result that the objects are stuck on to the liver. The liver is now trimmed with a scalpel into a rectangular shape and embedded in the usual way.

Minchin used this method for the stomachs of fleas. I have found it most successful for Echinoderm and other small eggs.

Or the following method may be used :-The organisms should be strongly fixed, then dehydrated and cleared, and brought into melted paraffin in a small watch glass. After a few minutes therein they are brought on a cataract needle on to a small block of paraffin, and arranged there with a heated needle and sectioned. They may be stained after fixation, or the sections may be stained on the slide, $\S 186$ or 187.

Entz (Arch. Protistenk., xv, 1909, p. 98) brings the objects from clove oil into clove oil collodion of the consistency of honey, then brings them in this into a funnel made of paraffin, and when they have collected at the bottom of this puts it into chloroform, which dissolves the paraffin and hardens the collodion.

Some Current Stains for Protozoa.-Below are given a number of special staining methods suitable for routine protozoological work.

1023. HeidenhaIn's Alcoholic Iron Hæmatoxylin.-(1) Fix in Schaudinn's solution for fifteen minutes.

(2) Place at once in 70 per cent. alcohol for at least one hour.

(3) Mordant for five hours or more in

50 per cent. alcohol . . . . . 10 parts.

4 per cent. aqueous iron alum solution . 1 part.

(4) Stain for twelve to twenty-four hours in

Heidenhain's hæmatoxylin

70 per cent. alcohol . . . . . 10 parts.

Heidenhain's hæmatoxylin-

Hæmatoxylin

Absolute alcohol

Distilled water .

Thymol .

1 part.

1 grm.

10 c.c.

90

1 crystal.

Dissolve the hæmatoxylin in the absolute alcohol and then add the distilled water.

(5) Differentiate in the same solution as was used in (3) for mordanting. 
(6) Wash thoroughly in several changes of 70 per cent. alcohol to remove mordant.

(7) Dehydrate and mount in Canada balsam.

1024. Dobell's Hæmatein Method (see Arch.f. Protistinkunde, 1914, p. 144.)-Films or sections are transferred from 70 per cent. alcohol into 1 per cent. solution of iron alum in 70 per cent. alcohol. This is most easily made in the way described by Hickson (Quart. Journ. Micr. Sci. 44, 1901, p. 470), 1 grm. of salt dissolved in 23 c.c. of warm water, then add 77 c.c. of 90 per cent. alcohol after cooling. They are mordanted in this for ten minutes, rinsed in 70 per cent. alcohol and transferred to a 1 per cent. solution of hæmatein in 70 per cent. alcohol. They are left in this for ten minutes and then differentiated, either in the iron alum or in acidified 70 per cent. alcohol $(0.6$ per cent. $\mathrm{HCl}$ in 70 per cent. alcohol). After differentiation wash in several changes of 70 per cent. alcohol and then pass up through the alcohols into any of the usual mounting media. Any alcoholic counterstain can be used, light green in 90 per cent. alcohol being very good. By this method the organism is stained a purple grey, and hard black and white contrasts are not obtained. The various nuclear and cytoplasmic constituents may be stained with individual intensity.

(The hæmatein method is good, when it works, but often it is a complete failure; certain amœbæ, for instance, are not stained at all by it.)

1025. Dobell's Modification of Mann's Methyl Blue, Eosin Stain.(1) Stain with Mann's methyl blue eosin mixture (see Lancet, p. 196) in distilled water till everything is overstained (two to eight hours).

(2) Rinse in distilled water and differentiate in 70 per cent. alcohol dried from water, containing a small quantity of Orange G-just enough to colour it. It is best to keep Orange $G$ in concentrated solution in 90 per cent. alcohol; add sufficient of this with a glass rod to clear 70 per cent. before differentiating. This weak Orange G cannot be used often as it gets discoloured very quickly.

(3) Dehydrate in absolute alcohol (not too long) and transfer to xylol (also not too long, as eosin comes out in time if left).

(4) Mount in balsam.

$\mathrm{By}$ this method permanent and pretty results are obtained, often quite as good or better than those got with Giemsa. The method is especially good after Bonin fixation, but it is important that all the picric acid is removed before staining.

1026. Giemsa Stain (Mrnchin's Method, Q. J. M. S., lx, 1915, p. 510). - Slides are washed in tap water and put in dilute Lugol solution ( 1 c.c. Lugol to 25 c.c. distilled $\mathrm{H}_{2} \mathrm{O}$ ) for ten minutes. After this, rinsed in tap water and put into a 0.5 per cent. watery solution of hyposulphite of soda for ten minutes. Next wash in a current of water for five minutes and then put into the stain. The distilled water used to dilute the Giemsa stain has to be neutralised in the way prescribed by Giemsa. 
A measured volume of the distilled water is taken and to it are added a few drops of hæmatoxylin solution ( 5 per cent. in dist. $\mathrm{H}_{2} \mathrm{O}$ ) sufficient to tint it. Then a weak solution (1 per cent. in dist. water) of potassium carbonate is added drop by drop until the colour of the tinted water changes from yellow-red to reddish purple. In this way the number of drops of the carbonate solution required to neutralise a given volume of the distilled water is found.

The slides are now placed in the stain, 1 drop Giemsa to 1 c.c. neutralised distilled water for one hour. Transfer to a weaker solution ( 1 drop stain plus 4 or 5 c.c. water) and leave overnight. Remove excess of stain by washing in water. Differentiate stain by carrying slides through different strengths of acetone mixed with xylol, beginning with 95 per cent. acetone and ending with pure xylol. Mount in dammar or Canada balsam.

(If these instructions are carefully followed the results are excellent, but care must be used.)

1027. Giemsa Method for Staining Gut Flagellates (J. G. Thомson).-

(1) Fix wet sat. perchloride, 2 parts; alcohol, 1 part.

(2) Wash in weak alcohol (circa, 50 per cent.).

(3) Wash in water (aq. dist.).

(4) Wash in water, to which is added a few drops of Gram's iodin.

(5) Wash in 1 per cent. solution of hypo.

(6) Wash in running water.

(7) Stain in Giemsa (1 drop, 1 c.c.) twelve hours.

(8) Differentiate in $(a)$ acetone, 95 per cent.; xylol, 5 per cent., for about five minutes ; then (b) acetone, 70 per cent. ; xylol, 30 per cent. ; (c) pure xylol to clear.

(9) Mount in Canada balsam.

1028. Iron Brazilin Stain (HIckson).--(1) Fix in Schaudinn's solution for fifteen minutes.

(2) Seventy per cent. alcohol for one hour.

(3) Mordant for four hours in a 1 per cent. solution of iron alum in 70 per cent. alcohol.

(4) Stain twelve to sixteen hours in a 1 per cent. solution of brazilin in 70 per cent. alcohol.

(5) Wash thoroughly in several changes of 70 per cent. alcohol.

(6) Dehydrate and mount in balsam.

1029. Fontana's Stain. - (1) Fix in Hugo's fluid for one minute. Acetic acid (B.P.)

1 c.c.

Formalin

20,

Distilled water

100,

(2) Wash thoroughly in tap water.

(3) Mordant. Slide should be gently warmed.

Tannic acid

Carbolic acid

Distilled water

5 grms.

1 c.c.

100 c.c. 
(4) Wash thoroughly in tap-water.

(5) Stain and warm gently: (a) 5 per cent. solution of silver nitrate in water; (b) ammonia. Add the ammonia until the precipitate redissolves in excess of ammonia. Then add more silver nitrate drop by drop until the precipitate reappears and remains constant.

(6) Wash thoroughly in tap-water.

(7) Dry rapidly in air.

1030. Sphærozoa.-Brandt (Fauna u. Flora Golf. Neapel, xiii, 1885 , p. 7) fixes with chromic acid of 0.5 per cent. to 1 per cent. (half an hour to an hour), or with a mixture of equal volumes of sea water and 70 per cent. alcohol with a little tincture of iodine for a quarter to half an hour, or with a 5 to 15 per cent. solution of sublimate in sea water.

KarawaIew (Zool. Anz., xviii, 1895, p. 286) fixes Aulacantha for twenty-four hours in equal parts of strong liquid of Flemming and acetic acid, and hardens for several days in pure liquid of Flemming.

See also Lo Bianco, § 1021.

1031. Sporozoa.-WASIELEWSKI (Sporozoenkunde, Jena, 1896, p. 153) studies them living in their natural medium, or in normal salt solution, or in a medium composed of 20 parts white of egg, 200 of water, and 1 of common salt. He fixes Gregarinæ and Coccidia with osmic acid, sublimate, or picro-sulphuric acid, and Myxosporidia with liquid of Flemming.

Schaudinn (Zool. Jahrb., Abth. Anat., xiii, 1900, p. 197) fixes Coccidia with a mixture of 2 parts of saturated aqueous sublimate and 1 of absolute alcohol, with, if desired, a trace of acetic acid.

Stempeli (Arch. Protistenk., xvi, 1909, p. 389) fixes caterpillars infected with Nosema in 2 parts of saturated sublimate with 1 of alcohol and a little acetic acid, and stains sections for as much as four days in Giemsa's mixture, rinses with alcohol and passes through xylol into balsam.

LÉGER (ibid., iii, 1904, p. 311) fixes cysts for a minute in " acetic sublimate," puts for a minute into absolute alcohol, and stains as a smear with hæmalum or iron hæmatoxylin.

Brasil (Arch. Zool. Expér., 4, iv, 1905, p. 74) fixes them for twenty-four hours in a mixture of 1 grm. picric acid, 15 c.c. acetic acid, 60 c.c. formol and 150 c.c. alcohol of 80 per cent., and stains paraffin sections in iron hæmatoxylin followed by eosin and orange G., or Lichtgrün and picric acid.

1032. Hæmatozoa.-Grassi (Att. Accad. Lincei, iii, 1900, p. 357) demonstrates the Malaria-parasites in the intestine, body-cavity and salivary glands of Anopheles by treating them with normal salt 
solution containing 2 per cent. of formol (pure formol produces swellings), or in a mixture of $1.5 \mathrm{grm}$. of salt and 250 c.c. of water with the white of an egg. He fixes with sublimate, makes paraffin sections, and stains with hæmalum or iron hæmatoxylin. He stains the Sporozoïtes by. making cover-glass preparations, which are allowed to dry, put for twenty-five minutes into absolute alcohol, and stained by the process of Romanowsky, $\$ \S 784,1008$.

For minute instructions for the application of this process to sections, see Giemsa, Deutsch. med. Wochenschr., xxxvi, No. 12, 1910 ; and Schuberg, ibid., xxxv, No. 40, 1909 (Zeit. wiss. Mik., xxvii, 1910 , pp. 160, 161 and 513).

For clinical methods, see CoLes, The Diseases of the Blood, London, J. and A. Churchill, 1905.

Bradford and Plimmer (Quart. Journ. Micr. Sci., xlv, 1902, p. 452) fix Trypanosomes in vapour of equal parts of acetic acid and 2 per cent. osmic acid, or with GuLland's formol and absolute alcohol, and stain with methylen blue and eosin, and mount in turpentine colophonium.

Hindle (Univ. Calif. Pub. Zool., vi, 1909, p. 129) makes smears on cover-glasses coated with albumen, fixes for five minutes in liquid of Flemming, passes through water up to absolute alcohol, then for ten minutes into alcohol of 80 per cent. with a good proportion of iodine in potassic iodide, then into 30 per cent. alcohol, and stains with iron hæmatoxylin or safranin, then with polychrome methylen blue, and lastly with UNNA's orange with tannin, and gets quickly through alcohol into xylol and balsam.

Mrnchin (Quart. Journ. Micr. Sci., liii, 1909, p. 762) makes coverglass smears, fixes them with vapours of osmic acid (with or without acetic acid), and mounts them dry, or in balsam after fixing in liquids and various stains, amongst these that of TworT. Halfsaturated solutions of neutral red and Lichtgrün are mixed, the precipitate dried and dissolved to about $0 \cdot 1$ per cent. in methyl alcohol with 5 per cent. of glycerin. Three parts of this are diluted with 1 of water, the smears stained for an hour, differentiated with UNNA's glycerin-ether, and mounted in balsam. This stain works best after fixation with sublimate.

Policard (C. R. Soc. Biol., lxviii, 1910, p. 505) stains Trypanosomes intra vitam by adding a drop of concentrated solution of neutral red to the edge of a drop of blood spread between slide and cover.

1033. Flagellata.-Lauterborn (Zeit. wiss. Zool., lix, 1895 p. 170) fixes Ceratium for about ten minutes in liquid of Flemming, puts into alcohol for twenty-four hours, brings back into water, 
bleaches if necessary with hydrogen peroxide, and stains with picrocarmine or Delafield's hæmatoxylin. He also embeds in paraffin, §1022, and stains sections with iron hæmatoxylin.

ZaChaRIAS (Zool. Anz., xxii, 1899, p. 72) fixes Uroglena, etc., with a mixture of 2 volumes saturated aqueous solution of boracic acid and 3 of saturated sublimate.

1034. Stains for Flagella.-The Romanowsky stain will give a red stain of the flagella of some forms.

The method of LöFrLer (Centralbl. Bakteriol., vi, 1889, p. 209 ; vii, 1890 , p. 625 ; Zeit. wiss. Mik., vi, 1889, p. 359 ; vii, 3, 1890, p. 368 ; Journ. Roy. Mic. Soc., 1889, p. 711 ; 1890, p. 678) is as follows. To 10 c.c. of a 20 per cent. solution of tannin are added 5 c.c. of cold saturated solution of ferrous sulphate and 1 c.c. of (either aqueous or alcoholic) solution of fuchsin, methyl violet, or "Wollschwarz." Cover-glass preparations are made and fixed in a flame in the usual way, special care being taken not to over-heat. Whilst still warm the preparation is treated with mordant (i.e. the above-described mixture), and is heated for half a minute, until the liquid begins to vaporise, after which it is washed in distilled water and then in alcohol. It is then treated in a similar manner with the stain, which consists of a saturated solution of fuchsin in anilin water (p. 177), the solution being preferably neutralised to the point of precipitation by cautious addition of $0 \cdot 1$ per cent. soda solution.

See also Liebetanz, Arch. Protistenk., xix, 1910, p. 23.

Bunge (Journ. Roy. Mic. Soc., 1894, p. 640 ; Zeit. wiss. Mik., xiii, 1896, p. 96$)$ makes the mordant by mixing 3 parts of the tannin solution with 1 of liquor ferri sesquichlorati diluted twentyfold with water, and lets the mixture ripen for some days exposed to the air, or (Journ., 1895, pp. 129, 248) adds to it a few drops of hydrogen peroxide, until it becomes red-brown, when it is shaken up and filtered on to the cover-glass and allowed to act for a minute. The cover-glass is then mopped up and dried, and stained with carbol gentian.

Koerner and Fischer (quoted from Encycl. mik. Techn., p. 514) make the mordant with 2 parts of tannin, 20 of water, 4 of ferrous sulphate solution of $1: 2$ strength, and 1 of saturated alcoholic solution of fuchsin. Warm, let it act for a minute, rinse and stain with anilin-water-fuchsin, or carbol fuchsin.

Similarly Eluis (Centralb. Bakt., xxi, 1903, p. 241 ; Journ. Roy. Mic. Soc., 1904, p. 249), but staining with Säureviolett, 1 part to 75 of alcohol and 75 of water.

Peppler (Centralb. Bakt., xxix, 1901, p. 376 ; Zeit. wiss. Mik., 
xviii, 1901, p. 222) makes the mordant with 20 parts of tannin in 80 of water, and 15 parts of 2.5 per cent. chromic acid added gradually. This mordant will keep for months.

Rossi (Arch. per le Sc. med., xxiv, 1900, p. 297 ; Zeit. wiss. Mik., xviii, 1901, p. 226) takes for the mordant a solution of 25 grms. of tannic acid in 100 of caustic potash of $0 \cdot 1$ per cent., which will keep indefinitely. The stain is Ziehl's carbol fuchsin, § 289. Coverglasses are prepared with a drop of culture, dried, and treated with 1 drop of the mordant and at the same time 4 to 5 of the stain, allowed to remain for fifteen to twenty minutes, washed, and mounted. See also Centralb. Bakt., xxxiii, 1903, p. 572 (Zeit. wiss. Mik., xix, 1903, p. 517).

Gemelli (Centralb.,.xxxiii, 1903, p. 316 ; Zeit. wiss. Mik., xix, 1903 , p. 516) mordants for ten to twenty minutes in 0.025 per cent. permanganate of potash, rinses and stains for fifteen to thirty minutes in a mixture of 20 parts 0.75 per cent. aqueous solution of calcium chloride and 1 part of 1 per cent. neutral red solution.

A method of Pitfield is described by Kendall, Journ. app. Mic., v, 1902, p. 1836 (Journ. Roy. Mic. Soc., 1902, p. 502). The mordant consists of 10 parts of 10 per cent. tannin solution, 5 parts of saturated sublimate solution, 5 of saturated solution of alum, and 5 of carbol fuchsin. Mordant for a minute with heat, and stain with a mixture of 2 parts saturated aqueous solution of gentian violet with 10 of saturated solution of alum.

VAN ERMengem (Journ., 1894, p. 405) fixes for a few minutes with a mixture of 1 part 2 per cent. osmic acid, and 2 parts 10 to 25 per cent. solution of tannin, washes, treats with 0.25 to 0.5 per cent. solution of nitrate of silver, then for a few seconds with a mixture of 5 parts gallic acid, 3 of tannin, 10 of acetate of soda, and 350 of water, then puts back again into the silver for a short time, then washes and mounts.

See also Stepinens, ibid., 1898, p. 685, and Gordon, ibid., 1899, p. 235, and the methods of Trenkmann (Centralb., vi, 1889, p. 433 ; Zeit. uiss. Mik., vii, 1890, p. 79); Brown (Journ. Roy. Mic. Soc., 1893, p. 268); Julten (ibid., 1894, p. 403) ; Sclavo (Zeit. wiss. Mik., xiii, 1896, p. 96); Hessert (ibid., p. 96) ; MUIR (Journ. Roy. Mic. Soc., 1899, p. 235); MCCrorie (ibid., 1897, p. 251; he stains for two minutes in a mixture of equal parts of concentrated solution of night-blue, 10 per cent. solution of alum, and 10 per cent. solution of tannic acid): ZetrNow (ibid., 1899, pp. 662, 664); Morton (ibid., 1900, p. 131); Welcke (ibid., p. 132); Levaditi, C. R. Soc. Biol., lix, 1905, p. 326 (for Spirochate pallida, RAMON's neurofihril stain); MEIRowSkx, Münch. med. Wochenschr., lvii, 1910, No. 27; KalB, ibid., No. 26 (Zeit. wiss. Mik., xxix, 1912, pp. 123, 124; both for Spirochoete). 


\section{CHAPTER XXXVII.*}

THE CULTIVATION OF TISSUE " IN VITRO" AND ITS TECHNIQUE.

1035. A culture of tissue consists of a special medium, natural or artificial, such as lymph or plasma, inoculated with small fragments of living tissues, and is characterised by an active growth of the cells of the fragment into the nutrient plasmatic or lymph medium. Cells wander out into the latter, and may live up to twenty days without any signs of necrobiosis.

The cultivation of tissues outside the body was first accomplished successfully by Ross Harrison, of Johns Hopkins University in the year 1907. This brilliant observer has demonstrated by a series of experiments that fragments of nervous tissue of the frog embryo, covered with fluid from the lymph sac of an adult frog, show growth of long nerve fibres (Harrison, Proc. Soc. Exper. Biol. and Med., iv, 1907, p. 140). Alexis Carrel, at about the same time, had been studying the laws of redintegration of tissues, and adapted Harrison's technique to mammalian tissues. M. Burrows, a pupil of Ross Harrison, at this period began to work on tissue-culture, and first used blood plasma instead of lymph. Subsequently Burrows adapted the technique of Harrison to the cultivation of tissues of the chick. In September, 1910, Carrel and Burrows, working in conjunction at the Rockefeller Institute, succeeded in cultivating, in vitro, the adult tissues of mammals, and thus began a series of contributions which have taught us many valuable facts regarding senesence and rejuvenescence and the pathology of tissues (CARRel and BuRRows, Jour. Exper. Med., xiii, No. 3, 1911).

Two methods of tissue-culture may be distinguished :-

(1) Hanging-drop or smear cultures (Harrison).

(2) Large plate cultures (Carrel and Burrows).

The former are useful for direct observation of living growing cells, the latter can be studied when fixed and cut into sections.

There has been a great deal of work done on tissue-culture, but most of it has been carried out by vertebrate pathologists and histologists. There seems little doubt that this field is a most promising one for zoologists as well as histologists. Many problems of gametogenesis and general cytology might be settled by recourse to tissue-culture, especially by the application of such methods to the cells of invertebrate animals, whose cytology had previously been examined in detail with the aid of modern techniques. 


\section{Precautions to Insure Complete Sterilisation of Apparatus.-}

It cannot be too strongly emphasised that the utmost precautions must be taken to insure complete sterility of all apparatus. Bacterial infections of the cultures are fatal. The worker who is not familiar with the minute precautions taken by surgeons and bacteriologists in sterilising instruments, apparatus, etc., is advised to become so before undertaking tissue-culture work. $A$ rigid asepsis is necessary for the preparation of any tissue-culture. These words are addressed especially to the zoologist who may undertake tissue-culture work ; dirty floors and benches, dirty walls and garments, and casual methods will all contribute towards failure. A clean, warm room should be set aside for making the cultures, another for making the various plasmas, and, if possible, another for incubators and incubator microscopes. The ordinary zoological or botanical laboratory is generally unsuitable for such delicate work.

Dr. Drew informs me that whether in vitro culture be carried out in a special laboratory or not, it is advantageous to use a specially constructed glass chamber to shield the cultures from chance con-• tamination. Such a chamber is supplied by Hearson's (§ 11), or can be made by any carpenter. The apparatus consists essentially of a glass box in a wooden or metal framework measuring about 2 feet 6 inches in length, 2 feet in width, and about 1 foot in height. The top is hinged so as to allow ready access to the interior for cleaning, etc. The side facing the worker is made of wood, with either two small wooden doors or a piece of thick sheet rubber pierced so as to allow the easy entrance of the hands. The box should have glass racks to contain the hollow ground slides and a glass table for the cover-glasses. The slides are cleaned and stored in absolute alcohol ; they are removed from this by means of forceps and are flamed in a Bunsen and quickly transferred to the glass racks; cover-glasses are cleaned in acid bichromate, washed in water till free from all trace of the acid, rinsed in distilled water, then in absolute alcohol, and stored in ether. They are removed by means of forceps and flamed and placed on the previously sterilised glass table. Here both slides and cover-glasses are protected from all falling dust, and can be manipulated easily with the hands through the openings in the case. The majority of failures occur through infection taking place, and the glass chamber reduces such a possibility very greatly.

103\%. Simple Culture Technique by means of Frog Lymph (HARRIson, op. cit., 1907).-Pieces of embryonic tịsues of frog embryos 
about $3 \mathrm{~mm}$. long are dissected out with clean instruments, removed to a coverslip, and covered by a drop of lymph freshly drawn from one of the lymph sacs of an adult frog. The coverslip is inverted over a hollow slide, and the rim sealed with paraffin wax. When reasonable aseptic precautions are taken, tissues will live under these conditions for from a week up to four weeks.

GoLDschmidT (Arch.f. Zellf., 1916) has investigated the sperm cells of Lepidoptera by tissue culture methods.

1038. Technique for Culture of Mammalian Tissues.-Preparation

- of the Animal for Procuring Plasma.-The animal is anæsthetised with ether, and must be kept just at the correct depth of anæsthesia. A. J. Walton (Journ. Path. and Bact., xviii, 1914), from whose article the following paragraphs are partly culled, recommends for this purpose a wide-necked bottle, with a closely-fitting cork pierced with two tubes of wide bore, both of which pass down to within $\frac{1}{2}$-inch of the surface of the ether placed in the bottle; one tube communicates with a tin funnel having a mackintosh flange; this tube also has a side tube, and the other tube is open to the air.

The animal's head is placed in the funnel, and, when the side tube is clamped, breathes air and ether vapour. By clamping either the side or the short tube the amount of air or ether can be suitably controlled.

The hair of the throat, is either shaved off, or removed by the application of a solution of sodium sulphide 3 ii ad $\mathrm{Oi}$, which rapidly dissolves it. The skin is then sterilised by painting with a 2 per cent. solution of iodine in spirit.

1039. Preparation of Instruments, etc.-Previous to the operation the following apparatus is sterilised:- Short test tubes, $2 \frac{1}{2}$ inches in length ; corks kept in stoppered bottles, to fit these tubes ; small glass cannulæ in olive oil ; three glass tubes, 3 inches by 1 inch ; several narrow-bore pipettes which are kept corked in the lastmentioned tubes, and which just before the operation on the animal, are removed from the tube by means of sterile forceps, dipped in a deep tin of molten paraffin, everted to allow the paraffin to run out, and when cool placed in another sterile tube.

Two small sterile test tubes, as mentioned above, are similarly treated in paraffin, and should be corked with sterile corks as soon as cool. These two tubes are placed in two other larger tubes made to fit the centrifuge, and ice is packed between.

Just before the operation, the instruments and some rubber teats to fit the pipettes are boiled in water for ten minutes. 
1040. Removal of Plasma.-When dogs, rabbits, cats, chickens, guinea pigs and rats are used, the carotid artery is ordinarily selected; an incision is made in the mid-line in the neck, and as soon as the skin is divided the edges are clipped to sterile towels. The carotid is exposed, its distal end ligatured and its proximal end clamped. A little sterile oil is placed on the artery, which is opened, and one of the cannule from the sterile oil is taken, inserted and tied in position; on releasing the clamp the blood flows freely. This is collected in the paraffined test tubes for centrifuging. The tubes should be in their ice-jackets; they are corked at once and immediately centrifuged for about five minutes ; they are then removed and placed in an ice box at $0^{\circ} \mathrm{C}$.

For human plasma one may remove blood from a vein by means of a needle pipette sterilised in olive oil.

After the centrifugilisation the supernatant plasma may be removed with pipettes coated in paraffin (§ 1039). It should be used immediately for making the cultures, but can be preserved for some time in a fluid condition if kept very cool. Chicken plasma can be so preserved for more than a week, human and dog plasma for a few days, while rat plasma always coagulates after a few hours (CARrel and Burrows, Journ. Exp. Med., 1911) ; when coagulation takes place the plasma is no longer of use.

Carrel and Burrows (Jour. Exper. Med., xiii, 1911) found that dilution of the plasma had a marked influence on the rate of growth of splenic tissue; normal plasma is not the optimum medium for growth of tissue ; the most favourable plasma for spleen culture contains twofifths distilled water, and slightly less for liver and heart, and generally for skin, too.

1041. Preparation of Tissues.-The tissues for cultures should be in normal condition, and are best when taken from the living animal or immediately after death. Positive results can still be obtained, however, when the tissues have been deprived of circulation for more than thirty minutes.

With a cataract knife and a fine needle, a small fragment of tissue is dissected from the animal and placed on a glass plate; the piece is rapidly cut into smaller pieces about the size of a millet seed and transferred to a perfectly clean sterile coverslip. This process must be carried out rapidly because some tissues die in even as short a time as ten seconds when exposed to the air (e.g., thyroid). To prevent this the tissue may be dissected in serum or Ringer.

1042. Preparation of Cultures. - For cultures of the hanging-drop type one uses a hollow ground slide of a sufficient depth to prevent 


\section{THE CULTIVATION OF TISSUE "IN VITRO."}

the drop of plasma from touching the bottom. The tissue is quickly placed on a coverslip, 2 drops of plasma from the paraffined pipette (§ 1039) are added and evenly and thinly spread around the tissue; this must be done before coagulation occurs. If the plasma is not spread evenly the tissue-culture will grow in many planes, and will be less easy to observe, manipulate, and to fix and stain. When the plasma is spread out the cover glass is inverted over a hollow slide of suitable depth; a little sterile vaseline may be placed at each side of the cell to assist adhesion preparatory to waxing down.

The latter process is done by brushing molten paraffin around the edge of the coverslip, and on the slide, to prevent drying. Immediately this has been done the preparation is transferred to an incubator. Carrell and Burrows use a small portable electric incubator which is used for carrying the finished cultures to a bigger incubator in the observation room.

For the large plate cultures the same technique is used. Tissue may be rapidly removed, cut into very small fragments, suspended in Ringer, and then spread on the cover of a flat glass-covered (Gabritschewski) box, and covered with plasma. The Gabritschewski boxes after several days' incubation (three to five days), are opened, and the plasmatic jelly cut out into blocks and preserved.

Or, instead of using Gabritschewski boxes, one may make the culture on large black plates, which must then be placed in glass boxes with cotton sponges soaked in water, in order to preserve the proper humidity. The boxes are then carefully sealed with paraffin and kept in such a position that the fluid products of the culture may drain to the bottom.

1043. Subculturing is generally difficult, the technique of cultivating of tissue cells in series being far from perfect. One extirpates a piece of the primary culture at its most active period, and transfers it to a fresh medium, growth often, but not always, beginning anew. Tertiary cultures are made in the same way. CARREL and BURRows (Journ. Exper. Med., xiii, 1911) find that a very good way is to cut out the middle of the old culture around the original piece of tissue, and then fill up the space with new medium. The old cells grow into the new plasma.

1044. Fixation and Staining of Cultures.-CARREL and BURRows (Journ. Exper. Med., 1911) remove the cover-glass to which the culture is adherent, and immerse in corrosive sublimate, acetic acid, or formalin, or the various preparations of chrome salts. Afterwards the culture is stained in hæmatoxylin of Benda, Heidengain or Weigert.

Dr. A. Drew informs me that he has found that the best fixatives 
for in vitro cultures are, 70 per cent. alcohol and 5 per cent. acetic, Flemming, and Bouin. In all cases the cultures on the slip should be first detached from the slide and placed in warm Ringer's solution $37^{\circ} \mathrm{C}$. for five minutes. They are then placed in the fixative. Alcohol-acetic gives the cleanest pictures. Staining is best done by Ehrlich's hæmatoxylin, Delafield's hæmatoxylin, iron hæmatoxylin, carmine or Giemsa. As counterstain either eosin or orange G. may be used.

1045. Artificial Culture Media.-Margaret R. Lewis and W. H. Lewis (Anat. Record, v, 1911, p. 277) have investigated tissue cultures of chick embryo cells made in artificial media. Eighty combinations of $\mathrm{NaCl}$, $\mathrm{CaCl}_{2}, \mathrm{KCl}$ and $\mathrm{NaHCO}_{3}$ and water, to form culture media have been proposed. It was possible to obtain growth in such media, in which either the $\mathrm{CaCl}_{2}$ or the $\mathrm{KCl}$ or the $\mathrm{NaHCO}_{3}$ was omitted, but not when the $\mathrm{NaCl}$ was left out. Such growths continue only for several days, and are never as extensive as those grown in plasma media.

More recently MARgaRet R. Lewis (Contrib. to Embryolog!, ix, 1920, Nos. 27-46) for tissues of chick embryos of four to twelve days' incubation uses "Locke-Lewis" solution (90 c.c. of $\mathrm{NaCl} 0.9$ per cent. $+\mathrm{KCl} 0.042$ per cent., $+\mathrm{CaCl}_{2} 0.025$ per cent., $+\mathrm{NaHCO}_{3} 0.02$ per cent., +10 c.c. of chicken bouillon +0.25 per cent. dextrose). The embryo was removed from the egg and placed in a petri dish containing 20 c.c. of the warmed solution. Pieces of tissue to be explanted were removed, washed through one or more changes of warm medium, and cut with sharp seissors into pieces about $0.5 \mathrm{~mm}$. in diameter; each piece was then placed in the centre of a coverslip, part of the drop drawn off, and the coverslip sealed on to a vaseline ring around the well of a hollow slide. Cultures thus prepared were kept in an incubator at $39^{\circ} \mathrm{C}$, and observations made in a warm box at $39^{\circ} \mathrm{C}$. 


\section{CHAPTER XXXVIII.}

\section{A GUIDE FOR STUDENTS OF MICROTOMY.}

1046. Three Examples for Beginners :- (1) The preparation of whole stained mounts of some small object (Daphnids).

(2) The preparation of sections of the muscle or an organ of a vertebrate.

(3) The preparation of an embryo (or tadpole) for the making of serial sections.

Example I.-From a pond or ditch obtain some water-fleas (Daphnia or Simocephalus); allow the jar to stand for several hours till the suspended material has settled. Capture some of the organisms as follows:- Take a piece of glass tubing some 8 inches in length; place a finger over one end, dip the other end under the water and by taking away the finger, suck up some of the Daphnids into the tube; put your finger over the end of the tube, remove the latter and transfer the organisms to a capsule or watch-glass about 2 inches in diameter. With a clean pipette carefully suck up most of the water, hardly allowing the animals enough to swim in; now add a fixative to kill the organisms (see $\S 2$ ), and to coagulate their protoplasm (§ 29) as rapidly as possible so as to leave the groups of cells forming the organs intact and in situ.

Use corrosive acetic acid ( $\S 63), 2$ per cent. acetic acid in saturated aqueous corrosive. Pour the fixative into the watch-glass or capsule, till it is full (the watch-glass or capsule contains about 15 to 20 c.c.). Place a glass square or plate over the capsule, and leave it for thirty minutes. The organisms become opaque, indicating the coagulation of the proteids of their cells.

With a pipette carefully remove as much of the fixing fluid as possible. Now that the organisms are killed, the mercury salt must be removed; unless the fixative is thoroughly removed, it will form masses of pin-shaped crystals at a later stage when the animals are being mounted in balsam.

To remove the corrosive sublimate, it is necessary to convert it into another substance which may be more easily washed away; this is effected by immersing the animals in some 70 per cent. alcohol which has been coloured light port-wine shade with tincture of iodine $(\S 63)$, whereupon the mercury bichloride becomes mercury iodide, which is very soluble in 70 per cent. alcohol. The iodine and alcohol mixture 
should be used until it no longer loses its colour, which indicates excess of iodine. The whole process should last several hours and may be carried on overnight.

The iodine and 70 per cent. alcohol are poured away, and the animals washed for several hours (a minimum of two) in at least two changes of 70 per cent. alcohol to remove as much of the iodine as possible. The objects are then transferred to 50 per cent. alcohol for one halfhour, then into 30 per cent., for the same time. They are brought down these grades in order that shrinkage may not occur when they are being transferred to stains containing little alcohol, or none at all.

Two stains may be tried, Mayer's acid hæmalum ( $\$ 248$ and 249), and Grenacher's alcoholic borax carmine ( $\$ 213$ and 233). The time that both these stains should be used depends almost entirely upon the accessibility of the cells of the object to the stain. Daphnids are covered by a chitinous shell, which though delicate tends to prevent instant penetration. It is a good thing to leave the animals in the stain for about five hours at least, and overnight preferably.

Take two clean eapsules, pour into one about 10 c.c. of borax carmine, into the other a similar quantity of the hæmalum. With a camel-hair brush or a pipette transfer some of the organisms to the stains and leave as directed above. See that the capsules are securely covered.

After some hours in the stain, the latter is poured away, and the process of differentiation ( $(203)$ is begun. The object of differentiation is to wash away superfluous stain from certain organs or parts of organs, in order that a contrast in depth of colour may be obtained in the various other organs and tissues. Both borax carmine and Mayer's acid hæmalum may be differentiated in acid alcohol (4 to 6 drops of $\mathrm{HCl}$ to 100 c.c. of 70 per cent. alcohol), which should generally be allowed to act at least for as long as the stain has been used, and, if necessary, longer. In both cases when differentiation has reached the right stage, the objects examined under a microscope have a transparent appearance, and such parts as the viscera and muscles should be well contrasted.

The borax carmine specimens are washed out for several hours in neutral 70 per cent. alcohol. They are then upgraded to 90 per cent. and absolute alcohol, two hours in each, or overnight in absolute alcohol, and cleared in cedar wood or clove oil for at least two hours, and then mounted in xylol balsam.

The hæmalum specimens have to be brought to an alkaline solution in order to "blue" the stain, and to get rid of all acid. Some workers "blue" the stain in 70 per cent. alcohol made slightly alkaline with ammonia or bicarbonate of soda, but the best results are obtained by downgrading the objects to tap-water, which is allowed to run over them gently till they go quite blue, which should occur for small objects within an hour. The animals are then gradually upgraded through 30 , 50,70 and 90 per cent., to absolute alcohol, and cleared as above described for borax carmine specimens. 
In order to obviate the differentiation stage, one may dilute both the borax carmine and the acid hæmalum till they are about one-third or one-half as strong; dilution of the borax carmine may be carried out with 50 per cent. alcohol (not methylated spirit) and with distilled water in the case of hæmalum. In these solutions the animals remain till sufficiently stained. But the best results are got by the overstaining and differentiation method.

1047. Example II.-From a frog remove a large leg or thigh muscle, and cut it into two pieces about as big as the nail of the little finger. If desired, the liver, a halved testis, or a kidney may also be used.

Transfer the material to a capsule containing at least 20 c.c. of Zenker's or Helly's fluids ( $\$ \$ 73,684$ ). Leave till next morning, and wash in running water under the tap for at least three hours, preferably overnight, then transfer to 50 per cent. alcohol for an hour ; then to 70 per cent. alcohol containing enough tincture of iodine to give the solution a light port-wine shade. Add more iodine as the colour disappears, prolonging the treatment overnight for large pieces. Pour away the alcohol, and add pure 70 per cent., in which the material is washed at least three hours. Transfer to 90 per cent. for several hours and leave in absolute alcohol overnight. Next morning it is safest to give the material another hour in a fresh change of alcohol absolute. Pour away a good deal of the alcohol and add about the same quantity of xylol or cedar oil. Shake, leave half an hour, and then transfer the material to pure xylol or cedar oil; leave half an hour. Pour away some of the xylol, either add chips of hard wax to cover the tissue, or add some of the stock xylol and wax mixture. Leave an hour in thermostat on the upper shelf, pour off, and add molten pure wax; leave one or two hours on the bottom shelf. Embed blocks (§§ 142, 143).

1048. Example III. Preparation of an Embryo for Serial Sections.Fix in Bouin's fluid corrosive acetic or picro-nitric, overnight ( $\$ 110$, $63,97)$. In the case of the first and last mentioned fixatives, the embryo is afterwards transferred to 30 per cent. alcohol (half-hour), 50 per cent. (two hours), and then washed for a day in several changes of 70 per cent. The corrosive acetic fixed specimens are similarly treated except that at this stage iodine solution is added to the 70 per cent. (or this may be done in 90 per cent.) alcohol till the corrosive sublimate is removed. Leave overnight in 90 per cent. alcohol (or at least three or four hours), and at least six hours in two changes of absolute alcohol (preferably overnight). De-alcoholisation and clearing must be done carefully as directed in $\S 591$, p. 269 . It is a good plan to bring embryos from absolute alcohol, through several gradually strengthening mixtures of alcohol and cedarwood oil-to pure cedar-wood oil, and then wash out in benzole. Embed in wax as described in $\S 591$, generally about one hour in benzol and wax, and two hours in pure wax. Embed blocks $(\S \S 142,143)$. Now read $\S \S 144$ to 151 . 
1049. General Plan of Procedure Applicable to Histological Specimens.

Anæsthetise animal, kill $1 \mathrm{t}$, quickly take out organ, cut pieces $1 \mathrm{~cm} \times 1 \mathrm{~cm} \times \frac{1}{\mathrm{a}} \mathrm{cm}$.

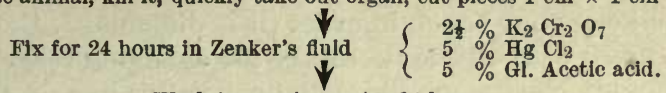

Wash in running water 24 hours.

Freezing method.

Paraffin method.

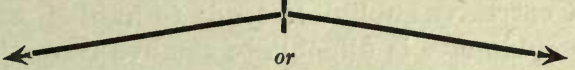

Preserve in 5\% Formalin

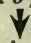

Wash in water

Impregnate with gum

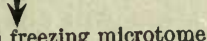

Cut sectlons with freezing microtome

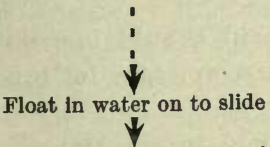

Stain with Picro-carmine 15 minutes Drain off and wipe away the stain around

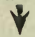

Mount in Farrant's medium.

\section{Celloidin method.}

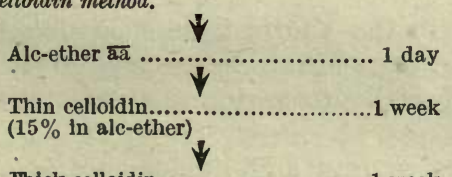

Thick celloidin

$(30 \%$ in alc-ether)

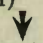

After evaporation, mount on block of vulcanised flbre

$\checkmark$

Harden celloldin in chloroform.........1-2 hours and then in $80 \%$ alc. 1-6 hours<smiles>[AlH2]</smiles>

Cut with razor (oblique) wetted with $80 \%$ alc.

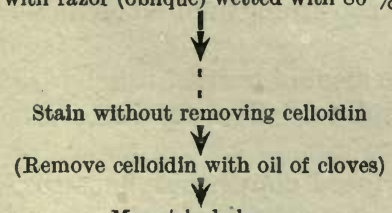

Mount in balsam.
Pass through increasing strengths of alc.

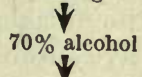

Remove $\mathrm{Hg}$ deposit with lodine in $80 \%$ alcohol (Preserve in $80 \%$ alcohol)

\section{$\stackrel{y}{\forall}$}

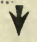

Pass through $90 \%$ alcohol.................1 day Dehydrate in abs. alc.

y

Clear in xylol or chloroform...From 1 to 2 hours $\sqrt{1}$

Pass through xylol satd, with paraffin wax 1 hour $\checkmark$

Impregnate with paraffin at $52^{\circ} \mathrm{C} . \ldots . .2$ hours and

Embed and make blocks with

Cut sections with microtome

!

[1 ${ }^{\circ}$ Apply fixative to slide or glycerine and albumen water]<smiles>[CH][CH]</smiles>

$2^{\circ} \quad$ Float section on drop of water on slide Warm gently to open out the section Wlpe away excess of water and dry in air

$3^{\circ} \quad$ Remove paraffin with xylol

$4^{\circ}$ Remove xylol with abs. alc.

$5^{\circ}$ Pass through 90 and $70 \%$ alcohol to water

$8^{\circ}$ Stain in Hæmatoxylin, etc., 5-15 minutes, etc.

$7^{\circ}$ Wash in water, 5 minutes -1 hour

Counter-stain in Eosin-1 minute, etc.

$9^{\circ}$ Remove excess with $90 \%$ alcohol

Dehydrate with abs, alc.

Clear in clove oil or xylol Mount in balsam. 
1050. General Rules and Hints for Students.-(1) Keep all your bottles and capsules as clean as possible.

(2) Try to keep your bench in order (it is difficult, J. B. G.).

(3) Keep notes of the time necessary for changing reagents.

(4) Thoroughly clean your slides and coverslips in acid alcohol before using. See addendum.

(5) Note that corrosive sublimate tends to harden material.

(6) Corrosive sublimate is difficult to remove from tissue unless you use iodine. If not properly removed you will find numerous pin-shaped crystals in the finished sections. $§ 63$.

(7) Corrosive sublimate attacks the surface of steel and other metals. Use quills, or wooden needles for manipulating tissue in sublimate.

(8) Watery stains after picric acid fixation will cause maceration if prolonged. $§ 93$.

(9) Unless very well washed out, picric acid should not be used in conjunction with thionin or toluidin blue. Precipitates form. Certain other dyes do likewise.

(10) Osmic acid crystals should be dissolved in the purest distilled water. Wash the tube with distilled water before you break it, removing label. Wash out capsules and bottles for osmic acid solutions in distilled water. Keep solutions in shade or dark. $\$ 27$.

(11) Osmic acid tends to harden yolk and certain other cell materials. The vapour of osmic acid is injurious to the eyes and nose.

(12) Osmic acid and fixatives containing it inhibit staining, but if necessary you can induce osmicated material to stain in delicate dyes by bringing sections down to distilled water and treating in a 25 per cent. solution of permanganate of potash for a short time. Permanganate also decolorises sections. See page 31 .

(13) Nitric acid tends to soften chitin and yolk, but it may inhibit staining a little. $\$ 97$.

(14) Imbed material in paraffin in the shortest time possible, for materials left in the thermostat longer than necessary go hard, especially from xylol ; this refers especially to vertebrate material and yolky embryos.

(15) Alcohol and chloroform dissolve fats and lipoids, acetic acid dissolves away lipins. Vegetable oils dissolve fats less readily than xylol or chloroform. Read $\$ 120$ et seq.

(16) Strong alcohol is bad for the finger nails and skin.

(17) When diluting stains with alcohol, use solutions made up by breaking down pure absolute alcohol. Do not use methylated spirit, as this generally precipitates the stain.

(18) You can soon learn to tell roughly the strength of alcohols by the smell.

(19) Don't use the dregs of the absolute alcohol bottle for dehydrating anything. The dregs are no longer absolute. Keep a waste alcohol bottle for used liquid.

(20) Some workers add a little bag of fused copper sulphate to their store bottles of absolute. This keeps the alcohol dehydrated.

(21) After fixation, when dehydrating and embedding a piece of tissue, an egg or an embryo, it is at its softest when in weak alcohol, and its 
hardest when in xylol or a clearing oil. Flatten or otherwise manipulate a fixed object, while it is still in weak alcohol, or it will break up ; but some objects may be dissected successfully in clove oil. $\$ 122$.

(22) Cells alter soon after death : formalin fixation is the best for corpse material. Carefully note $\S 31$.

(23) The organs of animals over-anæsthetised by chloroform or ether are often spoilt (especially in the vicinity of large blood vessels) and are sometimes useless even for general purposes. $§ 12$.

(24) Keep balsam or colophonium jar in the dark, or paint it black outside. Acid balsam soon removes stains from tissue; acid balsam is the micrologists' bête noire. §443a.

(25) After Zenker fixation sections may overstain in eosin.

(26) If finished sections have crystals in them this is due to improper washing out of fixative, or stain.

(27) Formaldehyde gas dissolves in water up to 40 per cent. The commercial formalin is acid and must be neutralised with magnesium or sodium carbonate kept in a little bag in the stock bottle. $\S 108$.

(28) Formaldehyde gas is injurious to the skin and mucous membrane of nose.

(29) If after staining in delicate dyes (e.g., methyl green), all the colour keeps coming out of the sections during passage through alcohols, try the following method:-Wipe superfluous water from around the sections, and dehydrate by dropping acetone on sections : then plunge into a jar of half acetone, half xylol, then pure xylol.

(30) For clearing embryos or pieces of tissue for whole mounts, cedarwood oil is better than xylol. $\$ 120$.

(31) If bubbles get under the coverslip they can often be removed by gently warming, or by placing slide under bell jar of an exhaust pump.

(32) If after mounting an object in balsam white or black lines and blotchy areas appear, this means that dehydration was not complete. Bring back through xylol to absolute alcohol.

(33) When, after embedding, the block is set aside for a time and it is found that the object is surrounded by a halo of white wax, this means that all the clearing oil was not removed and is now exuding from the object. Re-imbed in pure wax.

(34) When, after embedding, the material seems soft and tends to fall out of the wax, this indicates that dehydration was not complete, and possibly also that the time in pure wax was not long enough. Without efficient dehydration it is impossible to make good sections.

(35) If when cutting the sections curl up, it means that either the knife is blunt or the material has been overhardened during imbedding. Occasionally an incorrect slope of the knife may be the cause of curling.

(36) When the sections will not form a ribbon, this means that either the wax is too hard or the slope of the knife is not correct. If the wax is hard, place 1 drop of soft wax on each side of the block and flatten it out with a warm knife. Read carefully pp. 83 to 90 .

(37) The broad side of a block should be parallel to the knife.

(38) Some people use miniature drums for rolling up the wax ribbon. Laying them on a piece of foolseap does quite well. Avoid sticky 
paper. If sections accidentally adhere you can often release them by cautiously wetting the paper with absolute alcohol.

(39) Before placing sections on a slide, write with a diamond pencil the number of the slide and the material used. At a pinch, a glass wax-pencil may be used instead-

(40) If you have not used a diamond, it is always possible to tell on which side of the slide the section lies, simply by slightly tilting the slide and observing the shadow thrown on the other side of the glass.

(41) Finally, if your first attempts are failures, do not be discouraged - even the most skilful microtomists generally produce atrocities at their first attempts. Try again ! 


\section{APPENDIX.}

1051. Chemicals, Stains, and Apparatus.-Addresses of British firms from which it is recommended that these be obtained are given in $\S 11$.

1052. Cleaning Slides and Covers. - New ones should first be soaked in one of the following liquids: strong sulphuric, hydrochloric or nitric acid, or aqua regia, or a mixture of an ounce each of sulphuric acid and bichromate of potash with from 8 to 12 ounces of water, then washed first with water and lastly with alcohol, and dried with a clean cloth.

For used ones, if a balsam mount, warm, push the cover into a vessel with xylol or other solvent of the mount, and put the slide into another vessel with the same, leave for a few days, and then put into strong alcohol. If this is not sufficient, treat as for new ones. Some persons boil in lysol, which I do not find efficacious.

For the final treatment, see p. 121.

1053. Gum for Labels.-Labels stuck on glass often strip off. This may be avoided (Marpmann, Zeit. Angew. Mik., ii, 1896, p. 151 ; Journ. Roy. Mic. Soc., 1897, p. 84) by means of the following adhesive : 120 grms. of gum arabic are dissolved in a quarter of a litre of water, and 30 grms. of gum tragacanth in a similar quantity. After a few hours the tragacanth solution is shaken until it froths, and mixed with the gum arabic solution. Strain through linen and add 150 grms. of glycerin previously mixed with $2 \frac{1}{2}$ grms. of oil of thyme.

PeIRce (Journ. app. Mic., ii, 1899, p. 627 ; Journ. Roy. Mic. Soc., 1900, p. 404) finds that if the end of the slide be painted with a thin solution of balsam, it may be written on with ink when dry, and the record preserved by a second coat painted over it.

For other receipts see early editions. 


\section{INDEX.}

Names like Lo Bianco are given under the latter half of the name.

Abderhalden, 357

Acalephæ, 523

Acanthocephali, 513

Acephala, injection, 503

Acetate of lead, brain, 404

of potash, medium, 219

Acetic acid, fixation, 51 alcohol and sublimate, 53

and alcohol, 52

bichromate, 41 decalcification, 252

alum, carmine, 137

Aceto-carmine, 138

Acetone, 168

for dehydration, 4

fixation (Lucidol), 47, 59

and formol, 64, 494

and sublimate, 47

-chloroform, for narcotisation, 14

Achucarro, neuroglia, 490

Acid fuchsin, 171

for mitochondria, 320,321

and malachite green (Pianese), 175

and methyl green, 324

myelin, 452

and orange G, 172

Acid hæmalum, 153

hæmatoxylin, 155

magenta, 171

rubin, 171

Acidic dyes, 120

Acidophilous tissue, 128

Actinians, narcotisation, 13,521

Adamkiewicz, myelin, 452

Adenoid tissue, 249

Adsorption, 120, 122

Adurol, 469

Agar, sections between coverslips, 307

Agar-Agar, growing amœbæ on, 527

Agassiz and Whitman, 281

Agduhe, Bielschowsky, 431

Aguerre, 482

De Albertis, neuroglia, 485

Albumen, method of mounting sections, 113

mercurial mounting medium, 220

removal of from eggs, 260 et seq., 283 , 284

Alcohol, for dehydration, preservation, 4

narcotisation, 13

fixation, 56, 58

for maceration, 244
Alcohol-contd.

absolute, 58

table for dilutions, 57

and nitric acid for decalcifying, 253

Alcoholic, mercury bichloride, 46

hæmatoxylin, Apáthy and de Groot, 156

Heidenhain, 543

Dobell, 544

cochineal, 144

Alcyonaria, 522

Alcyonidium, 500

Alcyonium, 12, 521, 522

Alexander, 262

Alfieri, bleaching, 256

Alizarin, 184

and crystal violet, 322

nervous system, 408

Alkanna, 368

Allen, methylene blue, 192

Allen, Ezra, 304

chromic Bouin's fluid with urea, 306

on clearing, 307

Allen, E. J., menthol, 12

Allen and Browne, 27, 523, 524, 525

Allerhand, iron myelin method, 451

Alt, 408

Altmann, 20 et seq., 38

acid fuchsin picric acid method, 320 corrosion, 249

fat, 366

fluid, 37

Alum, aniline, 355

carmine, 136 and picric acid, 138

-nitric acid for decalcifying, 253

hæmatoxylin, generalities, 151

Aluminium hæmatein, 151

Alzheimer, 416,480

Amann, lactophenol, 222

Amato, 425

Ammonia, carmine, 140 chromate, 43

Ammonio-chloride of tin for myelin, Besta, 448

Ammonium, bichromate, brains, 403 sulphocyanide for maceration, 245 vanadate, Golgi method, $\mathbf{4 7 6}$

Amœbæ, cultures, 527

Amphibia, 275 et seq.

brains, 405

Champy's fluid, 37,318 
Amphioxus, 281

Amphipoda, embryology, 288

Amyl nitrite, 233

Amyloid, 133

Andeer, 254

Andres, actinida, 12, 13, 521

Andrews, avian embryology, 272

Andriezen, Golgi method, 465

Anemones, narcotisation, 12 See Actinians.

Anglade and Morel, Victoria blue neuroglia stain, 483,485

Anilin dyes, 159 et seq.

blue, 182

blue-black, 183, 408

blue and carmine, 213

red, 169

oil, 69

anilin oil water, 166

Anitschkow, 110

Annelids, 39

blood vessels, 510

Champy-Kull, 322

killing, 12

nerves, 510

Antedon, 519

Apáthy, 97, 98, 511

alcoholic corrosive, 46

hæmatoxylin, 156

bergamot oil method for celloidin sections, 117

embedding in oil of cedar, 78

Canada balsam, 226

cement for glycerine mounts, 231

gold, 198, 206, 207

glycerine gelatin embedding, 93

gum syrup medium, 221

hæmatin mixture, 155

methylen blue, 189, 192, 193

on knife tilt, 85

neurofibril methods, 416

nitric acetic for maceration, 247

picro-säurefuchsin, 177

series-on-knife method for celloidin sections, 118

theory of gold impregnation, 203

Apel, 512

Aqueous humour, 219

Araneida, 287

Arctiscoida, 509

Argentamin, 415, 452

Argyroneta ova, 287

Arndt, bone saw, 370

Arnold, 178, 297, 517

chondriosomes, 323

staining kidney, 394

Arnstein, 190, 193, 342

method for corpuscles, 342

Aronson, myelin, 448

Arsenic acid, decalcifier, 254

Arthropoda, 504 et seq.

embryology, 284 et seq.

fixation, 44, 47, 55

mounting whole, 504
Artifacts, 20, 304

Artificial fecundation, 258 et seq.

Artificial iodised serum, 219

Artom, ascaris ova, 290

Ascaris ova, 52, 289

Aschoff-Kiyono, 388

Ascidia, narcotisation, 12 buds, 282

general, 499

Ascoli, Cajal's method, 426

Asphalt varnish, 230

Asphyxiation, by boiled water, carbonic acid gas, 16

Assmann, blood stain, 384

Astacus, eye, 508

methylen blue, 192

nerve-endings, 344

Asteroidea, 519

Astrocytes, 479

Athanasiu and Dragoiu, 352

Atheson, 511

Athias, 467

Atta, ova, 287

Auerbach, 415

buds, 425

stain, 312

Augstein, 514

Auricularia, 520

Aves, embryology, 271 et seq.

Gerlach's window method, 271

Axis cylinder, 452 (methylene blue)

other stains, 454 et seq.

and dendrite, advice on special forms, 459

processes similar to Golgi methods, 475

rapid process, 458

Azoeosin, 313

Azoulay, 209

ammonium, vanadate process, 476

osmic acid methods (myelin), 451

Babcock, 102

Babes (safranin), 166, 167

Babkin, 396

Bacteria, in amcba cultures, 528

in tissue, 326,354

Ballowitz, 348

electric organ, 346

mammals, 264

reptiles, 275

Balsams, 225 et seq.

cedar wood, alcoholic, 226

method, section-grinding, 109

neutral, 226

Barnes, 514

Barrois, echinoderm larvæ, 520

Bartel, 482

Baryta-water, for maceration, 245

Basic dyes, 120

Basophil granules, nerve tissue, 410,414

"Basophilous " tissue, 128

Bastian, gold, 205 
Bataillon and Koehler, 308 ascaris ova, 289

Batchelor, 390

Bath (paraffin), 77

Baumgarten, 183, 213

Bayerl, decalcifier, 254 ossifying cartilage, 377

Bayliss, 125, 309 on dyes, 120 on specificity of stains, 134 vaso-dilators, 233

Beale, 232 digestion by pepsin, 248

Beard, raja embryos, 280

Beauchamp, 513

Beckwith, 309

Bedot, 524

Bees, brain, 508

Behrens, 182, 221, 344

mounting medium, 224 salmon embryos, 280

Bell, cement, 229, 366 fat, 367,369

Benario, blood, 381

Benda, 495

alizarin method, 322 copper hæmatoxylin, 157 crystal violet, 184

fatty acids, 368

"Flemming fluid," 319 iron hæmatoxylin, r48 neuroglia stain, 482 picro-säurefuchsin, 177 rapid myelin method, 448 safranin and light green, 181 secretion, granules, 315

Benecke, fibrils, 351,388

Van Beneden, acetic acid, fixation, 51 method against contraction, 12 acetic alcohol, 52

live mammal embryo in serum, 267 mammals, 265

tænia eggs, 289

and Neyt, ascaris ova, 290

Bengal rose, 180

Bengtsson, eggs of diptera, 285

Bensley, brazilin and water blue, (thyroid), 394

pancreas, 395

intestine, 393

Bensley-Cowdry, acid fuchsin methyl green stain, 324

Benzidine dyes, 388 et seq. teeth, 373

Benzoazurin, 170, 184 cartilage, 376

Benzol, 70 embedding, 78 peroxide, 59,382

Benzopurpurin, 179, 390

Benzoyl, green, 181

Bergamot oil, 68,323 for celloidin sections, 117

Bergh, annelids, 510
Bergonzini, 356

Berkeley, Golgi method, 466 liver, 393

rapid myelin method, 447

Berlese, Acarina, 504

Berlin blue, aqueous masses, 240 of Mayer, 240

injecting teeth, 372

mass, Brücke, 236

Bernard, mollusc, 504

Berner, 368

Bernheim, 206

Beroë, 524

Bertarellis, protozoa, 532

Best's carmine stain for glycogen, 295

Besta, ammonio-chloride of tin, 448

Cajal's method, 425

Golgi apparatus, method, 438

Bethe, 507

chitin, 506

methylen blue, 194

molybdenum-toluidin blue, neurolibrils, 417

Bettendorf, 516

Betz, hardening nerve, 403

Bevan Lewis, 183, 404

Lo Bianco, $14,15,37,510,514,515$, $516,518,519,524$

acetic fixation, 51

ascidia, 499

molluscoida, 500

chromo-sublimate, 48

corrosive acetic, 44

gephyrea, 512

mixture for narcotisation, 13

protozoa, 542

Bichloride of mercury. See under Mercury.

Bichoff, mammals, 264

Bichromate of ammonia, 43

of calcium, 43

of potash, 41

decoloration of, 41

maceration, 245

Muller's fluid, 42

and alcohol, 43

fixation, nervous system, 403 et seq. -osmic, 37

-platinic, 37

chromic-osmic, 37

and mercury, 48

-sublimate, Golgi method, 470

Bickfalvi, digestion, 249

Biebrich scarlet, 180, 313

Biedermann, methylen blue, 344

Bielaszewics, 150

Bielschowsky methods, 426 et seq.

Da Fano modifications, 432 et seq.

other modifications, 430 et seq.

silver method, for connective tissue, 352

and Bruehl, ear, 497

and Plien, cresyl violet, 414

Bigelow, Medusæ, 524 
Bile capillaries, 393

- Bilharzia, 516

Binet, 31

Bing, 64, 449

Biniodide of mercury mounting liquid, 224

Binnennetz, 316

Biondi, blood, 380

Bipinnaria, 520

Bismarck brown, 161, 169

for cartilaginous skeletons, 377

Bizzozero, 391

blood-platelets, 386

and Torre, blood, 382

Bjeloussow, gum arabic mass, 241

Bladder, frog, nerves, 349

Blastoderms, general, 260 of mammals, 267,268

Bleaching, Mayer's chlorine method, 255 sulphurous acid, hydrogen peroxide, chlorine, 31

Bles, frog embryology, 278

Bleu de Lyon, 183

Blochmann, 115, 500

cestodes, 516

frog embryology, 276

Blood, 379 et seq.

cells, mitochondria, 333

elective stain for reds, 388

fixation, 379 et seq.

fixing in bulk, 380

new Golgi body, 387

and iron salts, 300

platelets, 386

-serum media, 219

stains like methyl green, etc., 380

Blue gelatine mass, 236

lumière, 183

Blum, 401

Bobretzky, 286

Boccardi, 206

erythrosin and toluidin blue, 415

Bödecker, decalcification, 251, 373

Boeke, Bielschowsky, 430-431

Böhm, 206 and Oppel, 41, 219 reptile blastoderms, 274

Böhmer, hæmatoxylin, 154

Böhmig, 515, 517.

Bolina, 524

Bolton, 469, 447

Golgi method, 464

Bombyx mori eggs, 285

Bone, 369 et seq.

decalcification, 251

dry sections, 369 et seq

mounting, 370

non-decalcified, 369

soft parts, 371 et seq.

saw, 370

Bonnet, 269

Bonney, 178

Bonome, 480

Bonvicini, hardening human brain, 404
Borax carmine, 141

Bordeaux R., 178

Borgert, 75

Boring, ascaris ova, 290

Born, 262, 278

Borrel, 213

-method, 532

Borrel's blue, 534

Bouffard, Benzidine dyes, 388

Bouin, picro-formol, 62

frog larvæ, 278

salmon embryos, 280

Boule, Cajal's method for lumbricus, 425

Bouma, 376

Boveri, ascaris ova, 290

embedding of echinoderm ova, 260

picro-acetic, 55

Boyce and Herdman, copper, 300

Boycott, 507

Brachiopoda, 500

Bradford and Plimmer, 547

Braem, Bryozoa, 282

Brain, cat, dog, neurofibrils, 421.

See under Nervous System.

cat, man, preliminary fixation, 402 insect, 508

preliminary treatment, $397-399$

Branca, 168

sublimate formol, 63

Brandt, 546

glycerine jelly, 223

protozoa, 540

Brasil, 546

Brass, on embedding, 76 protozoa, 542

Braun, 514, 517

Braus, 26, 281

bile capillaries, 393

Brazilin, 211

for sponges, 526

Breglia, 449

Bremer, 181, 473

Bresslau, mesostomid ova, 288

Brilliant kresylblau, blood, 383

Bristol, 511

Brittle object, cutting of, 88

Brock, maceration, 245

Brodmann, 398

Bromide of soda or potash, Simarro, 419

Brookover, 442, 468

Golgi method, 465

Brown, 549

Brücke, digestion, 249

Bruel, dipterous eggs; 285

Brühl, corrosion, 249

Bruno, mucin, 391

Brunotti, gold gelatin, 93 gelatin embedding, 108

Brunswick black, 230

Bryozoa, 282, 500 heat-killing, 12

Buchner, glycogen, 296 nucleoli, 314 
Budge, injection, 241

Bugula, 500

Bujor, 523

Bulb, 424

Bullard, 367

Bumpus, 103

Bunge, fluid for iron stain, 299

flagella stain, 548

Burchardt, 139

paraffin, 92

chrome fixation, 40

protopterus brain, 405

Burzynski, 94

Burger, nemertina, 515

Burrows, 550

Busch, 29, 32, 214, 252

decalcification, 251

Marchi, 450

Butschli, 154

Buzzi, eleidin, 341

Cade, gastric glands, 393

Cajal, 435, 465, 176

double-impregnation, Golgi process, 461

avoidance of silver precipitates, 467

gold chloride-sublimate method of neuroglia, 487

Golgi apparatus method, 436

methylene blue diffusion process, 478 myelin, 450

nucleolini, 311

retina, 495

spirals and funnels, 440

Cajeput, oil of, 68, 103, 411

Calberla, 161, 181, 182

Bismarek brown, 161

liquid, 222

Calcium chloride medium, 219

Calyptoblastea, 523

Camsal balsam, 227

Canada balsam, 225 et seq.

Caoutchouc, cement of Miller, 230

Capitellidæ, narcotisation, 13, 510

Capsicum berries, 368

Carazzi, 256

Carbol-pyronin-methyl green, 172, 355

Carbolic acid (clearing), 69 fuchsin, 169

thionin (King), 168

Carbon, bisulphide for embedding, 77 Carter on, 372

insects, 507

tetrachloride, 77

Carleton, Golgi apparatus, 438

nucleolini, 311

Carmalum, 137

and indigo-carmine, 212

Carmine-generalities, 136

alcoholic stains, 141

aluminium chloride solution, 138

and anilin blue, 213

ammonia, soda, lithium, magnesia, 140
Carmine-generalities - contd.

and cochineal stains, 135

-gelatine masses, 234

Hoyer, 235

Fol, 235

Krause, 235

glycerine mass (cold), Beale, 238

Robin, 233

Grenacher's alum-carmine, 136

and malachite green, 213

for nervous system, 407

neutral alkaline, 140

blue, 183

Carminic acid, 135

Carnoy, 151, 178

acetic alcohol, 52

and Lebrun, frog eggs, 276 iron, 300

Carothers, Bouin and urea for insect chromosomes, 306

Carrel, 550 et seq.

Carter, J. Thornton, bone, 369 et seq. on post-mortem changes, 25 teeth sections, 371

Cartilage and bone, 376 silver, 200

skeletons, 377

Caryophyllia, 521, 522

Cassiopeia, 524

Castellarnau, 519

Castle, Ciona, 281

Castor oil, mounting medium, 228

Catois, methylene blue method for fishes, 478

Cattaneo, 541

Cattani, funnels and spiral filaments, 410

Caullery, 499

Causard, 509

Caustic soda bleacher, 257 potash or soda, corrosion, 250 maceration with, 244

Cavalié, electric organs, 346

Cavazzani, 215

Cedar wood oil, 66 et seq., 77 for minute dissections, 7

Cell inclusions, 316 et seq. granules, lymph and blood cells, 315

Celloidin embedding, 95 et seq. See also under Collodion. knife smeared in vaseline, 102 for injection, 241 sections, Apáthy's method, 118

Bolles Lee's method, 116

Summer's method, 116 staining, 102

Celloidinum inelasticum, 96

Cements and varnishes, 229 et seq.

Centrifuge, for oogenesis studies, 332

Centrosomes, 315

Cépède, 307

Cephalopoda, 282 eyes, 502

Ceratium, 547

Cercaria, 517 
Cerebellum, 424.

See under Nervous System. preliminary treatment, 399

Cerebrum, hardening, 399 Cajal's methods, 424

Cerfontaine, amphioxus, 281 Ascaris ova, 290 worm, 509

Certes, protozoa, 540

Cesaris-Demel, 383

Cestoda, 515 ova, 289

Chætopoda, 509 marine, 510

Chalicodoma, eggs, 286

Chambers, intra vitam stain, 310

Champy, 314 fluid, 37

iodide of osmium method, 331 trichloracetic, 53

Champy-Kull,mitochondrial method,321 fixation, Gatenby, 322

Chenzinsky, 181 blood stain, 383

Chick, embryology, 271 et seq. axis cylinder and dendrite, 459

Child, fish embryos, 279

Chilesotti, 407, 477

Chilopoda, blood, 381

China blue, spirals, funnels, 440

Chitin, 506 et seq.

bleaching, 256, 250

tests for, 507

Chiton, eggs, 284

Chitonidæ, 503

Chloral hydrate, for narcotisation, 14 jelly, 224

maceration, 248

mounting medium, 220

Chlorcarmine, 139

Chloreton, for narcotisation, 14

Chloroform, for killing, injurious in cytology, 11

clearing, 70

embedding, 76,78

vapour, collodion embedding, 98

Cholesterin, 356 et seq.

Chondriokonts, 316

Chondriome, 316

Chondriosomes, 316

Chorion, removal, 284 et seq.

Chromates, 40

Chromate masses, 237

Chromatin, microchemistry, 294, 308 et seq.

and enzymes, 308, 309

digestive fluids, 294

methyl green test, 293

Chrome salts, Burckhardt on, 40

Chromic acid, decalcification, 252, 254

fixation, 32,33

washing.out, 33

formol, 63

for hardening, 34
Chromic acid-contd.

and hydrochloric acid, decalcifier, 254

maceration, 246

nerve tissue, 404

and platinum chloride (Merkel), 39

Chromidia, 308, 309, 310, 316

Chromo-acetic acid, 35

Chromo-aceto-osmic decalcifier, 254

Chromo-formic, 35

Chromo-nitric acid (Perényi), 39 decalcifier, 254

Chromophility tests, 309, 310

Chromosomes, techniques for, Gatenby, 303 et seq.

Chromo-sublimate, 48

Chrysoidin, 364, 369

Ciacco, 206, 343

method, 369

(fat) tendon, 347

Ciaglinski, myelin, 452

Ciechanowski, bile capillaries, 393

Cilia, mollusc, 503 protozoa, 541

Ciliated epithelium, 339

Ciliates, general staining, 533

Cilimbaris, 353

Cinnamon (cassia) oil, 67

Cladocera, Haker, 288

Clasmatocytes, 356

Cleaning slides, 112, Appendix

Clearing, 5, 65

Clove oil, for minute dissections, 7, 67

Coal gas, for killing, 11

tar dyes (plasma), 171

Cobb, differentiator, 3

Cocaine, for narcotisation, 14

Coccidia, staining, 532

Cochineal, 135

alum carmine, 137

Cochlea, 375, 496 et seq.

Coe, Distomum, 289

Cœlenterata, 521

maceration, 524

nervous system, 522

Cœrulein S., 182

Cohnheim, gold, 204

Cold-blooded animals, injection, 241

Cole, gum mass, 110

Coleoptera, eggs, 286, 287

Collargol, 434

Collin, 510

and Lucien, 438

Collinge, 281

Collodion, êmbedding, 95 et seq.

alcohol hardening, 100

bone sections, 372

bath, 96

for celloidin sections (Weigert), 118

blocks, clearing in cedar oil, 104

clearing and mounting, 102

cutting, 101

dry cutting, 104

hardening, 98

newer method, 103 
Collodion-contd.

Obregia's syrup method of mounting sections, 115

older method, 96

preservation of blocks, 100

rapid process, 104

section mounting with albumen, 116

method of mounting paraffin sections, 115

and paraffin, 105

solution of, 97

Collodionisation, brittle objects, 88

Colloidal gold, 133

Colloidal-complex, 120

Colloids, 123

Colloxylin, 96

Colophonium, 226

and wax method, section-grinding, 109

Colucci, 226

Comatula larva, 520

Congelation masses, 109

Congo-Corinth, 179

Congo red, 121, 122, 124, 178 et seg.

nervous system, 408

myelin, 453

Conklin, Crepidula, 284

Connective tissue, 350 et seq. Bielschowsky, 434

Conser, 500

Contraction, prevention of, 11 acetic acid in, 12 corrosive sublimate in, 12

Cooling paraffin, 82

Copal method, 108

Copepoda, 288, 505

Copper, in tissue, 300

bichromate, 64

chloride and acetate fixation, 53 mounting fluids, 220

ferrocyanide injection mass, 234

formol, 64

nitrate, 54

sulphate and corrosive for nerve tissue, 405

fluid for frog eggs, 279

Corallin, 169

Corals, decalcification, 251

Cori, narcotisation mixture, 14,15 osmic solutions, 29

Cornea, 342 et seq. maceration, 247

fibres, maceration, -246

silver method, 198

Corning, 475

neurokeratin, 441

Corpuscles, Golgi, 347

Meissner, Krause, 342

Nissl, 180

tactile, 341

Herbst and Grandry, 342

Corrosion, 249 et seq.

Corrosive sublimate. See also under Mercury bichloride.

preventative of contraction, 12
Corrosive sublimate-contd washing out, 26

Cowdry, 304, 415 acid fuchsin stain, 324

Janus green, 332

Mitochondria, 338

Cox, 415, 434

Golgi process, 472 et seq. neurokeratin, 441

Cramer, 30

fatty substances, 356

osmic vapour method, 330

Feiss and Bullock, 366

Creases (paraffin sections), 88

Creighton, 297

Creosote (clearing), 69

Crepidula, 284

Cresyl violet, nerve, 414

Crinoidea, 519

Cristatella, 282, 500

Crustacea, 505 et seq.

Crystal violet, 184

for mitochondria, 322

Crystalline lens, 247, 343

Csokor, 230

bone saw, 371

Ctenophora, 524

Cucumaria, 518

Cultures, manipulation of amœbæ, 527

Cunningham, micro-injection, 270

Curare, 292

for narcotisation, 16

Curling of sections, 86

Curreri, 469

Cutting, paraffin, 86

tissue, 319

Cyclas ova, 284

Cytological methods, 292 et seq.

Cytoplasmic inclusions, 316 et seq.

Czokor, 137

Daddi, fat, 367

Dahlia, 162, 169, 333, 340

Dakin, 503

Dallinger, 530

Damar, 226

Davidoff, 524

Tunicata, 281

Dead cells, 131

Dealcoholisation, 65 et seq. or clearing, 5

Death, 25, 131

Decalcification, 251 et seq. teeth, 251 et seq. and 372 et seq.

Decapod, eyes, 508

Decapoda, ova, 288

Deecke, 404, 407

Deetjen, 387

Deflandre, fat, 369

Degenerate nerve, Marchi, 449

Dehydration, 2

by alcohol, acetone, methylal, aniline oil, 4

Dejerine, 407 
Dekhuyzen, 218, 386

liquids, 43

osmacet, 380

Delafield. hæmatoxylin, 154

Delage, Turbellaria, 517 sponge, 526

Delamare, 351

Dell'Isola, Golgi method, 463

Della Rosa, Indian ink mass, 240

Della Valle, Orchestia, 288

Deltapurpurin, 179

Demoor, 214

Dendrite stains, 454 et seq.

Dendrocœlum, 517

Dendy, Geonemertes, 515 sponges, 526

Denne, orientation of objects, 81 embedding method, 78 et seq.

Dentine, 370 et seq.

Dependorf, 374

Depigmentation, 256. See Bleaching.

Descemet's membrane, 343

Desiccation method for paraffin sections, 111

Desilification, 251 et seq., 255

Dewey, 373

Dewitz, 503

Dictyosome, 316

Dietrich, 366, 509

Digestion, 248 et seq., 308 and dissociation, 243 et seq.

Dimmer, 115, 119

Diomidoff, 405

Diptera eggs, 285

Disse, 377

Dissections-minute, cedar wood oil for, clove oil for, 7 glycerine for, 8

Distomum ova, 289

Dobell, modification of Heidenhain stain for protozoa, 544

Mann's stain, 544

Döderlein, 519

Dog brain, 402

Dogiel, 342

corpuscles of Herbst, 342

Meisser and Krause, 342

Grandry's corpuscles, 421

iris, 348

methylen blue, 189, 190 et seq . for epithelia, 195

tendon organs, 347

Donacia eggs, 286

Donaggio, 158 neurofibril methods, 417 et seq.

Donaldson, 405

fæces, 538

Doncaster, chromosome fixation, 305

Double-embedding collodion paraffin, 105,506

Double-staining in hæmatoxylin and acid fuchsin, 326

Downey, benzidine dyes, 388

Drasch, 204
Dreuw, 341

Drew, formol-chrome method, 325

manipulation of amoba cultures, 527

-protozoa, 526 et seq.

staining amœbæ, etc., 531

tissue culture, 551,554

Drew-Griffin live-slide, 530

Drew-Murray, connective tissue stain, 354

Driessen, 297

Drost, 504

Drüner, 26

Duboscq, blood, 381, 465

Dubreuil, connective tissue, 350

Duerden, cœlenterates, 521

Duerk, 354

Dunham's mixture (celloidin sections), 103

Durig, Golgi method, 464

Duval, 404

carmine and anilin blue, 213

collodion embedding, 95

orientation method for blastoderms, 273

silver, 200

Dyes, nature of, 120

electric charges, 122

Ear, inner, 496 et seq.

Eau de Javelle and Eau de Labarraque bleachers, 256

corrosion, 250

for eggs, 284, 285

for frog embryology, 276

Eberth and Runge, 469

Echinodermata, 518 et seq. larvæ, 520

Echinoderms, decalcification, 251

Echinoidea, 518 spines, 518

Edinger, 34

Edington, blood, 381

Egg-capsules, removal, 284 orthoptera, 286

Egg, white of, injection mass, 240

Eggs, Unio, 284

Ehrenbaum, grinding, 109

Ehrlich, 155

blood, 379

hæmatoxylin and eosin, etc., 214

Indulin-Aurantia-Eosin, 180

mast cell method, 356

methylen blue, 188 et seq.

neutral red, 179

triacid mixture, 175

Ehrlich-Biondi, 161, 173

and Lazarus, 297

Ehrmann, 340

Eichler, 498

Eisath, neuroglia granules, 486

Eisenberg, Nile blue, 368

Eisig, 13, 510

fluid, 40

maceration, 245 
Eismond, 539

Ekman, 500

Elasthæmatein, 353

Elastic tissue, 352 et seq.

fibres, of spleen, 394

Elderberries, Kappers, 409

Electric organs, 345

Eleidin, 341

Ellis, 548

Elschnig, 97

Embedding, gelatine masses, 92 et seq. collodion, 95

paraiffin, 76 et seq.

lead-gum, 106

boxes, 73

brass squares, 74

thimbles, 73

trays, 72

in vacuo, 80

Embryological methods, 258 et seq.

Embryonic cartilage, 376 et seq.

Embryos, Bielschowsky methods, 429 Cajal's methods, 424

fixation, $260,268,278$, etc. 275 .

Emery, aqueous carmine mass, 240

Encephala, 401, 404

Endothelium, silver, 200

Engelmann, 218

Enriques, 501

Entamcba, 538

Entire objects, preparation of, fixing agents for, best stains for, 7

Entz, 81, 543

protozoa, 541

Enzymes and chromatin, 308

Eosinophilous cells, $180,383,386$

Eosins, 180 et seq. methyl green, 181

methylen blue, 383 et seq.

Epeira eggs, 287

Ependyma cells, 479

Epidermis, digestion, 249

neurofibrils, 421

Epiploön, silver method, 199

Epithelia, silver method for, 198

Epithelium, maceration, 244, 245

Eppinger, 393

Erhard, 297

Erlanger, ascaris ova, 290

Erlicki's fluid, 42

Van Ermengem, 549

Ernst, 341

Erythrosin, 180

with toluidin or methylen blue for nerve tissue, 415

Eternod, 83

Ether method, for celloidin sections, 116 for narcotisation, 13

Eucaine, for narcotisation, 15

Euler, 309

Euparal, 227, 223, 369, 10

Evans, benzidine dyes, 388 micro-injection, 270

Everard, 214
Ewald, blood, 380

section washing apparatus, 3

Examination media, 216 et seq.

Eycleshymer, 98, 103

Eye, 493 et seq.

arthropod, 508 et seq.

bleaching, 255, 257

mollusca, 502

Fabre-Domergue, 220

protozoa, 540

Faded sections, treatment of, 6

Fæces, examination for protozoa, 538 smears, lucidol, 382

Fairchild, 3

Fajerstain, 342

hæmatoxylin, Golgi method, 476

Da Fano, 428, 434, 435

nervous system, 397 et seq.

axis cylinder stains, 454 et seq.

Bielschowsky method, generalities, $426-427$

modifications, 432 et seq.

Cajal's methods, 419 et seq.

advice on Cajal's methods, 424

modification of Cajal's method, 425

cobalt nitrate, Golgi apparatus method, 437

formaldehyde, Golgi methods, 463

Golgi preparations, on cutting, 467 on mounting, 468

Golgi-Cox method, 473

special treatment for Golgi-Cox preps., 474

neurofibrils, $416,417,418$

neuroglia methods, 479,484

Golgi's sublimate method, 470

Fanz, grinding bone, 370

Farrant's medium, 221

Fat and glycogen stain, 296

Fatty substances, Cramer and Gatenby, 356 et seg.

Fauré-Fremiet, centrifuge, 332 protozoa, 540

Faussek, cephalopoda, 283

Feist, 407

Felizat, 168

Ferreri, decalcifying, 255

Ferria, 352

Ferric and ferrous salts, 297 et seq.

Fettponceau, 367

Fibres of Sharpey, 375,376 ,

Fibrils, connective tissue, 350 et seq.

Fibrin, Weigert stain, 388

Fick, 184

kerato-hyalin, 340

Siredon, 277

Fieandt, neuroglia granules, 486

Fiedler, 525

Field and Martin, 105

Fiessinger, 297

Films, blood, 379

fixation, 381 et seq. 
Finotti, 408

Marchi method, 450

myelin, 452

Fischel, 505

chick embryos, 273

Fischer, 223, 344, 353

on coagulation, 20 et seq.

nucleoli, 314

trematodes, 516

Fischler, 368

Fish, 405

embryos, Bielschowsky (Paton), 430

eggs, 279 et seq.

methylen blue nerve method, 478

Fish, on clearing celloidin sections, 103

brain of Desmognathus, 405

decalcification, 253

Golgi method, 464

Fixation, in embryology, 259 et seq.

by immersion, 25

by injection, 26,397

by salts, 40

Fixing agents, cytological, 301

theory of, $18,19,131$

Flagella. stains for, 548

Flagellata, general stains, 533

Flatau, 402

Golgi, corrosive method, 472

Flattening sections, 90

Flechsig, 206, 449, 472

Flemming, 339

chromo-acetic, 35

chromo-aceto-osmic, 35

Dahlia, 169

decalcified bone sections, 375

orange method, 171, 177

picro-osmic, 56

safranin, 166

Flesch, 444, 498

blood, 380

Floyd, 508

Flustra, 500

Foà, 214

sublimate and bichromate, 49

Foettinger, 14, 515

Fol, 16, 542

mixture, 39

mounting watery sections, 116

carmine mass, 235

picro-chromic, 56

Fontana, tannin-silver protozoon stain, 545

Foot and Strobell, smears, 308

Formaldehyde, 60 et seq. and alcohol, 62

modifications of Golgi method, 463

mordant, 165

Formalin, blood, 381

for nervous system, 401

and picric, 62

chrome techniques, 323

and sodium chloride, for maceration, 244
Formalin-contd

Formol, chromic, 63

Formol-Müller, 63

nitric acid for decalcifying, 253

sublimate, 63

, vapour, blood, etc., 381

Formic acid, gold method, 204

Foster and Balfour, embryology, 271

Fræinkel, 297, 449

Francotte, 150, 289

Freeborn, 407

picro-nigrosin, 350

Freezing methods, 109

Frenkel, palladium and osmic, 50

Frenzel's mercuro-nitric, 47

Fresh cells, 293

Frey, 219

Friedenthal, hardening mass, 237

Friedländer, 524

Frog embryology, 275

eggs, removal of mucin, 276 et seq.

Gatenby's fluid for eggs, 277

methylen blue, 191

skin, 391

Fröhlich, 176

Frozen sections, 109

brain, 406

Fuchsin (basic), 169

See Acid Fuchsin.

carbolic, 169

Fürst, bleaching, 256

Fusari, 377

Gage, 101, 297

albumen and mercury mounting medium, 220

alum, nitric acid decalcifier, 253

celloidin sections, 117

clearing mixture, 69

maceration, 248

with formalin, 244

Galesescu, 482

Gallein, myelin, 448

Galli, China blue, 440

Garbini, 522

Gardiner, ova of polychœrus, 288

Garlic water, 115

Gaskell, 94

Gastric glands, 392 et seq.

Gastropoda, 283, 500

embryology, 283

eyes, 502

Gatenby, 308, 435

amœba culture, 536

benzidine dyes, 388 et seq.

centrifuge, 332

Champy-Kull fixation, 322

Cramer's osmic vapour method, 330

differentiation between cytoplasmic inclusions, 334 et seq.

Donacia egiss, 287

double stain for mitochondria, 326

(and Cramer) fatty substances, 356

on Fischer's theory, 20, 21 
Gatenby - contd.

on fixing agents, $22,23,24,25$

Flemming modification, 36

fluid for frog eggs, 277

on killing, 11

Limnæa embryology, 284

Mann-Kopsch method, 328

Mann-Kopsch-Altmann combination stain, 329

on methods of mammalian embryology, 263

mitochondria, etc., 316 et seq.

mitochondrial fluid, 319

new advances in embryological technique, 258

plan for cytological research, 337, 338

sponges, 526

technique for chromosomes, 303 et seq.

tissue culture methods, 550 et seq.

on Unna's oxypolarity theory, 21

and Woodger, 365

Gaule, desiccation method for paraffin sections, 111

solution, 46

Gaultheria, oil of, 68

Gavazzeni, 341

Geberg, 206

corpuscles, 342

Gedoelst, 249

neurokeratin, 441

Gee and Harrison, 124

Gehardt, reptile blastoderms, 274 lens, 343

Van Gehuchten, 413, 465

axis cylinder and dendrite, 459

fixing fluid, 415

ear, 498

Gelatin, embedding masses, 92

injection masses, 232

blue, 236

green, etc., 237

red, 234

yellow, 237

cement, 229

freezing mass, 110

and glycerine, 93

Gemelli, flagella, 549

Gentian blue, 183

violet, 162,167 et seq.

neutral, 396

for fibrin, 388

carbol, far flagella, 548

Geoffroy, mounting medium, 224

Gephyrea, 511

Gerlach (gold), 207

Gerota, 202, 398

brains, 402

Golgi method, 464

Gerould, 518

Giemsa, blood stain, 384 et seq.

for neuroglia, 487

for protozoa, $536,544,545$
Gierke, 197, 214

maceration, 245

Giesbrecht, 505

embedding, 78

Van Gieson (picro-säurefuschin), 176, 215

Giglio-Tos, blood, 382

Gilbert, 442

Gilson, bleaching, 256

chloral hydrate jelly, 224

copper formol, 64

mercurial mounting medium, 220

mercuro-nitric, 47

Sandarac mounting media, 227

rapid celloidin method, 104

Glands, 391 et seq.

Glaue, 514

Glia, 479 et seq.

Glochidia, 284

Glucose mounting medium, 221

Glycerine, gelatin and glycerine injection media, 233

gelatin, 93

and $\mathrm{HCl}$. decalcifier, 254

jelly, 223

for maceration, 247

for minute dissections, 8

mounting media, 222 et seq.

Glycerised blood-serum, 219

Glychæmalum, Mayer's, 153

Glycogen, 294 et seq.

and cell inclusions, 338

Goadby, 220

Goblet cells, 392

Gold, colloidal forms, 133

gelatin embedding, 93

gelatin mass, Tandler, Pearl, Mayer, Mozejko, 238

impregnation, generalities, 202 et seq. preservation of specimens, 208

Gold-size, 230

Gold-sublimate, Golgi method, 475

Goldmann, 131

benzidine dyes, 388 et seq.

Goldscheider and Flatau, 414

Goldschmidt, 552

Golgi, 26, 435, 438

axis cylinder and dendrite stains, 454 et seq.

sublimate method, 470 et seq. mixed process, 460

avoidance of precipitates, 466

cutting and manipulation, 467

mounting, 468

body in red cells, 387

Golgi method, modifications, 461

formaldehyde process, 463

theory of impregnation, 460

methods for funnels and spiral fila. ments, 439

bichromate-sublimate modifications, 472

gold, 207

osmio-bichromate mixture, 458, 516 
Golgi-contd.

processes for rejuvenation of overhardened tissue, 462

apparatus, generalities, 316

bone and teeth, 376

Kopsch techniques, 327 et seq.

Mann-Kopsch-Altmann method, 329

other methods (silver), 438

plants, 325

silver methods of Golgi, Veratti, Da Fano, Cajal, 435 et seq.

Sjövall method, 331

corpuscles of, 347

Golgi-Cox, 472

Da Fano's special mounting method, 474

making permanent preparations, 473

Golgi-Kopsch apparatus, 316

Golgi-Veratti, Golgi apparatus method, 435

Golodetz, 8, 210, 366

ana Unna, cholesterin, 341

Golovine, 179

Goodrich, 308, 377

modification of Kent's method, 541

Goodsiria, 282

Gordon, 180,549

Gorgonia, 521, 522

Goronowitsch, 280

Gothard, methylen blue, 414

Graeffe, paraffin solvents, 76

Graff, Hirudinea, 511

Turbellaria, 517

Graham, 390, 514

Gram, gentian violet, 167

Grandis and Mainini, 300

Grand-Moursel and Tribondeau, pancreas, 395

Grandry, corpuscles, 342

Grassi, hæmatozoa, 546

Gray, 498

Greef, 493

Green, B., 14

Green leucocytosis, oysters, 300

Gregory, micro-injection apparatus, 270

Grenacher, borax carmine, 136, 141

bleaching mixture, 257

eyes of mollusca, 502

Greppin, 469

Griesbach, 178, 182, 352

blood, 380

Grinding sections, 108

de Groot, alcoholic hæmalum, 156 iron carmalum, 139

Groselj, 522

Grosser, partially aqueous ink mass, 241

Grünpulver, 159

Grünstein, bladder, nerves, 349

Grynfeltt and Mestrezat, 256

Guanin, 301

Gudden, 465

myelin, 446

Gudger, salmon embryology, 280
Gulick, Ascaris ova, 290

Gulland, 379

blood fixing fluid, 381

Gum, and chloral hydrate mount, 221

glycerine, Allen, Langerhans, 221

mounting medium, 221

syrup masses, 110

Thus, 228

Gurwitsch, 150

Guyeisse, 392

Gymnoblastea, 522

Gymnotus, electric organ, 346

Haber and Guild, Cajal's method, 426

Hadzi, 523

Haecker, 301

Hæmacalcium, Mayer, 155

Hæmalum, 152

de Groot, 156

Hæmastrontium, Mayer, 156

Hæmatein, 145 et seq.

neurofibrils, 416

Hæmatoxylin, 145 et seq.

Benda and Heidenhain, 148, 149

Böhmer, 154

Delafield, 154

chemical nature of, 145

chrome, Hansen, 157

Heidenhain, 156

Schultze, 157

combination stains, 213

copper, Benda, 157

elastin, 353

Golgi-method, 476

iron, 148

Kleinenberg, 155

for iron in tissue, 298

mounting in glycerine, 152

osmium, Schultze, 158

phospho-tungstic, Mallory, 158

and picro-Säurefuchsin, 215

ripening, 146

and safranin, 214

and Säurefuchsin, 214

stock solutions, 146

tin, Donnaggio, 158

vanadium, 157

Hæmatoxyline noire, 151

Hæmoglobin, 300

Hæmosiderin, 300

Hair, 341

Halle and Born, orientation method, 98

Béla Haller's mixture, maceration, 247

Halliburton, 410

Hamann, 506, 513

Asteroidea, 519

Hamburger, 218

Argyroneta ova, 287

Hamilton, 404

congelation method, 110

Hammarsten, 357

Hanazawa, dentine, 371

Hance, fixation of mammalian chromosomes, 305 
Hansen, 140, 151, 176, 180

chrome-hæmatoxylin, 157

Hantsch, 223

Hardening, 27

injection mass, 237

nerve tissue, 398

Hardy, 123, 512

Hári, 391

Harmer, 202

Harris, 15, 154

hæmatoxylin, for elastin, 353 myelin, 449

methylen blue, 195

Harrison, Ross, tissue culture, 550 et seq.

Hart, 353

Harting, 219 gamboge glycerine mass, 239

Hartmann, 269 mammalian embryology, 265 opossum embryology, 267

Haswell, Temnocephala ova, 289

Hatschek, Amphioxus, 281

Haug, 251, 449 decalcifier, 254

Havet, 517 cœlenterates, 522

Hayem, blood fluid, 380

Heat, for killing, 12

Heckert, eggs of Distomum, 289

Heidenhain, 163, 173

alcoholic hæmatoxylin for protozoa, 543

carbon bisulphide embedding, 507

centrosomes, 315

chrome-hæmatoxylin, 156

theory of dyeing, 125

Ehrlich-Biondi, 173

gelatin glycerine jelly, 223

iron hæmatoxylin, 147 et seq.

vanadium hæmatoxylin, 157

Heinke and Ehrenbaum, 281

Heinrich, 352

Held, 150, 326

formol Müller, 63

method for marginal neuroglia, 484 methylen blue and erythrosin method, 415

Helix, 501 ova, 283

Heller, 209 and Gumpertz myelin, 451

Helly, sublimate and bichromate, 49

Henchman, gastropoda, 283

Henking, examination medium, 293 methods for arthropod eggs, 285 ova of Phalangida, 287

Henneguy, 38 acetic alum carmine, 137

albumen water method for section mounting, 114

chick, 273

fish embryology, 279

gastropod embryology, 283
Henneguy - contd. mammal blastoderm, 268 permanganate method, 165 treatment of faded sections, 6

Hennings, 505

eyes of arthropoda, 509

Insecta, 505

Hénocque, gold, 205

Herbst, 506

corpuscles, 342

Herdlicka, brain, 402

Hermann, 401

fluid, 38

pyrogallol method, 209

safranin and gentian, 168

L'Hérmitte and Guccione, 485

Herrick, Astacus ova, 288

Hertwig, silver for marine animals, 202

frog eggs, 278

maceration mixture, 246

Triton eggs, 277

Van Herwerden, nuclease, 309

Herxheimer, 184, 340

Scharlach R., 367

Hesse, eyes of heteropoda, 502

Hessert, 549

Heteropoda, 500

eyes, 502

Heyder, Arion embryos, 284

Heymons, 501

eggs of Orthoptera, 286

Hickson, 508

Brazilin, 211

eosin and hæmatoxylin, 214

maceration, 248

High refractive mounting liquids, 224

Hill, J. P., 274

clearing and embedding, 262, 269

manipulation of ova, 266

" marsupial mixture," 265

paper for reconstructions, 262

modification of picro-nitric, 55, 264

treatment and isolation of eggs of mammals, 264

Hill, 461, 466

myelin method, 448

Hindle, 547

Hippel, 495

Hirota, orientation, 274

Hirschfelder, 512, 513

Hirschler, 328

Donacia eggs, 286

protozoa, 541

Hirudinea, 511

killing, 12

methylen blue, 192

nervous system, 511

His, 38, 197

Histiocytes, 388

Histriobdella, 511

Hochstetter, injection, 241

Hoehl, 37, 249

Hofer, 15 
Hoffmann, 81, 390, 508, 517

chick embryos, 273

green, 182

Hogben, chromosomes, 303

Hoggans, histological rings, 199 perchloride of iron, 209

Hollande's chlorcarmine, 139

Holmes, Planorbis ova, 284

Holmgren, trophospongium, 439

Holothuria narcotisation, 12

Holothurioidea, 518

Homans, pancreas, 396

Homarus, 288

Honing, 84

Hopewell-Smith, teeth, 373

Hopkins, maceration, 246

Horn, hair, nails, 341

Hornowski, 351

Horny structures, maceration, 247

Hoskins, chick, 273

Hot water, for sudden killing, 12

Houser, 442

Hoyer, 75, 401

carmine mass, 235

gold, 205

Golgi method, 463

mounting medium, 221

mucin, 391

shellac mass, 242

silver method, 200

nitrate yellow gelatin mass, 237

Huber, 468

Hudson, 512

Human brain, 402, 404 neurofibrils, 421

embryos, 42

Hyatt, 108

Hydatina, 512, 513

ova, 288

Hydra, 521

methylen blue, 189

Hydrochloric acid, carmine, 143 for decalcification, 251 et seq. for maceration, 247

Hydrogen peroxide bleacher, 256

Hydroidea, general, 523

heat killing, 12

Hydroxylamin, for narcotisation, 15

Hymenoptera, eggs, 286

Hypochlorite of potash, corrosion, 250

of soda, corrosion, 250

Ide, 105

Idiozome, 316

Igacuschi, 393

Ilberg, 415

Imbedding, 71 et seq.

See also Embedding.

for amphibia, 276

in paraffin or collodion, 5

Imms, 504

Impregnation methods, 197 et seq.
Impregnations other than gold, silver, or osmium, see page 210.

iron sulphate, 210

palladium chloride, 210

perchloride of iron, 209

mass of gelatin, 237

Indian ink, injection of insects, 509

embryos, 270

mass, 240

India-rubber and paraffin, 92

Indifferent liquids, 217

media, 292

Indigo, 212

Indigo-carmine, 182, 212

with oxalic acid, 212

Indophenol, 367

Indulin, 409

aurantia-eosin, 180

and nigrosin, 182

Injection masses, purely aqueous, 240

partially aqueous, 240 et seq.

celloidin, etc., 241

Fol, Tandler, Beale, 238

gamboge glycerine, 239

gum arabic, 241

indigo carmine, Thoma, 239

milk, 241

warm, 232

cold, 238

methods, 232 et seq.

arthropods, 508

Hirudinea, 511

mammalian and other embryos, 270

mollusea, 503

"natural," 242

Innervation, bladder, 349

Insabato, 352

Insects, 504 et seq.

double embedding, 506

cärbon bisulphide, 507

mounting whole, $504-505$

Instruments, microscopes, microtomes, 8

Intercellular bridges, 339

Intestine, 393

Intranuclear rodlet of Roncoroni, 421

Intra vitam, methods, 292

stains, mitochondrial, 332

staining, alizarin, 130

Bismarck brown, 130, 162

Bolles Lee on, 129, 130

Congo red, 130

Fischel, 130

kidney, 394

Loisel, 130

methylen blue, 186 et seq.

neutral red, 130, 179

protozoa, 540

sulphorhodamin, 130

theoretical, 129 et seq.

In vacuo, embedding, 80

Inversion plasma stains, 184

Invertebrates, general methods, 499 et seq.

dendrites and axis cylinders, 454 
Invertebrates-contd. ganglia, Cajal, 425 nervous system, 416,417

Iodide of osmium, 331 of potash and biniodide, 'liquid, 224 for maceration, 244

Iodine, extraction of corrosive, 45 fæces examination, 538 fixation, .51 green, 182 method for glycogen, 295 vapour fixation, 51

Iodised serum, 219 for maceration, 243

Iridium chloride, 50

Iris, 348

Iron, 297 et seq. alum fixation, 50 Brazilin, 211 for protozoa, 545 carmalum, 139 carmine, 139 cochineal, 140 hoematoxylin, 147 et seq.

Benda, 148

Bütschli, 150

rapid method, 150

Weigert, 150 perchloride fixation, 50 sesquichlorate, myelin, 451

Isamin blue, 390

Islets of Langerhans, 395, 396

Isolation of single cyst, etc., 527

Israel, 180, 212

Iwanzoff, 518 electric organ, 346

Hatchett Jackson, 66

Jacoby, 183

Jacquet, leeches, 511

Jadassohn, 340

Jäderholm, 419

Jaenichen, 517

Jäger, 223

Jakimovitch, 201

Jander, 502

bleaching, 257

Janssens, 151,183

Janus green, 181, 332, 333 pancreas, 395

"Japanese" method of section mount. ing, 114

Jaquet, 510

Jelinek, 101

Jenner, blood stain, 383

Jensen, protozoa, 540

Joest, 509

Lindsay Johnson, 206

aceto-osmic; 51

collodion method, 101

eye, 494

fluid, 37

on metallic stains, 198
Johnston, 262, 415

nerves of Petromyzon, 405

Johnstone-Lavis and Vosmaer, section grinding, 109

Joliet, gum glycerine embedding, 107

Jones, 300

Jonescu, 508

Jordan, 66

Jörgensen, 309

nucleoli, 314

Joris, 434

Joseph, 197, 341

white-of-egg mass, 240

Juliusburger, Nissl, 413

Kadyi, 407

brain, 402

Kaes, myelin, 447

Kaiser, 170, 223, 513

glycerine gelatin, embedding, 93

myelin, 447

solution, 44

spinal cord, 408

Kalb, 549

Kallius, 455, 469

embryonic cartilage, 376

Golgi modification, 461

Kaplan, 477

myelin, 452

neurokeratin, 441

Kappers, elderberries, 409

Karawaiew, 546

anobium eggs, 287

Karger, 493

Karyosome, 310 et seq.

Kastschenko, 262

Selachian embryos, 280

Kató, Cajal's method, 425

Kattwinkel, 400

Katz, ear, 497

Kawamura, 366

Keibel, 270

Kent, iodine fixation, 51

Kenyon, 508

Keratohyalin, 340

Kernschwarz, 211

Graham Kerr, 119

reconstruction method, 26 L.

Kerschner, 205

Kidney, 394

Killing, amphibians, pithing, II

birds, lizards, newts, 11

by sudden heat, 12

by hot water, 12

large mammals, ether and chloroform for, coal gas for, 11

King, brain hardening, 405

Bufo eggs, 278

carbolic thionin, 168

Kingsbury, 368

Kingsley, Limulus ova, 288

Kionka, orientation method, 274

Kishinouye, spider eggs, 287 
Kizer, blood, 381

Klein, cornea, 343

Kleinenberg, hæmatoxylin, 155 picro-sulphuric, 55

Knife position, paraffin cutting, 83 slope, 85

\section{Knowen, 81}

Koch, 449

Von Koch, copal method, section grinding, 108

Kockel, 388 .

Kodis, 404 myelin, 442

Koerner and Fischer, tannin fuchsin flagella stain, 548

Kofoid, gastropod embryology, 283

Köhler, 516

Kölliker, mammal blastoderm, 268 embryology of mammals, 264 indigo-carmine for bone, 376

Kollman's fixative for fish eggs, 280

Kolmer, 26, 495

Kolossow, 32, 206, 209

modification of Cajal's axis cylinder stain, 462

prickle cells, 339

Kolster, stomach, 392

Kopsch, 386

osmic method, 327

Golgi method, 464

molluse eyes, 502

teleost embryology, 280

Korotneff, 13

Korschelt, 541 cephalopoda, 283

Kostanecki, mercuro-nitric, 48 and Siedlecki, Ascaris ova, 290 and Wierzejski, mollusc eggs, 284

Kotlarewski, 404

Kowalewsky, teleost eggs, 280

Kowalski, 425

Kozowsky, myelin, 446

Krause, 173, 182, 393, 482, 496 carmine mass, 235

salivary glands, 392

Krauss, 201

Krecker, 14

Kresofuchsin, 169

Kresyl blue, 383

Kresyl-echtviolett, 184

Kresyl violet, 184

Krogh, 415

Krohnthal, lead sulphide impregnation, 475

Kromayer, 184, 388 plasma fibrils, 339

Kronecker's serum, 218, 264

Krüger, 354

Harpactida ova, 288

Kühne, maceration, 247

Kuhnt, 496

Kükenthal, 14, 510

Lumbricus, 509

Kull, 392

\section{Kultschizky, 341}

bichromate and sublimate, 43

double embedding, 105

mucin, 391

myelin method, 447

preservation of tissue in ether or xylol, 4

rubin for neuroglia, 486

solution, 42

spleen, 393

Kupffer, 393

Kuskow, digestion, 249

Lachi, 401

Golgi method, 463

Lactic acid, 233, 254

Lactophenol, 222

Lævulose, for myelin preparations, 448

Laffont, 341

Lahille, 499

Lake, 132

Lakmoid, 393

La mbert, Epeira eggs, 287

Lamellibranchiata, 284, 500 et seq.

cilia, 503

eyes, 502

maceration, 247

pigment, 257

Lams, 265

Landois, maceration solution, 245

Landolt, 496

Lane, pancreas, 396

Lang, corrosive liquid, 46 mollusca, 501

Langdon, 510

De Lange, 450

Langerhans, 341

guin glycerine, 221

islets of, 395

Langeron, 59

Lanis, fixation of mammal eggs, 265

Lankester and Bourne, 508

Lansberg, 541

Lanthanin, 302

Larvæ, echinoderm, 520

Laslett, 447

Lattice fibres, 393,394

Laurent, 181

Lau terborn, 75,547

Lavdowsky, 192, 228, 498 maceration, 248

Laveran's solution, 534

Law, nerve-endings, 374

Lawrence, 223

Lead-gum embedding, 106

Lead sulphide, Golgi method, Krohnthal, 475

Leber, 495

Lebrun, Anuran embryology, 276

Lecithin. See under Fatty Substances, 356 et seq.

Bolles Lee, 407

decalcification, 251 


\section{Bolles Lee-contd.}

celloidin section mounting, 116 chick embryos, 272

chromosome stain, 303

on choice of stain, 134

dry cutting process, celloidin, 104

ear and eye, $493-498$

fish embryos, 279

fixation of ground cytoplasm, 301, 302 gold, 206

iron carmine, 139

Kernschwarz, 211

mounting fluid, 222

mounting Golgi preparations, 468

myelin stain, 442

osmic-pyrogallol method, 208

paper cell mounting method, 230

on staining nucleus intra vitam, 310

Tunicates, 499

sponges, 525

Leeches, 511

maceration, 247

nervous system, 511

Legal, alum carmine, picric, 138

Legendre, Golgi a pparatus, 438

Léger, sporozoa, 546

Legros, 201

Amphiox us, 281

Leiper, 514

Leishman, blood stain, 385 protozoa, 535

Lemon-juice gold method, 204

Von Lendenfeld, 525

Von Lenhossek, 342,455

Nissl bodies, 413

molluse eyes, 502

platinum chloride, 50

Lennhoff, methylen blue, nerve, 414 processes for nerve cells, etc., 476

Lennox, 496

Lens, eye, 343

Lenssen, 513 Hydatina, 288

Leontowitsch, 195

Lepidoptera ova, 286

Lepkowsky, 375 vessels in teeth, 373

Leuckhart, embedding boxes, 73

Leutert, 300

Levaditi, 549 blood smears, 383

Levi, fixation of manmal eggs, 265 mitochondrial method, 325

\section{Levulose, 221}

Levy, 389

Bevan Lewis, 408

Lewis, 389, 511 tissue culture, 555

Lewy, 450

Lich tgrün, 159

Liebetanz, 548

Liesegang, 407 Cajal's method, 426

Ligamentum nuchæ, digestion, 249
Light green, 181

Lillie, 332

Unio eggs, 284

Limax, 283, 501

Lime salts, 300

Limnæa ova, 284

Limulus, 288

Linville, molluse ova, 283

Lipin, 357

Lipoids, 357

List, 214, 314

Coccids, 506

Mytilus, 500

Sagartia, 522

Lithia, for picric fixed material, 54

Lithium carmine, 140

Little, Hydra, 521

Live slide, Drew and Griffin, 530

Liver, 393

molluse, 501

Lizard blastoderms, 275

Locke's solution, 218

Locy, spider eggs, 287

Loele, 390

Loewy, method for integument, 339

Löffler, stain for flagella, 548

Loisel, 367

fat, 369

sponge, 526

Long, constant temperature box for mammal eggs, 266

and Mark, fixing fluid for mammals, 266

mouse embryology, 263

Longhi, 542

Longworth, 342

Lönnberg, 516

Looss, 514, 516

corrosion of chitin, 250

Nematodes, 513

Lophomonas, 533

Lord, methylen blue, nerve 414

Löwit, blood fluid, 380 gold, 204

Loyez, 442

Lucidol, 308

in acetone, 59

blood, 382

Ludford, 308

Lugaro, 417

collargol, 434

Lugol's solution, 45

Lühe, 516

Luithlen and Sorgo, 414

Lumbricus epidermis, nerve, 421 nerve, 425

sections, 509

Lundvall, 377

Lustgarten, 169

Luxenburg, Nissl bodies, 413

Lymphatics, in teeth, 373 glands, 394

Lymph-spaces, methylen bluc, 105

Lysol, for maceration, 248 
Maas, 213, 249

sponges, 526

Macallum, 300, 301

iron methods, 297

Mac Bride, larvæ of Echinoderms, 520

Maceration, 243 et seq.

Béla Haller's mixture, 247

epithelium of molluses, 503

Ranvier, 244

Moleschott, 244

Landois, 245

Macklin, 271, 389

Maclean, 357

Macrophages, 388

Von Maehrenthal, 209

Magdala red, 169

for spleen fibres, 394

Magenta, 169, 171, 2.3

Magini, 477

Magnesia carmine, 140

Magnesium chloride or sulphate, for narcotisation, 15

Malachite green, 181,213

Malaria, 546

pigment, 300

Malassey, 218

Mall, 378

Mallory, 354

eosin and methylen blue, 181

neuroglia, 483

phospho-molybdic àcid hæmatoxylin, 157

phospho-tungstic, 158

säure-fuchsin and phospho-molybdic acid, 351

Weigert stain, 481

Mammalia, Cajal's methods, 424 eggs 266. See aiso under Ova.

Mammals, chromosomes, 305 et seq. clearing and embedding, 269 embryological methods, 263 fixation of whole tubes, 268 isolation of eggs, 263 et seq.

Manchester brown, 161

Manfredi, 206

Mann, 26, 31, 114, 155, 398, 468

chromo-sublimate, 48

fat, 366

extraction of corrosive sublimate, 45

Golgi-Cox, 473

Golgi method precipitates, 466

methyl blue eosin, 183

osmio-sublimate, 48

stain, for protozoa, 544

sublimate formol, 63

Mann-Kopsch method, 328

Marcacci, maceration, 247

Marcano, blood, 381

Marchi, degenerate nerve, 449 et seq. mollusca, 501

tendon organs, 347

Marcus, 446

Maresch, 352, 394

Marfori, 300
Marie, 400

Marina, 405

Marine animals, fixation of, 27

cell inclusions, 332

Mark, collodionisation, 88

reconstruction, 262

Marsh, 229, 230

Martin, 392

tracheæ, 508

Martinot ti, 167, 196, 354, 369, 386, 467, 408

Golgi method, 465

elastic tissue, 352

Mason, brains, 405

Massart, 214

Masson, connective tissue, 351

Mast cells, 354 et seq.

Unna's method, 356

Matschinsky, bone sections, 371

Matuszewski, 450

Maurice and Schulgin, 213

Mawas, 496

Maximow, 356, 389

Altmann method, 322

May-Grünwald, 384

Mayer, 15, 33, 155, 184, 297, 350

acetate of potash, 151

albumen water method for sections, 113

alcoholic cochineal, 144

aluminium chloride carmine, 138

benzol embedding, 78

bleaching, 256

carmalum, 137, 212

cartilage, 377

chitin, 508

cochineal stain, 135

decalcification, 253

desilification, 255

eye, 339

glychæmalum, 153

hæmacalcium, 155

hæmalum, 152 et seq.

hæmastrontium, 156

on hæmatoxylin staining, 145

iron staining, 298

methylen blue, 193, 196

mucin stains, 392

paracarmine, 142

picro-hydrochloric, 56

picro-magnesia carmine, 141

picro-nitric, 55

Plutei, 520

section stretcher, 87

triacid, 175

Maysel, 161

McClung, urea in fixing, 305

McClure, 502

McCrorie, 549

Medium of Farrant, 221

Medulla, hardening, 399

Medusæ, 523, 524

killing, 12

-maceration, 246 
Medusæ-contd.

narcotisation, 13

sections, 524

Mehnert, 275

Meirowsky, 549

Meisenheimer, gastropoda, 283

Melanin, 300

Melting point of paraffin, 91

Membrana, nictitans, 339

limitans, marginal neuroglia, 48.)

Membranes of eggs, 284, 285

Mendel and Bradley; 300

Menthol, for narcotisation, 12

Mercier, 449

Mercury, bichloride, 44

alcoholic liquids, 46 et seq.

and acetic acid, 44

extraction, 45

sodium thiosulphate, extraction by, 46

washing out, 45

and bichromate, 48

and formol, 63

mounting media, 220

nitric mixtures, 47

sublimate and salt, 46

Merkel, indigo-earmine, 212

Merkel's fluid, 39

Merton, 502

Merzbacker, 485

Mesostomidæ ova, 288

Messner, picrocarmine, 414

Metachromasy, 133

Metachromatic dyes, 133

Metagelatin vehicle, Fol, 238

Metallic stains, 197 et seq.

theory of, 197 et seq.

osmic acid, pyrogallol, 208

Metcalf, Chiton ova, 284

Methyl, aniline green, 159

blue, 183

with eosin (Mann), 183

nephridia, 511

green, 159 et seq.

for chromatin, 293

with eosin, 181

mixture for maceration, 247

salicylate oil, 68

violet, 162

for fibrin, 388

fluid for blood, 382

plasma fibrils, 340

B., 184

Methylen blue, 170, 186 et seq.

blood, 383

Cajal's diffusion process, 478

Distomum, 516

and eosin (Mallory), 181

eosin stains for blood, 383 et seq.

and erythrosin, Nissl granules, 415

fixation of stain, 192 et seq.

frog bladder, 349

intra vitam staining, 187, 344, 477
Methylen-contd.

for epithelia, lymph-spaces, 195

insect eyes, 508

method, cornea, 343

methods for sections, 194

modes of staining, 190

myelin, 449, 452

nerve endings, 344

methods, 477 et seq.

Nissl bodies, 411

polyehrome, 186

Säurefuchsin, 351

for skeletons, 377

Methylenazur, 186, 187

Meves-Flemming modification, 36

Victoria green stain, 333

Meyer, Berlin blue neurofibril method, 434

methylen blue nerve method; 477

myelin, 445

new celloidin method, 103

Mibelli, 352

Mica sheets, for mounting sections, 119

Michaelis, 133, 181

blood stain, 383

fat, 367

polychrome methylen blue, 187

Triton eggs, 277

Michailow, 190, 195

Microchemistry, 293 et seq.

Microglia, 493

Micro-injection, 270

Microscopes, 8

Microtome, 8

sliding, freezing, rotary, Jung, Minot, Tetrander, rocking, 9

Migula, 219

Miller, cement, 229

Minchin, albumen method for mammal eggs, 266

embedding protozoa, 542

hæmatozoa, 547

sponges, 525

Minervini, 353

Mitochondria, fresh examination, 332, $333,334, \mid 26,-361$

Gatenby on, 316

protozoa, 540

teeth and bone, $\mathbf{3 7 5}$

vital stains, 332

Mitrophanow, 183, 342

chick embryos, 273

integument, 339

myelin, 447

Mitsukuri, reptile blastoderms, 275

Mixed process of Golgi, 460

Mobius, 348

maceration medium, 246

Moerner, 377

Moleschott and Piso Borme, salt alcohol for maceration, 244

Molge, embryology, 277

Moll, cartilage, 376

Mollison, Alkanna, 367 
Mollusca, 282, 500 et seq.

embryology, 282 et seq.

to kill extended, 500

maceration of epithelium, 503

mucus glands, 504

narcotisation, 12

nervous system maceration, 247

Molluscoida, 500

Molybdenum-toluidin blue, neurofibrils, 417

Mönckeberg and Bethe, 31

bleacher, 256

Mondino, 472

Montanari, 419

Montgomery, 515

nucleoli, 314

ova of Theridium, 287

Monti, 477

gastric glands, 393

Mordant, 131

Mordanting, for coal tar dyes, 165

Moreaux, formol-picric-trichloracetic, 62

Morel, 175

Morgan, Ascidia, 282

cockroach eggs, 285, 286

frog embryology, 276, 278

Morphia, for narcotisation, 16

Moseley, 503

Mosse, 415 argentamin, 452

Mosso, 380

Mott, 129

Mounting media, giycerine, 222 resinous, 225

Farrant, Allen, Brun, 221 . sections, collodion paper method, 116 Mayer's albumen method, 113 watery, 116

Mozejko, 503 vaso-dilators, 233

Muchæmatein, 392

Mucicarmine, 392

Mucilage syrup mass, 110

Mucus, 391 et seq. glands, mollusca, 504

Muir, 549

blood, 381

Mullenix, 497

Müller, 392

Berlin blue mass, 240

fluid, 42

with formol, 63

for maceration, 245

silver method, 201

Mummery, embedding tooth germs, 372

nerve tissue of teeth, 373 .

J. A. Murray, Altmann's method, 321, 365

on glycogen stain, 296

connective tissue stain, 354

chrome-osmic method, 326

lead gum, 107
Muscle-fibres, maceration, 246

and tendon, 344, 347

spindles, 345

Myelin, ammonio-chloride of tin, Besta, 448

and axis cylinder stains, 452

Berkley rapid method, 447

bulk stain, 448

iron method, 451

Kultschitzky, 447

osmic acid, 450 et seq

stains, 442

silver nitrate, 451

Weigert stains, 442

Myers, 415

Mytilus, 500

Myxosporidia, 546

Nabias, gold chloride Golgi method, 476 mollusca, 501

Nageotte, 399, 448

Nails, 341

Nakahara, urea fixation, 305

Nakanishi, blood, 383

Nansen, 245

Naphtha, clearing, 77

Naphthalin, monobromide, 224

rose, 169

Naphthol, 390

Naphthylamin brown, 408

Naples water bath, 79

Narcotisation, anemones, Holothuria, Ascidia, Mollusca (menthol), 12

Medusæ, starfishes (chloroform), Actiniæ, Capitellidæ (ether alcohol), 13

Cristatella, 14

larvæ (chloretone), $1 \dot{4}$

Alcyonella, Bryozoa, Annellida, Mollusca, Nemertians, Actiniæ (chloral), 14

hydroxylamin, 15

magnesium chloride or sulphate, 15

morphia, curare, strychnin, prussic acid, 16

asphyxiation : tobacco, carbonic acid gas, soda water, 16

hydrogen peroxide, 17

Hydra (cocaine), 14

Vorticellidæ, Rotatoria, Vermes (cucaine), 15

Ascidia, Rhopalæa, 15

Ciona, 16

Medusæ, 16

snails, 16

menthol, nicotine, 12

chloroform, cocaine, eucaine, ether, alcohol, 13

methyl alcohol, chloreton, chloral hydrate, 14

Nasal mucosa, maceration, 247

Nathusius, 341

Nealey, bone sections, 372 
Nebenkern, 316

Nelis, brain fixation, 405

Nematodes, 513 et seq. corrosion of chitin, 250 ova, 289

Nemertina, 514 et seq. heat killing, 12

Nephridia, Histriobdella, 511

Nerve endings, beetle, 344

Bielschowsky, 426 et seq.

frog, 344

gold method, 344

insect, 345

methylen blue method, 344

silver method, 345

bichromate of silver, 345

of teeth, $373 \mathrm{et}$ seq.

staining, methylen blue, 189,191

Nervous system, Achucarro's tannin neuroglia method, 490

Apathy's neurofibril method, 416 axis cylinder, 452

axis cylinder and dendrite stains (Golgi and others), 454

Cajal's double-impregnation process, 461

Golgi bichromate - sublimate method, 470

processes similar to Golgi methods, 475

Cajal's method, advice as to choice of formula, 424

(Golgi), axis cylinder and dendrite, treatment of larvæ, $459-460$

axis cylinder and dendrite, formaldehyde modifications, 463

Berkley rapid myelin method, 447

Bielschowsky methods, introductory, 426

for sections, 427

for peripheral nerve fibres, 428

for pieces, 429

modifications, 430 et seq.

Cajal's gold chloride neuroglia method, 487

methods, special objects, 424

neurafibril methous, 419 et seq.

collodionising sections, 406

Da Fano's Bielschowsky methods, 432

Donaggio's neurofibril methods, 417

Fish's fluid, 402

fixation, 397

alcohol, 400

chromic salts, 403 et seq.

formalin, 401

general methods, 397

stains, 407

Golgi method, avoidance of precipitates, 466

cutting and mounting, 467 469

Gulgi internal apparatus, 435 et seq.
Nervous system-contd.

Golgi-Cox modification, 472

hardening, 398 et seq.

Held's method for marginal neuroglia, 484

injection fixation, 397

Kultschitzky, myelin, 447

maceration, 245, 246, 247

Marchi method, 449

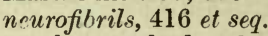

other methods, 434

neuroglia, 479 et seq.

Nissl substance, 410 et seq.

Pal method, 446

phosphomolybdic acid hæmatoxylin, 157, 158

polarised light, 398

preliminary fixation for Sauropsida, 405

Sand's neurofibril method, 434

sections, 405 et seq.

special cyfological methods, 410 et seq.

sustaining apparatus of medullary sheaths, neurokeratin, 439

Simarro's process, 419

Weigert's myelin stains, 442 et seq.

Nesteroffisky, 206

Nettovitch, 505

Neuberger, decalcifier, 254

Neubert, 297

Neukirch, 297

Neumayer, 262, 270

Neurofibril methods, 416 et seq. advice on, 424

Neuroglia, 479

Achucarro's tannin method, 496

Cajal's gold chloride sublimate method, 487

granules, 486

protoplasmic, 486

marginal, Held's method, 484

methods, 479 et seq.

staine, Anglade and Morel, 483

Da Fano, 484

Held, 484

Oppenheim, 486

Weigert, 480

Benda, 482

Mallory, 483

Neurokeratin, 439, 441

Neurosomes, 415

Neutral, balsam, 226

dye, 120

gentian, 396

red, 179 et seq.

blood, 382

granules, 389

kidney, 394

mucin, 392

pancreas, 395

Neutralisation of carmine mass, 235

Neuville, silver impregnation mass, 237 
Nicolas, 32

embedding in gelatin, 93

reptiles, 275

Nicolle, 168

Nicotine, for narcotisation, 12

Nigrosin, 170, 182, 408

Nikiforow, 102

Nile blue, connective tissue, 354 fat, 362,368

Nissl, $410,411,415,450,480$

brain hardening, 403

bodies, 410 et seq.

stains other than Nissl's, 412

granules, nature of, 130

methylen blue and erythrosin, 415

method, precautions against fading, 413

modifications, 413

Nitric acid, and acetic, for maceration, 247

bleacher, 257

and chlorate of potash, for maceration, 247

corrosion, 250

decalcification, 252,253

for fixation, 38

fixative for neurofibrils, 417

formol, 64

maceration, 247

nerve tissue, 404

Noack, 83

Noll, corrosion, 250

Nordmann, mast cells, $35 \tilde{5}$

Nosema, $\mathbf{5 4 6}$

Nowak, sublimate formol, 63

tactile corpuscles, 342

Nuclear stains, coal tar, 159 et seq.

Nuclease, 308

Nucleoli, Gatenby on, 308, 310

malachite green for, 181

Nucleolini, 311

Nucleus, "vital" stains, 310

Nudibranchs, 502

Nuttall, Cooper and Robinson, 506

Obersteiner, brain, 403

Obregia, 469

method for paraffin and celloidin sections, 115, 119

Obst, nucleoli, 314

Octopus, 501

Odenius, maceration, 247

Odier, Golgi method, 465

Oestergren, $13,515,517$

Ohlmacher, 167

alcoholio corrosive, 47

fluid, brain, 405

formaldehyde process, 165

myelin, 452

picric acid, 176

Oils, 65 et seq.

See also under definitive name, e.g.,

Cedarwood.

winter green, 271
Okajima, elective stain for reds, 388 fat, 368

Olmacher, 404

Olt, manipulation of frozen sections, 109

Opalina, 533

Ophiothrix, 519

Ophiuridea, 519

Oppel, lattice fibres, 393

Oppenheim, neuroglia granules, 486

Oppitz, 201

Orange G., 172, 173, 177, 274 ,
Orcein, 212

embryonic cartilage, 376

elastin, 353

method, Unna, 351

water blue, 340

Orchella, 211

Orcin, 212

Organic acids, fixation by, 51

Orientation methods, 273 et seq.

for blastoderms, 273

in embedding, 80 et seq.

Origanum oil, 68

for celloidin sections, 102

Orr, 451

Marchi method, 450

Orth's fluid, 260

Orthoptera eggs, 286

Orton, 503, 508

Osmacet, 380

Osmium chloride, 50

Osmium tetroxide, reduction, 29, 32, 124 acetic acid maceration, 246

bleaching, 31

restoration of staining, 31

for blood, 380

gold method (Viallane), 205

Kopsch method, 327

nerve tissue, 404

and fat, 356 et seq.

and picric, 56

pyrogallol method, 208

regeneration of, 30

fixation by vapour, 30

after-treatment, 30

sublimate, 48

vapour method, Cramer, 330

Ossifying cartilage, 254, 377

Osteoblastic areas, 378

Ostracoda, 505

Wo. Ostwald, 125

Ova, of Echinoderms, fixation, 260

fixation of mammalian, 265

frog's, 275 et seq.

Insecta, Pisces, 259

treatment of uterine eggs of mammals, 266

Ovary as index to pregnancy, 263

Ovens, paraffin, 79

Overhardened tissue, rejuvenation of, 462

Overton, 31,34

iodine vapour, 51

Oviatt and Sargent, 233 
Oxalic acid, for maceration, 247

Oxidised hæmatoxylin (Unna), 155

Oxidisers as fixers, 21

Oxydase reaction, 390

Oxygen, place in tissue, 390

Pacini, 220

blood, 380

Pal, 472

Paladino, 453

Palladium chloride, 50 decalcification, 252

Palythoa, 522.

Pansch, injection, 242

Pancreas, 395, 396 inclusions, 338

Pancreatin, digestion, 248

Paneth, cells, 392

Paper trays, embedding, 73

Papillæ foliatæ, 342

See also under Corpuscles.

Pappenheim, 356

hæmopoietic tissue, 386

mast cells, 355

panoptic stain, 383

pyronin and methyl green, 172

Paracarmine, 142

Paraffin, pure, 91

overheated, 92

sections, flattening, 90

clearing and mounting, 91, 111 et seq.

embedding, 76 et seq.

shaping block, 83 et seq. cooling, 82

bath, 77

Paramœcium, culture, 537

Paravicini, 418

Parietal cells, 393

Paris green, 160

Parker, 508

bleaching, 257

methylen blue, 194

and Floyd, sheep brain, 402

Parlodion, 95

Parmenter, Amblystoma, 306

Parolein, 228

Parthenogenesis, 259

Partington and Huntingford, 32, 364

Partsch, cochineal alum carmine, 137 decalcification, 254

Passarge and Krösing, 354

Paton, 272

Bielschowsky modification, 430

Patten, 286, 502

mollusca, 504

orthopterous eggs, 286

orientation during embedding, 81

Patterson, chick, 273

Pauropoda, 505

Pavlov, myelin, 446

Peabody, corrosion, 249

Pearl, worms, 509
Pedicellina, 500

Pekelharing, 297

Pelagic fish ova, 281

Penfield, Golgi apparatus, 438

Holmgren's trophospongium, 439

Pensa, reconstruction method, 261

Peppler, 549

Pepsin, digestion, 248

Peptic cells, 392

Peptonum siccum, 233

Perchloride of iron impregnation, 209

Perdrau, Bielschowsky method, 434

Perényi's fluid, 39

Pérez, fly pupæ, 286

Periplaneta eggs, 285

Permanganate of potash, bleacher, 31, 256

Henneguy's mordant, 165

for osmic solution, 29

Perophora, 282

Peroxide, of benzol, blood, 382

of hydrogen, bleaching, 256

for killing, 17

of magnesium, 256

of sodium, 256

Perrier, worms, 509

Perrin, 122

Perusini, 453

Peter, 140

reconstruction method, 262

yolk stain, 260

Peters, R. A., culture of Paramœcium, 537

Petromyzon, nerves, 405

Petronne, 387

Petrunkewitsch, Hymenoptera, 286 mercuro-nitric, 48

Pfitzner, 182

protozoa, 541

safranin, 166

Phalacrocera, 286

Phalangida ova, 287

Phenol, solution for fixation, 47

Phenylen brown, 161

Philippson, 339

Phloroglucin decalcifying mixtures, 254

Phloxin, 180

Phoronis, 512

Phosphatides, 356 et seq.

Phospho-molybdic acid hæmatoxylin, Mallory, 157

Phospho-tungstic acid fixative, 38

Phosphoric acid decalcification, 252, 254

Photographic negative varnish mount, ing medium, 228

Photoxylin, 95

Physa eggs, 284

Pianese, 175 methylen blue eosin, 181

Picraminic acid, 176

Picric acid, 54 decalcification, 252

plasma stain, 176 
Picric acid-contd.

and alcohol, 55

for maceration, 248

Picro-acetic, 55

Picro-carmine, 140 general nerve stain, 414

Picro-chromic, 56

Picro-hydrochloric, 56 decalcifier, 254

Picro-indigo-carmine, 212

Picro-nigrosin, 182, 213, 350, 408

Picro-nitric, 55 decalcifier, 254

Picro-osmic, 56

Picro-platinic, 56 with formol, 63

Picro-Säurefuchsin, 176, 350

Picro-sublimate, 48

Picro-sulphuric, 55

Pictet's liquid, 219

Pink salt, 418

Pintner, 29, 515

Pisces, embryology, 279 et seq .

Pitfield, flagella, 549

Pittock, reconstruction method, 262

Pizon, Ascidia, 282

Placenta, glycogen, 295

Planaria, 44, 517

Plankton, general preservative, 525

Planorbis ova, 284

Plants, Golgi apparatus, 325

Plasma celis, 354 et seq.

fibrils, 339

stains, 171 et seq.

Bolles Lee, 314

Plasmodium, 546

Plasmosome, 310 et seq.

Plastic reconstruction, 261

Plastochondria, 315

Platino-aceto-osmic, 38

Platinum chloride, 49

Platner, neurokeratin network, 441

Kernschwarz, 211

Pleschko, 194

Du Pléssis, 515

Pleurobrachia, 524

Pluteus, 520

Podwyssozki, 167 fluid, 36

Polaillon, perchloride of iron impregnation, 209

Polariscope, 332

for fat, 361 et seq.

in nervous system, 398

Policard, 547

Politzer, 496

Polumordwinow, 413

Polychœrus ova, 288

Polychrome methylen blue, mast cells, 355

nerve cells, 476

for nerve tissue, 414

Polychrome toluidin blue, 196

Polyclads ova, 289
Porifera, 525 et seq.

decalcification, 251

Post-chroming, 326

Post-impregnation for gold, 207 et seq.

Post-mortem changes, 25

Post-osmicating, 326

Potash method, for skeletons, 378

Potassium, 301

See under Bichromate.

bichromate, maceration with, 245

chlorate and nitric acid, for maceration, 247

iodide, brain, 404

permanganate, for maceration, 246

sulphocyanide, for maceration, 245

Pötter, 446

Pouchet, bleaching, 256

Pranter, 353

Precipitates, Golgi method, 466

Prenant, 181, 301, 497

Preservation media, 216

by alcohol, ether, xylol, or toluol glycerine mixtures, acetic acid, cedarwood oil, 4

Preyer, 13

Priapulus, 512

Prickle cells, 339

Primerose soluble, 180

Pritchard, 498

Progressive stains, coal-tar, 159

Propylic alcohol balsam, 227

Protease, 308

Protozoa, 526 et seq.

Borrel's blue, 534

Borrel method, 532

cell inclusions, see pages $316-338$

collection, 529

culture, 527 et seq.

of paramœcium, 537

current stains, 543 et seq.

determination of life cycle, 530

Drew-Griffin live slide, 530

embedding, 542

examination in a coloured medium, 540

of fæces, 538 et seq.

fixing and preserving, 541 et seq.

flagellates, 533, 547

general fixation and staining, 531 et seq.

general morphology, 531

hæmatozoa, 546

killing by heat, 12

iodine, $\mathbf{5 4 1}$

-Romanowsky methods, 535

immobilisation, 539

staining Coccidia, 532

ciliates, 533

hæmamœbæ, 533

intra vitam, 540

stains for flagella, 548

sporozoa, 546

sphærozoa, 546 
Protozoa-contd.

Taylor's culture method for amœbæ, 536

trypanosomes, $533,539,547$

Prowazek, 179

Prussian blue, aqueous mass; Ranvier, 240

acid cold, Beale, 239

cold, Beale, 239

glycerine mass, cold, Ranvier, 239

Ranvier, 236

reaction, 297 et seq.

Prussic acid, 16

Przesmycky, nucleus staining, 310

Pulmonata, nervous system, 501

Purcell, Atta ova, 287

eyes of Phalangida, 508

Purpurin, 212

Pusateri, tachiol, 425

Puschkarew, 542

Pyridine, 59

with lucidol, 59

nitrate-pyridine fixative, 418

Pyroligneous acid decalcification, 252

Pyronin, 172

and methyl green, 172

pancreas ducts, 395

Pyrosin B., 180

Pyroxylin, 97

Pyrrhol cells, 388

Pyrrol blue, 389

De Quervain, 26, 398

Quinolein blue, 182, 367

Raadt, blood stain, 385

Rabl, 341

chromoformic, 35

cochineal, 137

embryological fixation, 260

lens, 343

picro-sublimate, 48

platinum chloride, 49,50

platinic sublimate, 260

superheated paraffin, 89

teleost eggs, 280

Rabl-Rückhard method for salmon eggs, 280

Racovitza, mollusca, 504

Raffaele, 281

Raja, electric organ, 346

Rana, embryology, 277

Randolph, 14

Ranke, neuroglia method, 487

Ransom, 516

Ranson, Cajal's method, 426

Ranvier, 182, 340, 356, 393

alcohol, 58

bladder, nerves, 349

chromic acid maceration, 246

carmine gelatin mass, 234

cornea, 343
Ranvier-contd.

electric organs, 345

formic acid, gold, 204

eleidin granules, 341

eye of Triton, 494

lemon juice, gold, 204

maceration with alcohol, 244

nerve endings, gold, 345

silver method, 345

picro-carmine, 141

silver impregnation mass, 237

method, 198, 200

Vom Rath, picro-sublimate, 48

Rawitz, 38, 184, 502

brain hardening, 404

carmine, 138

indulin, 409

mucin, 392

picro-nitric, 56

V. Recklinghausen, silver, 200

Reconstruction from sections, 261

Red blood cells, elective stain, 388 gelatin mass, 234

Reddingius, 314

Redenbaugh, 15

Van Rees, 285

Refractive indices of media, 216

Regaud, formol-bichromate method, 323 myelin stain, 442

Regenerating nerve tissue, 421,425

Regressive stains, 162 et seq. coal tar, 159

Rehm, 227

Nissl method, 413

Reichenbach, decapoda ova, 288

Reinke, 177, 341

maceration, 248

orange method, 171

Rejsek, corrosion, 249

Rejuvenation of tissue, Golgi, 462 by veronal or chloral, 421

Remak and Goette's fluid, for frog eggs, 279

Renault, $214,343,390$

Rengel, 38

Reptilia, embryology, 274 et seq. brain, 405

Resins, 225 et seq.

Resorcin-fuchsin, 353

for embryonic cartilage, 377

Retina, 493 et seq. of arthropods, maceration, 248 bleaching, 255

maceration, 247

Retterer, smooth muscle, 348

tendon, 347

uterine eggs of mammals, 267

and Lelievre, 377

and Zenker, injection, 242

Retzius, 191

Rhumbler, 75

Ribbons, paraffin, 86 and 89 et seq.

Richards, 14

Rieder, 367 
Ringer's solution, 218

Rio-Hortega, Bielschowsky, 431 neuroglia, 490-492

Ripart and Petit's fluid (copper), 53

Ritter, 282

Robertson, 449,451 tsetse flies, 539 and Macdonald, 474

Robin, injection masses, 233 et seq. and Ranvier, 232

Robinski, silver, 200

Rochon-Duvigneaud, 495

Roessle and Yoshida, 394

Rollett, 343 maceration, 246

Romanowsky, stains, 384 et serq.

Roncoroni, rodlet of, 421

Roosvelt, 210

Rosaniline, 120

Röse, bone, 370

Rose B, 180

Rose de naphthalin, 169

Rosein, 169

Rosenstadt, 340, 509

Rosenthal, fat, 367

Rosin, 181, 413

Ross, blood, 383 thick film method, 534

Rossi, 449 blood, 380 tlagella, 549

Rossolimo, 451

Rotatoria, 512 Jennings, 288

Rothig, 169 axis cylinder stain, 453 mammals, 263 methylen-azur, 408

Rouget, 191, 200

Rousseau, 497 decalcification, 251 desilicification of sponges, 255 sponge, 526

Rousselet, 15, 512 preparing aqueous mounts, 230

Rubaschkin, neuroglia, 482

Rubin, 169 method for neuroglia, 486

Rubin S, 171

Ruffini, 347

Russell, glycogen staining, 296

Russo, Ophiothrix, 519

Ruzicka, 314

Ryder, 105

Sabin, micro-injection, 271

Saeff tigen, 513

Saffrosin, 180

Safranin, 165 et $s e q, 391$

elastic tissue, 352

and light green, 181

mucin, 391

myelin, 452

and wasserblau, 351
Saguchi, 396

pancreas cells, 338

Sahli, 226

brain hardening, 403

myelin, 452

Sainton, 400

Sala, 440

axis cylinder dendrite stain, 459

Salamandra, embryology, 277

Salicylic acid, fixation, 53

Saling, Tenebrio, 506 eggs, 287

Salivary glands, 392

Salkind, lead gum embedding, 106

Salmonidæ, embryology, 280

Rabl-Rückhard method, 280

Salts, for fixing, 40 solutions, 218 for maceration, 244

sublimate, 46

Samassa, 468, 525

Samter, 75, 288

Sanchez, 425

Sand, 482 neurofibril method, 434

Sandal-wood oil, 68

Sandarac, 228

media, Gilson, 227

Sanders, 473

Sankey, 408

Sansom, Carnoy modification, 53

Sanzo, fixing apparatus, 260

Sarcolemma, 344

Sata, fat, 367

Sattler, 201

Sảurefuchsin. See under Acid Fuchsin.

Saurerubin and Orange G, 393

Sauer, kidney, 394

Säureviolett, 181

Savini, 177, 415

Sazepin, 506

Scala, 542

Scarlet R. See Scharlach.

Scarpatetti, 408

Schäfer, 232

Schaffer, 251, 261, 300, 350, 356 bone, 370

decalcification, 252,254

thionin for bone and cartilage sections, 376

Schaper, 262

Scharlach R, 356 et seq. fat, 367

Herxheimer, 367, 368

Schaudinn, fluid, 546

Woodcock's modification, 541

Schaxel, 309

Schiefferdecker, 67, 68, 444

celloidin masses, 241

eye, 496

maceration mixture, 247

and Kossel, 356

Schlemmer, Bielschowsky, 431

Schmaus, 407 
Schmidt, gastropod embryology, 283

Schmorl, thionin for bone and cartilage sections, 376

Schneider, 300 aceto-carmine, 138

Schönemann, 262

Schreiber, Golgi method, 464

Schridde, 356

blood, 381

mitochondrial method, 325

Schrötter, 408, 449

Schuberg, malaria, 547

Schulemann, 389

Schultze, 219, 496

chrome hæmatoxylin, 157

frog embryology, 278

iodised serum, 219

muscle, 348

osmium hæmatoxylin, 158

oxydase reaction, 390

potash method, 378

tendon, 347

Schumacher, 354

Schürmayer, 539

Schütz, 425

Bielschowsky, 430

Schwalbe, 407

cochlea, 497

Schwarze, 517

Sclavo, 549

S. G. Scott, 119, 180, 498

blood, 381

Romanowsky stain, 386

neutral balsam, 226

standard hæmatoxylin stain, 312

Scott and Osborn, triton embryology, 277

Scyphistoma, 524

Secretion granules, 315

Section-grinding, 108

Sestions, crumpling, 88 flattening, 86, 90

mounting, Henneguy's method, 114

Seeliger, 520

Segregation granules, 389

Sehrwald, 468

Golgi methods and precipitates, 466

Seidenmann, 192

Seiler, alcoholic balsam, 226

carmine and indigo, 212

decalcifier, 254

Selachia embryology, 280 et seq.

Selenka, 269

Seligmann, 493

Sensory ganglia, 424

Serial section mounting, 111

Serum, for maceration, 243,244 media, 219

Severeanu, injection, 242

Shearer, 511

Sheep, brain, 402

Sheldon, 446

Shell, molluse, 503

Shellac, for brittle sections, 89 embedding, 108
Shipley, 271, 310

benzidine dyes, 389

and Macklin, trypan blue, 378

Shun Ichi Ono, glycogen, 338

Siebenmann, 498

Silver, carbonate, neuroglia method, 492 impregnation, double-staining, 202 for elastin, 354

fixation, 201

marine animals, 202

metallic stain, 198 et seq.

for nerve. See under Golgi, Ramon y Cajal, and Bielschowsky.

nitrate, reduction, 201

picrate, lactate, acetate, for staining, 201

Simarro, neurofibrils, 419

Siphonophora, 524

Siphunculus, 511

Siredon, embryology, 276

Sjövall, 435

formol and osmic acid, 331

Skeletons, cartilage, 377

Skin nerves, 341

Slides, cleaning of, $112^{\circ}$

Slow-worm eggs, 275

Small objects, embedding, 75

Smears, blood, 379

of gonads, 307 et seq.

lucidol fixation, 59

Smirnow, 341

Golgi method, 465

Smith, eyes of gastropods, 502 and Mair, 366

Lorrain Smith, Nile blue, 368

Smooth muscle, 348

Snails, asphyxiation, 16

Snake blastoderms, 274

Snessarew, Bielschowsky, 352

Soap masses, 92

Sobotta, 269,280

Amphioxus, 281

mammals, 263

Soda carmine, 140

Sodium chloride and alcohol, for maceration, 244

nitrite, 233

sulphalizarinate, myelin, 449

Solferino, 169

Solger, bleaching, 256

muscle, 344

salivary glands, 392

Solvents, for paraffin, 76

Soulier, maceration, 245

De Souza, pyridine, 59

Spalteholz, method of clearing, 270

Spee, 92,270

Sphærozoa, 546

Spicules, sponge, 526

Spielmeyer, 442

Spinal cord, 424

axis cylinder and dendrite, 459

hardening, 399

See under Nervous System. 
Spindles, fixation, 301

Spiral filaments, 439, 440

Spleen, 393

Sponges, 525

desilicification, 255

embryos and larvæ, 526

sections, spicules, 526

Sporozoa, 546

Spuler, 140

sublimate formol, 63

Squire, 173

blueing sections, 151

glycerine jelly, 223

Staining, adjective, 131

in bulk, 6

with earmine, theory of, 135

effect of heat, 124

electrolytes in, 131

hindrances to, aids to, 25

nature of, 121

progressive, 132

regressive, 132

removal of dyes, 125

substantive, 131

two kinds distinguishable, 128

unsafe criterion of chemical constitution, 133, 134

vessels, 6

Stappers, 505

Starfishes, narcotisation, 13

Statoblasts, 282

Stauffacher, cyclas eggs, 284

Steensland, 450

Stein, 497

decalcification, 251

Stempell, 546

Stephens, 549

Stephenson's high refactive mounting medium, 224

Stirling, maceration, 245

Stoeltzner, 300, 442

Stöhr, 214

Storch, 482

Strahuber, 477

Zur Strassen, 514

Ascaris ova, 290

S trasser, 262, 407

Stratum granulosum, 340, 341

Strecker, brain, 402

Streeter, myelin, 448

Van der Stricht, decalcification, 254 thysanozoon eggs, 289

Stricker, gum embedding, 108

Strong, 398, 449

brain of acanthias, 405

copper bichromate, 64

Golgi method, 463

iron alum fixation, 50

Stropeni, 355

Strychnin, for narcotisation, 16

Students, guide for, 556

Studnicka, 352

Stylaria, asphyxiation, 16

Styrax, 370
Styrax and liquidambar, 228

Sublamin, 49

Sublimate, bichromate, 43

Golgi method, 470

See under Mercury and Corrosive. substitution stains, 164

Suchannek, 3

anilin oil, 69

bergamot oil, 68

mounting medium, 227

Suchanow, Golgi apparatus, 438

Sudan III., for blood, 383

Daddi, 367

fat, 356 et seq.

Sulima, 14

Sulphonic acid, 121

Sulphuric acid, maceration, 247

Sulphurous acid, 43

bleacher, 256

decalcification, 253

for teeth, 372

Sumita, 300

Summers, method for celloidin sections, 116

Sumner, 279

Suschkin, chick embryos, 273

Suspensoids, 123

Sussdorf, 391

Sustaining apparatus, of medullary sheaths, 439

Sympathe tic ganglia, 424

Synapta, 518

Syrup media, 219

Szecsi, lucidol, $59^{\circ}$

for blood, 382

Szent-Györgi, eye, 494

Szütz, 185, 208

Tachiol, 425

Tactile corpuscles, 341 et seq.

hair, nerve endings, maceration, 247

Tadpoles, intra vitam staining, 179

Taenzer-Unna, orcein method, 353

Tafani, 498

Taguchi, Indian ink mass, 240

Tannin, for mounting, 220

Tannin-fuchsin, for flagella, 548

Tap water substitute, 151, 313

Tartuferi, 343

eye, 495

Taylor, Sister Monica, amœba culture method, $536-537$

chromosome methods, 303

Teeth, Carter, 369 et seq.

decalcification, 253

embedding through carbon bisulphide, 372

lymphatics, 373

soft parts, 371 et seq.

vessels, 373

Tegumentary organs, 339 et seq.

Teleost eggs, 279, 280

embryology, 259 
Teljatnik, 451

Tellyespiczky, acetic bichromate, 41

Temnocephala ova, 289

Tendon, 344,347

silver, 200

Tenebrio eggs, 287

Terpinol, 69

as mounting medium, 228

Test-cells of Ascidia, 282

Theory of dyeing, electrical, 124

of fixation, 18

of staining, 120

Theridium, 287

Thiersch, 212

Thin, 496

Thionin, 162, 168

cartilage, 376

for Golgi apparatus, 438

for intra vitam staining of nerve, 196 mucin, 391

pancreas, 396

for skeletons of cartilage, 377

Thionine phéniquée (Nicolle), 168

Thiophen green, 182

Thoma, decalcification, 253

indigo-carmine mass, 239

and Fromherz, corrosion, 249

Thome, 173

Thompson, Arthur, reconstruction method, 262

Thomson, J. G., Giemsa stain, 545

Thread cells, 521

Thyme, oil of, 68

Thymus, 394

Thyroid, 394, 395

Thysanozoon ova, 289

Tigroid substance, 410 et seq.

Timofejew, 196, 393, 405

Tirmann, 300

Tischatkin, 3

Tissue culture, 550 et seq.

Toison, staining fluid for blood, 382

Tolu balsam cement, 231

Toluidin blue, 169

for cartilaginous skeletons, 377

and erythrosin, 415

for intra vitam staining of nerve, 196 myelin, 449

Nissl granules, $412-413$

Toluol, 70

Tomaselli, neurofibrils, 418

Tonkoff, 183

Torpedo, electric organ, 346

Tortoise embryos, 275

Tower, Moniezia, 515, 516

Tozer, 513

Tracheæ, 508

Tracheata, 505

Trachymedusæ, 523

Trematodes, 516 et seq. ova, 289

Trenkmann, 549

Triacid mixture, 175

Trichina, 514
Trichlor-acetic, 53 decalcifier, 254

Trimming blocks, Eternod, 83

Triple stain, Bonney, 178

Triton (Molge), embryology, 277

Trophospongium, 439

True fats, 356

Trypan blue, 389,390

cartilaginous skeletons, 377 dental pulp, 373

violet, 390

Trypanosomes, 533, 539, 547

Trypsin, digestion, 248

Trzebinski, 405

Tschaskin, 389

Tschernyschew, 446

Tsetse flics, 539

Tullberg, 15

Tunicata, 499

killing, 12

ova, 281 et seq.

Turbellaria, 517 et seq. embryology, 288

Turpentine, clearing, 69

for mounting, 227

dissolving fat 322,325

Uexküll, 16

Underwood, 375

Unio, methylen blue, 192

Unmasking iron, 299

Unna, 32, 34, 350, 388, 391

carbol-pyronin-methyl green, 172

collodion mass, 96

half-ripe hrmatoxylin stock, 153

on hæmatoxylin staining, 145

keratin, 341

mast cells, 355

methylen blue and Säurefuchsin, 351

orcein method, 351

oxidised hæmatoxylin, 155

polychrome methylen blue, 186

Rongalit white, oxygen test, 390

safranin and wasserblau, 351

smooth muscle, 348

water-blue orcein, 339,340

Upson, 407,477

Uranium acetate, 54 nitrate silver method, for Golgi apparatus, 436

Urea and Bouin's fluid, 306

and chromosome fixation, 305

Urodele, embryology, 276

Ussow, cephalopoda, 282

Vanadium chloride process, 475

hæmatoxylin, 157

Varnishes and cements, 229 et seq.

Vaso-dilators, 232 et seq.

Vassale; 450

Weigert method, 444

and Donnagio, Golgi method, 465 
Vastarini-Cresi, embryonic cartilage, 377 silver, myelin, 451

Vejas, 408

Vejdovsky, 514

Venderovic, 407, 450)

Venetian soap, 411

Venice turpentine, 227

for cementing, 230

Ventral cord, insect, 508

Veratti, 468

Veretillum, 523

killing, 12

Verhoeff, 354

Vermes, embryology, 288 et seq. general, 509 et seq.

Vernon, 309

Vert d'Alcali, 160 Lumière, 159

Verworn, 14

Vesuvin, 161 mast cells, 355

Viallane, 509 osmic gold method, 205

Vialleton, cephalopoda, 282 silver impregnation of chick, 274

Victoria blue, 169 mucin, 392 neuroglia stain, 483 green, 181

Violet B, 184 of Lauth, 168

Virchow, 33, 280

Vital stains, benzidine, 390 mitochondrial, 332

See under Intra Vitam.

Scharlach VIII, myelin, $\mathbf{4 5 3}$

Vivante, bone, 375

Vivisection Acts, caution against, 265

Vogt and Tung, 511

Cestodes, 515

Cucumaria, 518

worms, 509

Volk, 17

Vosmaer, reconstruction method, 261 and Pekelharing, sponges, 526

Vosseler, wax feet, 243

Waddington, 13, 541

Waite, 288

Waldeyer, 498

decalcification, 252

Walsem, 449

Walton, tissue culture, 552

Ward, 16

Gephyrea, 511

Warnke, 407

Washburn, mollusc ova, 284

Washing out, after fixation in alcohol, formol, acetic acid, picric acid, nitric acid, corrosive, osmic, chrome, 26

liquids for, 26

Wasielewski, sporozoa, 546
Wasserblau, 183

blood-platelets, 386

and orcein, Unna, 340

and safranin, 351 *

spleen, 394

Wassermann, 300

Watasé, cephalopoda, 282

Watch-glass, embedding in, 74

Water-baths, paraffin, 79

Water-blue. See under Wasserblau.

Watery media, 217

Wax feet, 243 et seq.

Webb, gum mass, 110

Weber, 512

Siphonophora, 524

Wedl, 211

Weed, 271

Weidenreich, 341

blood, 380

Weigert, 177, 450,461

elastin stain, 353

fibrin stain, 388

hæmatoxylin, 150

method for celloidin sections, 118

myelin, formol material, 444 stains, 442 et seq.

neuroglia stain, 480

- picro-Säurefuchsin, 215

Weigert-Pal, myelin method, 446

Weigl, Mann-Kopsch method, 328

Weil, 109

bone, 370

teeth sections, 373

Wellings, intra vitam staining of teeth, 373

Wermel, 381

Werner, smooth muscle, 348

Wester, 507

Weysse, 269

Wharton's jelly, 391

Wheeler, eggs of Orthoptera, 286

White, bone sections, 370

Whitman, brains, 405

frog embryology, 276

pelagic fish ova, 281

Hirudinea, 511

Wickersheimer, 220

Widakowich, 270

Widmann, lens of Arachnida, 509

Van Wijhe, ammonia carmine, 141

cartilaginous skeletons, 377

Wilhelmi, 518

Will, reptile embryology, 275

Willebrand, blood stain, 383

Wilson, J. T., and Hill, J. P., 269

Wilson, Alcyonaria, 522

stain, 535

Wimmer, 482

Winiwarter, 177, 270

Wintergreen oil, for clearing, 271

Wislocki, 390

Von Wistinghausen, 155

De Witt, 34

Witte, pancreatin, 249 
Wittmaack, 497 myelin, 451

Wolff, 388,428

bladder, 349

Wolfrum, elastin stain, 353

Wollschwarz, 548

Wolter, 453

chloride of vanadium process, 475

Wolters, 376

myelin, 447

Woodcock, fæces, 538

and Wilson, Schaudinn fixation, 541

Woodger, 365

Woodland, 518, 521

Woodworth, reconstruction, 261

Woolf, 168

Worcester's liquid, 280

Worms, 509

Wright, blood platelets, 386

Romanowsky stain, 535

Wynn, 447

\section{Xylol, 70}

Yellow gelatin mass, 237

Yolk stain, Peter, 260

See under Fat and Cytoplasmic

Inclusions, 316 et seq.
Zacharias, 139, 505

acetic alcohol, 52

protozoa, 548

Zaleski, 300

Zander, 507

Zawarsin, cornea, 343

Zenker, fluid, 48

note on, 323

Zernecke, Ligula, 516

Zettnow, 549

Ziegler, decalcification, 253

teeth sections, 372

Zieglwallner, glycogen and fat stain, 296

Ziehen, gold sublimate, Golgi method, 475

Ziehl, carbolic fuchsin, 169

Zimmermann, 301, 352

Golgi preparations, 469 nucleoli, 314

Zinc, 300 chloride, 50

Zoantharia, skeletons, 522

Zograf, 542

Rotifers, 512

Zoja, 189

Ascaris ova, 290

Zosin, 453

Zschokke, 377

Zürn, 495

Zwaardemaker, safranin, 166 

THIS BOOK IS DUE ON THE LAST DATE STAMPED BELOW

AN INITIAL FINE OF 25 CENTS WILL BE ASSESSED FOR FAILURE TO RETURN THIS BOOK ON THE DATE DUE. THE PENALTY WILL INCREASE TO 50 CENTS ON THE FOURTH DAY AND TO \$1.0O ON THE SEVENTH DAY OVERDUE.

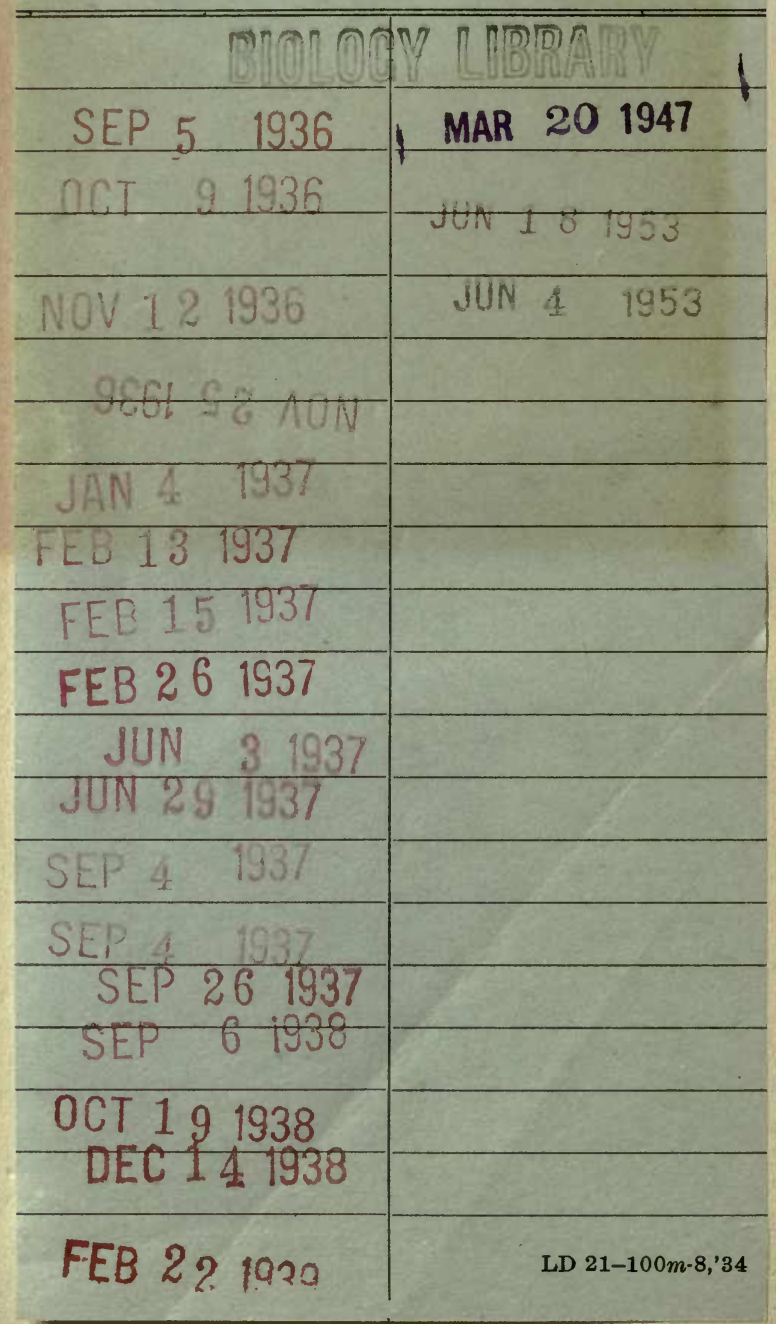




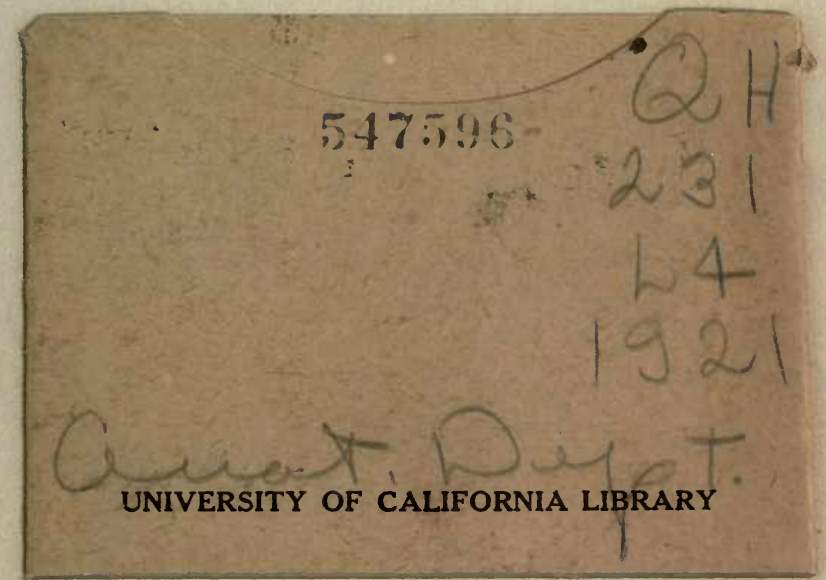

L. $=4$ isir

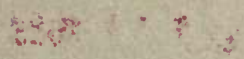

$2 \cdot 4 \cdot 1$ 


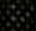

\section{\% 8 \%}

$\%$ \%

$8 \% 8 \%$

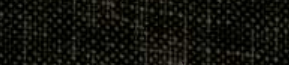

$8 \% 8 \%$

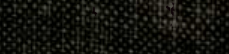

8.8.

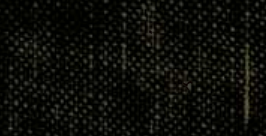

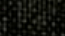

\%

影
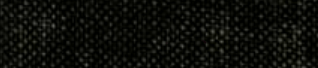